\title{
Integrating Score, Performance, and Medium in the work-concept: The HypeSax as a Case Study
}

\author{
By
}

Cristohper Ramos Flores

\begin{abstract}
A thesis submitted to the Victoria University of Wellington in fulfilment of the requirements for the degree of Doctor of Philosophy
\end{abstract}

Victoria University of Wellington 


\section{Supervisors}

Michael Norris - Jim Murphy 


\section{ABSTRACT}

This thesis presents a novel music-technology project, the HypeSax, which affords new roles to the saxophone and enhances its sound capacities. This document presents a discussion of the musical ideas and design criteria behind the development of this new instrument, addressing issues of embodiment that arise from the use of new technologies, and of what this new medium means in the discussion of the ontology of the musical work. This project is intended to research the medium through a case study, in which the medium becomes the central focus of my compositional decisions.

As part of this project, a body of new musical works, associated with the HypeSax, was created. These compositions and the creative process from which they originated are analysed in relation to the HypeSax, questioning if the musical work is limited to the composition or if other processes such as the development of the medium, which in this case is the HypeSax, can be considered part of its ontology.

The desire to understand and define the ontology of the musical work has led musicians, musicologists and philosophers to formulate multiple propositions that observe perspectives of creation and reception, as well as different ways in which these interact. This thesis proposes the integration of a new element in the conversation of the workconcept: the medium. The argument presented is that, in light of compositional practices in the twenty-first century, the creative work begins when musicians design instruments, software, audio setups, and other new technologies, actively transforming the medium through which their work works are created. Despite the fact that the medium has always been in close relation with the composition, performance and reception of the work, it has not been considered an element in the ontology of the work. Nevertheless, it becomes impossible to ignore the importance of the medium as new technologies facilitate its manipulation as a part of the creative process.

New works featuring the HypeSax are discussed, as well as how this novel medium provides the affordances and possibilities that allow the creation of said works. This case study serves to demonstrate the importance of the medium in the context of a new tripartite model of the work-concept where score, performance and medium are integrated, in a nonhierarchical structure, as one inseparable reality of music. 
This research was funded by the Mexican public institutions FONCA and CONACYT through the BECAS PARA ESTUDIOS EN EL EXTRANJERO program.

Esta investigación fue patrocinada por las instituciones publicas mexicanas FONCA y CONACYT bajo el programa de BECAS PARA ESTUDIOS EN EL EXTRANJERO.

\section{CULTURA I FONCA}

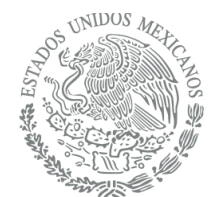




\section{ACKNOWLEDGEMENTS}

Many thanks to my supervisors who not only have been helpful during these past years, but have enriched my experience in New Zealand. Thanks for your guidance and encouragement . Thank you Michael for sharing your musical knowledge and ideas. Thank you Jim for sharing your technical expertise. I will treasure what I have learned from you. Thank you both for the fun meetings, it never felt like work, yet, I learned so much from you. Your work as artists and your dedication as educators is inspiring.

Thanks to the many people at the NZSM who supported and helped me explore music in new ways. Thanks for all of the musicians who participated in the recording of my Portfolio: Bella Anderson, Leah Thomas, Kate King, Gabriela Glapska, Jake Church, Thomas Friggens, Tristan Carter, Salina Fisher, Elliot Vaughn, Charley Davenport, Jake Baxendale, Glen Downie, and special thanks to Peter Liley who dedicated many hours to my music.

Thanks to my family and friends in New Zealand. Your support has made my $\mathrm{PhD}$ experience less difficult. Thanks Jim and Valerie for all you have done for me this time.

Gracias a mi familia y amigos en México. A pesar de la distancia, su apoyo y cariño siempre llegó hasta de este lado del mar. Gracias mamá por dar tanto de ti, gran parte de lo que soy lo debo a tu esfuerzo. Éste logro también es tuyo.

Thank you Jasmine for every walk, each conversation with you has had an impact in this dissertation. Your love and care has changed my life and is the force that I need to keep going. 


\section{CONTENTS}

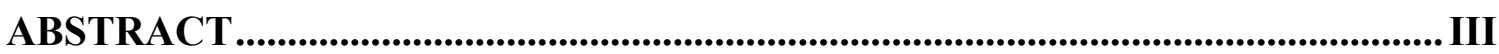

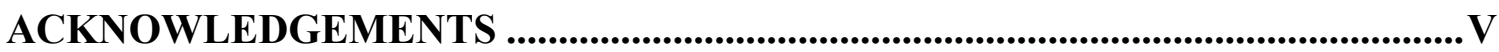

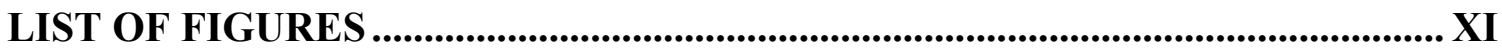

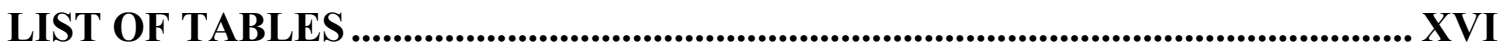

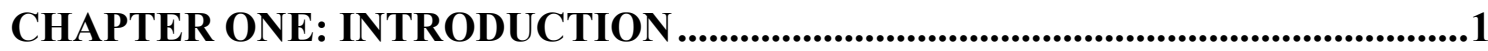

1.1. Personal background. The foundations of my musical journey. .........................2

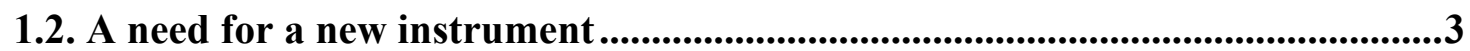

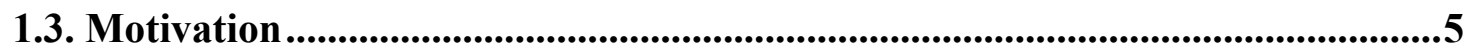

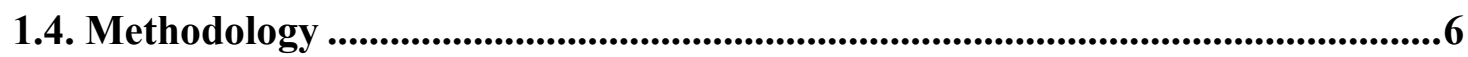

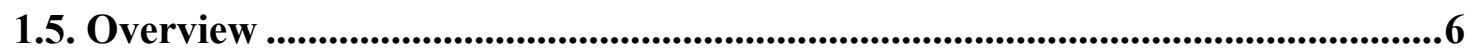

CHAPTER TWO: A WORK-CONCEPT FOR TWENTY-FIRST CENTURY

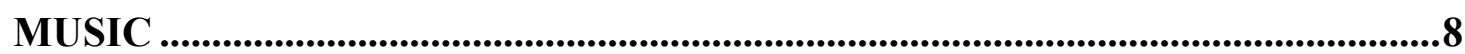

2.1. The issues in defining a musical work ........................................................9

2.2. Technology as a factor to define a musical work .................................................10

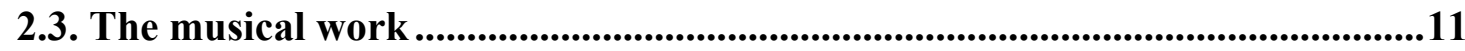

2.4. A new work-concept: The tripartite model .......................................................19

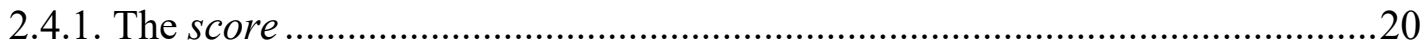

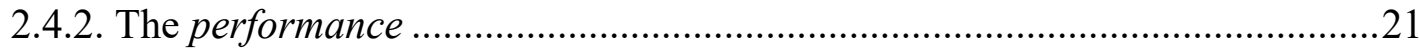

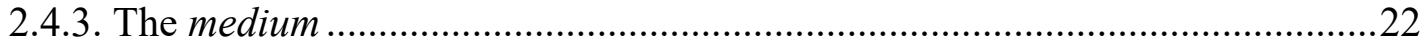

2.5. The work as the intersection between the elements of the tripartite model ...23

2.6. Reaching the audience with the aid of the medium .......................................26

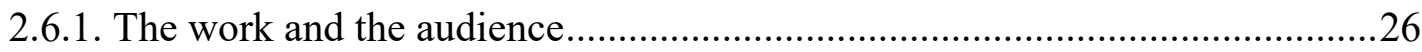

2.6.2. Two strategies that build bridges between the work and the listener..............29 
2.6.3. Embodiment and the medium

2.6.4. The medium as a carrier of grain and instrumentality in technologically devised music

2.7. The background of the medium as a creative exploration.

2.7.1. Air pressure measurement.

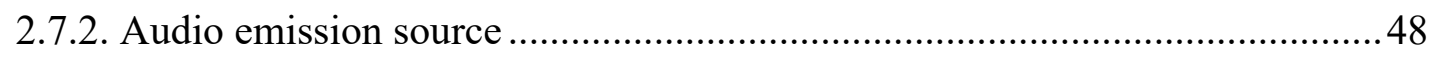

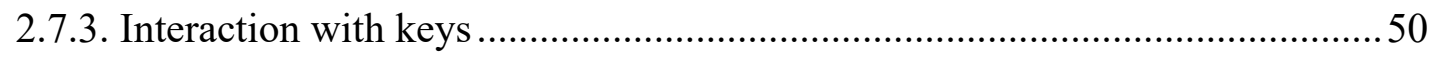

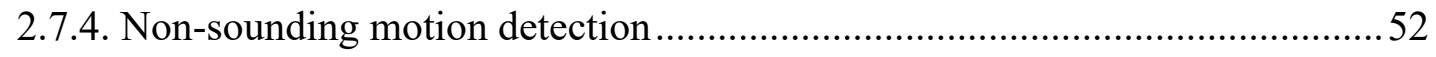

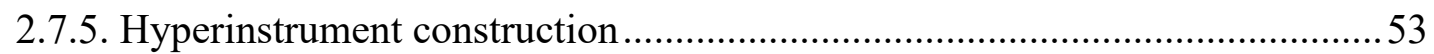

CHAPTER THREE: THE HYPESAX AS THE COMPOSED MEDIUM OF THE

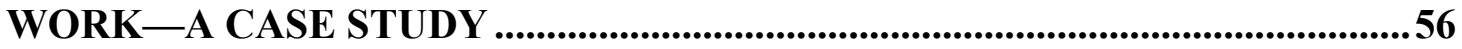

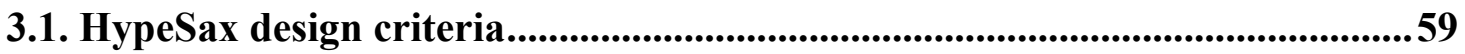

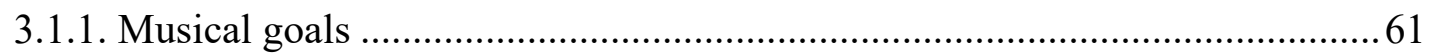

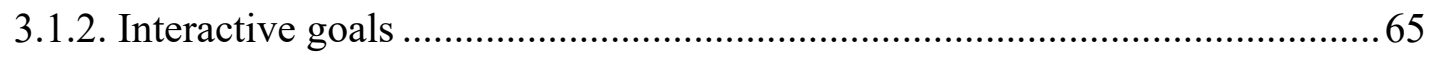

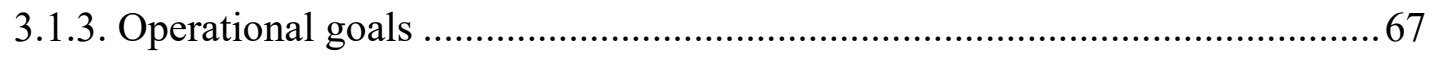

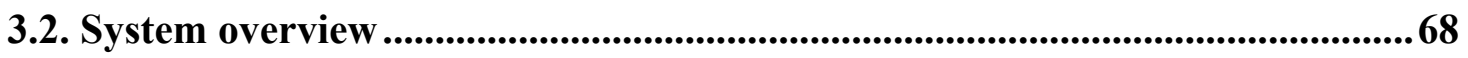

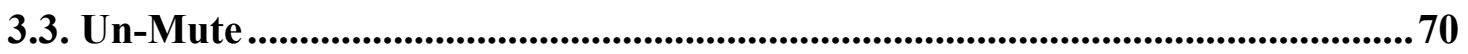

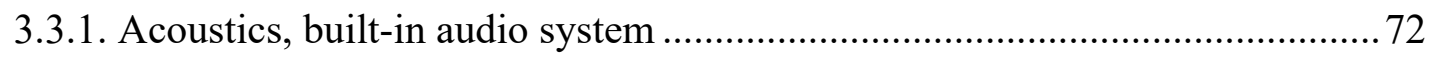

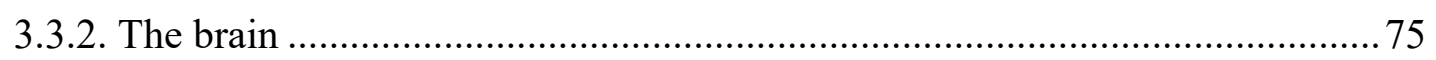

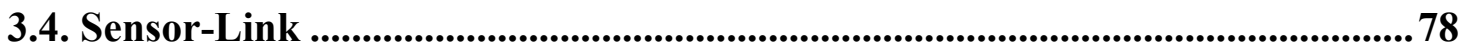

3.5. Fingers: touch sensors and momentary buttons ...........................................80

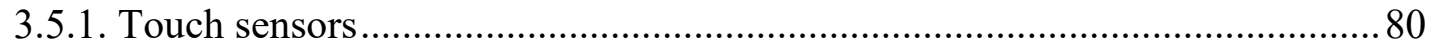

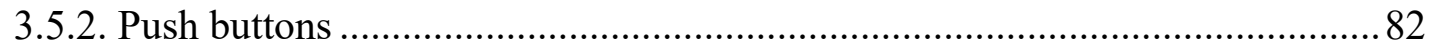

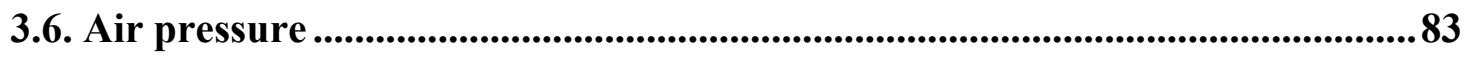

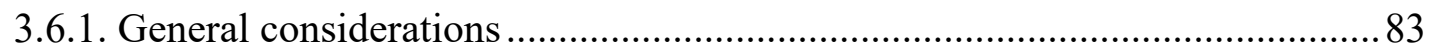

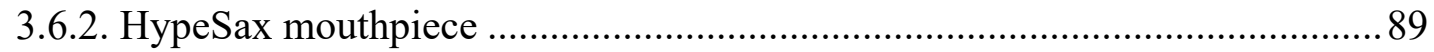




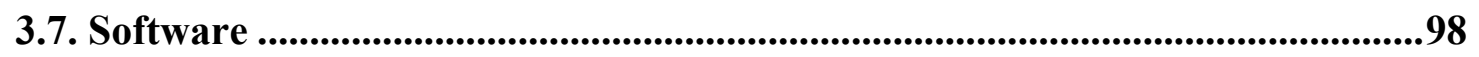

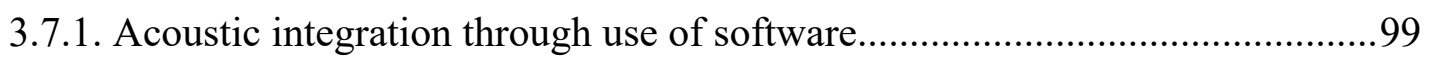

CHAPTER FOUR: A NEW MEDIUM-HYPESAX EVALUATION AND

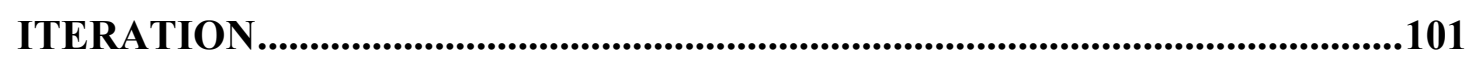

4.1. The HypeSax as hybrid medium: the bodyless sound and the mutability of

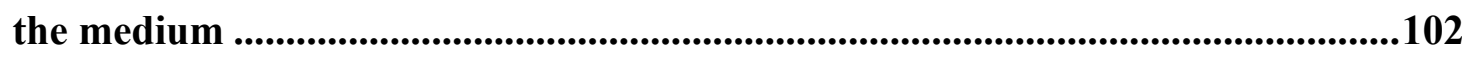

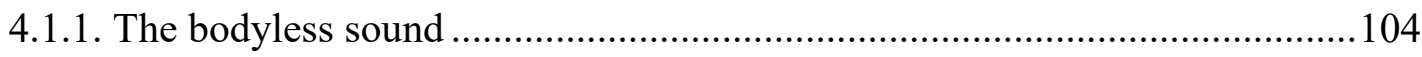

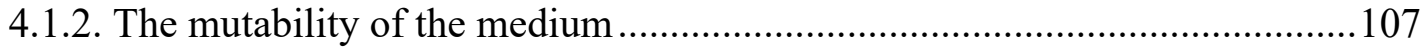

4.2. User-centred study ........................................................................................................109

4.3. User study design ...........................................................................................................110

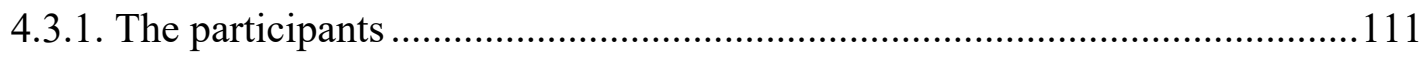

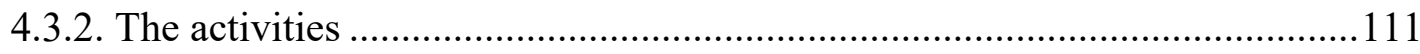

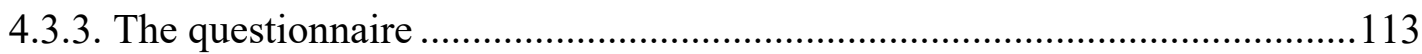

4.4. A new version of the HypeSax compatible with soprano and tenor

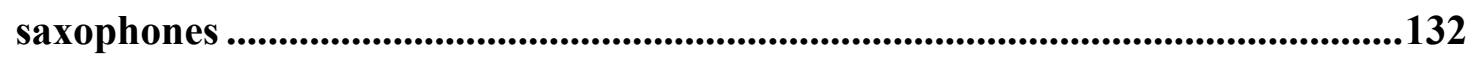

CHAPTER FIVE: A NEW MEDIUM FOR NEW WORKS...................................138

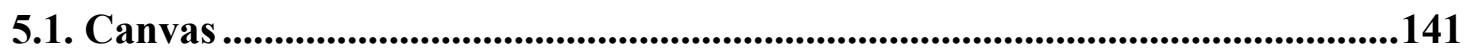

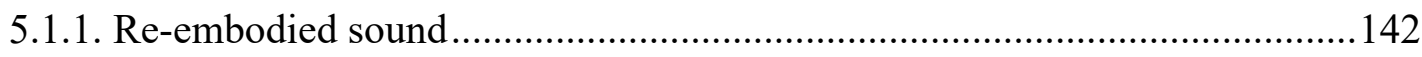

5.1.2. Data processing and software expansion: The Hub .................................. 148

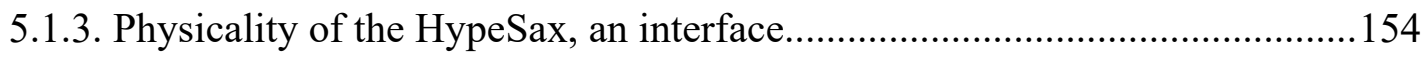

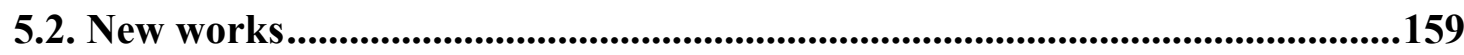

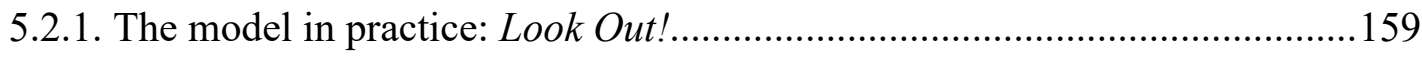

5.2.2. The score in my portfolio of works....................................................... 165

5.2.3. The performance and the gestural-instrumental technique in my works .....184

5.2.4. The HypeSax as the medium in new works...............................................191 


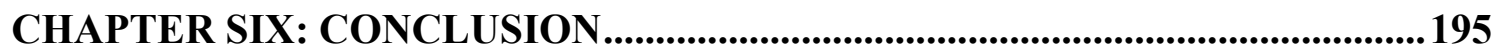

6.1. Summary and contributions ........................................................................196

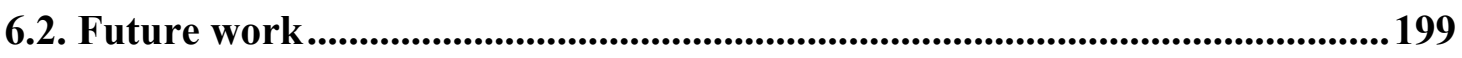

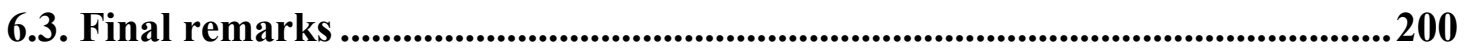

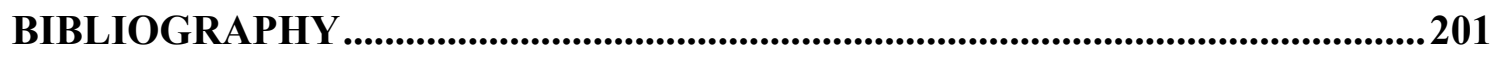

APPENDIX A: PARAMETER FOR 3D MOUTPIECE MODEL..........................212

APPENDIX B: GESTALT THEORY - EXPANDED DISCUSSION ....................214

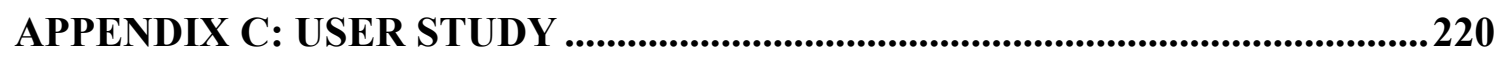

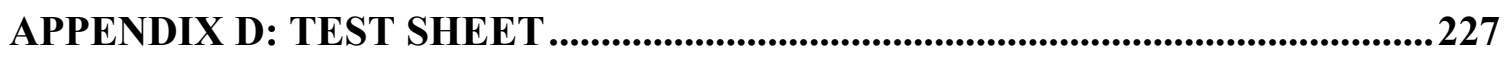

APPENDIX D: 'A 3D PRINTED HYBRID SAXOPHONE MOUTHPIECE FOR

DATA COLLECTION' ..................................................................................2231

APPENDIX E: 'HYPESAX. SAXOPHONE ACOUSTIC AUGMENTATION' ..240

APPENDIX F: LIST OF INSTRUMENTAL TECHNIQUES FOR GESTURALINSTRUMENTAL TECHNIQUE DEVELOPMENT ......................................247

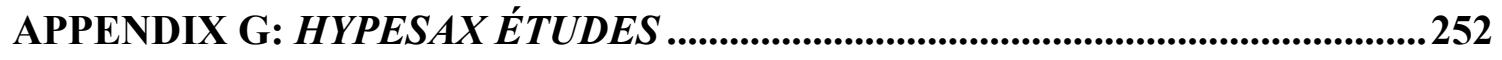

APPENDIX H: MÁS ALLÁ DEL DELIRIO ......................................................260

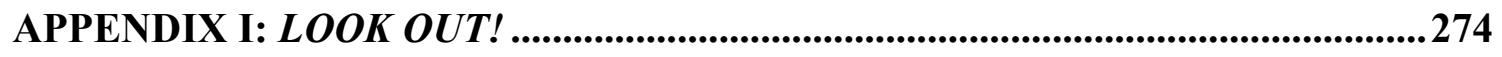

APPENDIX J: BREATHE IN, BREATHE OUT ...................................................2278

APPENDIX K: COUNTLESS SOULS ...............................................................292

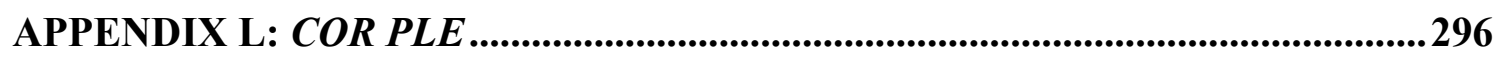

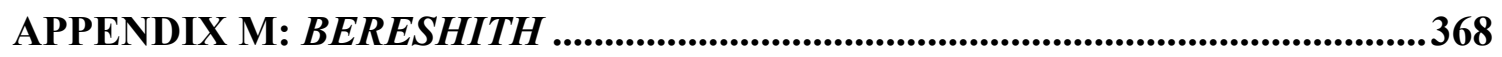

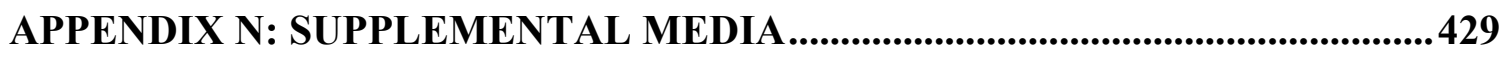




\section{LIST OF FIGURES}

Fig. 1. Classic schema for communication (A) contrasted against Molino's model (B). 12

Fig. 2. Frisk's interpretation of Ricœr's model. 13

Fig. 3. Interpretation of Vázquez's scheme of musical ontology.... 14

Fig. 4. Molino's scheme (A), Nattiez's reinterpretation of Molino (B), and Nattiez's final model (C).

Fig. 5. Proposed tripartite model in which the poietic and esthesic process flow in any direction.

Fig. 6. Representation of the most basic Gestalt psychology laws of perceptual organization.

Fig. 7. Temporal proximity allows association between strings in bb. 4-8, Cor Ple (top). Proximity of range produces association instruments in musical gesture, b. 5254, Bereshith (bottom). 32

Fig. 8. Young's Hyperbow. .45

Fig. 9. Dan Trueman using the R-Bow to play the BoSSA .45

Fig. 10. HIRN wind controller designed by Perry Cook. .48

Fig. 11. Rainforest IV at L'espace Pierre Cardin in Paris, 1976 .50

Fig. 12. Matthew Burtner's Metasaxophone featuring force sensitive sensors. .52

Fig. 13. Design criteria follow the tripartite model. .60

Fig. 14. Modular components of the HypeSax: Un-mute (A), Sensor-link (B), new keys for thumbs and new keys situated over custom-designed keycaps (C), and mouthpiece (D)

Fig. 15. Early prototype of the HypeSax. .72

Fig. 16. The Un-mute. .73

Fig. 17. Un-mute PCBs and electronic components. .77

Fig. 18. $X, Y$ and $Z$ Axes of the HypeSax's gyroscope in reference to the saxophone's natural performing position. 
Fig. 19. Sensor-link. .79

Fig. 20. Various iterations of the keycaps. .81

Fig. 21. 3D-Printed moulds and final keycaps made of VytaFlex 40 Polyurethane. 82

Fig. 22. Thumb buttons. 83

Fig. 23. Pressure sensor and probes mounted around the mouthpiece. 86

Fig. 24. Detail of the openings in the mouthpiece. 87

Fig. 25. SABRe's mouthpiece with mounted hose (a), pressure sensor (b), RF electronics (c) and battery (d). 88

Fig. 26. Comparison between the original 3D model and the modified version used in the HypeSax. .91

Fig. 27. Interior of the first iteration of the mouthpiece. .92

Fig. 28. At the top, a comparison of width of beak between first and second iteration. At the bottom, detail of the S-shaped conduit and the printed mouthpiece (sensor installed and sealed with silicone). .95

Fig. 29. Common air pressure sensor in a funnel-like casing and BMP180-GY68 (top). BMP180 on a cross-section rendered image of the mouthpiece showing the Sshaped air conduit (bottom). 97

Fig. 30. Data flow for acoustical integration. 100

Fig. 31. Electrophones and the six subcategories according to the Hornbostel-Sachs classification. .104

Fig. 32. Fourth exercise found in the 'test sheet.' The top staff (feedback) represents an approximate sounding pitch of acoustic feedback generated by the indicated fingering and gain control. The lower staff must be performed as usual. The result of combining both is a polyphonic sound of acoustic saxophone and feedback, which resemble each other. 113

Fig. 33. The assessment of hardware setup was favourable. 116

Fig. 34. The fingering chart (top) shows the extra keys where thumb buttons are labelled 1, 2 and 3, while touch buttons on keycaps are labelled with letters. Exercise one 
of the 'test sheet' (bottom) shows a melody and indications of combinations of key 1 and touch buttons to record and produce granular synthesis of the sound currently played. In bar 3 , beat 2 , for instance, the saxophonist plays the pitch D while, while holding key 'a', granular synthesis reproduces and holds the pitch A (from bar 2, beat 3 ) and D, holding key 'e'. These two sounds are elongated throughout bar 4 .

Fig. 35. Performers' (top) and composers' (bottom) answers to the same question......118

Fig. 36. Responses to questions 6 and 7 of Section B. 119

Fig. 37. Notation suggested by one of the survey participants 120

Fig. 38. Answers to question 6 of Section A.

Fig. 39. Answers to question 7 of Section A.

Fig. 40. Answers to questions 8 (top) and 9 (bottom) of Section A. 124

Fig. 41. Answers to question 11 of Section A. 125

Fig. 42. Common differences in the mouthpiece designs (dimensions have been exaggerated). 126

Fig. 43. Max patch developed to monitor (left panel) and record (right panel) air pressure data.

Fig. 44. Exercise 1 of the 'Test sheet' and recorded data obtained from four participants.

Fig. 45. Exercise 5. A and B present data recorded from classical performers, while $\mathrm{C}$ and D belong to Jazz saxophonists.

Fig. 46. Images A and B present data recorded from classical performers, while C and D belong to Jazz saxophonists. 130

Fig. 47. New PCB design. 134

Fig. 48. New alto saxophone keycaps design (top) and 135

Fig. 49. New Un-mute and adaptors. 136

Fig. 50. Five right thumb button states are indicated by distinctive patterns of illumination of the LEDs. A sixth state is indicated when LEDs are off. 137 
Fig. 51. Pardue's Svampolin. .145

Fig. 52. HypeSax's possible configurations of sound embodiment. 148

Fig. 53. Screenshot of Arduino and Max programs showing how data is handled. 150

Fig. 54. Screenshot of The Hub (top) and a partial screenshot of The Hub displaying setup instructions via 'setup help' button. .151

Fig. 55. OSC setup window. 153

Fig. 56. Bonger's human-machine interaction model. 155

Fig. 57. Bonger's model including multiple modalities. 156

Fig. 58. Adaptation of Bonger's model reveals the aspects of the relationship between performance and medium in the use of the HypeSax.

Fig. 59. Final modification to of the model identifying the intrinsic score. .158

Fig. 60. Look Out! Score displayed on MiraWeb. 161

Fig. 61. Four modules from Lookout! .164

Fig. 62. Cartesian $\mathrm{X} / \mathrm{Y}$ space allocating audio samples to be navigated though ancillary motion. 165

Fig. 63. bb.1-8, Breathe in, breathe out. 167

Fig. 64. bb. 17-24 of Breathe in, Breathe out, orchestrated using the principle of similarity. 169

Fig. 65. bb. 39-44, Breathe in, breathe out, the principle of similarity separates strings from HypeSax. 170

Fig. 66. The principle of cloud clang unifies instruments in b. 26 . 171

Fig. 67. b.37, inspiration for the orchestration of b.26. 172

Fig. 68. Waveform visualisation of Génesis from 0'00" to 1'30". 174

Fig. 69. Timbral transformation of the 'breathing' gesture in bb. 36-46 of Bereshith. .175

Fig. 70. Woodwind section of the 'breathing' gesture in bb. 35-40 of Bereshith..........176

Fig. 71. Simplified flowchart of hybrid synthesis. .178 
Fig. 72. b. 124, Cor Ple.

Fig. 73. bb. 62-64, Cor Ple. The graphic above the saxophone staff on b. 64 indicates the beginning of the hybrid synthesis.

Fig. 74. Chord found in b.1, Cor Ple (left) and flute harmonic series (right). 183

Fig. 75. bb. 16-18 of HypeSax Étude No.3. In b. 18 beat 1, ricochet technique is used, followed by hammering on beats 3 and 4 .

Fig. 76. Section of p. 4 of Más allá del delirio... desde un lugar secreto showing the sul ponticello technique adapted for the analogue gestural-instrumental technique on the HypeSax. 190

Fig. 77. Musical idea composed by one of the survey participants. Top staves show the written score. Bottom staff shows the score played where the synth pitches are randomly selected. 193

Fig. 78. Musical idea composed by one of the survey participants. 194 


\section{LIST OF TABLES}

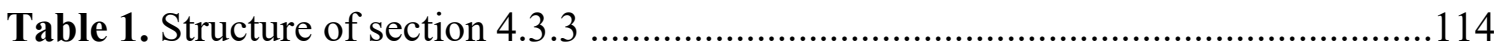

Table 2. Detail of data sent via [send] messages over Max ......................................151

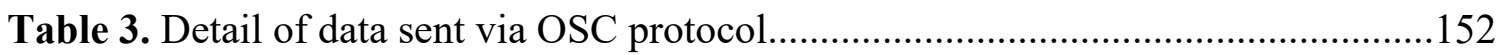

Table 4. Onset category of comparative list of instrumental technique access, where $X=$ available,$\quad /=$ similar technique is available, and $-=$ not available..............187

Table 5. Onset category of the potential HypeSax gestural-instrumental techniques

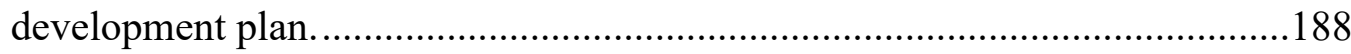





\section{1}

\section{INTRODUCTION}

[...] the potential trouble with the term 'work-concept' is that, by including 'work' as the first element in a compound expression, it tends to divert attention from the broader context towards the individual product, as well as implying that if the regulative control of the concept is missing, this product is liable to be different or even deficient in some way ${ }^{l}$

Michael Talbot

This thesis presents a body of original work, centred around the development of a major new-music technology development, a hyperinstrument called HypeSax. This instrument is a device that has been designed to augment the sounding possibilities of an acoustic soprano, alto or tenor saxophone. This hyperinstrument, and its development, led me to contemplate the relationship and ontology of various components of the musical works.

As a composer, understanding the result of my creative work, my compositions and how they relate to others, is fundamental. What my work is, how it is presented, and how it is received, to some degree, defines who I am. The idea of the 'work-concept' helps in understanding the result of my creative work. From my personal point-of-view, the workconcept can be used as a tool to understand and organise musical ideas.

\footnotetext{
${ }^{1}$ Michael Talbot, 'The Work-Concept and Composer-Centredness', in The Musical Work: Reality or Invention?, ed. by Michael Talbot (Liverpool University Press, 2000), pp. 168-86 (p. 170).
} 
In recent decades, a discussion of what defines a 'work', and of what the 'workconcept' is, has, in Reinhard Strohm's words, 'deeply influenced our musical culture; it is as "real" as any aesthetic idea can be, and many generations of musicians have believed in it'. ${ }^{2}$ While the idea of the work-concept serves to analyse and define the limits of a musical work, it can also 'impose' limits to what the work is, its ontology and the relationship between the work itself and the observer, whether a composer, performer, or the audience.

The goal of this dissertation is to establish an understanding of the relationship between musical ideas, performance and the instruments (or medium) which allow those ideas to become real music. To reach this understanding, a new work-concept model is proposed and explored through a case study research presented in this dissertation.

\subsection{Personal background. The foundations of my musical journey.}

My first forays into creativity in music began as a teenager in Mexico, playing with sound manipulation tools in early commercial DAWs. These first explorations into musique concrète and digital sound processing were highly influential for my future relationship with music. This early and free exploration served as an introduction to composition.

More than a decade later, as I was finishing a music degree in composition and regularly performing with the cello, the Mexican Centre for Music and Sonic Arts (CMMAS) was founded in my home town of Morelia, Mexico. I then took my first lessons in electronic music in 2007 at CMMAS with Rodrigo Sigal. At this point, most of the concepts I was learning seemed natural to me as I had explored them in an intuitive way for a long time.

In 2012, while studying at Wesleyan University, I became a member of Toneburst, the music department's laptop orchestra. This was my first experience performing with computers (software and virtual instruments) without the use of physical interfaces. Performing with this ensemble, I experienced the potential to access a wide horizon through live sound manipulation. However, performing live electronics using a computer

\footnotetext{
${ }^{2}$ Reinhard Strohm, 'Looking Back at Ourselves: The Problem with the Musical Work-Concept', in The Musical Work: Reality or Invention?, ed. by Michael Talbot (Liverpool University Press, 2000), pp. 128-52 (p. 128).
} 
resulted in a somewhat unsatisfactory experience. The lack of connection between physical gesture and aural response, which I had previously experienced as a performer playing acoustic instruments, was missing.

Today, thanks to those experiences, my approach to music composition is linked to my history as a performer of acoustic instruments and computers, and the possibilities offered by sound processing and synthesis, as well as the awareness of the importance of embodiment. In response to this, I decided to explore ways in which these ideas could inform my compositional processes. As a result, I urgently felt the need for a new instrument that could allow me to explore these ideas. I believe that this dissertation and case study described in it have the potential to benefit other composers and musicians by presenting a pathway to explore and develop different ways of music-making.

\subsection{A need for a new instrument}

Musical instruments such as the violin have been evolving over centuries. Other instruments such as new music software have only been around only for a few decades at most. Both the 'old' and the 'new' possess the potential to create and transform sound into musical works. Musicians, aware of this potential, have learned the possibilities and limitations, and have pushed the limits of these instruments expanding the sound palettes available for music creation. On the other hand, these instruments relate to the performers in very different ways: while the 'old' instruments require a physical interaction with performers' bodies through the manipulation of their mechanical systems, the 'new' instruments are capable of escaping the restraints of the physical world, potentially offering limitless possibilities. This relationship between the musician and the instrument has a strong impact on how we create and perceive music.

As a composer, I have developed a need to create music using both 'old' and 'new' instruments, as is the case with many composers today. It is easy to imagine how composers in the nineteenth century might have been excited about the possibilities of keyed woodwinds such as the Boehm flute system. These possibilities would have allowed them to create music that was not possible before. Similarly, today, some composers take advantage of new technologies and incorporate them into their creative process. New approaches to instrument-building take advantage of the qualities of 'old' and 'new' 
instruments by adapting existing instruments or even creating new ones. In the last few decades, researchers have been working on extending the physical possibilities of instruments by hybrids of acoustic and electronic instruments: so-called 'hyperinstruments'. To date, however, most of these hyperinstruments rely on an external computer and loudspeakers to achieve their sound, and, as such, the acoustic instrument itself becomes merely a controller. The consequence of following this paradigm is that the sound becomes disembodied from the instrument, without any feedback of the produced sound into the acoustics of the instrument.

I identified the potential to further investigate hyperinstruments and to refocus their design to create a direct link with the produced sound and provide a pathway for acoustic feedback into the instrument itself. I believe it is possible to create hyperinstruments that do not focus solely on controlling computer software, but are capable of integrating sonorous results into their own acoustics, or of taking unexpected roles in the compositional and performance processes.

The research presented in this thesis describes the development of the HypeSax, an instrument that augments a traditional acoustic saxophone, as a case study to explore the limits of what defines my musical work, and to explore a new work-concept model that integrates creativity, performance and the instruments (or medium) through which music is made. An important aspect of this project is to understand and develop instrumental techniques that have a logical and natural physical connection between the performance and the resulting sound, similar to the usual interaction between a performer and an acoustic instrument.

As part of this project, a set of pieces were composed for the HypeSax in order to explore the potential of integration of acoustic and electronically processed sound to achieve new saxophone expression, as well as to get a new insight of the work-concept. In addition, a non-HypeSax work was composed and instrumental techniques were investigated, prior to the completion of the HypeSax's development, in order to explore issues of sonic transformation in acoustic ensembles, and to evolve my own concepts and techniques when it came time to integrate the HypeSax into an acoustic ensemble setting. 


\subsection{Motivation}

Prior to this research I had not been preoccupied with the concept of embodiment. Nevertheless, I perceived a natural need to investigate this idea after performing with computers, an experience that, to me, lacked the physicality of playing a traditional instrument. I had also worked with and experienced the advantages of 'sound sculpting' in electronic music, which had influenced the way I think about music. This made me question the importance of the medium through which music is made, how we relate to the medium, and how this medium influences my compositional approaches. To investigate the medium, I decided to use the HypeSax development as a form of case study in how developing a hyperinstrument — a medium — might impact my own compositional and creative processes.

While issues of embodiment and the relationship between 'new instruments' ${ }^{3}$ and musicians, as well as 'new instruments' as composing tools have been widely discussed, the instruments themselves have rarely if ever been considered a fundamental part of the final musical work. While it is true that there is much work involved in building or manipulating instruments, the instruments themselves are considered mere tools for musicmaking, despite the fact that often the musical work cannot exist without the instrument, such as in the case of works for laptop ensemble compositions that rely on a software developed specifically for one piece. Considering this, the following questions arise:

- If a medium (instrument) built for a specific composition is to be considered part of the work, how can it be inserted in a new work-concept?

- How can the process of 'building the medium' become part of the composition process?

- How can hyperinstruments change the way we approach music composition in a meaningful way, not only by offering new affordances? (see

\footnotetext{
3 'New instruments' refers to electronic instruments such as software, hyperinstruments, robotic instruments, new electro-acoustic instruments or any instrumental construct powered in some way by electricity. I also want to make clear that, while there are countless cases of new instruments that do not need electronic components, computers, or electricity — such as those built by Harry Partch — in the context of this dissertation I will use this term mostly to refer to electricity dependent instruments.
} 
There are the essential research questions underpinning my development of the HypeSax, and the associated portfolio of creative works that are presented in this thesis.

\subsection{Methodology}

To find answers for the research questions that motivate this dissertation, I chose to conduct this research following the paradigm of case study research. This methodology has the advantage of offering a description, understanding, prediction and control of the studied case. ${ }^{4}$ In the particular case of this research, there is also a component of practicebased research, which acknowledges the practitioner's creative work as a valid site for research. ${ }^{5}$

The first step in this research was to reflect on my own compositional needs and how to address them. This was done through revisiting compositional strategies, ideas and musical works through compositions created before beginning the development of the HypeSax. The exploration of these ideas and strategies informed the HypeSax's design goals and development strategy. I formulated a new work-concept model that allows for the integration of the medium as a fundamental component of the musical work. This proposition is based on the acknowledgment that the medium, through which the musical work is realised, has a direct impact on the work itself. As the realisation of the importance of the medium in the creative process became a central focus of this investigation, quantitative characterisation of the development and a qualitative user study, as well as exploratory compositions, afforded the insight to address the research questions.

\subsection{Overview}

This thesis details my journey in understanding the implications of the work-concept in my compositional process. I use the development of the HypeSax as a case study to research the creative aspect of shaping the medium, and to investigate the potential

\footnotetext{
${ }^{4}$ Arch G. Woodside, Case Study Research: Theory, Methods and Practice (Emerald Publishing Limited, 2010), p. 1.

${ }^{5}$ Linda Candy, Practice Based Research: A Guide, CCS Report: 2006 (Sydney, Australia: University of Technology Sydney, 2006), p. 3.
} 
practical problems and solutions found in thinking about the medium as a part of the work. I explore the process of creating musical works at the same time as creating the medium itself. I describe compositional techniques developed to be used in my creative practice, while addressing questions related to embodiment, acoustically informed composition, and new technology as elements of my musical language.

I begin this dissertation by discussing current work-model concepts as well as proposing a new model that integrates the score, performance and medium. Following this, I discuss some compositional strategies that are part of my musical language as an examples of how composition techniques can be understood, seen and used through the proposed model. In Chapter Three, I present a detailed description of the HypeSax, its design criteria, technical description and development. Chapter Four presents a user study and a second iteration of the HypeSax design. In this chapter, the first glimpse of the potential of the proposed work-concept is revealed through the responses of the participants as the relationship between musicians and the medium becomes evident and influential in the creation of musical works. In Chapter Five I discuss the HypeSax and its role as a medium and compositional tool, and the potential it has to take different roles beyond being a musical instrument. In the second part of Chapter Five, I exemplify how the new medium influenced and was used in my recent works. Finally, in Chapter Six I present my conclusions and closing thoughts. 


\section{2}

\section{A WORK-CONCEPT FOR}

\section{THE TWENTY-FIRST}

\section{CENTURY MUSIC}

This chapter presents a discussion about a work-concept model that can be used to analyse the ontology of new musical works with technology, as well as other works, by introducing the concept of the medium. The model (described in section 2.2) aids in integrating current compositional practices such as developing software and hardware into the ontology of the musical work. This model, however, is not exclusive for works that include an element of new technology, as the medium is the source that produces or modifies sound such as an instrument, the reverberance of a room, or mechanical system. By considering the medium as an important element of a musical work, new relationships and understanding of the creative process and the realisation of the work may be revealed. This model seeks to offer a framework for exploration and understanding of the challenges faced by new composers in the context of twenty-first century technologies.

In the following pages of this chapter I discuss the importance of technology in new musical works. First, I introduce this chapter by discussing, from a personal perspective, the issues in defining what the musical work is. Following this, I analyse traditional conceptions of musical works. Then, I propose a new work-concept model. I argue that 
the inclusion of new technologies as an essential part of the composition craft has largely influenced the works of the of modern composers, and that our view of musical works should be discussed in the light of the relationship between music and technology from the perspective of the composer, the performer, and the audience. Finally, I analyse how new technology fits in the proposed model, and why I have developed a hyperinstrument to explore this model in my compositional practise.

\subsection{The issues in defining a musical work}

In Western art music there is a tolerated (indeed, necessary) contradiction between the knowledge that a musical performance is an operation dependent for its existence on an earlier, invisible operation (composition) and the fiction, in which executants and audiences share, that the performance is itself an act of creation. [...] The resistance by performers to the idea, proposed by Stravinsky and others, that the 'interpretation' of a musical score should be replaced by its mere 'reproduction' becomes very understandable in this light. ${ }^{6}$

Michael Talbot

While creating music is often portrayed as a linear process that begins with the composer's idea, my own experience has forced me to rethink this. Based on my experience playing the cello or performing with a laptop, I consider compositions to only be finished when they are performed. However, this conflicts with my compositional training, where I was taught to believe that our work is found in the unique design of the composition, not in its realisation, which can be full of inaccuracies or misinterpretations. Unfortunately, the complete sonic design cannot be accurately transmitted by oral or written means, and I do not have the capacity to play all instruments, objects, computers, or processed sounds that I imagine working together in my creation. This suggests that there is a barrier that stops my imagined work from being realised as I conceived it. This barrier becomes a filter when my design is translated into sound by the musicians during

${ }^{6}$ Talbot, p. 176. 
performance. This filter is effectively a new moulding force that gives my design a new shape.

What arises from this process of realising a score is a new musical entity that comprises elements of the original design, elements brought in by the performerprovided by their curation, technique, skills, and other characteristics unique to the instrument(s) such as specific timbre or quality of the instrument - as well as the acoustic response of the instrument or space in which the performance occurs. The final work, the one that is received by the audience, resembles the composer's original design, but is enriched by the nuances introduced by the elements involved in its realisation.

The transformation of the work is unavoidable. For this reason, in my later scores, I have been less specific with some events, such as using a line in the score to represent a melodic contour rather than specific notes, or providing a text describing an performative action, such as 'improvise rhythmic patterns', or giving performance notes such as 'the score is a suggestion, disregard it at will. While some music requires a precise notation, I find value in openness and un-specificity, seeking to engage and incorporate creativity from the performers. This, however, brings some question to mind: where does my work end and the performer's work start? And, what is the work? Is it what I compose or what is being interpreted?

\subsection{Technology as a factor to define a musical work}

The use of new technologies has made me rethink my relationship with composition and my musical works. Today, more than ever, music is easily accessible and can be easily accessed everywhere. Apart from the traditional ways in which one could encounter live music, we can take music everywhere thanks to recording technologies. At the same time, music accompanies many events, films, advertisements, video games, and many other activities. Most importantly, we have the option to choose, interact with, and even manipulate music in everyday situations (such as creating a ringtone for the cell phones without prior knowledge in audio manipulation). This new relationship with music has changed the way we think about it. The role that new digital and smart technologies play in the relationship between music and composers, performers, and audience is so important that it has become fundamental. Some scholars have already explored the effects of new 
technology in music from different perspectives, such as exposing audiences to 'good music' through curatorial practices on radio, ${ }^{7}$ the importance of technologies for popular musicians, ${ }^{8}$ the introduction of computers in contemporary composition, ${ }^{9}$ and many other examples that show that music is being transformed by technology. Considering this, perhaps we should start thinking of new technologies as an integral part of modern music and our relationship with it.

In the next section, I present different points-of-view of what the musical work is. Then, in section 2.4, I propose a model for the ontology of music that introduces the medium as a fundamental element of the work, allowing new technologies to be considered as integral parts of musical works. With these ideas as reference, it will become easier to understand the role that technology can play in music, and how the use and development of new technologies can become part of the creative process and the musical work as a whole.

\subsection{The musical work}

A considerable amount of literature has been published on what the musical work is and how we relate to music through the lenses of those conceptions. In the past few decades, musicians, musicologists, and philosophers, such as Lydia Goehr, Michael Talbot, Roger Scruton, Lawrence Dreyfus, Paul Ricœr, Jean Molino and Jean Jacques Nattiez, have engaged in defining the musical work. These views are at the centre of the current accepted discourses. Notably, in his book Music and discourse: toward a semiology of music, Jean Jacques Nattiez asserts that 'the essence of a musical work is at once its genesis, its organisation, and the way it is perceived'. ${ }^{10}$ With this statement Nattiez refers to a strategy of production (poiesis), the materiality of the work or neutral level (trace or immanent

\footnotetext{
${ }^{7}$ T. W. Adorno, 'A Social Critique of Radio Music', The Kenyon Review, 18.3-4 (1996), 229.

${ }^{8}$ Leslie C. Gay, Jr., 'Acting up, Talking Tech: New York Rock Musicians and Their Metaphors of Technology’, Ethnomusicology, Vol. 41, No.1, 1998, 81-98.

${ }^{9}$ Barry Truax, 'Paradigm Shifts and Electroacoustic Music: Some Personal Reflections', Organised Sound, 20.1 (2015), 105-10.

${ }^{10}$ Jean Jacques Nattiez, Music and Discourse: Toward a Semiology of Music (Princeton, N.J: Princeton University Press, 1990), p. ix.
} 
level), and a strategy of perception (esthesis), implied in the theory described by Jean Molino in 'Musical Fact and the Semiology of Music'. ${ }^{11}$ Molino's proposition, mirrored by other authors like Roland Barthes, ${ }^{12}$ is that the work is not an intermediary in a unidirectional process between composer and listener, as has been suggested by the analogue elementary structure of communication defended by Umberto Eco. ${ }^{13}$ In contrast, Molino's model (see Fig. 1) implies that a symbolic form (for example a symphony) is the 'result of a complex process of creation [...][that] is also the point of departure for a complex process of reception that reconstructs a "message" '. ${ }^{15}$

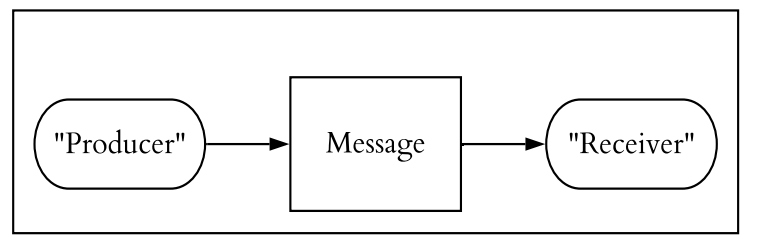

A

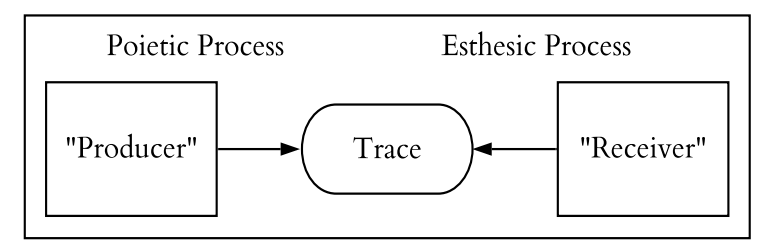

B

Fig. 1. Classic schema for communication (A) contrasted against Molino's model (B). ${ }^{16}$

Paul Ricœur also disputes the traditional model of communication where the author transmits in one direction. He argues that the author disengages from the work during the writing stage. At this point, the act of writing forces the author to focus on the act of reading, effectively introducing the concept of interpretation. ${ }^{17}$ This presupposes a modification of Molino's model where the author (the composer) is involved in both the poietic and esthesic processes, as illustrated by Frisk in his 2006 EMS Conference paper,

${ }^{11}$ Jean Molino, 'Musical Fact and the Semiology of Music', trans. by J. A. Underwood, Music Analysis, 9.2 (1990), 105 (pp. 128-32)..

12 See 'III Syntagm and System' in Elements of Semiology Roland Barthes, Elements of Semiology, trans. by Annette Lavers and Colin Smith (Hill and Wang, 1968), pp. 58-88..

${ }^{13}$ See 'Chapter 1: Signification and Communication' in A Theory of Semiotics Umberto Eco, A Theory of Semiotics (Bloomington, Indiana: Indiana University Press, 1976), pp. 32-47..

${ }^{15}$ Nattiez, p. 17.

${ }^{16}$ (A) and (B) are originally presented in Molino's 'Musical Fact and Semiology of Music' (p.106) with the word 'sender' instead of 'producer'. However, Nattiez adapts this model using the word 'producer' in Music and Discourse: Toward a Semiology of Music (p.16)

${ }^{17}$ Paul Ricœur, From Text to Action (Northwestern University Press, 1991), pp. 106-10. 
where the author is in charge of the constructive and reproductive process of the text (see Fig. 2). ${ }^{18}$

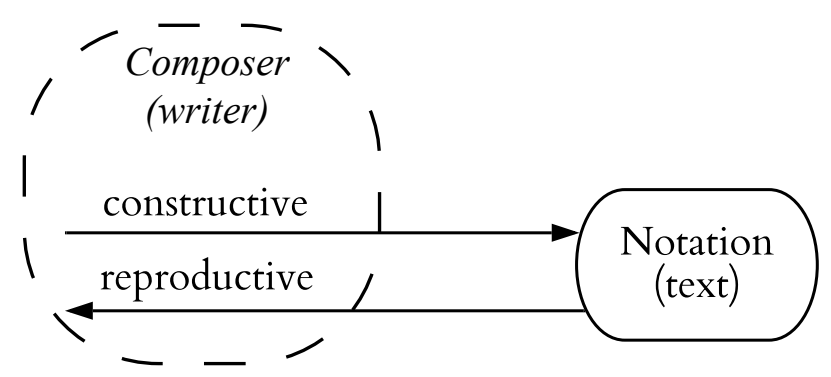

Fig. 2. Frisk's interpretation of Ricœr's model. ${ }^{19}$

Horacio Vaggione supports this two-way stream of actions in his article 'Some Ontological Remarks about Music Composition Processes', ${ }^{20}$ stating that 'action and perception are principal components' in the ontology of the work. In his vision, an 'action/perception feedback loop' validates the musical process in which the work is created. In this way, Vaggione suggests that the composer evaluates the work from the perspective of the performer either by 'performing' it virtually or, at least in the case of electronic music, have the music be reproduced as it is being designed.

Similarly, in his article 'Rhizome and Gesturality in Atonal Music', ${ }^{21}$ composer and theorist Hebert Vázquez — who introduced me to the issues of musical semiotics - argues that music has the power of being the 'meeting point' between the composer and the audience. As a first step, the composer also takes the place of the audience, and becomes a metaphorical listener. ${ }^{22}$ This metaphorical listener has a conversation with their self during the creative process, where an internal dialogue helps shape the score. As a

${ }^{18}$ Henrik Frisk and Stefan Östersjö, 'Negotiation the Musical Work. An Empirical Study on the InterRelation between Composition, Interpretation and Performance', in Terminology and Translation (presented at the EMS: Electronic Music Studies Network, Beijing, 2006), p. 13.

${ }^{19}$ Frisk and Östersjö, p. 3.. Dashed line added to avoid confusion about the flow. Frisk and Östersjö's model does not present this line.

${ }^{20}$ Horacio Vaggione, 'Some Ontological Remarks about Music Composition Processes', Computer Music Journal, 25.1 (2001), 54-61.

${ }^{21}$ Translated from the original title in Spanish: 'Rizoma y Gestualidad en la Música Atonal'

${ }^{22}$ Vázquez, in the same article, expresses his belief that excluding the 'metaphoric listener' from the compositional process can be possible, but the resulting music has very little possibilities of resonating with a wider audience. 
consequence, the music that the composer writes is curated and predisposed to find affinity and have a close dialogue with a second audience of similar taste and background. ${ }^{23}$

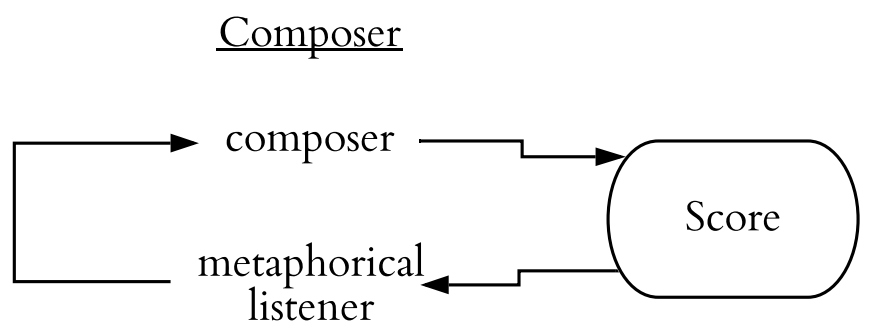

Fig. 3. Interpretation of Vázquez's scheme of musical ontology

Extending Vázquez's idea of the music as 'meeting point' - and overlapping my initial worries about viewing the work from the point-of-view of the listener-the conversation continues now between the composer and the second listener, the real audience sitting at the concert hall. While this conversation does not traditionally result in changes to the score, ${ }^{24}$ the musical work 'breathes and lives' only while it is being performed as the audience enters this dialogue. After it has been performed, the work is recognised as a whole and is valued. It must be clarified that none of these authors, nor my own point-ofview (which is discussed in the next pages), imply that the score (the text) is necessarily a 'message' in a metaphor of communication. Therefore, the use of words such as 'conversation' or 'dialogue' are used without any linguistic connotations such as sign, signifier or signified ${ }^{25}$ assigned to the score. Music, however, seems to be in the centre of the relationship between the creator and the audience. Understanding what the role music

${ }^{23}$ Hebert Vázquez, 'Rizoma y Gestualidad En La Música Atonal', Revista Pauta. Cuadernos de Teoría y Crítica Musical, Vol. XXIII, No. 95 (2005), p. 91.

${ }^{24}$ The exception are works designed to respond directly to the audience such as in the case of interactive installations or performances, or subsequent revisions made by the composers based on feedback.

${ }^{25}$ Ferdinand de Saussure proposed the concept of sign as a two-part mental linguistic unit which encompasses signifier and signified. The signifier is the abstract representation of something, for instance the word 'flower'. The signified is the idea or concept of that thing, for instance the idea of the flower. The sign unifies these two concepts into a meaningful unit that represents the actual thing, in this example the sign 'flower' evocates the idea of the flower and represents a real flower. See: Ferdinand de Saussure, Course in General Linguistics, ed. by Charles Bally and Albert Sechehaye, trans. by Wade Baskin (McGraw-Hill Book Company, 1966), pp. 65-71. 
occupies in that relationship is requires a discussion about the concept of the musical work. ${ }^{26}$

Many have theorised the concept of musical work. Lydia Goehr, for instance, in 'Being True to the Work', argues in that the idea of the musical work as a 'composer's unique, objectified expression, a public and permanently existing artifact made up of musical elements ${ }^{27}$ has its origins in the early $1800 \mathrm{~s}^{28}$. Before then, Goehr explains, pieces of music were not necessarily thought of as finished products. Many compositions were created to be performed once (if they were composed for a specific event) or a handful of times at most, but never did the composers believe that their music would live longer than themselves. Those works were commonly not even completely finished after the composer drew the final bar line. Often, composers did not have full control of what they could compose or the instruments they should write for. The orchestras, established ensembles such as string quartets or wind quintets, or even instruments were not defined in the way they are today. This meant that the scores would have to be adapted for their performance. ${ }^{29}$ If they were performed a second time, these pieces would most likely be adapted to the new conditions (instruments characteristics or performers available), and would be different from that of the original score or previous realisations. ${ }^{30}$

In other cases, such as avant-garde music, jazz, Indian raga and film music, the workconcept is forced to be expanded in its meaning, as in these cases the music does not necessarily meet the criteria of the composer-centric paradigm established during the Romantic period. The concept becomes open in nature, as its 'understanding is affected by extraneous influences [...] as a consequence of conceptual migration and social

${ }^{26}$ Referred to as the 'work-concept' in musicology.

${ }^{27}$ Lydia Goehr, 'Being True to the Work', The Journal of Aesthetics and Art Criticism, 47.1 (1989), 55 (p. 1).

${ }^{28}$ Goehr, 'Being True to the Work'; Lydia Goehr, The Imaginary Museum of Musical Works: An Essay in the Philosophy of Music, Rev. ed (Oxford: Oxford Univ. Press, 2007).

29 There was also not a clear distinction between rehearsals and performance. There did not used to be a dedicated space to listen to music, and 'music was not so much listened or attended to, as it was worshipped, danced, and conversed to.'Goehr, The Imaginary Museum of Musical Works, p. 192.

${ }^{30}$ Goehr, The Imaginary Museum of Musical Works, pp. 176-204. 
change' ${ }^{31}$ In other words, we can assume, based on this perspective, that what defines the limits of the musical work can be changed according to the context in which this work lives.

In The Aesthetics of Music, Roger Scruton focuses on the ontology of the work by identifying 'the sound pattern intended by the composer, which is realised in performance by producing sound events. This sound pattern defines the salient features of the musical work, and can be written down in the form of a score' ${ }^{32}$ Under this argument, the relationship the work has with the performance takes on a new level of importance. The performance attempts to bring out the salient features of the sound pattern designed by the composer. There are features of the performance, however, that are not — and cannot bedefined in the pattern represented by the score. According to Sergiu Celibidache, Mahler believed that in the score one can find 'everything, but not the essential'. ${ }^{33}$ The performer then has an important role to play in the aesthetic experience, translating the design of the work to the 'breathing entity' that talks to the listener. This resonates with Celibidache's belief that music can only exist as a dynamic phenomenon. ${ }^{34}$ If one considers this as a truth, the question of what the work is becomes more difficult to respond. The concept of work as a dynamic phenomenon becomes more evident in music where the tradition involves improvisation over a set design (such as in the case of jazz), to realise the design according to a specific performative tradition (such as in Javanese music), to perform a design that is not fully determinate (such as some avant-garde compositions), or to follow other criteria that are difficult or impossible to represent using traditional music notation.

Other authors, such as Laurence Dreyfus, Peter Walls, Gabriel Solis, or Laurenz Lütteken, approach the work-concept or the true self of the work ${ }^{35}$ from historical,

${ }^{31}$ Goehr, 'Being True to the Work', p. 62.

32 Roger Scruton, The Aesthetics of Music (Oxford University Press, 1999), p. 109.

${ }^{33}$ Was steht in der Partitur? Alles, nur das Wesentliche nicht! in Jan Schmidt-Garre, Celibidache - You Don't Do Anything - You Let It Evolve (Arthaus Musik, 1991).

${ }^{34}$ Miguel Astor, Aproximación Fenomenológica a la Obra Musical de Gonzalo Castellanos Yumar (Caracas, Venezuela: Comision de Estudios de Posgrado, Facultad de Humanidades y Educación Universidad Central de Venezuela, 2002), p. 64.

${ }^{35}$ Werktreue, or the notion of being true to the work is a concept developed-and used in the language of musical criticism - by E.T.A Hoffman, which provides value to music and musical activities without considering any extra-musical aspects. 
philosophical, or pragmatic perspectives. Considering the different perspectives of what the work-concept is, one can suggest that the work is not found in its materiality, its sounds or representations, but on how the score presents itself and 'speaks' to an audience. Goehr suggests that 'the concept of a musical work is intimately tied to a conception of the complex relationships obtaining between the composer, the score, and the performance, as these are expressed on several levels: within musical and aesthetic literature; and in terms of institutional codes'. ${ }^{36}$ This suggestion elevates the interpretation, in the sense of performance, and in the sense of understanding the work (both from the performer's and the audience's perspective) to a level of importance similar to the score.

Mirroring Goehr's observation of the importance of the role that performance has in the work-concept, Nattiez suggests a modification of Molino's model. Nattiez reinterprets Molino's scheme and adapts it with musical terms. Then, he inserts an extra step which is necessary in the case of Western art music, in which a text (score) is traditionally interpreted by a performer (see Fig. 4). ${ }^{37}$

${ }^{36}$ Goehr, 'Being True to the Work', pp. 56-57.

${ }^{37}$ Nattiez, p. 73. 


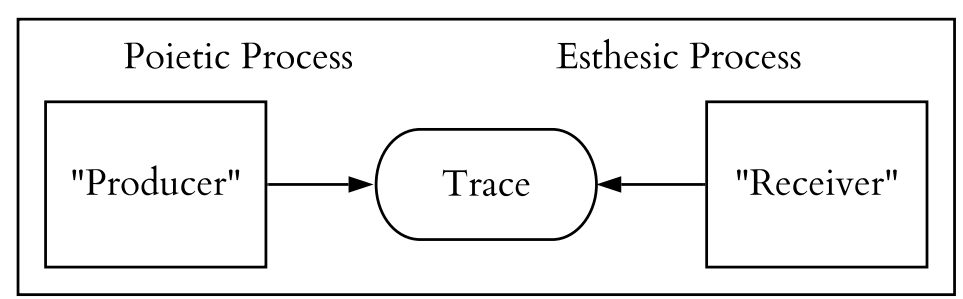

A

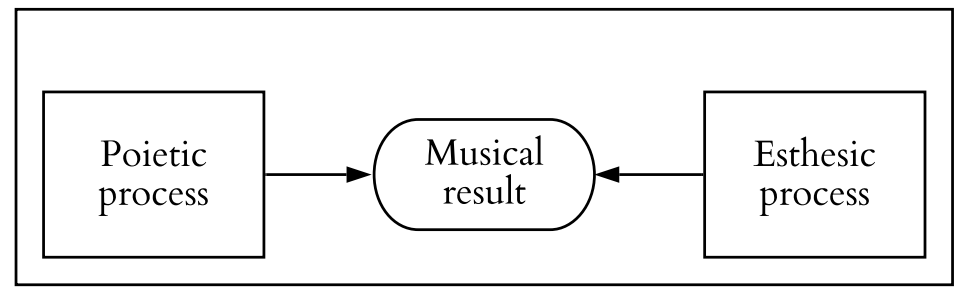

B

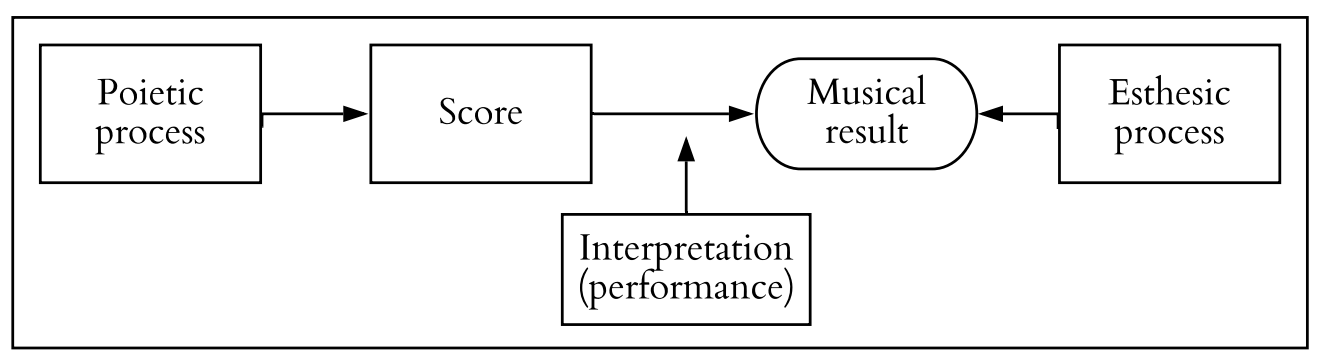

$\mathrm{C}$

Fig. 4. Molino's scheme (A) ${ }^{38}$ Nattiez's reinterpretation of Molino (B), and Nattiez's final model (C). ${ }^{39}$

Despite the fact that Nattiez recognises the importance of the interpretation, he declares to be more 'inclined (in the context of Western music) to apply [semiological] analysis of the neutral level to the graphic sign alone, because that sign precedes interpretation'. ${ }^{40}$

Neither the schemes of music ontology nor the models of work-concept that I have presented consider the materiality of sound, despite the fact that it is essential both for the ontology and the concept of the work. It is true that some of the schemes presented were not formulated specifically to refer to music, but rather to respond to linguistic and semiotic issues. However, once they are translated to music - or any other form of art, except perhaps literature - it is of upmost importance to consider the materiality of the

\footnotetext{
${ }^{38}$ Molino, p. 106.

${ }^{39}$ Nattiez, p. 73.

${ }^{40}$ Nattiez, p. 72.
} 
work. In the ontology of the work, composers enter the 'action/perception feedback loop' only thanks to the possibility of understanding the materiality of the sound which is being sculpted. The composer writing a piece for guitar cannot enter this loop if the instrument's capabilities and limitations are not being considered. The guitar, in this case, provides the materiality of the sound, as well as the physical limitations with which the performer can sculpt that materiality according to the composer's design. There are cases where a deeper level of abstraction in composition can disregard the materiality of sound; for instance, in the case of some imitative counterpoint works, such as a canon, in which the lines are not assigned to a specific voice or instrument. Nevertheless, past the point of the composition, once this canon is performed, certain decisions have to be made based on the materiality of the sound that will be used in realisation of the canon.

As the discussion about the work-concept continues, authors have and continue to propose changes and adaptations to it according to their interests or needs. Upon reflecting on the perspectives presented in this section and the idea of considering new technology as part of modern music (discussed in section 2.1), I wonder about how the relevance of technology for the work-concept. Technology_old and new-is what enables musicians to make music. ${ }^{41}$ A piano concerto cannot exist without a piano, and electronic music could have not existed without electricity and electronic technologies. The medium influences music, perhaps a new work-concept model can be useful.

\subsection{A new work-concept: The tripartite model}

Previous models fail to consider the materiality of the work, preventing us from acknowledging and embracing the physicality of music-making and the curation of the actions and medium, through which sound is produced, as part of that creative process, and, as a consequence, part of the work itself. While composers and performers are engaged with an idiomatic music practice and awareness of sound, certain compositional practices of great creative value (such as software and musical instrument development, circuit bending, or creative use of acoustic space, amongst many others) do not have a prominent place in the current musical work models. I believe it is necessary to create a

\footnotetext{
${ }^{41}$ Although there are some primitive instruments that don't require any technology such as conch shells, ram's horns or the human voice.
} 
new model that can aid in expanding our conception of musical work, allowing for creative aspects that are not musical, but that have a direct impact in sound, to be integrated as part of the work-concept. I identify three essential components that are fundamental and should be considered in the formulation of a new model: the score, the performance, and the medium. The work-concept model I propose integrates these three components, for which reason I call it the tripartite model.

Note that the words score, performance and medium should be written in italics when referring to the three elements of the tripartite model - as they are written in this dissertation. For instance, score (non-italics) can refer to musical notation, while score represents an element of the tripartite model. Score, performance and medium are discussed and exemplified in the following sections.

\subsubsection{The score}

The score is the work that the composer initially imagines, despite how well it can be communicated through notation or other means. The score represents the composer's design, both in its dynamic (the creative act) and static states (the product).

The score is the process and abstract product of creation, which can be recorded in many ways. It is not the record of the composition but instead is what it represents. The score (non-italics) is the representation, while the score (what is represented) is the essence of the work.

In a piano sonata, the score is the notation of the composition, while the score is the structure, the harmonic progression, the melodies, articulations, and every other musical aspect, as well as the interactions between all of these elements. In a musique concrète composition, the score is not found in the recording itself, the tape, the vinyl, or MP3 file, but in the way in which the sounds have been organised by the composer. A piece of software, for instance a SuperCollider code, is not the score. The way in which sound and music have been design and fixed in the code is the score. In an interactive installation, the score is the hard-coded design - of conditions and reactions to those conditions - that establishes the relationship between the users and the response of the components of the 
installation. In the interactive installation Urban Lights Contacts, ${ }^{42}$ for instance, a user gets in direct contact with a sensor. Then, other users are invited to touch the first user or create a chain of contact leading to the initial user. Lights are triggered and a soundscape is generated based on the amount of contact between users. The score in this work is the predesigned sounds, sound and light behaviour, and their reaction to the user's interaction with the sensor through their own contact. The score (non-italics), on the other hand, is the set of instructions that describe how to connect and set up the sensor, lights and speakers, and the instructions that tell the users how to interact.

\subsubsection{The performance}

The performance comprises three different stages: first, the virtual performance that the composer imagines while designing the work (as suggested by Vaggione and Vázquez). This is rooted in the composer's experience as a performer, or their knowledge of the instruments and how performers would interact with them. The second stage is a collaboration with performers, in which changes occur to the work. Note that the first and second stages are often blurred into one as the composer can be a skilled performer. The final stage is the actual live performance (or recording) of the work.

For a piano sonata, the tree stages of performance are: 1) the composer's previous knowledge and exploration of the instrument, as well as how the imagined composition would feel under the hands of the performer and sound with one or another piano or acoustic space. This stage also includes the composer playing the in-progress-composition. 2) Then, the composition is workshopped with a pianist. 3) Finally, the third stage is the performance in a real concert, where the acoustics of the room, the audience, and the performer's physical and mental state influence the music as it is transported from the abstract to the real world.

In the musique concrète composition the three stages are: 1) the recollection of sounds and the act of manipulating them, 2) playing back the sounds as a process to achieve the desired sound, and 3 ) listening to the final recording.

42 Urban Light Contacts is an interactive work by Grégory Lasserre and Anaïs met den Ancxt. More information about this work can be found at http://www.scenocosme.com/urban_lights_contacts_e.htm 
For the composition encoded in software, for instance the SuperCollider code, the stages are: 1) designing an overall structure of the code making correct use of the programming language, 2) running the code and listening to the result in order to make appropriate changes or debug, and 3) running the code and performing in front of a live audience.

An interactive sound installation would potentially present the three stages in the following way: 1) the composer conducts small-scale tests of the interactions and reactions of the potential users and elements of the installation, 2) builds - possibly in collaboration with other specialists such as welders, electricians, or carpenters-runs and tests the installation, and 3) the users interact with the installation.

\subsubsection{The medium}

The medium represents the materiality of sound - the physical and acoustic qualities of the sound - and the physical object that produces the sound. It is normally determined by an instrument. This could be a musical instrument (acoustic or electronic), the human voice, synthesiser, software, an object, or any other body used to produce or modify sound. Under specific circumstances the medium can also be found in the qualities of the acoustic space in which the work is being performed, which can also be a core element of the composition; for instance, the use of large reverberant spaces in the works of Oliveros, Dempster and Panaiotis - the Deep Listening Band. ${ }^{43}$

Considering the examples previously discussed, one can say that the medium in the piano sonata is the piano, its shape, size, strings, quality of the wood, and many other characteristics, as well as the room where it is found as it extends and transform the perception of the sound through reverberation. All of these have a profound effect on the work, both during the compositional process and the performance. In other words, the medium is the sound of the piano, as well as the piano as an object with specific physical properties and the mechanisms that make the piano produce sound-the keys or pedals mechanisms, for instance.

\footnotetext{
${ }^{43}$ Pauline Oliveros, 'Acoustic and Virtual Space as a Dynamic Element of Music', Leonardo Music Journal, 5 (1995), 19-22.
} 
For the musique concrète piece one finds the medium in: 1) the recording techniques, 2) the audio storage medium (non-italics) — tape, vinyl, digital, or any other-, 3) the audio processing techniques and tools, 4) and the mode of reproduction-for example the sound system and its technical characteristics, or loudspeaker array.

The medium in an interactive installation comprises the components of the installation-software, sensors, cameras, magnetic fields, textures, speaker arrays, or room, for instance-as well as the users as their interaction with the installation is the catalyst for the development of the piece.

More and more, technology is taking the role of the medium. And as it finds a more relevant place in the life of the modern composer (who often designs software and hardware devices for their works), we need to redefine our concept of the 'musical work' in order to include this element, as is explored in the new hyperinstrument developed for this $\mathrm{PhD}$ project and presented in subsequent chapters.

\subsection{The work as the intersection between the elements of the tripartite model}

Considering how important the score, the performance and the medium are for the work to exist, I propose a concept of music as a tripartite model, where the work is found in the intersection of the aforementioned concepts fused as a unity. In this model we can apply the concepts previously described, including both the ontological schema and the workconcept, allowing for a free flow between all of its elements. The poietic (creative) and esthesic (receptive) processes can proceed in any direction, in a constant feedback loop. Similar to Molino's scheme, the work - analogous to the trace - serves as a condition for the poietic process to flow from the score towards the performance, or to flow towards the medium. This flow, in any of these directions, triggers an esthesic process back to the score. This bifurcation of the poietic process and eventual flow backwards could also occur if the poietic process begins in the performance, or in the medium. 


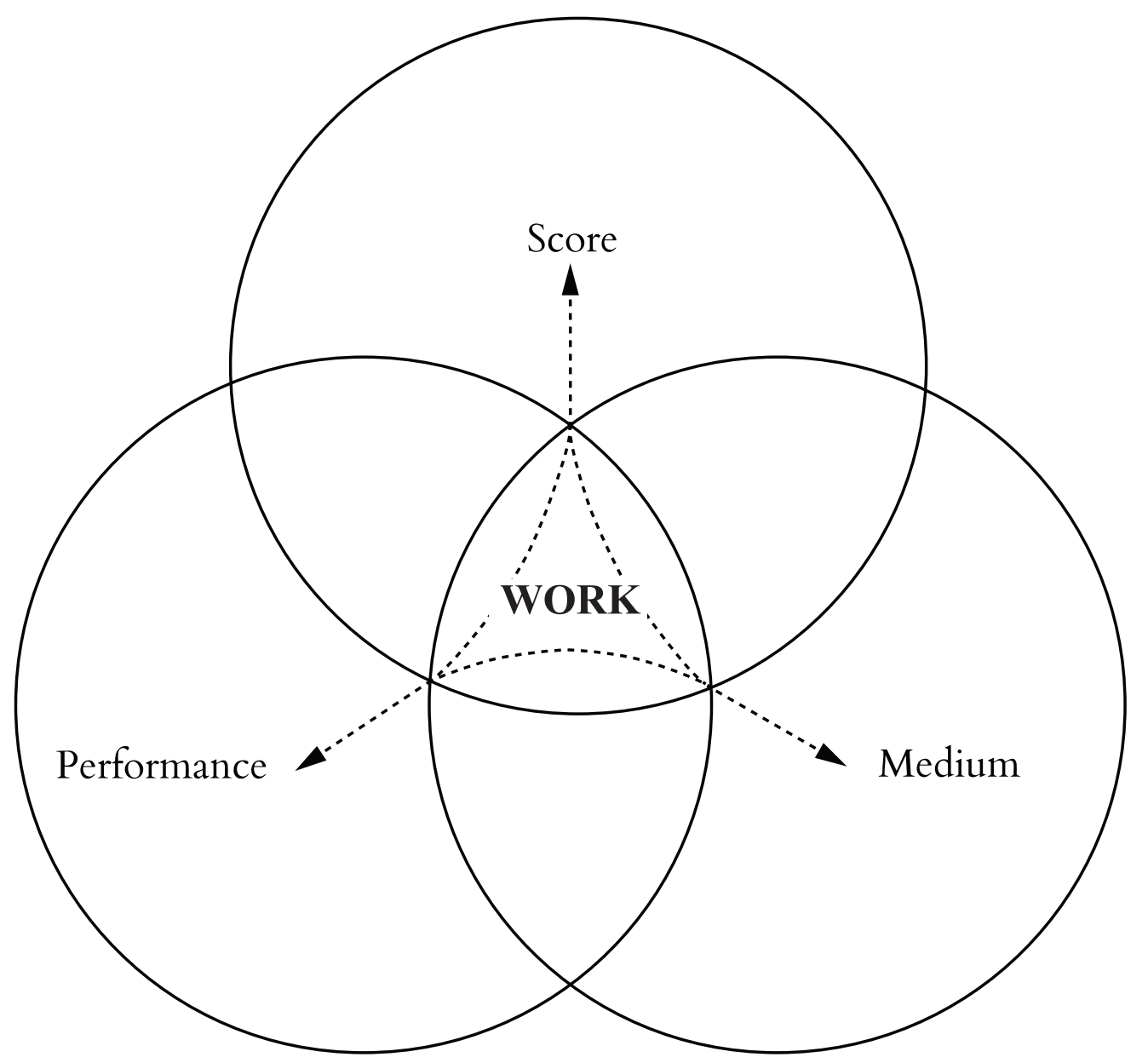

Fig. 5. Proposed tripartite model in which the poietic and esthesic process flow in any direction.

To visualise the tripartite model in action, we can imagine a hypothetical composer, performer and medium joined together by one work. This hypothetical composer, who studied guitar early in her formative years at the conservatory, is invited write a composition for a collaboration between a new music ensemble and a traditional music ensemble from Veracruz, Mexico. The instrumentation for the piece includes a clarinet, piano, violin and cello (new music ensemble), another violin, harp, and three jaranas ${ }^{44}$ (traditional music ensemble). The composer is familiar with all instruments, except for the jaranas. Most importantly, the composer is not familiar with the style of veracruzana music. The composer usually begins the act of composition by imagining and designing the score. Nevertheless, since this is the composer's first experience with the jarana, she

${ }^{44}$ Jaranas are instruments derived from the first baroque guitars that arrived in Mexico during the colony times. 
begins by exploring the sounds of the instrument. This means that the genesis of this particular composition is found in the medium. The instrument-with five strings usually tuned G-C-E-A-G, of which the central three strings are doubled (eight strings in total) tuned either in unison or octaves, and transposed according to the size of the instrumentsuggests harmonies in response to the 'guitar chords' (fingerings) the composer tries first. She also observes the limits in range possible in chordal contexts. At this point, we can suggest how the model works for the ontology of this work, by observing that the poietic process commenced in the medium and, in the flow of the model, moved towards the performance, passing through the potential first stages of the work. The esthesic process then pushed the flow back from performance to the medium, and from there, the composer moved towards the score with the first generation of ideas of what the work will be.

The composer then has a session with the jarana players. She brings a score ready to be performed. The performers try the score but find that the composer is asking for extended techniques used in the contemporary guitar works. The performers suggest changes to the score, especially to the section containing the extended techniques, since these cannot be realised in the jarana as they would on the guitar. The composer, in response, suggests trying a different extended technique, based on her previous exploratory session, rather than her experience with the guitar. Here we can observe that the poietic process begins in the score, moving towards the performance, then to the medium, to the score, and finally going backwards to medium, performance and score. This example illustrates how the flow of the poietic or esthesic process can begin at any of its elements, move in any direction and offer a complex feedback system.

More possibilities could be explored in this hypothetical situation if we consider the rest of the elements that will be part of this work: nine performers, nine completely different instruments (considering that all three jaranas are different, and the difference in skills and performing styles of both violin players coming from different traditions), aspects of improvisation embedded in the veracruzana music performing style, and many other factors. In the case of the model used as a work-concept, the relationships between the elements is similar, as the work cannot escape these three elements, nor exist without any of them.

While the proposed model satisfies me as composer and performer, I wonder if this paradigm can encompass all that a musical work can be. What about the perspective of the 
audience? Is it satisfied by this understanding of the work? Would I as an observer see a finished work in that? Would creating music that follows this model help reinforce the power of 'being a meeting point', as Vázquez describes it? The proposed tripartite model does not integrate reception, but I believe that the medium can be the element that allows the model to extend towards the audience, hence accepting reception as an important component of the work.

\subsection{Reaching the audience with the aid of the medium}

Vázquez proposes that music has the power of being a 'meeting point'. If this is accepted as truth, then the proposed model should include reception and consider the relationship that a potential audience might have with the work, as well as the critical responses the work might have over time. This model gives the work a role in which it is precisely the meeting point of the score, performance, and medium. As detailed in section 2.3, the score is directly linked to the composer, while the performance is linked to both the composer and the performer. On the other hand, the audience is not directly linked to the work in this model, it is only linked through the 'metaphorical listener' (the composer). As stated in the previous section, this model was created when thinking about my own relationship with the work, as I observe it from the point-of-view of the composer, the performer, or the audience. How would this model then include the audience's point-of-view? To address this issue, the relationship of the work and the audience should be discussed.

\subsubsection{The work and the audience}

Milton Babbitt, in his essay 'Who cares if you listen?', proposed that the 'serious' composer

would do himself and his music an immediate and eventual service by total, resolute, and voluntary withdrawal from this public world to one of private 
performance and electronic media, with its very real possibility of complete elimination of the public and social aspects of musical composition. ${ }^{45}$

In this controversial essay, nevertheless, Babbitt establishes a difference between the audience and the 'specialist-audience'. For Babbitt, the specialist, for whom this 'serious' music seems to be for, is a reflection of the composer (the metaphorical listener). These specialists - fellow professionals - might have a way to 'connect' with this music by means of understanding it, just like a physicist would understand a complex physics problem. However, Babbitt argues, the average audience (which in the physics metaphor can barely understand high-school-level-math) struggles to 'connect' with the sounding world offered by the work of the 'serious' composer.

Even when it is not the case of all contemporary serious music, in general terms, Babbitt argued that there was an unprecedented divergence between the musical works and a broader audience in the twentieth century, due to the fact that this music broke with previous canons after reaching new levels of sophistication. ${ }^{46}$ Previous music - and popular music - , on the other hand, was accepted and, to some degree, was intuitively understood by a broader audience. This music, even when it was as sophisticated as new music, already had established two important cornerstones to facilitate a strong relationship with the audience: tonality and form. Even though the technicalities of these two elements are not usually known - at least not in the same degree as a specialist would - by the majority of the audience, they can be clear enough for an audience to be able to follow the musical discourse. Tonality provides tension and release, and the interplay between these (with occasional surprises) provides music with a tool to keep the audience engaged. In a similar manner, the structure offers a clear path to be followed by the listener, playing with his expectations and whether or not these are met.

In Structural Functions of Music, Wallace Berry puts it this way:

Almost since music began there have been, in given style contexts, complexes of pitches in linear and vertical arrangements by which listeners are

\footnotetext{
${ }^{45}$ Milton Babbitt, 'Who Cares If You Listen', High Fidelity Magazine, 1958, pp. 38-40, 126-27 (p. 126).

${ }^{46}$ Babbitt, p. 38.
} 
conditioned to expect certain responsive succeeding events. These are sometimes conducive to a range of expectations, sometimes very specific in their implications [...] It is thus virtually impossible to exaggerate the significance of tonality in the structure of Western music. ${ }^{47}$

In contrast, by not relying on tonality and common formal schemes, new music has struggled to 'connect' with large audiences in the same way as popular music does.

To address this, some composers have utilised other strategies such as musical gestures $^{48}$ (see section 2.6.2.2) or conventions, ${ }^{49}$ which help the listener to follow their music. It must be clarified that it is not being suggested that using these strategies to connect with the audience is necessarily the intention of the composers, but merely that these strategies are very helpful for the audience to follow the musical discourse without the need to become a 'specialised' listener.

A direct link to Barthes' 'Death of the Author' can be drawn here. In this article, Barthes, in fact, advocates for the separation of the author from the text, in order for the reader to be free to access an open reading of the text, one that does not presuppose the intentions of the writer, but is open for the reader's own approach. ${ }^{50}$ In a similar way, the hope is that the listener could be able to find their own ways of approaching a musical work by holding onto one of these strategies - which can be as effective and immediate as tonality or form - without the need to understand what they consist of (whether a specific pitch-class set is being explored, a complex spectral process has been used to develop a harmonic world, the most delicate but obscure musical signifier being quoted intertextually, or any other technical trick that could have been used by the composer).

${ }^{47}$ Wallace Berry, Structural Functions in Music, Dover Books on Music, Music History (New York: Dover, 1987), p. 178.

${ }^{48}$ For instance, the repeating and evolving ensemble gesture in Romitelli's Professor Bad Trip: Lesson I that is presented for the first time in b. 7, marked by the flute attack. See: Fausto Romitelli, 'Professor Bad Trip: Lesson I' (Ricordi, 1998).

${ }^{49}$ For example the use of high-pitch dissonant strings for a suspenseful effect such as the opening of Threnody to the Victims of Hiroshima. See: Krzysztof Penderecki, 'Threnody to the Victims of Hiroshima' (Polskie Wydawnictwo Muzyczne, 1961).

${ }^{50}$ Roland Barthes, 'The Death of the Author', in Image, Music, Text, ed. by Stephen Heath (London: Fontana Press, 1987), pp. 142-48. 
These strategies to which I have been referring are varied and proliferated in the twentieth century. Some strategies that are easily recognised by the broader audience include: those utilised in minimalist music, such as short musical fragments that repeat many times while undergoing a slow development; the use of borrowed musical elements from other traditions such as rock and pop; musical quotations; Gestalt theory elements (see section 2.6.2.1 and APPENDIX D); and musical gesture. The following subsections focus on two specific strategies that I explore in my own compositional practice: musical organisation based on Gestalt theory, and musical gesture. These two strategies can be very useful as they can exist in any kind of music, regardless of genre, compositional technique, style, or tradition.

\subsubsection{Two strategies that build bridges between the work and the listener}

The tripartite model fails to fully integrate the reception of the work-concept considered in most previous models such as that of Goehr's or Nattiez's. It only considers reception through the composer acting as a 'metaphorical listener.' The model is an analytical tool which may suggest a defined approach to the creative act. It does not, however, limit the interaction of the work with the external factor of reception, either as an analytical tool or as a suggestion of the creative approach to the work. The reason why it remains open to reception is because the medium - which represents the materiality of music (sound) and its configuration (see section 1.3) — acts as a gate out of the model, allowing for reception to be integrated into the model. In other words, reception is not possible if there is not a medium to translate the musical work designed by the composer and realised by the performer without the presence of the medium. In this sense, the compositional strategies devised by composers can help provide the configuration aspect of the medium. Let us now analyse two strategies that can help the medium act as an effective gate to the audience: Gestalt theory and musical gesture. These strategies, which are part of my compositional language, have been used to develop part of the portfolio of works accompanying this dissertation (discussed in section 5.2). This portfolio of works features a hyperinstrument called HypeSax (described in Chapter 5 and section 4.4), developed as a case study to explore the medium and its relevance in the tripartite model. 


\subsubsection{Gestalt theory}

Gestalt psychology is founded on the idea that many elements of an image can be grouped together to be conceived as a whole. This way, our brain does not need to analyse every single leaf, branch and trunk to understand this whole as a tree. The brain, in effect, looks for a pattern and mentally 'groups' all of these parts to conceptualise the tree as a unity, without needing to observe every single detail. To describe the way in which we tend to group visual objects, Gestalt psychology describes a set of or principles - or laws —of perceptual organisation: no grouping, proximity, similarity of colour, similarity of size, similarity of orientation, common fate, symmetry (or order), parallelism, continuity, closure, common region, and figure versus ground, amongst others (see )..$^{53}$

In a similar manner, these principles help us understand and organise sounds in a musical composition. Christian von Ehrenfels argued that our brain has the capacity to recognise a melody despite the fact that it can be presented with different pitches, timbres or speed, as long as the relationship of its elements is maintained. In this way, a melody can be identified due to a unique 'Gestalt quality'. ${ }^{54}$

In his 1961 thesis Meta (+) Hodos, ${ }^{56}$ James Tenney brought the Gestalt principles to bear on the discipline of musical analysis, as he sought to describe works by Ives, Webern, Bartók, Schönberg, Ruggles, and Varèse, with new analytical tools that would be equally effective on works by all of these composers. To facilitate the transition from Gestalt psychology to musical analysis, Tenney proposed the use of the terms: 'clang' to identify a sound, sound configuration, or musical idea; 'element' as the constituent parts of the clang; and 'sequence' for structures comprising a chain of clangs recognised as a unified whole. He later adds the terms 'segment', formed by sequences, and finally, 'section', which encompasses sequences. ${ }^{57}$

\footnotetext{
53 Johan Wagemans and others, 'A Century of Gestalt Psychology in Visual Perception: I. Perceptual Grouping and Figure-Ground Organization.', Psychological Bulletin, 138.6 (2012), 1172-1217 (p. 1180).

${ }^{54}$ Wagemans and others, p. 1175.

${ }^{56}$ This thesis was published, along with posterior writings, in 1980 as Meta + Hodos and META Meta + Hodos

57 James Tenney and Larry Polansky, 'Temporal Gestalt Perception in Music', Journal of Music Theory, 24.2 (1980), 205-41.
} 


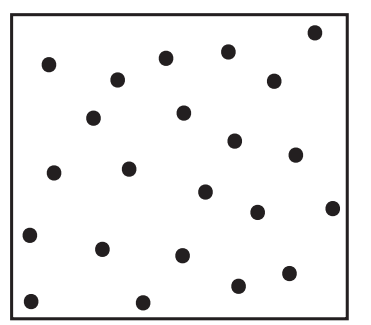

No grouping

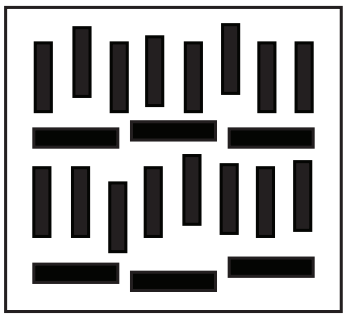

Similarity of orientation

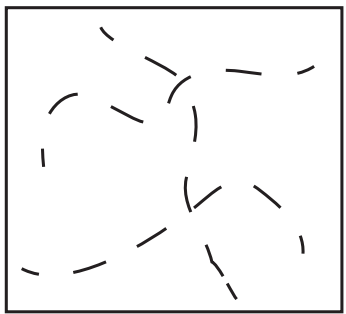

Continuity

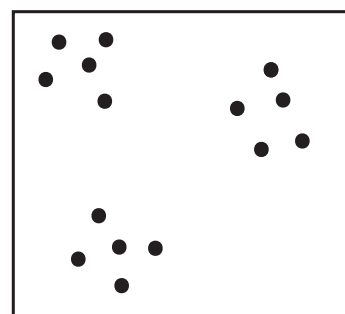

Proximity

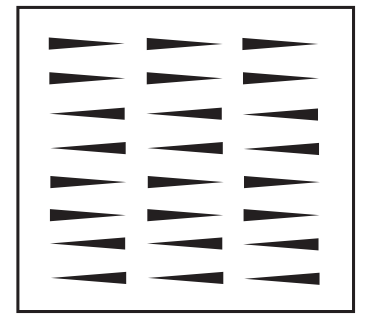

common fate

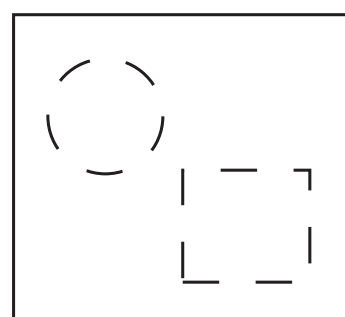

Closure

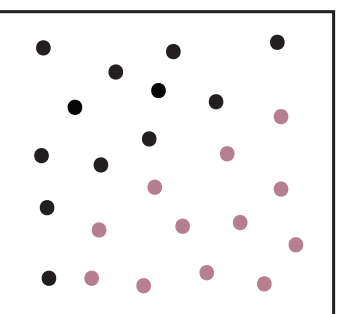

Similarity of colour

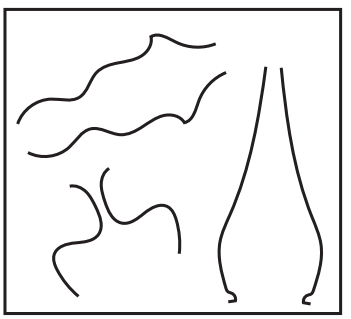

Symmetry

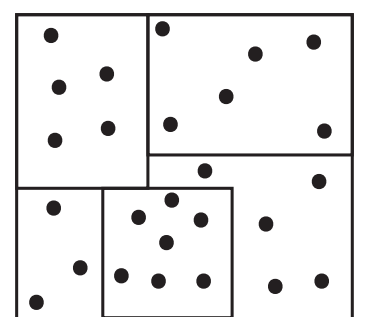

Common region

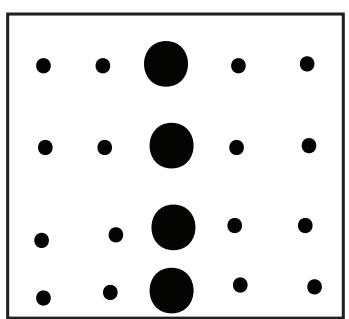

Similarity of size

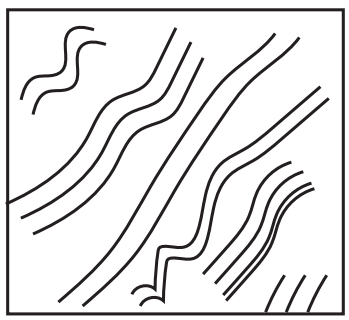

Parallelism

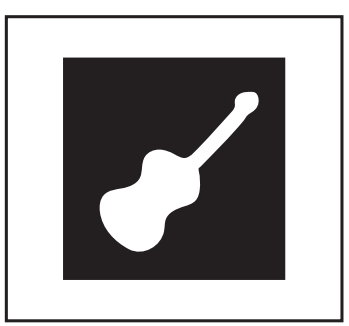

Figure/Ground

Fig. 6. Representation of the most basic Gestalt psychology laws of perceptual organization.

Tenney adapted some of the principles ${ }^{58}$ of Gestalt theory and proposed the use of proximity, similarity, Intensity, repetition, objective set and subjective set, referring to them as 'factors of cohesion and segregation' 59 for their potential to suggest groupings of elements and/or segregation from elements which do not follow the same principle. In addition, in my 2014 Master of Arts thesis, I proposed the addition of a seventh principle to this list, the cloud clang ${ }^{60}$ which serves to identify_ or manage, if seen from the creative point-of-view - densities produced by multiple elements that, due to their relationship, cannot be perceptually broken down into singular elements.

${ }^{58}$ Tenney refers to these principles as factors of association

${ }^{59}$ James Tenney, Meta + Hodos and META Meta + Hodos, ed. by Larry Polansky (Oakland, CA., 1988).

${ }^{60}$ Cristohper Ramos, 'Sound, Shape, and Interactivity: Sound Maze and Other Recent Works' (Wesleyan University, 2014), p. 22. 
These principles can be used as creative or analytical tools, by adapting each one of them to different musical aspects. For instance, using the proximity principle, musical ideas can be organised according to the proximity of temporal events, or proximity of range (see Fig. 7). An expanded discussion on Gestalt theory in music is presented in APPENDIX D. These factors or principles of cohesion and segregation have been used to develop strategies that highlight salient features of the HypeSax sound (developed for this research) and its influence in organisational strategies to develop structure and orchestration in works such as Breathe in, breathe out (discussed in section 5.2.2.1)
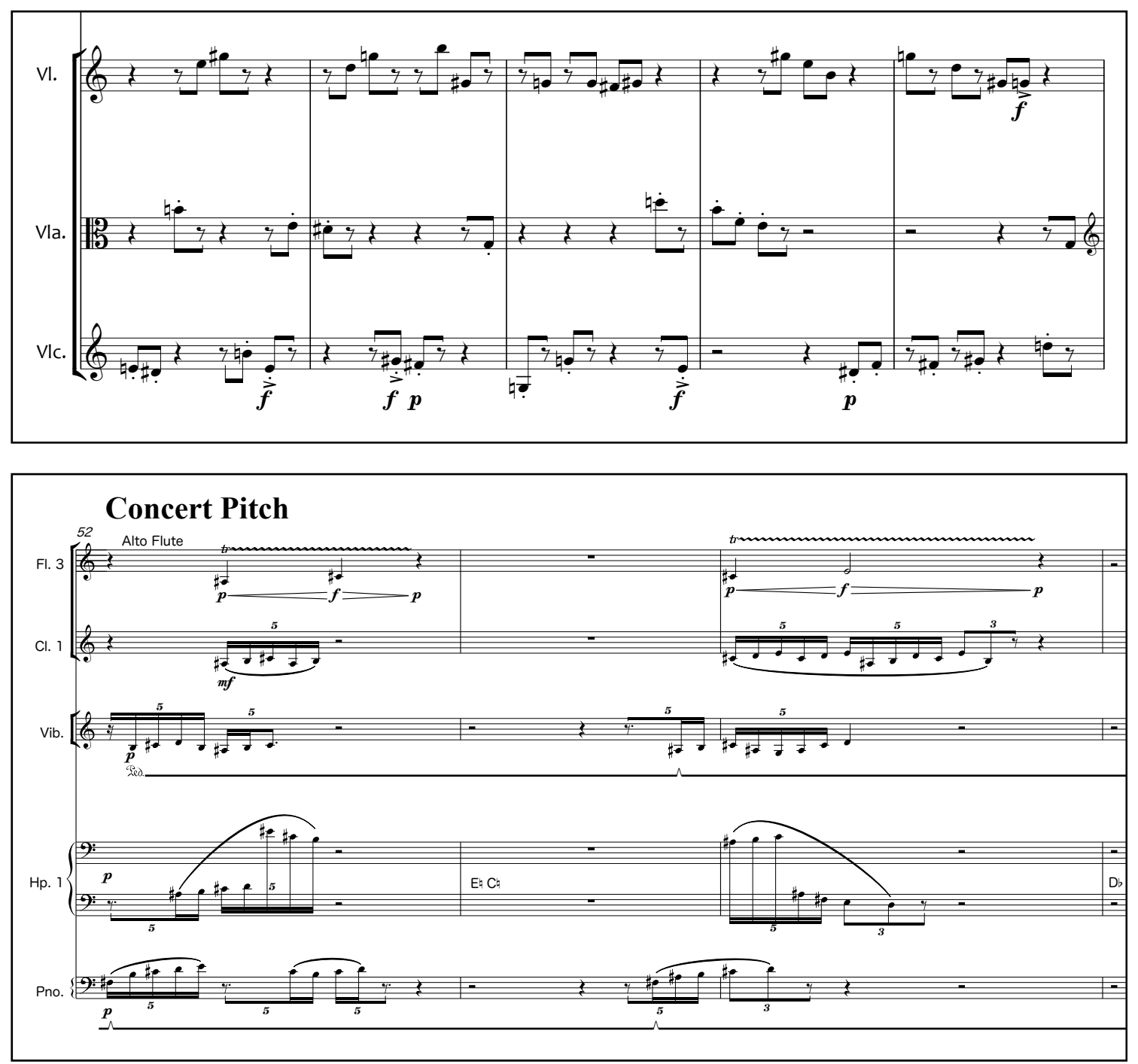

Fig. 7. Temporal proximity allows association between strings in bb. 4-8, Cor Ple (top). Proximity of range produces association instruments in musical gesture, b. 52-54, Bereshith (bottom). 


\subsubsection{Musical gesture}

The use of musical gestures is another strategy that I have used in my compositional practice. I find this strategy to be especially relevant for the works developed for this project due to the fact that a musical gesture is able to relate a sequence of musical events with a sequence of performative physical actions. This was important to me as the performance aspect of the tripartite model relates to the medium through embodiment, an important consideration in the design of the HypeSax.

The musical gesture, similar to the Gestalt theory principles, does not rely on defined pitches or harmonic organisation. This strategy results from the interaction of simple elements that complement each other, potentialising immediacy of musical discourse for the audience. Hebert Vázquez asserts that '[...] the musical gesture is a complex texture, which is expressed by the integrative contrast of its elements, represented by simple textures. The recurrence, transformation and development of the gestural unity provides the atonal musical discourse with an auditive referential point, which grants coherence and continuity'. ${ }^{62}{ }^{63}$ Musical gesture, or its parts, can also be recognised by using one or more of the Gestalt principles as described by Tenney. Specifically, the principles of similarity and repetition can give the musical gesture a strong sense of uniqueness, which establishes the gesture as an important element inside the musical work.

The musical gesture is a construct formed by multiple elements, presented as consecutive events. The simple fact that events are presented sequentially, however, does not make the sequence a gesture. This status is only recognisable when the sequence of events is presented multiple times, even when the elements in these iterations of the sequence are altered - as they usually are. The condition for being a musical gesture is met if the alterations of the elements of the sequence conserve the original relationship of the elements of the gestural unity. For example, in my work Bereshith for orchestra which alludes to the creation according to the book of 'Genesis' (See section 5.2.2.2 and

62 Translated from Spanish: '[...] el gesto musical es una textura compleja, que se expresa por medio de la contraposición integradora de sus elementos, representados por texturas más simples. La recurrencia, transformación y desarrollo de la unidad gestual provee de un punto de referencia auditiva al discurso musical atonal, confiriéndole coherencia y continuidad.'

${ }^{63}$ Hebert Vázquez, Fundamentos Teóricos de La Música Atonal (Mexico: Universidad Nacional Autonoma de México, Fondo Nacional para la Cultura y las Artes, 2006), pp. 220-21. 
Appendix M), I make use of a musical gesture to represent the presence of God. The gesture consists of a dynamic sequence that describes an arch from $\boldsymbol{p}$ to $\boldsymbol{f}$ and back to $\boldsymbol{p}$. This simple gesture, designed to emulate the physical sequence of inhaling and exhaling, is repeated constantly throughout the composition. This sequence is constantly transformed, however, changing instrumentation, pitch, duration, and relative dynamic levels (for instance $\boldsymbol{p p}$ to $\boldsymbol{f f}$ to $\boldsymbol{p p}$ ). Despite these constant transformations (see Fig. 69), the gesture can always be recognised.

The term 'musical gesture', as described before, is useful for musical analysis or creative practice from a traditional point-of-view. The term 'gesture', however, can be interpreted in various ways, and there is not one unique definition - as claimed by Oded Ben-Tal in 'Characterising Musical Gestures'. ${ }^{64}$ Of the two most commonly discussed interpretations, the first grants the term a role of musical concept - similar to 'theme' or 'structure' - as described by Vázquez (and presented in the epigraph of the current section). The second interpretation takes the perspective of gesture as a bodily act. In this second sense, the sequence of events is not a sequence of musical elements, but a sequence of physical movements required to transfer energy to a sounding body. This refers, therefore, to functional aspects of music-making. The two meanings complement each other, through a process of embodiment (discussed in the following section), in the tripartite model of the work, creating a stronger and more meaningful discourse that can help achieve the goal of providing the work with the power of being a 'meeting point' between composer and listener.

Philosopher Maurice Merleau-Ponty states in Phenomenology of Perception that:

It is through my body that I understand other people; just as it is through my body that I perceive 'things'. The meaning of gesture thus 'understood' is not behind it, it is intermingled with the structure of the world, outlined by gesture, and which I take up on my own account. It is arrayed all over the gesture itself. ${ }^{65}$

\footnotetext{
${ }^{64}$ Oded Ben-Tal, 'Characterising Musical Gestures', Musicae Scientiae, 16.3 (2012), 247-61.

${ }^{65}$ Maurice Merleau-Ponty, Phenomenology of Perception, trans. by Colin Smith (Psychology Press, 2002), p. 186.
} 
Perhaps for this reason both the Gestalt theory and musical gesture (empowered by both of its meanings) can be powerful signifiers in a dialogue between the composer and the listener. After exploring Gestalt principles and gesture (in previous works such as Bereshith), and reflecting on their potential, I decided to use them to explore the medium in my compositional practice, and its relationship to the score and performance (this exploration is presented in Chapter 5). The relationship between the medium and performance, however, is a special one, as through gesture physical action and sounding event are linked. In the following section, I analyse the importance of embodiment in the interaction of the score and performance, and how the medium is situated in this relationship.

\subsubsection{Embodiment and the medium}

Vilém Flusser in 'Gesture and Affect: The Practices of a Phenomenology of Gestures' argues that 'gestures are movement of the body that express an intention' ${ }^{66}$ Intention can be a very subjective word, but in any case, an intention expects a response. Merleau-Ponty suggests that there is a sort of language implicit in gesture when he considers that 'the communication or comprehension of gestures comes about through the reciprocity of my intentions and the gestures of others' ${ }^{67}$ This quality of quasi-language is in many respects similar to that found in music, of which the possibility of it having sufficient elements to be a language has been explored by authors such as Robin G. Collingwood, ${ }^{68}$ Willson Coker, ${ }^{69}$ amongst others who are cited — and challenged — by Ann Clark in her article 'Is Music a Language?'. ${ }^{70}$ However, language implies meaning, sign, signifier and signified, which is why the suggestion that music can be a language collapses.

Noam Chomsky, however, in 'Human Language and Other Semiotic Systems' states that:

\footnotetext{
${ }^{66}$ Vilém Flusser and Nancy Ann Roth, 'Gesture and Affect: The Practice of a Phenomenology of Gestures', in Gestures (University of Minnesota Press, 2014), pp. 1-9 (p. 1).

${ }^{67}$ Merleau-Ponty, p. 148.

${ }^{68}$ R.G. Collingwood, The Principles of Art (Oxford University Press, 1938).

${ }^{69}$ Willson Coker, Music \& Meaning: A Theoretical Introduction to Musical Aesthetics (The Free Press, 1970).

${ }^{70}$ Ann Clark, 'Is Music a Language?', 1982, 11.
} 
To determine whether music, or mathematics, or the communication system of bees, or the system of ape calls, is a 'language', we must first be told what counts as a 'language'. If by language is meant 'human language', the answer will be trivially negative in all cases. If by language we mean 'symbolic systems' or 'system of communication', then all of these examples will be languages, as will numerous other systems $[\ldots]^{71}$

Under this view, one can envision a system of codes embedded in a musical work which can act as a 'system of communication' between the composer and the listener through the elements in the tripartite model.

In his book Embodied Music Cognition and Mediation Technology, ${ }^{12}$ and his essay 'Music, Gesture, and the Formation of Embodied Meaning', ${ }^{73}$ Marc Leman presents multiple views by which it becomes easier to understand how musical gesture can suggest meaning. According to him, the suggestion of meaning can be provided by factors such as behavioural resonance (or 'entrainment', as described by Clayton ${ }^{74}$ ) in which rhythmic synchrony is achieved by means of empathy between two subjects, in this case performer and audience, or between performers of an ensemble. This phenomenon of physical and biological origins has a great effect in the reciprocity between the performer and the audience, and it "may contribute to the "magic" atmosphere that facilitates direct involvement with what happens on the scene. ${ }^{75}$ Nevertheless, this 'spell' cannot be tampered with by introducing 'too much awareness,' as involving the mind in thinking and reasoning seems to break this line of communication established by entrainment. In other words, the implied meaning is one that is not (or should not be) thought as something fixed by the intention of the composer or performer. Leman makes it completely clear that this

${ }^{71}$ Noam Chomsky, 'Human Language and Other Semiotic Systems', in Speaking of Apes: A Critical Anthology of Two-Way Communication with Man, ed. by Thomas Albert Sebeok and Jean UmikerSebeok, Softcover reprint of the original 1st ed. 1980 (New York London, 2012), p. 12 (p. 430).

${ }^{72}$ Marc Leman, Embodied Music Cognition and Mediation Technology (Cambridge, Mass.: Mit Press, 2008), pp. 1-26.

${ }^{73}$ Marc Leman, 'Music, Gesture, and the Formation of Embodied Meaning', in Musical Gestures: Sound, Movement, and Meaning, ed. by Rolf Inge Godøy and Marc Leman, 2009, p. 28 (pp. 127-29).

${ }^{74}$ Martin Clayton, Rebecca Sager, and Udo Will, 'The Concept of Entrainment and Its Significance for Ethnomusicology', ESEM CounterPoint, 1 (2004), 83.

${ }^{75}$ Leman, Embodied Music Cognition and Mediation Technology, p. 5. 
suggestion is a subjectivist approach in which '[previous] experiences can provide a basis for speculative interpretations of how music feels and what it means'. ${ }^{76}$

Another important factor for suggesting meaning through musical gesture comes from signification. In this case, unlike with entrainment, reasoning is involved. By previously introducing a verbal description or contextualisation, the musical content is placed under a new light that offers new possibilities for engagement and interpretation (of meaning). These potential interpretations often link subjective experiences with a broader and historical context. In the words of Robert Hatten: 'The linkage between sound and meaning, though mediated by forms, is also mediated by habits of association that, when stylistically encoded, produce correlations, and when strategically earned (inferred through a stylistically constrained interpretive process) produce interpretations. ${ }^{77}$ While cultural and historical context does not imply clarification of musical meaning, it facilitates access to music. This is why new music can benefit from good program notes, which help the listener to engage with it when no historical or cultural reference (such as form or tonality) is present.

An action-based factor, also described by Leman, implies that physical energies (namely movement, perceived visually or sonorously) have an impact on the body and mind. This impact brings signification through gesture. Corporeal articulations provide meaning through a process that involves accessing memory and empathy. The listener's musical involvement through corporeal engagement opens the possibility of interacting with music in many ways: these include interactions based on mimesis, interactions based on goal-directed gestures that are culture-dependent, or those involving responses based on emotive, affective or expressive capabilities, based on social interaction.

Often, physical gestures can express properties of music. These gestures, learned throughout our lives, gain, lose or replace their meaning according to our experiences. Movement such as lifting a hand can imply pitch going up or volume rising. In a different context, however, it can be a cue between musicians, which predisposes the listener to

\footnotetext{
${ }^{76}$ Leman, Embodied Music Cognition and Mediation Technology, p. 12.

${ }^{77}$ Robert S. Hatten, Musical Meaning in Beethoven: Markedness, Correlation, and Interpretation, Advances in Semiotics (Bloomington: Indiana University Press, 1994), p. 275.
} 
expect an event that might be of significance to the overall shape of the work. This movement, the expectation it triggers, and its consequence speak to the listener and reinforces engagement. The formation of meaning takes place when synaesthetic or kinaesthetic transformations are present thanks to these interactions. As stated before, only suggestions of meaning will exist in reality, but the listener will grant meaning to the interactions observed. In synaesthetic transformation, physical properties of the sound, such as frequency, duration, spectral density, and loudness, are assigned a representation that links to space, visual and tactile metaphors such as 'large', 'heavy' and 'rough'. Through kinaesthetic transformation, the dynamics of sound properties afford impressions such as movement, gesture, tension, and release of tension. ${ }^{78}$

Gesture has been widely discussed by musicologists, ethnomusicologists, psychologists, philosophers, and other specialists including those focused on interactive technologies. For the purpose addressing how, through gesture, the medium can become an important component of the musical work, however, I will concentrate on a concept of gesture that includes the interpretations discussed above: gesture as a musical entity, gesture as a movement, and gesture as a movement that embodies a special meaning. These three interpretations are similar to François Delalande's figurative gesture, effective gesture, and accompanist gesture. The figurative gesture responds to the intrinsic musical aspects, while effective gesture refers to the physical motion that produces the sound. Finally, the accompanist gesture involves the whole body of the performer, not only the parts of the body directly interacting with the instrument, with non-sounding movements that often take the role of signifiers, such as cues. The body swinging in entrainment, expressive facial articulations, or others which can be read by the listener to articulate the musical discourse. ${ }^{79}$

The medium, as proposed in the tripartite model, represents the object or set of objects through which the musical work is realised. Of course, in this model, the medium in itself has no extrapolation in the work and does not have the power to give the score the category

\footnotetext{
${ }^{78}$ Leman, 'Music, Gesture, and the Formation of Embodied Meaning', p. 128.

${ }^{79}$ François Delalande, 'Le geste, outil d'analyse : quelques enseignements d'une recherche sur la gestique de Glenn Gould', Analyse Musicale, 1988.
} 
of the work without the performance. It is through gesture that performance empowers the medium. In a similar way, the performance alone does not have the possibility to translate the score out of the imaginary and place it in the real world. In this way, the three are intimately linked in the act of realisation of the musical work.

If gesture is directly linked to the body, then gesture is directly linked to the medium. In his article 'Meaning in Musical Gesture', Fernando Iazzeta suggests that the body, considered to be the medium in the tripartite model, is the interface that translates ideas into physical signs operating by similarity, causality or convention. ${ }^{80}$

When the gesture operates by similarity, temporal or special relations are drawn. For instance, if the first violinist of a string quartet after a pause projects a loud inhale sound accompanied by a large movement of the arm and bow up in the air, the other members of the ensemble will interpret this as a suggestion to continue with greater dynamic intensity, through the principle of similarity. By the same token, reducing the size of such gestures might suggest soft dynamics.

Causualty in gestures operate as a response to a given circumstance. One example could be when the lighting on stage is not adequate, making it difficult to see the first violinist. He might be forced to exaggerate his movements to portray cues, with possible unmatching responses by the other musicians.

Convention, on the other hand, is previously constructed and shaped by factors such as culture and context. If the string quartet was presented with a score that evokes unfamiliar instruments, such as the haegeum, and the performers had not heard or studied the instrument, it is possible that they would not play the wide vibrato usual in the performative practice of that instrument, replacing it with more Western-influenced fiddle gestures, thus changing the intended sound of the score. Gesture is therefore very important, and its absence can cause difficulties for the work to 'connect' with the audience.

${ }^{80}$ Fernando Iazzetta, 'Meaning in Musical Gesture', in Trends in Gestural Control of Music, ed. by Marcelo M. Wanderley and M. Battier (Paris, France: IRCAM-Centre Pompidou, 2000), pp. 259-68. 
Iazzetta states that:

Actions such as turning knobs or pushing levers are current in the use of today's technology, but they cannot be considered gestures. Also, to type a few words in a computer's keyboard has nothing to do with gesture since the movement of pressing each key does not convey any special meaning. It does not matter who or what performed that action, neither in which way it was performed: the result is always the same. ${ }^{81}$

This assertion puts electronic music into a difficult position, as making electronic music has traditionally meant that the interface tends to hide a process through which the sound is produced. Therefore, pressing a button is a gesture with no intrinsic meaning, with a multitude of possibilities. Furthermore, if the musical work is acousmatic, the problem becomes greater. This issue has been around at least since the appearance of musique concrète. Codes, however, slowly become part of our common language and, as we get exposed to codes that fit a specific context, we can learn and give meaning to gestures. For instance, the gesture of turning a knob in a kitchen relates to turning an appliance on. On the other hand, if at a night club we see a DJ turning a knob, we expect a specific musical result.

In this section I discussed the importance of embodiment, an important aspect of the ontology of the medium. But when it comes to instruments that rely on electronic means, embodiment needs to be observed from a different angle, our relationship with technological devices is different than our relationship with acoustic instruments. In the next section I discuss issues of embodiment in electronic music.

\subsubsection{The medium as a carrier of grain and instrumentality in technologically devised music}

Today, almost seventy years after Pierre Schaeffer composed his first work of musique concrète, new tools, such as computers, high-performance recording systems, and powerful software, allow composers to study the phenomenon of sound in a different way, even allowing real-time manipulation of sound. With all of these new paradigms, new

${ }^{81}$ Iazzetta, p. 260. 
problems have also arisen, such as the disembodiment of sound being generated by 'virtual sources', the lack of connection between performative gestures and electronic audio processes (such as the action of pressing a button which might not correspond with a complex sounding event triggered by that action), and mutations of sound through electronic processes. The unique quality that physicality gives to sound, and the grain, as Barthes calls it, ${ }^{82}$ are at the centre of these questions.

Live performance is fundamental for the dialogue between the creator and audience thanks to the personal aspect that physicality gives to sound. The grain of the instrument (or voice), which 'is not - or is not merely - the timbre; the significance it opens cannot better be defined, indeed, than by the very friction between the music and the something else, which something else is the particular language'. ${ }^{84}$ The grain, the unrepeatable characteristic of live sound and live performances, is 'the outcome not just of the physical nature of the instrument, but of its physical limitations' ${ }^{85}$ One cannot separate this physicality without affecting the perception of the grain, and thus affecting the dialogue.

Unfortunately, the grain is hidden when the process that gives music this characteristic is not evident. In the case of live electronic music, it becomes more difficult to recognise the grain, the uniqueness of the performance, when the medium does not have obvious limitations and most, if not all, of its processes happen 'inside a box.' John Croft says, in his article 'Theses on liveness', that:

[...] the limits of an instrument are essential to its being perceived as an instrument at all. A loudspeaker can, in principle, produce any sound; on an instrument, almost all sounds are impossible, and of those that are possible, some are more difficult to produce than others, and this difficulty is patent in the act of performance. This is surely why performance engages us in a way

\footnotetext{
${ }^{82}$ Roland Barthes, Image, Music, Text, ed. by Stephen Heath (London: Fontana Press, 1987), p. 181.

${ }^{83}$ The idea of the grain can be interpreted in different ways: it can be found somewhere between the texture and the timbre of the singing voice, or perhaps between the language and the voice, or the bodily expressions that accompany a musical gesture and the meaning of poetry in the song. In any case, the grain comes from the unique materiality of the sound and the emergence of pleasure that one experiences while listening to a particular performance.

${ }^{84}$ Barthes, Image, Music, Text, p. 185.

${ }^{85}$ John Croft, 'Theses on Liveness', Organised Sound, 12.01 (2007), 59-66 (p. 65).
} 
that cannot be accounted for in terms of the sound alone: the difficulty, the impossibilities, the encounter with limits, the finitude of the instrumental performance resonates with wider human experience. ${ }^{86}$

I believe it is important to address this issue, especially when making music through electronic means, as the sound is designed and generated in a virtual environment, and performers usually interact with sound using generic devices that are not necessarily designed to respond to a physical gesture that corresponds to a musical gesture. Interfaces that were not designed for musical purposes, such as a computer keyboard for instance, can mediate a physical gesture with a process that is not seen or heard by the listener, with any number of sounding results. While in such cases, the interface takes the role of the instrument, it nevertheless lacks true instrumentality.

David Burrows, in his article 'Instrumentalities', observes that a key feature of a musical instrument is to act as mediator between the performer's body and the sound they produce. He uses the concept of instrumentality to describe the purpose of the musical instrument, theorising the concept of 'transitional object' ${ }^{87}$ For Burrows, instrumentality is the capacity of the instrument to be the means of physical expression, the mediator 'between the material and the immaterial' ${ }^{88}$ Philip Auslander, on the other hand, argues that an important aspect of instrumentality is found on skill and technique involved in instrumental performance. ${ }^{89}$ In other words, effort is a key feature of instrumentality. Philip Alperson contrasts these ideas with the 'intentionality' of the instrument and says that

the character, so to speak, of musical instruments - their typical uses, the way they have come to be played and thought of in the history of music-is often

\footnotetext{
${ }^{86}$ Croft, p. 62.
}

${ }^{87}$ Based on the 'transitional objects' concept by D.W. Winnicott, which represents 'childhood articles such as blankets or stuffed toys that have a constructively ambiguous status between the small child's self and his or her emergent sense of otherness' David Burrows, 'Instrumentalities', The Journal of Musicology, 5.1 (1987), 117-25 (p. 121)..

${ }^{88}$ Sarah-Indriyati Hardjowirogo, 'Instrumentality. On the Construction of Instrumental Identity', in Musical Instruments in the 21st Century, ed. by Till Bovermann and others (Singapore: Springer Singapore, 2017), pp. 9-24 (p. 15).

${ }^{89}$ Philip Auslander, 'Lucille Meets GuitarBot: Instrumentality, Agency, and Technology in Musical Performance', in Musical Instruments in the 21st Century, ed. by Till Bovermann and others (Singapore: Springer Singapore, 2017), pp. 297-314. 
rooted in the technical development of the physical instrument and its corresponding musical possibilities. ${ }^{90}$

This means that there is instrumentality implicit in the intention embedded in the object by the luthier, programmer, performer or composer, as instruments-especially those 'interfaces' used in electronic and digital music-making-cannot be reduced to mere material objects when they are used to make music.

Croft, who supports the idea that 'the difficulty, the impossibilities, the encounter with limits, the finitude of the instrumental performance resonates with wider human experience', proposes a set of 'conditions for instrumentality' ${ }^{91}$ in instruments or interfaces mediating the performer's actions and the hidden processes embedded in electronic or digital mediums:

- The response of the computer must be proportionate to the performer's action.

- The response must share some energetic and morphological characteristics with the performer action.

- The onset of the response must be synchronous with the performer's action

- There must be a timbral continuum, affinity, or fusion between the untreated instrumental sound and the response of the electronics.

- The relationship between the performer and the computer must be stable.

- The relationship must be scrutable.

- The relationship must be learnable by the performer.

- The mapping must be sufficiently fine-grained.

Croft's conditions resonate with the ideas of Burrows, Auslander and Alperson. In addition, he finds instrumentality to be threatened by the disembodied sound coming out of a loudspeaker rather than the instrument itself. He draws attention to the aspect of 'liveness' in live performance-more specifically live electronics. Croft justifies the

\footnotetext{
${ }^{90}$ Philip Alperson, 'The Instrumentality of Music', Journal of Aesthetics and Art Criticism, 66.1 (2008), 37-51 (p. 41).

${ }^{91}$ Croft, p. 64.
} 
proposed conditions with the link between the performer's actions and the computer's response-although this could be the case with any instrument that does not necessarily rely on a computer - which would strengthen instrumentality and its 'resonance with a wider human experience'.

\subsection{The background of the medium as a creative exploration}

While this thesis presents the first attempt to consolidate the medium as an essential component of the musical work, especially considering new creative practices-building instruments and software - the genesis of this idea comes from recognising a long history of creative minds exploring and building new mediums. The creation and development of musical instruments is a large field of research that extends beyond the scope of this dissertation. However, it is worth discussing the prior state of the art in the augmented instruments field, as well as mentioning projects that have influenced the development of the HypeSax

Many kinds of electronically augmented instruments - also described as meta or hyper instruments - have been designed for many years. Tod Machover, led the first major research on this topic when the digital era became accessible for artists. His work began at IRCAM with a research on 'the adaptation of computer to the needs of sophisticated real-time musical performance' (1978) as well as 'investigating the technical and scientific problems of collecting, analysing, and interpreting musical data' after the establishment of the MIDI standards (early 1980s). ${ }^{93}$ Machover's work continued over the following years at MIT, leading to the creation of systems named hyperinstruments, 'a combination of machine-augmented instrumental technique, knowledge-based performance monitoring, and intelligent structure music generation.' ${ }^{94}$

A new field of research was created with the work of Machover and the Hypersintrument Group at the MIT Media Lab. This filed encompasses any research involving the use of acoustic instruments - or any of their parts - in conjunction with new technologies, aiming to expand the performative and sounding affordances that traditional

\footnotetext{
93 Tod Machover, Hyperinstruments. A Progress Report 1987-1991 (Cambridge, 1992).

94 Tod Machover, 'The Extended Orchestra', in PEYSER, J. (Ed.), The Orchestra: Origins and Transformations. (New York, NY: Billboard Books, 1986).
} 
instruments provide. Diana Young, for instance, developed the Hyperbow, an electronically augmented bow capable of collecting data of the bowing technique. This data was then mapped to control various parameters needed to process audio (see Fig. 8). 1

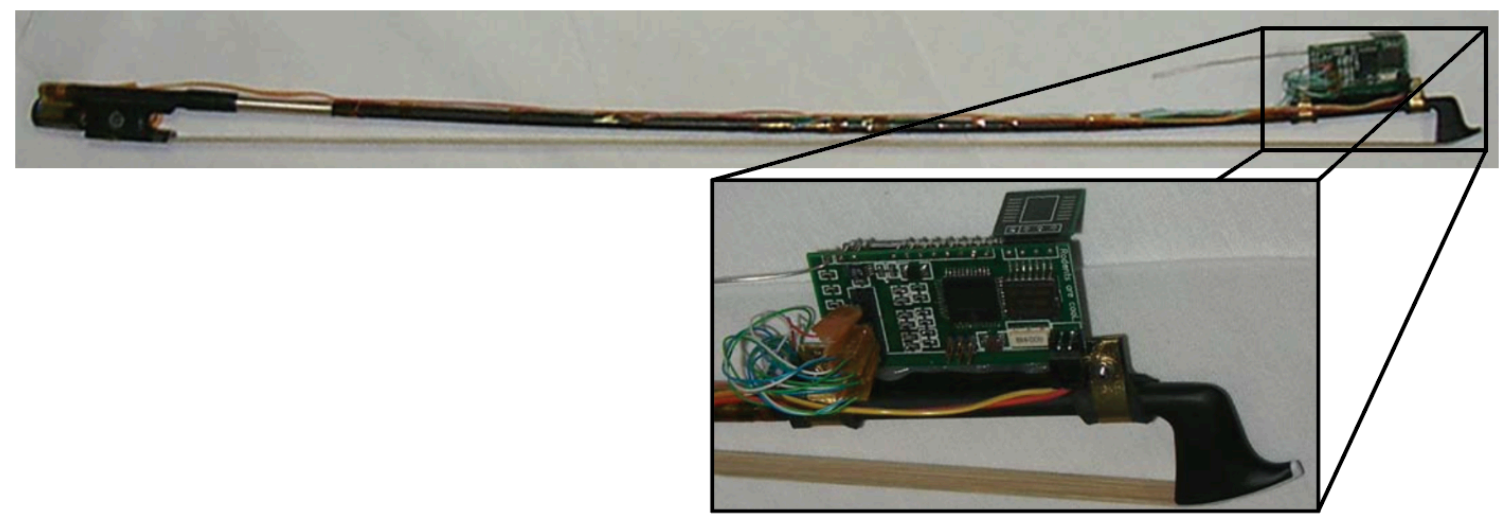

Fig. 8. Young’s Hyperbow.

The Hyperbow is just one of the first examples of instrument augmentation which had a great influence in other research such as the R-Bow, designed by Dan Trueman and Perry Cook, developed as in the scope of a research in which the traditional concept of the violin was reimagined (see Fig. 9). Trueman and Cook's project also delved in developing a novel approach for sound emission with the invention of the BoSSA (discussed in section 5.1.1). ${ }^{95}$

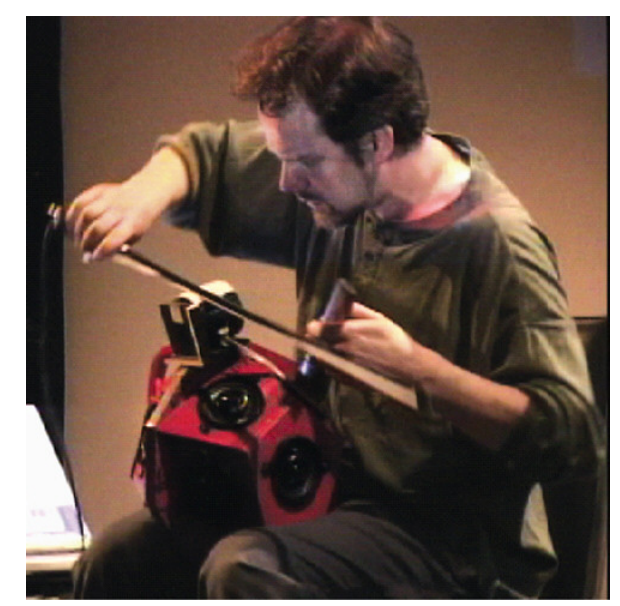

Fig. 9. Dan Trueman using the R-Bow to play the BoSSA.

95 Perry Cook and Dan Trueman, 'BoSSA: The Deconstructed Violin Reconstructed', Journal of New Music Research, 29.2 (2000), 121-30. 
Out of the Hyperinstrument Research Group at MIT, other seminal instruments and approaches inspired future generations. Hand tracking devices, for instance, were developed for Machover's Bug-Mudra in order to track performing gestures-similar to those found in conducting practices - to shape sound. This approach was necessary as instruments developed at the moment did not allow for continuous tracking of motion. This need put in a new perspective the importance of embodiment in performance, as well as the possible avenues for mapping gestures into parameter control.

Following the development of these early instruments in the $1980 \mathrm{~s}$, the field of research took off and slowly grew along with the development of new powerful and cheaper technologies that appeared in the following decades. Slowly, a community of researchers and enthusiast was formed. The New Interfaces for Musical Expressions Conference (NIME) is a proof of the rich life of the field. The proceedings of this annual conference are shared globally through its digital archive. ${ }^{98}$ This repository is one of the most important resources for a field that is alive and blossoming. ${ }^{99}$

Many composers have pushed the boundaries of composition by exploring the usage of 'new technologies.' Beethoven, for instance, dedicated enough of his time to explore the recently created pianoforte. His exploration of the instrument, as well as his opinions on the development were greatly influential in the work of the pianoforte builders of the time, pushing the development of the modern piano. Composers can push technology, and in the same way, technology can take music in unexpected directions. The invention of the valve systems for brass instruments and the implementation of the Boehm system on woodwinds, for instance, changed the sound of the orchestra.

In the case of the development of the HypeSax, some previous projects influenced the approach to measure the air pressure in woodwind and brass instruments while dealing with air disturbance, audio emission source, interaction with keys, non-sounding motion detection (also known as ancillary motion) and aspects of construction.

\footnotetext{
98 'NIME | Archive of NIME Proceedings' <http://www.nime.org/archives/> [accessed 29 October 2017].

${ }^{99}$ A NIME Reader. Fifteen Years of New Interfaces for Musical Expression, ed. by Alexander Refsum Jensenius and Michael J. Lyons (Cham: Springer International Publishing, 2017), p. ix.
} 


\subsubsection{Air pressure measurement}

A fundamental interaction with wind instruments consists in introducing a stream of air into the instrument's body. Measuring the air pressure without disturbing the air flow has proven to be very difficult, as any disruption can have negative effects on the sound. Several approaches have been used by researchers such as:

- Yoichi Nagashima and Tanami Tono Ito, who developed an augmented shō using an air pressure sensor placed in a tube that replaced one of the original decorative bamboos of the instrument. ${ }^{101}$

- Andrey da Silva's investigation of the use of flute air jet as a musical control variable, placing air pressure sensors in front of the flute's embouchure. ${ }^{102}$

- Francisco García's study of blowing pressure profiles in recorders demonstrated that the modification of the mouthpiece of a wind instrument can also help in measuring air pressure.

These projects were also influenced by previous controllers-such as Damien Pusset's MIDI flute developed at IRCAM, Bleauregard by Beauregard, ${ }^{103}$ or Perry Cook's HIRN wind instrument controller ${ }^{104}$ (see Fig. 10) — that, even though did not include a traditional acoustic instruments into their designs, set the basis for the development of air pressure measurement. Yamaha Corporation patented hybrid wind instrument designs, by Naoyuki Onozawa and collaborators, ${ }^{105}$ also influenced the approaches taken in the design of the Hypesax.

${ }^{101}$ Eduardo Reck Miranda and Marcelo M. Wanderley, New Digital Musical Instruments: Control and Interaction beyond the Keyboard, The Computer Music and Digital Audio Series, v. 21 (Middleton, Wis: A-R Editions, 2006), p. 23.

${ }^{102}$ Andrey R. Da Silva, Marcelo M. Wanderley, and Gary Scavone, 'On the Use of Flute Air Jet as a Musical Control Variable', in Proceedings of the 2005 Conference on New Interfaces for Musical Expression (National University of Singapore, 2005), pp. 105-8.

${ }^{103}$ Gerald Beauregard, 'Rethinking the Design of Wind Controllers' (unpublished MA Thesis, Dartmouth College, 1991).

104 Perry R. Cook, ‘A Meta-Wind-Instrument Physical Model, and a Meta-Controller for Real Time Performance Control', in 1992 International Computer Music Conference (presented at the Computer Music Conference, San Jose, California: Computer Music Association).

${ }^{105}$ N. Onozawa and K. Fujita, 'Hybrid Wind Instrument Selectively Producing Acoustic Tones and Electric Tones and Electronic System Used Therein', 2006 $<$ https://www.google.com/patents/US7049503>. 

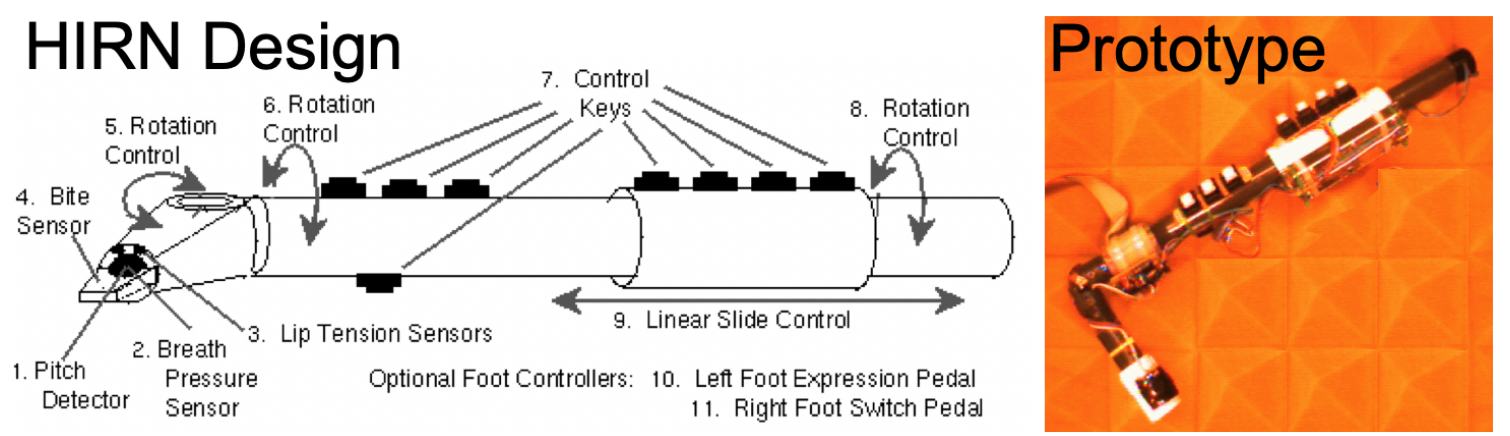

Fig. 10. HIRN wind controller designed by Perry Cook. ${ }^{104}$

\subsubsection{Audio emission source}

The fluctuation of air and the modification of the air column is what produces a pitched sound. This sound irradiates outwards from the wind instrument's bodies. This is the paradigm of traditional acoustic instruments. Nevertheless, with the invention of electronic instruments, as well as amplification through loudspeakers, an alternative to the model of natural sound emission coming out of the instrument appeared. Loudspeakers provide an extension of the sound emission source while potentialising its capabilities. At the same time, this invention also presents new avenues for reflection on how the instrument is perceived.

With the emergence of electronically mediated music, new possibilities of emission and transformation of sound arose. A traditional instrument-while it can have multiple oscillators, for instance multiple strings - produces sound that is emitted from one single source: the instrument itself. Meanwhile, with the use of amplification via loudspeakers (for example an electric guitar and amplifier), the sound can be emitted through multiple sources, depending of the sound setup, going from the instrument plus one channel (loudspeaker) to multichannel arrays, which allow for the instrument to expand its presence in space.

Creative approaches to sound radiation and the effects of the manipulation of the source have been explored in works such as Rain Forrest by David Tudor, where a prerecorded soundscape is reproduced through what he called 'instrumental speakers', a set 
of objects that - through the use of mounted transductors-acted as loudspeakers. ${ }^{106}$ Similarly, Alvin Lucier created works which challenged the traditional model of sound radiation. Lucier's Music on a Long Thin Wire, ${ }^{107}$ allows for acoustic phenomena of feedback to become a fundamental element of his work, while on I'm Sitting in a Room, ${ }^{108}$ Lucier explores the effect of sound degradation as the quality of the resonance of the room take over. Equally important is the work of John Driscoll which, in multiple compositions, focus on the resonance in sculptural materials, architectural resonance, and feedback. ${ }^{109}$ These kind of approaches set a paradigm in sound source expansion and the implication of different audio setups, which is further discussed in section 5.1.1.

106 John Driscoll and Matt Rogalsky, 'David Tudor's Rainforest : An Evolving Exploration of Resonance', Leonardo Music Journal, 14 (2004), 25-30.

${ }^{107}$ Alvin Lucier and Douglas Simon, Chambers: Scores by Alvin Lucier (Middeltown, CT: Wesleyan University Press, 1980), p. 160.

${ }^{108}$ Lucier and Simon, p. 30.

109 John Driscoll, 'Resonance: From the Architectural to the Microscopic', Leonardo Music Journal, 22 (2012), 25-33. 
This content is unavailable due to copyright

(c) 1976 Ralph Jones Click here to visit source image

Fig. 11. Rainforest IV at L'espace Pierre Cardin in Paris, 1976.

New hybrid instruments also employ hybridity in sound emission. By mounting transducers and loudspeakers directly on the acoustic instruments, it is possible to combine acoustically and electronically produced sound. Examples of similar setups are further discussed in section 5.1.

\subsubsection{Interaction with keys}

Another important aspect of interaction with musical instrument is the mechanical process through which pitch is modified. In the case of wind instruments, being the air stream the main variable for pitch control (in combination with the oscillator that modulates the air column such as lips or reeds), the mechanical process of elongating the pipes in which the air column vibrates is central. Various techniques to capture data on the mechanics of pitch control have been explored by authors such as: 
- Barry Verco and Lawrence Beauregard. They developed a MIDI flute in the early 1980's at IRCAM, using optical sensors on the keys. ${ }^{110}$

- As a follow up to Verco and Beauregard, Michael Ducoureau, Michael Starkier and Beauregard developed a final version of the MIDI flute featuring Hall effect sensors placed on rings around the keypads. ${ }^{111}$

- Following these approaches, over the following decades, multiple projects implemented similar techniques. Notable examples are: Sølvi Ystad and Thierry Voinier' LMA flute ${ }^{112}$, Cléo Placio-Quintin's hyper-flute, ${ }^{113}$ the SABRe designed by a team led by Matthias Müller, ${ }^{114}$ or Sarah Reid's augmentation of the trumpet using the MIGSI ${ }^{115}$ (built in the tradition of Cook/Morril). ${ }^{116}$

- Other approaches using force sensor resistors include the works of Matthew Burtner (Metasaxophone, see Fig. 12), ${ }^{117}$ Henrique Portovedo (augmented saxophone), ${ }^{118}$ or Euyshick Hong et al. (hybrid saxophone). ${ }^{119}$

The HypeSax, inspired by these projects, features capacitive touch sensors, which have not been used previously in augmented woodwind instruments. A further discussion is found in section 3.5 .

${ }^{110}$ Barry Vercoe, 'The Synthetic Performer in The Context of Live Performance', in ICMC ' 84 Proceedings, 1984.

${ }^{111}$ Miranda and Wanderley, p. 45.

${ }^{112}$ Ystad Sølvi and Vionier Thierry, 'Design of a Flute Interface to Control Synthesis Models', in ICMC '99 Proceedings (presented at the ICMC 1999, Beijing, 1999).

${ }^{113}$ Cléo Palacio-Quintin, 'The Hyper-Flute', in Proceedings of the 2003 Conference on New Interfaces for Musical Expression (Montreal, 2003), pp. 206-7.

${ }^{114}$ Sébastien Schiesser and Jan C. Schacher, 'SABRe: The Augmented Bass Clarinet.', in NIME, 2012.

${ }^{115}$ Sarah Reid and others, 'Minimally Invasive Gesture Sensing Interface (MIGSI) for Trumpet', in Proceedings of the International Conference on New Interfaces for Musical Expression, 2220-4806 (Brisbane, Australia: Queensland Conservatorium Griffith University, 2016), XVI, 419-24.

${ }^{116}$ Perry R. Cook, 'Principles for Designing Computer Music Controllers', in Proceedings of the CHI'01 Workshop on the New Interfaces for Musical Expression (Seattle, 2001), pp. 3-6.

${ }^{117}$ Matthew Burtner, 'The Metasaxophone: Concept, Implementation, and Mapping Strategies for a New Computer Music Instrument', Organised Sound, 7.02 (2002), 201-13.

${ }^{118}$ Henrique Portovedo, Paulo Ferreira Lopes, and Ricardo Mendes, 'Saxophone Augmentation: An Hybrid Augment System of Gestural Symbiosis', 4.

${ }^{119}$ Euyshick Hong, Guanyu Zhu, and Wendel Moreira Duarte, 'Dr. Saxophone: Hybrid Saxophone Interface', 2016 3rd International Conference on Systems and Informatics (ICSAI), 2016, 1149-53. 


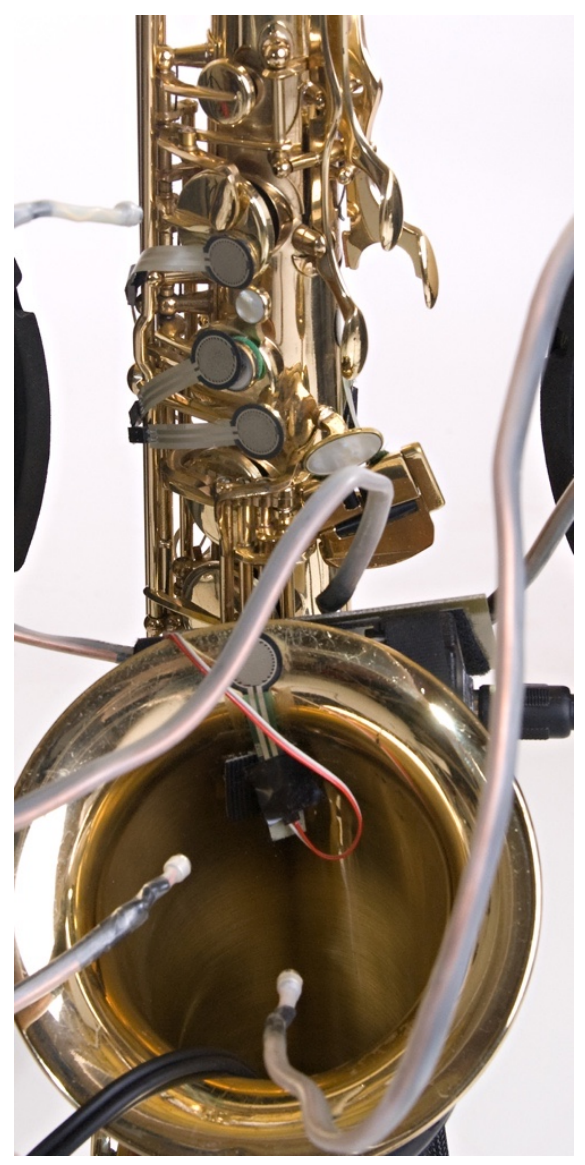

Fig. 12. Matthew Burtner's Metasaxophone featuring force sensitive sensors. ${ }^{117}$

\subsubsection{Non-sounding motion detection}

Motion is the basis of interaction between the performer and the instruments. ${ }^{120}$ Nevertheless, multiple actions that do not have a direct influence in sound production accompany every performance. These motions, known as ancillary motion, can become an important feature of the performance-which is an element considered equally important in the tripartite model proposed in this thesis-due to embodiment.

Multiple controllers, as well as augmented instruments, have taken advantage of ancillary motion as another way to control sound synthesis parameters. John Melo et al., for instance, make use of the motion sensors found in the Wiimote in order to communicate capture motion data using a Pure Data application named Gest-O. This data is used to

${ }^{120}$ Although there are some exemptions to this paradigm such as Alvin Lucier's Music for Solo Performer where wave brain activity is amplified in order to interact with percussion instruments. 
control parameters of sound synthesis. The Wiimote's electronic plaque (including all sensors) is placed over instruments such as saxophones and trumpets. This way, as the performer plays the instrument in a traditional fashion, the ancillary motion is used to control synthetic sound production. ${ }^{121}$

Multiple techniques have been employed to capture non-sounding motion. These include the use of tilt sensors, ${ }^{122} 123$ camera-based systems, ${ }^{124}{ }^{125}$ gyroscopes, ${ }^{126}{ }^{127}$ and many other sensors according to the particular modes of performance of each instrument.

\subsubsection{Hyperinstrument construction}

Most of the projects that influenced electronic the design of the HypeSax, as well as the techniques for data collection, were developed in an academic context. However, when it comes to the construction of the housing of the sensors, DIY approaches were highly influential.

In recent years, 3D printing technology has allowed new developments in design throughout a wide spectrum of fields. In the field of music and acoustics, this new technology has also opened new possibilities allowing for accessories, instruments, resonators, replacement parts, protective cases and other related items to be developed. Perhaps the importance of 3D printing technologies for musical research lies on the fact that these new printers can produce prototypes and final products with a relative ease of construction, unlike working in the old-fashioned way by handcrafting the instruments or parts out of natural materials (typically wood). It is well known that luthiers, woodwind

${ }^{121}$ Jonh Melo, Daniel Gómez, and Miguel Vargas, 'Gest-O: Performer Gestures Used to Expand the Sounds of the Saxophone', in Proceedings of the International Conference on New Interfaces for Musical Expression (Ann Arbor, Michigan: University of Michigan, 2012).

122 Palacio-Quintin.

${ }^{123}$ Sergi Jordà, 'Afasia: The Ultimate Homeric One-Man-Multimedia-Band', 7.

${ }^{124}$ H. Morita, S. Hashimoto, and S. Ohteru, 'A Computer Music System That Follows a Human Conductor', Computer, 24.7 (1991), 44-53.

${ }^{125}$ Graziano Bertini and Paolo Carosi, 'Light Baton: A System for Conducting Computer Music Performance', in Proceedings of ICMC 1992 (presented at the ICMC, San Francisco, 1992).

${ }^{126}$ T. Kanamori and others, 'Gesture Sensor in Virtual Performer', in Proceedings of ICMC 1993 (presented at the ICMC, San Francisco, 1993).

127 Joseph A Paradiso, 'The Brain Opera Technology: New Instruments and Gestural Sensors for Musical Interaction and Performance', 31. 
and brass instruments makers, as well as other professionals in similar fields, learn their craft over many years, and a musician or researcher is usually not skilled to build musical instruments, repair them, build new parts or developments, therefore slowing down any projects in this field. Not only are the new 3D printers allowing more professionals to access easy and fast prototypes, but their low price has opened the doors for the DIY amateur enthusiast.

There are documented projects of printed models that have furthered the developments of musical instruments and acoustic investigation. The authors catalogue these $3 \mathrm{D}$ printed models in six categories: protective accessories (shells, cases, pads, caps, etc), repairments (knobs, string pegs, bridges and other small part replacements), enhancements (pickup holders, shoulder rests, inserts, etc.), replicas, customizations and innovative designs. Many of these models, mostly from DIY projects, are shared as creative commons in specialized webpages such as thingiverse.com, yeggi.com, grabcad.com and many others. Other scholarly projects focus on researching musical and acoustic issues rather than practical ones, such as producing microtonal flutes ${ }^{128}$ or a multi-sized shank in a trumpet mouthpiece for acoustic inquiry. ${ }^{129}$ The HypeSax design and construction take advantage of the $3 \mathrm{D}$ printing technologies with an approach that fits in the category of customizations, enhancements and innovative designs. The influences of 3D print technologies in the design and construction of the HypeSax are thoroughly discussed in chapter 3 .

There are many issues and questions that had been addressed and even overcome with the development of technology. Nevertheless, new approaches on thinking about the interaction between instrumentalists and instruments, composers and instruments, and even audience as participants can be explored. New technologies, sensors, communication protocols, actuators, 3D printing and other techniques and materials used for building instruments are arising every day making us ask ourselves in which new ways we can

${ }^{128}$ Matthew Dabin and others, '3D Modelling and Printing of Microtonal Flutes', in Proceedings of the International Conference on New Interfaces for Musical Expression, 2220-4806 (Brisbane, Australia: Queensland Conservatorium Griffith University, 2016), XVI, 286-90.

${ }^{129}$ Sasha Leitman and John Granzow, 'Music Maker: 3D Printing and Acoustics Curriculum', in Proceedings of New Interfaces for Musical Expression 2016 (presented at the NIME 2016, Brisbane, Australia, 2016). 
develop our instruments, create new instruments and combine old instruments with new technologies, but most importantly which new directions music could take. Reflections on this, as well as discussions on how the current state of the art has influenced the design of the HypeSax continue in the following chapters as elements hardware and software design are presented and compared to previously known projects.

In the following chapters, I describe my own approach to the medium, putting into consideration the ideas discussed in this chapter. This is a case study that explores the potential of 'composing' the medium as part of the compositional practice-which has become common for composers who develop software, mechatronics, sound artists, amongst others - in the context of the tripartite model. 


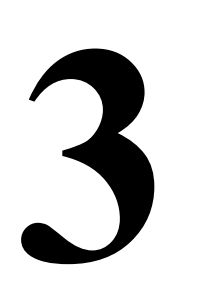

\section{THE HYPESAX AS THE COMPOSED MEDIUM OF THE WORK -A CASE STUDY}

As described in the previous chapters, I am seeking to rethink the compositional paradigm by adopting the proposed score-performance-medium tripartite model (see 2.4). In this instance, I developed a hyperinstrument, the 'HypeSax', as a case study to research the creative aspect of shaping the medium and investigate the potential practical problems and solutions found in thinking about the medium as a part of the work.

In its current state, the HypeSax features modular system that includes an audio system (a sound card, microphone, and speaker), motion sensors, touch sensors, a custom mouthpiece able to collect data of air pressure during performance, and USB capabilities that allow it to communicate with other devices. The different modules-described and discussed later in this chapter-are the Un-Mute, Sensor-Link, Keycaps and buttons, and a custom mouthpiece. These modules can be attached to commercial saxophones, providing them with electronic capabilities. The HypeSax, working along with computer software, offers the possibility of processing and synthesising sound, as well as communicate with third party devices and software which can, in turn, interact with the data obtained from the HypeSax sensors during performance. 
Currently, the HypeSax does not have an embedded audio signal processing software - a characteristic that will be implemented in the future. All of the signal processing happens remotely through the use of a USB port which sends sensor data and audio. Once audio is processed externally it is imput back into the HypeSax system via the same USB connection. This is one of the main characteristics of the instrument, since the inclusion of an onboard audio system offers the possibility to feed synthesised audio directly into the body of the instrument. The aim of this system is to couple acoustic sound produced by the saxophone with synthesised sound produced artificially by the HypeSax working together with specialised software and/or other devices (this is further discussed in section 3.7, and chapter 5). Images and videos found at www.hypesax.com show the instrument and some of its capabilities.

I chose to work with the saxophone because it is an instrument that shares a high degree of connection with the body: the performer plays the instrument with both hands; many facial muscles are required to control embouchure; breathing and lung capacity are involved are involved in performing with the saxophone; and it can be carried around while playing. Not only does the instrument provide many opportunities to explore embodiment, but it also produces sound by shaping an air column, not by vibrating its body, which makes it a very interesting case when it comes to discuss the relationship between physical medium and sound. I also considered the potential opportunities to collaborate with other musicians in this project. As a foreigner in New Zealand, with no previous connections with the local community, I was relying on my wife's connection to the New Zealand music scene. She, being a saxophonist that had previously lived in Wellington, was my connection to a community of local saxophonists. I also considered the opportunities for further ways to augment woodwind instruments past this research.

Developing woodwind hyperinstruments is fertile field, as not too many developments have been produced. While there are some commercial woodwind electronic instruments, none of them offer acoustic capabilities. The only exceptions are the latest version of the 
SABRe Multi Sensor ${ }^{130}$ and the Varitone. ${ }^{131}$ Both are non-destructive devices that can be attached to the saxophone in order to augment its capabilities. These devices, however, do not offer some of the characteristics that I was hoping to explore: the SABRe does not offer acoustic integration with the instrument; the Varitone does not offer the possibility of embodiment. Other devices, such as Matthew Burtner's Metasax, ${ }^{132}$ have been built but are not available in the market.

The HypeSax is a device designed to be attached to a saxophone in order to bestow the instrument with digital capabilities. Its design aims to make it feel natural and wellintegrated with the design and acoustics of the saxophone. In order to achieve this goal, the design of the HypeSax takes into account the main aspects of the interaction of the performer with the instrument for sound production and expressivity. In this interaction, parts of the performer's body are used in different ways. Some parts play a bigger role in the production of sound, while others are more important for communicating performative aspects and intention. While the design of the HypeSax considers some of the key aspects of such use of the performer's body, it should be noted that this project does not intend to focus on the body of the performer alone, but on its interaction with the saxophone.

This chapter includes a thorough description of the HypeSax, with a full description of the device and the process of its design and development. First, I discuss the design criteria which is concern with fulfilling three main goals: musical goals, interactive goals, and operational goals. Then, I present three sub-sections detailing these three general goals. Finally, the HypeSax is described through a series of sections describing its components, arguing how they relate to the relate to the design goals.

${ }^{130}$ Originally developed for a bass clarinet in a research project at the Institute for Computer Music and Sound Technology (ICST), the newer version of this instrument consists of a module that can be attached to a saxophone of clarinet neck. It collects ancillary movement data which can be repurposed for musical performance.

'SABRE | SABRE Multisensor', SABRe < https://www.sabre-mt.com/sabre-multisensor $>$ [accessed 9 January 2019].

${ }^{131}$ The Varitone is an 'effect box' for the saxophone. It was developed and sold by Selmer in the late 19070s. It is not currently available for purchase.

'Selmer VARITONE - Sax On, El Mundo Del Saxo'<https://sax-on.com/selmer-varitone/> [accessed 13 August 2019].

132 Burtner, 'The Metasaxophone'. 
More details on how this device addresses the issues of embodiment and its use in the creative portfolio that accompanies this dissertation are found in chapters 5 and 6.

\subsection{HypeSax design criteria}

In order to develop the HypeSax as an example of the medium in the proposed workconcept, a set of criteria was established with the purpose of developing a device that would expand a traditional saxophone, converting it into a hybrid instrument that keeps its acoustic and performative capabilities and, at the same time, can work as an electronic instrument. The design criteria were established based on previous experiences developing electronic instruments and software. I was looking to create a connection between the acoustic instrument and the electronic components, so I considered three main aspects for the design: performing with the instrument required a degree of physical activity similar to playing an acoustic instrument; that it could be removable and non-destructive; and that it could be easily re-configured for new compositions. While there are many aspects that could have been considered to establish the designed, these aspects provide a good starting point to seek answers to the questions.

The criteria for the HypeSax design are categorised into three groups:

- Musical goals (those that motivate this development) that focus on the sounding result and its integration in the context of the musical work,

- Interactive goals, which refer to the way in which the performer interacts with the new sounding possibilities of the saxophone through the use of the HypeSax, and

- Operational goals, those that relate to the physical design, construction, and assemblage of the HypeSax with the saxophone.

Although these goals have been separated into different categories, all of them are intimately related, and can be analysed from musical, interactive and operational points of view. The decision to group them in these categories, however, responds to a view of the hierarchical process in which the three elements of the proposed tripartite structure have an effect on the work. The score (the voice of the composer) is the foundation and initial instigator of the work. The performance then acts as a moulding force that reshapes the work. And finally, the medium is the filter that responds to the actions of the performer 
allowing for the work to exist in the world, out of the imaginary of the composer. This response of the medium is a reaction: it pushes back but not necessarily against the performer. This reaction is due to the qualities of the medium: the weight of the string, its tension, the amount of rosin used, or any other particular feature.

The design criteria observe the aforementioned three components, and thus have a similarity to the tripartite model. The musical goals correspond to the score, while the interactive goals are more easily understandable from the point-of-view of the performance, and the operational ones are analogous to the medium (see Fig. 13).

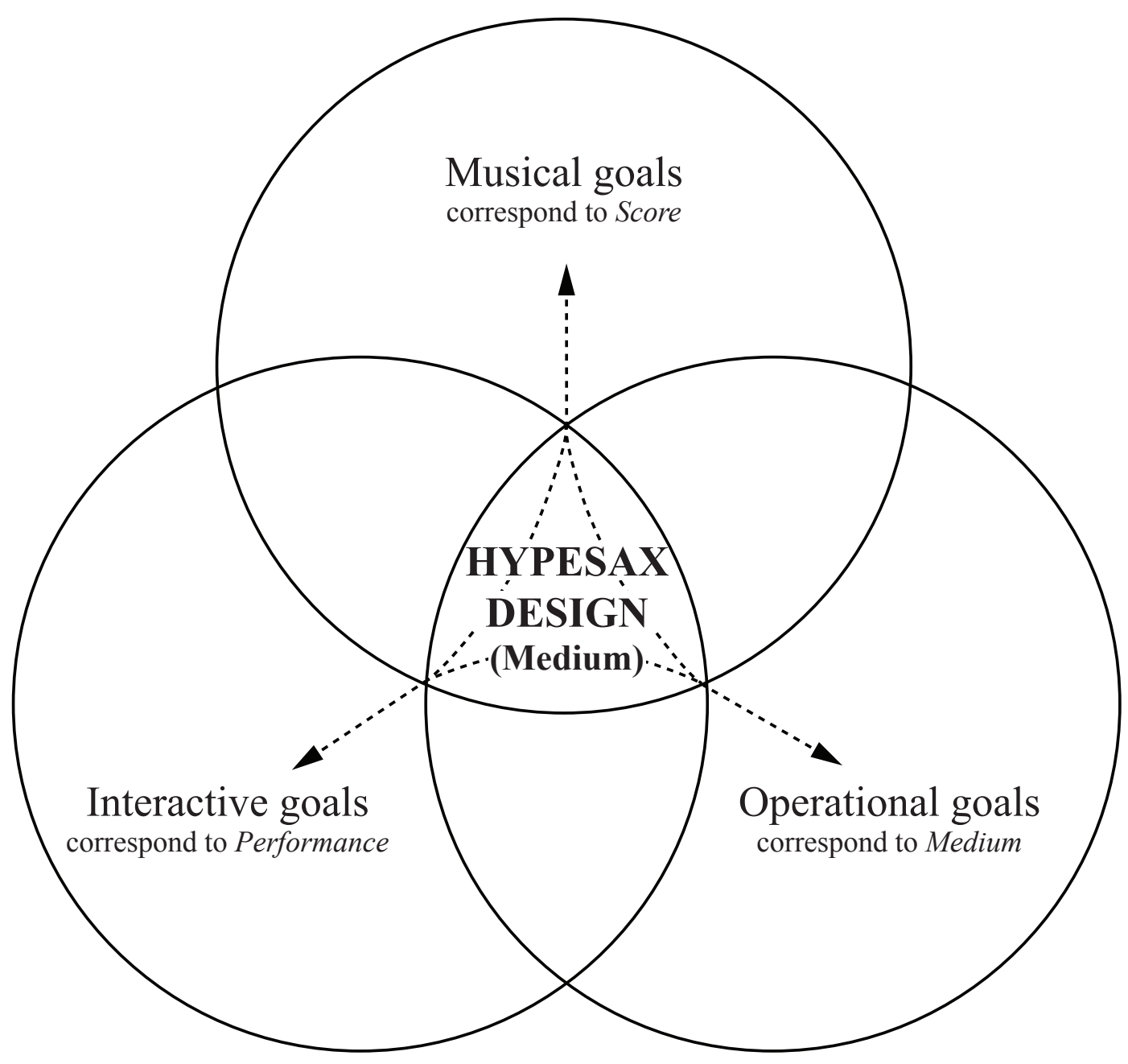

Fig. 13. Design criteria follow the tripartite model.

These three categories further subdivide to focus on more specific goals in the following way: 


\section{- Musical goals}

- Allow performative nuances.

- Offer variety in the relation performer-instrument-sound while maintaining instrumentality.

- Provide the possibility of performing gestural-instrumental techniques.

\section{- Interactive goals}

- Afford maximum possible control of electronic sound while maintaining a natural performative practice.

- Retain the dominance of the performative practices.

- Achieve hybridisation of acoustic and electronic sound.

- Provide virtual physicality.

- Offer performance perfectibility.

\section{- Operational goals}

- Produce a design that fits or that can be easily modified to fit any model or make of the instrument.

- Offer optional modularity.

- Provide full compatibility with commercial or custom-made software.

While the operational goals are described in depth in this chapter, the interactive aspects are further examined and exemplified in Chapter 4, while musical ones are discussed in chapters 5 and 6 .

\subsubsection{Musical goals}

As discussed in section 1.2, to realise the music that composers imagine it can sometimes require more capabilities than those offered by traditional instrumental performance. Creative solutions have been devised in order to approximate the musical idea to its materialization, such as skilled use of orchestrations, special techniques or unusual instrument adaptations. A notable case is the development of extended techniques on the piano during the twentieth century, with cases such as John Cage's prepared piano Sonatas 
and Interludes ${ }^{133}$ or George Crumb's piano amplification to bring out the delicate sonorities of the piano in his Makrokosmos series. ${ }^{134}$ This is not a new practice, and we can find cases where the composers asked for 'unusual' techniques in order to portray a specific musical result, such as in the use of the col legno technique in the seventeenth century in works by Hume (1605), Farina (1627) and Biber (1673), ${ }^{135}$ which became more popular after Berlioz's use of this technique, alluding to fantastical images in his Symphonie Fantastique of 1830. The threshold between what is conventional and the aforementioned creative ways of performing is hard to define, it sometimes is blurred and even erased by time. The snap or fingernail pizzicato, also known as Bartók pizzicato by its association with Bartók’s string quartets, was once considered an unusual technique, whereas today when it is commonly found in new compositions.

In the last century, besides the extensive exploration in instrumental techniques, new technologies allowed composers to explore novel ways of integrating the manipulation of sound as part of their compositional strategies. As described in section 2.6.4, the use of electronic sound production and digital sound processing became standard techniques in the palette of modern composers. At the same time, issues of embodiment had to be considered from new perspectives, as the relationship between sound and the actions that produce sound reached different horizons with the use of electronic and digital technologies. However, this fresh relationship also inspired new ways of understanding instrumentality. In the words of Matthew Burtner in his article 'Making Noise: Extended Techniques After Experimentalism':

With the integration of [new] technology into instrumental performance, a very interesting switch gradually took place in orchestration. In the context of synthesized sounds, extended techniques are useful for blending acoustic and electro-acoustic media. The two are idiomatically integrated through the implementation of extended techniques. In the early years, composers utilized

\footnotetext{
${ }^{133}$ John Cage, Sonatas and Interludes (Edition Peters, 1960).

${ }^{134}$ George Crumb, Makrokosmos Volume I (C. F. Peters, 1972); George Crumb, Makrokosmos Volume II (C. F. Peters, 1973); George Crumb, Makrokosmos Volume III 'Music for a Summer Evening' (C. F. Peters, 1974); George Crumb, Makrokosmos Volume IV 'Celestial Mechanics’ (C. F. Peters, 1979).

135 David D. Boyden, Col Legno (Oxford University Press, 2001), I.
} 
extended techniques to expand the pallet of sounds beyond the confines of traditional modes. But in a world of electro-acoustic screeching, beeping, glitches, blips, and drones, instrumental extended techniques take on new meaning. They are employed precisely because they are instrumental. In fact . . . they capture the essence of that particular instrument, drawing out its unique timbre, its grain. These techniques are no longer an "other," disassociated from the instrument as some noise invasion. They are rather part of the sonic context of the instrument. ${ }^{136}$

Agreeing with Burtner's words, I can say that instrumental extended techniques have been successful in part because of their instrumentality (see section 2.6.4) and the potential affordances and grain they provide.

The affordances, following Leman's description in Embodied Music Cognition, are action-relevant values that arise from the perception of an object that draw upon an actionoriented bias. ${ }^{137}$ In other words, affordances are the properties of an object that suggest a way of interaction with that object: a string instrument suggests that it should be played by interacting with the strings. Beyond the realm of that suggestion, through the use of extended techniques, a performer could choose to make sound by banging on another part of the instrument.

The grain, analysed in 2.3.4, is perhaps one of the most important elements when it comes to realising a musical work, as it provides a strong sense of connection between the score, performer and audience. As Roland Barthes defines it in his essay 'The Grain of the Voice':

The "grain" is the body in the voice as it sings, the hand as it writes, the limb as it performs. If I perceive the "grain" in a piece of music and accord this "grain" a theoretical value... I inevitably set up a new scheme of evaluation which will certainly be individual - I am determined to listen to my relation

\footnotetext{
${ }^{136}$ Matthew Burtner, 'Making Noise: Extended Techniques after Experimentalism', NewMusicBox, 2005 $<$ https://nmbx.newmusicusa.org/making-noise-extended-techniques-after-experimentalism/> [accessed 26 November 2018].

${ }^{137}$ Leman, Embodied Music Cognition and Mediation Technology, p. 52.
} 
with the body of the man or woman singing or playing and that relation is erotic - but in no way "subjective". ${ }^{138}$

In other words, the grain provides an effective way for the music to become a powerful meeting point between composer and audience through the performance and the relation between the performer and the medium (see sections 2.6.3 and 2.6.4). I suggest, then, that even when the grain seems to be found mostly in the realisation of the work, it exists first as an intention in the composer's mind. In response to this, the design criteria of the HypeSax seeks to offer the possibility of providing the performer with a way of incorporating the grain into the electronic sound produced by the instrument. This, of course, becomes very difficult to pin down, as the very essence of the grain is the fact that it cannot be programmable or codifiable. However, actions that allow for the grain to exist can be considered in order to establish the musical goals of the design criteria:

1. Performative nuances. Music is full of contrasts in dynamics, pitch, timbre, rhythm, and envelopes. In order to allow the instrument to make music with these elements, it is important to provide the possibility of detecting nuances in the interaction with the instrument and scaling them to be mapped correctly with the musical intention.

2. Variety in the relation performer-instrument-sound while maintaining instrumentality. Based on Croft's analysis of the paradigms of this relationship, ${ }^{139}$ the design seeks to provide the different modes (backdrop, accompanimental, responsorial/proliferating, environmental, and instrumental) while preserving the conditions for instrumentality (see section 2.6.4).

3. Gestural-instrumental techniques. A set of instrumental techniques with their corresponding sounding consequences. These can be unique to the instrument or 'translated' from other instruments, ready to provide new expressive possibilities. (see section 5.2.3)

\footnotetext{
${ }^{138}$ Barthes, Image, Music, Text, p. 188.

${ }^{139}$ Croft, p. 62.
} 
A detailed description of how the musical goals of this design considerations influenced the final development is presented in Chapter 5, where I present an analysis of the portfolio developed for this project.

\subsubsection{Interactive goals}

The way in which the performer relates to the instrument is an important factor that determines many aspects of the work. Firstly, in the tradition of Western art music, composers tend to write music in a way that works idiomatically, that is, sympathetically with the instrument's design. This establishes a set of rules and limitations that the composer must follow. If composers were to ignore the instrument's design and its limitations, it would be very likely that the performability of the work could be compromised despite the efforts of the performer.

Secondly, the performer has an established a relationship with the instrument. After many years of practising, the human body adapts to the musical instrument in different ways, from developing a kind of extended proprioception to more bodily adaptations such as muscle development from carrying the instrument, an increase of lung capacity, the development of calluses on contact points, as well as other physical alterations. This pairing of bodies has an effect on the performer musical thought processes. Luc Nijs et al., in the paper 'The Musical Instrument as a Natural Extension of the Musician', state that:

The merging of musician and musical instrument implies that the musician no longer experiences a boundary between himself and the instrument. The instrument is felt from within and has become like an organic component of the body. ${ }^{141}$

Finally, the construction of the instrument itself and some of its characteristics, such as resonance, material response, and other variable components (strings, reeds, beaters, and others) determine certain aspects of its playability.

${ }^{141}$ Luc Nijs, Micheline Lesaffre, and Marc Leman, 'The Musical Instrument as a Natural Extension of the Musician', in Music and Its Instruments (presented at the The 5th Conference of Interdisciplinary Musicology, Paris: LAM-Institut jean Le Rond d'Alembert, 2013), pp. 132-33. 
To address these in the development of the HypeSax, the paradigms for the relation between performer, instrument, and electronic sound, described by Croft, as well as his proposed conditions for instrumentality (discussed in sections 2.6.3 and 2.6.4) were considered as the foundational reflections that influenced the following set of interactive goals of the design criteria:

1. Allow maximum possible control of electronic sound while maintaining a natural performative practice. It is of upmost importance to offer a non-obstructive design that allows for the use of regular instrumental techniques and permits the integration of new hybrid-instrumental techniques. Achieving this goal ensures that the integrity of the relationship that the saxophonist has developed with the saxophone through many years of practice.

2. Retain the dominance of the performative practices. While new affordances are provided, the original capabilities of the instrument should not be compromised.

3. Afford hybridisation. Integration of the electronics with the acoustics of the instrument allows for better conditions of instrumentality.

4. Provide virtual physicality. While the instrument itself is responding to physical actions through the use of sensors, the sounding consequences should imitate the natural response of an instrument, by providing resistance and virtual physicality.

5. Offer performance perfectibility. The design of the HypeSax also aims to avoid compromising the playability of the saxophone or creating discomfort for the performer, despite the new components grafted around the instrument's body. Nevertheless, the level of difficulty required to perform with this hyperinstrument beyond the possibilities offered by the standard saxophone implies a further level of skills in the process of hyper-specialisation, as described by Jonas Braasch in Hyper-specializing in Saxophone Using Acoustical Insight and Deep Listening Skills. ${ }^{142}$

${ }^{142}$ Jonas Braasch, Hyper-Specializing in Saxophone Using Acoustical Insight and Deep Listening Skills, Current Research in Systematic Musicology (Cham: Springer International Publishing, 2019), VI, p. 1 $<$ https://doi.org/10.1007/978-3-030-15046-4>. 
The consequences of establishing these design criteria are discussed and exemplified in chapters 4, 5, and 6 , where the results of an evaluation of the HypeSax are examined along with iterations of the instrument.

\subsubsection{Operational goals}

Previous hyperinstruments (or augmenting devices) have been developed with the goal of expanding the expressive capabilities of an instrument. Most of those designs belong to one or both of the following paradigms: 1) the hyperinstrument works as controller mounted on an instrument, meaning that there is no acoustical integration with its host, as the resonant body of the instrument is not involved in sound making process; 2) the device is built around the instrument, which means that it is unique to that instrument and nontransferable to a second instrument. The outcome of both of these paradigms is a disembodied instrumentality and/or a design that is difficult to duplicate and share with multiple performers.

In order to circumvent the previously described paradigms in hyperinstruments, the HypeSax has been designed with the following operational goals in mind:

1. A design that fits, or can be easily modified to fit, any model or make of the instrument. Not all instruments have the exact same design, shapes or measurements. In the case of saxophones, for instance, different models tend to be curved with different angles, or even be straight. Some models include extra keys that facilitate performance, and instrument designs change over time. At the same time, some models are reinforced with key and structural protections. All of these facts make the physical construction of different models and makes unique.

2. Modularity. This gives the flexibility of use of the sensors. It also allows for an easier and faster installation and replacement of components when necessary.

3. Full compatibility with commercial or custom-made software. Remaining open to be programmable removes creative barriers and allows users to rethink the way in which the HypeSax works in conjunction with personalised software.

Up until this point, the three groups of design considerations have been presented following the hierarchical order in which they are posited. Nevertheless, the actual process of development of the HypeSax followed an inverse hierarchy, as it is necessary first of 
all to build the instrument, followed by testing, and finally its use in a musical context. This implies that the operational goals had to be met first, followed by the interactive goals; only after that could the musical goals be achieved. For this reason, and in order to present a sequential narrative of the development, the next section discusses the details on how the operational goals were met. Then, Chapter 4 presents a user study and an iteration of the design, and Chapter 5 describes how the design influenced my creative process, continuing the analysis of how design criteria goals were met.

\subsection{System overview}

Because of the operational goals of design criteria, the HypeSax was designed to be an attachment that can be fitted on any ordinary alto saxophone. The system is modular and can work with some or all of its components attached to the saxophone. It consists of four components: a) The Un-mute, a brass-mute-like device (detailed in section 3.3); b) Sensorlink, designed to allow connections between the Un-mute and some of the HypeSax sensors, as well as with computer and MIDI devices (see section 3.4); c) triggers activated by touch sensors - mounted on the so-called keycaps - and buttons (detailed in section 3.5); d) and a customised mouthpiece (described in section 3.6) (see Fig. 14).

The system is capable of retrieving data using six touch sensors, placed on the saxophone to work as additional keys, and three push buttons used normally as triggers or switches. These touch sensors and buttons were chosen to comply with two of the design goals; avoiding any alterations to the traditional performative practices (see section 3.1.2) while allowing compatibility with different saxophone models (see section 3.1.3). The touch sensors are situated on top of some of the keys in order to be easily reached without changing the natural position of the hand, and due to the size of the keys, no more than six sensors can be used. In a similar way, there are only three buttons (that are activated using the thumbs) due to available space where they are located close to the thumb rests. Other sensors included in the system are a gyroscope and accelerometer inside the Un-mute, as well as an air pressure sensor mounted on the mouthpiece to monitor the actions of the performative practices. Also found inside the Un-mute are a microphone to capture and process audio, and a self-contained audio system (soundcard and speaker). The way in which all of these components work within the system is described in detail in the following sections. 


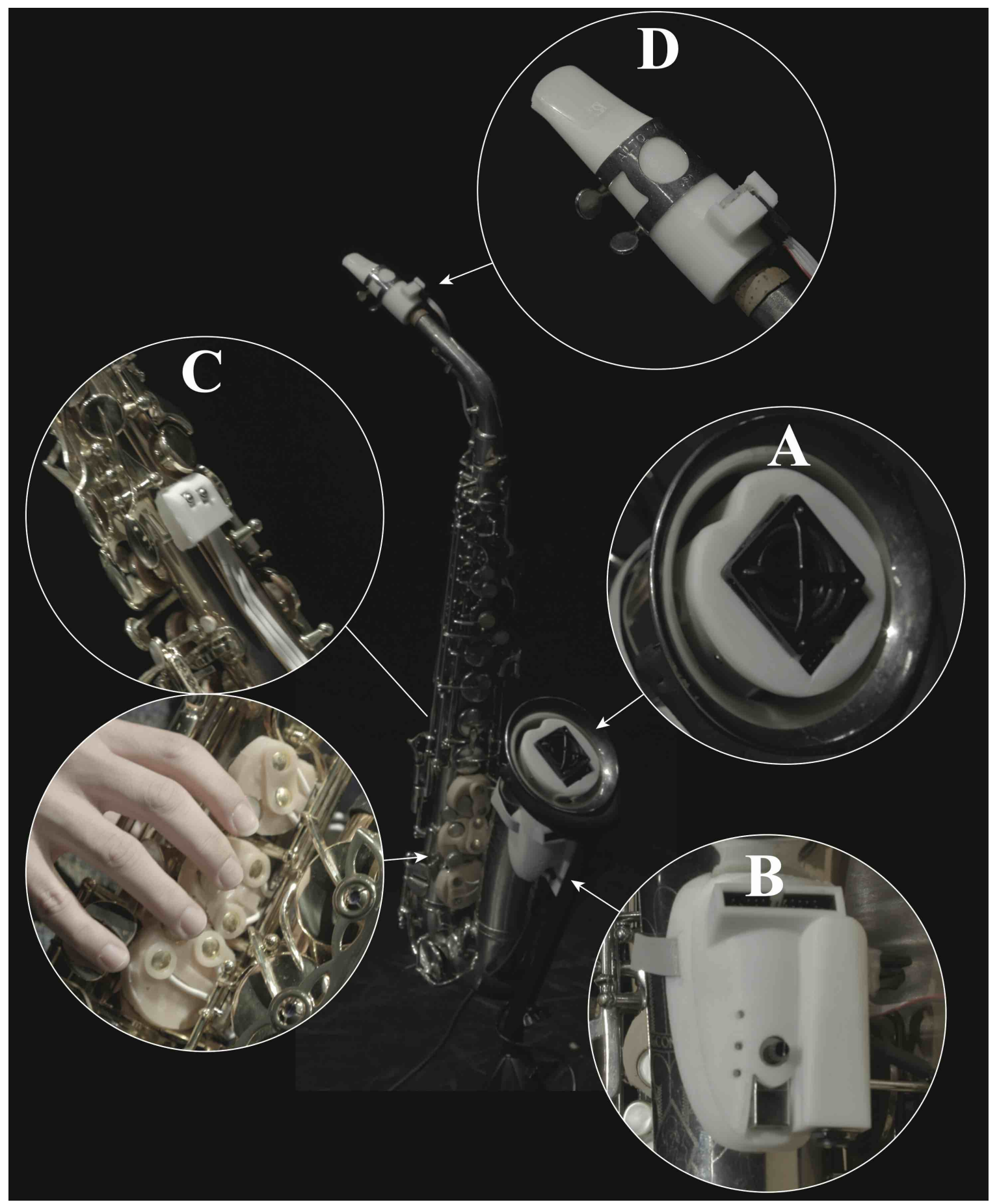

Fig. 14. Modular components of the HypeSax: Un-mute (A), Sensor-link (B), new keys for thumbs and new keys situated over custom-designed keycaps (C), and mouthpiece (D). 


\subsection{Un-Mute}

Augmented instruments often work with a hemispherical speaker located near the performer, following Cook and Trueman's approach to 'reorient the relationship between performer, sound source and listener' in electronic music performance. ${ }^{143}$ However, a goal of this project is the integration of the electronics with the acoustics of the instrument (see section 3.1.2). A number of research projects include the augmentation of the instruments' sound field combining acoustic and electronically generated sound, such as the IMAREV project, ${ }^{144}$ Smart Instruments, ${ }^{145}$ Juan Arroyo's hybrid string instruments ${ }^{146}$ or the Active Instruments. ${ }^{147} 148$ Unlike the paradigm established by Cook and Trueman, these projects approach instrumental augmentation by mounting sound actuators (speakers or surface transducers) directly on the instruments. This configuration allows for the use of the instruments' resonant chambers, which imposes acoustic characteristics of the instrument onto the electronic sound. At the same time, this location of the drivers facilitates the recognition of the sound source as embodied in the instrument. These aspects allow for fully integrated acoustic/electronic hybridisations.

In order to achieve integrated hybridisation (one of the design goals, see section 3.1.2), the HypeSax includes a speaker inserted inside the saxophone in order to make use of the acoustic chamber of the instrument and blend it into the system. A 3D model of an enclosure was designed to hold the speaker inside the bell of the saxophone. This enclosure also holds other electronic components. This mute-like enclosure and its components is called Un-mute. Rather than muting the sound of the instrument, it expands the sounding

${ }^{143}$ Cook and Trueman.

$144 \mathrm{http}: / /$ instrum.ircam.fr/imarev

$145 \mathrm{http} / / /$ instrum.ircam.fr/smartinstruments

${ }^{146}$ Fabien Houlès, Le Premier Quatuor à Cordes Hybride, l'example de Smaqra de Juan Arroyo, L'Harmattan (Paris, 2017).

$147 \mathrm{https}$ //www.nordiskkulturfond.org/en/inspirational-projects/acoustically-active-augmentedinstruments/

148 Otso Lähdeoja, 'Active Acoustic Instruments for Electronic Chamber Music', in Proceedings of the International Conference on New Interfaces for Musical Expression, 2220-4806 (Brisbane, Australia: Queensland Conservatorium Griffith University, 2016), XVI, 132-36. 
capabilities of the saxophone by collecting sensor data and outputting new sounds. It is the heart and brains of the HypeSax.

While the Un-mute is similar to a brass mute in shape, unlike mutes that are designed to filter and decrease the energy of overtones and spectral components, it enriches the sound of the saxophone by incorporating new digitally processed audio to the final sound. There is a down side, however, as the playability of lower range of the instrument is affected. This is due to the fact that the Un-mute is long enough to reach and partially block the opening of the low $\mathrm{B} b$ key when inserted in the bell, making it difficult to impossible to play the lowest note. The concept of an 'un-mute' is an expansion of the active mute designed by Meurisse et al. ${ }^{149}$ and Hong et al.'s hybrid saxophone. ${ }^{150}$ This concept can potentially be adapted to fit in different instruments, and its acoustic impact will depend on many aspects including the shape of the instrument, the acoustic response of the speaker, the position of the speaker (facing inwards or outwards relative the body of the instrument), use of multiple speakers, audio capture system and processing, microphones, and other elements. Further study of its effects on the resulting sound of any adaptation is necessary.

In the case of Meurisse's research, an active mute is used to address the negative effects of the use of a traditional mute, which is designed to alter the sound, but at the same time affects the playability of the lower register of the instrument as it 'decreases the input impedance magnitude at the playing frequency' by introducing a second impedance peak. By using an active mute system, the playability is restored by using a system that analyses the effect of the second impedance peak and actively supresses it via modifications of the phase. In a similar way, the HypeSax project aims to provide a system that analyses the acoustics of the saxophone in real time, in order to actively introduce signal that could alter the saxophone sound for creative purposes. In early prototypes of the HypeSax, the Un-mute was not part of the design and the electronics were attached outside the bell (see Fig. 15). This approach, however, does not allow for the active integration of the introduced signal and the acoustics of the instrument

\footnotetext{
149 Thibaut Meurisse, Adrien Mamou-Mani, René Caussé, and others, 'An Active Mute for the Trombone', The Journal of the Acoustical Society of America, 138.6 (2015), 3539-48.

${ }^{150}$ Hong, Zhu, and Duarte.
} 


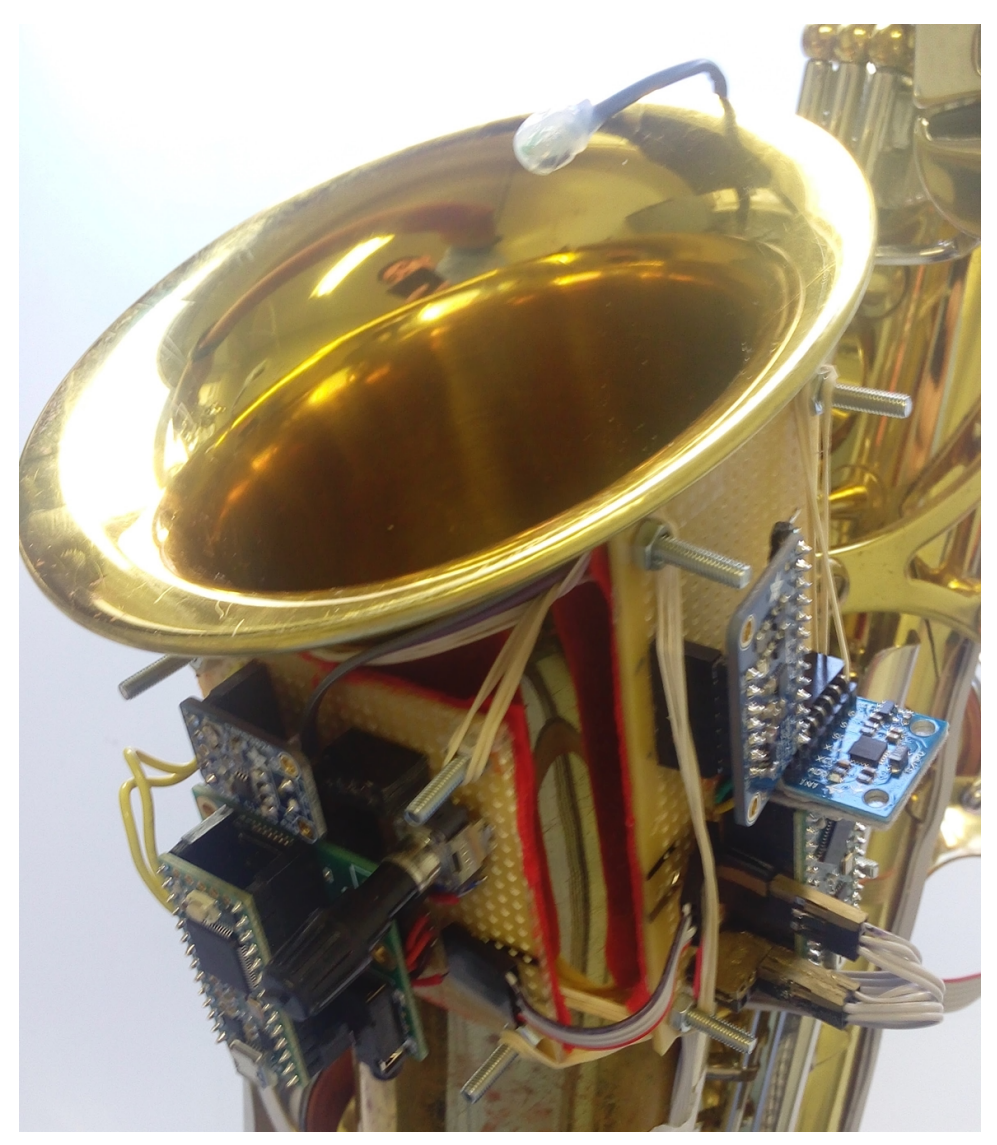

Fig. 15. Early prototype of the HypeSax.

\subsubsection{Acoustics, built-in audio system}

The enclosure was designed to avoid negative effects on the air column inside the saxophone, such as stopping the continuity of air flow, disruption of the air column in nodal points or even stop the air flow at all. A dip was included at the top of the Un-mute to allow air flow while playing in the low register of the instrument with most of the keys closed (see Fig. 16). 


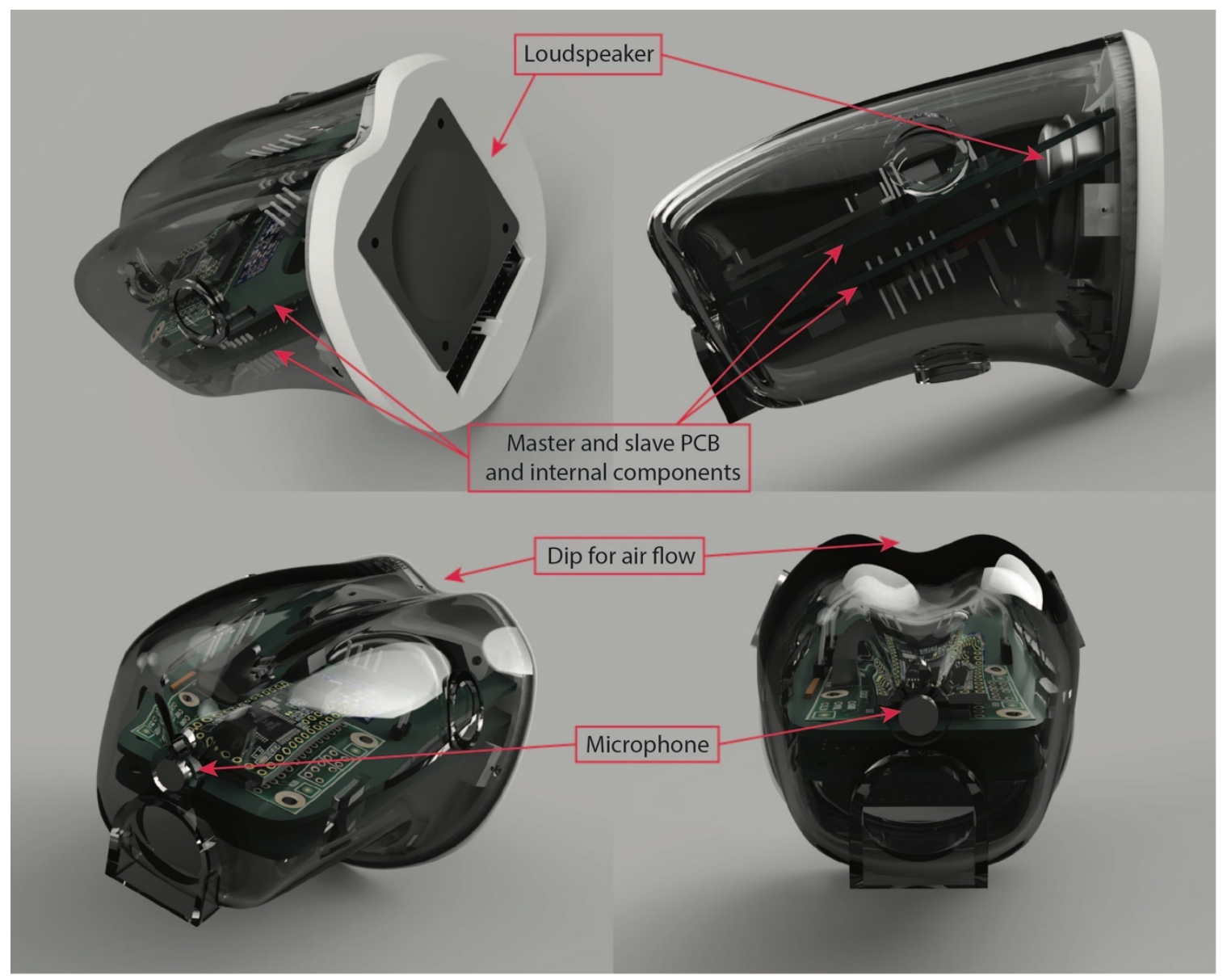

Fig. 16. The Un-mute.

In the back of the enclosure there is an opening that allows for the free flow of air pressure produced by the back of the cone of the speaker, which faces the front of the enclosure and out of the bell of the saxophone. The back opening is partially covered to stop moisture from entering the enclosure, potentially damaging the electronic components.

The Un-mute's audio system features an audio board adaptor for Teensy, a mono 2.4W Class D audio amplifier PAM8302A, a $4 \Omega 5 \mathrm{~W}$ speaker, and an electret microphone. These components were selected from a large list of possible candidates because their size allows them to fit inside the Un-mute. The individual characteristics that make these components ideal for the HypeSax are described in the next paragraphs.

The Teensy audio board adaptor facilitates handling audio I/O's using a Teensy 3.2 or a more powerful Teensy microcontroller board. It also features an onboard SD card slot which can be useful in order to store samples or record audio during performance. With this adaptor, the Teensy is also capable of handling some signal processing independently 
from an external device such a computer. Another favourable aspect of working with this adaptor is the Audio System Design Tool developed to handle the Teensy Audio Library, a GUI available online at the Teensy developer's website. ${ }^{151}$

One of the design challenges was provided by the fact that the saxophone tends to be a loud instrument. To pair the energy of its sound, it is necessary to amplify the signal that the Un-mute produces. Various amplifying developments were considered. In the first instance, stereo amplifiers were ruled out due to the requirement of hybridization, and the construction of the saxophone. Saxophones are conical and behave as closed conical pipes. One of the outcomes of this design is the directionality of the instrument. Utilizing a multichannel system would go against this natural aspect of the saxophone. If a multichannel setup is desired for creative reasons, this can be achieved via external speakers using the USB audio capabilities of the audio adaptor. The potential output gain was also considered, but due to the size of the cavity of the saxophone's bell (which is where the Un-mute sits) a large speaker could not be used. In fact, a more powerful amplifier is not necessary, as the PAM8302A already has enough power to drive the speaker above distortion levels.

The speaker chosen for the HypeSax is a Peerless PMT-40N25AL01-04 by Tymphany. This model has a design advantage over other similar speakers in that it features a protective polycarbonate frame. More importantly, it offers a spectral bandwidth that ranges from $100 \mathrm{~Hz}$ to $20 \mathrm{kHz},{ }^{152}$ which is enough to cover the entire range of an alto saxophone. It has a different response and power level capacity throughout the range. However, its average $4 \Omega$ impedance and $5 \mathrm{~W}$ of power make it powerful enough to match the saxophone's energy, at $\boldsymbol{m} \boldsymbol{f}$ or quieter, throughout the range of the instrument. Although this seems a mismatch with the instrument's power, it is what is currently possible to achieve with a speaker that is small enough to fit in the instrument's bell. This limitation can also be overcome with the use of external speakers if necessary.

151 https:/www.pjrc.com/teensy/gui/index.html

${ }^{152}$ Approximate range of the notes produced by the saxophone. However, the instrument can produce sounds out of this range through the use of extended techniques such as key clicks, reed slaps, subharmonics, and others. 
Finally, a miniature electret microphone is situated in the back of the Un-mute to capture audio from inside the saxophone chamber, allowing for a close audio capture setup that can potentially be used to amplify micro-sounds from the interior of the instrument. This allows the HypeSax to meet some Croft's conditions for instrumentality (see section 2.6.4). Dynamic, ribbon and condenser microphones were all considered. Dynamic microphones are better suited for loud explosive sounds due to their moving coils, which makes them ideal for a saxophone, although other kinds of microphones might be better for capturing subtleties such as breathing or small mechanical sounds. Ribbon microphones would be better for these situations but are not ideal for close miking and are also more fragile, so they would not be suitable for being housed inside a saxophone. On the other hand, a condenser microphone typically has a roll-off response curve above $10 \mathrm{kHz}$ and is extremely durable in comparison to a ribbon microphone. In the end, I chose a condenser microphone of the electret type for the Un-mute, which does not require phantom power, but can be biased with $2 \mathrm{~V}$. Its $4 \mathrm{~mm}$ size reduces the typical peak in frequency response situated over the $5 \mathrm{KHz}$, making it stable in most of the range of an alto saxophone. All of these characteristics make it a suitable device to be used in the Unmute.

The close placement of the microphone (facing inwards the saxophone body) and the speaker (facing outwards of the bell) about $7 \mathrm{~cm}$ away from each other is one of the reasons why the speaker faces forward and not directly into the opening of the saxophone. The back opening of the enclosure (see Fig. 16) however is big enough for the speaker's sound to travel back into the instrument in order to introduce new audio into the body of the saxophone. In the same way, the introduced audio is affected (filtered) by the resonance of the saxophone's acoustic chamber, allowing acoustical integration between acoustic sound and synthesis. Feedback can become present if there is a free signal flow and the levels are not calibrated. Nevertheless, feedback can become an interesting feature of the Un-mute that can be exploited as a creative device (see section 5.2.2.1).

\subsubsection{The brain}

Two PCB boards were designed in order to connect two microcontrollers, as well as the audio system and some of the sensors. These are housed inside the Un-mute (see Fig. 17). For this reason, the HypeSax cannot work without the Un-mute, as it contains the most 
important electronic components of the HypeSax. The system works with one Teensy LC board (slave) and a Teensy 3.2 board (master). The Teensy 3.2 is connected to the audio board adaptor. Its main functions are to retrieve audio using the microphone, send the audio to a laptop via USB, and receive the audio signal that is outputted using the speaker mounted on the Un-mute.

The HypeSax features six touch keys mounted over the keys of the saxophone (see section 3.5.1), and an Adafruit 12-key capacitive touch sensor board MPR121 is mounted on the PCB. Limited space between the instrument's keys led to the decision to use only six of the twelve inputs. Even when only six keys are used, however, a 12-key breakout board was used in order to facilitate sharing code with future iterations of the HypeSax for bigger saxophones (tenor or baritone) which might feature more keys.

In order to capture data on non-sounding motion such as swinging, cueing, and other torso movements characteristic of a saxophonist's performative practices, an accelerometer and gyroscope board (GY-521 MPU6050) is also located inside the Unmute. This location was chosen as the saxophone bell is possibly one of the best locations for it, given it is right at the centre of the instrument. This location helps in keeping track of any movement in x, y or z planes more accurately (see Fig. 18). Although this is an operational feature, it responds to the first and second interactive goals (see section 3.1.2). Locating this board in a different part of the saxophone might not favour equal results in all axes. If the board was located near the mouthpiece it would have very little movement as that zone of the instrument is the closest to the mouth, which needs to have a stable connection with the instrument, and therefore experiences less movement. On the other hand, locating the board at the bottom of the instrument, towards the curve of the 


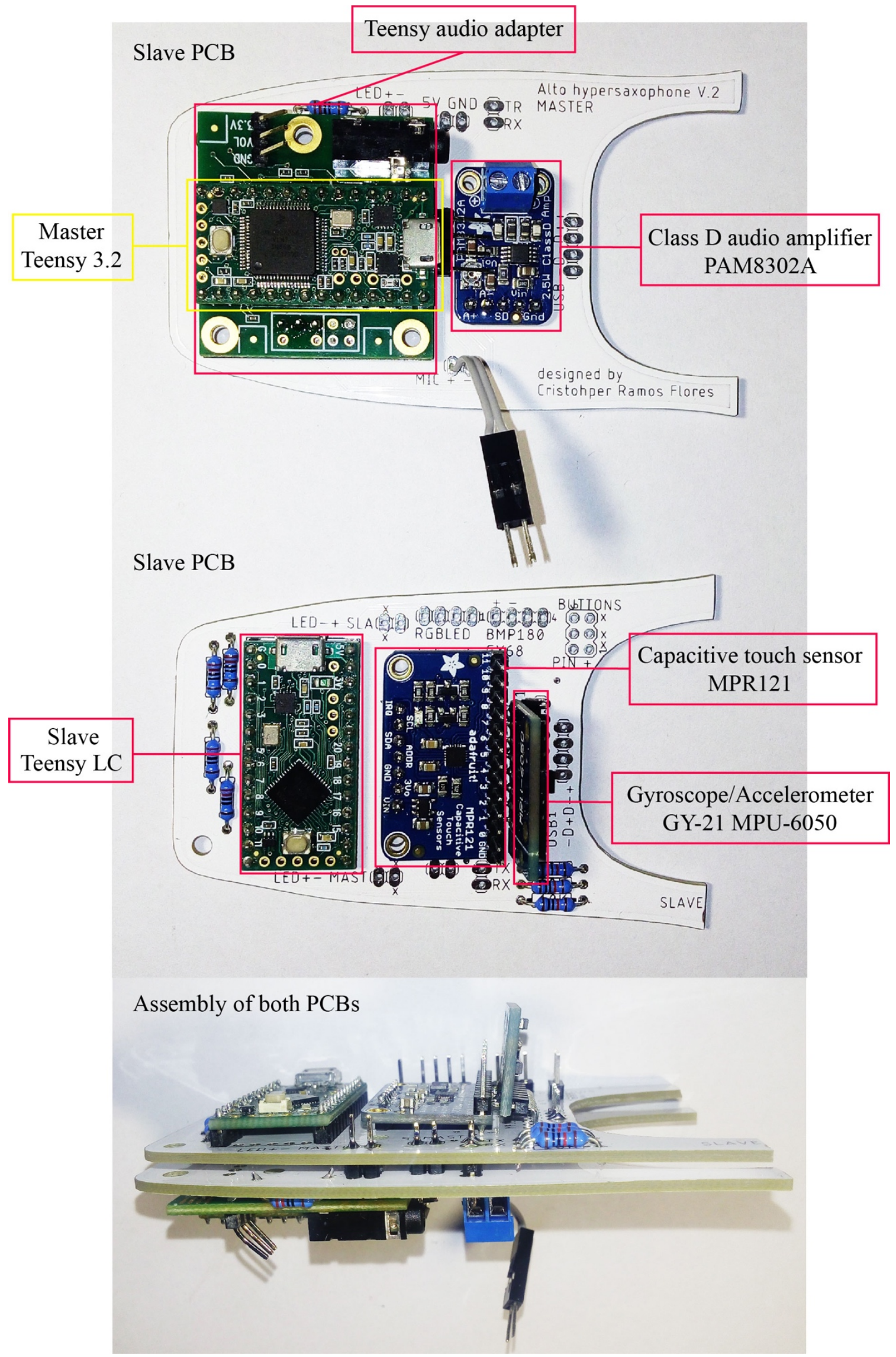

Fig. 17. Un-mute PCBs and electronic components. 
instrument might increase the readings on the $\mathrm{X}$ and $\mathrm{Y}$ axes due to a leverage effect. In addition, situating this board inside the Un-mute allows for less obstruction with the mechanism of the saxophone than if it was placed externally, and it would be less exposed to possible damage. Finally, situating it in the same bottom region, but internally, would have a more prominent impact in the acoustics of the instrument, and less alteration to the air flow (this is further discussed in section 3.3).

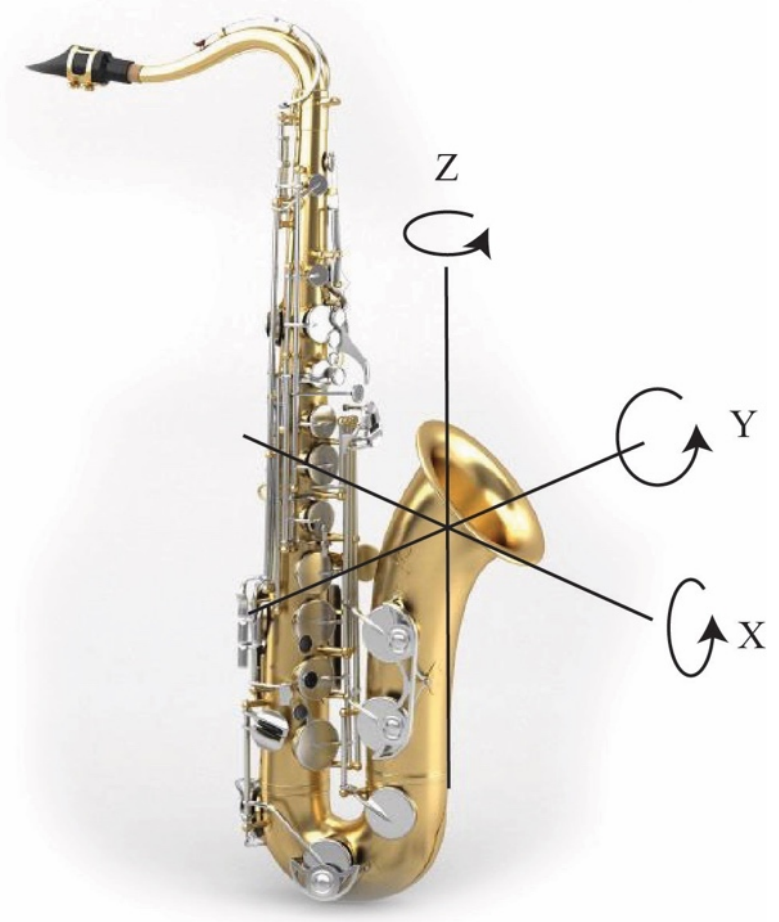

Fig. 18. $X, Y$ and $Z$ Axes of the HypeSax's gyroscope in reference to the saxophone's natural performing position.

The slave microcontroller is in charge of collecting data from all of the sensors and sending it to the master Teensy via serial communication. These two microcontrollers are connected via the PCBs previously described and depicted in Fig. 17. This design fits inside the Un-mute and features a port which allows connection to the Sensor-link which is located outside the bell of the saxophone.

\subsection{Sensor-Link}

As described in the previous section, the Un-mute holds the slave microcontroller that retrieves data from a variety of sensors. Some of these sensors are located in the 
mouthpiece, over the saxophone keys, and near the thumb rests. In order to connect those sensors to the slave microcontroller, the Sensor-link was developed. It consists of a custom PCB that features sensor inputs, a type B USB port, LED indicators for visual feedback, a gain knob and a standard $6.35 \mathrm{~mm}$ jack to connect an expression pedal for gain control. The Sensor-link is the connector route where signal and data flows between the HypeSax and the computer.

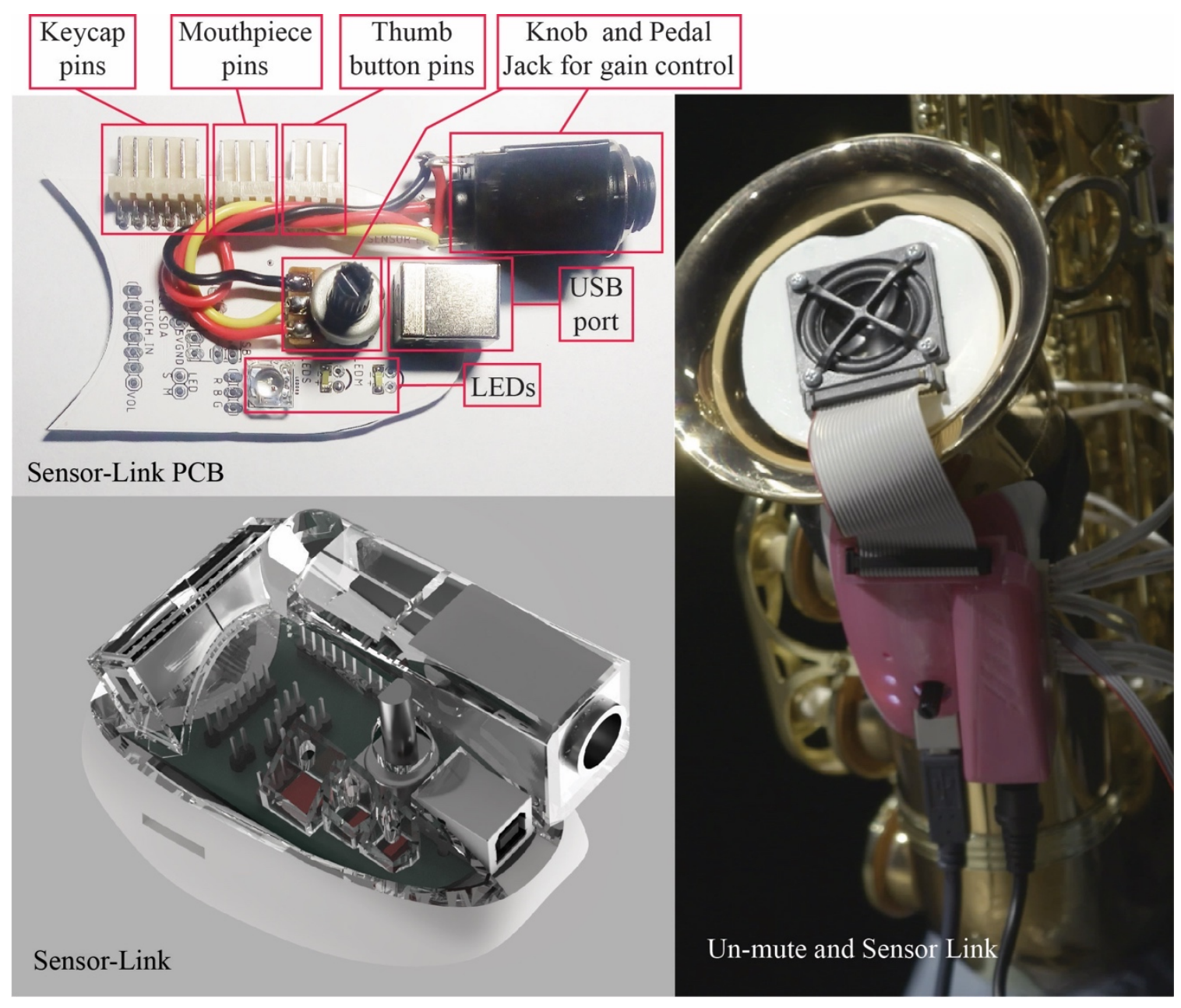

Fig. 19. Sensor-link.

Alternative configurations to achieve the necessary connections are possible. For example, the connecting ports could be located directly on the front face of the Un-mute. However, that design would make it difficult to fit all the components inside the Un-mute. On the other hand, using some of the area of the Un-mute's front face to allocate sensor ports would result in less space for a speaker, which would have an impact on the audio capabilities of the system. For these reasons the Sensor-link is necessary. In addition, it 
provides a better configuration of cable connection for the USB and pedal jack ports facing down, preventing potential stress on connected cables produced by gravity.

\subsection{Fingers: touch sensors and momentary buttons}

From the point-of-view of the interactive goals (see section 3.1.2), it is of paramount importance to maintain the traditional performative practices of the saxophonist, while providing new possibilities by digitally manipulating sound. The most intuitive response to this (from my perspective, as well as from the approach of previous projects such as the Metasax, ${ }^{153}$ the SABRe ${ }^{154}$ and its predecessor ${ }^{155}$ or Palacio-Quintin's hyper flute ${ }^{156}$ ) is to situate some kind of discrete or continuous sensor over or near the parts of the instrument where the fingers make contact. These sensors help the saxophonist interact with the signal processing. Complex systems, such as the one implemented in the SABRe, monitor all keys to identify fingering by using Hall Effect sensors. ${ }^{157}$ Such a design is highly effective but requires a complex setup process which makes the design inconvenient, possibly explaining why the commercial version of the SABRe (Multi-Sensor) does not include this fingering tracking system. ${ }^{158}$ This exemplifies the justification for the first and second operational goals.

\subsubsection{Touch sensors}

In the case of the HypeSax, easy setup and high compatibility with many saxophone models is a priority. To address this, a series of 3D models of saxophone keycaps were designed to allocate terminals coming from the MPR121 capacitive touch sensor board installed inside the Un-mute (see section 3.3.2). There are six terminals mounted on three keycaps which fit over the F, E and D keys. The 3D models are parametric. The parameters of the models in the computer files-

\footnotetext{
${ }^{153}$ Burtner, 'The Metasaxophone'.

${ }^{154}$ Schiesser and Schacher, 'SABRe'.

${ }^{155}$ Sébastien Schiesser and Caroline Traube, 'On Making and Playing an Electronically-Augmented Saxophone', in Proceedings of the International Conference on New Interfaces for Musical Expression (Paris, France, 2006), pp. 308-13.

${ }^{156}$ Palacio-Quintin.

157 Schiesser and Schacher, 'SABRe'.

$158 \mathrm{https}: / /$ www.sabre-mt.com/sabre-multisensor
} 
such as shape, and size — are variables that can be changed in order to adapt the models to fit any size and configuration of saxophone keys (see Fig. 20).

The position of the terminals mounted on the keycaps is close enough to the resting buttons of the keys to allow for a comfortable experience while performing. With this configuration, the performer can either close the keys by pressing the key buttons or by pressing on the touch pads.
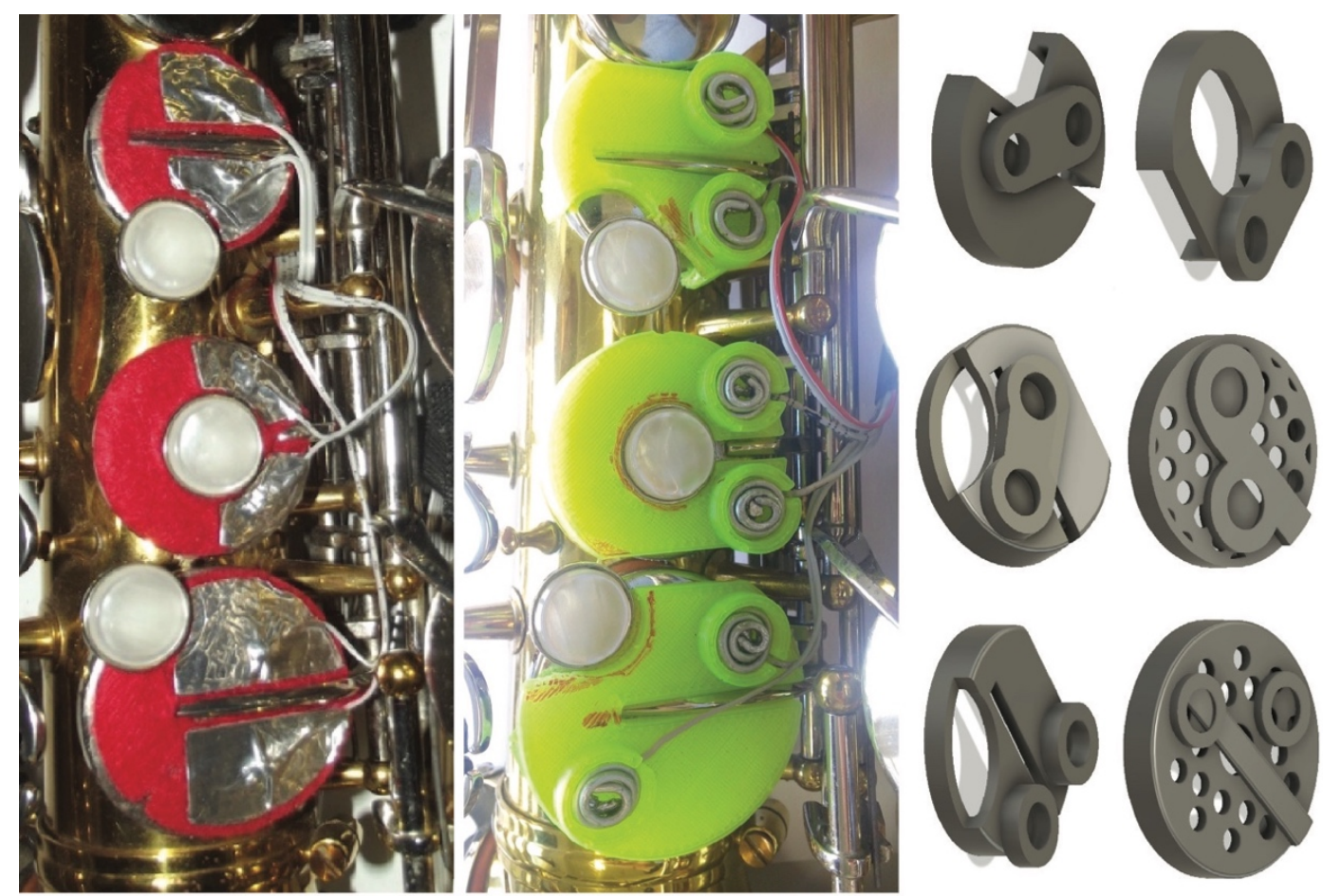

Fig. 20. Various iterations of the keycaps.

These sensors can also be configured to detect proximity or the amount of area touched, which could be used to detect different amounts of pressure. This allows for nuances of interaction such as not touching or closing the key, touching the key but not closing it, touching the key while closing it, or closing the key with different levels of contact. These nuances, provided by an operational capacity of the sensor, allow the HypeSax to meet some of the musical goals established for this design (see section 3.1.1).

Depending on the shape of the performer's fingers and/or the angle of the finger in reference to the saxophone body, up to two touch pads can be touched by one finger at a time. The protuberances on the keycaps holding the terminals also help the player to avoid touching them by mistake. There is also a possibility of activating the touch sensors by only touching the terminals without pressing or closing the keys. 
In the original design of the keycaps, these were going to be 3D-printed, but upon making some of them it became evident that the nature of the plastics used to print the models could potentially bend the keys and damage the integrity of the saxophone. To avoid this problem, it was decided to use the 3D models to create moulds in order to create negatives of the keycaps which could be filled with another material to create a final positive version of the models. Different materials were considered, ranging from commercial silicones, caulk, and various kinds of soft plastics. Finally, a polyurethane VytaFlex 40 by Smooth-On was used due to its properties of toughness and flexibility.

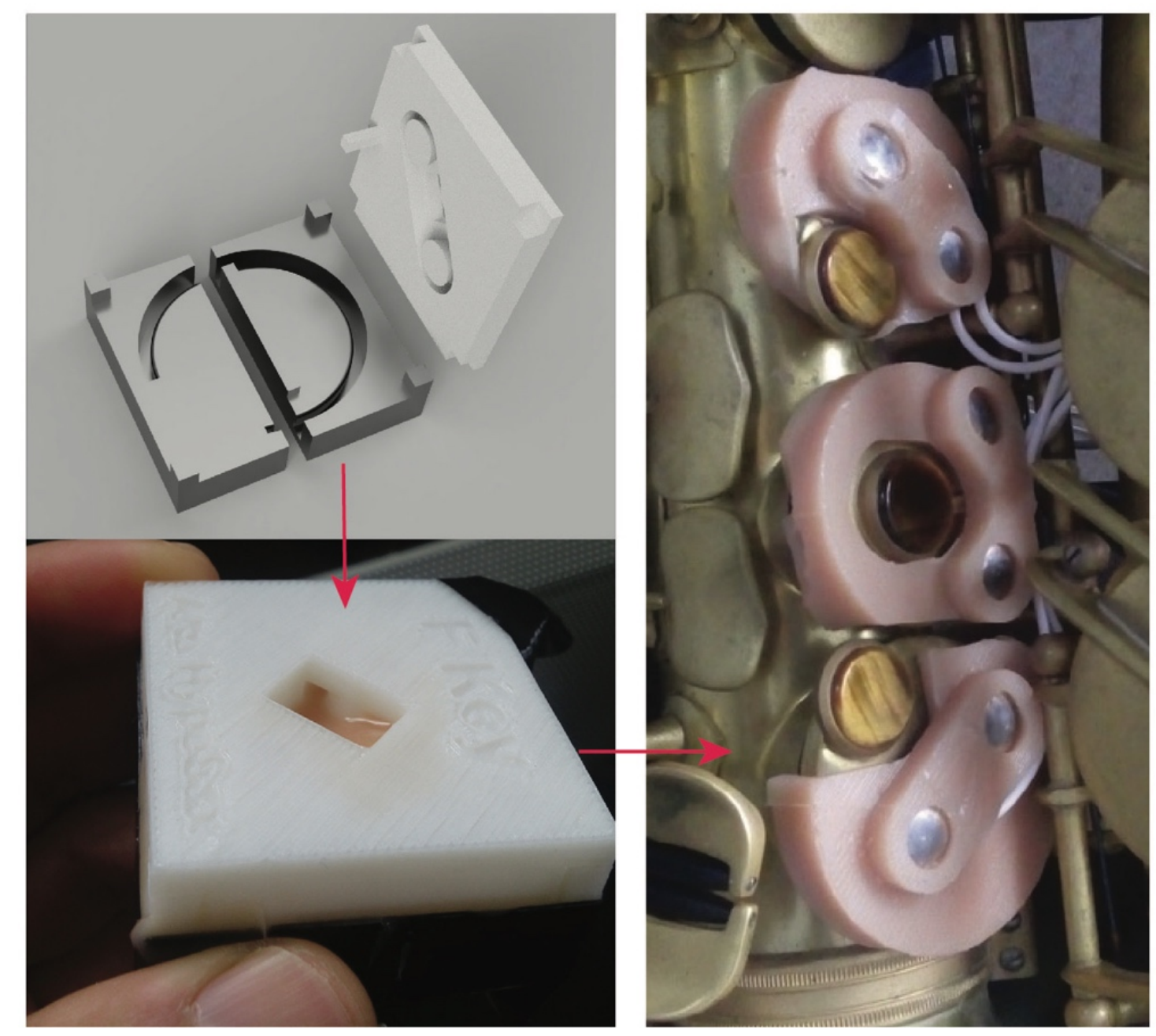

Fig. 21. 3D-Printed moulds and final keycaps made of VytaFlex 40 Polyurethane.

\subsubsection{Push buttons}

Three push buttons are included in the HypeSax design. Two of these buttons are mounted on the saxophone below the left thumb rest, and the third button is mounted next to the thumb hook 
to be easily accessible with the right thumb (see Fig. 22). Unlike the case of the terminals mounted on the saxophone keys, the thumbs interact with push buttons as the weight of the saxophone pushes against the thumbs, which produces unwanted interactions with touch sensors. This makes the mechanical system of the push buttons a more reliable approach. Custom PCBs and 3D-printed housings were designed to mount these thumb buttons on the saxophone.

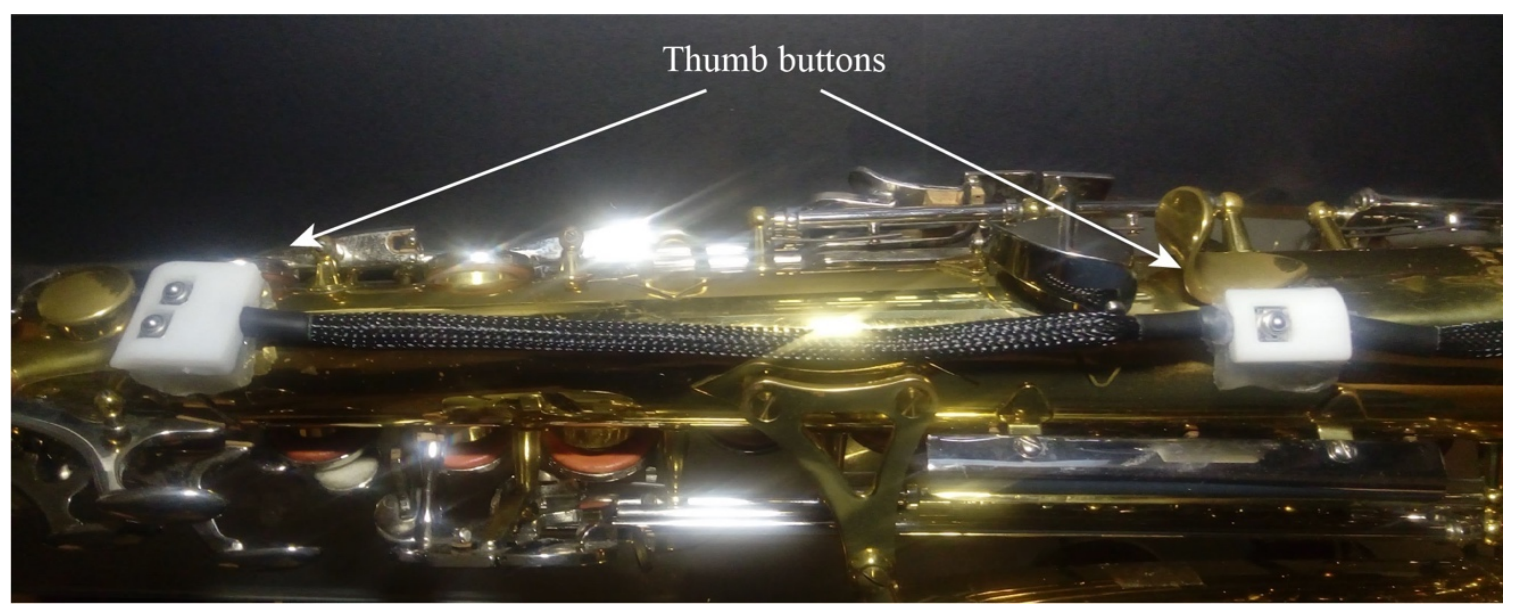

Fig. 22. Thumb buttons.

\subsection{Air pressure}

\subsubsection{General considerations}

The saxophone's main mode of sound production is by forcing a vibrating air column through the body of the instrument. To achieve this, a reed is mounted on the mouthpiece of the instrument. When air is blown into the instrument with sufficient force it pushes the reed and closes it against the mouthpiece. A portion of the air stream that enters the body reflects off the end of the resonator (the bell or any open key) due to the pressure difference (end effect). This partial reflection pushes the reed in the opposite direction, forcing the reed to open before it is closed again by the force of the air blown into it by the performer. This creates a cycle that effectively becomes a harmonic oscillator. ${ }^{159}$

\footnotetext{
${ }^{159}$ Braasch, VI, p. 14.
} 
As part of the sound production system, the resonator (the bore of the instrument) plays a very important role, as its shape and size regulate the cycle at which the reed vibrates. This is what defines the pitch, by 'channelling the energy of the air-stream into the frequencies that resonate' within the body of the instrument out of the entire noise-like spectrum introduced by the air-stream. ${ }^{160}$

The complexity of this system is extended out of the saxophone into the mouth of the performer, where many other factors play roles with varying degrees of importance, ranging from inadequacy of air flow continuity, misplacement of tongue, teeth or cheeks, and many other aspects such an embouchure that does not seal properly. The details of the physics of the sound production and the effects of the variations in the different parameters in the sound production system of woodwind instruments, in particular the saxophone, have been studied by authors such as Backus, ${ }^{161}{ }^{162}$ Benade, ${ }^{163}$ Braasch, ${ }^{164}$ Chaigne, ${ }^{165}$ Fletcher, ${ }^{166}$ Photinos, ${ }^{167}$ Wolfe, ${ }^{168}$ and many others. While a substantial study of the specific details of the system is beyond the scope of this dissertation, the conclusions at which I arrived, based on the works of the aforementioned authors and my own experimentation, are the basis for many of the design considerations for the HypeSax development.

The sound production system of the saxophone should preferably not be tampered with, as interfering the delicate balance of its mechanics would cause the sound to lose energy, change its quality or even prevent the system from producing any pitched sound

${ }^{160}$ Panos Photinos, Musical Sound, Instruments, and Equipment, 2017, pp. 11-13.

${ }^{161}$ John Backus, 'Input Impedance Curves for the Reed Woodwind Instruments', The Journal of the Acoustical Society of America, 56.4 (1974), 1266-79.

162 John Backus, 'The Effect of the Player's Vocal Tract on Woodwind Instrument Tone', The Journal of the Acoustical Society of America, 78.1 (1985), 17-20.

${ }^{163}$ A. H. Benade, 'On Woodwind Instrument Bores', The Journal of the Acoustical Society of America, 31.2 (1959), 137-46.

${ }^{164}$ Braasch, VI.

${ }^{165}$ Antoine Chaigne and Jean Kergomard, Acoustics of Musical Instruments, Modern Acoustics and Signal Processing (New York, NY: Springer New York, 2016).

${ }^{166}$ Neville H. Fletcher and Thomas D. Rossing, The Physics of Musical Instruments, 2. ed., [Nachdr.] (New York, NY: Springer, 2010).

167 Photinos.

${ }^{168}$ J Wolfe and others, 'Some Effects of the Player's Vocal Tract and Tongue on Wind Instrument Sound', Proceedings of the Stockholm Music Acoustics Conference 2003 (SMAC 03), 2003, 4. 
at all. As a consequence, it is of utmost importance to design the HypeSax in such a way that none of its components would negatively affect sound production. While this was largely achieved, having minimal effects on the system, there is an impact on the sound production in the lower range of the instrument, due to the presence of the Un-mute.

The first and most important point of interaction the performer has with the saxophone is through the mouthpiece and reed. Previous woodwind hyperinstruments and research projects have addressed the necessity of retrieving data to understand the changes of air pressure and reed vibrations produced during performance. Some designs take advantage of this data to control parameters during sound synthesis, audio processing or any number of non-audio-related software processes.

In research undertaken at McGill University in $2005{ }^{169}{ }^{170}$, it was shown how difficult but useful it can be to gather airflow data from a flute to later reuse it as a musical control variable. In the case of the flute, however it is possible to mount sensors outside the body of the flute, since the performer's mouth is not in direct contact with the instrument, thus avoiding any corruption the airflow or sound production system. In this case, air pressure sensors were mounted around the mouthpiece to collect data (see Fig. 23). This system can be unreliable, however, since flute performance practices sometimes require a change of angle in which the air is blown into the instrument, resulting in variation of the air pressure in the sensor.

169 Andrey R. Da Silva, Marcelo M. Wanderley, and Gary Scavone, 'On the Use of Flute Air Jet as a Musical Control Variable', in Proceedings of the 2005 Conference on New Interfaces for Musical Expression (National University of Singapore, 2005), pp. 105-8 $<$ http://dl.acm.org/citation.cfm?id=1085969> [accessed 4 May 2017].

${ }^{170}$ Gary Scavone and Andrey R. Silva, 'Frequency Content of Breath Pressure and Implications for Use in Control', in Proceedings of the International Conference on New Interfaces for Musical Expression (Vancouver, BC, Canada, 2005), pp. 93-96. 


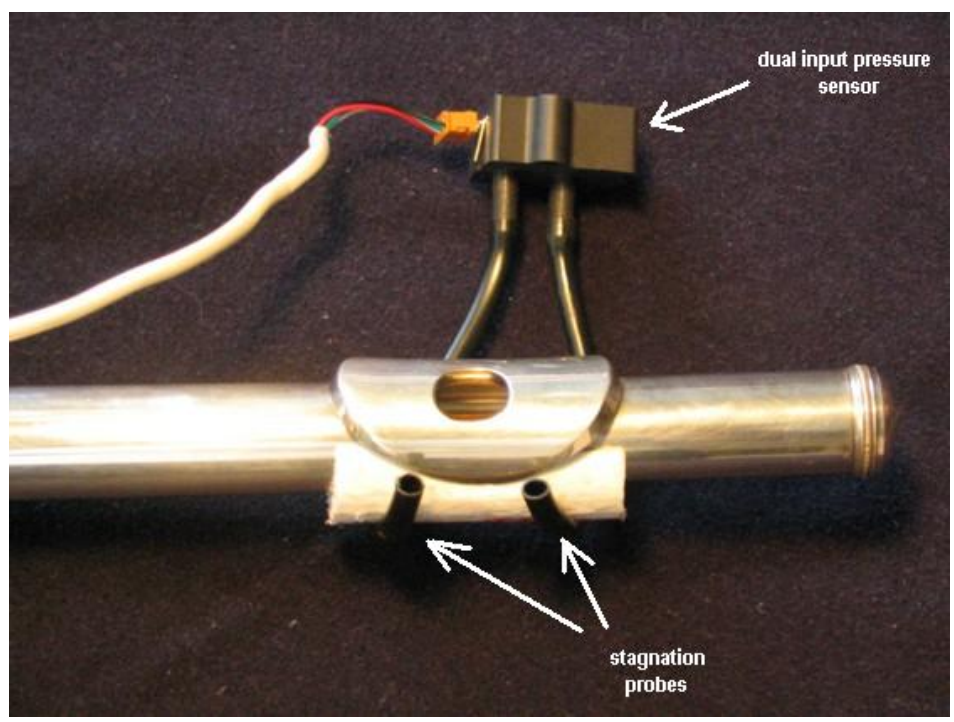

Fig. 23. Pressure sensor and probes mounted around the mouthpiece. ${ }^{171}$

Other projects aiming to measure the energy of the air stream utilise a microphone that gathers information on the quality and dynamics of noise produced by blown air to obtain comprehensible data of the energy carried by the air stream. The use of microphones to obtain information on the air blown has been very successful in projects such as Ocarina by Smule, an iOS app which has been used by thousands of people around the globe. ${ }^{172}$ While this is an approach that could potentially be used successfully with some woodwind instruments, it would not be possible on reed instruments as a microphone cannot be inserted inside the mouthpiece or the body of the instrument. Considering the fact that the saxophone focuses the energy of noise, turning it into a pitched sound with a high degree of harmonicity ${ }^{173}$, placing a microphone at any of the point where the air is released out of the instrument would not be a feasible approach to analysing the energy content of the air stream.

Another way to achieve accurate acquisition of data was explored in a joint effort between Universitat Pompeu Fabra and Stanford University, where it was demonstrated

${ }^{171}$ Da Silva, Wanderley, and Scavone, 'On the Use of Flute Air Jet as a Musical Control Variable'.

${ }^{172}$ Ge Wang, 'Designing Smule's Ocarina: The IPhone's Magic Flute.', in NIME, 2009, pp. 303-7 $<$ https://www.researchgate.net/profile/Ge_Wang9/publication/253074606_Designing_Smule's_Ocarina _The_iPhone's_Magic_Flute/links/00b495̄2b01c00ecaf9000000/Designing-Smules-Ocarina-TheiPhones-Magic-Flute.pdf $>$ [accessed 29 June 2017].

${ }^{173}$ Photinos, pp. 11-13. 
that modifications to the mouthpiece of an alto recorder to redirect a portion of the air flow into an air pressure sensor was possible ${ }^{174}$ by creating conduits in the body of the mouthpiece to redirect a portion of the air stream into a sensor (See Fig. 24).

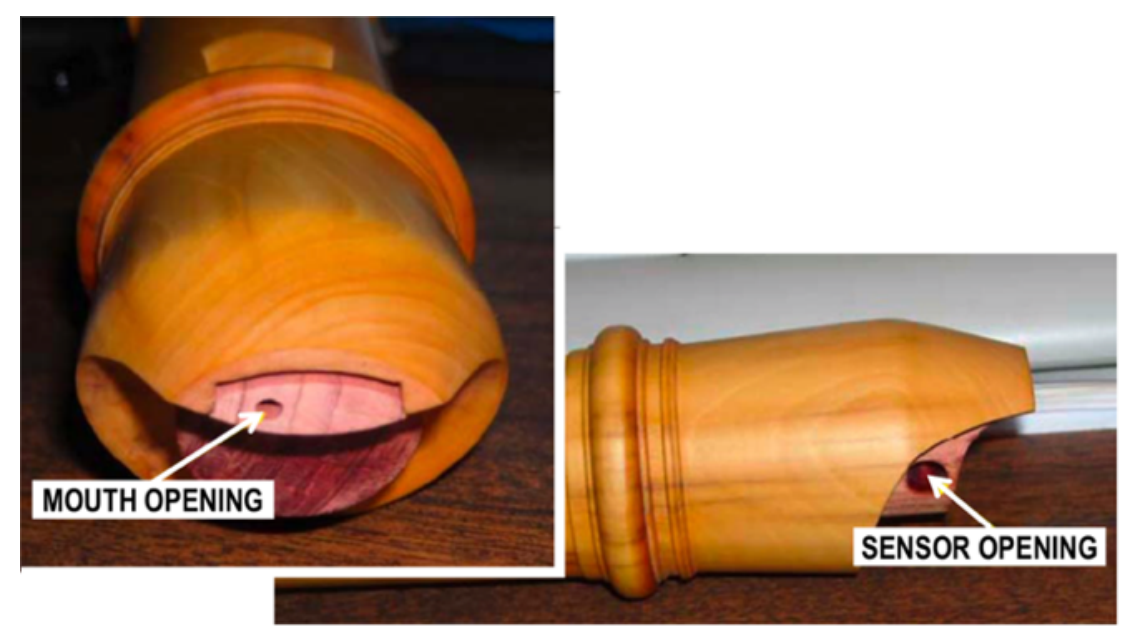

Fig. 24. Detail of the openings in the mouthpiece. ${ }^{175}$

Projects such as the SABRe ${ }^{176}$ deal with problems related to reed instruments. In this project, the authors developed a system in which a small hose mounted on top of the mouthpiece redirects a portion of the air stream into an air pressure sensor, obtaining accurate readings. In this case, the sensor, being mounted on top of the mouthpiece (as shown in Fig. 25), is close enough to the air stream source (player's mouth) to ensure that no energy (pressure) is lost before the sensor reading is done. The SABRe's air pressure data acquisition system was an ideal solution to retrieve data for the HypeSax without compromising sound production.

${ }^{174}$ Francisco Garcia and others, 'Acquisition and Study of Blowing Pressure Profiles in Recorder Playing.', in NIME, 2011, pp. 124-27

$<$ http://mfile.narotama.ac.id/files/Umum/JURNAR\%20STANFORD/Acquisition\%20and\%20study\%2 0of\%20blowing\%20pressure\%20profiles.pdf $>$ [accessed 4 May 2017].

${ }^{175}$ Garcia and others.

${ }^{176}$ Sébastien Schiesser and Jan C. Schacher, 'SABRe: The Augmented Bass Clarinet.', in NIME, 2012 $<$ http://vhosts.eecs.umich.edu/nime2012/Proceedings/papers/193_Final_Manuscript.pdf $>$ [accessed 4 May 2017]. 


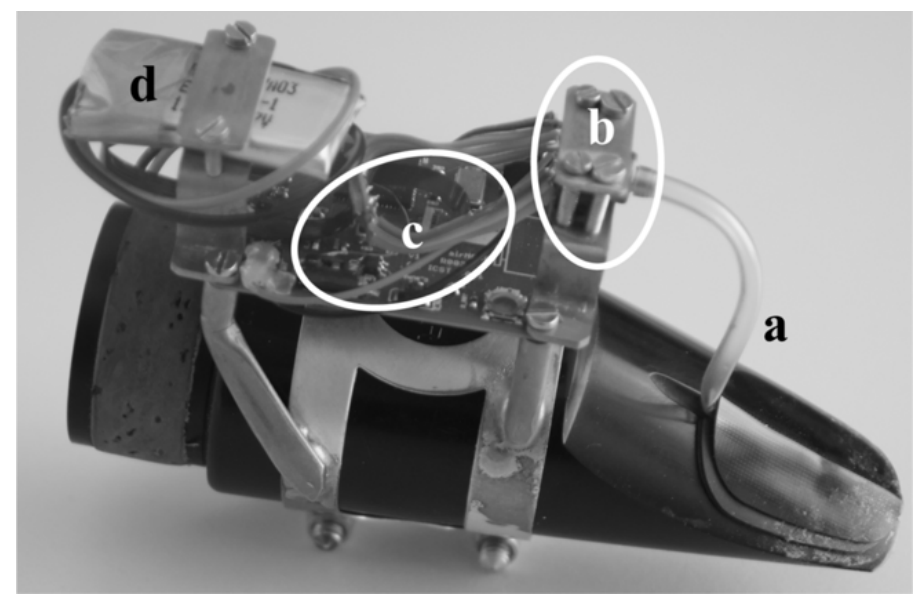

Fig. 25. SABRe's mouthpiece with mounted hose (a), pressure sensor (b), RF electronics (c) and battery (d). ${ }^{177}$

While many other projects 178179180181182 as well as commercial woodwind controllers deal with air pressure in different ways with successful results, I based my design concepts on that of SABRe and García et al.'s modified flute recorder mouthpiece, ${ }^{183}$ as well as some aspects of the design of a patented hybrid saxophone developed by Yamaha ${ }^{184}$ (a product that has not yet been commercialised), to feature a barometer sensor that collects data on the air pressure variations during performance.

Making a musical instrument requires both knowledge about the instrument as well as knowing how to work or machine different materials and operate specialised equipment. Fortunately, digital fabrication opens the possibility to design instruments in a virtual world. 3D modelling has become a gateway into the instrument maker world for many DIY enthusiasts, and a powerful tool for rapid prototyping in the case of professional

\footnotetext{
${ }^{177}$ Schiesser and Schacher, 'SABRe'.

${ }^{178}$ Baptiste Bergeot and others, 'Response of an Artificially Blown Clarinet to Different Blowing Pressure Profiles', The Journal of the Acoustical Society of America, 135.1 (2014), 479-90.

${ }^{179}$ Sidney Fels and others, 'Evolving Tooka: From Experiment to Instrument', 2004, 6.

180 Onozawa and Fujita.

${ }^{181}$ Perry R. Cook, 'A Meta-Wind-Instrument Physical Model, and a Meta-Controller for Real Time Performance Control'.

182 Gary P. Scavone, 'THE PIPE: Explorations with Breath Control', in NIME, 2003.

${ }^{183}$ Garcia and others.

184 Onozawa and Fujita.
} 
makers and researchers. Amit Zoran, for instance, modelled and printed a fully functional transversal flute. ${ }^{185}$ Similarly, Valerio Lorenzoni et al. ${ }^{186}$ designed and 3D-built a set of saxophone mouthpieces that feature varying dimensions and shapes of bore and baffle. Influenced by that project, I studied the effect of the geometry of the mouthpiece, ${ }^{187}$ as well as the effect that using different materials and technologies to fabricate these $3 \mathrm{D}$ models would have on the acoustics of the saxophone. These projects proved that making professional level mouthpieces through digital fabrication not only offers a valuable tool for research, but also provides a gateway to personalizing and making instrument parts of high standard, such as the commercial 3D-printed mouthpieces produced by Syos, ${ }^{188}$ a company founded by Dr. Pauline Eveno. With this in mind, I developed the HypeSax mouthpiece.

Considering the importance of the mouthpiece on the resultant sound of the saxophone, a hybrid saxophone mouthpiece was designed for the HypeSax, with the purpose of being able to both be used to perform and to collect performance data in real time. The collected data can be used in different situations that may include information gathering for performance study or more practical goals such as controlling diverse parameters inside software capable of communicating via serial or MIDI protocols.

\subsubsection{HypeSax mouthpiece}

The first step in developing the mouthpiece was to consider the fact that introducing an object inside a saxophone causes disturbance in the air column, thereby changing the quality of the sound, affecting the intonation, producing 'squeaks', or even potentially stopping the sound altogether. Based on past projects (listed above) I decided to work with

\footnotetext{
${ }^{185}$ Amit Zoran, 'The 3D Printed Flute: Digital Fabrication and Design of Musical Instruments', Journal of New Music Research, 40.4 (2011), 379-87.

${ }^{186}$ Valerio Lorenzoni, E. L. Doubrovski, and J. C. Verlinden, 'Embracing the Digital in Instrument Making: Towards a Musician-Tailored Mouthpiece by 3D Printing', in Proceedings of the Stockholm Music Acoustics Conference 2013, SMAC 2013, Stockholm (Sweden), 30 July-3 August, 2013, 2013.

${ }^{187}$ S. Carral, V. Lorenzoni, and J. C. Verlinden, 'Influence of Mouthpiece Geometry on Saxophone Playing', Proceedings of the 3rd Vienna Talk on Music Acoustics" Bridging the Gaps", Vienna, Austria, 16-19 September 2015, 2015.

188 https://www.syos.co/en
} 
a 3D design that would feature an alternate conduit in the body of the mouthpiece, which redirects a portion of the air column to a barometer sensor to measure the air pressure without affecting the air stream flowing through the mouthpiece and final air column inside the saxophone.

\subsubsection{3D Model}

The first iteration of the mouthpiece was an adaptation of the Alto Saxophone Mouthpiece designed by Thingiverse user Allanrps. ${ }^{189}$ The original design is based on a Yamaha beginner mouthpiece, with similarities including bore length and shape, facing width, tip opening and basic length measurements, featuring a flat baffle and squared chamber.

The adaptation keeps the basic shape of the interior with the same dimensions in the chamber of the mouthpiece, facing width and bore shape, which ensures that the air column running through the chamber behaves in the same manner as in the original design. The changes made to the design include a shorter tip opening of $1.8 \mathrm{~mm}$ (original is $2 \mathrm{~mm}$ ) and a thicker beak that includes a small conduit running through the body of the mouthpiece to an added section located on the back, in which a sensor is mounted to measure the air pressure. Fig. 26 shows a comparison between the original design by Allanrps and the adaptations made to it for the HypeSax, where the original version of model can be seen on the left, next to the new version on the right.

189 https://www.thingiverse.com/thing:14495 


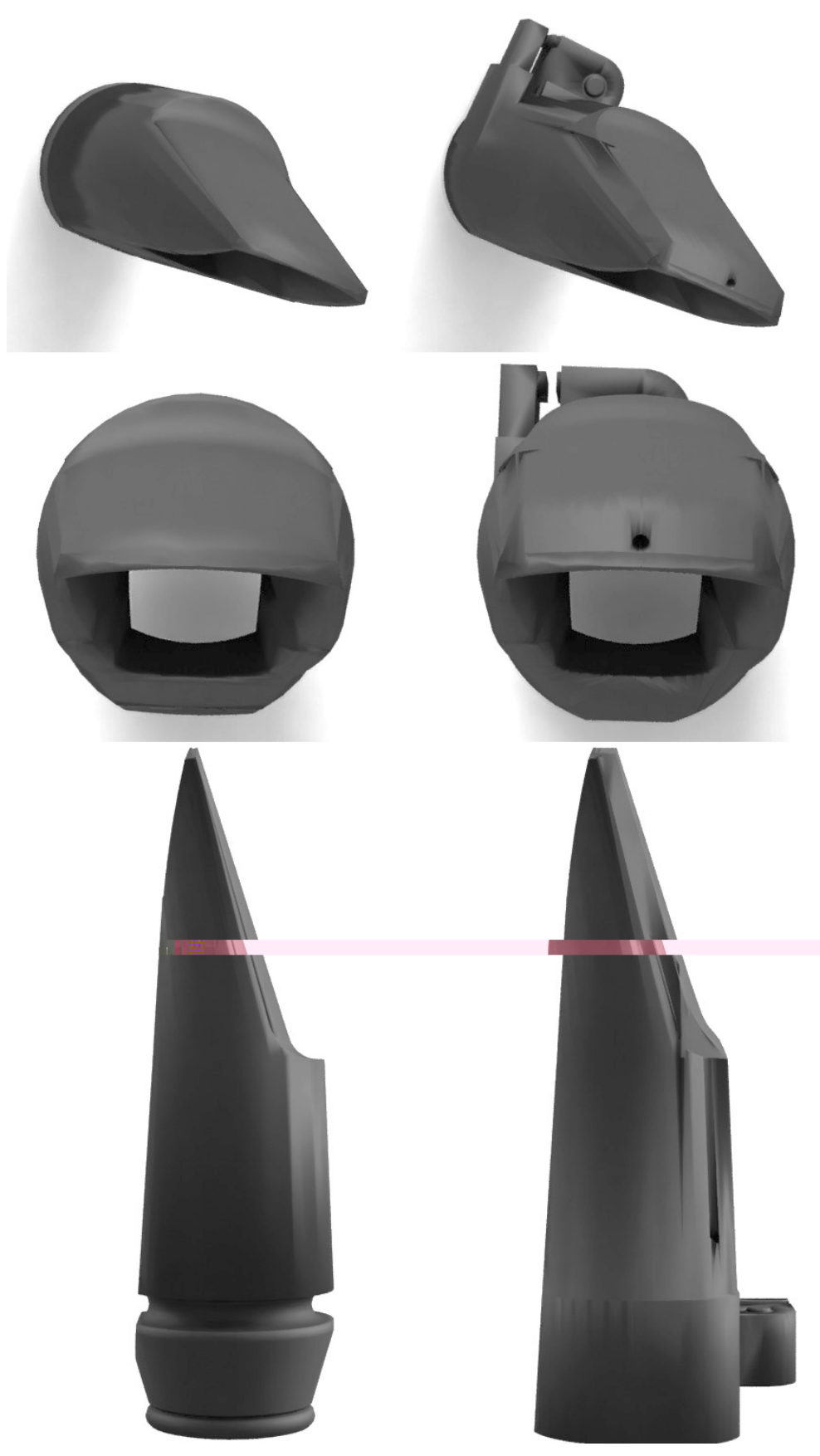

Fig. 26. Comparison between the original 3D model and the modified version used in the HypeSax.

The conduit was designed to allow comfortable playing with no disturbance to the air stream or air column. To achieve this goal, a minimal amount of material was added to the top of the beak of the mouthpiece to accommodate the conduit. In total, $2 \mathrm{~mm}$ of thickness was added to the beak, of which a $1 \mathrm{~mm}$ radius cylindrical opening is found at the front/centre of the beak, where the structure is weaker because of its dimensions. Further back, the conduit opens more to fit the dimensions of the sensor at its back end (see Fig. 
27). A small side canal was also designed to allow moisture and saliva to escape the system. Further tests demonstrated that the second canal was not necessary, as pressure tends to be lost through it, and it does not release enough moisture to be considered of any help, as the system in general does not to accumulate liquids.

The 3D model can be printed in a variety of materials, including organic-based materials or plastic and polymer-based materials, many of which resemble ebonite and hard rubber, common materials used in the fabrication of mouthpieces. For the first printing trials, an Up Mini filament deposition modelling 3D printer was used. ABS (acrylonitrile butadiene styrene) was used due to its durability, ease of use and price, as well as the fact that is not toxic. Fig. 27 shows slices of the mouthpiece where, the top image depicts the chamber and conduit are in white. The bottom image shows the conduit and side canal in grey. The area designed to position the sensor breakout is coloured in black.
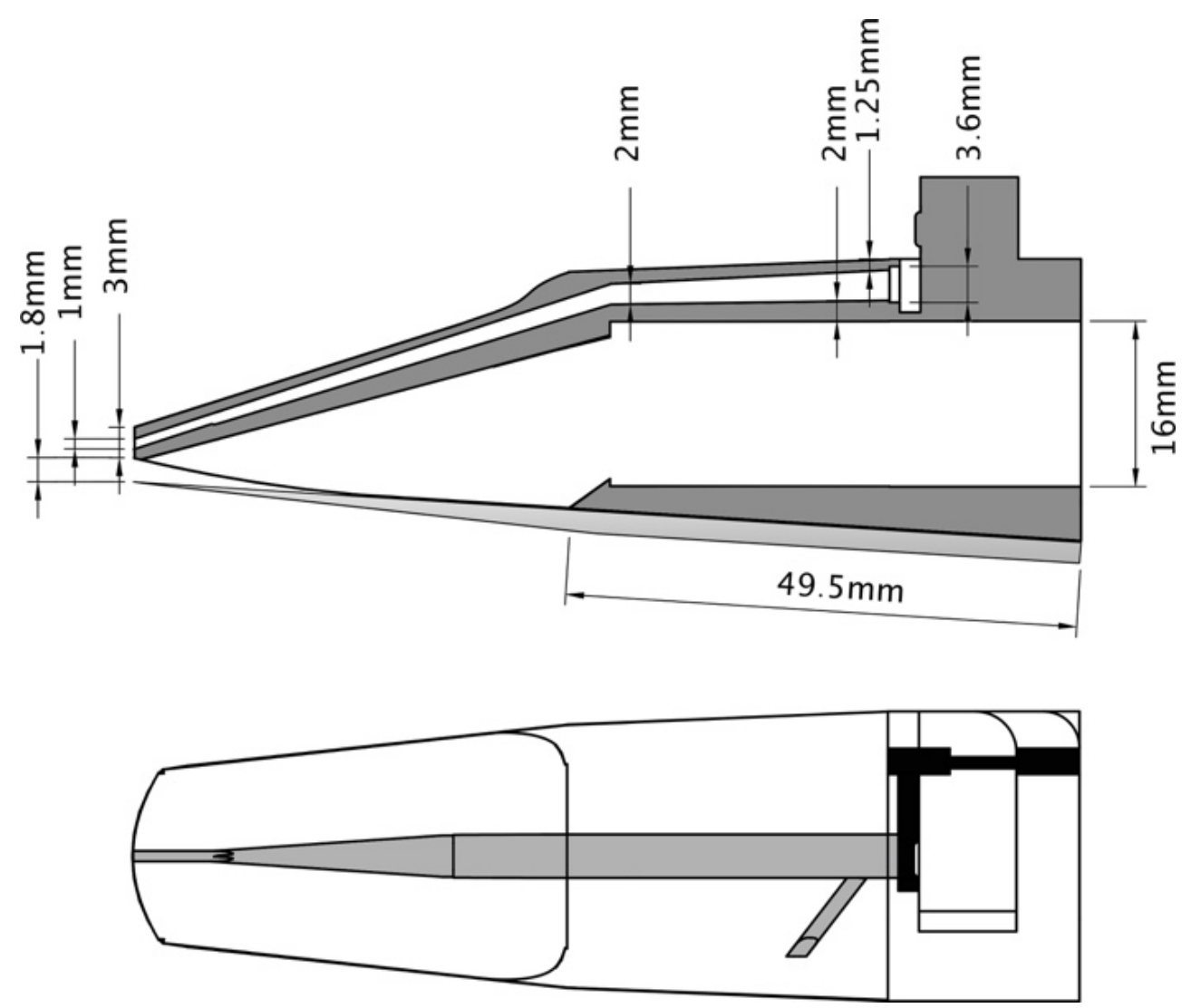

Fig. 27. Interior of the first iteration of the mouthpiece. 
The evaluations made to this mouthpiece showed that this model would produce a sound with quality comparable to a commercial mouthpiece (see section 4.3.3.3 and Appendix D). However, this design was improved thanks to a pilot study made with saxophonists Peter Liley and Jasmine Lovell-Smith, whose opinion pointed at the fact that extra material at the top of the beak would increase the difficulty in having a perfectly sealed embouchure. The three to five extra millimetres (depending on the position of the mouth over the beak) forces the lips to open on the sides of the mouth. To prevent this, the performer has to tighten the lips and cheek muscles, creating unwanted tension, and eventually a tired and loose mouth. A second mouthpiece following the same concept was designed to address this issue.

For this new iteration, a parametric design ${ }^{190}$ was created using the Fusion 360 CAD modelling software. To make this model, it was necessary to learn about the acoustic effects of mouthpiece geometry. There are many sources available that cover this subject (some already cited in previous sections) but specific details of the design of each mouthpiece are not disclosed by commercial companies or private makers. Again, the online DIY community spread over many websites became an important source. The two most important and reliable sources found were Theo Wanne's website ${ }^{191}$ and Syos' blog, ${ }^{192}$ while the most useful was provided by Johan Jonker ${ }^{193}$ through his workshop's website and email correspondence. Based on the information found on these websites and Jonker's Measuring a mouthpiece blog post, ${ }^{194} \mathrm{I}$ created a list of forty-five parameters (see Appendix A) to be used in my parametric 3D mouthpiece model. Eight more parameters were necessary to create the section of the mouthpiece that houses the barometer, extending the list of parameters to 53 (these parameters are not included in the

${ }^{190}$ In a parametric design all dimensions are specified as variables. This allows for adaptability of the design by updating the variables as needed. Shapes and dimensions can be adapted while maintaining certain constrains that forces the components of the models to retain specified relationships.

191 Theo Wanne is a well-regarded mouthpiece maker company that offers an informative resources section on its website www.theowanne.com.

192 Syos is a 3D-printed mouthpiece company founded by Pauline Aveno, who holds a PhD in Acoustics from Université Pierre et Marie Curie. The company's website also offers useful and reliable information on its blog section (https://www.syos.co/en/blog).

193 Johan Jonker is a professional woodwind instruments repairer and maker based in Houten, Netherland.

194 https://sax.johanjonker.net/measuring-a-mouthpiece 
accompanying Appendix A as the variations in design only occur in the mouthpiece, but the sensor remains the same).

The second model allows customization of the geometry, which allows adjustability to the preferences of the performer. Nevertheless, the most important feature of this iteration is the relocation of the conduit that redirects a portion of the air column. In this case, the conduit is still located on the front of the beak, but shifted to one of its sides, rather than on the top. From there it runs in an S shape through the beak to the opening in the back, where the sensor is situated as in the first iteration. 


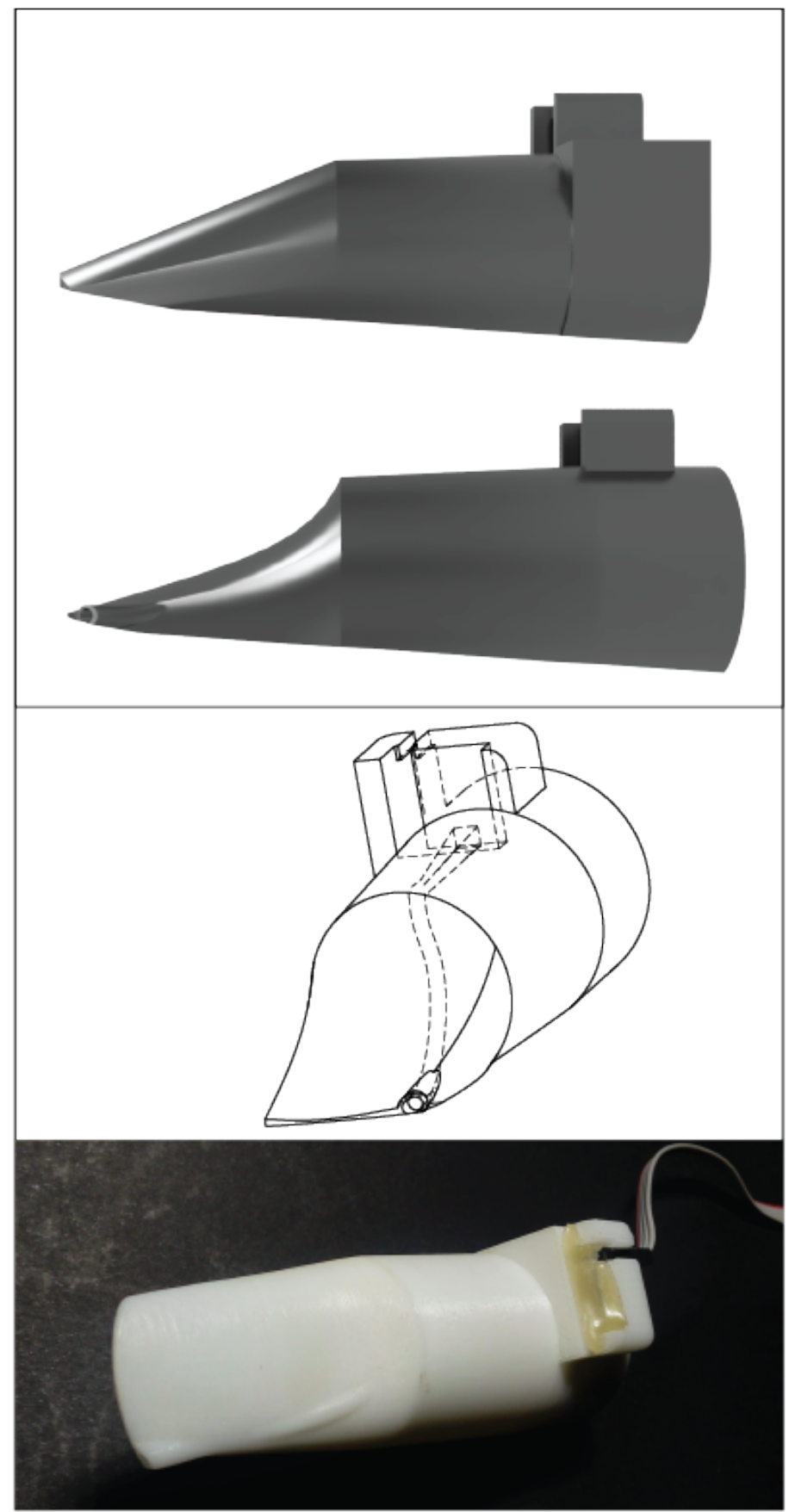

Fig. 28. At the top, a comparison of width of beak between first and second iteration. At the bottom, detail of the S-shaped conduit and the printed mouthpiece (sensor installed and sealed with silicone).

\subsubsection{Sensor}

To measure the air pressure, various kinds of sensors were considered. Unlike previous projects, this one has the advantage of $3 \mathrm{D}$ design customization which allows the opportunity to fit any reasonably sized sensor. The chosen sensor is a BMP180 designed 
by Bosch as a barometric pressure sensor commonly used in applications such as indoor navigation, GPS-enhancement for dead-reckoning, or slope detection, in sport devices (to obtain altitude profile for instance), weather forecast, vertical velocity indication (rise/sink speed) and others. ${ }^{195} 196$ Considering the applications for which the BMP180 was designed, this is not the most obvious choice of sensor. Nevertheless, due to the design of the GY68 breakout board on which is commonly mounted, it was easy to fit this sensor in the mouthpiece and match it with the end of the alternate air conduit.

The BMP180's most relevant features are: $300-1100 \mathrm{hPa}^{197}$ pressure range, an average current consumption of $650 \mu \mathrm{A}$ that can go down to $3 \mu \mathrm{A}$, supply voltage VDD of $1.8 \mathrm{~V}$ to $3.6 \mathrm{~V}$, a relative accuracy pressure of $\pm 0.12 \mathrm{hPa}$, and the use of the $\mathrm{I} 2 \mathrm{C}$ protocol at a 3.4MHz transfer rate. ${ }^{198}$

Unlike most sensors designed to measure air pressure, the BMP180 does not feature a funnel-like structure to redirect the air into it, which becomes unnecessary with the conduit built into the mouthpiece 3D model (see Fig. 29). The BMP180 can be purchased on its own or mounted on different breakout boards. For this project, the GY68 breakout was chosen because on this design the 'hole' that captures the air in the sensor is situated close to one of its edges, which makes it convenient to introduce it in the body of the mouthpiece and reach the end of the conduit. Other breakout boards place the sensor on the centre of the PCB, meaning that it would be necessary to carve into the model to reach the air conduit, this could compromise the structure of the mouthpiece.

The breakout pins help secure the sensor in place once they are connected to standard $2.54 \mathrm{~mm}$ connectors. The GY68 breakout design is perfect for handling and replacing the sensor if necessary as it only needs to be pushed into the opening in the mouthpiece designed to allocate it. Therefore, any user can replace it without the assistance of a technician and at a very low cost (usually under USD\$5)

\footnotetext{
195 https:/www.bosch-sensortec.com/bst/products/all_products/bmp180

${ }^{196}$ Bosch Sensortec, 'BMP180 Digital Pressure Sensor Data Sheet', 2015.

${ }^{197} \mathrm{hPa}$ (Hectopasqual) is a metric measurement unit of pressure equivalent to a millibar. It is commonly used to measure atmospheric pressure.

${ }^{198}$ BOSCH, 'BMP180 Digital Pressure Sensor Data Sheet', $2015<$ https://aebst.resource.bosch.com/media/_tech/media/datasheets/BST-BMP180-DS000-121.pdf>.
} 


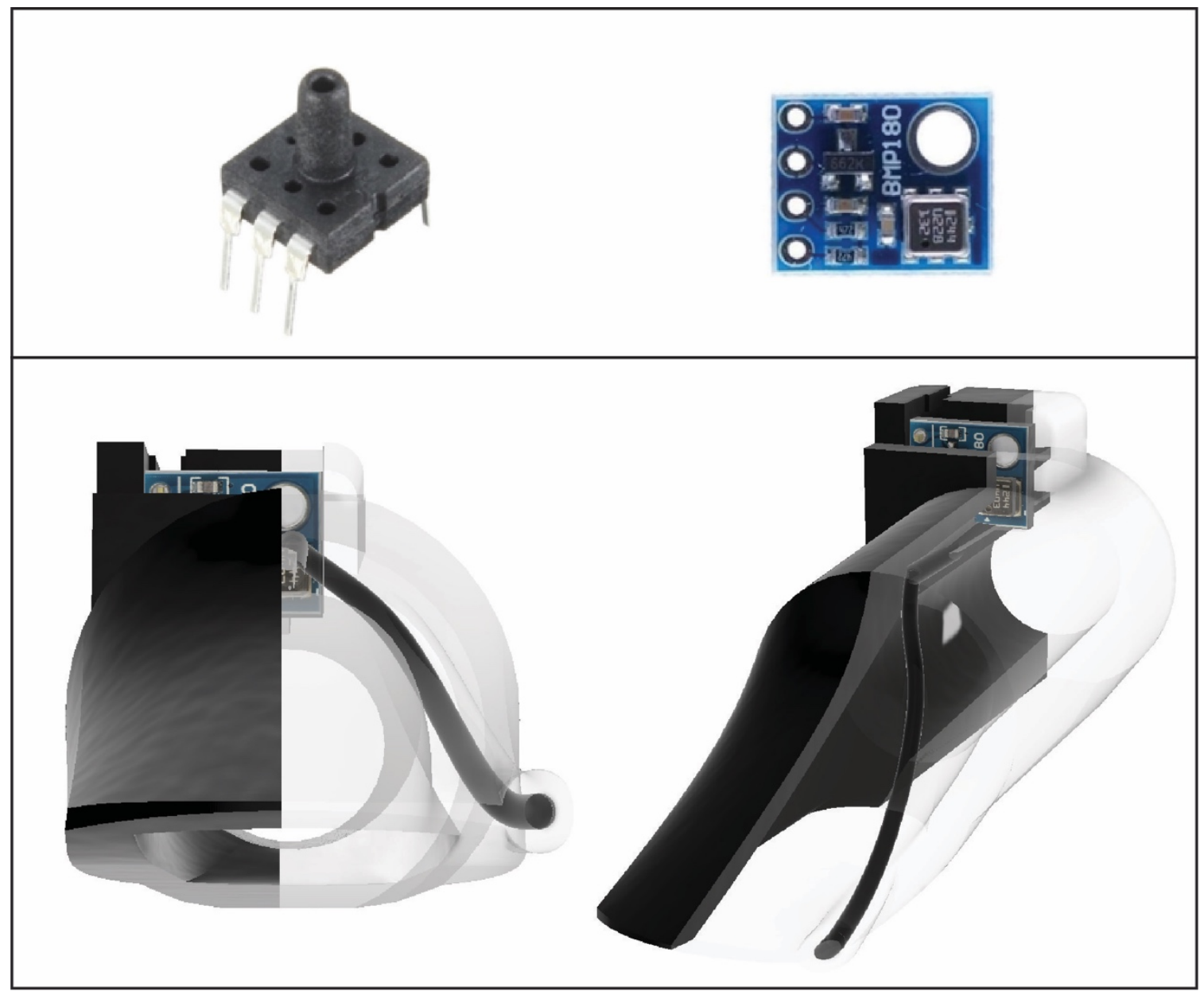

Fig. 29. Common air pressure sensor in a funnel-like casing and BMP180-GY68 (top). BMP180 on a cross-section rendered image of the mouthpiece showing the S-shaped air conduit (bottom).

\subsubsection{Board and On-board software}

The barometric sensor is connected to a Teensy LC board, ${ }^{199}$ sending data using the I2C protocol to a second Teensy board which handles data and audio. The program loaded on the board self-calibrates when the system is powered on. To calibrate, it firstly determines the ambient temperature, and then sets a current pressure (actual atmospheric pressure) as a baseline value, setting that value to zero. Once these parameters have been established, every iteration of the program loop compares the new results to the baseline value,

199 For more information on the Teensy LC, visit the developer's website at https://www.pjrc.com/teensy/teensyLC.html 
outputting positive numbers for any value greater than the baseline, and negative numbers to values under the atmospheric pressure (expressed in hectopascals).

The sensor is affected by temperature in a manner similar to woodwind instruments. To address this, the program performs a new calibration every five minutes to compensate for temperature changes that occur due to breathing and blowing into the mouthpiece. This calibration is done if there is a relative stability of values $( \pm 3)$ in 330 consecutive samples. This stability assumes that the performer has not played a note for ten seconds. The BMP180 is also capable of measuring temperature, which is one of the parameters used to perform the calibration. The sample rate is 33 readings per second, which can be changed according to the needs of the project: a faster sample rate could be beneficial to detect attacks more precisely and avoid latency in musical contexts. Another fact to be considered when deciding on a sample rate is the delay that occurs between the moment when the performer starts blowing and the sonic event, which depends on dynamics, expressivity, the hardness of the reed, the calibration of the instrument's mechanics (to avoid air leaks) and other factors. Further investigation must be done to address this issue.

\subsection{Software}

The HypeSax has a USB port that allows communication with a computer or a MIDI device thanks to the high flexibility of Teensy boards. For this project, the slave board is set up as a Serial device since its only function is to gather information from the sensors, organise it and send it to the master board. The master board is set up to work as a Serial/MIDI/Audio device. With this configuration, the HypeSax is able to send MIDI messages for full compatibility with MIDI devices or commercial software. It can work as an audio card to record and output audio (using the audio board adaptor), as well as send and receive audio signals through the USB port to a computer. It also communicates with a computer using the Serial protocol.

To take advantage of the latter feature, a server application was developed in the Max audio software development environment to receive, decode and organise the messages sent from the HypeSax. Once communication is established, the application shows a visual representation of the sensors' activity. The user can then re-route the data using either MIDI, OSC, Serial or Max's send/receive messages (when using the patch version of the application). 
The potential of the HypeSax as a controller allows for adaptability for the creative needs of a composer or performer. While most hyperinstruments have hardware augmentations that act as controllers mounted on an instrument, many of them lack true integration between the electronics and the instrument, as the synthesis remains as an additional output through an external source (speaker), rather than being integrated with the acoustics of the instrument.

The ideal for the HypeSax project is for it to be able to work as a standalone device without the need to communicate with a computer (such as guitar pedals). While the onboard software can handle data and process audio, further software development is needed for the HypeSax to perform intense signal processing. Meanwhile, computer software is used to achieve integration and will be featured in future releases.

\subsubsection{Acoustic integration through use of software}

As described in section 3.3, the Un-mute features a speaker that is mounted inside the bell of the saxophone. This allows for a new approach to hybridisation of acoustic and electronic sound. Recent developments such as Juan Arroyo's hybrid string quartet ${ }^{200}$ or the IMAREV project ${ }^{201}$ have demonstrated the way in which active instruments allow for an interesting approach to sound treatment through acoustic augmentation. To achieve this augmentation, special software capable of real-time audio analysis, sensor data analysis and audio processing has to be developed.

Any software developed to work with the HypeSax must follow the following data flow: first data is collected from the performance via HypeSax sensors. Next, this data is sent to the computer using the built-in USB port. Software then analyses audio and sensor data to process the signal. The signal is then sent back to the HypeSax and played back into the body of the saxophone to achieve audio hybridisation. In some cases, the software might allow feedback for a special audio treatment (see Fig. 30). More details on software that has been developed for the HypeSax are discussed in chapters 4 and 5 .

\footnotetext{
200 Houlès.

201 http://instrum.ircam.fr/imarev
} 


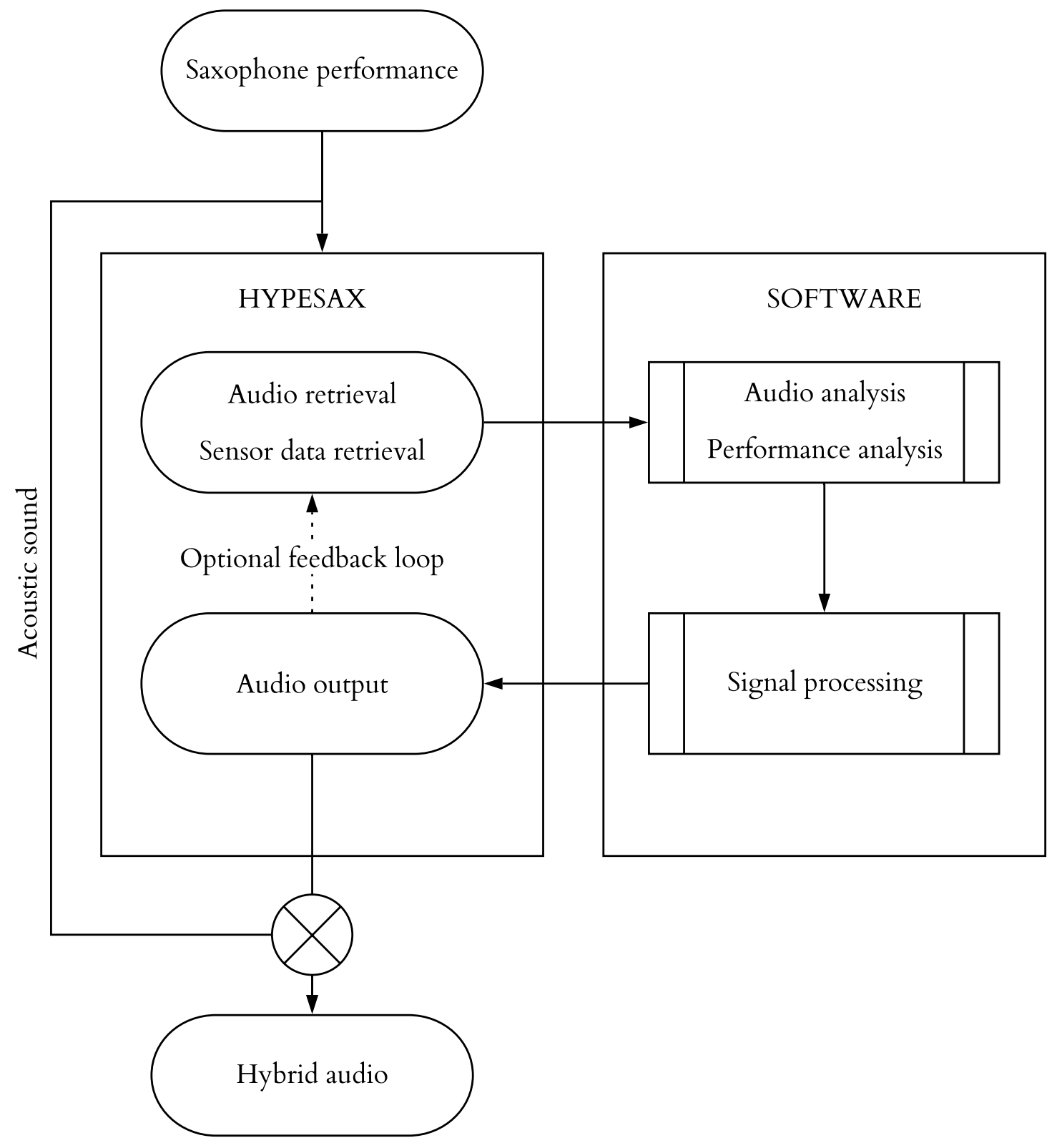

Fig. 30. Data flow for acoustical integration.

This chapter has served to present a thorough description of the HypeSax, its development and influences. Extra information about the mouthpiece can be found in my article 'A 3D Printed Hybrid Saxophone Mouthpiece for Data Collection' for the 2017 Australasian Computer Music Association Conference (ACMC) (see Appendix D) or my article 'HypeSax; Saxophone Acoustic Information for the NIME 2019 Conference (see Appendix E) In the following chapter I focus on evaluating this hyperinstrument based on a survey filled by potential users. I also present variations of the HypeSax, developed for tenor and soprano saxophones, based partly on the responses collected on the survey. 


\section{4}

\section{A NEW MEDIUM -HYPESAX EVALUATION AND ITERATION}

In order to have a broader view of the HypeSax as the medium, it was necessary to evaluate its potential. This was addressed by composing a portfolio of works and developing a user study. While the portfolio and musical ideas are discussed in Chapter 5, this chapter focuses on both discussing the potential use of the HypeSax as an adaptable medium and on presenting the results of a user-centred evaluation of the HypeSax.

This chapter has been organised as follows: first, I analyse how the development of a personalised instrument, in this case the HypeSax, emphasises the role of the medium in the ontology of the musical work. Then, I describe the user study designed to evaluate the efficacy of the HypeSax as the medium in the tripartite model. Following that, I present the findings of the user study. And, finally, I describe two new versions of the HypeSax, in this case for tenor and soprano saxophone, developed upon consideration of the feedback from the user study. 


\subsection{The HypeSax as hybrid medium: the bodyless sound and the mutability of the medium}

The tripartite model was theorised as a tool to approach modern compositional practiceswhere the composer develops software, hardware, any kind of instrument, or modifies an existing medium as part of the creative process — or to analyse the ontology of works from a quasi-rhizomatic ${ }^{202}$ perspective. The HypeSax then, in this context, takes the place of a malleable tool through which the medium can be reshaped in the creative process. In other words, the HypeSax is not intended to be an instrument, a mere object, but to be a canvas for creativity.

Every musical instrument is a canvas for creativity, especially in the hands of the expert musician, composer or performer. Nevertheless, the number of sounds that can produced using traditional acoustic instruments is limited due to the physicality of the their construction. A wooden recorder, for instance, can only offer tones within a specific range. These tones can vary in spectral content depending on the performing technique. With the use of more complex or 'extended' techniques, the recorder can offer a new range of sounds, for instance by striking its body with the fingernails or rubbing a stick against its body and over the tone holes (alla güiro). In this case, all of the sounds carry the sounding qualities of the of the wood used to build the recorder, plus qualities of a second object used to produce sound with the recorder such as air, fingernails, or stick.

The physicality of an instrument and the way in which we interact with such instruments give each sound a unique identity defined by a set of characteristics. These characteristics - explored by Pierre Schaeffer in his Treatise on Musical Objects ${ }^{203}$-are

202 The term 'rhizomatic' is used in A Thousand Plateaus by Gilles Deleuze and Félix Guattari, referring to a root system, in order to describe theory and research that that allows for multiple points of entry and exit of data as well as its representation and interpretation. In this case, the term 'quasi-rhizomatic' refers to the possibility of analysis and understanding the ontology of the musical work from different views that can have an influence on each other.

203 Pierre Schaeffer described and catalogued the characteristics of what he calls 'sound objects' in his Treatise on Musical Objects, more specifically on Book 3: Correlations Between The Physical Signal And The Musical Object; Book Four: Objects And Structures; And Book Five: Morphology And Typology Of Sound Objects Pierre Schaeffer, Treatise on Musical Objects: An Essay across Disciplines, trans. by Christine North and John Dack (University of California Press, 2017).. In this book, Schaeffer introduced the concept of typomorphology of sound, an analytical tool referring to the typology and the morphology of sound. The difference between these two is one of function, 'typology seeks to identify 
better understood under the concept of spectro-morphology proposed by Denis Smalley. This concept encapsulates the spectrum of a sound, consisting of the totality of its perceptible frequencies, and their relationship through the various stages of transformation of sound as it unfolds in time. The concept observes sound from its temporal origin to its end, describing its changes in dynamics (or morphology), and timbre (spectral typology). ${ }^{204}$ The unique spectromorphology of each sound produced by a musical instrument is determined by its the materials and construction, as well as the performing technique used to play it. These characteristics cannot be changed through traditional means. The musical instruments that fall under this paradigm can be classified under the Hornbostel-Sachs system in the following categories: idiophones, membranophones, chordophones, and aerophones. This catalogue groups instruments according to the ways in which they produce sound. All of the aforementioned categories include instruments that produce sound by vibrating their body, one of their components, or a column of air running through its body. In contrast, electrophones form a fifth category that incorporates instruments that can generate sound beyond the constraints of the physicality of their construction. ${ }^{205}$

Electrophones took an important role in the past century, as these instruments have allowed for new sounds and powerful outputs (which are especially useful for large concert venues and setups). Electrophones helped define new genres such as electronic music and experimental academic music, or popular genres like rock, pop, and many others. Electrophones not only changed the sound of music - and perhaps the taste of massive audiences - but also set new paradigms that helped us think about sound more deeply. The

and isolate sonic objects from a sound continuum, and then to compare and classify them. Morphology seeks to qualify (or describe) the objects'. Carlos Vicente de Lima Palombini, 'Pierre Schaeffers TypoMorphology of Sonic Objects' (Durham Univeristy, 1993), p. $66<\mathrm{http}: / /$ etheses.dur.ac.uk/1191/>.. In typology sounds are classified as balanced (not too complex, not too simple) or unbalanced and its sustainment or iteration. The morphology is divided in seven categories: mass, harmonic timbre, grain, allure, dynamic, melodic profile, and mass profile.

${ }^{204}$ More on Denis Smalley's concept can be found in 'Smalley, Denis. 1986. "Spectro-Morphology and Structuring Processes." In The Language of Electroacoustic Music, edited by Simon Emmerson. London: Palgrave Macmillan.'

205 The Sachs-Hornbostel classification system was originally designed and published by Erich Moritz Hornbostel and Curt Sachs in Zeitschrift für Ethnologie in 1914. This system was updated and published in 2011 by the Musical Instrument Museums Online Project (MIMO). This revision is available online at:

http://www.mimo-international.com/documents/Hornbostel\%20Sachs.pdf 
synthesiser, for instance, has allowed experts and non-experts to explore spectromorphology of sound, by liberating the instrument from the physicality of its body. On the other hand, by decoupling sound and body, electrophones have created a new paradigm that never existed before: a sound with no body.

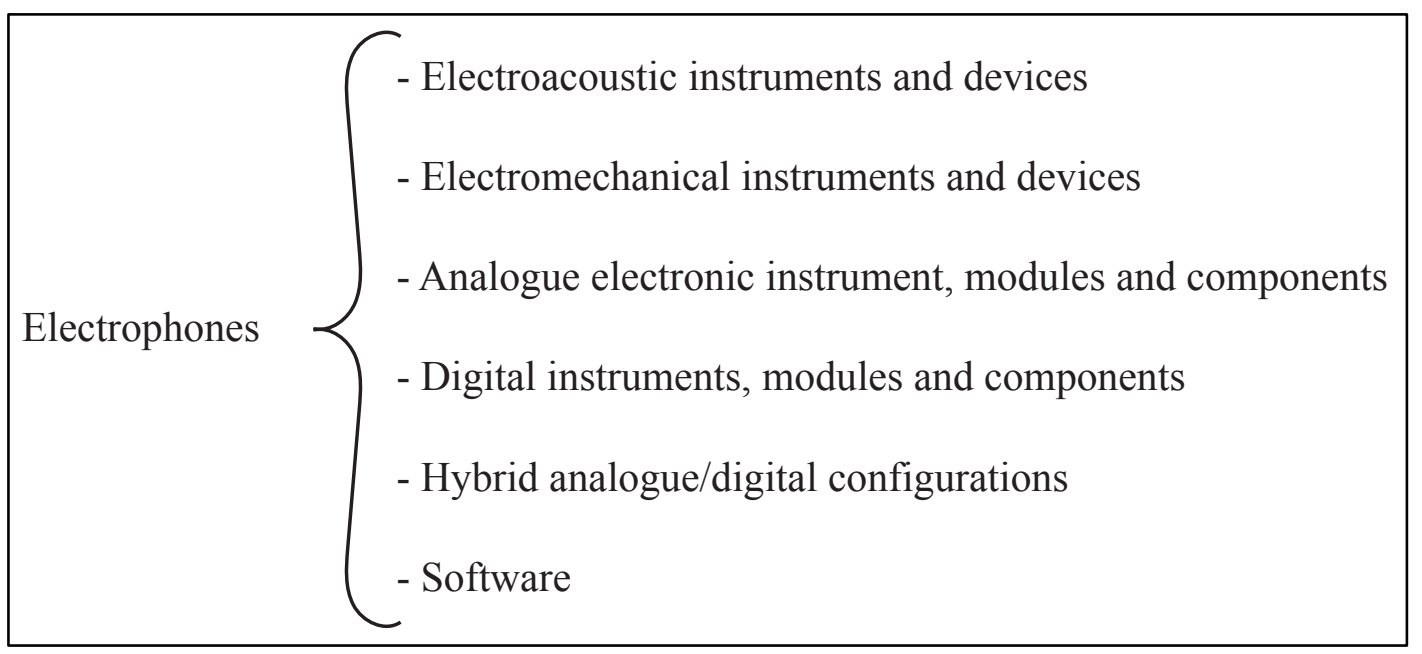

Fig. 31. Electrophones and the six subcategories according to the Hornbostel-Sachs classification.

It becomes necessary to clarify that 'Software,' as included in the list, refers to a standalone software application running in a computer, for instance, and not for example to the software running in a microcontroller inside an instrument such as a digital instrument.

\subsubsection{The bodyless sound}

According to the Hornbostel-Sachs system, electrophones are:

Instruments that use materials generating acoustic sounds, mechanicallydriven signal sources, electronically stored data or electronic circuitry to produce electrical signals that are passed to a loudspeaker to deliver sound. ${ }^{206}$

Despite of the original action that triggers their sound, electrophone tend to rely on loudspeakers to output a sonorous event. Unlike the instruments classified in the other

${ }^{206}$ MIMO Consortium, 'Revision of the Hornbostel-Sachs Classification of Musical Instruments by the MIMO Consortium', 2011, p. $21<$ http://www.mimointernational.com/documents/Hornbostel\%20Sachs.pdf $>$. 
categories, electrophones' sounds do not necessarily maintain a constraint related to the relationship between the material, construction and performance. Thanks to the invention of the loudspeaker (and other kinds of transducers), 'non-physical' electronic or virtually designed sounds can be produced, and sounds of physical origin can be reproduced without the presence of the vibrating body or instrument that would otherwise generate that sound. Additionally, the development of different recording techniques - and more recently electronic media and computers - made it possible to understand and explore sound in a different way, allowing for the manipulation of sound to be reproduced through speakers. This paradigm produces a unique consequence: a bodyless sound. This concept refers only to the fact that sounds produced by the speakers (or other electronic mediation), even when they can have a physical origin, can have the sound qualities of a body that is not present. A synthesiser, for instance, can produce a variety of timbres without making any physical modification to the instrument. Its sound is not subject to its physicality. Similarly, a recording of a violin can be processed and, while it has a physical origin, once it is reproduced, there is not body of a "modified violin" present. I should clarify that, while the speaker itself is the body that is producing the sound, I do not see it as the body of the sound since its physicality does not shape the characteristics of the sound - although the characteristics of the speaker will, to some degree, affect some aspects of the sound.

As discussed in sections 2.6.3 and 2.6.4, embodiment has problematised the ontology of the musical works when it comes to the creative process. However, freeing sound from the constraints of a physical correspondence can actually broaden creative possibilities. The most notable cases of creative approaches that take advantage of the bodyless sound are musique concrète and electronic music. Musique concrète consists of recorded sounds that are manipulated and reorganised in such way that their bodily characteristics can be completely lost and replaced. Electronic music, produced with analogue or digital oscillators signal generation, is intrinsically bodyless. The 'un-natural' origin of electronic sounds gives the possibility of freely shaping their spectromorphology, giving these sounds a level of malleability that sounds produced with physical instruments do not possess.

Although the Hornbostel-Sachs system clearly situates hyperinstruments (including the HypeSax) in the broad category of electrophones, it becomes more difficult to find a 
place for hyperinstruments in one of its subcategories. In this classification system there are six subcategories of electrophones:

- Electro-acoustic instruments and devices. Modules and configurations of acoustic, vibratory mechanisms and electronic circuitry such as transducers and amplifiers. The acoustic or mechanical vibration is transduced into an analogue fluctuation of an electric current.

- Electromechanical instruments and devices. Configurations of (electrically excited) silent, mechanical moving parts with encoded patterns, and electronic circuitry. The movement enables the encoded patterns to be transduced into an analogue fluctuation of an electric current

- Analogue electronic instruments, modules and components. Continuously varying electrical signals are passed to a loudspeaker to produce sound. The electrical signals are generated using electronic circuitry. Modules and configurations containing analogue fully electronic devices used to produce, process and communicate electronic sound signals and/or sequences of signals

- Digital instruments, modules and components. Electrical signals are generated in the form of quantised sequences of pulses. These are converted to continuous signals that activate a loudspeaker. Modules and configurations containing devices to digitally design and process electronic sound signals and/or sequences of signals

- Hybrid analogue/digital configurations. Devices with analogue oscillators and digital filters etc.

\section{- Software $\boldsymbol{S}^{207}$}

At this sub-level, this system fails to situate hyperinstruments, since these augmented instruments often produce and process audio signals simultaneously and in multiple ways. This system is not adequate for hyperinstruments because it focuses on how the sound is

${ }^{207}$ MIMO Consortium, pp. 21-24. 
produced, but not of how the user interacts with the sound. If the focus is on interactivity, then it becomes of utmost importance to think of hyperinstruments as instruments augmented with the use of one or multiple controller configurations. While controllers can be classified in the subcategory of electromechanical instruments, they do not have a fixed physical gesture-to-audio relationship. The quality of controllers allows hyperinstruments to interact with any device that can be classified as electrophone in any of its six subcategories, or any other device that is not built to produce sound; for instance, a set of blenders that could be activated by the actions of the performer. In other words, as a controller, a hyperinstrument can interact directly with acoustic sounds, mechanicallydriven signal sources, electronically stored data or electronic circuitry in order to produce sound. In this way, while the HypeSax can be classified in the subcategory of electroacoustic instruments, it is a saxophone augmented with a controller that gives it the possibility to further extend its capabilities through digital instruments and software (via Serial, MIDI and OSC communication protocols). With these possibilities, the HypeSax and other hyperinstruments can be reprogrammed to produce different sounds without the constraints of their physicality, and allow for a constant renovation of its intrinsic bodyless sound.

\subsubsection{The mutability of the medium}

In the tripartite model, the medium is an important element because it has a direct effect on the work due to its physical 'responsiveness' (analysed in section 3.1). By losing the body of the instrument, the medium is no longer focused on the instrument. When the limits of the physicality of the body of the instrument are overcome, the medium is now only limited by the decisions taken by its designer. A synthesiser, for example, offers a set of characteristics and configurations previously decided by its maker, but these characteristics can be set up, adjusted and renovated by the user. The musician, for instance, can set up the way in which the synth responds to physical actions and the sounds it produces by adjusting envelopes. Being able to make these decisions gives the work a unique malleability. This characteristic is what I call the mutability of the medium. It provides another level of creative engagement with the work, where 'redesigning' or creating the medium becomes a creative process that can take part at any stage in the tripartite model. 
Through mutability we can find the medium involved at the score or at the performance stage of the tripartite model. At the same time, despite the fact that mutability brings new affordances, it comes with issues of embodiment and its own limitations specific to each situation and state of the medium. Often, new constraints are inflicted by this 'creative' process. It is common to hear multiple compositions that sound very similar due to the restrictions imposed to the newly-created or redesigned medium: this is exemplified by composers who use the same software to apply the same techniques to their works.

At this point, it is important to remember the function of the tripartite model to avoid any confusions of the role of the medium. The model offers an analytical tool and possibly a perspective from which to organise the creative process. In the model, the medium has a quasi-rhizomatic relationship with the score and performance. Any musical instrument can be the medium, and any decisions made at the score or performance level in relationship to how the instrument is used tend to be restricted by its physicality. Hence, the medium is not opened to the creative process, only to its reception (which in turn can provide the possibility for the poietic and esthesic process to enter a feedback loop, as explained in section 2.4). While this is the case in any idiophone, membranophone, chordophone, and aerophone, the mutability offered by electrophones allows the creative process to be acted upon directly in the medium.

In the specific case of the HypeSax - and other augmented instruments - the mutability of the medium blends with the bodily characteristics of the saxophone. The HypeSax provides the saxophone with a new identity and instrumental agency through mutability. Analysing the hybridity aspects of the HypeSax and its adaptability to software, it can be argued that the HypeSax takes the saxophone to a new state of the medium, maintaining the original identity and agency of the saxophone while providing it with mutability, thus allowing the use and transformation of HypeSax to be inserted in the score and the performance as another element of the poietic process.

This section has served to analyse how creating an instrument, either by developing or manipulating hardware or software, gives the medium an enhanced role in the ontology of the work. In the next section, I describe a user study that offers an insight into the reception of the HypeSax design. This study seeks to determine whether this design helps the saxophone reach that new role in the ontology of the work in the eyes of the user. 


\subsection{User-centred study}

The HypeSax was designed in the spirit of the multi-directional tripartite model, where score, performance or medium can provide the 'initial spark' for the work. Similarly, the 'initial spark' for the HypeSax design (the medium) could have originated from a creative or performing perspective, or with a specific sound goal. A specific sound was not considered as paradigm to design of the HypeSax, since the intention of developing the HypeSax was to provide a malleable tool which could be reprogrammed to offer an openended potential for new sounds. Deciding to develop a design based on an intended sound would have gone, to some degree, against the idea of providing mutability. The creative and performing perspectives were thus combined to approach the initial idea and design of the HypeSax in an intuitive way based on my personal experience and the needs of my creative process, as described in the introduction chapter. As a consequence, the HypeSax was designed and developed before being presented to the user, rather than presenting iterations to the user which developed in a final product. While a formal user study took place after the completion of a prototype HypeSax, expert saxophonists Jasmine LovellSmith and Peter Liley were approached periodically during the initial design phase. Their feedback provided particular insight into ergonomics-related matters.

According to Kathy Baxter et al. in Understanding Your Users: A Practical Guide to User Research Methods, Tools, and Techniques, user experience research can be broad and holistic due to its subjectivity. It often "seeks to gather "user requirements" for the design of technologies [...] or evaluate the usability of an existing technology. User requirements refer to the features/attributes a product should have or how it should perform from the users' perspective'. ${ }^{208}$ Considering that the HypeSax was not developed to fulfil a series of technical goals but to offer possibilities for the composer and performer to engage with the medium in a new way, a study, centred on the user experience, was designed. This study does not focus on testing the HypeSax as a product, but on analysing its potential role in the context of the tripartite model from the perspective of the user, and its latent use as a creative canvas. For this reason, the study did not enquire about the usability of the HypeSax aiming to develop a perfect product. It sought to understand the

${ }^{208}$ Kathy Baxter, Catherine Courage, and Kelly Caine, Understanding Your Users: A Practical Guide to User Research Methods, Tools, and Techniques (Elsevier Science \& Technology, 2015), p. 4. 
perception, ideas, wants and concerns of the user in order to situate the concept of the HypeSax (current development or future iterations) in the tripartite model. The results from this user study were expected be informative and open, seeking to observe the HypeSax as a medium in the tripartite model, rather than to point and give solutions to problems found in the design. Nevertheless, the responses aided in finding design problems. This information translated in the development a new version of the HypeSax that can be used with soprano, alto and tenor saxophones, with the potential to be used with any other saxophone (see section 4.4).

The user study consisted of inviting participants to test the HypeSax and respond a survey-based questionnaire. Its results provided insight into whether the musical, interactive and operational design considerations were fulfilled by the HypeSax presented to the users. The design criteria goals presented in 3.1 are: musical goals focused on the potential sounding result and its use in musical context; interactive goals which refer to the way in which the performer interacts with the HypeSax; and operational goals focused on physical design, construction and assemblage. At the same time, qualitative data obtained was useful to observe the role of the medium having an influence in the score and performance, these findings are discussed in Chapter 5 (see section 5.2.4).

\subsection{User study design}

The goal of developing the HypeSax is to explore the consequences of crafting a new medium in the creative process, in which embodiment, physicality of sound, and bodyless sound $^{209}$ are involved. This means that, based on the tripartite model, an insight in the relationship between the medium and both the score and performance needed to be analysed. This study is, thus, centred on observing the response of the composers and performers - analogous to score and performance, respectively - to the HypeSax. To do this, potential users were invited to participate in a series of activities and answer a questionnaire. This study was conducted with the approval from the Standing Committee of Human Ethics Committee at Victoria University of Wellington (Reference No. 0000026089).

\footnotetext{
${ }^{209}$ Synthesised sound that is introduced into the saxophone using the Un-Mute
} 


\subsubsection{The participants}

The study was realised with a set of people who possess the following characteristics:

- Have familiarity with the saxophone construction.

- Have familiarity with the saxophone: saxophone techniques and fingerings.

- Have an understanding of the actions involved in controlling the embouchure.

- Basic understanding of the relationship of the performer and the saxophone.

Two kinds of participants were invited: saxophonists and composers. Due to the specialised nature of the HypeSax, participants were selected on the basis of:

- Having experience performing the saxophone for at least three years (saxophonists),

- Having obtained a degree in composition and having composed at least one work for saxophone (composers).

In total, twelve saxophonists and five composers took part in this user study, of which two of these participants acted as both saxophonists and composers. While the sample size is not large enough to represent a global community of saxophonists and composers interested in working with augmented instruments, the results were helpful in informing and guiding my research. While this sample size won't allow for wide-ranging statistical significance to be derived from the data obtained, the size is sufficient to provide meaningful heuristic guidance.

\subsubsection{The activities}

A set of activities for saxophonists and composers were design for this study:

- Saxophonists were presented with a ten-minute-long video that described the HypeSax, its construction, assemblage and main capabilities. ${ }^{210}$

- As a second step, they were asked to assemble the HypeSax onto a saxophone. This is done in order to assess whether the design presents any

${ }^{210}$ While the participants could have discovered some of the characteristics of the HypeSax, I decided to offer the video aiming to have more efficient testing sessions. 
initial barriers that would prevent the user from approaching the HypeSax intuitively, a condition that facilitates engagement with creativity.

- Following this, they were asked to set up the saxophone to work with The Hub, a software developed to facilitate connection between the HypeSax and a personal computer (see section 5.1.2). This is an important aspect to asses, since facilitating this step can potentially allow users to engage with the HypeSax via connecting to a previously known software.

- Next, they were asked to test the mouthpiece, both to obtain information on the response of the mouthpiece, and to give the users opportunity to get used to the unique design and feel of the mouthpiece (see section 3.6.2).

- Then, they were presented with the 'test sheet,' a score presenting a series of musical exercises aiming to demonstrate some of the HypeSax's capabilities. This score presents 5 exercises that allow the user to manipulate all of the sensors included in the HypeSax, as well as the acoustic feedback generated by the Un-mute and manipulation of the tube's length (see Fig. 32 or Appendix C). The instrumental techniques available for the tests included: Audio feedback control, polyphony through combining natural saxophone sound and feedback, timbral modification through reinforcement of certain partials, ricochet effect through recognition of slap tongue, and synth control through air pressure control. More details about these techniques can be found in section 5.2.3. These techniques were chosen for this test as, by playing these techniques, all of the sensors are tested. This provided an insight of the general state of the design.

- Finally, they were asked to answer a questionnaire, aiming to collect information relevant to evaluate and improve the HypeSax.

- Composers were also asked to watch the ten-minute-long video.

- Next, they were asked to write a short musical idea which could have been a gesture. This work was to be no more than one minute long. Feedback from a saxophonist was offered in case it was necessary. They were asked to submit their composition in no more than three months. This activity 
aimed to: a) asses how easy it is to develop a musical idea for an unknown instrument that offers the possibility of transforming according to the needs of the creator, b) observe if embodiment would be a preoccupation for the composers and asses the relation of medium-performance from the perspective of the creator experimenting with the HypeSax, and c) to generate potential musical ideas that could drive the development of the HypeSax in new directions.

- Finally, they were asked to answer a questionnaire.

Alto Sax.

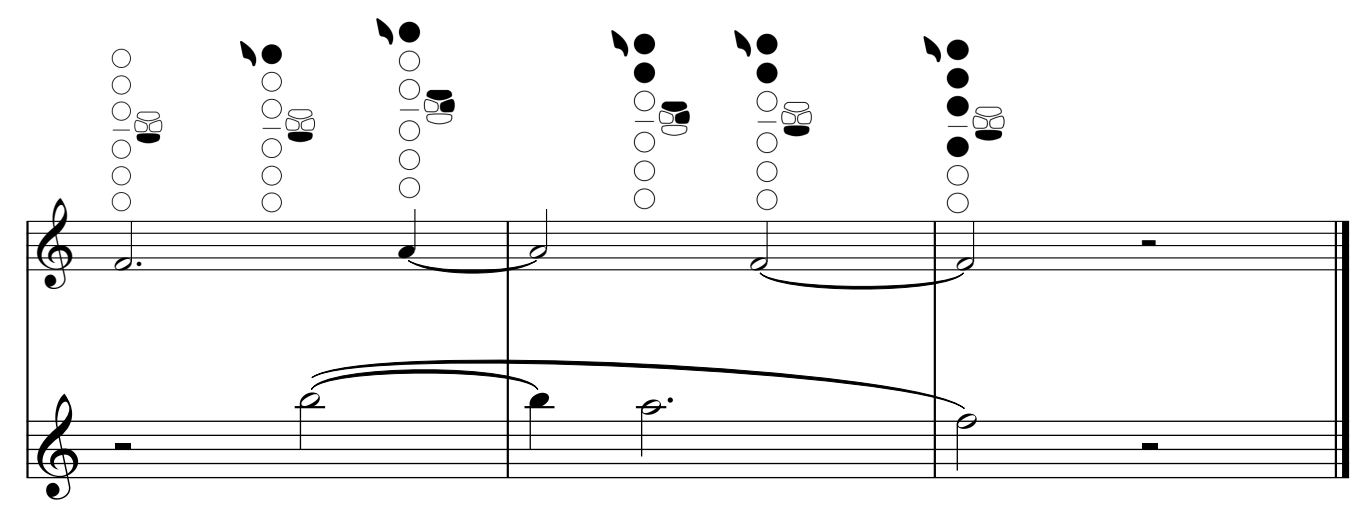

Fig. 32. Fourth exercise found in the 'test sheet.' The top staff (feedback) represents an approximate sounding pitch of acoustic feedback generated by the indicated fingering and gain control. The lower staff must be performed as usual. The result of combining both is a polyphonic sound of acoustic saxophone and feedback, which resemble each other.

\subsubsection{The questionnaire}

The questionnaire, intended to obtain the perspective of the two kinds of participants, contains two sections: Section A was exclusive to saxophonists, while Section B was to be responded by composers. Participants could act as saxophonists and composers, responding to both questionnaires. It was necessary to make a distinction between the answers of the performers and composers not only because their experience and relationship with the saxophone is different, but because the HypeSax is intended to fit in the tripartite model. This implies that different perspectives require different questions in order to be able to analyse the answers from the perspective of the score and the performance in relation to the medium. 
Both sections A (for saxophonists) and B (for composers) open with a set of questions intended to identify the profile of the participant. This is followed by a set of questions aiming to observe the fulfilment of the design criteria: musical, interactive, and operational goals. The order in which the questions were presented was not intended to be hierarchical or biased, the order simply follows the flow of the activities previously realised in the study. These questions were to be answered by selecting a number in a Lickert 5-point scale. The final set of questions open the possibility for participants to share any comments or suggestions for the future of the HypeSax. In the following sections I discuss the purpose of each question and summarise the answers. All questions and full answers can be found in Appendix B.

In the following subsections of section 4.3.3, I present the results of the survey. The questions, designed to evaluate the HypeSax according to the design criteria presented in section 3.1, as well discussions about the results, are presented in the order described in Table 1.

Table 1. Structure of section 4.3 .3

\begin{tabular}{|c|}
\hline Operational considerations questions and responses \\
\hline Questions \\
\hline Results \\
\hline Musical considerations quedtions and responses \\
\hline Questions \\
\hline Results \\
\hline Interactive considerations questions and responses \\
\hline Discussion on interactivity \\
\hline Questions and results \\
\hline Reflexions on results \\
\hline
\end{tabular}




\subsubsection{Operational considerations questions and responses}

The questions pertaining to the operational considerations help evaluate the success of the design in terms of the construction of the HypeSax. These questions are:

\section{For saxophonists:}

- 4. From 1 (very difficult) to 5 (very easy), how difficult did you find setting up the HypeSax hardware?

- $\quad$ 5. From 1 (very difficult) to 5 (very easy), how difficult did you find setting up the HypeSax software?

- 10. What (if anything) would you change about the design of the hardware? Cosmetic comments are acceptable but comments on ergonomics or ease of setup are preferred.

\section{For composers:}

- 4. If you used a different software from the one loaded onboard or the provided computer software, from 1 (not at all) to 5 (very) how difficult was it to make the HypeSax communicate with the chosen software?

- 5. Did you require additional help using the HypeSax together with another software?

In general, the results show that dealing with hardware was not difficult, and that the approach to augmenting the saxophone through using an attachable modular system seems to be successful. On the other hand, the results of dealing with the hardware depended greatly in the previous experience participants had using hardware. Nevertheless, the interaction with the hardware changed during the time the survey was in progress, as an update to Max forced me to make changes on The Hub. ${ }^{211}$ In the new version of the software, a help guide was included, pointing the steps to set up the hardware (see Fig. 54), thus making question five of Section $\mathbf{A}$ (for saxophonists) as well as questions four

211 The Hub, created in Max, is the software designed to facilitate reading data from the HypeSax in order to visualise the incoming data. The data collected by The Hub can also be re-routed to be used in other Max patches or to control third party software (see section 5.1.2). 
and five of Section B irrelevant, considering that the majority of the participants responded to the survey after the update was made.

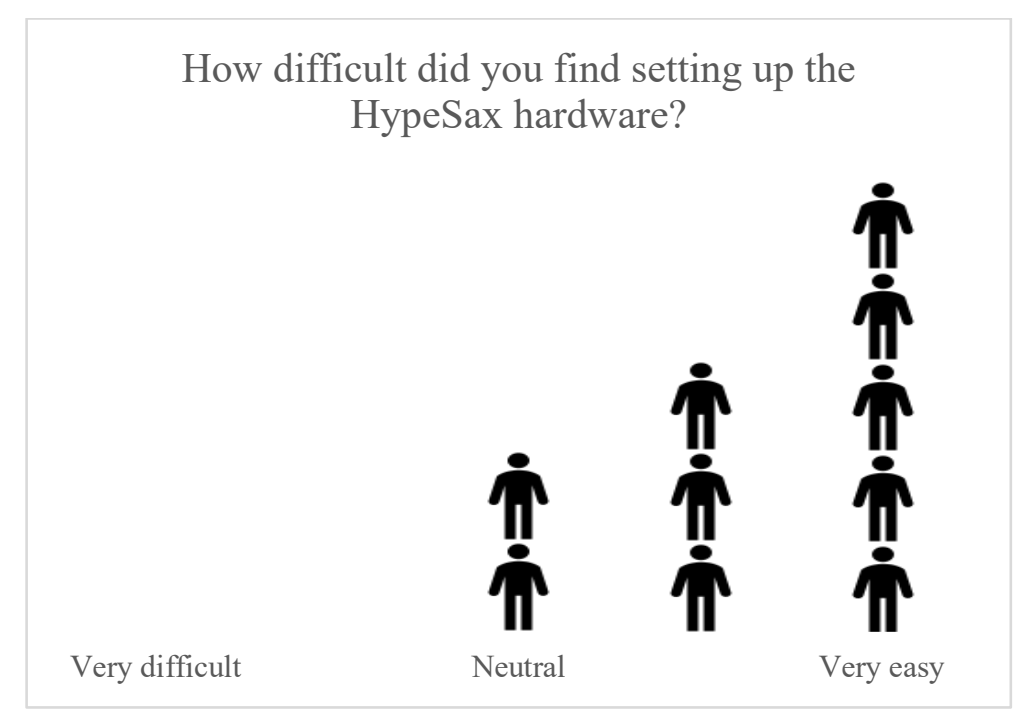

Fig. 33. The assessment of hardware setup was favourable.

\subsubsection{Musical considerations questions and responses}

In Section A (for saxophonists) of the questionnaire, two questions relate to the musical design criteria. These questions are also found in Section B:

12. From 1 (not at all) to 5 (very), how easy to understand and useful did you find the musical notation found on the "Test Sheet"? (see Fig. 32, Fig. 34, or Appendix C).

13. Do you have any suggestions about how to improve the suggested notation? Please attach your suggestions on a new page. ${ }^{212}$

${ }^{212}$ The numbering of the questions reflects their position in the questionnaire. 


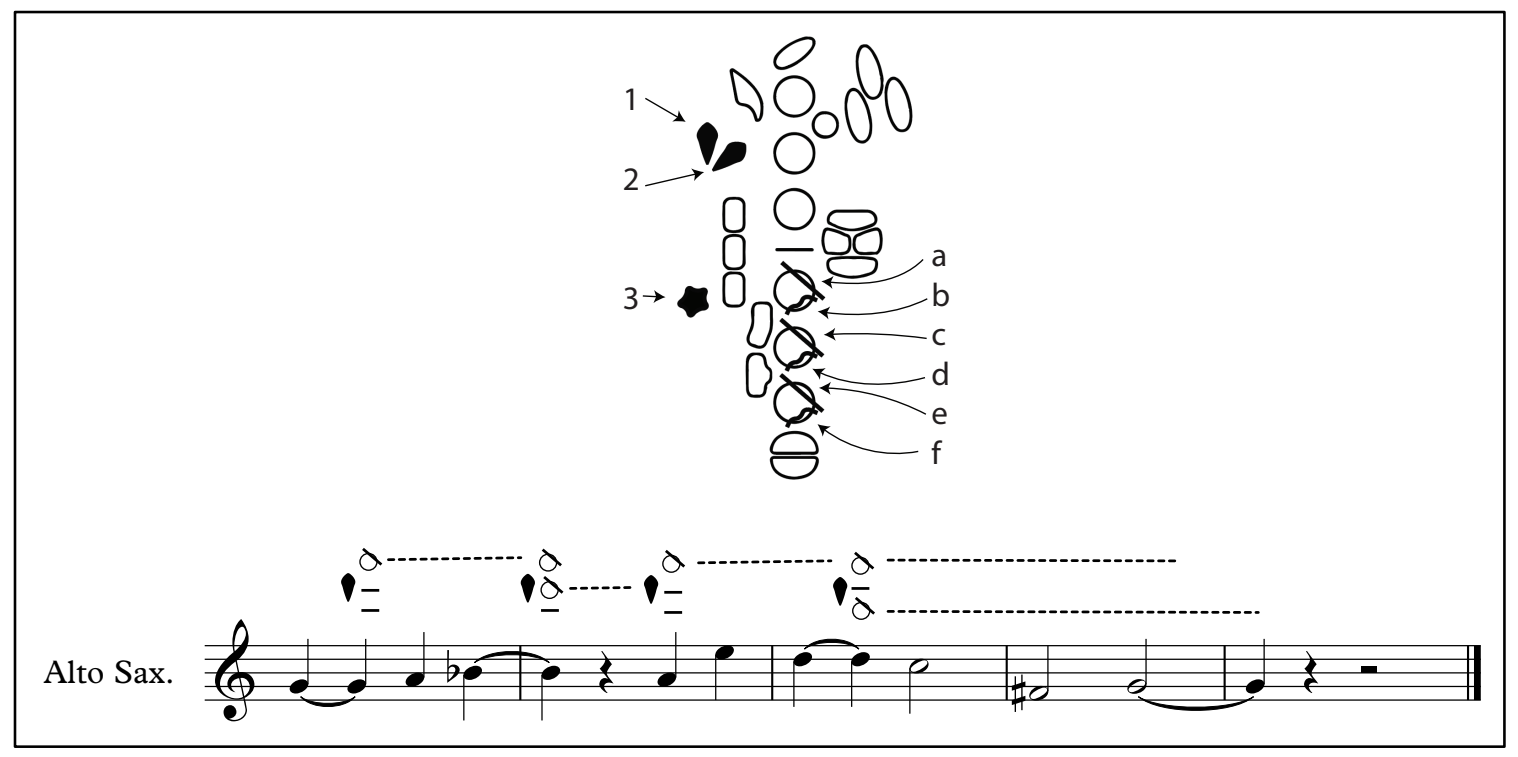

Fig. 34. The fingering chart (top) shows the extra keys where thumb buttons are labelled 1, 2 and 3, while touch buttons on keycaps are labelled with letters. Exercise one of the 'test sheet' (bottom) shows a melody and indications of combinations of key 1 and touch buttons to record and produce granular synthesis of the sound currently played. In bar 3, beat 2 , for instance, the saxophonist plays the pitch D while, while holding key ' $\mathrm{a}$ ', granular synthesis reproduces and holds the pitch A (from

bar 2, beat 3) and D, holding key 'e'. These two sounds are elongated throughout bar 4 .

On the surface, these questions seem to only address the notation. However, considering that notation is a way to communicate a musical idea, by presenting these questions, these questions present an invitation to engage with a creative approach to performing. Question twelve served as an introduction to engage with the thought of portraying a musical idea. Question thirteen sought responses that could provide an insight into how the performer can engage with musical goals of the design, such as performative nuances, the performerinstrument-sound relationship or their interest in gestural-instrumental techniques.

Answers to question twelve show a tendency towards accepting the proposed notation (see Fig. 35). Nevertheless, only four out of twelve participants engaged with it by giving a suggestion in question thirteen. This contrasts with the responses made by the composers to the same questions, where the results show that not only did they disagree with the effectiveness of the notation, but their suggestions in response to the following question show engagement on how to convey a musical idea. To a certain degree, one could assume that composers might prefer to indicate how to realise a musical idea via a clear notation, while performers might not be as interested to notate every detail, possibly due to the fact that their approach is more based on embodied experience. Interestingly, the two 
participants who responded the questionnaire acting as both performers and composers answered this question with a neutral response (three).

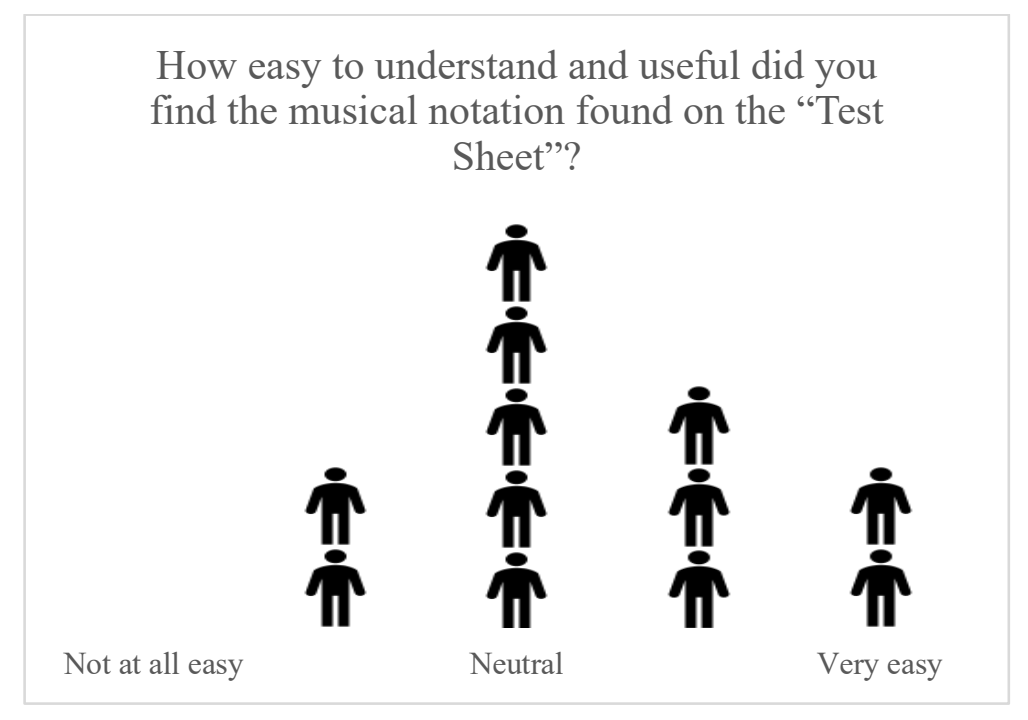

How easy to understand and useful did you find the musical notation found on the "Test Sheet"?

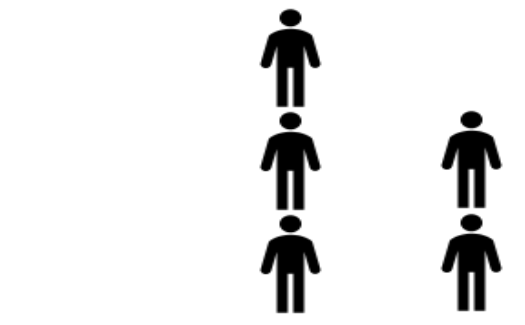

Not at all easy

Neutral

Very easy

Fig. 35. Performers' (top) and composers' (bottom) answers to the same question.

Section B (for composers) presents five questions related to the musical design criteria:

6. From 1 (not at all) to 5 (very) how difficult was it to integrate the new instrumental techniques to your composition?

7. From 1 (not at all) to 5 (very), how likely is it that you will want to write another composition using the HypeSax or a similar development?

Questions six and seven are designed to evaluate the current sounding possibilities offered by the HypeSax software and their possible future. As showed in Fig. 36, the results show a general acceptance and interest to use the HypeSax and integrate it into the participants compositional palette. 


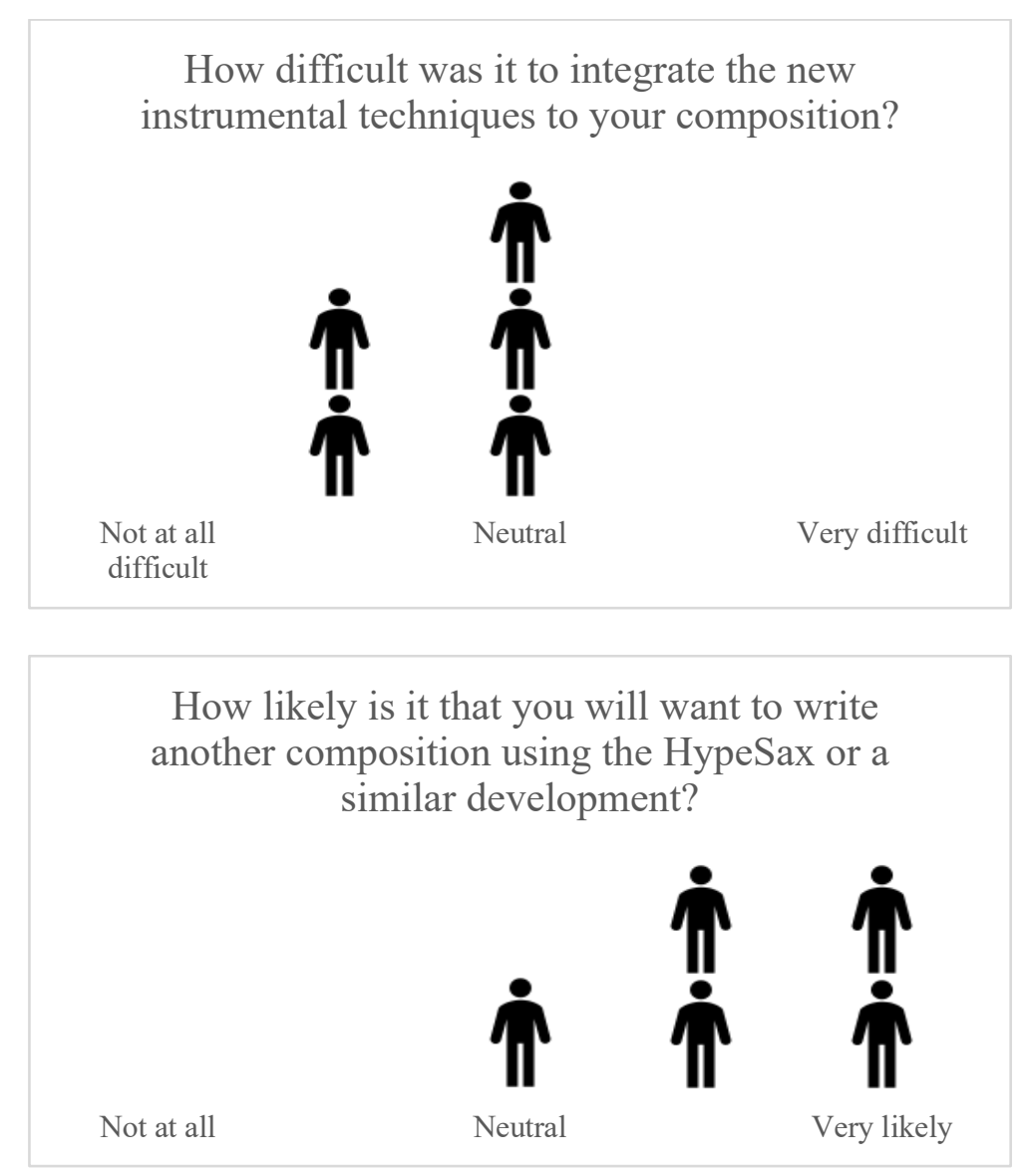

Fig. 36. Responses to questions 6 and 7 of Section B.

8. What other instrumental techniques or sound processes would you like to achieve using the HypeSax in potential future compositions?

This question opened the possibility for the participants to offer an insight of their perspective of what is missing or which direction the HypeSax could take in a musical context. The responses for this question were quite varied and reflected a general interest in applying usual sound effects such as reverb, delays, or distortion to the sound palette of the saxophone. "It would be great if I had preset effects like guitar pedals that can be turned on and off easily" said one of the participants. Suggestions also listed control of other musical devices, as well as interaction with other media and audio-visuals.

9. From 1 (not at all) to 5(very), how easy to understand and useful did you find the musical notation found on the "Test Sheet"? 
10. Do you have any suggestions about how to improve the suggested notation? Please attach your suggestions on a new page.

Finally, questions nine and ten are the same of questions twelve and thirteen of Section A, however, the results point in a different direction, possibly due to the composers' experience conveying a musical idea through notation (see Fig. 36). The suggested notation demonstrates interest in communicating performative nuances more clearly, with suggestions that resemble more of a tablature, where actions are described along with the expected sonic result, as exemplified in Fig. 37.

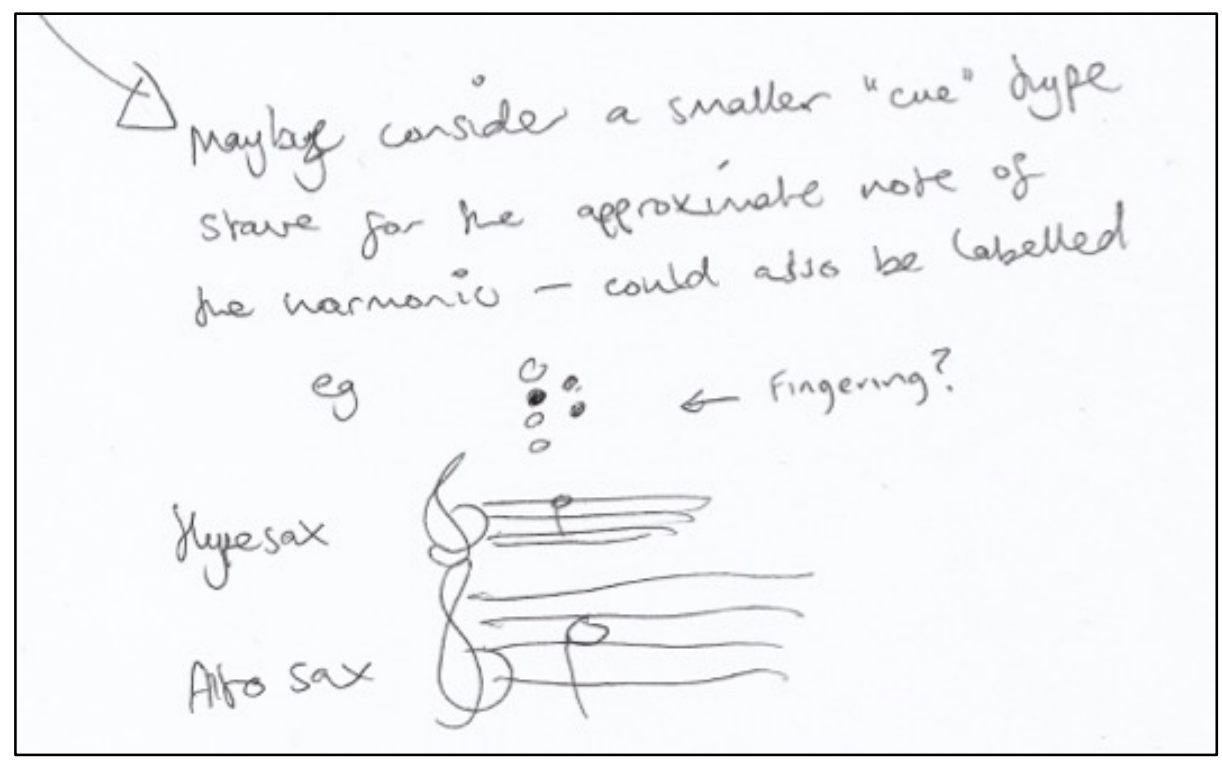

Fig. 37. Notation suggested by one of the survey participants.

Difficulty involved in mastering the techniques can prevent fine control. This can possibly be addressed by developing software updates and by integrating visual or haptic feedback which can help the performer learn and master new techniques, an approach that has already been proved to be effective. ${ }^{213}$

With the possibility for connecting to third party software and re-programming, the instrumentality can be maintained while offering variety in the relation between performer, instrument and sound. Gestural-instrumental techniques, on the other hand, offer a new approach to the saxophone from a quasi-choreographic sounding perspective. However,

${ }^{213}$ Qianwen Chen, 'A HAPTIC PARTIAL-GUIDANCE SYSTEM FOR FLUTE TUTORING'

(Dartmouth College, 2017), p. 6. 
further development and integration of user concerns, specifically on software, is needed. A detailed description of gestural-instrumental techniques is found in section 5.2.3.

\subsubsection{Interactive considerations questions and responses}

The interactive goals of the design criteria focus on the way the performer interacts with the new sounding possibilities of the saxophone through the use of the HypeSax. For this reason, no questions related to this domain were included in Section B (for composers) of the questionnaire. These questions can only be answered upon performing the "Test Sheet", where the exercises presented address specific interactive goals described in section 3.1.2. These questions provide practical insight into the concept of embodiment applied through the development and reception of the HypeSax, as observed from the perspective offered by Marc Leman on Musical Gestures and Embodied Cognition. ${ }^{216}$

The basic concepts of musical embodiment described by Leman are: the body as a mediator, the gesture/action repertoire, the action-perception coupling, and the link between subjective experiences and emotions. The concept of the body as mediator refers to the function that our bodies have to bridge perception of an external stimuli or event (in this case it could be a sound, a musical gesture, a physical gesture that produces a sounding event) and the subjective experience. The gesture/action repertoire is our own catalogue of learned experiences of how the external stimuli or events relate to our experience of it through our exterioception, proprioception and interioception. The action-perception coupling encapsulates two mechanisms: the sensorimotor loop, which informs and controls the actions of a performer based on the perception of the sound being produced (for instance slightly increase the air pressure to fine-tune the pitch on a saxophone), and the action-perception loop where a set of pre-learned actions is the controller of the production of sound (for example knowing and executing the required use of tongue, lips and lungs muscles to perform a slap tongue). This action-perception coupling also informs our perception in order to assume an action that could be the origin of a sounding event (for example the learned experience of performing articulation in woodwind instruments can be used to assume the ins and outs of the realization of phrasing in a musical passage).

\footnotetext{
${ }^{216}$ Marc Leman, 'Musical Gestures and Embodied Cognition', Actes Des Journées d'Informatique Musicale (JIM 2012), 2012, 3.
} 
Finally, the link between subjective experiences and emotions helps create a connection between the performer and listener through intentionality, expressiveness and empathy based on the action-perception coupling (for instance, an audience moving their bodies to the groove of a jazz ensemble).

In the case of this study, I was interested in observing the action-perception coupling, as it is under this concept where the performance aspect of the model enters in a feedbackloop through interaction and response with the medium. Embodiment is, thus, a very important factor in the successful placement of the HypeSax in the tripartite model.

The questions related to interaction presented in Section $\mathbf{A}$ are presented next, but a discussion on the findings will be presented towards the end of this section:

\section{From 1 (very difficult) to 5 (very easy), how difficult did you find} performing the preprogramed instrumental techniques?

This question aims to evaluate the current state of the unification of software/hardware under the preprogrammed instrumental techniques. At the same time, it provides an insight on whether the hybridisation between acoustic and electronic sound has been achieved (see Fig. 38).

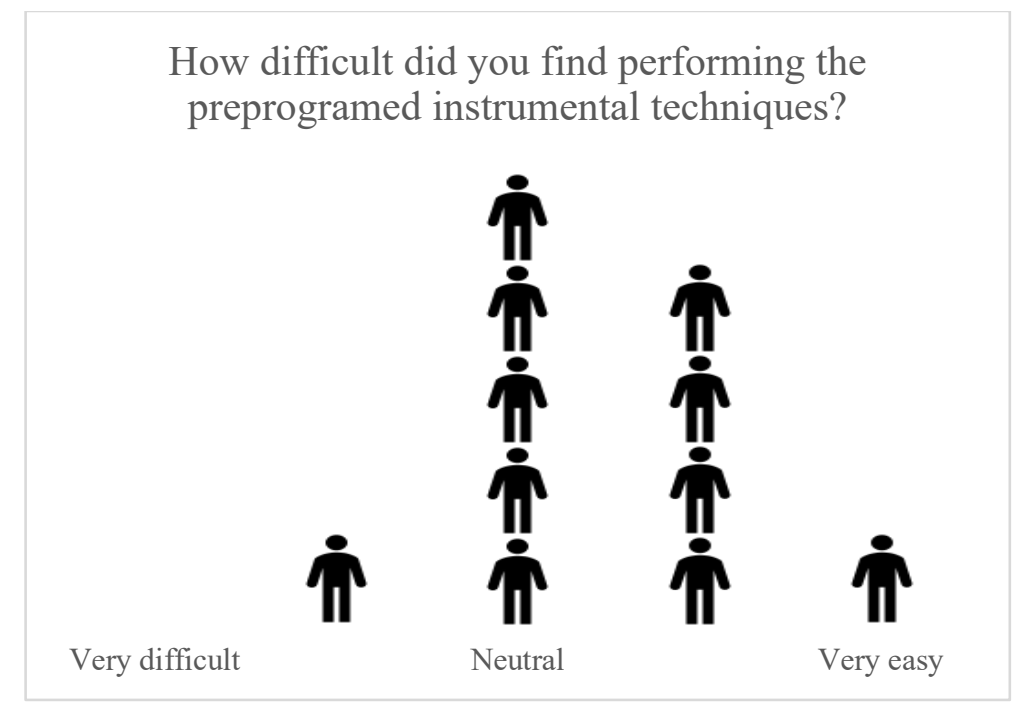

Fig. 38. Answers to question 6 of Section A.

7. From 1 (not at all) to 5 (very), how responsive and reliable did you find the HypeSax? 
Question seven moves away from the possibility of software error or miscalibration by addressing only the responsiveness of the hardware. This also allows for the observation of the possibility of performance perfectibility (see Fig. 39).

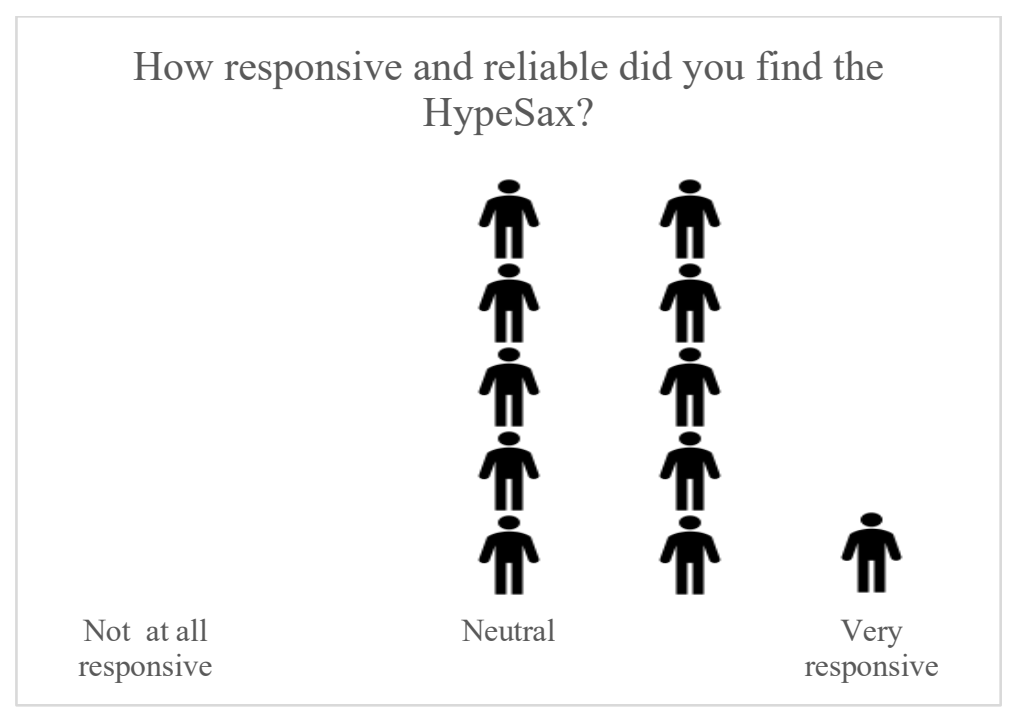

Fig. 39. Answers to question 7 of Section A.

8. Putting aside the fact that using the HypeSax stops the sound of the lowest note of the saxophone, from 1 (very) to 5 (not at all), how much did the HypeSax hinder performance of the instrument's regular techniques?

9. From 1 (not at all) to 5 (very), how comfortable was it to play the saxophone using the HypeSax?

These questions explore the possibility that HypeSax can affect the playability of the saxophone, assessing whether the design goals of 'affording maximum possible control of electronic sound while maintaining a natural performative practice' and 'retain the dominance of the performative practices' are met. 
How much did the HypeSax hinder performance of the instrument's regular techniques?

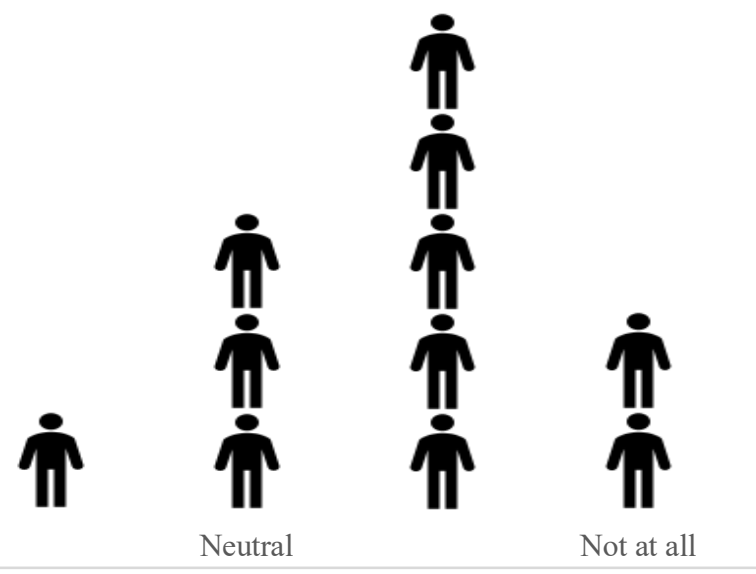

How comfortable was it to play the saxophone using the HypeSax?

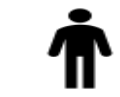

Not at all comfortable

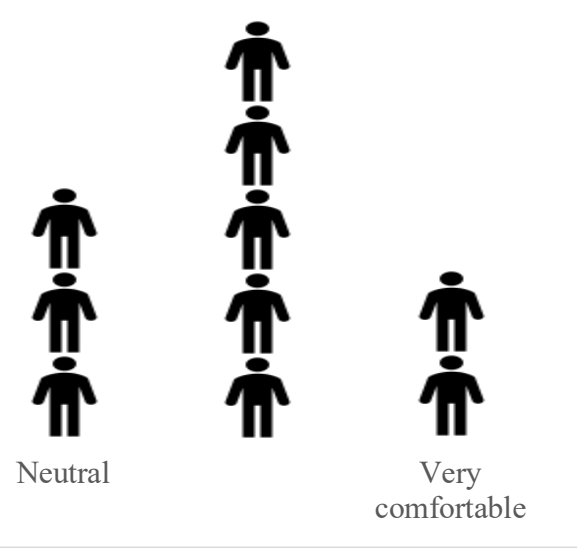

Fig. 40. Answers to questions 8 (top) and 9 (bottom) of Section A.

11. From 1 (not interested) to 5 (very interested), how interested would you be in performing a composition using the hypersaxophone?

Finally, the answer to question eleven shows a general interest in the HypeSax. 


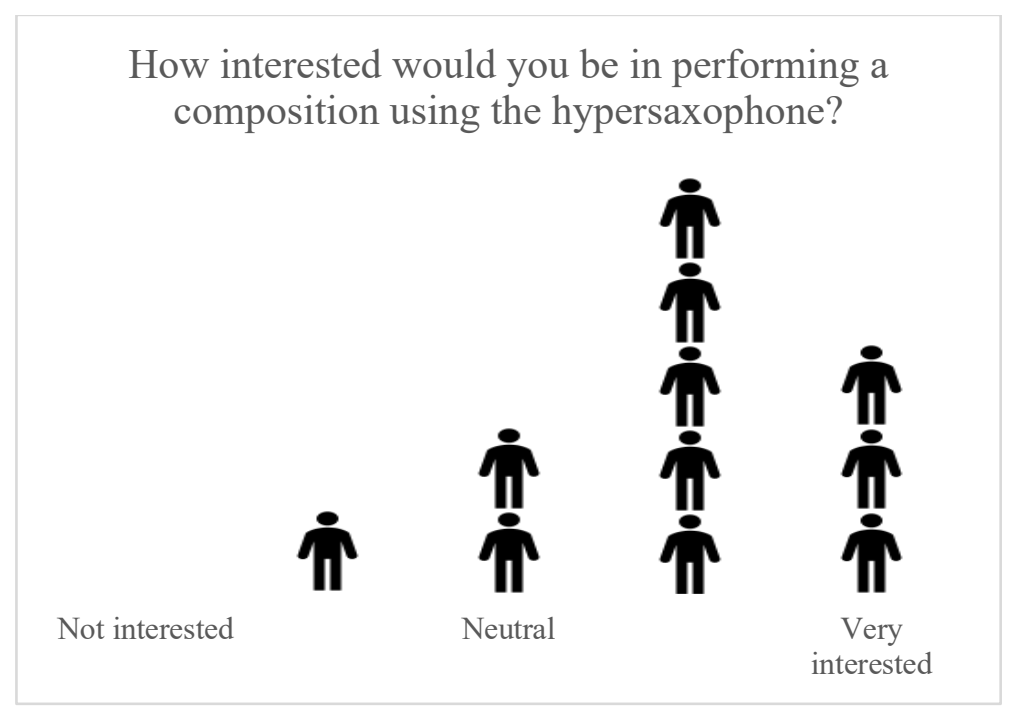

Fig. 41. Answers to question 11 of Section A.

The responses to these questions that refer to interactivity demonstrate that the design will benefit from additional development, and further iterations should observe multiple changes in the design in order to facilitate achieving Leman's basic concepts of embodiment. More specifically the problems listed in the responses are:

1. Difficulty of playability with the mouthpiece.

2. Touch buttons out of reach.

3. Thumb buttons out of reach.

4. Irresponsiveness of touch buttons.

Comments listed by the participants as well as conversations during and after the tests reveal the reasons for these problems:

The mouthpiece is one of the most important components for the sound production in the saxophone. Multiple elements found in the design of a mouthpiece allow for different characteristics of playability. Three of the most important elements that can affect playability are the tip opening, chamber size, and baffle type. Performers normally get used to the specific combination of these elements in the design of their mouthpieces. Jazz performers, for instance, tend to prefer a large tip opening which provides a loud sound, appropriate for the jazz ensemble. In contrast, classical saxophonists prefer a smaller tip opening which, rather than offering a loud sound, allows for a more precise control in dynamics. 


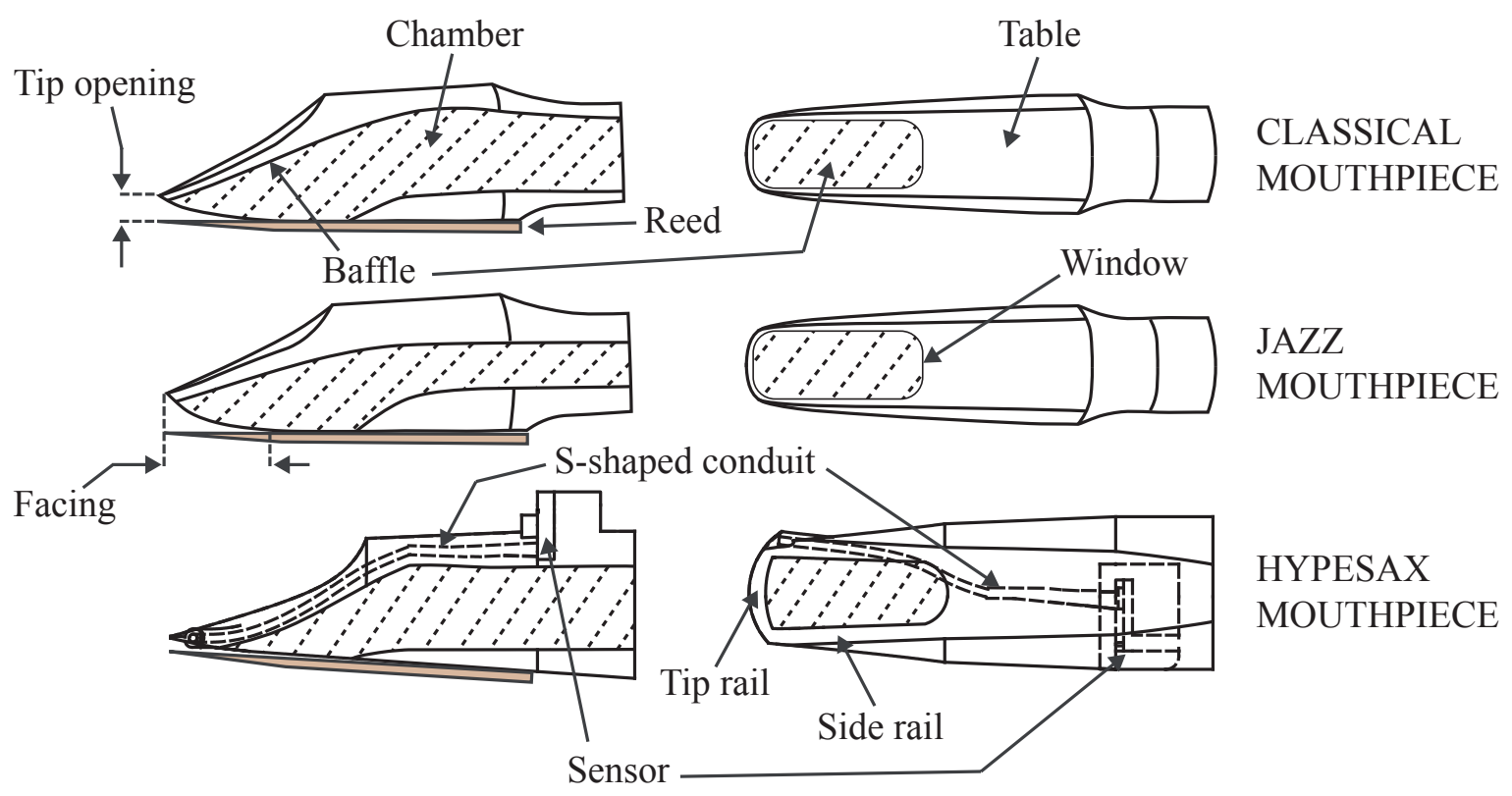

Fig. 42. Common differences in the mouthpiece designs (dimensions have been exaggerated).

All of the participants used the same mouthpiece, which featured characteristics similar to a classical mouthpiece, modelled with the same characteristics of the Selmer Concept mouthpiece. It is not surprising to find that the responses relating to the mouthpiece were in line with the musical training of each participant. This makes sense since each performer trains the different muscles necessary to produce sound on the saxophone depending on the characteristics and specific resistance of their mouthpiece. This was easily observed during the test. In the first part of the 'Test sheet', participants were asked to play seventeen simple exercises in order to introduce them to the sensation of the new mouthpiece. A Max patch recorded audio and air pressure data (depicted in Fig. 43). 


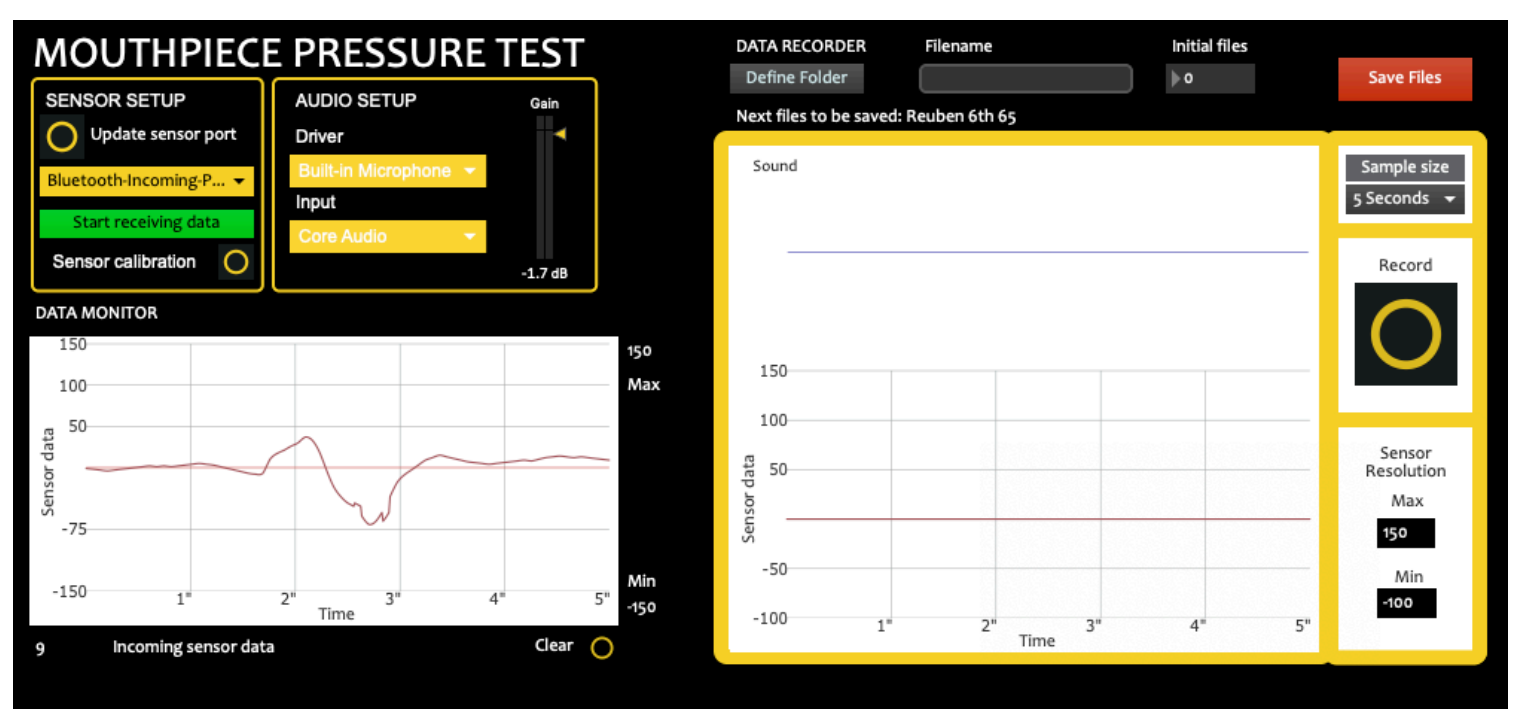

Fig. 43. Max patch developed to monitor (left panel) and record (right panel) air pressure data.

As stated before, the sample size of the survey was not large enough to obtain statistical data, but the collected data reveals a correlation between effort in performing and sound production using a 'classical mouthpiece.' Rather than to serve as a precision data acquisition tool, the software was intended to provide high-level insight into mouthpiece usage. The software used allows to record 5", 8" or 10" per exercise, and the graphic representing the air pressure data can be adjusted in range before the test, but the graphic representing the audio cannot be changed. This explains the inconsistencies in the comparative images shown in Fig. 44 and Fig. 45 (scales in both $\mathrm{X}$ and $\mathrm{Y}$ axis varies). Amongst other variables, the participants utilised reeds with the same characteristics but not the same one, the tests were performed on different days with different weather conditions, which renders the results unsuitable for quantitative research. For these reasons, this study did not seek to evaluate the mouthpiece thoroughly, but was intended as a high-level visual validation of the relationship between the air pressure and the sound production.

Fig. 44 presents the first exercise of the mouthpiece test section found in the 'Test Sheet', where participants were asked to play the lowest F note (transposed as D for the alto saxophone). It is possible to observe that classically trained performers (A and B), who are used to mouthpieces featuring small tip opening and chamber, were able to produce a stable sound at $\boldsymbol{m} \boldsymbol{f}$ using less pressure than saxophonists of jazz tradition, only needing pressure in the range of 50-70 $\mathrm{hPa}$. In contrast, Jazz saxophonists (C and D) 
required pressure in the range of 90-130 $\mathrm{hPa}$ to obtain similar results. They also expressed discomfort due to the resistance presented by the mouthpiece.

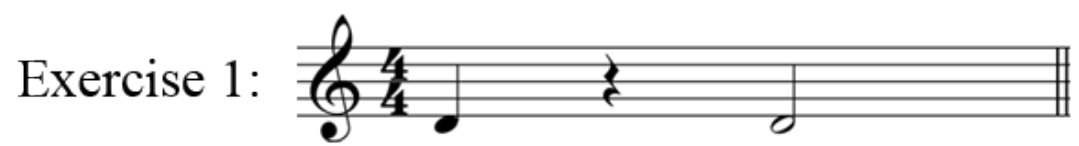

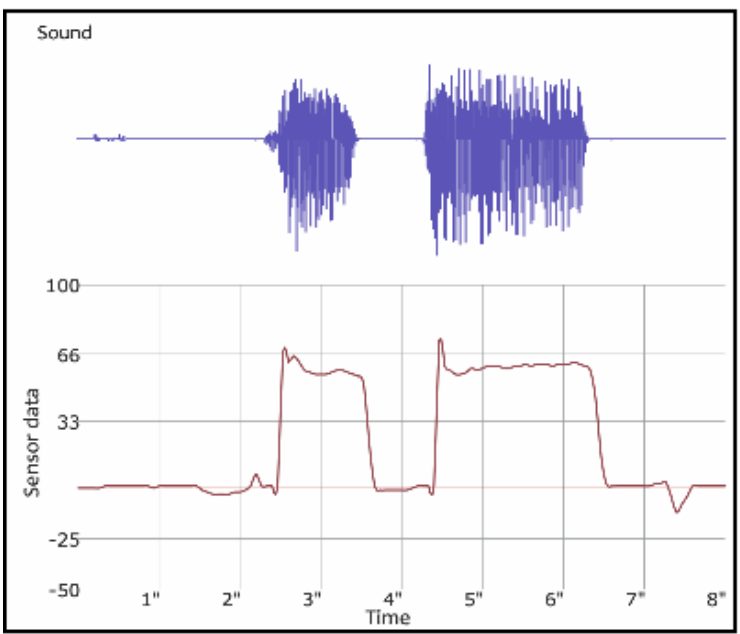

A

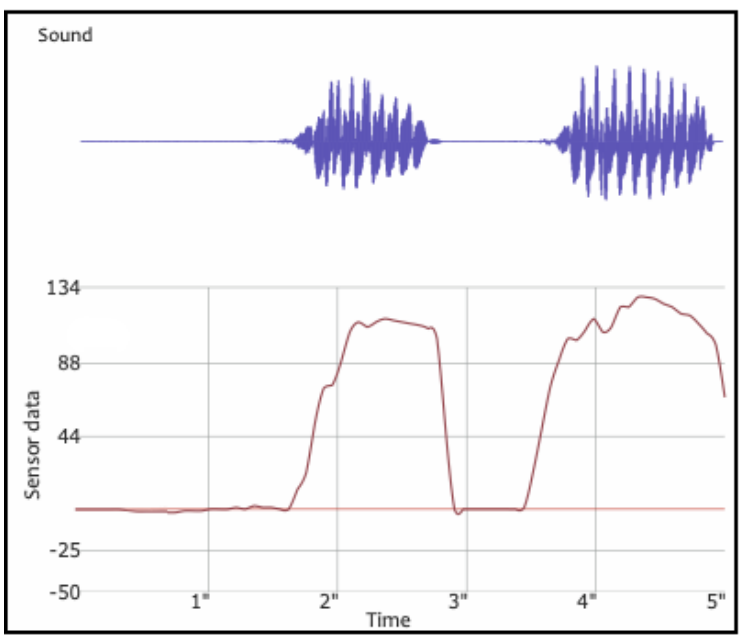

$\mathrm{C}$

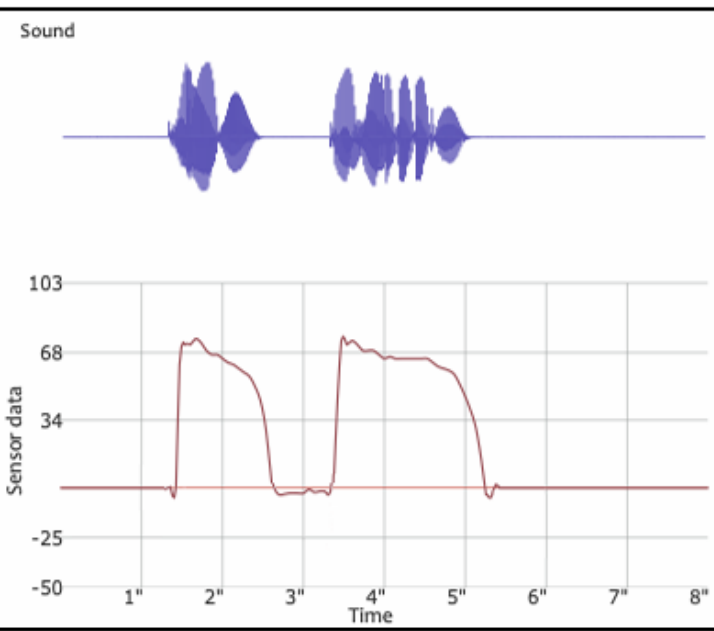

B

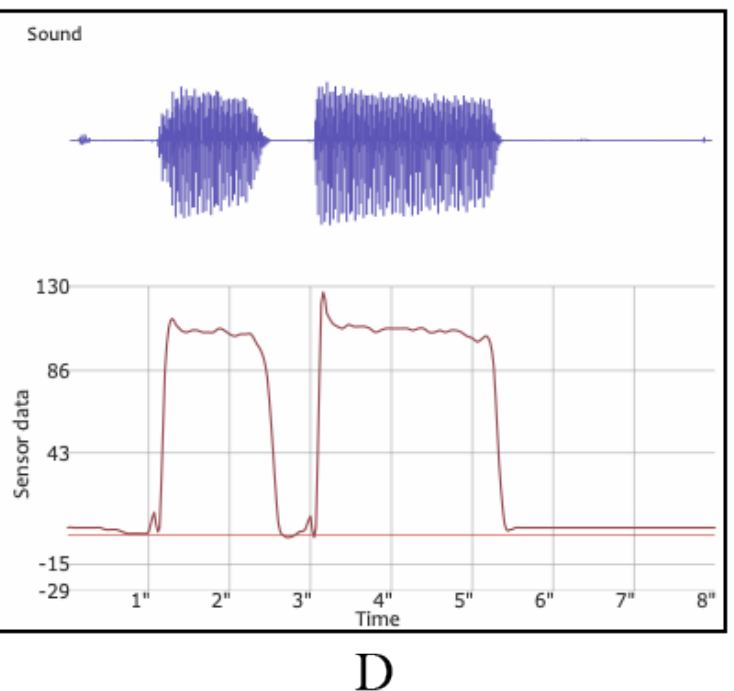

$\mathrm{D}$

Fig. 44. Exercise 1 of the 'Test sheet' and recorded data obtained from four participants. 


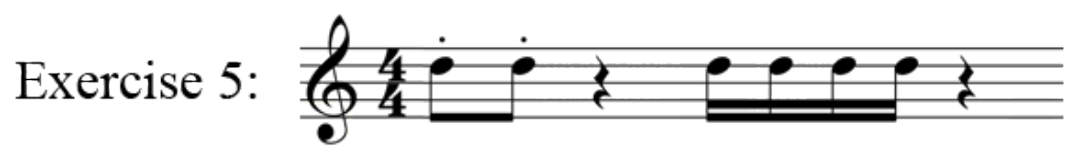

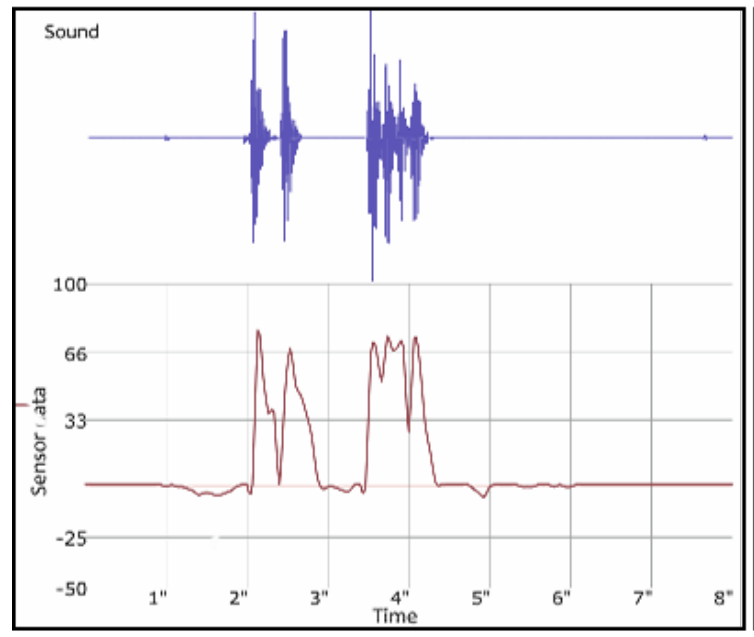

A

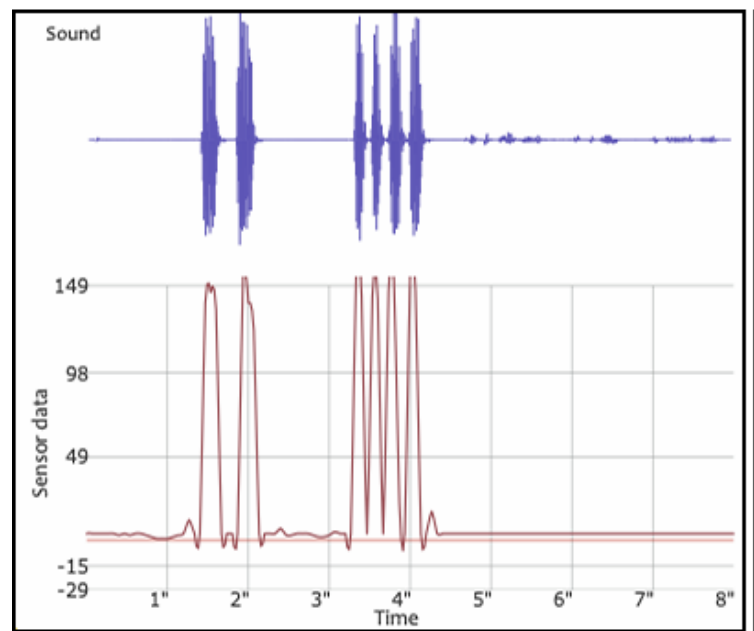

$\mathrm{C}$
Sound

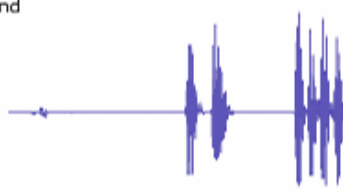

100

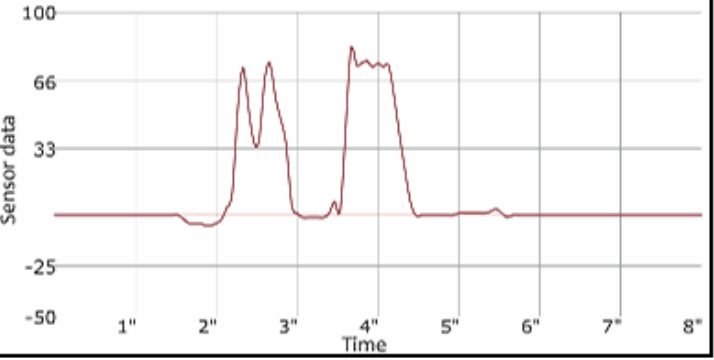

$\mathrm{B}$

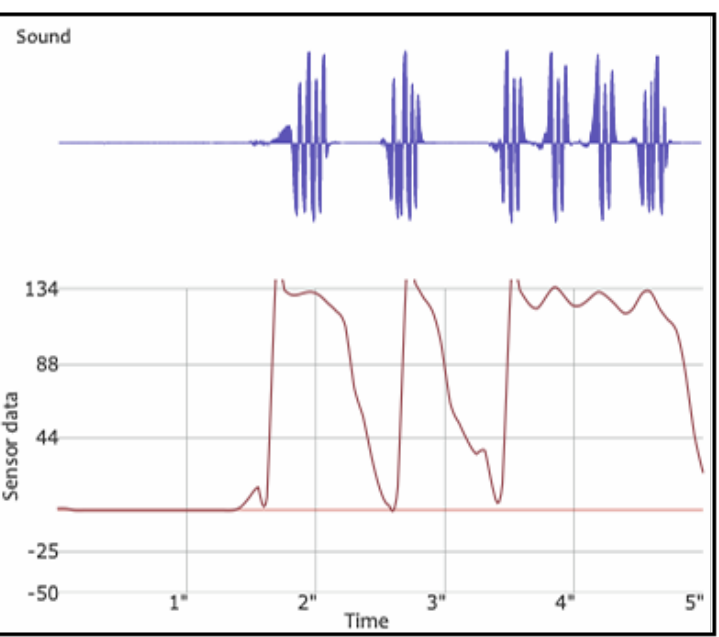

$\mathrm{D}$

Fig. 45. Exercise 5. A and B present data recorded from classical performers, while C and D belong to Jazz saxophonists. 
flz. $\quad$ flz.

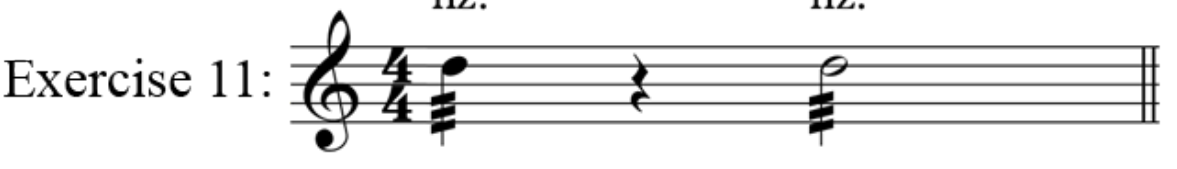

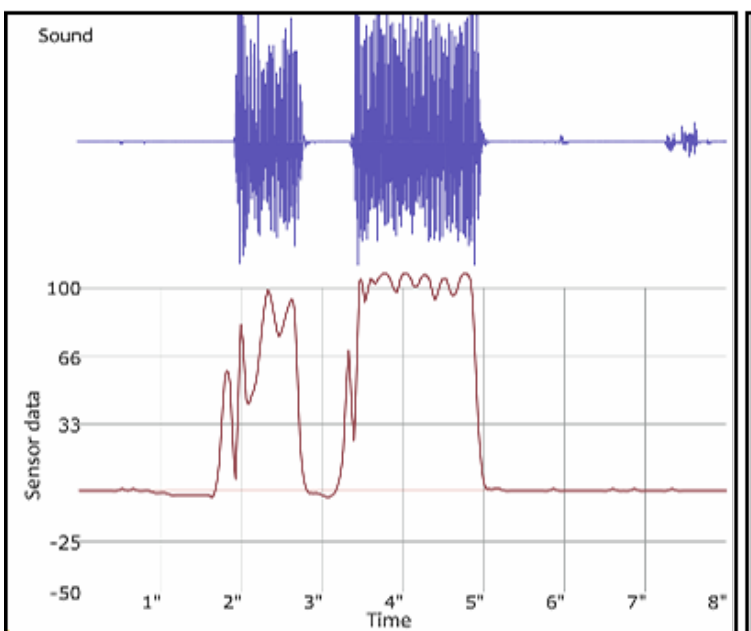

A

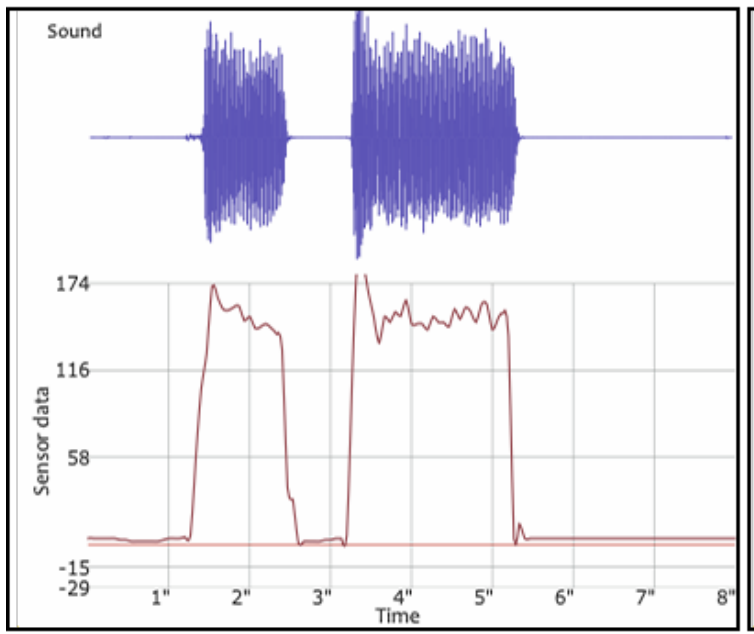

C

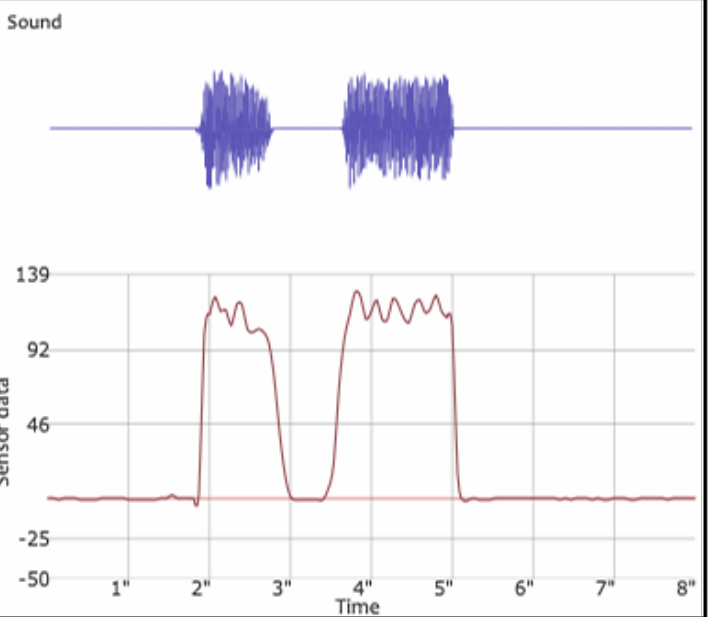

B

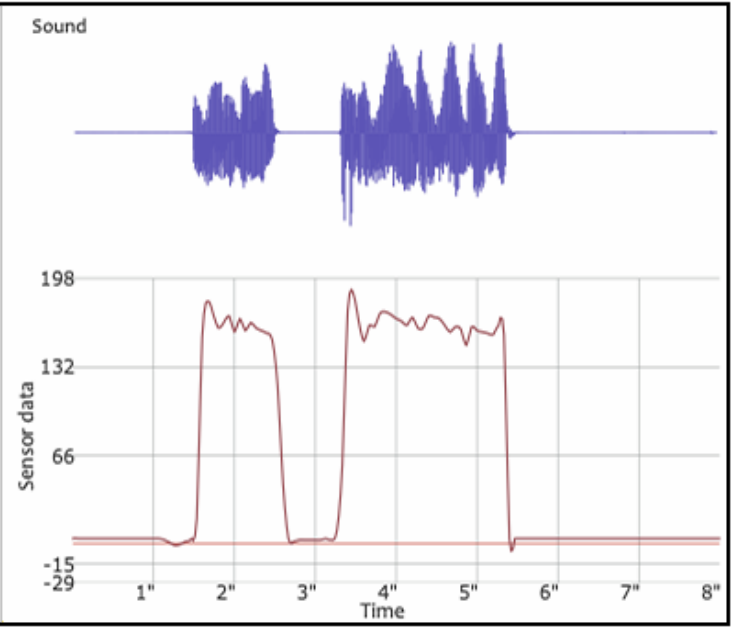

D

Fig. 46. Images $A$ and $B$ present data recorded from classical performers, while $C$ and $D$ belong to Jazz saxophonists.

The results of these tests help in visualising a possible explanation as to why certain participants found it more difficult to use the mouthpiece than others. Nevertheless, a more 
thorough research project similar to that by Backus, ${ }^{217} 218$ Fritz et al., ${ }^{219}{ }^{220}$ or Gazengel and Eveno, ${ }^{221}$ amongst others, which consider other factors such as the effects of the reeds and the performer's muscles, as well as controlled testing conditions, would be necessary to understand the relationship between certain mouthpiece characteristics and each performer. Despite the lack of accuracy of the results, there were observable differences between the classical and jazz saxophonists, showing similar results between the performers of each group regardless of the exercises (see Fig. 45). In the case of exercise 11 , for instance, the dynamic levels represented in the graphic seem more varied due to the noise coming from muscle vibration necessary to produce the flutter tongue effect, but the pressure levels show the same relationship observed in the previous cases (see Fig. 46).

Ergonomics are very important to facilitate the action-perception coupling. One participant, with particularly small hands, could not reach the thumb button for the right hand while reaching the touch buttons placed over the D, E and F keys. Other participants found that reaching the right thumb button forced them to not close the keys properly due to the necessary extension of the hand. They suggested placing the button on the right side of the hook rest, rather than to the left as is the case in the original design.

Finally, touch buttons would become irresponsive due to particularly dry hands. After questioning the participants, I found that those who had consumed water in the last hour did not have problems interacting with the touch buttons, unlike those who had not been drinking water recently. This is due to the fact that these buttons work as terminals for the MPR121 capacitive touch sensor. The fingers touching these terminals provide an induced capacitance to ground, which triggers the signal to which the HypeSax responds. However,

${ }^{217}$ Backus, John. 1974. "Input Impedance Curves for the Reed Woodwind Instruments." The Journal of the Acoustical Society of America 56

${ }^{218}$ Backus, John. 1985. "The Effect of the Player's Vocal Tract on Woodwind Instrument Tone." The Journal of the Acoustical Society of America 78

${ }^{219}$ Wolfe, J, A.Z. Tarnopolsky, N.H. Fletcher, L.C.L. Hollenberg, and J. Smith. 2003. "Some Effects of the Player's Vocal Tract and Tongue on Wind Instrument Sound." Proceedings of the Stockholm Music Acoustics Conference 2003

${ }^{220}$ How do Fritz, Claudia, and Joe Wolfe. 2005. "How Do Clarinet Players Adjust the Resonances of Their Vocal Tracts for Different Playing Effects." The Journal of the Acoustical Society of America

${ }^{221}$ Gazengel, Bruno, and Pauline Eveno. 2018. “Caractérisation Expérimentale de Becs de Saxophone Ténor à l'aide d'un Musicien Artificiel Fonctionnant En Aspiration.” 
the reduced moisture in the fingers can reduce their conductivity. Nevertheless, after playing for a few more minutes, the fingers of the performer tend to expel oils which help with conductivity. It also became evident that rubbing the fingers against the material chosen to build the keycaps (see 3.5.1), urethane Vitaflex 40, seems to cause a loss of skin oils, causing dryness again minutes later. To address this issue, it is necessary to replace the material used for the keycaps or to re-adjust the sensitivity of the MPR121 sensor.

After observing the preferences of the participants, one can assume that it is best to not assume that performers will be able to interact with all of the HypeSax components, and a discussion and ergonomics and mouthpiece setup must precede any performance to address any potential disruptions in the action-perception coupling. This is an aspect that had already been expected, for which reason all of the 3D models used in the design are composed of structures defined by parametric values. This facilitates making changes upon receiving feedback from a performer, adapting the ergonomics in accordance to the user's needs.

In conclusion, the survey was very helpful in visualising the way in which the users can relate to HypeSax and integrate it into their relationship with a musical work. The results show that this can be possible by either providing new avenues for creativity or allowing new ways of embodying the bodyless sound produced digitally. At the same time, the opinions of the participants revealed weaknesses, rights and wrongs of the design. Based on the findings, a new version of the HypeSax was developed.

\subsection{A new version of the HypeSax compatible with soprano and tenor saxophones}

In general, the survey showed a positive response from potential users. The participants expressed their ideas on how the instrument could be improved and what kind of sounds they would like to be able to make with it. Based on this feedback, a number of changes to the design were implemented. The new iteration of the HypeSax tries to eliminate the problems listed in the previous section, hoping to free the user from dealing with ergonomic issues and irresponsiveness. In this way, musicians can focus on performing, improvising, and composing with a tool that enables performance and creativity rather than represent a challenge. 
With the new iteration, I tried to address issues that became clear with the user study. The latest version of the design considers the following:

- The original design does not give saxophonists the option to swap to another to a different size saxophone.

- Uncomfortable position of thumb buttons.

- Some performers struggled with the keycaps.

- Resistance of mouthpiece.

- Plug-and-play capability and ease of software interaction.

The new iteration features the same components presented in the original design, but with changes to facilitate the use of the HypeSax:

- The system remains as a modular design.

- New PCB and embedded software eliminate the need for two microcontrollers and the Sensor-link.

- The new Un-mute features attachments that allow the HypeSax to fit soprano, alto and tenor saxophones.

- The right thumb button is used to scroll through a bank six modes of use of the touch buttons. This button is now placed to the right of the rest hook for easier access.

- New keycaps fit in most saxophones and feature a less intrusive design that allows for it to be rotated to avoid collisions with any parts of the saxophone keys. The new design also is more reliable and presents no issues with conductivity.

- The update version of the 3D mouthpiece model is fully customisable.

As in the original design, the system is modular: thumb buttons, touch buttons and mouthpiece are not necessary in order to access control of sound processing. The gyroscope/accelerometer and sound system, including the jack for gain control through the use of an expression pedal, are housed inside the Un-mute. The MPR121 12-key capacitive touch sensor is still included inside the Un-mute, still maintaining the use of only six keys (see Fig. 47). The unused keys are free to use with minor modifications to the face of the Un-mute. This allows for further extension if desired by the user. Future 
iteration for a tenor or larger saxophones could take advantage of all twelve keys (see Fig. 48).

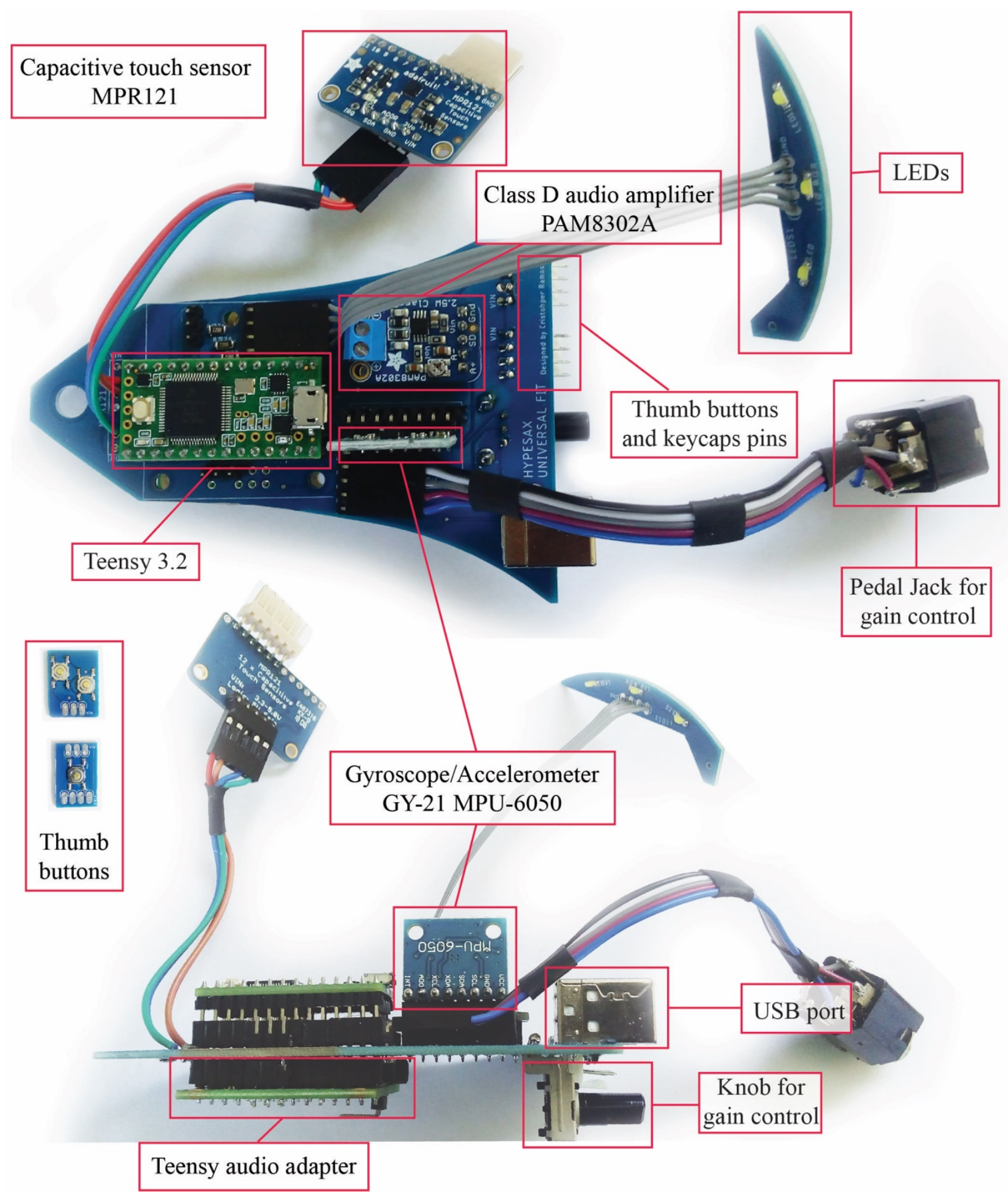

Fig. 47. New PCB design. 


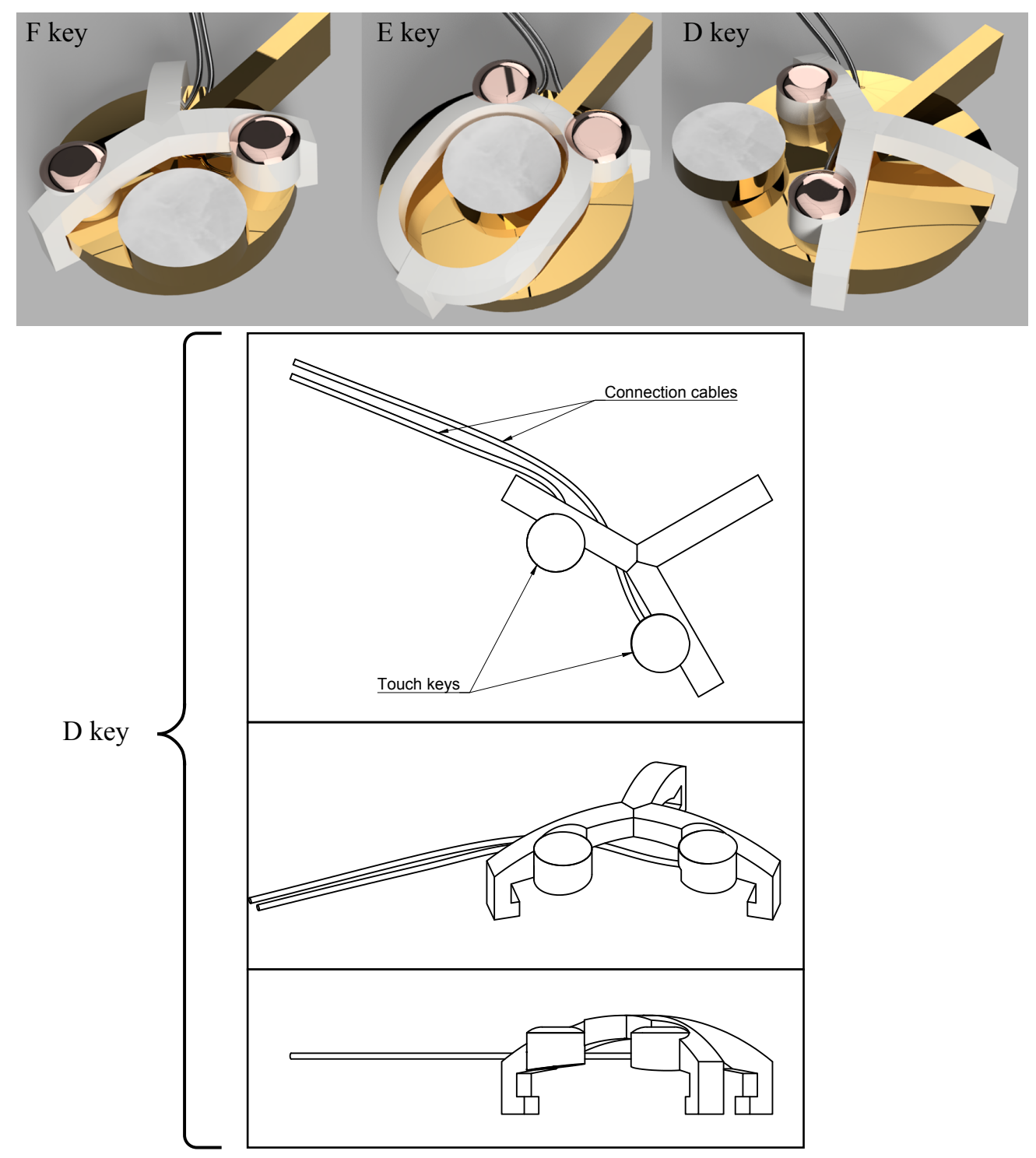

Fig. 48. New alto saxophone keycaps design (top) and drawing detail of alto Hypesax D key keycap (bottom).

The electronic components communicate directly to only one Teensy board which handles sensor data and audio. This is done in order to facilitate reprogramming of the system, unlike the previous design that required the system to be disassembled in order to access the slave Teensy board which handled the sensor data. On the other hand, the Teensy 4.0 board was released recently, featuring a dramatic improvement in processing power that is almost fifteen times faster in many benchmarks than the previous 3.2 version used in the HypeSax. This board is still not used in the new version of the HypeSax since the audio board needs to be updated to be pin-compatible. Version 4.0 and the revision of the audio 
board available at the time of development can be used together but special connections are required. These connections would require extra space not available inside the Unmute. A future iteration of the PCB can take advantage of the full power of the Teensy 4.0 working with a new revision of the audio board by making minimal changes to the PCB, without having to change the entire design of the Un-mute and the way the rest of the sensors communicate with the microcontroller.

Many performers play multiple instruments of the saxophone family. For this reason, the new Un-mute was designed to fit inside a straight soprano saxophone. Two adaptors were design to change the diameter of the Un-mute offering a flexibility to fit it inside an alto or tenor saxophone (see Fig. 49). This design can potentially be used with larger saxophones, but it would be preferable to have a different speaker and amplifier to offer a wider gain range to be used with larger instruments.
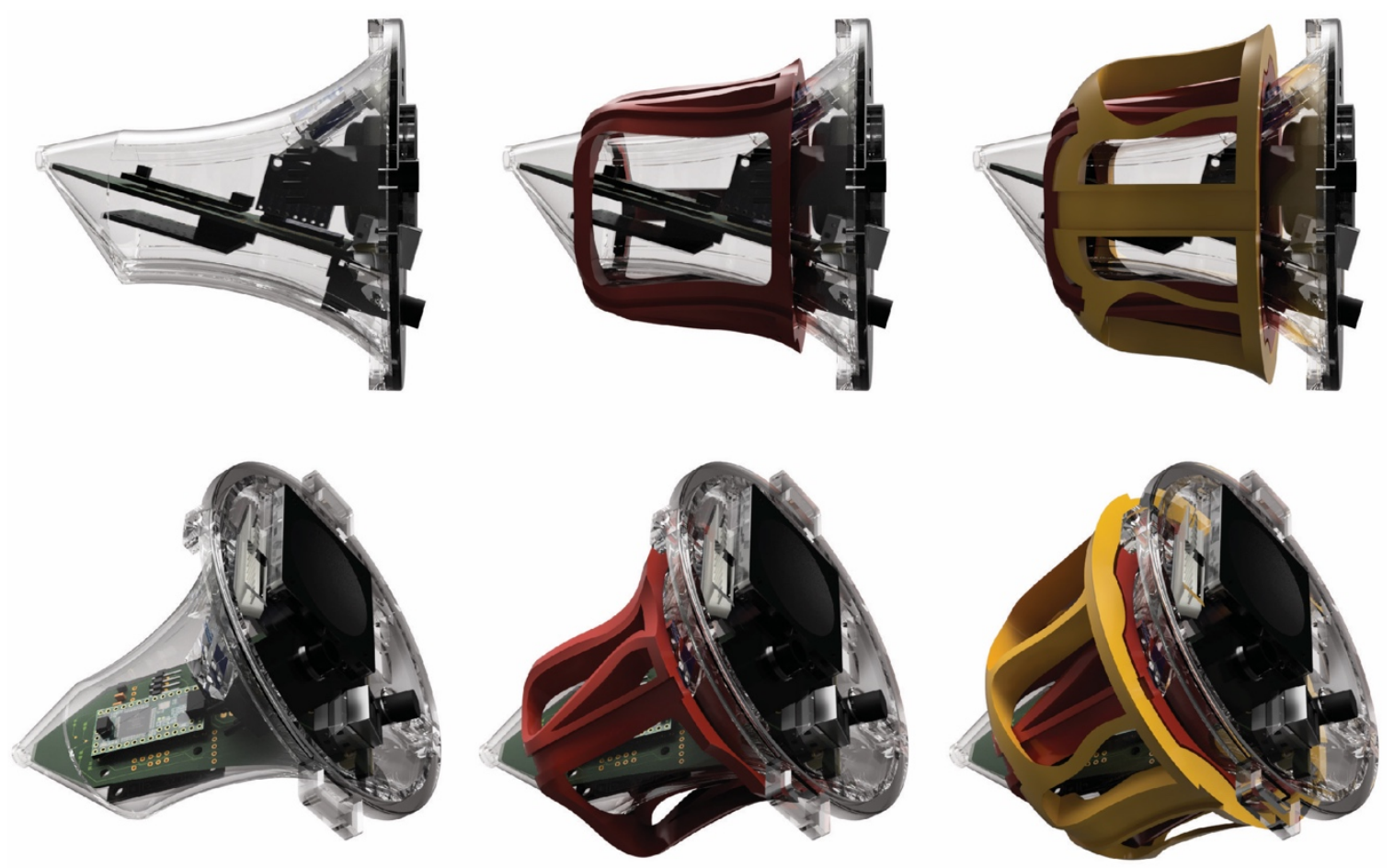

Fig. 49. New Un-mute and adaptors.

While the two thumb buttons for the left hand remain had a minor ergonomic redesign, the right button did not receive an ergonomic update except for the fact that it now attaches to the right of the thumb hook. This button is now used to scroll through a bank of six modes of use of the touch buttons. This means that, rather than writing discrete values $0-1$ on the serial port, the use of this button now sends a message that scrolls through the numbers 0 - 
5 in order to access presets for the touch buttons, effectively offering 36 functions, 3 per each key. Three LEDs placed inside the Un-mute indicate the state of this button (see Fig. 50). This multiplies the possibilities offered by the touch buttons. With this, six buttons are more than enough and perhaps repeating the function of three of the keys (both touch buttons mounted on the F key have the same function, both touch buttons on the E key have the same function, and both touch buttons on the D key have the same function) would allow access to more than enough number of functions (18). By repeating the role of the keys, the user has access to the better ergonomics, as it doubles the possibilities to reach the same function facilitating performance (see Fig. 48).
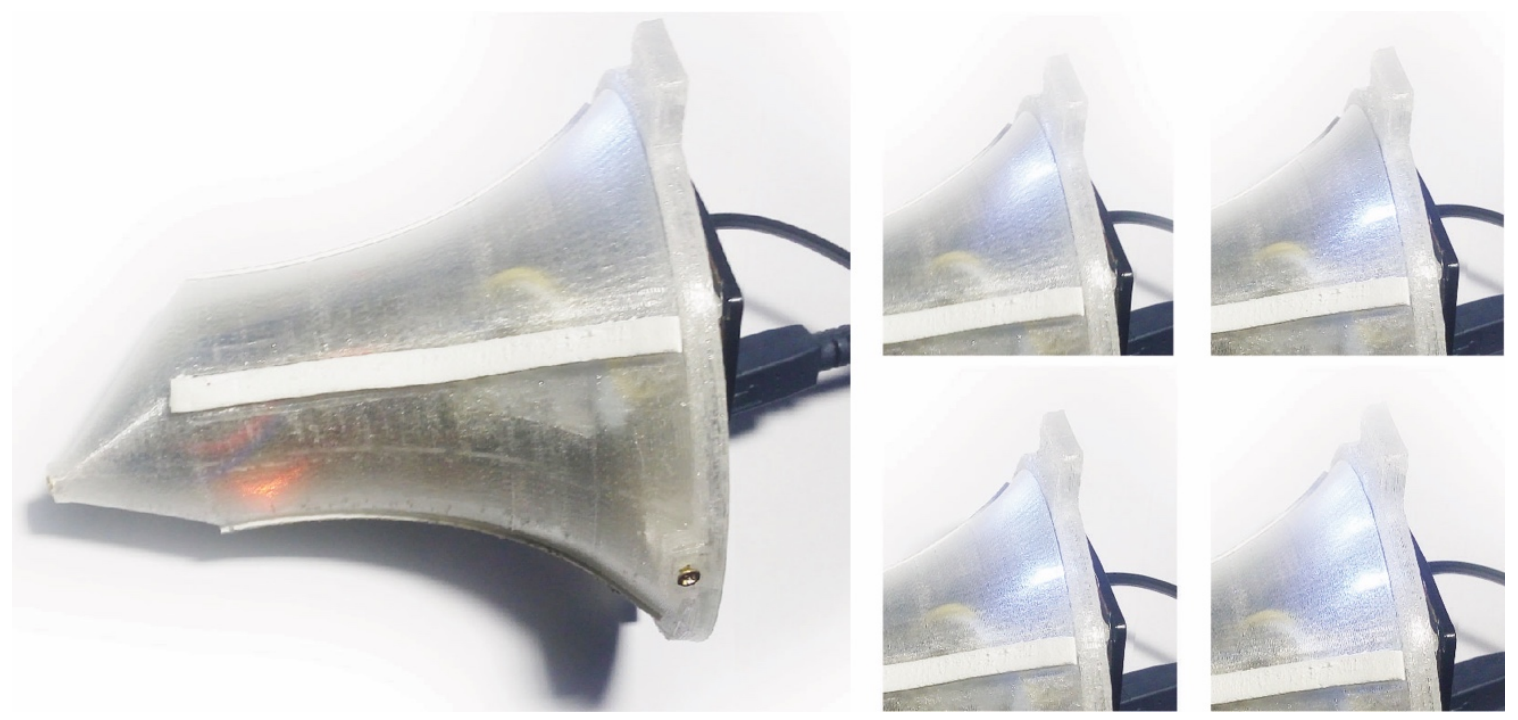

Fig. 50. Five right thumb button states are indicated by distinctive patterns of illumination of the LEDs. A sixth state is indicated when LEDs are off.

Ideally, this iteration would have been tested and assessed by multiple users as a last part of the project. Unfortunately, due to COVID-19 pandemic, it became impossible to do further tests with multiple users before the end of this research project.

The tests, survey, feedback and new iteration of the HypeSax represent a further step towards having a novel instrument that allows us to explore the medium in an innovative way as a creative tool. In the following chapter, I discuss the way in which the development of the HypeSax has allowed me to develop musical ideas through the exploration and manipulation of the medium. 


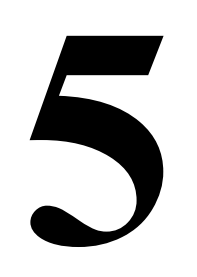

\section{A NEW MEDIUM FOR NEW WORKS}

The fifth chapter is concerned with the development of a series of new musical works, and how the HypeSax was integrated into my creative process, providing new avenues for expressivity. In this chapter, I discuss how becoming aware of the importance of the medium for the work and integrating it as a fundamental element of the tripartite model has influenced my creative process. This chapter is divided in two main sections: Canvas and New Works. The first section describes some of the strategies developed to approach my creative ideas using the HypeSax. The second section demonstrates the use of the HypeSax in eight works composed for various ensembles featuring the new hyperinstrument. In order to begin this self-reflection, I will relist the definitions of the key concepts previously presented in this dissertation. In this chapter, these concepts are continuously discussed in relation to my works.

The tripartite model, presented in section 2.4 , proposes that a musical work should be observed as comprising three elements: score, performance and medium. The score is the musical idea that is usually communicated through a score regardless of its format or representation, in some cases it might be stored in a non-graphical way such as in the case of electronic music. The performance is the stage at which the score is confronted with the plasticity and physicality of music-making. The performance can take place in an imaginary way, such as the composer's reflection on the act of performing the work, a collaborative way, such as when composer works with performers, or during the realisation of the work through a live performance or recording. Finally, the medium is 
found in the materiality of the sound, through the vibrating body and physical configuration that affects the sound. The medium is usually an instrument, acoustic space or virtual space. The tripartite model is discussed in the context of my composition named Look out!, for improvising ensemble, in section 5.2.1.

As a way to explore the possibility of integrating compositional strategies I already feel comfortable in the context of new works featuring the HypeSax, I utilised concepts from Gestalt theory. Developed in the field of psychology and adapted for the field of music by James Tenney, Gestalt theory states that, in order to simplify and easily process vast amounts of information, the brain tends to group elements of a whole to visualise comprehensible shapes and structures. In music, grouped elements can form elements such as melodies, harmonies, rhythmic patterns, motifs, timbres or textures, easily organised and understood by our internal cognitive processes (see section 2.6.2.1).

I was also interested in exploring the musical gesture along with the development of the HypeSax. Musical gesture, as discussed in section 2.6.2.2, has been explored by many authors. However, for the discussions found in this dissertation, I narrow the possible definitions to the following two:

- Musical gesture as a set of sounding elements presented sequentially, with or without variation, that allow for the recognition of a complex musical element, such a group of rapid ascending notes followed by five short attacks randomly throughout the full range of the instrument.

- Musical gesture as a sequence of bodily actions required to transfer energy to an instrument in order to make music, such as pressing a string with a finger and sliding it along the length of the string while bowing with the other hand in order to produce a glissando.

With the musical gesture, issues of embodiment arise due to its intrinsic relation to the physicality of sound. As with the concept of music gesture, embodiment has also been defined from multiple perspectives (see section 2.6.3). For the purposes of this dissertation, I use the term embodiment to refer to the following:

- Embodiment as the 'body' of the sound. In other words, the sound belonging to a source body; for instance, the sound of a guitar produced by a guitar, or the same sound produced by a loudspeaker. In this case, the sound is embodied by two 
different bodies, the guitar and the speaker. Embodiment in this sense encompasses the unique qualities of the physicality of an instrument, including both its qualities and limitations.

- Embodiment as sound production action. This includes any action required to produce sound as well as any accompanying motion. The latter, also known as expressive or ancillary movements, has 'an intrinsic relationship with the music, representing a link between the music and the expressive intention of the musician'. ${ }^{222}$

- Embodiment as perception. In this sense, embodiment refers to the observer's experience of music. For example, the perception of the duration of a note by observing the 'end cue' of a musician that could elongate more than the actual sound, or a video of a musician with mismatching audio that provokes the observer to perceive aurally what the video shows. ${ }^{223}$ Another example is found with entrainment, which creates a rhythmic synchrony by means of empathy. Similarly, embodiment can present itself through mimesis, engaging cognitive processes and past experiences.

The first of this list, embodiment as the 'body' of sound, can be transferred to a different sound through the use of new technology. This produces what I call the bodyless sound: sound produced by a non-natural source. In other words, sound produced with the use of an electromechanical system, driving the vibrations of a body with the use of a transducer, such as the use of a loudspeaker (see section 0 ). This sound can be natural in its genesis, such as a recording, or artificial, such as an oscillator. ${ }^{224}$

If sound is produced artificially, free from the physicality of a body, it can be manipulated in multiple ways affording what I call mutability of the medium: the capacity of a medium to change its spectromorphology or be redesigned without changing

${ }^{222}$ Manfred Nusseck and Marcelo M. Wanderley, 'Music and Motion-How Music-Related Ancillary Body Movements Contribute to the Experience of Music', Music Perception: An Interdisciplinary Journal, 26.4 (2009), 335-53.

${ }^{223}$ Michael Schutz and Scott Lipscomb, 'Hearing Gestures, Seeing Music: Vision Influences Perceived Tone Duration', Perception, 36.6 (2007), 888-97.

${ }^{224}$ While it is true that a clarinet reed, for instance, is an oscillator, in this case I am referring to electrically driven oscillators. 
its physical construction or configuration, taking on new sounding characteristics or behaviours according to the needs of the performer or composer (see section 4.1.2). Mutability of the medium, however, can also refer to the fact that medium can change its role, acting, for instance, as musical instrument, decision maker, data collector or sound manipulator (exemplified throughout this chapter).

These key concepts will be discussed further, in the next pages of this chapter, in the context of the HypeSax as a creative tool and the portfolio of works composed during this research.

\subsection{Canvas}

Bernard Sève, in Bords de l'œuvre musicale, uses the metaphor of the frame as the physical limits of a picture to describe the edges (bord) of the musical work. ${ }^{225}$ Although Sève's article is concerned with the musical work in the context of its realisation in the paradigm of a classical music concert, I find the physicality of this metaphor useful in analysing the impact of a hyperinstrument in the creative process, since hyperinstruments provide traditional instruments with new capabilities beyond the restrictions of their physicality. The sound of a hyperinstrument, like any other electrophone, can be redesigned (see section 4.1.2). With this potential to become something different, hyperinstruments are similar to a painter's canvas. A canvas has a defined shape, limits, and texture, where strokes and colours can form a new creation. Similarly, a hyperinstrument has limits, defined possibilities, physical and technical characteristics. They can be the space through which sounds and timbres can be reconfigured even before a concrete musical idea is realised with these new sounds. The ability to sculpt - or paint, in the canvas metaphorsound, via electronic means, opens up new horizons of possibilities, where the composer not only composes with sounds but composes the sounds, ${ }^{226}$ a feature found at the core of electronic music. Being able to address these points-of-view is the foundational motive to pursue the project described in this dissertation. Along with the HypeSax, I have developed strategies to take advantage of this 'creative space'. At the same time, the exploration of

\footnotetext{
225 Bernard Sève, 'Bords de l'œuvre musicale', Aisthesis, 3.2 (2011), 29-45.

${ }^{226}$ Jean-Claude Risset, 'Sculpting Sounds with Computers: Music, Science, Technology’, Leonardo, 27.3 (1994), 257-61.
} 
this 'canvas' has influenced my approach to composition, the way I think about the relationship between performers and medium, and other compositional aspects, such as the role of the solo instrument in relation to the ensemble, or timbre, pitch and rhythm, which I discuss in the following pages.

\subsubsection{Re-embodied sound}

As discussed in section 4.1, with the use of new technology we have the capacity to generate sound without the presence of a body to produce it. We can also create new sounds or modify existing sounds. These bodyless sounds rely on different kinds of transducers to emerge into the physical world and be perceived. These transducers generate sound in response to an electrical input signal by transforming that signal into mechanical movement. This movement is amplified by exciting an external object or one of its components. Surface transducers, for instance, are placed over a surface in order to couple with it and more efficiently radiate sound. Other kinds of systems, like the loudspeaker, include a diaphragm or moving component that amplifies the vibrations of its driver assembly, removing the need to interact with an external object.

Sound is a physical phenomenon, however, and it requires a physical medium in which to exist. In order to bring a bodyless sound to our physical world, a transformation occurs through which the bodyless sound takes the characteristics of the materials and techniques used to generate a real sound, re-embodying the sound into a new body. David Tudor's Rainforest is one of the most iconic examples of re-embodiment of sound in a musical work. In this work, he explores the transformation of sound through the physical properties of different structures and materials throughout the four versions of the piece. ${ }^{227}$ Rainforest exemplifies the way in which the medium becomes an important element in the ontology of the work. It also demonstrates how the creative process can be driven by the manipulation of the medium.

In the case of electrophones, the tendency is to rely on a 'bodyless sound system', in which a loudspeaker is separated from the instrument from which the sonic result of the

227 More information on the different versions of Rainforest can be found at https://davidtudor.org/Works/rainforest.html 
performance emerges. This can be both beneficial and problematic depending on the situation: electric guitars in a rock concert, for instance, might benefit from the use of an external PA directed at the audience, while using a PA facing the audience and no monitor speakers might make synchronisation between performers difficult. To address the issues of sound disembodiment, multiple solutions have been developed ranging from careful PA setups to clever loudspeaker arrays. An example of these solutions are hemispherical loudspeakers originally developed by Dan Trueman - the BoSSA ${ }^{228}$ - with further iterations detailed by Scott Smallwood in 'Don't Forget the Loudspeaker'. ${ }^{229}$ In these cases, while the system remains "bodyless", the effect obtained is that the sound is perceived as emerging from the instrument, and in multiple directions as it would naturally happen to some degree with an acoustic instrument.

Some electrophones include integrated loudspeakers, such as electronic keyboards that can include simple one-loudspeaker setups, or more complex ones such as the Yamaha's CVP-809GP Clavinova piano which features multiple speakers situated in a grand piano body, producing a more natural sound. ${ }^{230}$ Cook and Colby's squeezeVox (Bart and Lisa), for instance, presents a design in which an accordion has been modified to fit and work using an embedded electronic system to control vocal synthesis models. In this case, while the instrument retains its original appearance and, to some degree, mode of performance, an electronic system, that includes an onboard speaker, is in charge of producing the sound. ${ }^{231}$ Similarly, Cook's Nukulele'elua ${ }^{232}$ takes the body of an ukulele. This instrument features two speakers, one facing outward and the other one facing towards the performer. It also features two FSRs allocated over the finger board and

${ }^{228}$ Cook and Trueman.

${ }^{229}$ Scott Smallwood and others, 'Don't Forget the Loudspeaker - A History of Hemispherical Speakers at Princeton, Plus a DIY Guide', in Proceedings of the International Conference on New Interfaces for Musical Expression (presented at the NIME 2009, Pittsburg, PA, 2009), pp. 110-15.

230 More information about the Yamaha CVP Clavinova piano series can be found at https://usa.yamaha.com/products/musical_instruments/pianos/clavinova/cvp-series.html

${ }^{231}$ Perry Cook and Colby Lieder, 'SqueezeVox: A New Controller for Vocal Synthesis Models', in Proceedings of ICMC 2000, 2000.

${ }^{232}$ Perry R Cook, 'Remutualizing the Instrument: Co-Design of Synthesis Algorithms and Controllers', in Proceedings of the Stockholm Music Acoustics Conference, August 6-9, 2003 (SMAC 03), Stockholm, Sweden (presented at the SMAC 03, Stockholm, Sweden, 2003), p. 4. 
bridge. The performer interacts with these sensors as if pressing and plucking the strings. While the instrument retains the looks of the acoustic ukulele, the way it produces sound has been replaced by an electronic system. ${ }^{233}$

Hyperinstruments have traditionally been built under the bodyless sound system paradigm. In recent developments, however, there has been a tendency towards hybrid designs. Some examples include the hybrid string quartet developed by Juan Arroyo, where microphones and transducers are installed by a luthier inside the instruments. The signal captured by the system is then processed by a computer and output back into the instrument's body, turning it into the source from which the sound emerges. ${ }^{236}$ Other similar augmented instruments make use of the body resonance to amplify the processed signal, ${ }^{237}$ of which Laurel Pardue's Svampolin stands out, taking the concept of reembodiment to a new level. In Pardue's instrument, an electric 'stick' violin provides the input signal, which is processed through a Bela board and output via a transducer mounted directly on a violin body with no fingerboard or strings. All elements, fingerboard, Bela board, transducer and violin body are assembled together as one violin. However, unlike in the case of a real violin, there is not a direct acoustic interaction between the strings in the stick violin and the violin body, as a thick layer of foam separates the stick violin and the violin body (see Fig. 51). The result is an instrument that feels and responds like a violin but has the capability of sounding like any other instrument through synthesis embodied in a real violin setup. ${ }^{238}$

${ }^{233}$ Perry R Cook.

${ }^{236}$ Houlès, pp. 29-32.

${ }^{237}$ For more examples of augmented instruments amplifying audio signal through transducers coupled to the instruments' bodies see:

http://instrum.ircam.fr/category/creation-et-rercherche/controle-actif-dinstruments/

https://anr.fr/Project-ANR-11-PDOC-0010

https://medias.ircam.fr/x8f183b_le-projet-anr-imarev-instruments-de-musiq

Benacchio, Simon, Adrien Mamou-Mani, Baptiste Chomette, and René Caussé. 2012. 'Active Control Applied to String Instruments', in Proceedings os the Acoustics 2012 Nantes Conference (presented at the Acoustics 2012 Conference, Nantes, France, 2012)

Overholt, Dan, Edgar Berdahl, and Robert Hamilton. 2011. 'Advancements in Actuated Musical Instruments, Organised Sound, 16.02 (2011)

${ }^{238}$ Laurel S Pardue and others, 'Separating Sound from Source: Sonic Transformation of the Violin through Electrodynamic Pickups and Acoustic Actuation', in Proceedings of the International 


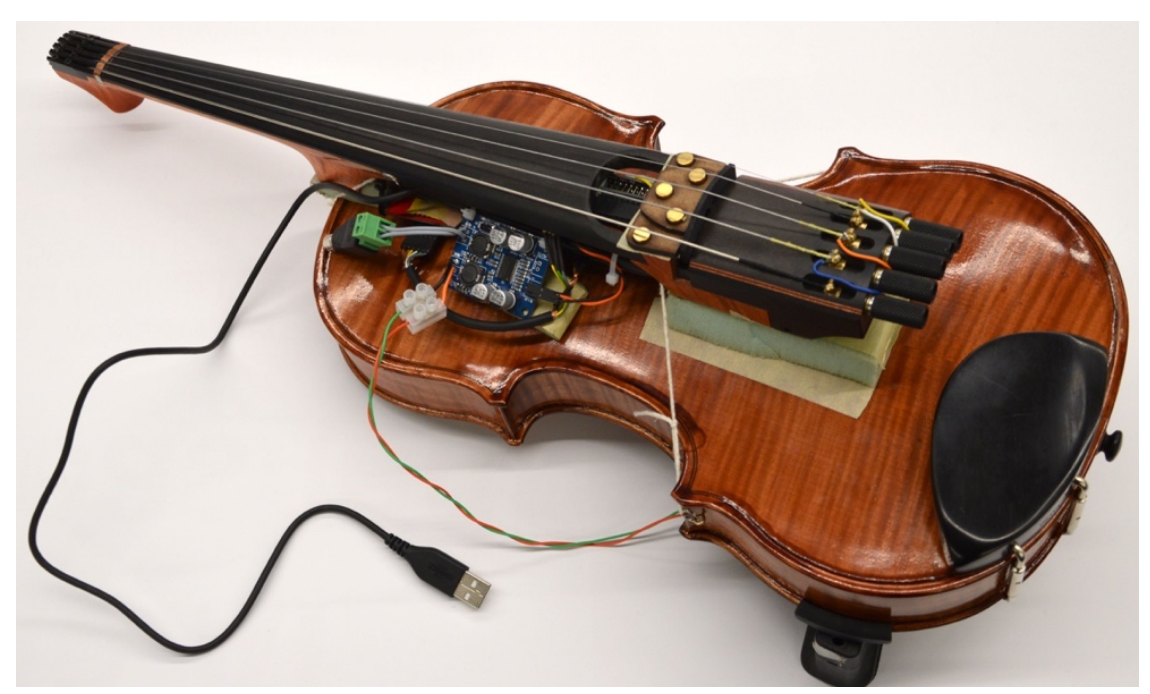

Fig. 51. Pardue's Svampolin. ${ }^{238}$

In the case of aerophones, the production of sound is not based on the vibration of the body or one of the elements of the instrument, but on the air column moving through the body of the instrument. With the first prototypes of the HypeSax, I tried mounting surface transducers outside the bell of the saxophone. While this setup was successful from the perspective of the performer, for the observer the result was underwhelming. The performer is physically joined to the saxophone by closing and pressing the mouth and teeth against the mouthpiece, which transfers the vibration of the saxophone body directly to the performer's auditory system. This configuration, however, prevents the hybridisation of synthesised and acoustic sound using transducers mounted on the body of the instrument in order to make it vibrate, as in the case of Arroyo's hybrid string quartet or Pardue's Svampolin, since the body of the saxophone is not an effective resonator. As a consequence, pushing air through the saxophone is the only way to effectively produce sufficient sound. Complex setups have been developed to produce sound with aerophones for research ${ }^{239}$ and creative ${ }^{240}$ purposes. Unfortunately, these systems override the ability

Conference on New Interfaces for Musical Expression (presented at the NIME 2019, Porto Alegre, Brazil, 2019), pp. 272-77.

${ }^{239}$ For example Bergeot et al.'s Pressure Controlled Artificial Mouth Bergeot and others. created to research the effects of blowing pressure profiles on the clarinet.

${ }^{240}$ For example the acoustic impedance convertor developed by Godfried-Willem Raes for the $<$ Asa $>$, $<\mathrm{Fa}>$ or $<\mathrm{Ob}>$ robots Godfried-Willem Raes, 'Logos Foundation - Godfried-WIllem Raes' Automatons and Robots', Www.Logosfoundation.Org $<$ https://www.logosfoundation.org/instrum_gwr/automatons.html > [accessed 14 May 2018]. 
of a performer to play the acoustic instrument at the same time. If a saxophonist is to perform the acoustic instrument at the same time as the synthesised sound, it is necessary to insert sound waves directly into the instrument using a loudspeaker, rather than inserting a secondary air column.

The IMAREV project, directed by Adrien Mamou-Mani at IRCAM, amongst other achievements, allowed its collaborators to explore multiple ways of introducing synthesis into the body of acoustic instruments. As part of this project, Thibaut Meurisse studied the effects of applying active control to wind instruments. Active control consists of 'a way of modifying the way in which a mechanical system vibrates, with the help of sensors, actuators and a controller'. ${ }^{241}{ }^{242}$ Meurisse developed a trombone mute ${ }^{243}$ and a simplified bass clarinet ${ }^{244}$ seeking to apply the concept of the active control. Meurisse and the IMAREV team were able to demonstrate that it is possible to change the perceptual sonorities of wind instruments by applying active control techniques through the use of a microphone, loudspeaker and an analysis/synthesis system. This approach, used in combination with Paul Clift's Acoustic Aggregate Synthesis, allows for the transformation of the bass clarinet timbre, simulating the timbre of other instruments through a hybridisation of acoustic sound and synthesis. ${ }^{245}$ This hybridisation is perceptually possible only when the source of the synthesis emanates from the acoustic instrument, or, in the case of smaller instruments that cannot house a loudspeaker, such as a flute, in close proximity.

${ }^{241}$ Thibaut Meurisse, 'Contrôle actif appliqué aux instruments de musique à vent' (Université Pierre et marie Curie, 2014), p. 4.

${ }^{242}$ Original in French: 'Le contrôle actif est un moyen de modifier la façon de vibrer d'un système mécanique à l'aide de capteurs, d'actionneurs et d'un contrôleur'.

243 Meurisse, Mamou-Mani, Caussé, and others.

244 Thibaut Meurisse, Adrien Mamou-Mani, Simon Benacchio, and others, 'Experimental Demonstration of the Modification of the Resonances of a Simplified Self-Sustained Wind Instrument Through Modal Active Control', Acta Acustica United with Acustica, 101.3 (2015), 581-93.

${ }^{245}$ Paul Clift, 'Department of Music Columbia University, New York, NY', in Proceedings of the International Computer Music Conference (presented at the ICMC 2012, Ljubljana, 2012), pp. 120-23; Paul Clift, Adrien Mamou-Mani, and René Caussé, 'Extending Brass \& Woodwinds with Acoustic Aggregate Synthesis', in Proceedings of the International Computer Music Conference (presented at the ICMC 2015, Denton, 2015), p. 7; Paul Clift, 'Acoustic-Aggregate-Synthesis | Paul Clift', Www.Paulclift.Net $<$ http://www.paulclift.net/aas/> [accessed 26 May 2020]. 
In 2014, Marco Suárez Cifuentes composed Libellule for the first hybrid bass clarinet (a product of the IMAREV project) and ensemble, which feature multiple possibilities of configurations of sound re-embodiment. In his $\mathrm{PhD}$ thesis, Suárez Cifuentes describes these configurations in terms of spatialisation. The piece calls for an octophonic loudspeaker array surrounding the audience, plus a quadrophonic array placed behind the ensemble. An audio system is also mounted in the bell of the hybrid bass clarinet, which is situated on a podium in front of the ensemble in the middle of the stage. This complex audio setup allows for the musical gesture to be transformed from solo-acoustic-clarinet to solo-hybrid-clarinet. Orchestrations of the gestures are also possible while diffusing the audio in multiple ways throughout the space. ${ }^{246}$

Similar variability of transformation in the sound re-embodiment configuration is also possible with the HypeSax. As explained in section 3.3, the Un-mute provides a solution for integrating a sound system into the body of the saxophone, while allowing for the extension of its sounding capabilities to be produced out of the instrument through a USB connection to a second sound system. In this way, the hybrid capabilities of the HypeSax offer three different configurations of sound embodiment: a) embodied sound, b) disembodied sound, and c) bodily extended sound (see Fig. 52). All of these configurations allow for multiple combinations of acoustic, synthesised or hybrid sound embodiment (resembling the multiple combinations used in Libellule).

${ }^{246}$ Marco Antonio Suárez Cifuentes, 'Corps, gestes, perceptions Interrelations, articulations et poétiques de l'espace instrumental, acoustique et électro-acoustique' (Conservatoire National Supérieur de musique et danse de Paris, 2017), pp. 80-81. 


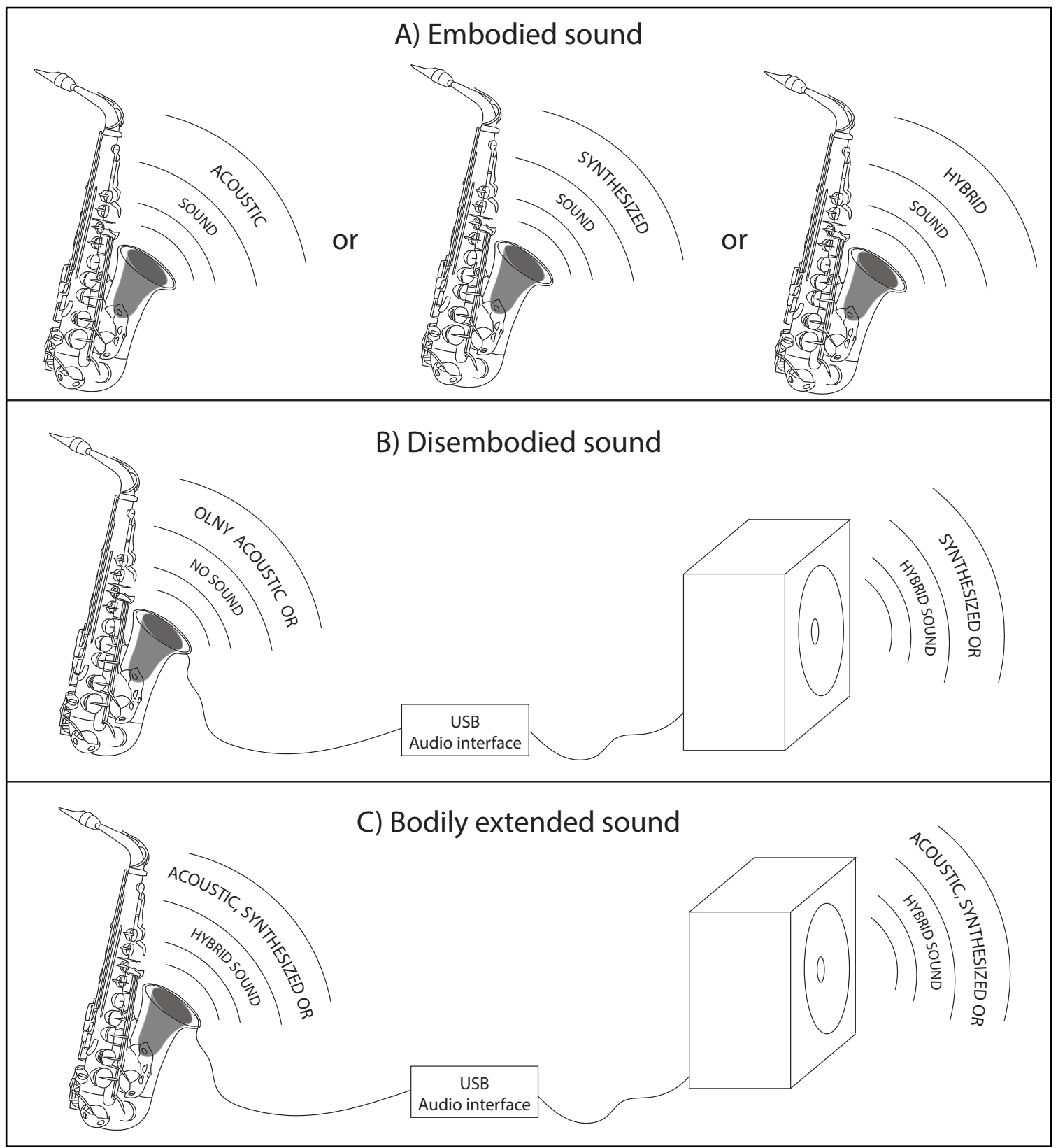

Fig. 52. HypeSax’s possible configurations of sound embodiment.

\subsubsection{Data processing and software expansion: The Hub}

Once the hardware was developed and characterised, the next step was to find ways to process the data obtained by the sensors in the HypeSax. The microcontrollers used in the HypeSax are capable of communicating simultaneously with external devices using serial, audio and MIDI protocols. A Max patch called The Hub was developed to facilitate reading and using data from the HypeSax's sensors. This patch is capable of reading the computer's serial ports and configuring the audio engine to work directly with the 
HypeSax working as a sound card, which can be useful when setting up a third Max patch. It can also process and send data over the OSC protocol.

The master microcontroller collects data from all sensors and creates a 58-characterlong messages that is sent over the serial port. This message includes 'space' characters which are used in the Hub to break the message into a series of short messages that can be easily converted to other communication protocols. The HypeSax scales the original values from the sensors to a series of three-digit numbers, for each analogue sensor, and one-digit numbers, for discrete values (see Fig. 53). This mapping is necessary in order to maintain a consistent size of the full message being sent, thus avoiding data loss or losing synchronisation on arrival to The Hub. While larger numbers were tested, in practice, no noticeable difference can be perceived beyond the 0-255 range.

In order to use the Hub, users must first set up the HypeSax components. ${ }^{247}$ Next, they must connect the HypeSax to the computer using the USB port. Once the LEDs are illuminated, the HypeSax is communicating with the computer. At this point, the user can follow the instructions available on the Hub by pressing the 'setup help' button. This button displays a set of steps necessary to set up the HypeSax in order to read data, calibrate data, and set up the audio interface. After this, adjusted serial data are converted into values that are sent via [send] messages to Max patches. The Max messages send a range of values according to the characteristics of each sensor. These messages are listed on Table 2.

${ }^{247}$ For more details on how to set up the HypeSax, visit www.hypesax.com or the HypeSax YouTube channel. 

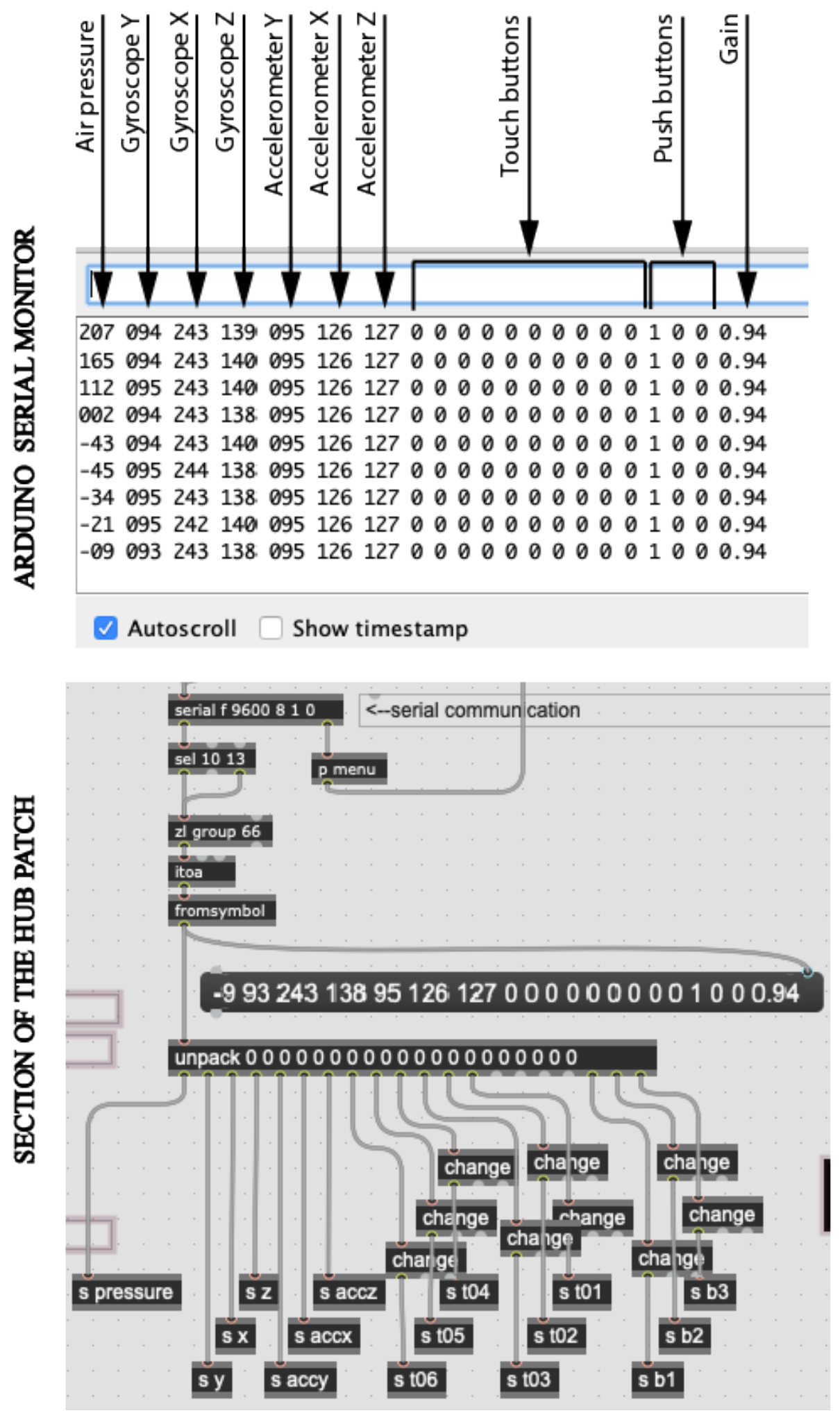

Fig. 53. Screenshot of Arduino and Max programs showing how data is handled. 


\section{THE HUB}
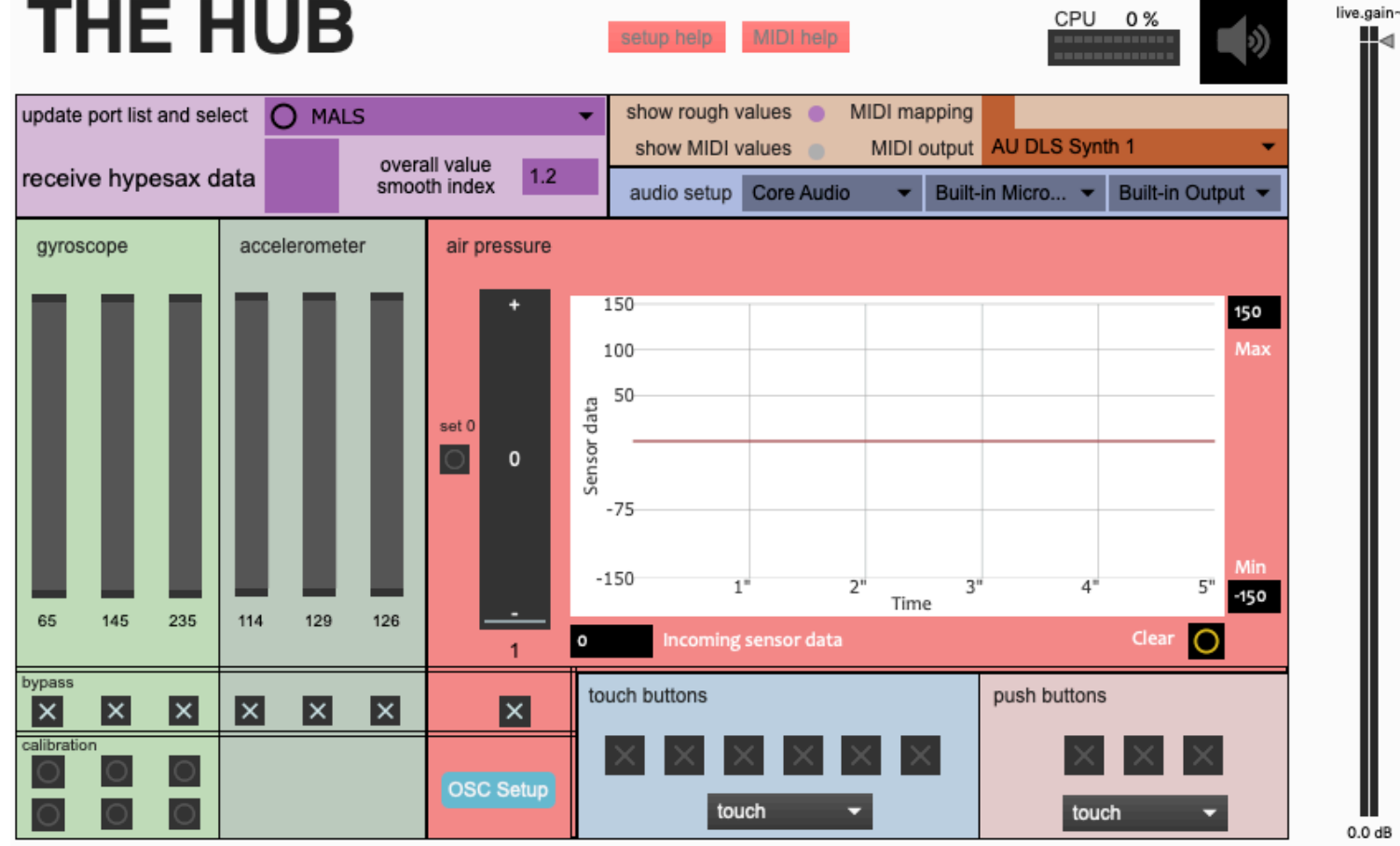

\section{THE HUB}
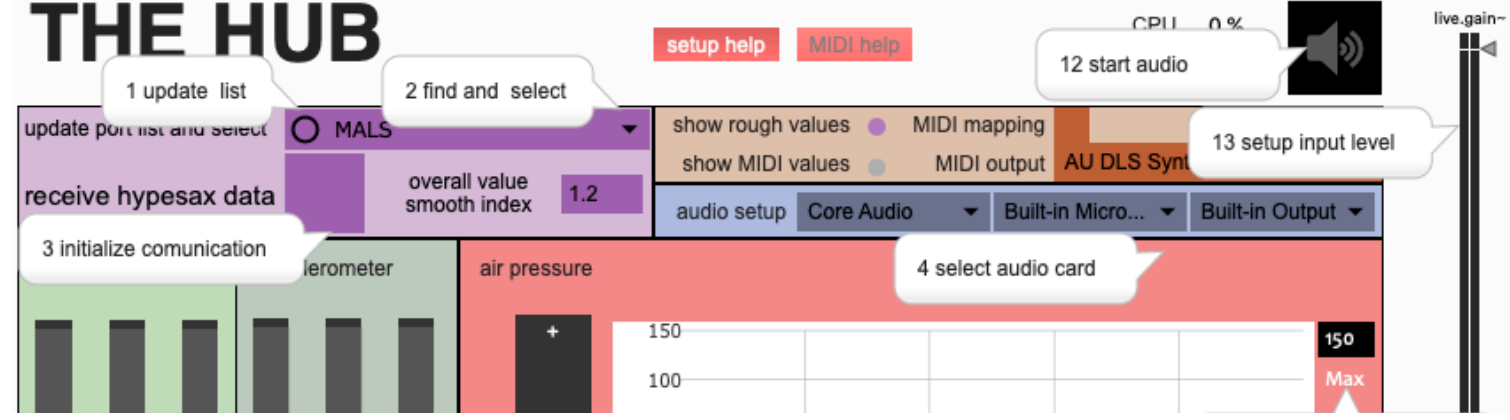

Fig. 54. Screenshot of The Hub (top) and a partial screenshot of The Hub displaying setup instructions via 'setup help' button.

Table 2. Detail of data sent via [send] messages over Max

\begin{tabular}{|c|c|c|}
\hline Description & Message & Value range \\
\hline Air pressure & [send air] & -150 to 150 \\
\hline Gyroscope Y & [send gyy] & 0 to 255 \\
\hline Gyroscope X & [send gyx] & 0 to 255 \\
\hline Gyroscope Z & [send gyz] & 0 to 255 \\
\hline Accelerometer Y & [send acy] & 0 to 255 \\
\hline Accelerometer X & [send acx] & 0 to 255 \\
\hline Accelerometer Z & [send acz] & 0 to 255 \\
\hline Keycap button 1 & {$[$ send t1] } & 0 or 1 \\
\hline
\end{tabular}




\begin{tabular}{|c|c|c|}
\hline Keycap button 2 & {$[$ send $\mathrm{t} 2]$} & 0 or 1 \\
\hline Keycap button 3 & [send t3] & 0 or 1 \\
\hline Keycap button 4 & [send t4] & 0 or 1 \\
\hline Keycap button 5 & {$[$ send t5] } & 0 or 1 \\
\hline Keycap button 6 & {$[$ send t6] } & 0 or 1 \\
\hline Thumb button 1 (HypeSax v.1) & {$[$ send b1] } & 0 or 1 \\
\hline Thumb button 1 (HypeSax v.2) & [send b1] & 0 to 5 \\
\hline Thumb button 2 & {$[$ send b2] } & 0 or 1 \\
\hline Thumb button 3 & [send b3] & 0 or 1 \\
\hline
\end{tabular}

These values are scaled to either 0 to 127 for continuous data, and 0 or 127 for discrete data when the option 'show MIDI values' is selected. Pressing the 'MIDI help' button displays a set of instructions to convert the serial data into MIDI values and send them through a virtual instrument to any third-party software or device. All of the values can be 'soloed' to facilitate mapping for use by other software applications.

In the same way, pressing the 'OSC setup' opens a new window to set up OSC communication. The messages sent over OSC are organised as shown on Table 3.

Table 3. Detail of data sent via OSC protocol

\begin{tabular}{|c|c|c|}
\hline Description & Message & Value range \\
\hline Air pressure & hypesax/air + value & -150 to 150 \\
\hline Gyroscope Y & hypesax/gyy + value & 0 to 255 \\
\hline Gyroscope X & hypesax/gyx + value & 0 to 255 \\
\hline Gyroscope Z & hypesax/gyz + value & 0 to 255 \\
\hline Accelerometer Y & hypesax/acy + value & 0 to 255 \\
\hline Accelerometer X & hypesax/acx + value & 0 to 255 \\
\hline Accelerometer Z & hypesax/acz + value & 0 to 255 \\
\hline Keycap button 1 & hypesax/t1 + value & 0 or 1 \\
\hline Keycap button 2 & hypesax/t2 + value & 0 or 1 \\
\hline Keycap button 3 & hypesax/t3 + value & 0 or 1 \\
\hline Keycap button 4 & hypesax/t4 + value & 0 or 1 \\
\hline Keycap button 5 & hypesax/t5 + value & 0 or 1 \\
\hline Keycap button 6 & hypesax/t6 + value & 0 or 1 \\
\hline
\end{tabular}




\begin{tabular}{|c|l|c|}
\hline Thumb button 1 (HypeSax v.1) & hypesax/b1 + value & 0 or 1 \\
\hline Thumb button 1 (HypeSax v.2) & hypesax/b1 + value & 0 to 5 \\
\hline Thumb button 2 & hypesax/b2 + value & 0 or 1 \\
\hline Thumb button 3 & hypesax/b3 + value & 0 or 1 \\
\hline
\end{tabular}

The OSC configuration window offers the option to set up an outgoing port (see Fig. 55). Messages sent to this port have no effect on the Hub or the HypeSax, but it can be used to send information between multiple programs or devices passing through the Hub, which ensures synchronization and avoids timing issues. More detailed information on how to use the Hub can be found directly on the software, which can be downloaded from www.hypesax.com, where instructional videos as well as musical examples can be found.

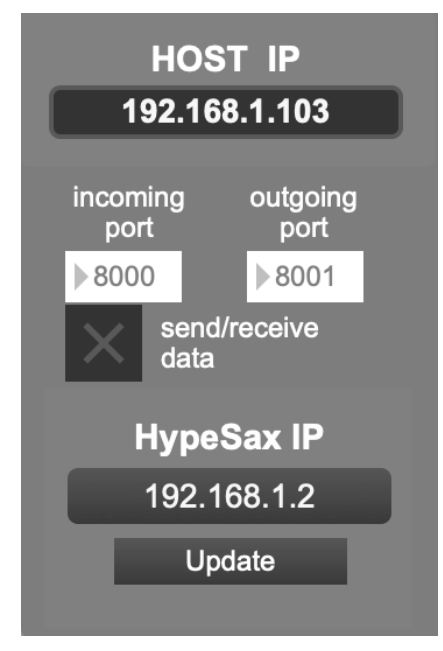

Fig. 55. OSC setup window.

In section 2.4, the concepts of the score and medium are discussed, stating that the software is not the score, but it can be the medium. It is clear that the Hub is not the score. Section 2.4.3 also states that the medium is what creates or modifies the sound. So, considering this statement, one can observe that the Hub is not the medium, as its function is not to create or modify sound. What it does is to collect data and translate it to values recognisable by MIDI, OSC and Serial communication protocols. In a way, it could be considered an extension of the HypeSax - which is the medium-but the Hub is an independent piece of software which could very well work with another controller that outputs Serial or MIDI data. The HypeSax on its own does not rely on the Hub, as the 
Serial data it outputs could be managed by another software. Similarly, the outcoming MIDI data can be received and handled by third party software or any MIDI device. The Hub, as its name suggests it, is the centre where all the activity data from the HypeSax can be found. There, data is converted, re-mapped, and routed according to the needs of the user. The Hub does not fit in the tripartite model, as it serves only as a tool to facilitate managing data, similarly to a piece of paper serving to facilitate the notation of a piano sonata.

\subsubsection{Physicality of the HypeSax, an interface}

While the HypeSax offers capabilities for manipulating synthesised sound that can be output in multiple sound embodiment configurations, the HypeSax is also a physical instrument. It does not rely on its physicality to produce sound, but its physicality determines certain limitations. The most important aspect of the physicality of the HypeSax is the way in which it functions as an interface, defining the relationship between the user and sound.

Bert Bonger, in his article 'Physical Interfaces in the Electronic Arts,' describes a human-machine interaction model consisting of three elements: a human, interaction and system (machine). The interaction encapsulates a two-way communication feedback loop that allows the human and system to act, and then react to each other (see

Fig. 56). Bonger also states that multiple modalities, or communication channels, can be involved in this feedback loop (see Fig. 57). 
5.1 Canvas

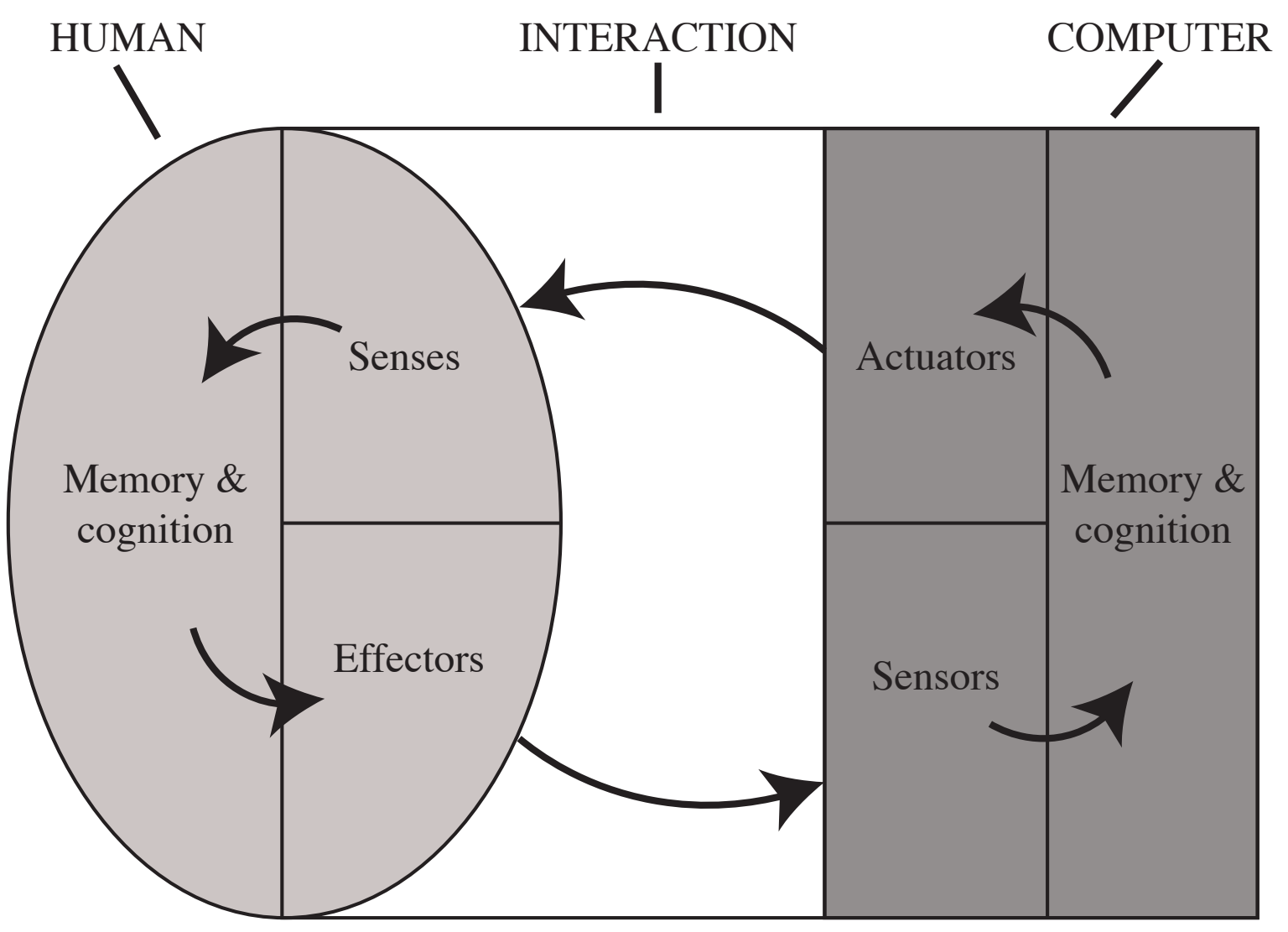

Fig. 56. Bonger's human-machine interaction model. 


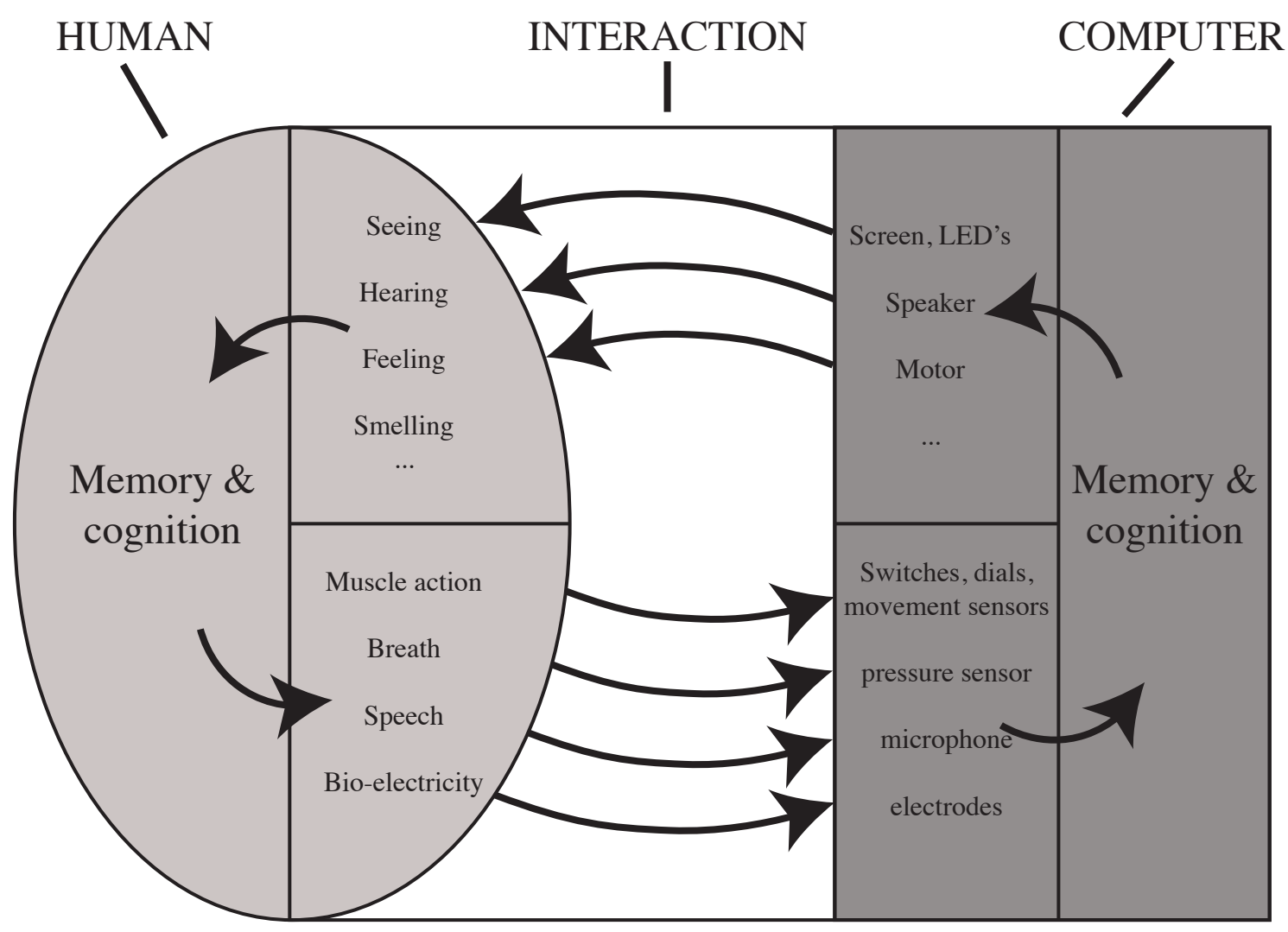

Fig. 57. Bonger's model including multiple modalities. ${ }^{248}$

Taking Bonger's model as a paradigm, it becomes easier to establish a better understanding of the HypeSax as the medium and its relationship with the performance element of the tripartite model. By replacing 'human' in Bonger's model with the 'performer', and the computer with HypeSax, one can visualise the multiple modalities involved in the feedback loop. In

Fig. 58, new modalities of interaction, in the proposed adaptation of Bonger's model, become evident. While it is clear that some of these modalities exist in the relationship between a performer and a saxophone, there are modalities that are new. For instance, while traditionally ancillary motion has no sounding result, with the aid of the HypeSax this motion can be captured and used to generate sound. If it had been previously decided that the HypeSax would generate signals in response to certain physical ancillary action,

248 Bert Bongers, 'Physical Interfaces in the Electronic Arts', Trends in Gestural Control of Music, 2000, 30 (pp. 44-45). 
then intrinsically, the score could also be embedded in the software running with the HypeSax.

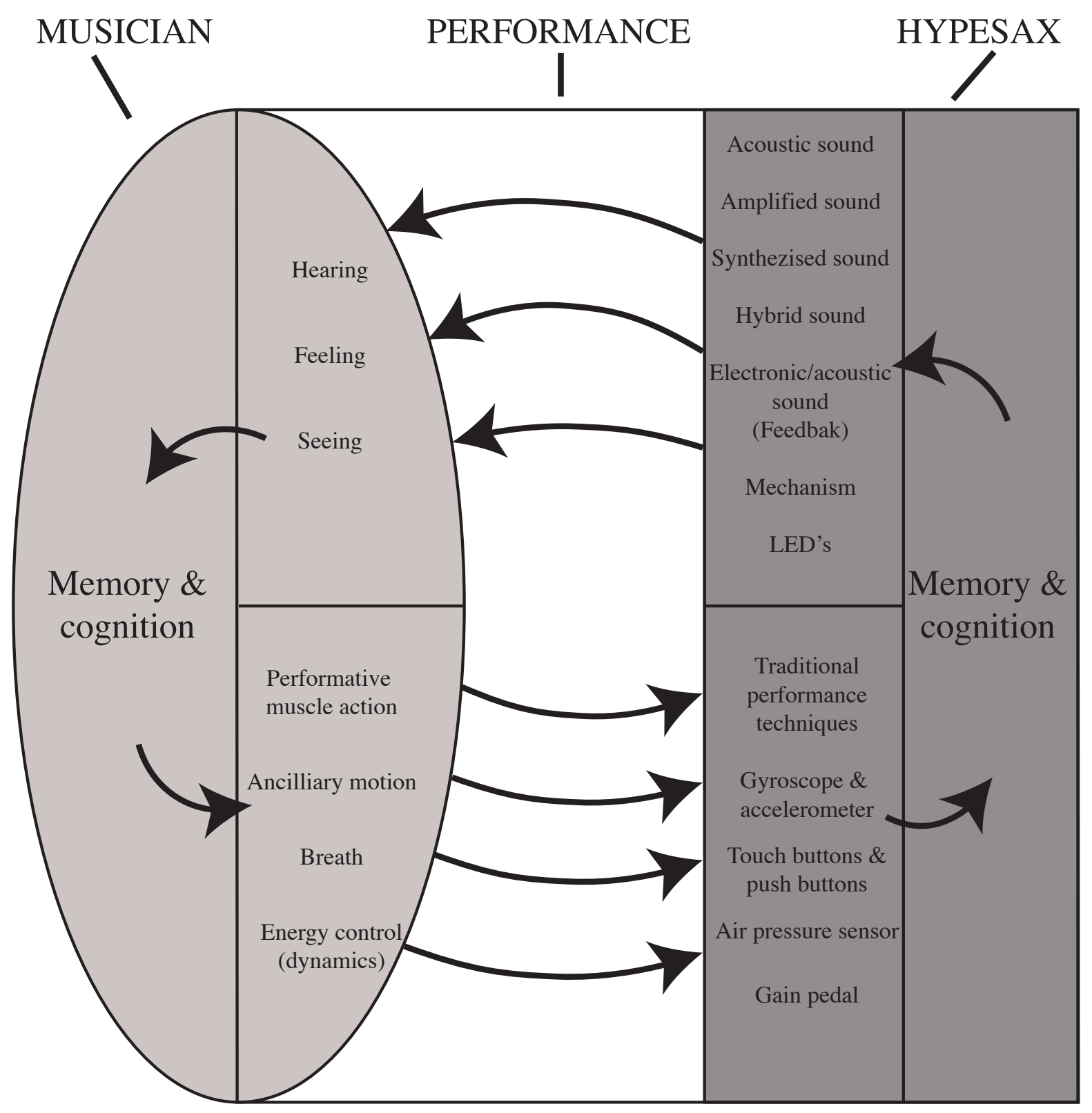

Fig. 58. Adaptation of Bonger's model reveals the aspects of the relationship between performance and medium in the use of the HypeSax. 
In this model, the musician represents both the performer and the composer. As stated in 2.4 , the score represents the composer's musical design. So, if we observe the flow in this model we can find that the score element can occupy a place next to the memory and cognition of the musician, which takes the score as motor and reference which activates and maintains the feedback loop. In the same way, if it occurs in generative, algorithmic and other kinds of self-generative compositions, the HypeSax could have a relevant role in the generation or manipulation of the score. With this in mind, Bonger's model can then have a final modification (see Fig. 59).

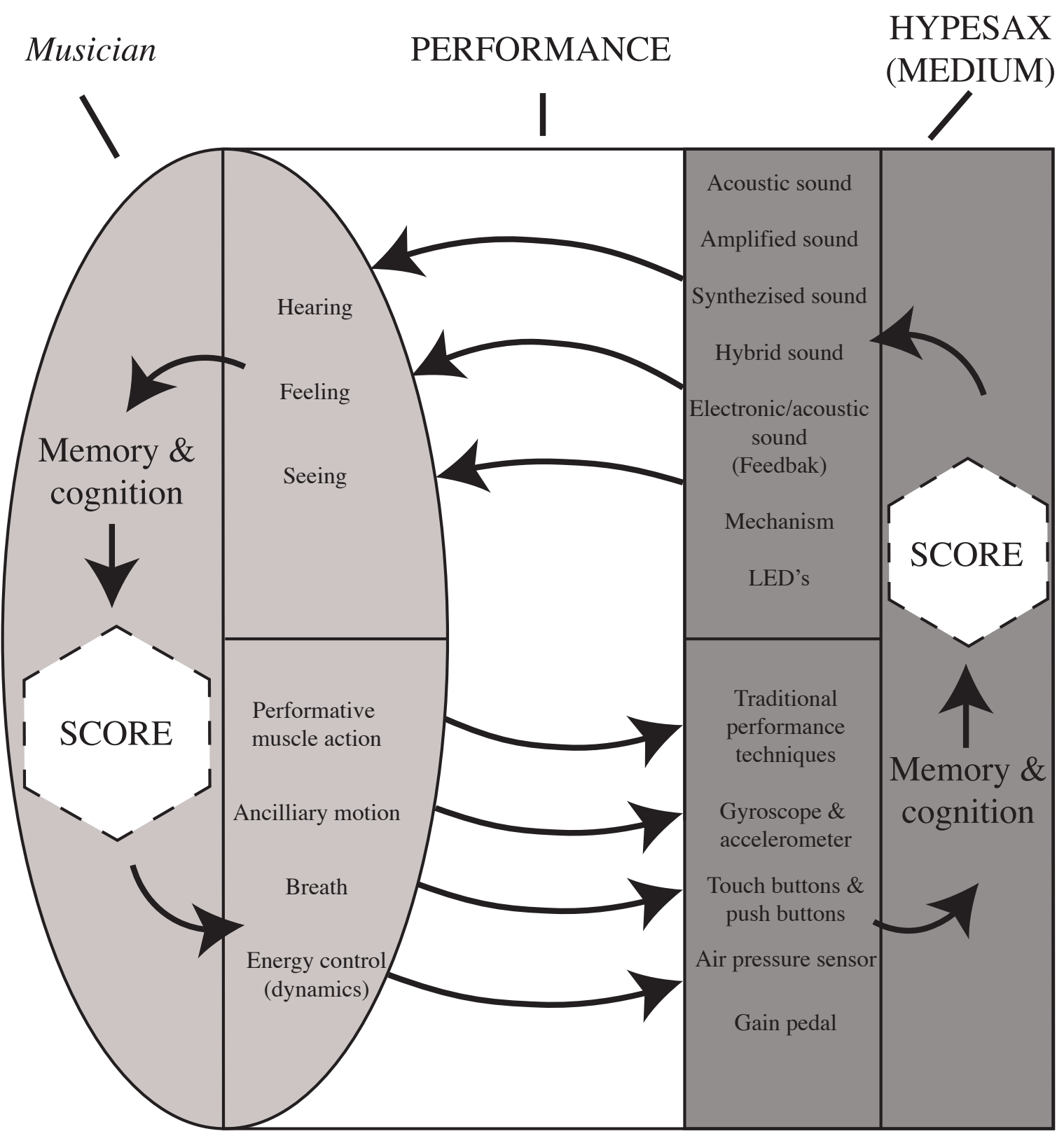

Fig. 59. Final modification to of the model identifying the intrinsic score. 
The adaptations made to Bonger's model aids in visualising how the elements of the tripartite model of the musical work fit in a workflow where causes and consequences are identifiable, and how the HypeSax (or any other hyperinstrument) can facilitate this workflow. I will exemplify this model in the next section, where I also discuss how my own creative process was benefited by the use of the HypeSax, and how, at the same time, it was influenced and pushed in unexpected directions by the process of development and experimentation with this new medium.

\subsection{New works}

Up to this point, I have presented some of the musical ideas that represent my personal approach to composition. Now, in order to observe how these ideas and the HypeSax come together in my works, I will focus on describing the process through which I composed a portfolio of works during the time I was developing the HypeSax. To begin this section, I will continue with a discussion on the adaptation of Bonger's model. To clearly exemplify how this model fits in a real-life scenario, I will present a discussion of my piece Look Out! then, I will continue with a discussion on compositional strategies using other works of the portfolio as examples.

\subsubsection{The model in practice: Look Out!}

The creation of this piece was inspired by the work that Wellington-based ensemble Noveltones had been presenting in the past two years. The members of this ensemble are Blair Latham (bass clarinet), Jasmine Lovell-Smith (soprano saxophone), Tristan Carter (violin) and Tom Callwood (double bass). Latham, Lovell-Smith and Callwood have a strong Jazz background, while Carter graduated from a classical music programme. Their professional work as composers and performers has allowed them to delve into a multiplicity of musical styles and music scenes. The music that the Noveltones has developed as an ensemble has been enriched by the varied background of its members. While none of the compositions in their repertoire has been collectively designed, these compositions allow for their musicianship and musical personalities to flourish into a 
unique non-idiomatic ${ }^{249}$ ensemble sound. These compositions feature scores that allow for a degree of improvisation similar to that found in the jazz tradition but with elements of aleatoricism and indeterminacy that allow for their varied musical backgrounds to surface during performance. I was interested in collaborating with them to explore the performance aspect of the tripartite model through observing their response to my musical ideas.

While composing this piece, I was able to fully observe the tripartite model in my own creative process by following the workflow described in Fig. 59 (modified Bonger's model). This composition began with the premise that the performers had a specific 'personal sound'. The first step was to try to appropriate the performance aspect of the model to my own work. After a few attempts at writing musical passages that resembled the Noveltones's sound, I realised that the variety of their backgrounds and their nonidiomatic approach to improvising as an ensemble was almost impossible to reproduce, as their sound is in constant renewal. I decided that, rather than providing specific determined musical passages, I should take advantage of their strong sense of musicality and offer an improvisatory framework where their personal sound could be expressed, and the performance could be a moulding force in the composition. The role of the HypeSax would be to participate as a fifth member of the ensemble, not performing, but monitoring and suggesting ways to navigate the score, similarly to a director in a conducted improvisation.

This composition was written for an improvising ensemble of four performers, one of which plays the HypeSax, while the other instruments are unspecified. The unspecified parts allow for other ensembles of different instrumental configurations to engage with the piece, allowing for multiple instrumental techniques and musical backgrounds to be inserted in the composition. The score for Lookout! is not fixed. It is interactive and is embedded in a Max patch. The score is streamed over a network using MiraWeb ${ }^{250}$ so that performers can follow it using a personal device such as a tablet, laptop, or phone by visiting the website to which the HypeSax Max patch is streaming the Mira objects (see

\footnotetext{
${ }^{249}$ In this case I am using Derek Bailey's definition of idiomatic that, rather that expressing ergonomic aspects of instrumental performance, points at specific styles of playing (or improvising) in a given genre Derek Bailey, Improvisation Its Nature and Practice in Music (Da Capo Press, 1992)..

${ }^{250}$ MiraWeb is a Max package that allows to communicate with Mira objects over an internet browser.
} 
Fig. 60). Look Out! requires the use of the Un-mute, since the role of the HypeSax is to monitor the ancillary motion of the saxophonist via gyroscope and accelerometer. Using the incoming data, the Max patch looks for ancillary movement, gestures and patterns in the saxophonist performance, which are used to generate a sort of internal entrainment of the system and follow cues. The thumb buttons are necessary to indicate to the HypeSax that the ensemble is ready to move on to a new section of the piece. The Max patch receives information from the HypeSax in order to suggest a page of the score, as well as a harmonic world based on audio analysis of the saxophone.

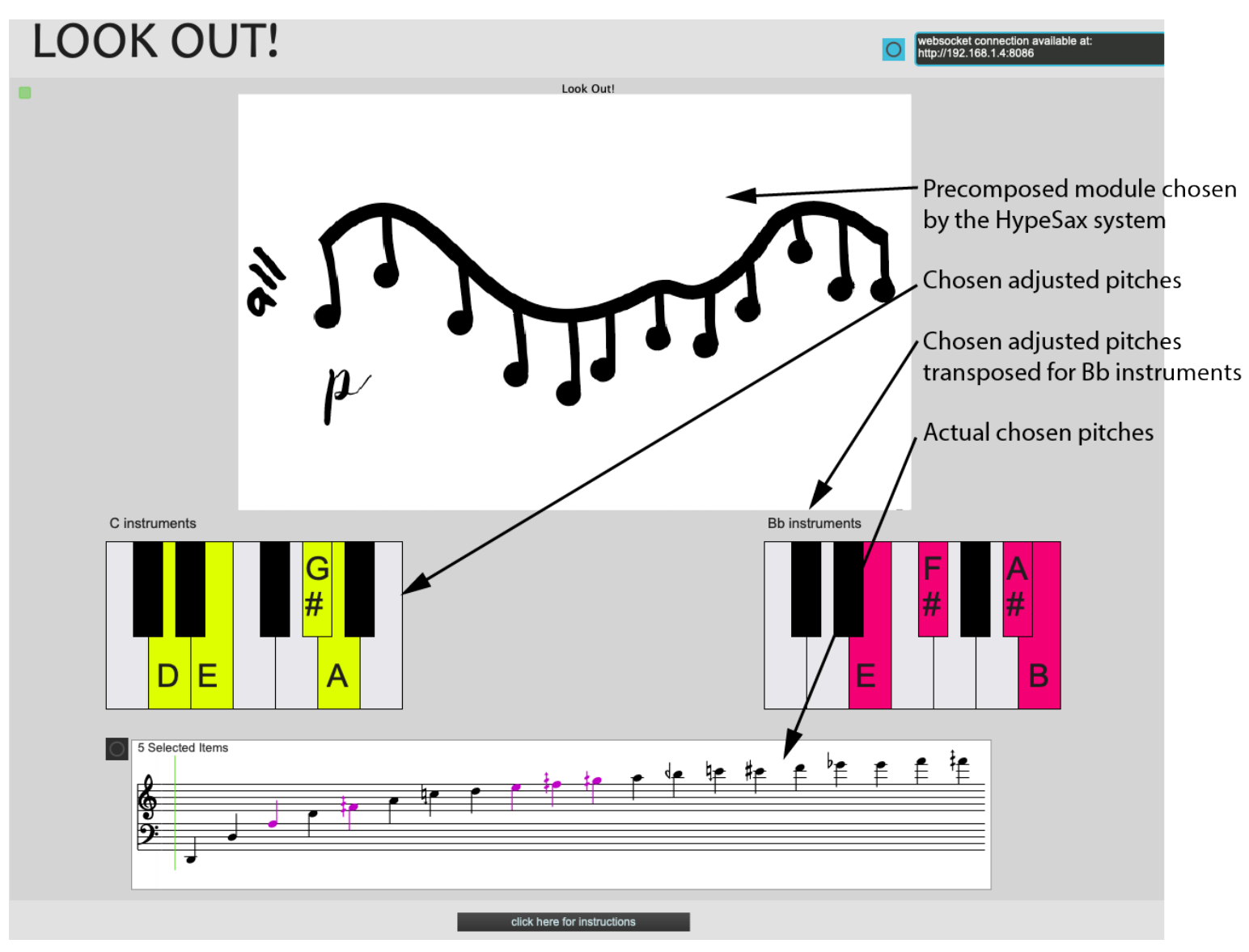

Fig. 60. Look Out! Score displayed on MiraWeb.

The ontology of Look Out! demonstrates the tripartite model when the composition is observed in the following way:

1) The 'Noveltones sound' (performance) inspire an indeterminate score. 
2) The score is precomposed and interactive, allowing for a loop of decisions taken by the system and the performers reacting to each other

3) The performers re-compose the piece by providing it with actual sounds which were not previously defined.

4) The HypeSax system monitors the performance and suggests new directions in which the piece can be taken.

5) The performers react to the suggestion, and the loop continues proceeding to revisit the three elements of the tripartite model until the performers decide to end the piece.

It can be observed that each element, the score, performance and medium, have a great degree of influence in the final piece. This dynamic composition feeds off the three elements and reinvents itself every time, appropriating decisions made by the composer, the performers and the medium, and integrating the three different sound worlds.

The piece begins with one note chosen by the HypeSax player (see Fig. 61, top left image). The rest of the instruments join in with the same pitch (or its equivalent in the appropriate octave for the instrument). The HypeSax player then calls for a suggestion for a new event by pressing button 2 (left thumb push button). The patch, which is continuously detecting the fundamental pitch of the saxophone, chooses randomly up to six pitches out of the first nineteen harmonics of the pitch being played by the saxophone when the button is pressed. The fundamental and the chosen six pitches are then adjusted to fit in one octave, and microtones are rounded in order to show a set of notes that can be displayed in a keyboard. A musical staff is display showing the harmonic series and the selected pitches with the appropriate accidentals, rounded from the nearest quartertone. With this information available, the performers are free to choose one of the proposed notes by the system and then move slowly towards it. They can choose to move towards a pitch class and decide in which octave to place it, or choose and play a specific pitch shown in the staff (quartertones are a possibility). When the musical gesture is completed, the leader-HypeSax player — calls for a new section (or module) of the piece using button 2 . The system chooses the next module randomly but considering the multiple parameters that are being monitored, such as average motion of the saxophone or relative loudness. Preferably, the HypeSax player should be aware of the current harmonic landscape in order 
to choose a new pitch when calling for a new section, as this pitch will be the basis for the generation of the pitch class set that will be used in the next module. This potentially allows for harmonic compatibility between both sections.

In a similar way, performers keep moving through a set of graphic representations that suggest the exploration of three categories of events: sequences of musical gestures and rhythmic patterns for the entire ensemble, or an indications of an ancillary movements, to be realised by the HypeSax player, over which the ensemble creates atmospheres using the suggested harmony (see Fig. 61). Performers decide when to move forward to the next module of the score, 'fading' into it when they consider it to be convenient. The score does not impose defined materials, but it is open to interpretation by offering only a suggestion of events, devoid of specific pitch material.

Each category of actions found in Look Out! is presented with a different colour: black for the musical gestures, blue for rhythmic patterns and red for ancillary motion. The musical gestures present notes, beams and stems that suggest a change of pitch and relative duration of the notes. The performers are free to realise these musical gestures at whatever tempo they choose. The pitches proposed by the system are used to generate a background atmosphere, while the musical themselves gestures can be realised by using those pitches or by freely choosing to use scales, pitch-class sets or any other system. This allows for the musician's personal musical background and language to be integrated in the composition. In some cases, these graphics may indicate different actions for each instrument. In Fig. 61, four of the possible modules are presented: on the top left, the opening musical gesture; top right a musical gesture where with indications for every instrument; bottom left square shows a rhythmic pattern; and bottom right shows an indication to realise an ancillary movement. 

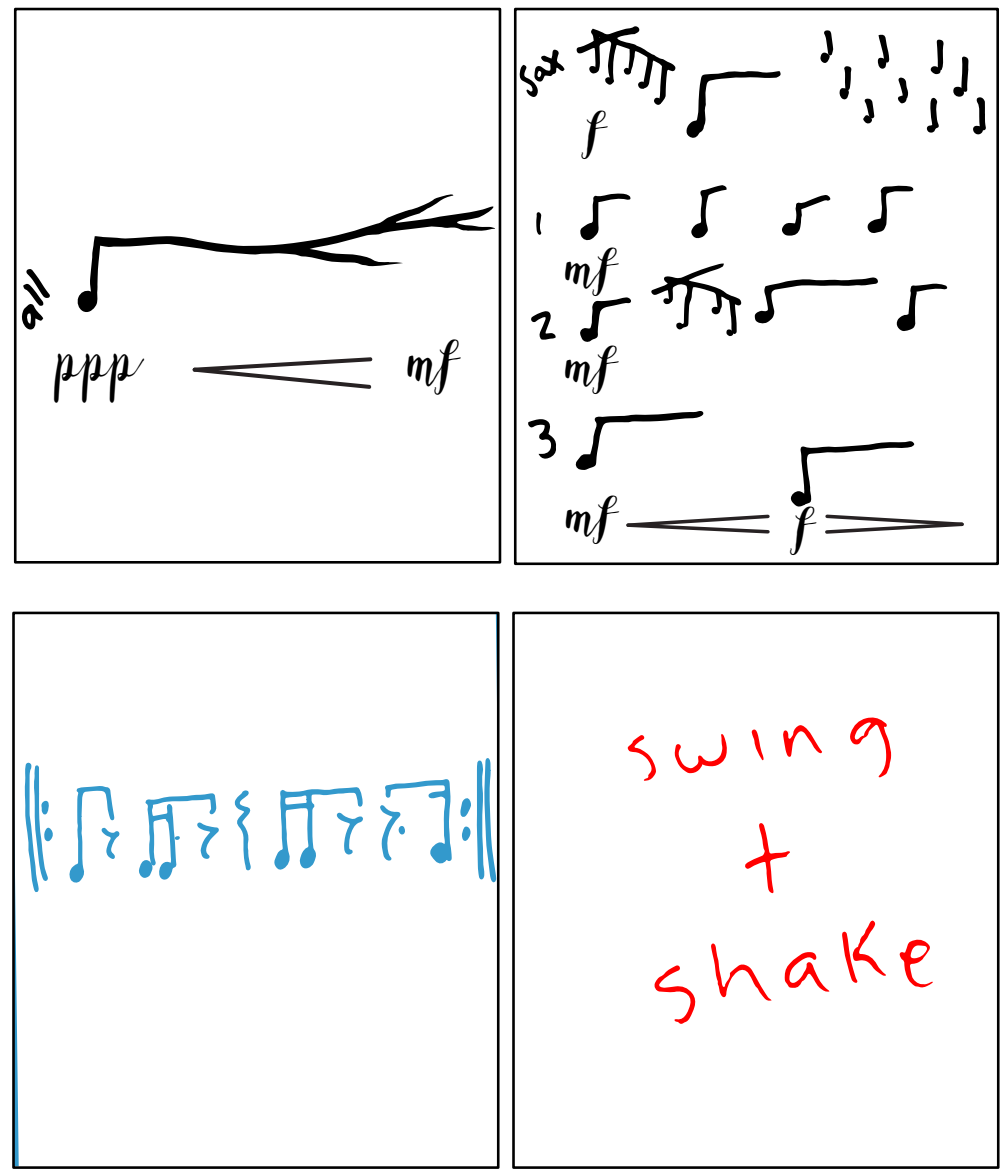

Fig. 61. Four modules from Lookout!.

The rhythmic patterns do not specify whether the performers should be synchronised or if the pitch content should be static; this allows for the creation of either a synchronous or asynchronous texture.

Ancillary movement indications are completely abstract. When these indications appear, the HypeSax player is meant to explore the response of the software to the ancillary movement. At this point, the Max patch activates a concatenative synthesis module that is previously loaded with - in this version for the Noveltones - samples of saxophone and strings. The data coming from the HypeSax is used to navigate a Cartesian X/Y map of the samples created using the iMuBu package in order to generate concatenative synthesis. An indication such as 'Swing + Shake' (see Fig. 61) uses the gyroscope in the Un-mute to navigate the sample pool. The performer is free to swing and shake the HypeSax in any possible way while exploring the virtual $\mathrm{X} / \mathrm{Y}$ space where the samples are scattered. The rest of the performers are expected to fill the acoustic space with an atmosphere generated 
using the proposed pitch-class set or imitating the resulting sound gestures generated by the synthesis.

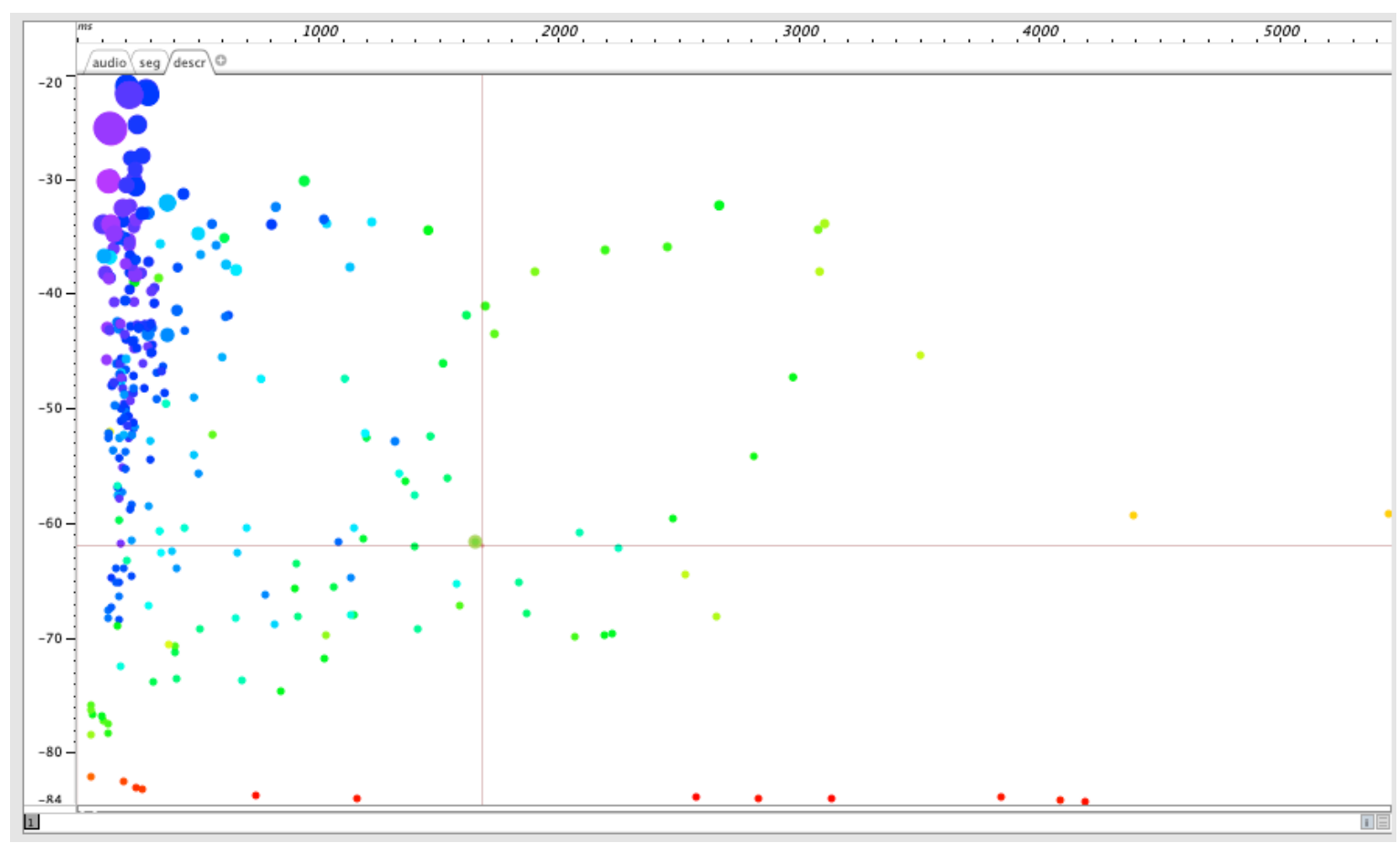

Fig. 62. Cartesian $\mathrm{X} / \mathrm{Y}$ space allocating audio samples to be navigated though ancillary motion.

In this example, I focused on the relationship between the three elements. To better understand how developing the HypeSax and thinking deeply about the medium has changed my understanding musical works, and why I am proposing the tripartite model, below I analyse how the three elements are approached independently in my portfolio of works.

\subsubsection{The score in my portfolio of works}

In Section 2.6.2, I mentioned that the tripartite model does not integrate reception, which, I believe, is an important aspect of the ontology of the work. In the same section, I suggested that the model can be extended to integrate reception via the medium's power to translate the musical work into an actual sounding realisation of the composition. I listed two compositional strategies that can be transferred to any pitch organisational system (tonal, pitch class set theory, spectral approaches, etc.) which I have used in my portfolio: Gestalt theory and musical gesture. In this section I focus on analysing how the medium can enable paradigms to implement these compositional strategies. 


\subsubsection{Gestalt theory in Breathe in, breathe out}

In Section 2.6.2.1, I described James Tenney's adaptation of Gestalt theory to describe our cognitive relation with the musical phenomena. According to his approach, the principles of similarity, intensity, objective set, subjective set, and cloud clang help our brains organise the sounds we hear from a composition in order to be able to follow and understand it. This is, perhaps, where audience reception begins.

Before I began the current thesis, I had already integrated the concepts of Gestalt theory into my compositional practice. I was interested in exploring the possibility of developing new strategies using these compositional tools in conjunction with the awareness of the medium, by taking advantage of the hybrid voice of the HypeSax, combining acoustic and electronic feedback.

Breathe in, breathe out, for alto HypeSax and string quartet, was composed using the concepts of Gestalt theory. In this composition, the relationship between the HypeSax and the strings is explored in four different ways:

- the HypeSax and strings acting together as a meta-instrument;

- the HypeSax and strings act as contrasting elements, taking respectively the roles of foreground against background;

- the contrasting of rhythmic elements against holophonic textures; ${ }^{251}$

- following the paradigm of the melody-accompaniment relationship.

It is worth mentioning that I am interested in creating an 'ethos' for the HypeSax, by normalising its use in different musical contexts. In the case of Breathe in, breathe out, the HypeSax is part of a 'traditional' ensemble, acting as another instrument, unlike in the case of Look Out!, in which the HypeSax adopts a role of decision-maker. I would like to see the HypeSax integrated in multiple musical scenarios, and not only being part of an

${ }^{251}$ In Panayiotis Kokoras words, holophonic musical texture is 'formed by the fusion of several sound entities which lose their identity and independence in order to contribute to the synthesis of a whole'.

Panayiotis A. Kokoras, 'Towards a Holophonic Musical Texture', in Proceedings of the Nternational Computer Music Conference 2005 (presented at the International computer music conference: ICMC 2005 free sound, International Computer Music Association, 2005), p. 3. 
experimental electronic scene, as commonly occurs with new technology-driven instruments.

The unique voice of the HypeSax, mixing acoustic sound and electronic feedback, was the inspiration for Breathe in, breathe out, a work in which instrumental passages present orchestrations of that unique hybrid sound in juxtaposition with the HypeSax itself exploring its own acoustic characteristics, while also transitioning between acoustic and electronic sound. The first section of the piece from bb. 1-24, for instance, was composed with the idea of decoupling the different elements that give the HypeSax its unique voice: the human element, the acoustic element, and the electronic element. The piece begins with two bars filled with nothing more than breath sound. These bars also serve to establish the pulse, which is based on the length of a cycle of breath. This is the initial human element - breathing in and breathing out - that is the basis for generating sound with the saxophone. From bb. 3-6, the HypeSax performer is asked to either attack notes with short articulations, slap tongue or key sounds, or a combination of thereof. The strings complement these gestures by orchestrating the attacks and emulating their resonance.

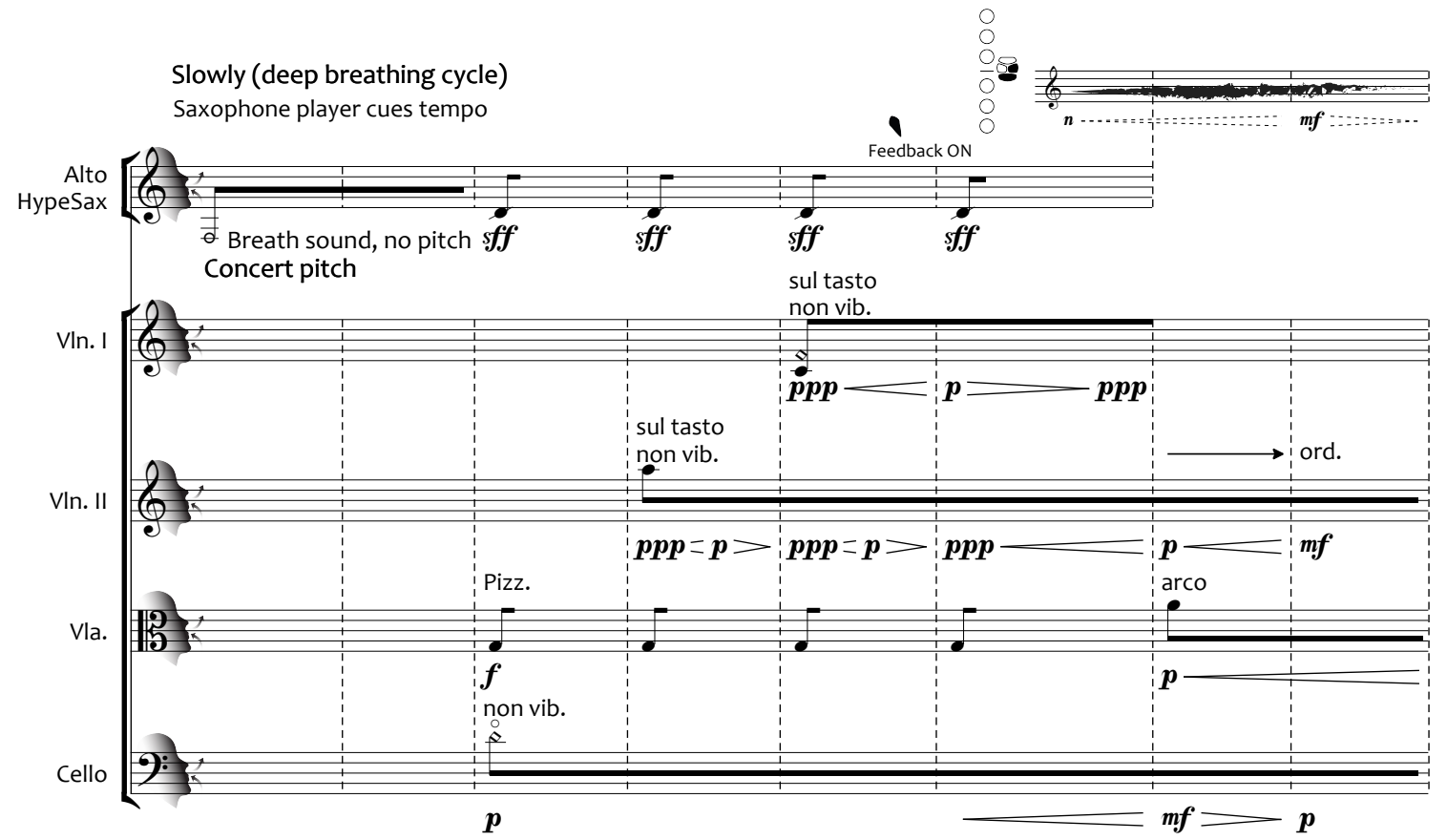

Fig. 63. bb.1-8, Breathe in, breathe out.

Following this, the HypeSax takes advantage of the feedback that can be produced using the free signal flow between the microphone and speaker mounted on the Un-mute, 
presenting the electronic element of the piece in a controlled manner using specific fingerings that help tuning the feedback sound.

Gestalt principles are used to organise gestures by presenting musical ideas inspired by the HypeSax sound and pushing them to the 'foreground of our listening experience', ${ }^{252}$ building structural points throughout the piece. In the first section, more notably in bb. $17-$ 24, I used the principle of similarity in order to expand the sound of the HypeSax using the string quartet: first, the medium mutates by fading out of an acoustic saxophone F5 note into the same pitch generated by feedback (see Fig. 64). The sound of the feedback in this register is very similar to that of the saxophone, which allows for a smooth transition. The score presents a fingering for the F5 note plus a tremolo between the low $\mathrm{B} b$ and low $\mathrm{C}$ keys, which, together with the gain control using the pedal, activate a feedback sound with a wide vibrato around the same F note the saxophone was previously playing. The key sound produced by the tremolo is complemented with finger-tapping on the cello. At the same time, the second violin and viola introduce a noise element, via overpressure, to imitate a jarring 'breathing' noise. Finally in b. 20, the cello finger-tapping moves on to a ricochet-col legno battuto technique, which in b. 21 , takes over and completes the expansion of the HypeSax sound. The similarity between the sounding result of the multiple techniques and the characteristics of the HypeSax sound used in this section allow for a sonorous blending that results in coherent sound. The transition between what is presented in bb. 17 and 24 would be very contrasting with no continuity if the process of transformation through the principle of similarity presented between bb. 17-20 did not exist. The mutability of the medium going from saxophone to HypeSax to meta-instrument was possible through the use of the principle of similarity (listen to Breathe In Breathe Out.wav 0:00-2:20).

${ }^{252}$ In Gestalt theory, elements of an image can appeal our attention in the form of a figure standing up and leaving the background level to be perceived as the foreground, helping our brains understand form and structures. 


\section{Concert pitch}
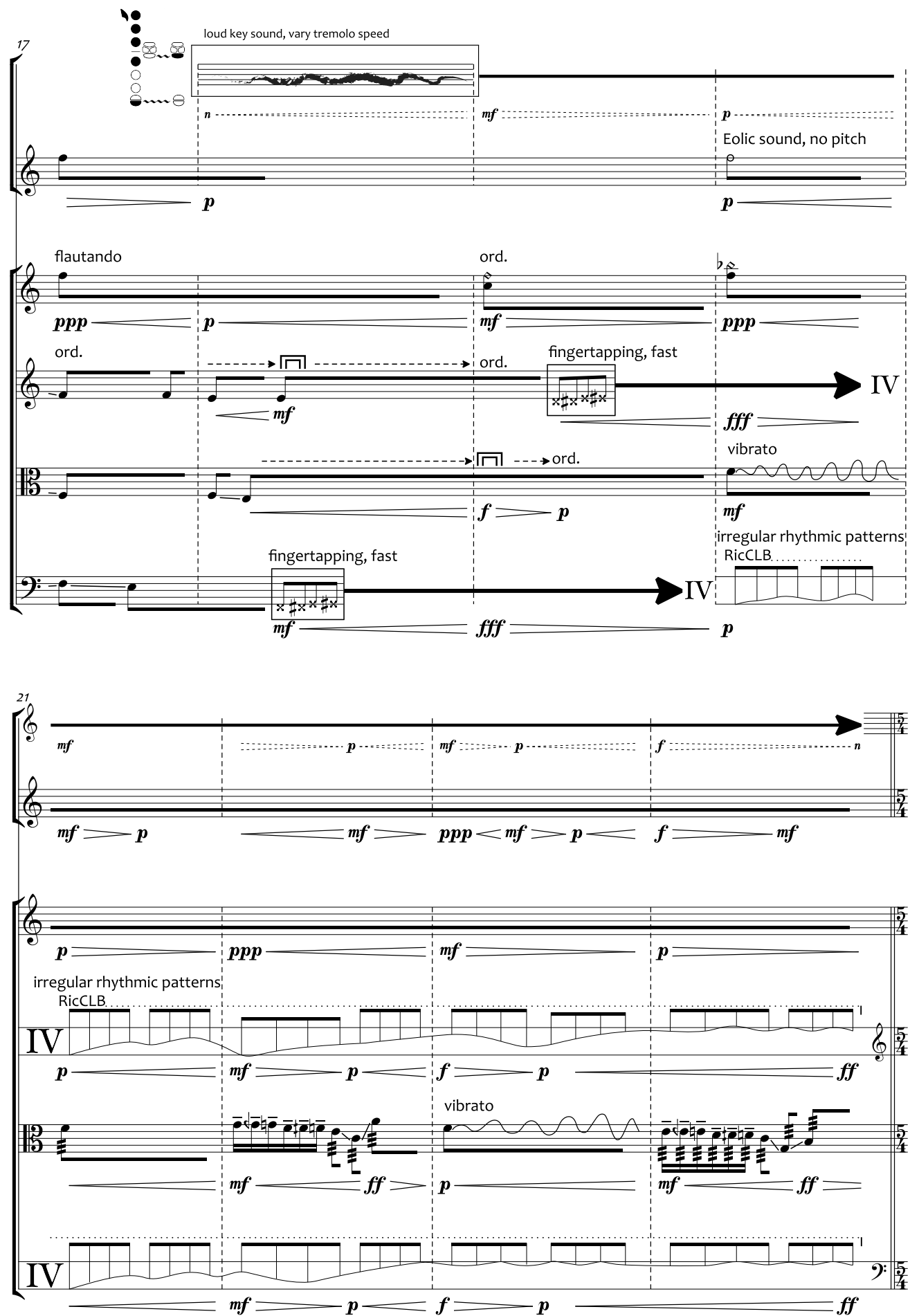

Fig. 64. bb. 17-24 of Breathe in, Breathe out, orchestrated using the principle of similarity.

The principle of similarity is also used in this piece to separate elements rather than joining them. In bb. 41-42, for instance, the strings are perceived as a unity by their gestural similarity, contrasting with the HypeSax that behaves in a completely different way. 

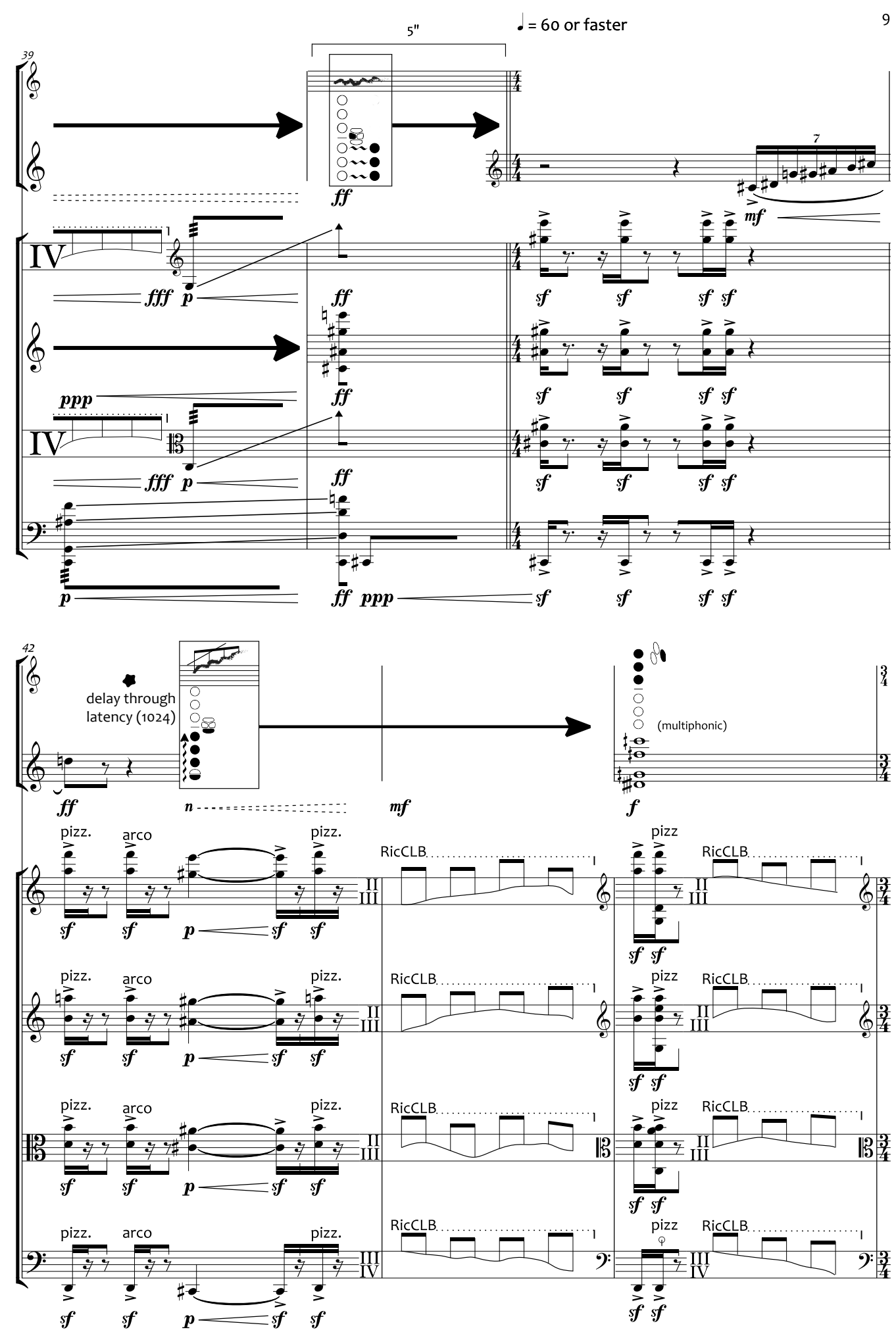

Fig. 65. bb. 39-44, Breathe in, breathe out, the principle of similarity separates strings from HypeSax.

While this section does not directly make use of the HypeSax's capabilities, it was the experimentation with the HypeSax and its unique control of feedback that inspired many 
of the passages of this composition. In b. 26, for instance (see Fig. 66), the strings are asked to play a module, in which they move at varying speeds between a fundamental note and two of its harmonics. The desynchronisation between the instruments makes it very difficult to know which instrument is playing what. The cello plays a background D4 which helps amalgamate the string sounds. The saxophone, in the first pass through this bar, is playing indeterminate notes with a half-breath sound, which also helps integrate it with the overall sound of the ensemble (listen to Breathe In Breathe Out.wav 5:00-5:20). The principle of cloud clang is used in this passage, by presenting an oversaturation of elements. It becomes almost impossible to perceive one of the elements as being in the foreground of our perception, which results in the blending of all elements as one, regardless of their dissimilarity. This bar is a reinterpretation of the effect presented in $b$. 37, where the unaccompanied HypeSax creates a similar texture using feedback by continuously sweeping through the lower keys of the saxophone (see Fig. 67).

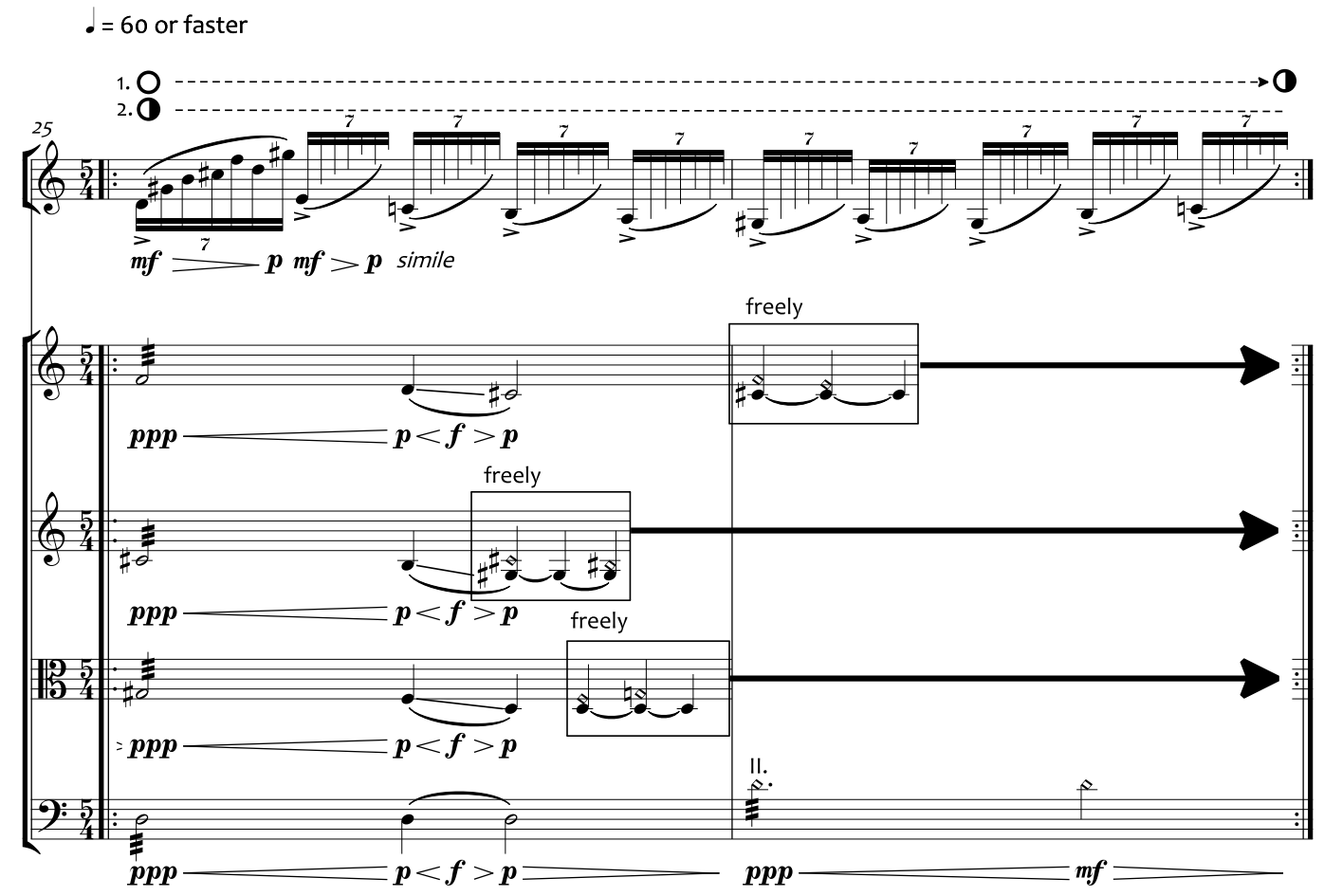

Fig. 66. The principle of cloud clang unifies instruments in b. 26 . 


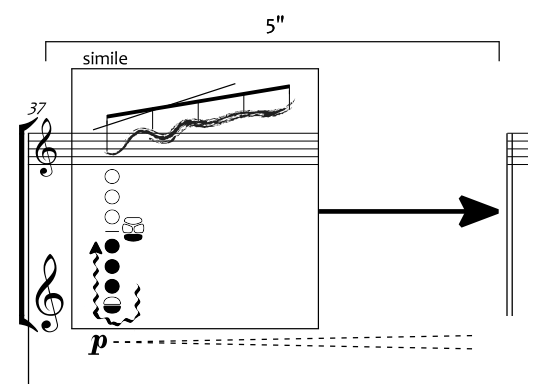

Fig. 67. b.37, inspiration for the orchestration of b.26.

As exemplified by the relationship between bb. 37 and 26, the principle of the objective set was used in this composition to create a logic that refers back to the nature of the HypeSax. The principle of the objective set, which help us understand a musical idea through referencing previous ideas or similar ideas such as the use of a theme and its development in a sonata, was used to construct the narrative of the piece. First, the sounding elements of the HypeSax are separated in bb. 1-24. These elements are used throughout the composition, referring back to the opening section of the work. All of the materials used for this composition were the result of a thorough exploration of feedback. Some of these materials were also used to develop another one of the pieces in my portfolio of works, HypeSax Étude No.2 (see Appendix G). In this way, by quoting materials from that composition, the subjective set principle is used in Breathe in, breathe out. This also helped to create a continuity in my exploration of the HypeSax as a new medium.

Directly or indirectly, the medium gave me an opportunity to explore and present sounding possibilities that might not exist if the HypeSax had not been a creative playground during the composition process. This ludic aspect that arose from the exploration of the instrument, which is reflected in my compositions, might be helpful in developing a relationship with the listener through the use of the Gestalt theory principles as compositional tools throughout my entire portfolio, with varying degrees of importance in the compositional process. It is my hope that, by appealing to this idea of organising sounding events according to how they may be perceived, it could become easier to understand and make sense of the composition as a whole.

As proposed before, through the medium and its relationship with the performer, the tripartite model is open to including reception as an element of the ontology of the work. Considering the complete sensory experience of the listener, I also explored both musical 
and bodily gesture - taking advantage of the unique embodiment capabilities of the HypeSax - in my portfolio of creative works. In the next section, I discuss how these concepts of gesture were integrated into my compositions.

\subsubsection{The extent of the musical gesture}

The development of the HypeSax not only resulted in a new instrument, but the design and development process also influenced my way of thinking about sound and its role in the ontology of the musical works. As I began my research into the design of the instrument, I considered new approaches to composition and music-making through the use of the potential new instrument that I was about to develop. One of these possible approaches was to analyse sound in real-time and transform it, combining acoustic audio and processed signal, to achieve some sort of additive hybrid synthesis. Similar techniques have been used in spectral composition, where it is common to find works in which the electronics complement a complex instrumentation, and vice versa, as in the case of works such as Tristan Murail's Terre d'Ombre or Désintégrations.

Although the HypeSax was not yet built, I decided to begin my exploration of sound with a piece that would become an important precursor to forming a relationship between microstructure of sound and the macrostructure of the harmonies I developed. I was sure that beginning my research working with acoustic instruments, which I already knew, while developing and thinking about the future hyperinstrument would generate ideas and goals that would be transferable to the HypeSax. The first work I composed was Bereshith for large orchestra, in which I explored different approaches to 'additive instrumental synthesis'. ${ }^{253}$ The piece is based around an orchestration of an acousmatic work that I composed in 2006, Génesis. This composition, built using synthesis and musique concrète techniques, would propose a sounding world that would require me to explore

${ }^{253}$ Additive instrumental synthesis is a common spectral composition technique that takes 'the concept of additive synthesis, building up complex sounds from elementary ones, using them metaphorically as a basis for creating instrumental sound colours (timbres). The sound complexes built this way are fundamentally different from the models on which they are based, since each component is played by an instrument with its own complex spectrum. Thus the result is not the original model, but a new, much more complex structure inspired by that model.' - Joshua Fineberg, 'Sculpting Sound. An Introduction to the Spectral Movement: Its Ideas, Techniques and Music.' (Columbia University, 1999), pp. 22-23. 
compositional strategies different from the techniques, such as using pitch-class set theory, that I had used for many years. I did not plan to fully orchestrate Génesis; instead, I decided to take it as a model, following its structure, using similar gestures found in the different sections of this piece and, through spectral analysis of relevant moments of the piece, developing a 'harmonic world' from the frequencies of the extracted partials. I also integrated sounds of the orchestral instruments to the harmonic world extracted from Génesis. For instance, I orchestrated the main musical gesture from Génesis - originally a pulsating breathing sound created by a cymbal sample played backwards (see Fig. 68) based on a recording of a B1 note played on a timpani. This sound would be the basis to create a central pitch throughout many points in the composition, such as the beginning and the end.

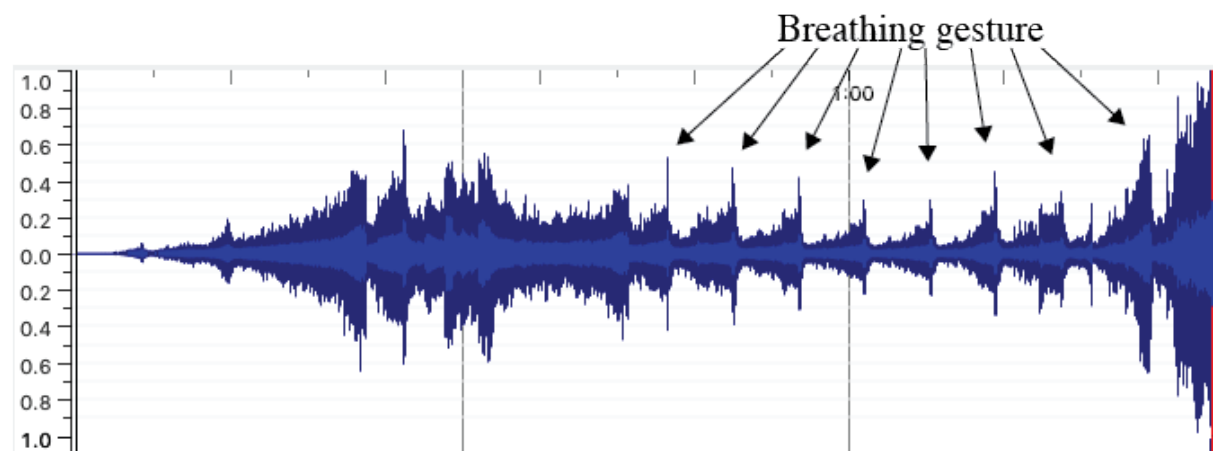

Fig. 68. Waveform visualisation of Génesis from 0'00" to 1'30".

The 'breathing' gesture provides continuity and unifies the piece. In order to orchestrate this gesture, I analysed the spectral content of the timpani recording using Spear, ${ }^{254}$ filtering out overtones with too short a duration or that fell below a gain threshold. Finally, I exported a SDIF file to Open Music and extracted a complex chord. In this way, the medium, the orchestra instruments, provided the score with content. With this initial material I set myself up for an exploration of the medium which, in this case, was the orchestra constantly reconfiguring, through multiple re-orchestrations of this gesture, a 'divine presence' represented by the constant 'breathing'. This is how I envisioned future

${ }^{254}$ Spear, developed by Michael Klingbeil, is an application for audio analysis, editing and synthesis, which attempts to represent a sound with many individual sinusoidal tracks (partials), each corresponding to a single sinusoidal wave with time varying frequency and amplitude. For more information visit: http://www.klingbeil.com/spear/ 
transformations of the saxophone timbre with the development of the HypeSax, except that I would use hybrid synthesis rather than orchestration.

The 'breathing' gesture is devoid of defined pitches, tempo, dynamics or timbres. The sequence of dynamics are the only elements that make this gesture. In the narrative of Bereshith, I orchestrated multiple instances of this gesture, presenting variations of the chord obtained from the timpani, beginning with its most simplified form, represented by a $B$ played by Clarinet 1 in the opening bar, and eventually presenting the chord in more complex forms. In bb. 36-46, for instance, a sequence of these gestures is presented. In Fig. 69, a reduction of the variations of this chord, presented between bb. 36-46, shows the harmonic content of each instance of the chord, which can be heard as the breathing gesture once the dynamics are applied as indicated in the score (see Fig. 70, full score can be found as Appendix M).

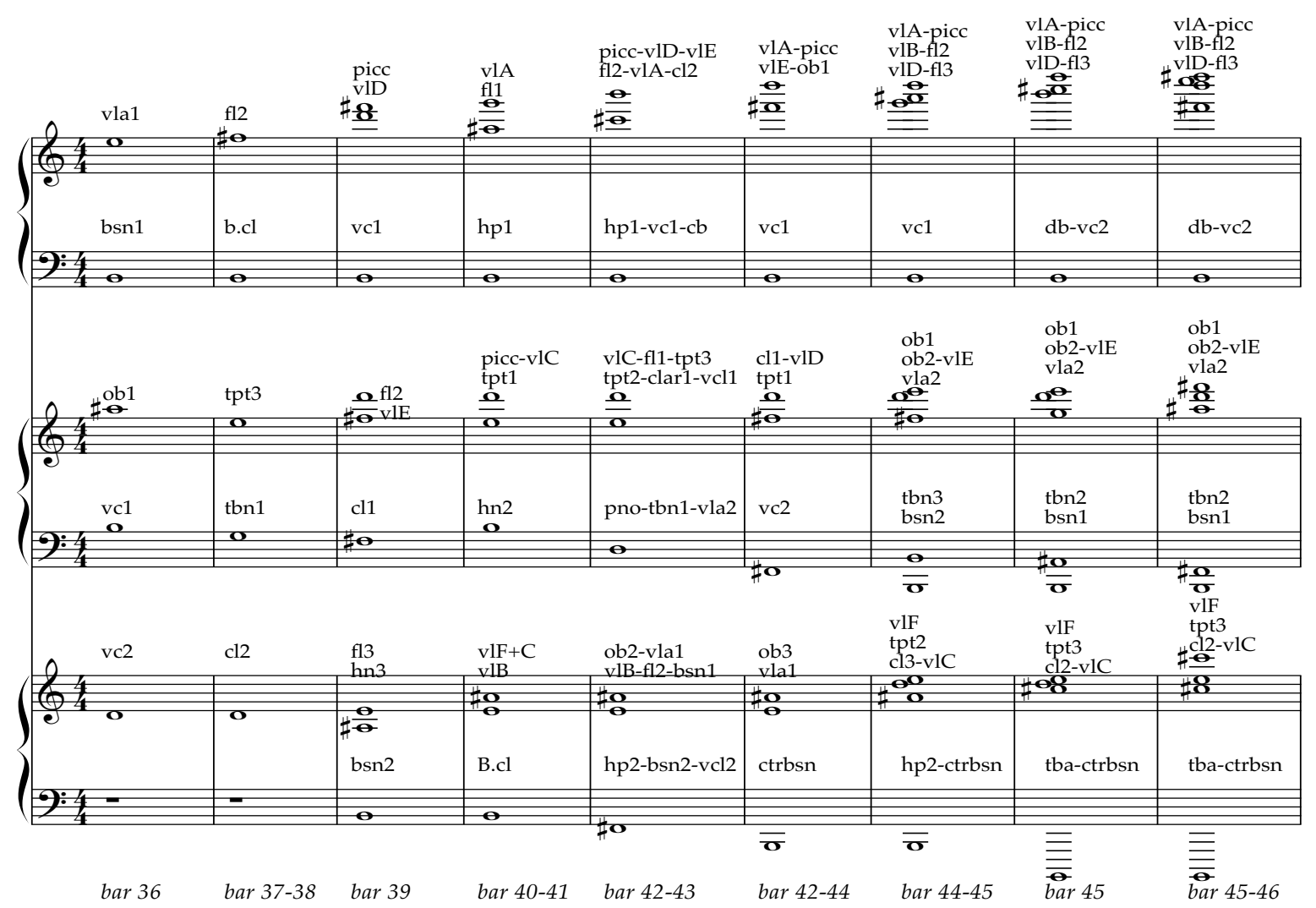

Fig. 69. Timbral transformation of the 'breathing' gesture in bb. 36-46 of Bereshith. 


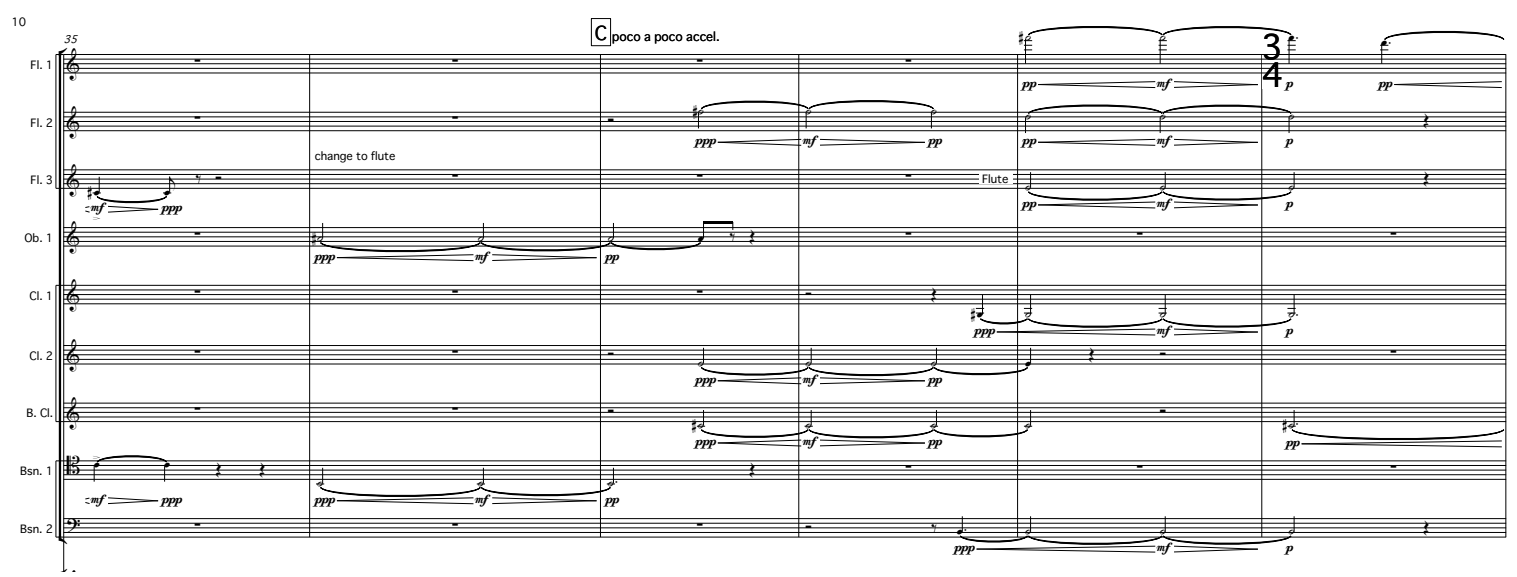

Fig. 70. Woodwind section of the 'breathing' gesture in bb. 35-40 of Bereshith.

Developing a compositional approach to the mutation gesture served as a study that helped me imagine multiple pathways to explore the medium. At the same time, I was able to generate harmonic content and textures through the exploration of sound. In similar ways, by exploring the medium, throughout the portfolio of works, I found suggestions for musical ideas, harmonies, textures and structure. For instance, a process similar to the mutation of this gesture was used in some of the subsequent compositions such as in Cor Ple, in which I followed a similar process in order to achieve harmonic continuity through expanding the idea of timbre transformation (explained below). In the case of Bereshith, the compositional approach taken helped me create macro structures through additive instrumental synthesis, but I was interested in the microstructures. For this purpose, the new hyperinstrument I was working on, the HypeSax, would be an ideal tool which would allow me bring similar strategies to the level of sound sculpting.

Cor Ple, for alto HypeSax and mixed ensemble, was conceived as an exploration of the timbre of the instruments in the ensemble, following a similar process to the one used to generate harmonic content in Bereshith. In this case, however, I started exploring the possibility of the mutation of the saxophone's timbre into the timbre of the other instruments of the ensemble, and applying this idea to multiple aspects of this piece, overlapping multiple views of the same material reorganised from different perspectives, in the same way that images, forms and structure are reconfigured in Cubist works.

I composed Cor Ple as a tribute to Cristòfor Taltabull's Madrigal (1946), a piece for SATB that cemented my love for choral music, while I was working on the first prototypes of the HypeSax. After developing the mutating breathing gesture, I was eager to try to do 
the same with a hybrid system on the saxophone, using a unique musical gesture to mutate the saxophone's timbre to the point where it would sound similar to another instrument. I came up with a strategy to transform the timbre of the saxophone. This strategy consists of:

1. As a preparatory step, analysing a recording of an instrument - any instrument - playing a given pitch, in order to obtain data about its overtones and relative energy of the first harmonics. Upon empirical experimentation and after considering the low energy of higher harmonics, it became clear from the beginning that any high harmonics beyond the 32 nd would not be audible against the sound of the saxophone. For this reason only 32 harmonics are analysed. Then, after repeating this process with multiple instruments, create a set of models based on this data. The relative energies of the components and their internal balance (relative prominence) change as sound unfolds in time. At the same time, these parameters are different throughout the range of an instrument, so I decided to use a D4 pitch at $\boldsymbol{m} \boldsymbol{f}$ to generate the initial models.

2. Analysing the saxophone sound, in real time, in order to obtain data about its relative energy of the first 32 harmonics.

3. Comparing the different energy levels between the saxophone's harmonics and the previously generated models, and calculate the necessary additional energy in the saxophone's timbral components to approximate the parameters of the models.

4. Finally, based on the calculation, synthesising a signal that would reinforce some partials of the saxophone, via the Un-mute, in order to obtain a hybrid timbre. This timbre should resemble the timbre of the instrument used as a model in the comparison (see Fig. 71). 


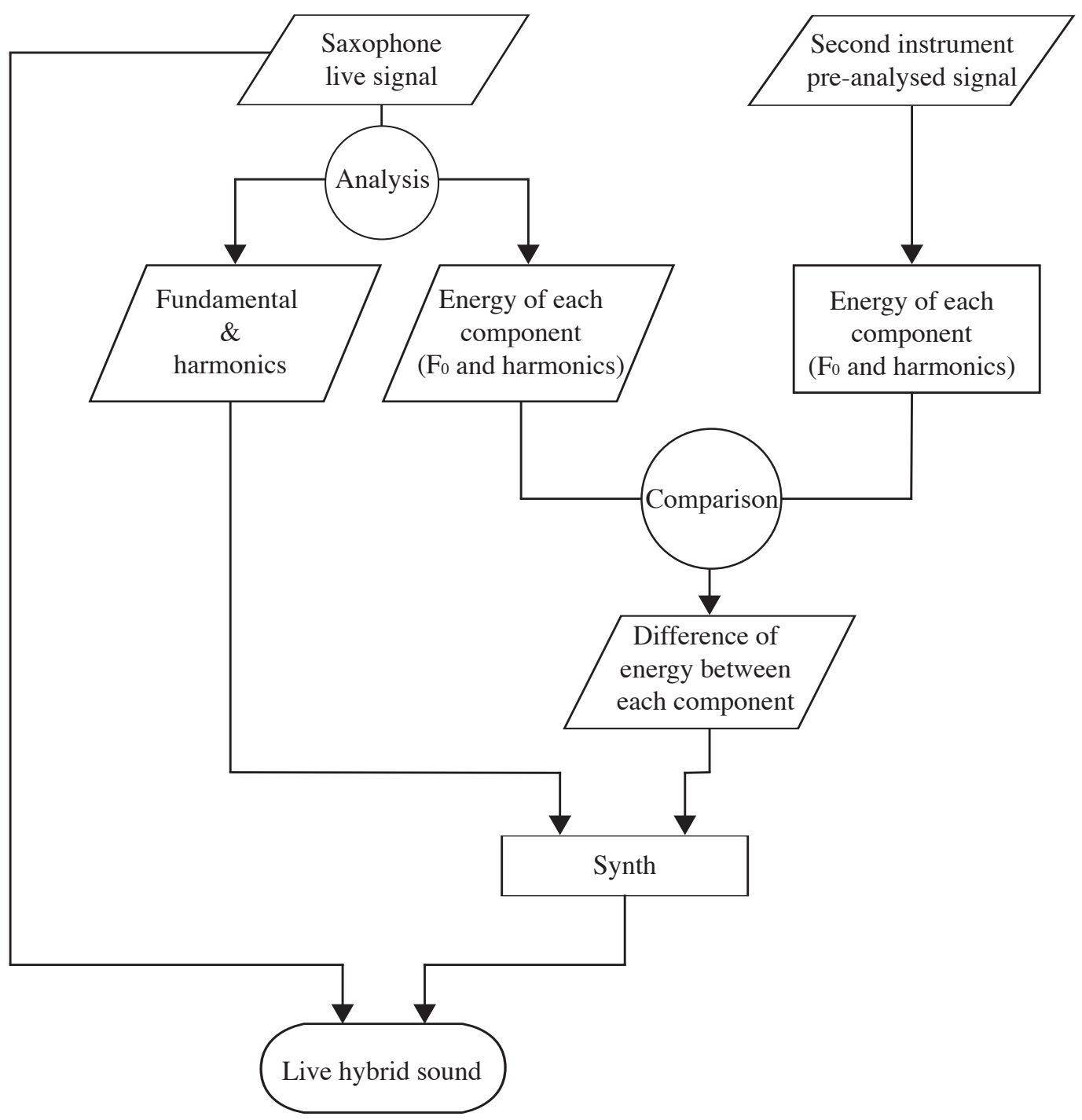

Fig. 71. Simplified flowchart of hybrid synthesis.

I developed a Max patch, called Morpher, that followed this plan. I implemented three different synths: 1) tracking $\mathrm{F}_{0}$ using the $z s a$ descriptors library ${ }^{255}$ and multiplying $\mathrm{F}_{0}$ to calculate the harmonics to feed the ioscbank 256 object; 2) tracking $\mathrm{F}_{0}$ as well as the

${ }^{255}$ Zsa descriptors library for real-time analysis was developed by Mikhail Malt and Emmanuel Jourdan. See Mikhail Malt and Emmanuel Jourdan, 'Zsa.Descriptors: A Library for Real-Time Descriptors Analysis', in Proceedings of SMC 20008 (presented at the SMC 20008, Germany, 2008), p. 4. and Mikhail Malt and Emmanuel Jourdan, 'Real-Time Uses of Low Level Sound Descriptors as Event Detection Functions Using the Max/MSP Zsa.Descriptors Library’, in Proceedings of SBCM 2009 (presented at the SBCM 2009, Recife, Brazil, 2009), p. 12.

${ }^{256}$ The ioscbank object is an oscillator bank than handles multiple oscillator frequencies and magnitudes. 
harmonics to feed ioscbank $\sim$; and 3) tracking $\mathrm{F}_{0}$ and harmonics to feed $f f f b \sim^{257}$ and filter the original input signal using a high $\mathrm{Q}$. The results of these approaches to hybrid synthesis varied depending on multiple factors such as dynamics, performance technique, 'breathiness' of tone, register, or the characteristics of the model to which the saxophone is matched. For this reason, these three synths gives better results in different situations, and choosing which synth to use requires some exploration of the musical context at hand.

Later in my research, after having developed the first functional version of the HypeSax and the Morpher, I encountered Paul Clift's Acoustic Aggregate Synthesis (AAS), described in his ICMC2012 paper 'Acoustic-Aggregate-Synthesis'. In it, Clift identified five levels of 'efficacy' of hybridisation:

1. Either nothing is diffused (i.e. all detected components from template $^{258}$ are present in the incoming signal at a greater intensity) OR little to no fusion occurs (i.e. synthesis is not achieved: two distinct sound sources remain perceptible);

2. some fusion occurs but the result is not in any way evocative of the instrumental-template;

3. a high level of fusion occurs but the result is only minimally evocative of the instrumental-template;

4. complete fusion between sound-source \& synthesis with a moderate to high degree of evocation of the instrumentaltemplate;

5. a convincing reproduction of the secondary, acoustically modelled timbre is achieved. ${ }^{259}$

\footnotetext{
${ }^{257}$ The $f f f b \sim$ object is a fast-fixed filter bank which works as a multiband equaliser.

${ }^{258}$ In this paper, Clift refers to the instrumental models as 'templates'.

${ }^{259}$ Clift, 'Department of Music Columbia University, New York, NY', p. 122.
} 
In his paper, Clift presents a sample table of 'efficacy' where the results show that matching 'simple' input signal (such as clarinet) to more 'complex' ${ }^{260}$ models (such as flute) has better results than matching 'complex' input signal (such as flute) to 'simple' signals (such as clarinet). Unlike Clift's AAS, which was developed to be used to match any instrument to any other instrument, the Morpher was designed specifically for the saxophone as the main instrument to be transformed. The main difference between AAS and my software is that I calibrated the system to listen only to the saxophone, filtering out unwanted signal that could compromise accurate tracking of $\mathrm{F}_{0}$ and partials. Clift also uses different models according to the range or different dynamics. However, the empirical results that I obtained are very similar, and the 'complexity' of the saxophone's spectrum and relative loudness makes hybridisation difficult with some instruments. It is, however, relatively easy to achieve hybridisation at $\boldsymbol{m} \boldsymbol{f}$ or quieter dynamics.

After developing the Morpher, I decided that the mutating gesture in Cor Ple would only happen over long tones and would need to be carefully orchestrated, as faster passages or complex orchestrations would hide the gesture. The gesture can be found in the piece in bb. 124 (see Fig. 72), 129 and 132, where the only other instruments participating are the clarinet, playing long tones, and the small gong, which is raised in and out of water, producing a glissando effect on its overtones which is perceptually similar to the mutation effect on the saxophone. The indication given in the score is to 'morph freely'. To do this, the performer must choose one of the instrumental models already loaded on the Morpher by pressing one of the combinations of buttons described in the score notes, as well as choosing how long the morphing will take.

${ }^{260}$ While it is not correct to categorise a specific timbre as simple or complex, by using these words I am referring to the perceptual presence of the partials that are more evident in some instruments than others. 


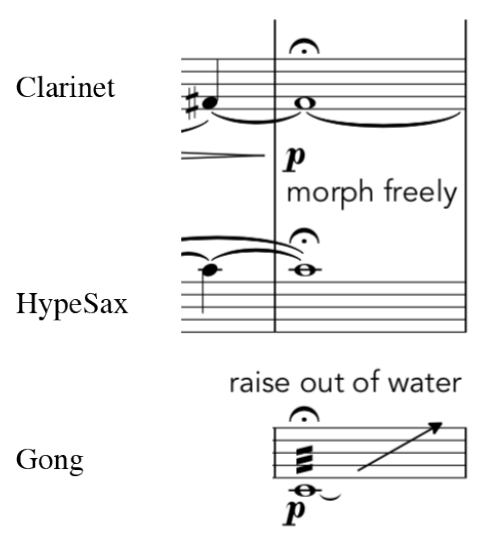

Fig. 72. b. 124, Cor Ple.

While a few mutations occur prior to these passages, they are not so evident in the ensemble context. However, they mark moments in the harmonic world of the piece, as these mutations make evident the origin of the harmonic zones. In bb. 64-69, for instance, the HypeSax is asked to press thumb button 1 while swinging the saxophone left and right. This physical gesture is mapped to activate the hybrid synthesis and sweep through the partials, changing their gain, producing a sort of controlled flanger effect. The synth, in b.64, calls for a model based on the horn. At the same time, the harmonic texture of the ensemble begins to shift to chords based on the horn spectrum. In b. 64 the HypeSax begins a mutation gesture by sweeping through partials of the horn model. In Fig. 73, the piano staff (bottom, not part of the actual score) shows the chord based on the analysis of the saxophone that is used from bb. 55-63, and the chord based on analysis of the horn, in $b$. 64. 


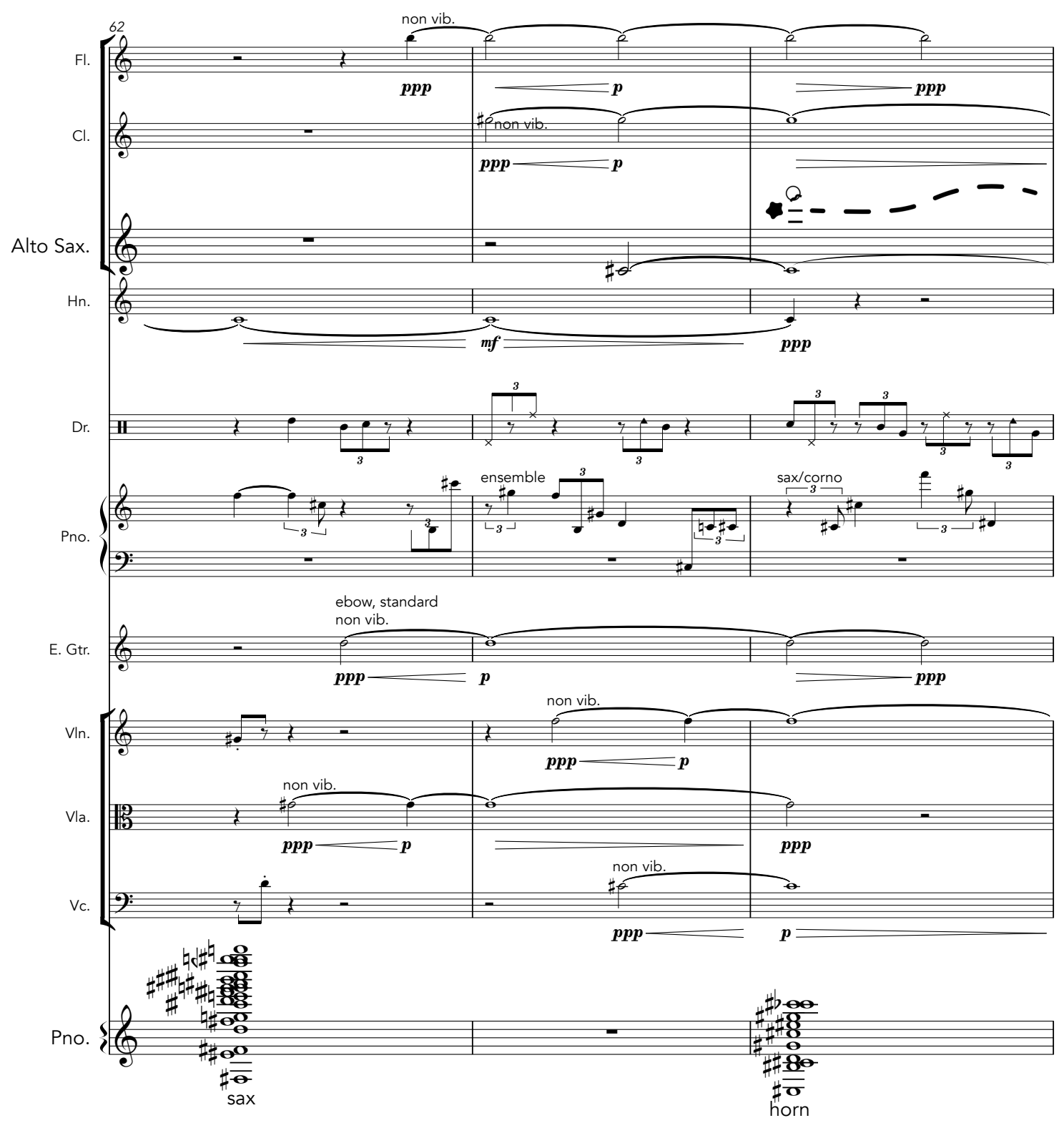

Fig. 73. bb. 62-64, Cor Ple. The graphic above the saxophone staff on b. 64 indicates the beginning of the hybrid synthesis.

As mentioned before, it was my intention to explore the medium and to use the results of that exploration in multiple aspects of the piece. In the same way that I built the models for the synth, I extracted the chords out of the timbres of the instruments of the ensemble. Unlike the models used in the synth, which look for the partials of the saxophone's fundamental frequency, the analysis to generate the chords was not heavily filtered, 
allowing for 'artefacts' ${ }^{261}$ in the harmonic series, resulting in complex sonorities. This is evident at the beginning of the piece where the piano chord, based on an analysis of the flute spectrum, includes notes that are not part of the flute's harmonic series. The notes on this chord are an approximation of the harmonic series that resulted from the 'artefacts' allowed in the analysis (see Fig. 74). This chord is then used with the rest of the ensemble to create different textures throughout bb. 1-44, and transposed according to the flute's pitch (as fundamental frequency). The flute, as well as the rest of the instruments at different moments of the piece, play lines taken from Cristòfor Taltabull's Madrigal (1946). The notes in these lines are used as the fundamental frequencies for the chords, generating a harmonic thread. Cor Ple - from Catalan for 'full heart', as a tribute to the lyrics in Taltabull's composition ${ }^{262}$ - is a 'Cubist' reinterpretation of Madrigal, where melodic lines and harmonies are blended as a result of my exploration of the medium, in this case the saxophone and its timbre potentially mutating into timbres that resemble the rest of the instruments of the ensemble.

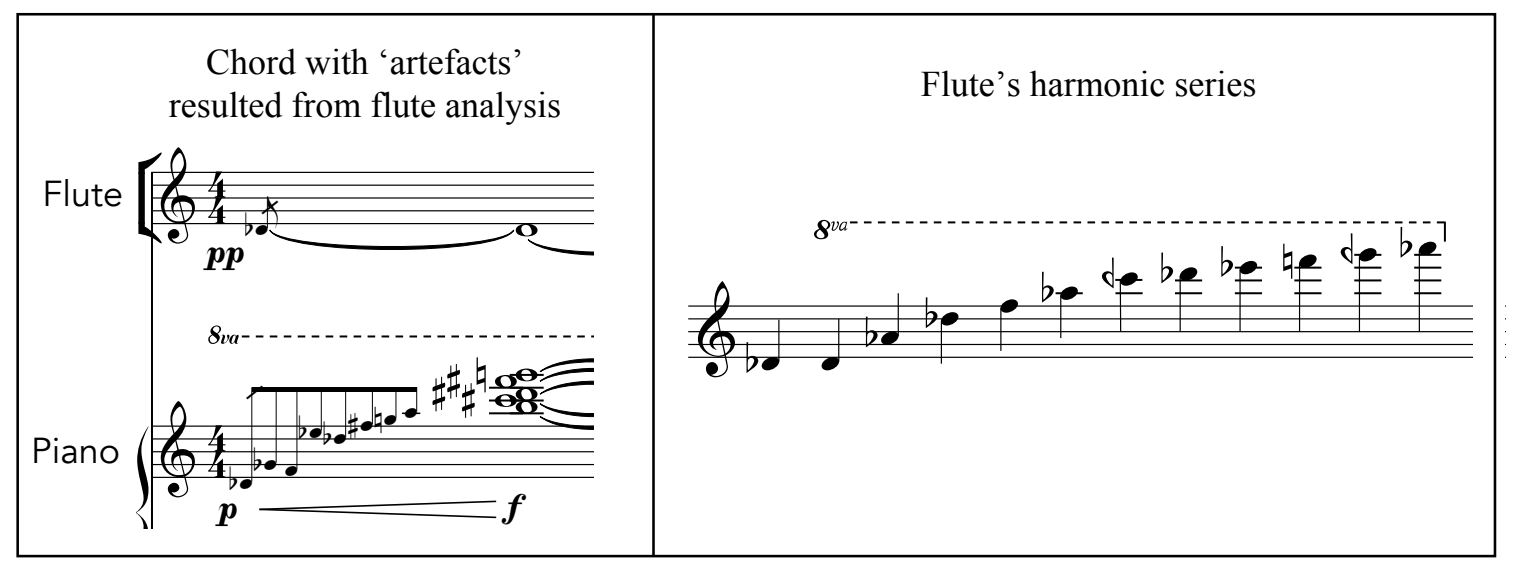

Fig. 74. Chord found in b.1, Cor Ple (left) and flute harmonic series (right).

While I utilised different musical gestures in my portfolio of works, the mutating gesture in the saxophone is possibly the best example of what can be done utilising hybrid synthesis, decoupling the physicality of the instrument, re-embodying the sound, and

261 These 'artefact' notes are likely to be prominent overtones that result from the nature of the instrument and performance. In the case of the flute, for instance, they could be present due to vibrato produced by changing air pressure or predominant components of air sound.

262 'Mon cor tinc pres catiu en mig d'un verd boscatge' = My heart is imprisoned in the middle of a green forest. Text by Pere Serafí, 1505-1567. 
exploiting the mutability of the medium. Similar musical gestures are used in traditional instrumental music, such as the use of the bouche technique on the horn, or the use of the 'wah-wah' (harmon) mute on brass instruments. Every instrument has a technique, or extended technique, that allows for it to be transformed away from its ordinary tone. Yet, none of these extended techniques can go to the extremes of emulating another instrument in the same way that hybrid synthesis has the potential to do.

A common technique used to change the timbre of a string instrument consists of moving the contact point of the actuator to different regions of the strings. Examples of this technique in the case of bowed instruments, for instance, are sul ponticello or sul tasto. Because these techniques rely on the nodes of the strings and the different levels of tension and range of movement of the strings throughout their length, special physical gesture must be used to execute them - the sonic gesture is tied to the physical gesture. Typically, the relationship between the physical and sonic gesture cannot be transferred to other media (instruments). For instance, to achieve the attenuation of lower partials and the boosting of higher partials that occurs in sul ponticello, a woodwind player would have to use a completely different physical gesture. A flute player, for instance, might change the angle or speed of air, while a brass player, on the other hand, would perhaps tighten the lips. This relationship between a physical gesture and an instrumental technique is what I call gestural-instrumental technique. This relationship is at the core of the relationship between a performer and the instrument, and, indeed, at the core of performance.

\subsubsection{The performance and the gestural-instrumental technique in my works}

I was intrigued by the possibility of transferring gestural-instrumental techniques that are available on other instruments to the saxophone. In order to do this, I considered the importance of the relationship between the performer and the medium. The relationship between musicians and their instruments is reinforced over many years of practice, as they master techniques and skills. It is these qualities that provide performers with the necessary tools to make the realisation of a work unique and personal. Considering the importance of how musical instruments are used to make music, it is assumed that good musicians should be well versed in the capabilities and limits of the instruments they are using. When composing and performing (especially in the case of improvisation), the degree of skill, knowledge and personal experience with an instrument has a direct influence on the music 
that is being created. In other words, when it comes to realising the music, the imagination that triggers the creative process is limited or shaped by the physical possibilities of the composite performer/instrument. ${ }^{263}$ This relationship is very important as to some extent the instrument becomes a sort of extension of the body of the performer, creating an 'integrated composite musical entity'. ${ }^{264}$ It can be argued that embodiment is an important factor in music-making, and that considering this factor in the creative process not only informs the creator but might offer new compositional frameworks.

It is interesting to consider the possibility that a musical score, besides representing a musical idea, is to some degree a set of instructions on how to realise a musical work. While an instruction such as 'run' can be understood and performed by most people, another instruction such as 'fly' does not yield the same result within humans. Similarly, instructions in a musical score would ideally call for actions that the composite performer/instrument could execute. Furthermore, for an action expressed in a score to be feasible, there should be an established relation between the physical gesture and the resulting sonic event. This relationship, described by David Wessel and Matthew Wright in 'Problems and Prospects for Intimate Musical Control of Computers' as 'one-gestureto-one-acoustic-event' paradigm, puts the performer 'in direct contract with the physical sound production mechanism'. ${ }^{265}$

Julio d'Escriván, in 'To Sing the Body Electric,' states that the 'one-gesture-to-oneacoustic-event' paradigm has changed for younger generations who relate to a new paradigm where technology allows for musical complexity to be exerted with less apparent effort. Whereas a precise, skilful and often difficult combination of physical gestures relate to complex sonic events, in the world of computer music, a simple gesture can result in a complex response. This does not mean that the work of computer music practitioners does not require any effort, many hours of research, and development of software and hardware

\footnotetext{
${ }^{263}$ Performer/instrument refers to 'what triggers the production of sound'/'what produces the sound', for example guitarist and guitar, computer and speakers, or algorithm and robot.

${ }^{264}$ Franziska Schroeder, 'Bodily Instruments and Instrumental Bodies: Critical Views on the Relation of Body and Instrument in Technologically Informed Performance Environments', Contemporary Music Review, 25.1-2 (2006), 1-5 (p. 3).

${ }^{265}$ David Wessel and Matthew Wright, 'Problems and Prospects for Intimate Musical Control of Computers', Computer Music Journal, 26.3 (2002), 11-22 (p. 11).
} 
are necessary to achieve what they do. D’Escriván questions whether effort plays an important role in performance and how it may affect perception. He concludes his article asserting that both traditionally skilful performance and effortless performance are valid in the context of the present times where a generational divide is tied to the development of the digital era. ${ }^{266}$

In the case of the HypeSax, the hybridity that I was looking for was not only a blending of the sounds, but also a hybridity in physical-gestural practices. The coupling of the HypeSax to the saxophone, without permanent modifications, allows for skilful performance to remain intact as the saxophone maintains its performative characteristics. At the same time, new affordances of 'effortless' performance are easily accessed through the sensors, allowing the performer to control a digital extension of the instrument. The hybridity of physical-gestural practices made me think of the potential for the development of new associations between the gesture and the musical event. I realised that the gesturalinstrumental technique, where a set of physical gestures relates to an instrumental technique, could be used by combining the traditional performative practices and the digital performative practices in order to access new possibilities.

When I was composing Bereshith, I took some time to examine the palette of instrumental techniques available for the orchestral instruments, while also exploring the characteristics of multiple gestural-instrumental-techniques. I made a catalogue of instrumental techniques and the instruments that can play them with the view that, through hybrid synthesis, the saxophone could imitate the instrumental techniques typically unavailable for the saxophone. The list presents instrumental techniques and identifies what technique are available for families of instruments (see Table 4). The techniques are classified in five categories: onset, continuant, termination ${ }^{267}$ (according to their effect on

${ }^{266}$ Julio d'Escriván, 'To Sing the Body Electric: Instruments and Effort in the Performance of Electronic Music', Contemporary Music Review, 25.1-2 (2006), 183-91.

${ }^{267}$ In Denis Smalley's words, 'every note must start in some way; some may be sustained or prolonged for a time and some may not; every note stops. These three linked temporal phases I refer to as onset, continuant and termination'.

Denis Smalley, 'Defining Transformations', Interface, 22.4 (1993), 279-300 (p. 112). 
the spectromorphology of sound), ${ }^{268}$ pitch, and miscellaneous (such as projection and directionality of sound, or mobility of the instrument). Some of the techniques can be placed in multiple categories - for example, harmonics and subharmonics, belong to both continuant and pitch categories - which might make the list seem less useful. The purpose of this list, however, was merely to serve as a reference, and the list would still be useful even without the categories. This list includes the most typical techniques and avoids special techniques or techniques with a potential indeterminate sound result such as bowing the tailpiece, which may have varying results.

Table 4. Onset category of comparative list of instrumental technique access, where $\mathrm{X}=$ available, / = similar technique is available, and - = not available.

\begin{tabular}{|c|c|c|c|c|c|}
\hline \multicolumn{6}{|c|}{ TECHNIQUES: MULTIPLE INSTRUMENTS } \\
\hline TECHNIQUE & IDIOPHONES & MEMBRAPHONES & CHORDOPHONES & AEROPHONES & ELECTROPHONES \\
\hline \multicolumn{6}{|c|}{ onset } \\
\hline Articulation & $\mathrm{X}$ & - & $\mathrm{X}$ & $\mathrm{X}$ & $\mathrm{X}$ \\
\hline Pizzicato & - & - & $\mathrm{X}$ & $\mathrm{X}$ & $\mathrm{X}$ \\
\hline Bartók pizz & - & - & $\mathrm{X}$ & - & $\mathrm{X}$ \\
\hline Key clicks & - & - & - & $\mathrm{X}$ & $\mathrm{X}$ \\
\hline Ricochet & $\mathrm{X}$ & $\mathrm{X}$ & $\mathrm{X}$ & - & $\mathrm{X}$ \\
\hline Legno battuto & $\mathrm{X}$ & $\mathrm{X}$ & $\mathrm{X}$ & - & $\mathrm{X}$ \\
\hline Slap tongue & - & - & - & $\mathrm{X}$ & $\mathrm{X}$ \\
\hline $\begin{array}{l}\text { Mult.Attack- } \\
\text { Tremolo }\end{array}$ & $\mathrm{X}$ & $\mathrm{X}$ & $\mathrm{X}$ & $\mathrm{X}$ & $\mathrm{X}$ \\
\hline Hammering & $\mathrm{X}$ & $\mathrm{X}$ & $\mathrm{X}$ & - & $\mathrm{X}$ \\
\hline Knocking & $\mathrm{X}$ & $\mathrm{X}$ & $\mathrm{X}$ & - & $\mathrm{X}$ \\
\hline Spiccato & 1 & I & $\mathrm{X}$ & 1 & $\mathrm{X}$ \\
\hline Tongue ram & - & - & - & $\mathrm{X}$ & $\mathrm{X}$ \\
\hline
\end{tabular}

Instrumental techniques are possible thanks to the 'one-gesture-to-one-acoustic-event' paradigm, limiting each instrument to a set of techniques due to that physical relationship between the physical gesture and the sonic event. To address this limitation, multiple strategies could be used, taking advantage of the hybrid physical-gestural practices,

${ }^{268}$ As described in Section 4.1, the concept of spectromorphology concept encapsulates the spectrum of a sound, consisting of the totality of its perceptible frequencies, and their relationship through the various stages of transformation of sound as it unfolds in time. 
incorporating the traditional skilful performance and the 'effortless' performance that the electronic component of the HypeSax provides. In this way, new gestural-instrumental techniques could be developed in order to allow the saxophone to access techniques that are not accessible through typical performative practices. I began exploring new gesturalinstrumental techniques by creating a table that lists the techniques accessible to the saxophone and potential ways to access techniques unique to other instruments (exemplified in Table 5).

Table 5. Onset category of the potential HypeSax gestural-instrumental techniques development plan.

\begin{tabular}{|c|c|c|c|c|c|}
\hline \multicolumn{6}{|c|}{ TECHNIQUES: SAXOPHONE } \\
\hline TECHNIQUE & $\begin{array}{l}\text { POSSIBLE } \\
\text { ON SAX }\end{array}$ & $\begin{array}{c}\text { POSSIBLE ON HYPESAX, } \\
\text { HOW? }\end{array}$ & $\begin{array}{c}\text { PHYSICAL GESTURE ON } \\
\text { HYPESAX }\end{array}$ & MAPPING & $\begin{array}{l}\text { PROGRAM } \\
\text { NAME }\end{array}$ \\
\hline \multicolumn{6}{|c|}{ onset } \\
\hline Articulation & YES & - & & & \\
\hline Pizzicato & YES & - & & & \\
\hline Bartók pizz & $\mathrm{NO}$ & $\begin{array}{c}\text { Synthesised 'hit' } \\
\text { synchronised with attack }\end{array}$ & $\begin{array}{c}\text { Play a loud-short note and at } \\
\text { the same time push/ press } \\
\text { button }\end{array}$ & $\begin{array}{l}\text { Use any } \\
\text { button }\end{array}$ & Bartok \\
\hline Key clicks & YES & - & & & \\
\hline Ricochet & $\mathrm{NO}$ & $\begin{array}{l}\text { Record buffer, detect attack } \\
\text { and repeat the last attack } \\
\text { multiple times with envelope }\end{array}$ & $\begin{array}{l}\text { Play slap tongue or forceful } \\
\text { attack, and quickly press/ } \\
\text { touch } 2 \text { buttons, one after } \\
\text { another. The entire event } \\
\text { should take no longer than } \\
\text { 300ms }\end{array}$ & $\begin{array}{l}\text { Air pressure } \\
\text { to detect } \\
\text { attack, use } \\
\text { any } \\
\text { combination } \\
\text { of buttons. }\end{array}$ & Ricochet \\
\hline Legno battuto & $\mathrm{NO}$ & $\begin{array}{c}\text { Play staccato, add } \\
\text { synthesised attack with } \\
\text { dynamics based on air } \\
\text { pressure. Use a button to } \\
\text { activate function }\end{array}$ & $\begin{array}{l}\text { Play staccato normally and } \\
\text { synchronise touching/ } \\
\text { pressing a button }\end{array}$ & $\begin{array}{l}\text { Use any } \\
\text { button }\end{array}$ & Legno_b \\
\hline Slap tongue & YES & - & & & \\
\hline $\begin{array}{l}\text { Mult.Attack- } \\
\text { Tremolo }\end{array}$ & YES & - & & & \\
\hline Hammering & $\mathrm{NO}$ & $\begin{array}{l}\text { amplify audio signal, only if } \\
\text { contact mic is used }\end{array}$ & activate function & $\begin{array}{l}\text { Use any } \\
\text { button }\end{array}$ & Martellato \\
\hline Knocking & NO & $\begin{array}{l}\text { amplify audio signal, only if } \\
\text { contact mic is used }\end{array}$ & activate function & $\begin{array}{l}\text { Use any } \\
\text { button }\end{array}$ & Knock \\
\hline Spiccato & $\begin{array}{c}\text { SIMILAR } \\
\text { TECHNIQUE } \\
\text { AVAILABLE } \\
\end{array}$ & - & & & \\
\hline Tongue ram & YES & - & & & \\
\hline
\end{tabular}

I developed some Max patch snippets ${ }^{269}$ that trigger a specific sounding in response to a specific gesture monitored by the HypeSax. The hammering (hammer-on technique)

${ }^{269}$ In Max, a snippet is a patcher file programmed with the intention to be re-used in different project. Snippets usually accept input and output data, audio, or video signal. 
snippet, for instance, activates a pre-recorded slap tongue by activating the 'hammering' function via thumb button 1 , and then closing the D, E or F keys by pressing directly on one of the six touch buttons. The effect is a combination of a popping sound from the closing key and the pre-recorded slap tongue. Hammer-on technique allows guitarists to play notes continuously without requiring the right hand. Similarly this gesturalinstrumental technique on the HypeSax facilitates continuity of notes, allowing the mouth to rest (see Fig. 75).

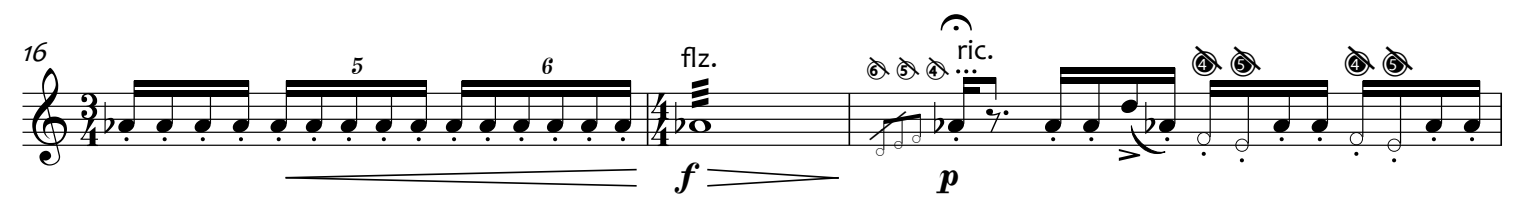

Fig. 75. bb. 16-18 of HypeSax Étude No.3. In b. 18 beat 1, ricochet technique is used, followed by hammering on beats 3 and 4 .

An example of the use of these snippets in my portfolio is found in Más allá del delirio... desde un lugar secreto for tenor HypeSax and piano, where I implemented an adaptation of the ordinario to sul ponticello techniques using a new gestural-instrumental technique. This new technique consists of emulating the physical gesture required to play sul ponticello on the cello. To play this technique, the violoncello is situated in an upright position in front of the performer. The performer bows the string, adding motion towards the bottom of the string. Similarly, in the emulated version for the HypeSax, the saxophone is situated in an upright position in front of the performer. Then, while playing a long tone, the performer sweeps through the touch buttons, moving the right hand towards the bottom of the instrument. The system recognises the sequence of events, a discrete activation of touch buttons in a specific order, and filters the incoming signal in order to boost the higher harmonics, emulating the sounding effect of ordinario to sul ponticello (see Fig. 76). So far I have developed snippets for Bartók pizz., ricochet, col legno battuto, martellato, knocking, polyphony, subharmonics and overpressure techniques. ${ }^{270}$

Gestural-instrumental techniques require a special set of motions which must be learned by the performer. These techniques, like any other, are represented with symbols

${ }^{270}$ Video examples are available at www.hypesax.com or the HypeSax YouTube channel 
that vary in complexity. In the case of the adaptations of techniques, I argue it is necessary to use a new symbol to represent these adaptations, to avoid confusion since the original symbol is associated with another instrument. This eventually becomes unnecessary once the association between physical gesture and the original symbol (used in the other instrument) is learned. In Más allá del delirio... desde un lugar secreto, for example, transitions to a sul ponticello sound are represented by two symbols: $\rightarrow$ sul pont, and the symbols representing the keys (labelled in Fig. 76). The issue of representation problematises the score (notation), since it does not accurately represent the score (element of the tripartite model).

Touch buttons placed over F, E, and D keys

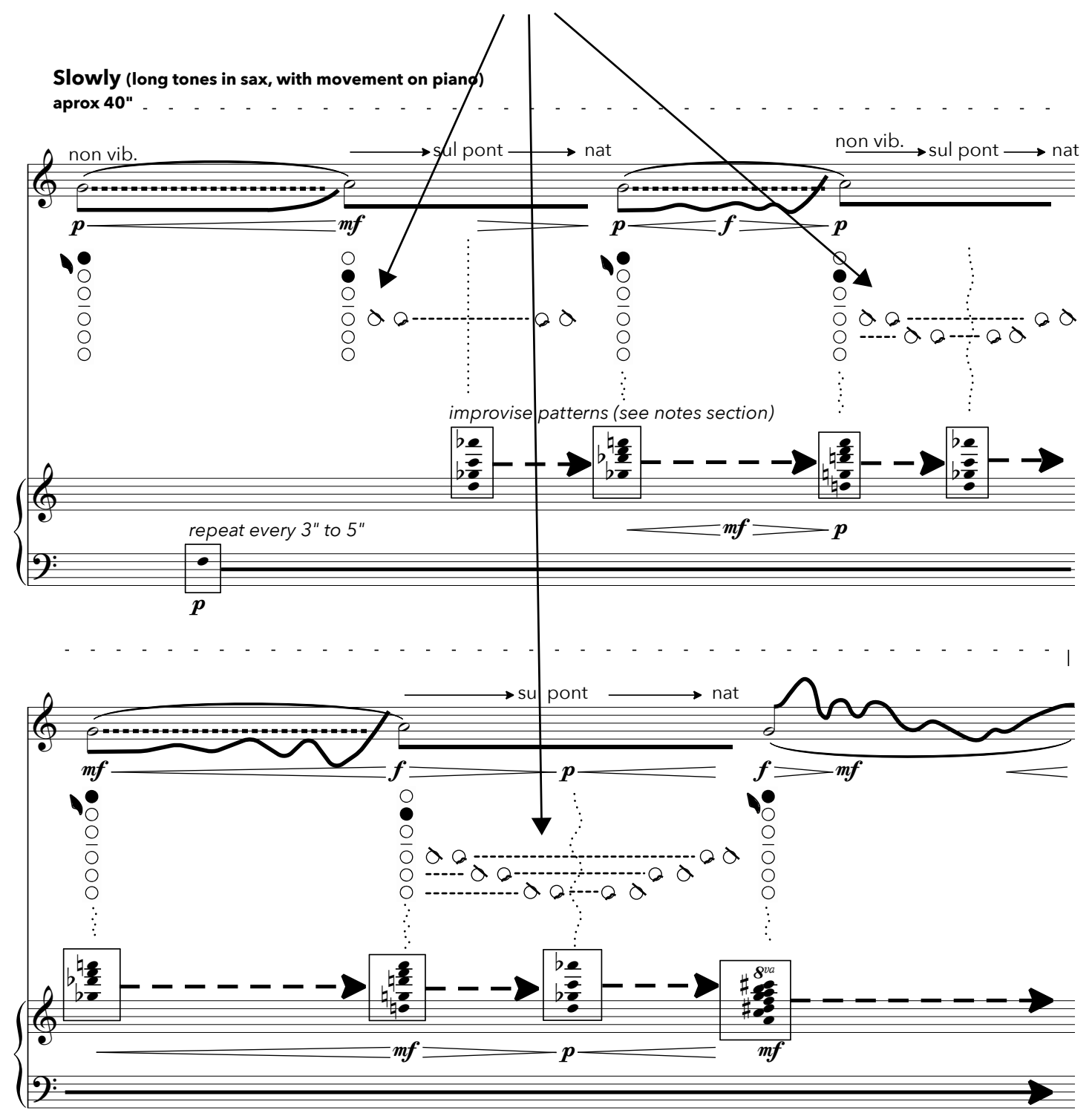

Fig. 76. Section of p. 4 of Más allá del delirio... desde un lugar secreto showing the sul ponticello technique adapted for the analogue gestural-instrumental technique on the HypeSax. 
The score intended by the composer typically reaches performers via some kind of graphic representation that has been agreed upon according to a tradition, style or genre of the music. The only exception is music that is passed on orally. In contrast, some compositions might not be notated using traditional musical conventions due to circumstances such as performative conventions (such as gamelan music), instrumental design (such as the UPIC system), or scores that are open to multiple interpretations (such as Cornelius Cardew's Treatise). In these cases, composers are forced to develop alternative notational strategies. In Look Out!, for instance, the score offers freedom of interpretation. The composition can be interpreted using tonal, atonal or any harmonic language, following any rhythmic approach, slow or fast. In addition, the instruments are not indicated. Look Out! is an improvisational framework. To some degree, many of my compositions are opened to improvisation, and to be taken in a ludic way. While it is clear that not every composer would agree with this, I argue that there is validity in giving performers the option to decide to change elements of the score, such as the octave of a phrase or specific pitches, as long as the musical gestures and relationships between the elements of the composition remain intact. The work does not exist if it is not realised by performers, so by letting performers reshape the score, the work can be renewed in interesting ways. Often, performers decide to play compositions in one way or another as they need to adjust their performance to the specific requirements of their instrument, such as when different keywork designs of a saxophone facilitate performance using a special key, subverting performance to the specificities of the medium.

\subsubsection{The HypeSax as the medium in new works}

Much has been said about the HypeSax in previous sections, from design and construction (Chapter 1) to software and strategies that make its use possible (sections 3.7 and 5.1), as well as examples of how it has influenced my compositional approach and its use in my works (section 5.2). To close this chapter, however, it is important to remind the reader that the development of HypeSax is a case study to explore the concept of the medium in the context of the proposed tripartite model.

During the period of this research, I composed multiple pieces featuring the HypeSax. In some compositions I was able to take advantage of the new acoustic capabilities of the saxophone, using the existing mechanical characteristics of the instrument to explore 
control of feedback, a new voice in the saxophone (see Appendix J and listen to Breathe In Breathe Out.wav). I utilised the HypeSax as a controller working both with my own software as well as commercial software, to apply audio effects to the acoustic sound of the saxophone (listen to Countless Souls.wav, in in which the HypeSax is connected to Ableton Live). I explored the possibility of transformation of the saxophone's timbre through hybrid synthesis. In all three movements of Más allá del delirio, for tenor HypeSax and piano, where the signal from the HypeSax is outputted via a surface transducer placed inside the piano, I explored multiple re-embodiment configurations. The use of gestural-instrumental techniques in pieces such as HypeSax Étude 3 allowed rhythmic fluidity while facilitating performance (see Appendix G, listen to Etude for HypeSax No1.wav). In Look Out!, rather than acting as a music instrument, the HypeSax system monitored the saxophone's sound and saxophonist's ancillary motion, and decided the structural direction of the composition, becoming both a kind of collaborator and, to some degree, taking on the role of director.

There is no doubt that an instrument such as the HypeSax offers multiple creative options due to its mutability, which offers a wide spectrum of possibilities. Surveyed composers, for instance, approached the use of the HypeSax in similar ways as I did, but also offered new ideas. Two of the composers proposed radical ways to use utilise the instrument's capabilities by using the HypeSax as a multiplayer instrument, and reinventing the instrument as multiple instruments: one of the participants proposed the idea of having two performers interacting with one HypeSax. One of these performers plays the HypeSax as a traditional saxophone. A second instrumental part is played by a second performer using a synthesiser embedded in a computer program set to -inf gain. The notes of the synthesiser are played by pressing the 'space bar,' ' $y$ ', 'u,' ' $\mathrm{i}$,' and 'o' keys. This software monitors the movements of the saxophonist who should only play if no sound is coming out of the synthesiser (via Un-mute). The synthesiser performer can then decide to use incoming data from the Un-mute's gyroscope's $\mathrm{X}, \mathrm{Y}$ or $\mathrm{Z}$ to control the gain of the synthesiser by pressing the 'e' key on the computer keyboard multiple times, in the same way as in a video game when 'effort to recover from an injury' requires pressing a key as fast as possible. Every time the synthesiser takes over the score, the pitches assigned to each keyboard key are shuffled as well as the parameter (X, Y or Z) assigned to the 'e' key. In this way, the synthesiser player follows a tablature score which results in 
unexpected melodic lines. The saxophone player, not knowing what parameter is controlling the gain, should then explore multiple actions flipping, turning or shaking the saxophone in order to take control of the synthesiser gain which goes down to -inf if successful. At that point, when no sound is coming out of the Un-mute, the saxophonist continues to play the score. Then, the computer performer must try to take control of the gain again. In this way, as both performers struggle to follow the score and take control as leaders, a new score composed by sections of the two parts is heard (see Fig. 77).

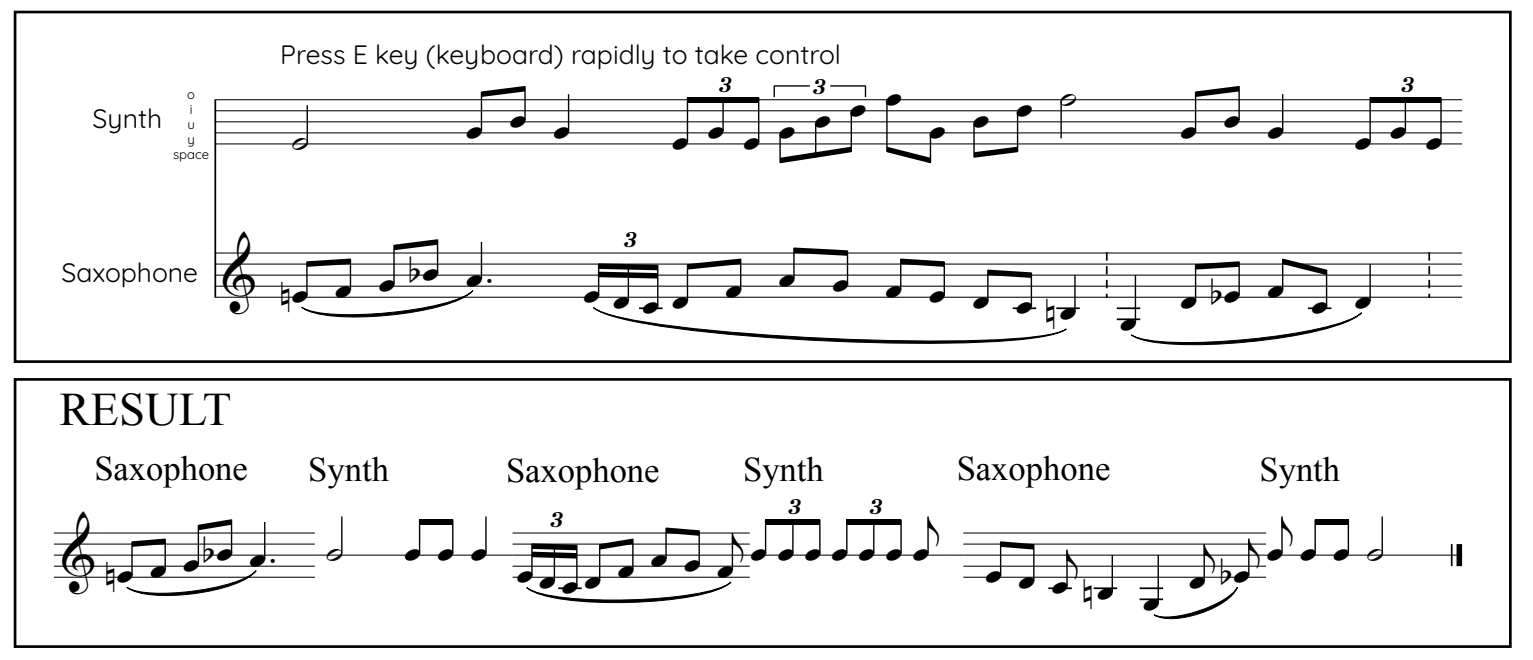

Fig. 77. Musical idea composed by one of the survey participants. Top staves show the written score. Bottom staff shows the score played where the synth pitches are randomly selected.

Another participant re-invented the saxophone and decoupled its components in order to create a small ensemble played by one or two performers. The mouthpiece is to be played on its own with the reed attached. The variations in air pressure, detected by the sensor, activate a synth (Max patch) that generates a drone that resembles the sound of a tanpura. Meanwhile, the touch buttons in the keycaps are used to trigger samples of drums, following Indian tala patterns. In this way, the HypeSax turns into three instruments in a short musical passage influenced by classical Indian music that can be realised by one or two performers (see Fig. 78). 

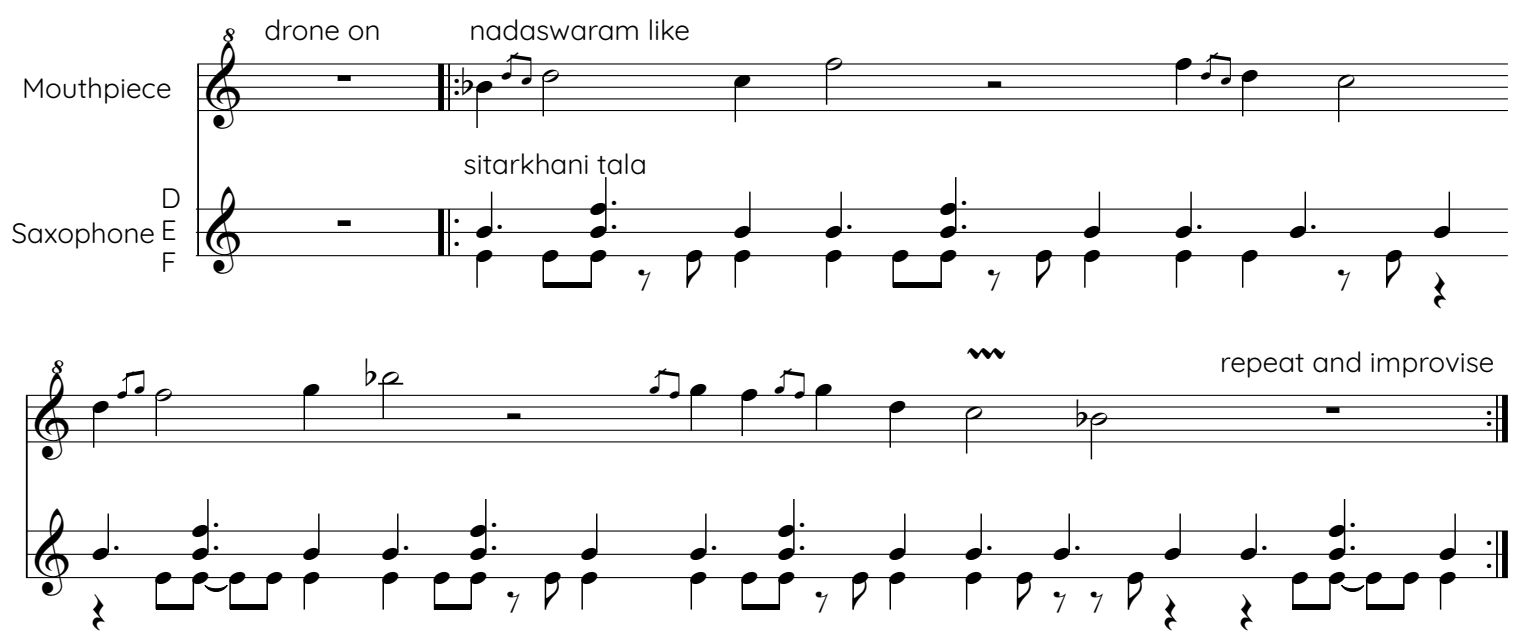

Fig. 78. Musical idea composed by one of the survey participants.

The HypeSax became a playground in which my creativity found a unique place. Not only did I find new opportunities to create and control sound as part of my musical discourses with the HypeSax, but, perhaps most importantly, it made me change the way I think about music. This hyperinstrument can change its role from that of a musical instrument, and can be used instead for sonification, decision-making, or as a synthesiser, controller, or multi-instrument. It could potentially take on many other roles as well. This mutability allows the HypeSax escape the category of an instrument, allowing for a dynamic relationship between itself and the composer or performer. 


\section{6}

\section{CONCLUSION}

This thesis presents the development of the HypeSax as a case study. The development of the HypeSax allowed for the exploration of the medium as an essential part of the creative process in the portfolio of new works composed for this thesis, and the development and evaluation of new performing techniques (including the gestural-instrumental techniques) that connect a physical performative gesture with an acoustic-synthetic sonorous result. The portfolio of works composed during this research helped in analysing the medium as an active tool that produces and transforms sound, the basis for music-making. The mutability of the HypeSax not only offers new sounding possibilities, but experimenting with it allowed me to realise that the medium can take multiple roles, and is able to act as an acoustic, electronic or hybrid instrument; as data collector and/or data processor; as a controller; and/or as a collaborator.

The research presented in this thesis proposes a new way of relating with musical works by presenting a model for the work-concept which comprises the score, performance and medium. In the light of the twenty-first century, re-visiting the traditional work-concept model becomes meaningful as musicians have easy access to new technologies which have changed the way in which we make music. More than ever before, composers and performers are able to create instruments or modify their instruments (or medium of sound production) through the use of new technologies. Digital fabrication, sensors, communication protocols, actuators, 3D printing and other techniques and materials can be used to build instruments. Software is constantly being written and improved upon by individuals and professional developers in order to create music in innovative ways. The way in which we create music is changing at a fast pace, and 
composers often develop new tools, musical instruments, software, robots or engineer a device necessary to perform their compositions. The line that separates the creation of musical work and the creation of the 'instrument' is therefore becoming blurred with these new creative practices. Considering the intrinsic connection between these two acts of creation and their consequences for the musical work, the tripartite model becomes essential to understand our new relation with music.

After developing the HypeSax, it became clear to me that the potential found in hyperinstruments - as well as the use of new technologies - pushes the boundaries of the traditional ways in which musicians interact with instruments to make music. Machover and Chung state that instrumental augmentation with new added technology 'pays close attention to the learnability, perfectibility, and repeatability of redefined playing technique'. ${ }^{271}$ From the point-of-view of the performer, it is exciting to imagine ways in which the skills developed over many years playing one particular instrument can be expanded with new possibilities afforded by hyperinstruments.

\subsection{Summary and contributions}

The first chapter of this thesis introduced questions that arose from my relationship with musical works as a composer and as a performer using traditional instruments and new technologies:

- If a medium (instrument) built for a specific composition is to be considered part of the work, how can it be inserted in a new work-concept?

The case study presented demonstrates that the degree of decision-making involved in developing a hyperinstrument is directly influenced by the composer's musical ideas and goals, as well as the relationship between the performer and the instrument. The close interaction, necessary to make music, between all three elements justifies the need to understand the musical work as a joint effort between these three aspects. The tripartite model allows for an interaction that flows in between the three actors of the musical work

271 Tod Machover and Joseph T. Chung, 'Hyperinstruments: Musically Intelligent and Interactive Performance and Creativity Systems', in Proceedings of the 1989 International Computer Music Conference (presented at the ICMC89, San Francisco, 1989). 
in a rhizomatic way. This model does not set a hierarchical structure, remaining open to music from multiple traditions that are not centred on the composer, being centred instead either on performance, as happens in jazz, or the medium, as can be the case in generative music or interactive sound installations (see section 5.2.1 where the model is analysed with Look Out!).

- How can the process of 'building the medium' become part of the composition process?

This research shows how, through the process of development of the medium, the composer can engage with the work in new different ways:

a) The composer can design music that uses the instrument in a traditional way, building a composition that uses the pre-established sounding palette of the instrument. At the same time, the composer is able to modify those sounds and create new ones through digital enhancement.

b) While the relationship between the performer and the instrument remains as usual, the composer is able to engineer new ways in which the performer interacts with the medium, potentially affording new expressive possibilities for the performer.

c) Through multiple embodiment configurations, the composer is able to explore sound space in new ways.

d) The physical limitations of the instruments can be blurred, which allows for the composer to access new potential for mutability of the medium as a potential for new compositional approaches

'Building the medium' offers the composer new perspectives that have an enormous influence in the micro - and macro-structures of a composition, providing a new framework for decision-making during the process of composition.

- How can hyperinstruments change the way we approach music composition in a meaningful way, not only by offering new affordances?

The enhanced mutability that hyperinstruments offer allows the medium to take on new roles. The medium has the potential to no longer act as a passive tool but to participate in the creative process through interactivity, by acting and reacting, using potential 'smart' 
capabilities afforded by its new technology. While these 'smart' capabilities are still defined by the designer of the medium, the final results are decided by the system.

In my personal experience with the HypeSax, I came to the realisation that the medium can be a dynamic and ever-changing collaborator in the creative process. The HypeSax is not only an instrument to be played, it is an instrument to be redesigned, and through this constant transformation, new musical ideas will arise, almost as if the HypeSax itself had proposed them. This realisation came to me slowly, in small steps, as I developed and explored the instrument, and as I came to understand what the medium is and its importance in the tripartite model.

The realisation and exploration of the medium came together with some contributions to the fields of composition, instrument-building and new musical interface design. The main contributions afforded through this case study include:

a) A new work-concept model that integrates the medium as an important element of the musical work. This tripartite model serves as a tool to visualise the extent of a musical work. It can also be considered as a model to follow during the compositional process, allowing for a free multidirectional flow of the dialog between the score, performance and medium, influencing and enriching the final work.

b) A new framework for hyperinstrument design, analogous to the proposed tripartite model, in which musical, interactive and operational design goals are considered and evaluated in an iterative design process.

c) A study of how hybrid synthesis can be achieved and its potential use in musical works.

d) An investigation into the potential expansion of instrumental techniques through the gestural-instrumental techniques concept and their exploration through hyperinstruments.

e) Research into the mutability of the medium, afforded by hyperinstruments, and its potential for diverse compositional practices.

f) Exploration of multiple embodiment configurations in a hyperinstrument and their implications in the creative process and audience reception. 
g) A novel software application, The HUB, that represents a paradigm in hyperinstrument software through the use of a master server. This software receives incoming data from a multitude of sensors communicating to a micro-controller. The data is then adapted for the needs of the composer in order to communicate via MIDI, OSC, Serial or Max send/receive messages to other programs, allowing composers to establish an ecosystem of applications that can be used in multiple compositions.

h) A new, flexible paradigm in hyperinstrument design, that uses nondestructive augmentation techniques and parametric design principles to allow it to be adapted to work with multiple instruments of different sizes. In the case of the HypeSax, the augmentation components can be easily adapted to work with soprano, alto and tenor saxophones, regardless of their model, to provide electronic capabilities.

\subsection{Future work}

While developing the HypeSax has arrived at a base level of maturity as an instrument, further developments might be necessary in order to achieve a more powerful sound system that can better exist alongside the saxophone in order to achieve true hybridity of sound across the entire dynamic range.

While the HypeSax was developed for creative purposes, its potential for use as a diagnostic tool was revealed during this project. Specifically, the mouthpiece has the potential to describe how certain techniques are achieved by changing the air pressure. Offering a visualisation of the variations in air pressure might be helpful as a pedagogical tool, to correct and improve students' performance of specific techniques. Similarly, the keycaps could be adapted, and new keycaps could be created to monitor all keys of the instrument; such a system would facilitate obtaining data that would be helpful in monitoring the interaction with the instrument through fingering variations. The pedagogical potential of the HypeSax is worthy of further exploration.

There is also the potential to further explore and develop new gestural-instrumental techniques, and to provide the saxophone with access to techniques that are traditionally only available for other instruments. While the HypeSax was developed to augment the 
saxophone, similar developments and design approaches could be used to enhance other woodwind instruments. The effect of hybridity can also be extended to idiophones, membranophones, and chordophones.

While the tripartite model has been successful in the scope of this research project and its case study, further research in the implications of the model, applied to different approaches to creativity, might afford more useful information that can help us situate the role of new technology in musical creativity, and potentially art in general, in the twentyfirst century.

\subsection{Final remarks}

This project took me on a journey that led to unexpected results. I initially intended to build new instruments and address the need I felt for embracing embodiment while still accessing the capabilities of digital sound processing. Nonetheless, this research made me realise the importance of the medium and the fact that musical works should not have to be (and are not) fixed constructs, as well as the fundamental and active role that developing the medium can play in the entire concept of the creative process. Musical works are dynamic and, just like sounds themselves, both evolve over time and are renewed on every realisation; and the performance and medium therefore become a creative force in the midst of musical practice.

This raises the question of whether the composer is the sole author of a musical work. I do not think there is a definite answer, as the level of involvement the performers and the medium have in a work may vary. However, I believe that the composer should remain open to the possibility of allowing performers and medium to share authorship of the work.

The work presented in this thesis has contributed to the fields of composition, instrument building, hyperinstruments, sound software design and, through a case study, sets precedents for new paradigms of instrumental design, placing the medium at the centre of the ontology of the creative work. 


\section{BIBLIOGRAPHY}

'Acoustically

Active

Augmented

Instruments' $<$ https://www.nordiskkulturfond.org/en/inspirational-projects/acoustically-activeaugmented-instruments $/>$ [accessed 15 January 2019]

Adorno, T. W., 'A Social Critique of Radio Music', The Kenyon Review, 18.3-4 (1996), 229

Alperson, Philip, 'The Instrumentality of Music', Journal of Aesthetics and Art Criticism, 66.1 (2008), 37-51

'Alto Saxophone Mouthpiece by Allanrps' < https://www.thingiverse.com/thing:14495> [accessed 3 September 2017]

Astor, Miguel, Aproximación Fenomenológica a la Obra Musical de Gonzalo Castellanos Yumar (Caracas, Venezuela: Comision de Estudios de Posgrado, Facultad de Humanidades y Educación - Universidad Central de Venezuela, 2002)

'Audio System Design Tool for Teensy Audio Library' $<$ https://www.pjrc.com/teensy/gui/index.html $>$ [accessed 28 November 2019]

Auslander, Philip, 'Lucille Meets GuitarBot: Instrumentality, Agency, and Technology in Musical Performance', in Musical Instruments in the 21st Century, ed. by Till Bovermann, Alberto de Campo, Hauke Egermann, Sarah-Indriyati Hardjowirogo, and Stefan Weinzierl (Singapore: Springer Singapore, 2017), pp. 297-314

Babbitt, Milton, 'Who Cares If You Listen', High Fidelity Magazine, 1958, pp. 38-40, $126-27$

Backus, John, 'Input Impedance Curves for the Reed Woodwind Instruments', The Journal of the Acoustical Society of America, 56.4 (1974), 1266-79

_ ' 'The Effect of the Player's Vocal Tract on Woodwind Instrument Tone', The Journal of the Acoustical Society of America, 78.1 (1985), 17-20

Bailey, Derek, Improvisation Its Nature and Practice in Music (Da Capo Press, 1992)

Barthes, Roland, Elements of Semiology, trans. by Annette Lavers and Colin Smith (Hill and Wang, 1968)

_ Image, Music, Text, ed. by Stephen Heath (London: Fontana Press, 1987)

- ' 'The Death of the Author', in Image, Music, Text, ed. by Stephen Heath (London: Fontana Press, 1987), pp. 142-48

Baxter, Kathy, Catherine Courage, and Kelly Caine, Understanding Your Users: A Practical Guide to User Research Methods, Tools, and Techniques (Elsevier Science \& Technology, 2015)

Beauregard, Gerald, 'Rethinking the Design of Wind Controllers' (unpublished MA Thesis, Dartmouth College, 1991)

Benacchio, Simon, Adrien Mamou-Mani, Baptiste Chomette, and René Caussé, 'Active Control Applied to String Instruments', in Proceedings of the Acoustics 2012 Nantes Conference (presented at the Acoustics 2012 Conference, Nantes, France, 2012), p. 7 
Benade, A. H., 'On Woodwind Instrument Bores', The Journal of the Acoustical Society of America, 31.2 (1959), 137-46

Ben-Tal, Oded, 'Characterising Musical Gestures', Musicae Scientiae, 16.3 (2012), 24761

Bergeot, Baptiste, André Almeida, Bruno Gazengel, Christophe Vergez, and Didier Ferrand, 'Response of an Artificially Blown Clarinet to Different Blowing Pressure Profiles', The Journal of the Acoustical Society of America, 135.1 (2014), 479-90

Berry, Wallace, Structural Functions in Music, Dover Books on Music, Music History (New York: Dover, 1987)

Bertini, Graziano, and Paolo Carosi, 'Light Baton: A System for Conducting Computer Music Performance', in Proceedings of ICMC 1992 (presented at the ICMC, San Francisco, 1992)

'BMP180' <https://www.bosch-sensortec.com/bst/products/all_products/bmp180> [accessed 4 July 2017]

Bongers, Bert, 'Physical Interfaces in the Electronic Arts', Trends in Gestural Control of Music, 2000, 30

BOSCH, 'BMP180 Digital Pressure Sensor Data Sheet', $2015<\mathrm{https} / /$ aebst.resource.bosch.com/media/_tech/media/datasheets/BST-BMP180-DS000121.pdf>

Bosch Sensortec, 'BMP180 Digital Pressure Sensor Data Sheet', 2015

Boyden, David D., Col Legno (Oxford University Press, 2001), I

Braasch, Jonas, Hyper-Specializing in Saxophone Using Acoustical Insight and Deep Listening Skills, Current Research in Systematic Musicology (Cham: Springer International Publishing, 2019), VI <https://doi.org/10.1007/978-3-030-15046-4>

Burrows, David, 'Instrumentalities', The Journal of Musicology, 5.1 (1987), 117-25

Burtner, Matthew, 'Making Noise: Extended Techniques after Experimentalism', NewMusicBox, $2005<\mathrm{https} / / / \mathrm{nmbx}$.newmusicusa.org/making-noise-extendedtechniques-after-experimentalism/> [accessed 26 November 2018]

, 'The Metasaxophone: Concept, Implementation, and Mapping Strategies for a New Computer Music Instrument', Organised Sound, 7.02 (2002), 201-13

Cage, John, Sonatas and Interludes (Edition Peters, 1960)

Candy, Linda, Practice Based Research: A Guide, CCS Report: 2006 (Sydney, Australia: University of Technology Sydney, 2006)

Carral, S., V. Lorenzoni, and J. C. Verlinden, 'Influence of Mouthpiece Geometry on Saxophone Playing', Proceedings of the 3rd Vienna Talk on Music Acoustics" Bridging the Gaps", Vienna, Austria, 16-19 September 2015, 2015

Chaigne, Antoine, and Jean Kergomard, Acoustics of Musical Instruments, Modern Acoustics and Signal Processing (New York, NY: Springer New York, 2016)

Chen, Qianwen, 'A HAPTIC PARTIAL-GUIDANCE SYSTEM FOR FLUTE TUTORING' (Dartmouth College, 2017) 
Chomsky, Noam, 'Human Language and Other Semiotic Systems', in Speaking of Apes: A Critical Anthology of Two-Way Communication with Man, ed. by Thomas Albert Sebeok and Jean Umiker-Sebeok, Softcover reprint of the original 1st ed. 1980 (New York London, 2012), p. 12

Clark, Ann, 'Is Music a Language?', 1982, 11

Clayton, Martin, Rebecca Sager, and Udo Will, 'The Concept of Entrainment and Its Significance for Ethnomusicology', ESEM CounterPoint, 1 (2004), 83

Clift, Paul, 'Acoustic-Aggregate-Synthesis | Paul Clift', Www.Paulclift.Net $<$ http://www.paulclift.net/aas/> [accessed 26 May 2020]

, 'Department of Music Columbia University, New York, NY', in Proceedings of the International Computer Music Conference (presented at the ICMC 2012, Ljubljana, 2012), pp. 120-23

Clift, Paul, Adrien Mamou-Mani, and René Caussé, 'Extending Brass \& Woodwinds with Acoustic Aggregate Synthesis', in Proceedings of the International Computer Music Conference (presented at the ICMC 2015, Denton, 2015), p. 7

Coker, Willson, Music \& Meaning: A Theoretical Introduction to Musical Aesthetics (The Free Press, 1970)

Collingwood, R.G., The Principles of Art (Oxford University Press, 1938)

Cook, Perry, and Colby Lieder, 'SqueezeVox: A New Controller for Vocal Synthesis Models', in Proceedings of ICMC 2000, 2000

Cook, Perry R., 'A Meta-Wind-Instrument Physical Model, and a Meta-Controller for Real Time Performance Control', in 1992 International Computer Music Conference (presented at the Computer Music Conference, San Jose, California: Computer Music Association)

, 'Principles for Designing Computer Music Controllers', in Proceedings of the CHI'01 Workshop on the New Interfaces for Musical Expression (Seattle, 2001), pp. 3-6

Cook, Perry R, 'Remutualizing the Instrument: Co-Design of Synthesis Algorithms and Controllers', in Proceedings of the Stockholm Music Acoustics Conference, August 6-9, 2003 (SMAC 03), Stockholm, Sweden (presented at the SMAC 03, Stockholm, Sweden, 2003), p. 4

Cook, Perry, and Dan Trueman, 'BoSSA: The Deconstructed Violin Reconstructed', Journal of New Music Research, 29.2 (2000), 121-30

Cope, David, Techniques of the Contemporary Composer, Schimer, 1997

Croft, John, 'Theses on Liveness', Organised Sound, 12.01 (2007), 59-66

Crumb, George, Makrokosmos Volume I (C. F. Peters, 1972)

—, Makrokosmos Volume II (C. F. Peters, 1973)

—, Makrokosmos Volume III 'Music for a Summer Evening' (C. F. Peters, 1974)

—, Makrokosmos Volume IV ‘Celestial Mechanics’ (C. F. Peters, 1979) 
Cycling74/Miraweb (Cycling '74, 2020) <https://github.com/Cycling74/miraweb> [accessed 24 June 2020]

Da Silva, Andrey R., Marcelo M. Wanderley, and Gary Scavone, 'On the Use of Flute Air Jet as a Musical Control Variable', in Proceedings of the 2005 Conference on New Interfaces for Musical Expression (National University of Singapore, 2005), pp. $105-8$

, 'On the Use of Flute Air Jet as a Musical Control Variable', in Proceedings of the 2005 Conference on New Interfaces for Musical Expression (National University of Singapore, 2005), pp. 105-8 <http://dl.acm.org/citation.cfm?id=1085969> [accessed 4 May 2017]

Dabin, Matthew, Terumi Narushima, Stephen Beirne, Christian Ritz, and Kraig Grady, '3D Modelling and Printing of Microtonal Flutes', in Proceedings of the International Conference on New Interfaces for Musical Expression, 2220-4806 (Brisbane, Australia: Queensland Conservatorium Griffith University, 2016), XVI, 286-90

'DAVID TUDOR: Rainforest' < https://davidtudor.org/Works/rainforest.html> [accessed 26 March 2020]

Delalande, François, 'Le geste, outil d'analyse : quelques enseignements d'une recherche sur la gestique de Glenn Gould', Analyse Musicale, 1988

Driscoll, John, 'Resonance: From the Architectural to the Microscopic', Leonardo Music Journal, 22 (2012), 25-33

Driscoll, John, and Matt Rogalsky, 'David Tudor's Rainforest : An Evolving Exploration of Resonance', Leonardo Music Journal, 14 (2004), 25-30

Eco, Umberto, A Theory of Semiotics (Bloomington, Indiana: Indiana University Press, 1976)

d'Escriván, Julio, 'To Sing the Body Electric: Instruments and Effort in the Performance of Electronic Music', Contemporary Music Review, 25.1-2 (2006), 183-91

Fels, Sidney, Linda Kaastra, Sachiyo Takahashi, and Graeme McCaig, 'Evolving Tooka: From Experiment to Instrument', 2004, 6

Fineberg, Joshua, 'Sculpting Sound. An Introduction to the Spectral Movement: Its Ideas, Techniques and Music.' (Columbia University, 1999)

Fletcher, Neville H., and Thomas D. Rossing, The Physics of Musical Instruments, 2. ed., [Nachdr.] (New York, NY: Springer, 2010)

Flusser, Vilém, and Nancy Ann Roth, 'Gesture and Affect: The Practice of a Phenomenology of Gestures', in Gestures (University of Minnesota Press, 2014), pp. 1-9

Frisk, Henrik, and Stefan Östersjö, 'Negotiation the Musical Work. An Empirical Study on the Inter-Relation between Composition, Interpretation and Performance', in Terminology and Translation (presented at the EMS: Electronic Music Studies Network, Beijing, 2006), p. 13 
Fritz, Claudia, and Joe Wolfe, 'How Do Clarinet Players Adjust the Resonances of Their Vocal Tracts for Different Playing Effects', The Journal of the Acoustical Society of America, 118.5 (2005), 3306-15 <https://doi.org/10.1121/1.2041287>

Garcia, Francisco, Leny Vinceslas, Josep Tubau, and Esteban Maestre, 'Acquisition and Study of Blowing Pressure Profiles in Recorder Playing.', in NIME, 2011, pp. 12427

$<$ http://mfile.narotama.ac.id/files/Umum/JURNAR\%20STANFORD/Acquisition $\% 20$ and $\% 20$ study $\% 20$ of $\% 20$ blowing $\% 20$ pressure $\% 20$ profiles.pdf $>$ [accessed 4 May 2017]

Gay, Jr., Leslie C., 'Acting up, Talking Tech: New York Rock Musicians and Their Metaphors of Technology', Ethnomusicology, Vol. 41, No.1, 1998, 81-98

Gazengel, Bruno, and Pauline Eveno, 'Caractérisation Expérimentale de Becs de Saxophone Ténor à l'aide d'un Musicien Artificiel Fonctionnant En Aspiration', in CFA '18 LE HAVRE (presented at the Congrés Français d'Acoustique, Le Havre, France, 2018)

Goehr, Lydia, 'Being True to the Work', The Journal of Aesthetics and Art Criticism, 47.1 (1989), 55

, The Imaginary Museum of Musical Works: An Essay in the Philosophy of Music, Rev. ed (Oxford: Oxford Univ. Press, 2007)

Hardjowirogo, Sarah-Indriyati, 'Instrumentality. On the Construction of Instrumental Identity', in Musical Instruments in the 21st Century, ed. by Till Bovermann, Alberto de Campo, Hauke Egermann, Sarah-Indriyati Hardjowirogo, and Stefan Weinzierl (Singapore: Springer Singapore, 2017), pp. 9-24

Hatten, Robert S., Musical Meaning in Beethoven: Markedness, Correlation, and Interpretation, Advances in Semiotics (Bloomington: Indiana University Press, 1994)

Hong, Euyshick, Guanyu Zhu, and Wendel Moreira Duarte, 'Dr. Saxophone: Hybrid Saxophone Interface', 2016 3rd International Conference on Systems and Informatics (ICSAI), 2016, 1149-53

Houlès, Fabien, Le Premier Quatuor à Cordes Hybride, l'example de Smaqra de Juan Arroyo, L'Harmattan (Paris, 2017)

Iazzetta, Fernando, 'Meaning in Musical Gesture', in Trends in Gestural Control of Music, ed. by Marcelo M. Wanderley and M. Battier (Paris, France: IRCAM-Centre Pompidou, 2000), pp. 259-68

'IMAREV | Acoustique Instrumentale' < http://instrum.ircam.fr/imarev/> [accessed 15 January 2019]

Jensenius, Alexander Refsum, and Michael J. Lyons, eds., A NIME Reader. Fifteen Years of New Interfaces for Musical Expression (Cham: Springer International Publishing, 2017)

Jonker, Johan, 'Measuring a Mouthpiece' <https://sax.johanjonker.net/measuring-amouthpiece $>$ [accessed 31 August 2017]

Jordà, Sergi, 'Afasia: The Ultimate Homeric One-Man-Multimedia-Band', 7 
Kanamori, T., H. Katayose, S. Simura, and S. Inokuchi, 'Gesture Sensor in Virtual Performer', in Proceedings of ICMC 1993 (presented at the ICMC, San Francisco, 1993)

Kokoras, Panayiotis A., 'Towards a Holophonic Musical Texture', in Proceedings of the Nternational Computer Music Conference 2005 (presented at the International computer music conference: ICMC 2005 free sound, International Computer Music Association, 2005), p. 3

Lähdeoja, Otso, 'Active Acoustic Instruments for Electronic Chamber Music', in Proceedings of the International Conference on New Interfaces for Musical Expression, 2220-4806 (Brisbane, Australia: Queensland Conservatorium Griffith University, 2016), XVI, 132-36

Leitman, Sasha, and John Granzow, 'Music Maker: 3D Printing and Acoustics Curriculum', in Proceedings of New Interfaces for Musical Expression 2016 (presented at the NIME 2016, Brisbane, Australia, 2016)

Leman, Marc, Embodied Music Cognition and Mediation Technology (Cambridge, Mass.: Mit Press, 2008)

- 'Music, Gesture, and the Formation of Embodied Meaning', in Musical Gestures: Sound, Movement, and Meaning, ed. by Rolf Inge Godøy and Marc Leman, 2009, p. 28

-, 'Musical Gestures and Embodied Cognition', Actes Des Journées d'Informatique Musicale (JIM 2012), 2012, 3

Lorenzoni, Valerio, E. L. Doubrovski, and J. C. Verlinden, 'Embracing the Digital in Instrument Making: Towards a Musician-Tailored Mouthpiece by 3D Printing', in Proceedings of the Stockholm Music Acoustics Conference 2013, SMAC 2013, Stockholm (Sweden), 30 July-3 August, 2013, 2013

Lucier, Alvin, and Douglas Simon, Chambers: Scores by Alvin Lucier (Middeltown, CT: Wesleyan University Press, 1980)

Machover, Tod, Hyperinstruments. A Progress Report 1987-1991 (Cambridge, 1992) , 'The Extended Orchestra', in PEYSER, J. (Ed.), The Orchestra: Origins and Transformations. (New York, NY: Billboard Books, 1986)

Machover, Tod, and Joseph T. Chung, 'Hyperinstruments: Musically Intelligent and Interactive Performance and Creativity Systems', in Proceedings of the 1989 International Computer Music Conference (presented at the ICMC89, San Francisco, 1989)

Malt, Mikhail, and Emmanuel Jourdan, 'Real-Time Uses of Low Level Sound Descriptors as Event Detection Functions Using the Max/MSP Zsa.Descriptors Library', in Proceedings of SBCM 2009 (presented at the SBCM 2009, Recife, Brazil, 2009), p. 12

, 'Zsa.Descriptors: A Library for Real-Time Descriptors Analysis', in Proceedings of SMC 20008 (presented at the SMC 20008, Germany, 2008), p. 4

Melo, Jonh, Daniel Gómez, and Miguel Vargas, 'Gest-O: Performer Gestures Used to Expand the Sounds of the Saxophone', in Proceedings of the International 
Conference on New Interfaces for Musical Expression (Ann Arbor, Michigan: University of Michigan, 2012)

Merleau-Ponty, Maurice, Phenomenology of Perception, trans. by Colin Smith (Psychology Press, 2002)

Meurisse, Thibaut, 'Contrôle actif appliqué aux instruments de musique à vent' (Université Pierre et marie Curie, 2014)

Meurisse, Thibaut, Adrien Mamou-Mani, Simon Benacchio, Baptiste Chomette, Victor Finel, David B. Sharp, and others, 'Experimental Demonstration of the Modification of the Resonances of a Simplified Self-Sustained Wind Instrument Through Modal Active Control', Acta Acustica United with Acustica, 101.3 (2015), 581-93

Meurisse, Thibaut, Adrien Mamou-Mani, René Caussé, Benny Sluchin, and David B. Sharp, 'An Active Mute for the Trombone', The Journal of the Acoustical Society of America, 138.6 (2015), 3539-48

MIMO Consortium, 'Revision of the Hornbostel-Sachs Classification of Musical Instruments by the MIMO Consortium', $2011<$ http://www.mimointernational.com/documents/Hornbostel\%20Sachs.pdf>

Miranda, Eduardo Reck, and Marcelo M. Wanderley, New Digital Musical Instruments: Control and Interaction beyond the Keyboard, The Computer Music and Digital Audio Series, v. 21 (Middleton, Wis: A-R Editions, 2006)

Molino, Jean, 'Musical Fact and the Semiology of Music', trans. by J. A. Underwood, Music Analysis, 9.2 (1990), 105

Morita, H., S. Hashimoto, and S. Ohteru, 'A Computer Music System That Follows a Human Conductor', Computer, 24.7 (1991), 44-53

Nattiez, Jean Jacques, Music and Discourse: Toward a Semiology of Music (Princeton, N.J: Princeton University Press, 1990)

Nijs, Luc, Micheline Lesaffre, and Marc Leman, 'The Musical Instrument as a Natural Extension of the Musician', in Music and Its Instruments (presented at the The 5th Conference of Interdisciplinary Musicology, Paris: LAM-Institut jean Le Rond d'Alembert, 2013), pp. 132-33

'NIME | Archive of NIME Proceedings' <http://www.nime.org/archives/> [accessed 29 October 2017]

Nusseck, Manfred, and Marcelo M. Wanderley, 'Music and Motion-How Music-Related Ancillary Body Movements Contribute to the Experience of Music', Music Perception: An Interdisciplinary Journal, 26.4 (2009), 335-53

Oliveros, Pauline, 'Acoustic and Virtual Space as a Dynamic Element of Music', Leonardo Music Journal, 5 (1995), 19-22

Onozawa, N., and K. Fujita, 'Hybrid Wind Instrument Selectively Producing Acoustic Tones and Electric Tones and Electronic System Used Therein', 2006 $<$ https://www.google.com/patents/US7049503> 
Overholt, Dan, Edgar Berdahl, and Robert Hamilton, 'Advancements in Actuated Musical Instruments', Organised Sound, $16.02 \quad$ (2011), 154-65 $<$ https://doi.org/10.1017/S1355771811000100>

Palacio-Quintin, Cléo, 'The Hyper-Flute', in Proceedings of the 2003 Conference on New Interfaces for Musical Expression (Montreal, 2003), pp. 206-7

Palombini, Carlos Vicente de Lima, 'Pierre Schaeffers Typo-Morphology of Sonic Objects' (Durham Univeristy, 1993) <http://etheses.dur.ac.uk/1191/>

Paradiso, Joseph A, 'The Brain Opera Technology: New Instruments and Gestural Sensors for Musical Interaction and Performance', 31

Pardue, Laurel S, Kurijn Buys, Michael Edinger, Dan Overholt, and Andrew P McPherson, 'Separating Sound from Source: Sonic Transformation of the Violin through Electrodynamic Pickups and Acoustic Actuation', in Proceedings of the International Conference on New Interfaces for Musical Expression (presented at the NIME 2019, Porto Alegre, Brazil, 2019), pp. 272-77

Penderecki, Krzysztof, 'Threnody to the Victims of Hiroshima' (Polskie Wydawnictwo Muzyczne, 1961)

Photinos, Panos, Musical Sound, Instruments, and Equipment, 2017

Pomerantz, James R., 'Visual Form Perception: An Overview', in Pattern Recognition by Humans and Machines: Visual Perception, ed. by Eileen C. Schwab and Howard C. Nusbaum (Elsevier, 2016), pp. 1-30

Portovedo, Henrique, Paulo Ferreira Lopes, and Ricardo Mendes, 'Saxophone Augmentation: An Hybrid Augment System of Gestural Symbiosis', 4

Raes, Godfried-Willem, 'Logos Foundation - Godfried-WIllem Raes' Automatons and Robots', Www.Logosfoundation.Org $<$ https://www.logosfoundation.org/instrum_gwr/automatons.html $>$ [accessed 14 May 2018]

Ramos, Cristohper, 'Sound, Shape, and Interactivity: Sound Maze and Other Recent Works' (Wesleyan University, 2014)

Reid, Sarah, Ryan Gaston, Colin Honigman, and Ajay Kapur, 'Minimally Invasive Gesture Sensing Interface (MIGSI) for Trumpet', in Proceedings of the International Conference on New Interfaces for Musical Expression, 2220-4806 (Brisbane, Australia: Queensland Conservatorium Griffith University, 2016), XVI, 419-24

Ricœur, Paul, From Text to Action (Northwestern University Press, 1991)

Risset, Jean-Claude, 'Sculpting Sounds with Computers: Music, Science, Technology', Leonardo, 27.3 (1994), 257-61

Romitelli, Fausto, 'Professor Bad Trip: Lesson I' (Ricordi, 1998)

'SABRE | SABRE Multisensor', SABRe <https://www.sabre-mt.com/sabre-multisensor $>$ [accessed 9 January 2019]

de Saussure, Ferdinand, Course in General Linguistics, ed. by Charles Bally and Albert Sechehaye, trans. by Wade Baskin (McGraw-Hill Book Company, 1966) 
Scavone, Gary P., 'THE PIPE: Explorations with Breath Control', in NIME, 2003

Scavone, Gary, and Andrey R. Silva, 'Frequency Content of Breath Pressure and Implications for Use in Control', in Proceedings of the International Conference on New Interfaces for Musical Expression (Vancouver, BC, Canada, 2005), pp. 93-96

Schaeffer, Pierre, Treatise on Musical Objects: An Essay across Disciplines, trans. by Christine North and John Dack (University of California Press, 2017)

Schiesser, Sébastien, and Jan C. Schacher, 'SABRe: The Augmented Bass Clarinet.', in NIME, 2012

- 'SABRe: The Augmented Bass Clarinet.', in NIME, 2012 $<$ http://vhosts.eecs.umich.edu/nime2012/Proceedings/papers/193_Final_Manuscr ipt.pdf> [accessed 4 May 2017]

Schiesser, Sébastien, and Caroline Traube, 'On Making and Playing an ElectronicallyAugmented Saxophone', in Proceedings of the International Conference on New Interfaces for Musical Expression (Paris, France, 2006), pp. 308-13

Schmidt-Garre, Jan, Celibidache - You Don't Do Anything - You Let It Evolve (Arthaus Musik, 1991)

Schroeder, Franziska, 'Bodily Instruments and Instrumental Bodies: Critical Views on the Relation of Body and Instrument in Technologically Informed Performance Environments', Contemporary Music Review, 25.1-2 (2006), 1-5

Schutz, Michael, and Scott Lipscomb, 'Hearing Gestures, Seeing Music: Vision Influences Perceived Tone Duration', Perception, 36.6 (2007), 888-97

Scruton, Roger, The Aesthetics of Music (Oxford University Press, 1999)

'Selmer VARITONE - Sax On, El Mundo Del Saxo' <https://sax-on.com/selmervaritone/> [accessed 13 August 2019]

Sève, Bernard, 'Bords de l'œuvre musicale', Aisthesis, 3.2 (2011), 29-45

Smalley, Denis, 'Defining Transformations', Interface, 22.4 (1993), 279-300

- 'Spectro-Morphology and Structuring Processes.', in The Language of Electroacoustic Music, ed. by Simon Emmerson (London: Palgrave Macmillan, 1986)

Smallwood, Scott, Perry Cook, Dan Trueman, and Lawrence McIntyre, 'Don't Forget the Loudspeaker - A History of Hemispherical Speakers at Princeton, Plus a DIY Guide', in Proceedings of the International Conference on New Interfaces for Musical Expression (presented at the NIME 2009, Pittsburg, PA, 2009), pp. 110 15

'SmartInstruments $\quad$ Acoustique Instrumentale' $<\mathrm{http}$ ://instrum.ircam.fr/smartinstruments/> [accessed 15 January 2019]

Sølvi, Ystad, and Vionier Thierry, 'Design of a Flute Interface to Control Synthesis Models', in ICMC '99 Proceedings (presented at the ICMC 1999, Beijing, 1999)

'SPEAR Homepage' < http://www.klingbeil.com/spear/> [accessed 20 July 2020] 
Strohm, Reinhard, 'Looking Back at Ourselves: The Problem with the Musical WorkConcept', in The Musical Work: Reality or Invention?, ed. by Michael Talbot (Liverpool University Press, 2000), pp. 128-52

Suárez Cifuentes, Marco Antonio, 'Corps, gestes, perceptions Interrelations, articulations et poétiques de l'espace instrumental, acoustique et électro-acoustique' (Conservatoire National Supérieur de musique et danse de Paris, 2017)

'Syos Saxophone Mouthpieces | What Sound Are You Looking For?', Shape Your Own Sound $<$ https://www.syos.co/en $>$ [accessed 7 August 2019]

Talbot, Michael, 'The Work-Concept and Composer-Centredness', in The Musical Work: Reality or Invention?, ed. by Michael Talbot (Liverpool University Press, 2000), pp. $168-86$

Tenney, James, Meta + Hodos and META Meta + Hodos, ed. by Larry Polansky (Oakland, CA., 1988)

Tenney, James, and Larry Polansky, 'Temporal Gestalt Perception in Music', Journal of Music Theory, 24.2 (1980), 205-41

Truax, Barry, 'Paradigm Shifts and Electroacoustic Music: Some Personal Reflections', Organised Sound, 20.1 (2015), 105-10

Vaggione, Horacio, 'Some Ontological Remarks about Music Composition Processes', Computer Music Journal, 25.1 (2001), 54-61

Vázquez, Hebert, Fundamentos Teóricos de La Música Atonal (Mexico: Universidad Nacional Autonoma de México, Fondo Nacional para la Cultura y las Artes, 2006)

, 'Rizoma y Gestualidad En La Música Atonal', Revista Pauta. Cuadernos de Teoría y Crítica Musical, Vol. XXIII, No. 95 (2005)

Vercoe, Barry, 'The Synthetic Performer in The Context of Live Performance', in ICMC '84 Proceedings, 1984

Wagemans, Johan, James H. Elder, Michael Kubovy, Stephen E. Palmer, Mary A. Peterson, Manish Singh, and others, 'A Century of Gestalt Psychology in Visual Perception: I. Perceptual Grouping and Figure-Ground Organization.', Psychological Bulletin, 138.6 (2012), 1172-1217

Wang, Ge, 'Designing Smule's Ocarina: The IPhone's Magic Flute.', in NIME, 2009, pp. 303-7

$<$ https://www.researchgate.net/profile/Ge_Wang9/publication/253074606_Desig ning_Smule's_Ocarina_The_iPhone's_Magic_Flute/links/00b4952b01c00ecaf900 0000/Designing-Smules-Ocarina-The-iPhones-Magic-Flute.pdf $>$ [accessed 29 June 2017]

Wertheimer, Max, 'Experimentelle Studien über das Sehen von Bewegung.', in Zeitschrift für Psychologie and Physiologie der Sinnesorgane, ed. by S Exner and G E Müller, 1912, pp. 162-265

Wessel, David, and Matthew Wright, 'Problems and Prospects for Intimate Musical Control of Computers', Computer Music Journal, 26.3 (2002), 11-22

Wolfe, J, A.Z. Tarnopolsky, N.H. Fletcher, L.C.L. Hollenberg, and J. Smith, 'Some Effects of the Player's Vocal Tract and Tongue on Wind Instrument Sound', 
Proceedings of the Stockholm Music Acoustics Conference 2003 (SMAC 03), 2003, 4

Woodside, Arch G., Case Study Research: Theory, Methods and Practice (Emerald Publishing Limited, 2010)

Zoran, Amit, 'The 3D Printed Flute: Digital Fabrication and Design of Musical Instruments', Journal of New Music Research, 40.4 (2011), 379-87 


\section{APPENDIX A: PARAMETER FOR 3D MOUTPIECE MODEL}

This appendix lists the parameters used in the Fusion 360 CAD modelling software to create the parametric model of the 3D-model of the HypeSax mouthpiece. These parameters can be used to generate variations of a common saxophone or clarinet mouthpiece of any size or preferences.

\begin{tabular}{|l|l|}
\hline NAME OF & DESCRIPTION \\
PARAMETER & Length of the table \\
\hline 1_table & Maximum diameter of the barrel \\
\hline d_barrel_max & Length of the shank \\
\hline 1_shank & Length of the cork part on the shank (clarinets only) \\
\hline 1_cork_clr_only & Diameter of the shank \\
\hline d_shank_bottom & Diameter of the shank on the barrel side (saxophones only) \\
\hline d_shank_top & Diameter of the shank in the middle (saxophones only) \\
\hline d_shank_mid & Position of middle of shank in reference to the bottom of barrel \\
\hline pos_d_shank_mid & M diameter of the cork part of the shank (clarinets only) \\
\hline d_shank_cork & Width of the table on the tip side \\
\hline w_table_tip & Width of the table on the shank side \\
\hline w_table_shank & Height of the tip opening \\
\hline h_tip & Length of the facing \\
\hline 1_facing & Diameter of the tip (adjust to mouthpiece) \\
\hline distance_to_r_tip & Angle of the table compared the centre line of the mouthpiece \\
\hline p_table & Minimum diameter of barrel \\
\hline d_barrel_min & Diameter of the tip \\
\hline r_tip & $\begin{array}{l}\text { Angle of the beak compared to the bottom of mouthpiece } \\
\text { (where shank begins) }\end{array}$ \\
\hline p_beak_outside & Angle of the beak compared to the table \\
\hline p_beak_inside & $\begin{array}{l}\text { Length of the beak measured along the centre line of the } \\
\text { mouthpiece }\end{array}$ \\
\hline 1_beak & Diameter of the curvature of the beak (difficult to measure) \\
\hline r_beak & Distance to centre of beak radius \\
\hline distance_to_r_beak & Thickness of the beak at the tip \\
\hline t_beak_tip & Width of the window at the tip side \\
\hline w_window_tip & Width of the window at the barrel side \\
\hline w_window_barrel & Diameter of window at barrel \\
\hline r_window_barrel & Distance to the centre from the bottom of window barrel \\
\hline distance_to_r_windo \\
w_barrel & Dength of the window \\
\hline 1_window & Length or depth of the bore \\
\hline d_bore & \\
\hline 1_bore &
\end{tabular}




\begin{tabular}{|l|l|}
\hline p_baffle & $\begin{array}{l}\text { Angle of the baffle compared to the centre line of the } \\
\text { mouthpiece }\end{array}$ \\
\hline 1_baffle & Length of the baffle measure along the roof \\
\hline pos_d_barrel_min & $\begin{array}{l}\text { Position of the minimum diameter of barrel at the top, in } \\
\text { reference to position of d_barrel_max }\end{array}$ \\
\hline pos_d_top_of_beak & $\begin{array}{l}\text { Position of the top of the beak in order to create circles to attach } \\
\text { the barrel shape to }\end{array}$ \\
\hline $\begin{array}{l}\text { d_top_of_beak_for_- } \\
\text { shape }\end{array}$ & Diameter of continuation of the body at top of beak \\
\hline d_mid_point_baffle & Mid-point where baffle knee is found \\
\hline d_chamber_bottom & Point where chamber begins, in reference of top of bore \\
\hline d_chamber_top & Point where chamber ends, in reference of top of bore \\
\hline roudness_top & Roundness of chamber at the roof \\
\hline roudness_bottom & Roundness of chamber at the window \\
\hline chamber_size & Size of chamber \\
\hline knee_of_baffle & Knee of baffle measured in mm in reference to the beak \\
\hline $\begin{array}{l}\text { position_chamber_b } \\
\text { ottom }\end{array}$ & $\begin{array}{l}\text { Position of bottom of chamber in mm in reference to the bottom } \\
\text { of the shank }\end{array}$ \\
\hline $\begin{array}{l}\text { position_chamber_to } \\
\text { p }\end{array}$ & $\begin{array}{l}\text { Position of top of chamber in mm in reference to the bottom of } \\
\text { the shank }\end{array}$ \\
\hline d_shape_of_beak & Diameter of circle that shapes the front of the beak \\
\hline
\end{tabular}




\section{APPENDIX B: GESTALT THEORY - EXPANDED DISCUSSION}

Gestalt psychology was originally developed by researchers such as Max Wertheimer, Kurt Koffka, and Wolfgang Köhler. ${ }^{272}$ Gestalt theory states that in order to understand and relate to the world, our brains tend to simplify a large amount of information that they receive through the means of visual stimuli. This is done in order to accelerate and facilitate our relationship with the world. ${ }^{273}$ Gestalt psychology is founded on the idea that many elements of an image can be grouped together to be conceived as a whole. This way, our brain does not need to analyse every single leaf, branch and trunk to understand this whole as a tree. The brain, in effect, looks for a pattern and mentally 'groups' all of these parts to conceptualise the tree as a unity, without needing to observe every single detail. To describe the way in which we tend to group visual objects, Gestalt psychology describes a set of laws of perceptual organisation: no grouping, proximity, similarity of colour, similarity of size, similarity of orientation, common fate, symmetry (or order), parallelism, continuity, closure, common region, and figure versus ground, amongst others (see Figure 1). ${ }^{274}$

Even though this theory is based on visual aspects, the psychological concept of 'Gestalt' - a German word meaning 'form', 'pattern' or 'configuration'—has its origins in aural phenomena. Christian von Ehrenfels observed in 1890 that our brain has the capacity to recognize a melody despite the fact that it can be presented with different pitches, timbres or speed, as long as the relationship of its elements is maintained. He argued that any of the representations of that melody could be identified as the same due to a unique 'gestalt quality'. ${ }^{275}$ Despite the fact that this organisation of the laws has been criticised and objected to due to the fact that the laws are 'disorganised, [...] [with]

\footnotetext{
${ }^{272}$ Max Wertheimer, 'Experimentelle Studien über das Sehen von Bewegung.', in Zeitschrift für Psychologie and Physiologie der Sinnesorgane, ed. by S Exner and G E Müller, 1912, pp. 162-265.

${ }^{273}$ Wagemans and others, pp. 1176-77.

${ }^{274}$ Wagemans and others, p. 1180.

${ }^{275}$ Wagemans and others, p. 1175.
} 
APPENDIX B: Gestalt theory - Expanded discussion

considerable variation in the names the laws are given, their description, how they are grouped, and how many of them there are', ${ }^{276}$ these laws, nevertheless, have been fundamental in establishing an alternative way of understanding music. James Pomerantz, for instance, invites us to stop giving these concepts the role of 'laws', and instead call them 'principles', as due to the aforementioned inconsistencies they cannot be considered universal, and thus are not candidates to be referred to as laws. For the sake of accuracy, I will describe them as 'principles' from this point on.

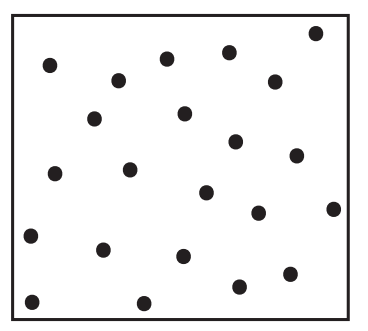

No grouping

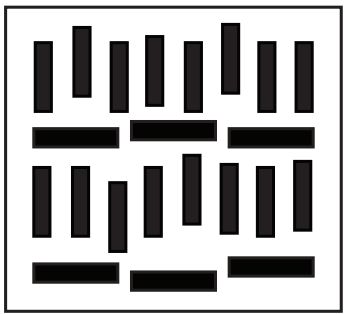

Similarity of orientation

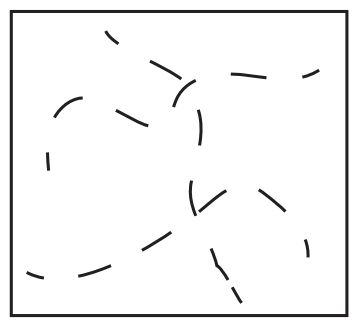

Continuity

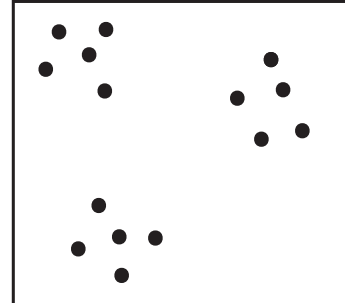

Proximity

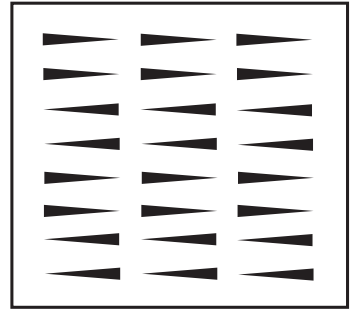

common fate

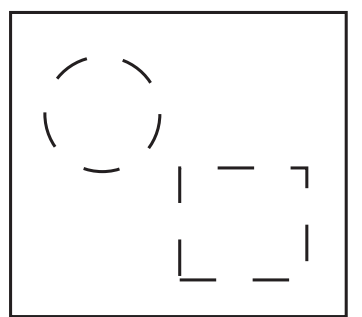

Closure

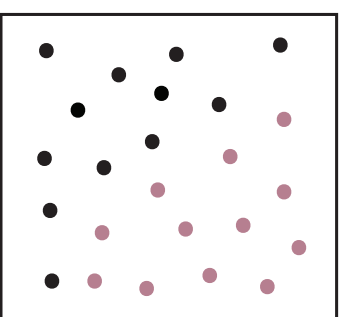

Similarity of colour

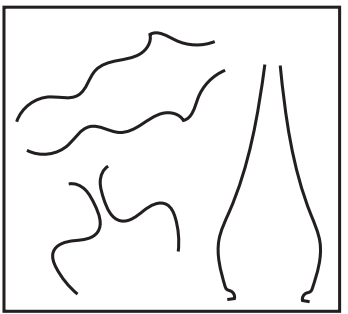

Symmetry

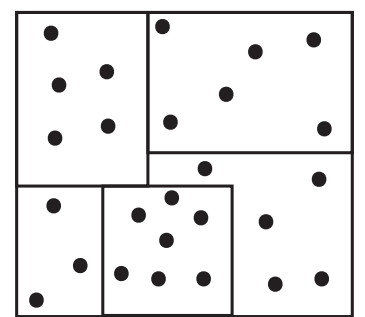

Common region

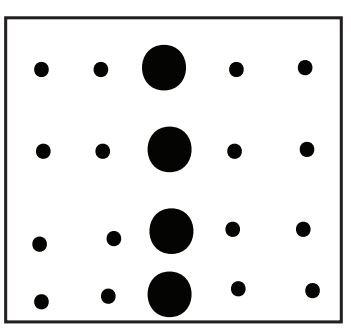

Similarity of size

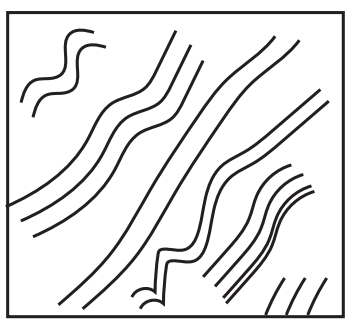

Parallelism

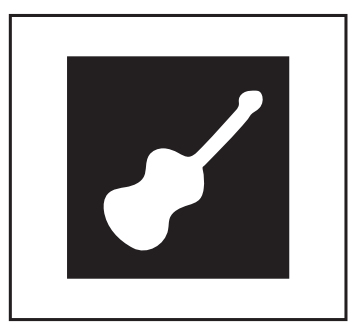

Figure/Ground

Figure 1. Representation of the most basic gestalt psychology laws of perceptual organization

In his 1961 thesis Meta (+) Hodos, ${ }^{277}$ James Tenney brought the gestalt principles to bear on the discipline of musical analysis, as he sought to describe works by Ives, Webern,

276 James R. Pomerantz, 'Visual Form Perception: An Overview', in Pattern Recognition by Humans and Machines: Visual Perception, ed. by Eileen C. Schwab and Howard C. Nusbaum (Elsevier, 2016), pp. 1-30 (p. 6).

277 This thesis was published, along with posterior writings, in 1980 as Meta + Hodos and META Meta + Hodos 
Bartók, Schönberg, Ruggles, and Varèse, with new analytical tools that would be equally effective on works by all of these composers. To facilitate the transition from gestalt psychology to musical analysis, Tenney proposed the use of the terms: 'clang' to identify a sound, sound configuration, or musical idea; 'element' as the constituent parts of the clang; and 'sequence' for structures comprising a chain of clangs recognised as a unified whole. He later adds the terms 'segment', formed by sequences, and finally, 'section', which encompasses sequences. ${ }^{278}$

Tenney adapted some of the principles ${ }^{279}$ of Gestalt theory and proposed the use of proximity, similarity, Intensity, repetition, objective set and subjective set, referring to them as 'factors of cohesion and segregation' 280 for their potential to suggest groupings of elements and/or segregation from elements which do not follow the same principle. In addition, in my 2014 MA thesis, I proposed the addition of a seventh principle to this list, the cloud clang ${ }^{281}$ defined below. These principles can be used as creative or analytical tools, by adapting each one of them to different musical aspects. For instance, using the proximity principle, musical ideas can be organised according to the proximity of temporal events, or proximity of range (see Figure 2).

- The principle of similarity can help create associations by similarity of behaviour; for instance, when a series of accented staccato notes sound during a slurred string orchestra section, one would tend to hear those notes as one single group that stands out and is brought to the foreground of the musical texture. Similarity can also have the potential to give identity to a musical construct; for instance, a passage with string pizzicati followed by an ascending glissando, is imitated by an analogous passage of short staccato notes followed by a rapid ascending scale on a flute. Both musical passages can contain completely different pitches, intervals, timbre, and duration, yet still be associated and recognisable by the principle of similarity.

\footnotetext{
278 Tenney and Polansky.

${ }^{279}$ Tenney refers to these principles as factors of association

280 Tenney.

${ }^{281}$ Ramos, p. 22.
} 

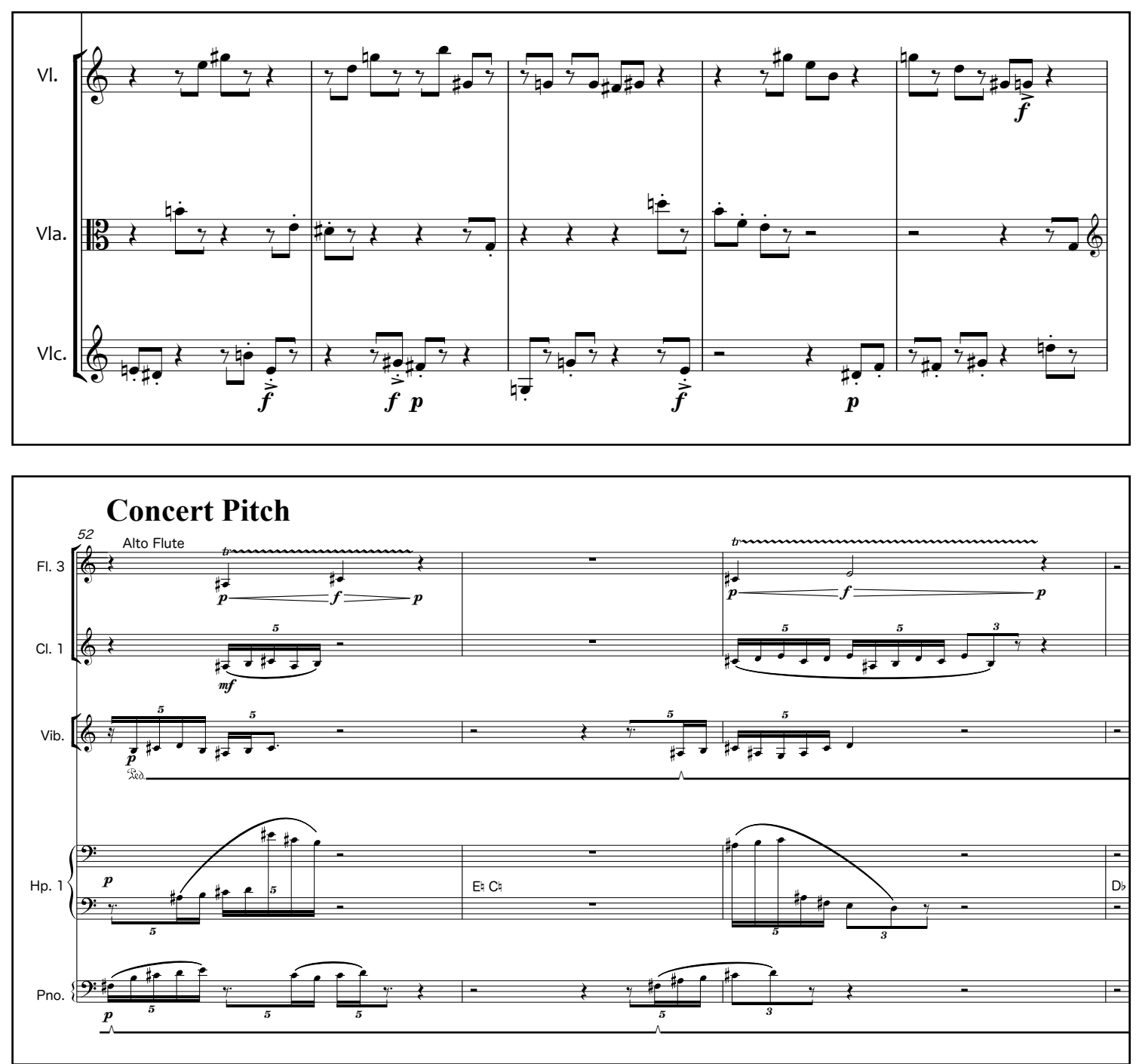

Figure 2. Temporal proximity allows association between strings in bb. 4-8, Cor Ple (top). Proximity of range produces association instruments in musical gesture, b. 52-54, Bereshith (bottom).

- The principle of intensity can be useful in organising rhythm, dynamics, densities, or any other characteristic. Even when this principle is clear enough to suggest how it might work, intensity is possibly the most complex principle to define as it can take many forms. To facilitate the usage of this principle, Tenney theorised the 'parametric profile' which can be expressed as a line in a Cartesian plane, where $\mathrm{X}$ represents time, and Y represents a parameter. In Figure 3, one can see how the same parametric profile can be used to describe the changes in intensity that occurs as a result of chances in dynamics, pitch range and tempo, potentially bringing such events to the foreground of the listener's attention, helping to create a musical discourse. 


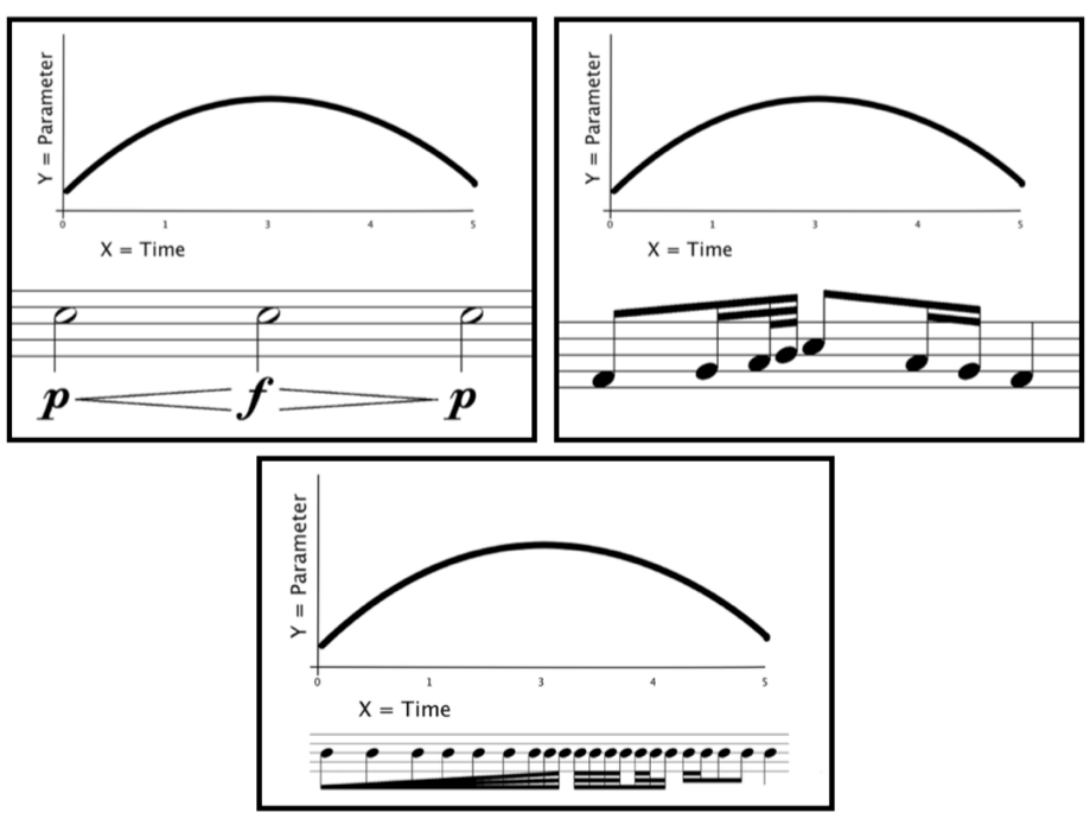

Figure 3. Parametric profile expressing dynamics, pitch and rhythm.

- The principle of repetition requires the identification of a clang (an identifiable musical idea) which then can be repeated to create associations in the memory of the listener.

- The objective set and subjective set refer to previously known musical materials. These two principles depend on the experience, memory and expectations of the listener. They are in essence the same, except that the former makes reference to an element found in the work, while the latter exists when referencing another work. The objective set refers to expectations created by the work. Tenney exemplifies how this principle can be identified in three cases: 1) the hemiola (presented, for instance, by one instrument in a string quartet during a passage otherwise homophonic) which, going against the already established pulse and accentuation, segregates the texture and pulls the attention of the listener to that element; 2) the establishment of musical referents such as a tonal centre; or 3) references to thematic ideas that have been previously presented. On the other hand, the subjective set relies on the listener's previous experiences, for instance when a traditional Cuban rhythmic pattern is used, or in the case of intertextuality quoting musical material from another composition, where, in order to be a factor of cohesion and segregation must be previously known by the listener. 
APPENDIX B: Gestalt theory - Expanded discussion

- Finally, my proposed concept of the cloud clang serves to identify-or manage, if seen from the creative point-of-view - densities produced by multiple elements that, due to their relationship, cannot be perceptually broken down into singular elements. This concept is easily exemplified by the kinds of textures generated by techniques such as Ligeti's 'micropolyphony', in which linearities, root movement or other musical elements cannot be discerned due to the saturation of multiple independent parts in the texture..$^{282}$

These factors or principles of cohesion and segregation have been used to develop strategies that highlight salient features of the HypeSax sound (developed for this research) and its influence in organisational strategies to develop structure and orchestration in works such as Breathe in, breathe out (discussed in section 5.2.2.1)

282 David Cope, Techniques of the Contemporary Composer, Schimer, 1997, p. 103. 


\section{APPENDIX C: USER STUDY}

This appendix lists user study questions and answers. This user study is discussed in Chapter 4. The user study consisted in two sections intended to investigate the response of potential HypeSax users and to collect data that could be used to improve the instrument's design. Section A was designed to be responded by saxophonists. Section B was designed to be responded by composers. In total, twelve saxophonists and five composers took part in this user study, of which two of these participants acted as both saxophonists and composers. This user study was approved by the Victoria University of Wellington Ethics Approval Committee (Reference No. 0000026089).

\section{Section A (for saxophonists)}

Please answer the following questions or circle one of the options.

1. What instrument of the saxophone family do you normally play?

Nine participants responded alto saxophones their main instrument. Two participants usually play soprano saxophone. One participant mainly plays tenor saxophone.

2. Do you play another instrument outside the saxophone family? Please list which instruments you play.

Seven participants play other instruments.

3. Have you ever played an instrument using live electronics?

Half of the participants have performed using live electronics.

4. From 1 (very difficult) to 5 (very easy), how difficult did you find setting up the HypeSax hardware?

Five participants indicated that it was very easy (5) while three participants responded 3 and two participants answered with a neutral response (3).

5. From 1 (very difficult) to 5 (very easy), how difficult did you find setting up the HypeSax software? 
One participant found it very difficult (1), followed by one participant with a neutral response (3), three participants responded 4, and two participants found it very easy (5). The rest of the participants responded to the questionnaire after a software update version made this question irrelevant due to automation.

6. From 1 (very difficult) to 5 (very easy), how difficult did you find performing the preprogramed instrumental techniques?

One participant struggled performing the techniques and responded 2, six participants had a neutral response (3), four participants were able to perform the instrument and execute the preprogramed instrumental techniques (4), and one participant found the task very easy (5)

7. From 1 (not at all) to 5 (very), how responsive and reliable did you find the HypeSax? One participant thought that the HypeSax was very responsive (5), five participants answered with 4 and six participants responded 3.

8. Putting aside the fact that using the HypeSax stops the sound of the lowest note of the saxophone, from 1 (very) to 5 (not at all), how much did the HypeSax hinder performance of the instrument's regular techniques?

Two saxophonists felt very comfortable playing the HypeSax and responded 5, six participants responded 4, three participants answered 3, and one saxophonist believed that the HypeSax really affected his performance and gave the lowest score of the group (2).

9. From 1 (not at all) to 5 (very), how comfortable was it to play the saxophone using the HypeSax?

One participant responded very negatively to the HypeSax (1), while the rest of the participants responded more positively. Three participants gave responded 3, six saxophonists responded 4, and two participants felt very comfortable (5).

10. What (if anything) would you change about the design of the hardware? Cosmetic comments are acceptable but comments on ergonomics or ease of setup are preferred.

- Participant 1. 'It takes too long to set up, a plug-and-play approach would be preferable. Also a snap system for the components would be useful.' 
- Participant 2. 'Touch sensors should be closer to the key buttons'

- Participant 3. 'The mouthpiece is quite resistant. More touch sensor response from the buttons on keys would be useful, it was hard to know if gentle touch triggered things or not'

- Participant 4. 'Depending on the saxophone, it is possible that changing the placement of the right hand buttons could help - I was almost already pressing it in a normal playing position. This design did make it easier to access however. I would require a significantly modified mouthpiece - I couldn't push par enough on the neck.'

- Participant 5. 'The thumb buttons are a bit far from where the thumbs rest, specifically the ergonomics of the right thumb should be corrected.'

- Participant 6. 'I found it difficult to reach some of the hypesax keys. The thumb rest in particular got in the way and hindered playability. This mostly due to the size of my hand and the fact that I play soprano.'

The rest of the participants did not respond to this question.

11. From 1 (not interested) to 5 (very interested), how interested would you be in performing a composition using the hypersaxophone?

Three people were very interested in using the HypeSax (5), six participants expressed being interested in keep using the instrument (4), two more were neutral (3) and one person was not very enthusiastic about using the HypeSax (2).

12. From 1 (not at all) to 5 (very), how easy to understand and useful did you find the musical notation found on the "Test Sheet"?

Two participants found the notation clear and easy to understand (5), three responded 4, five more were neutral (3) and two of the participants found didn't find the notation useful (2).

13. Do you have any suggestions about how to improve the suggested notation? Please attach your suggestions on a new page.

Participant 1: 'Maybe consider a smaller 'cue' type stave for the approximate note od harmonic [feedback] - could also be labelled' (see Fig. 37). 
Participant 2: 'This notation is helpful but it would be better to combine it with tablature style indications. It's difficult to know how to play what the score suggests, maybe after a few days playing it would be easier. A tablature would save time.'

Participant 3: 'For effects such as the feedback it would be better to just draw lines and let the performer figure out ways to play the necessary notes'.

Participant 4: 'I recommend using text when possible. For instance 'bell up' instead of a line. It can be confusing to know if I'm supposed to rotate the saxophone to one side or the other, lift it or whatever else if lines are used for everything, even if the lines look different.'

There were no additional suggestions to improve the notation.

14. Please add any additional comments below.

Participant 1. 'I would like it if it had presets useful for pop music.'

Participant 2. 'Program more variety of effects on the sensor which could add harmonic or rhythmic accompaniment, such as a drum kit.'

Participant 3. 'A ready-to-use user interface would be great, plug-and-play!'

Participant 4. 'I found it cumbersome to play, in part because I hadn't played the alto saxophone in the past 10 years. The set-up was very resistance and my stubby fingers had a hard time ergonomically. I found I was battling the horn and my focus was on that, which was distracting from testing.'

There were no additional comments.

\section{Section B (for composers)}

Please answer the following questions or circle one of the options.

1. Do you play the saxophone?

Only two of the participants play the saxophone.

2. Please list what instruments you play.

Two participants play saxophone and clarinet. The other three participants play the piano. 
3. Do you perform, or have you ever composed using computers/live electronics?

All participants have performed and composed using live electronics.

4. If you used a different software from the one loaded onboard or the provided computer software, from 1 (not at all) to 5 (very) how difficult was it to make the HypeSax communicate with the chosen software?

Due to a software update this question became irrelevant before the participants responded, as the updated version of the software included guided assistance. None of the participants responded to this question.

5. Did you require additional help using the HypeSax together with another software?

Due to the updated version, this question also became irrelevant.

6. From 1 (not at all) to 5 (very) how difficult was it to integrate the new instrumental techniques to your composition?

Two of the participants found it easy to use the instrumental techniques and answer with a 2. The other three participants were neutral, placing their response in the middle of the scale (3).

7. From 1 (not at all) to 5 (very), how likely is it that you will want to write another composition using the HypeSax or a similar development?

The responses were, in general, positive with two participants rating their likeliness a 5, two more a 4, and one participant rated it a 3 .

8. What other instrumental techniques or sound processes would you like to achieve using the HypeSax in potential future compositions?

Participant 1. 'It would love it if it had pre-set effects such as guitar pedals do, easy to tun on and off'.

Participant 2. 'It would be great if it was possible to manipulate pre-recorded audio while performing. For instance transposing or reversing a sample according to the performance'.

Participant 3. 'It should be used to make a lot of messy noise with it'. 
Participant 4. 'I think you can install more mics and use it to perform micro sounds. I can imagine how it would sound and I think it would give more possibilities to the saxophone. You could use it as a percussive instrument or just to make really great noise with the breath sound and key clicks'.

Participant 5. 'I see a lot of potential in this. I would spend hours using it with [super]collider, it just It needs to be louder. Just concentrate in making it have a big sound and let the composers come up with their own sounds. Awesome job'.

9. From 1 (not at all) to 5 (very), how easy to understand and useful did you find the musical notation found on the "Test Sheet"?

Two participants were neutral about this question, their answer was 3. The other two participants, who are saxophonists and responded Section A, were neutral (3).

10. Do you have any suggestions about how to improve the suggested notation? Please attach your suggestions on a new page.

11. Please add any additional comments below.

Participant 1. You should make something for another instrument and have them play together controlling each other.

Participant 2. Since you're already tracking the movements of the performer and the sound, is there a way that can be stored and used to play another instrument remotely? Maybe you could have another hypersax be played remotely, or have a saxophonist record his performance and then play a 'solo duo'.

There were no more comments.

12. Do you agree to share your composition fully or partially as an example in a thesis chapter or published journal/article?

Two of the participants agreed to share their musical ideas. These were discussed in section 5.2.4. 


\section{APPENDIX D: TEST SHEET}




\section{Test sheet}

\section{Polyphony}

Alto Sax

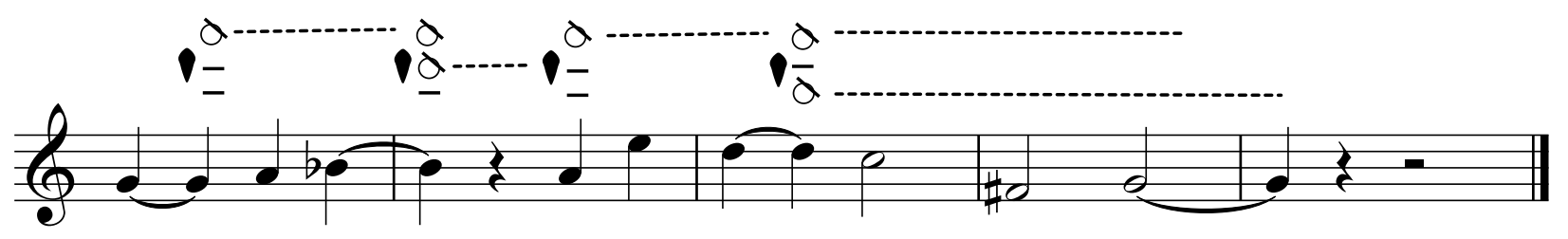

\section{Slaptongue/Ricochet}

play a slap tongue, then press thumb button 1 , and then press thumb button 3 play this sequece as fast as possible

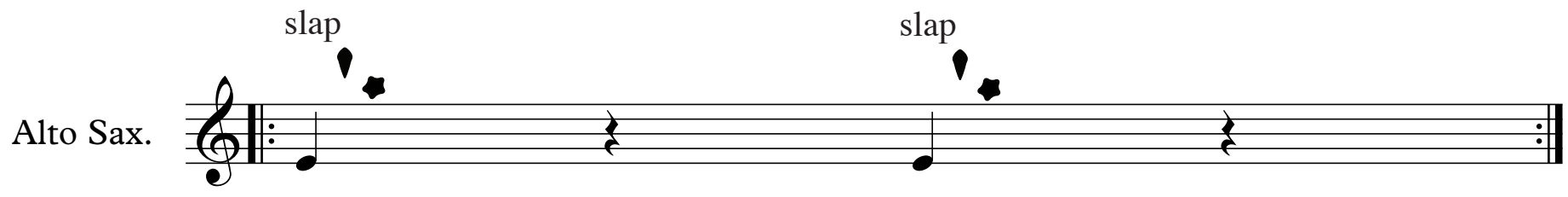

\section{Inharmonicity}

use touch buttons freely to add overtones the timbre of the saxophone

Alto Sax.

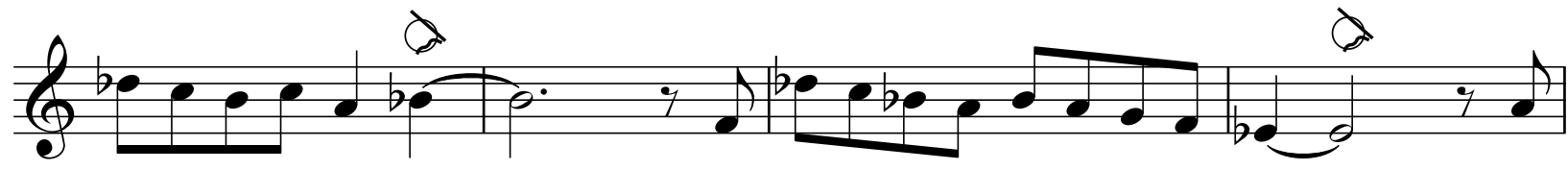

Alto Sax.

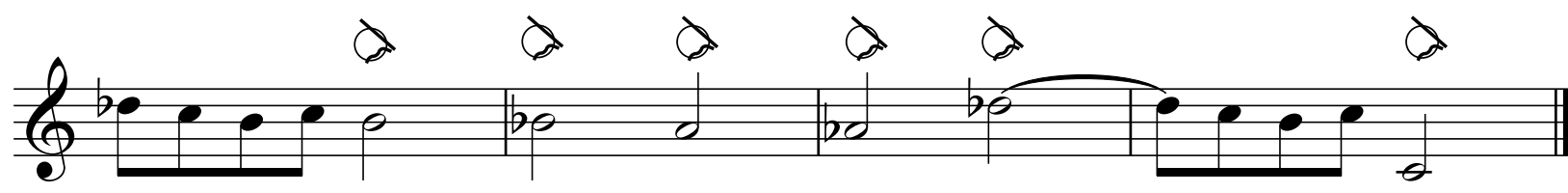


Feedback polyphony
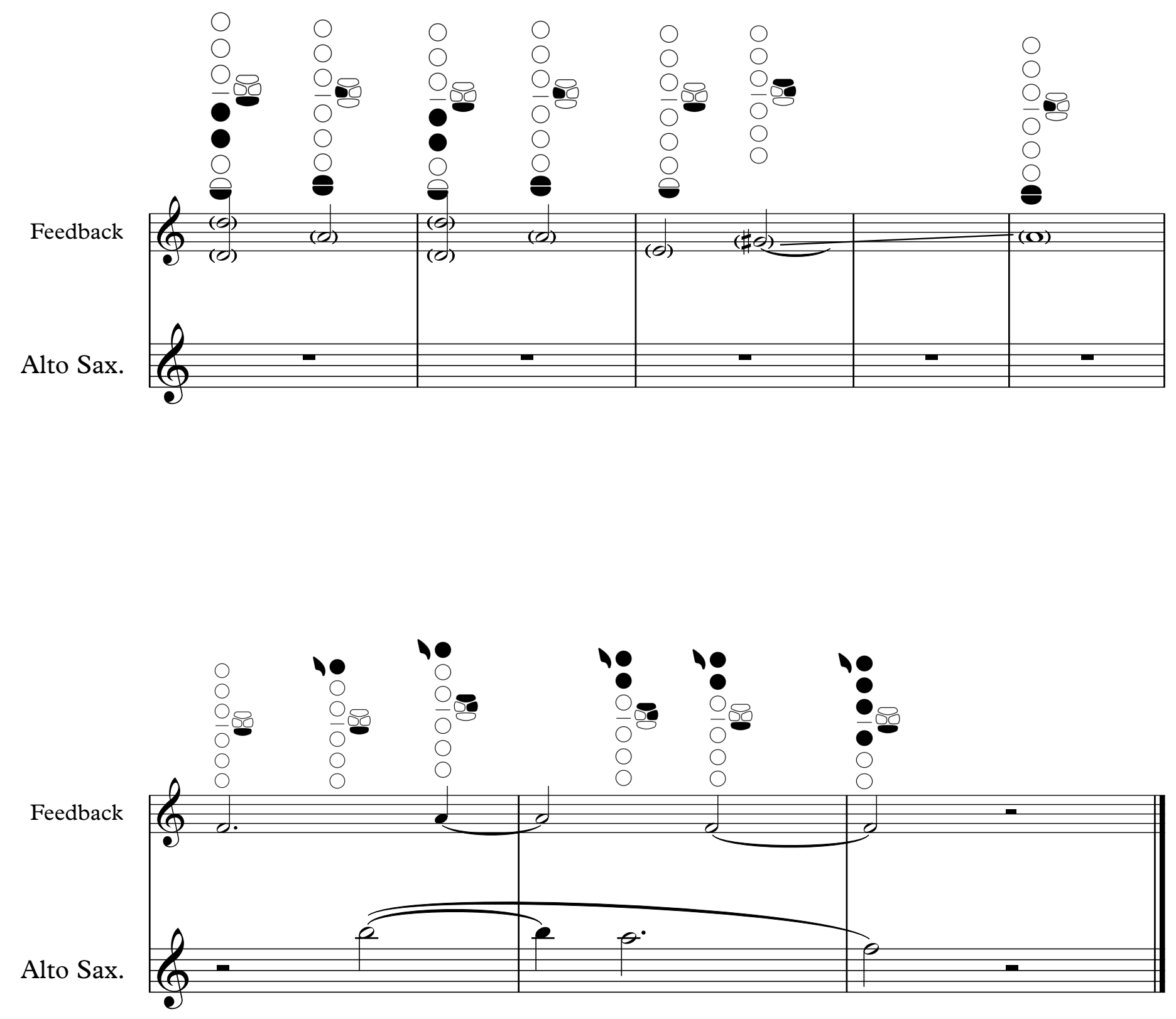

Overpressure

play any note and press thumg button 2, then change the air pressure 


\section{Mouthpiece Test}

$$
d=60
$$

$$
1
$$

2

3

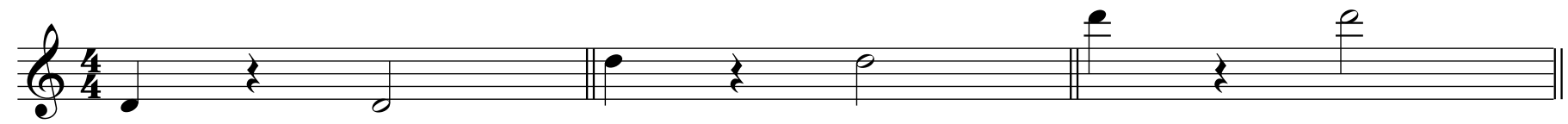

4

5

6

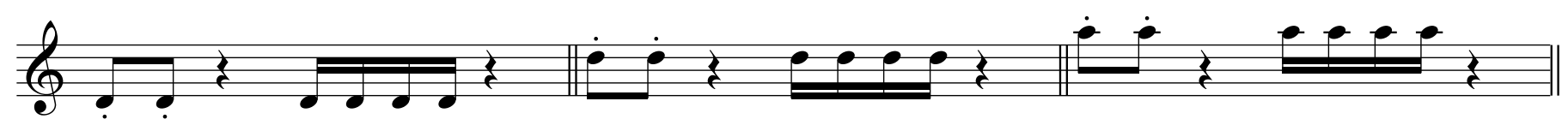
7
8
9
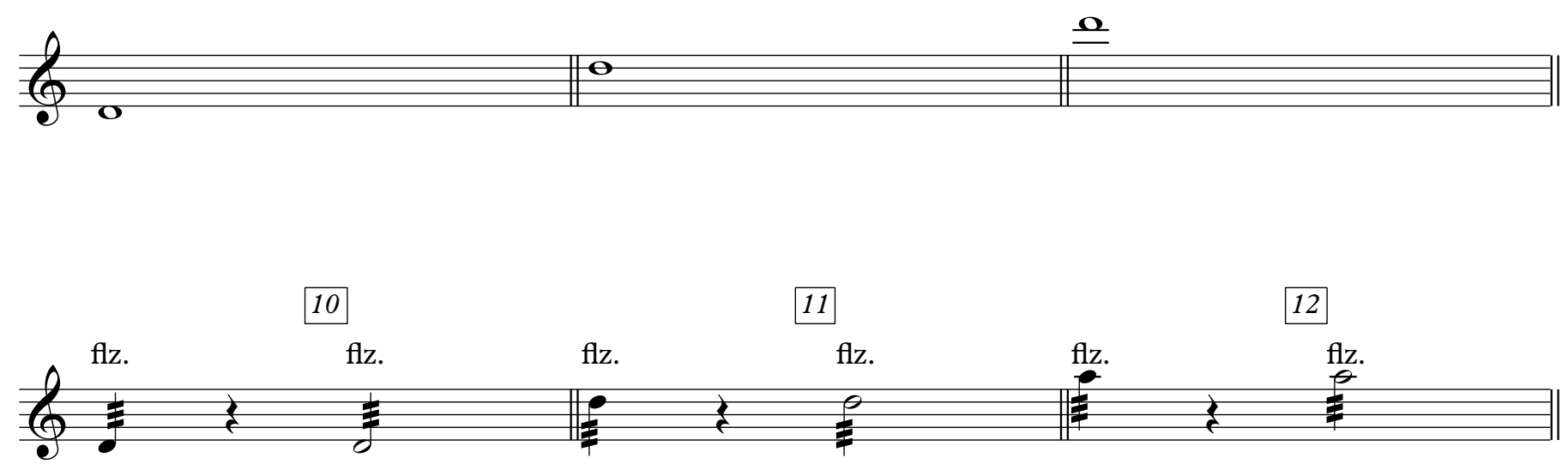

13

14

15

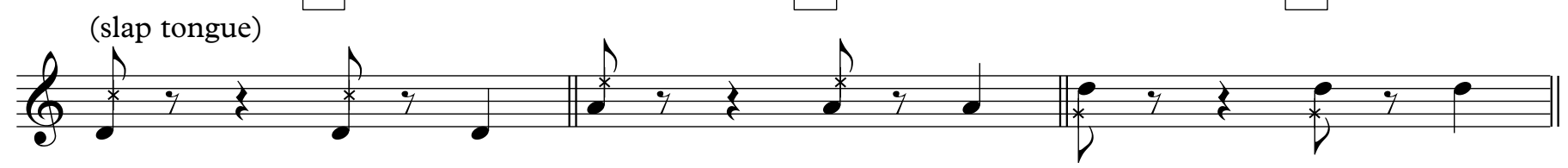

16

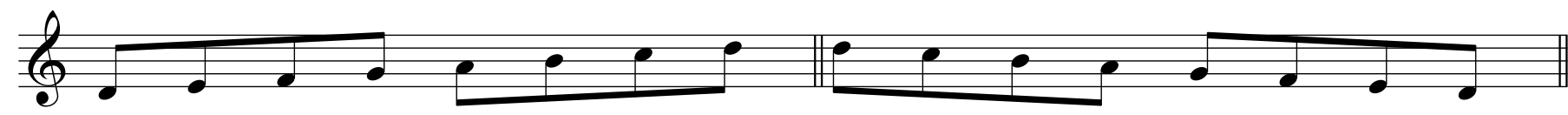




\section{APPENDIX D: 'A 3D PRINTED HYBRID}

SAXOPHONE MOUTHPIECE FOR DATA COLLECTION' 


\section{A 3D PRINTED HYBRID SAXOPHONE MOUTHPIECE FOR DATA COLLECTION}

\author{
Cristohper Ramos Flores \\ New Zealand School of Music \\ Victoria University of \\ Wellington
}

\author{
Jim Murphy \\ New Zealand School of Music \\ Victoria University of \\ Wellington
}

\author{
Michael Norris \\ New Zealand School of Music \\ Victoria University of \\ Wellington
}

\begin{abstract}
In the past few decades there have been explorations on hybridization of acoustic and electronic instruments in order to achieve new musical possibilities. These kind of hybrid instruments, also known as hyperinstruments, rely on the use of new technologies, sensors, cameras, microcontrollers, and other devices, as well as computers and software, to collect and process data to generate new audio. This paper documents the authors' own exploration on hyperinstruments and the search to access new instrumental techniques for the saxophone, and offers a detailed description of the design of a $3 \mathrm{D}$ printed saxophone mouthpiece which combines acoustic and electronic characteristics to collect data from the performance, aiming to control live electronics. The mouthpiece's performance is characterised and the potential to use the collected data for pedagogical purposes is also considered.
\end{abstract}

\section{INTRODUCTION}

In recent years, 3D printing technology has allowed new developments in design throughout a wide spectrum of fields. In the field of music and acoustics, this new technology has also opened new possibilities allowing for accessories, instruments, resonators, replacement parts, protective cases and other related items to be developed. Perhaps the importance of $3 \mathrm{D}$ printing technologies for musical research lies on the fact that these new printers can produce prototypes and final products with a relative ease of construction, unlike working in the old-fashioned way by handcrafting the instruments or parts out of natural materials (typically wood). It is well known that luthiers, woodwind and brass instruments makers, as well as other professionals in similar fields, learn their craft over many years, and a musician or researcher is usually not skilled to build musical instruments, repair them, build new parts or developments, therefore slowing down any projects in this field. Not only are the new 3D printers allowing more professionals to access easy and fast prototypes, but their low price has opened the doors for the DIY amateur enthusiast.

There are documented projects of printed models that have furthered the developments of musical instruments and acoustic investigation. The authors catalogue these $3 \mathrm{D}$ printed models in six categories: protective accessories (shells, cases, pads, caps, etc), repairments (knobs, string pegs, bridges and other small part replacements), enhancements (pickup holders, shoulder rests, inserts, etc.), replicas, customizations and innovative designs. Many of these models, mostly from DIY projects, are shared as creative commons in specialized webpages such as thingiverse.com, yeggi.com, grabcad.com and many others. Other scholarly projects focus on researching musical and acoustic issues rather than practical ones, such as producing microtonal flutes (Dabin et al. 2016) or a multi-sized shank in a trumpet mouthpiece (Leitman and Granzow 2017) for acoustic inquiry.

This current project takes advantage of the $3 \mathrm{D}$ printing technologies with a model that fits in the category of customizations, aiming to collect data on the air pressure blown into the saxophone. This mouthpiece, along with other developments, will be used in the creation of a hypersaxophone with the goal of allowing saxophonists to access new instrumental techniques.

This paper examines previous developments by other authors, and the influence those projects had on the development of the mouthpiece. Detailed information on the design and tests is also discussed to offer an overview of the project and its potential, and possible future uses of the mouthpiece.

\section{RELATED WORK}

In recent decades, new enhancements have been added to musical instruments, generating two new categories of musical instruments: hybrid instruments and hyperinstruments. Hybrid instruments are those instruments that combine different materials in their construction, characteristics of two or more instruments, or different ways of producing sound (e.g. electroacoustic instruments). On the other hand, in hyperinstruments the audio output is processed by a logical system loaded in a computer or microprocessor. There have been many examples in which hyperinstruments or hybrid instruments have been developed by means of combining traditional acoustic instruments and new technological developments such as sensors, microprocessors and computers.

In previous works, researchers have demonstrated that it is possible to collect data on different aspects of instrumental performance through the use carefully designed systems. As Matthew Burtner described it, these 
systems "convert the performance data into a continuous data stream" (Burtner 2002) which could be used to control digital audio processing, to study the performative practices, to understand the acoustic phenomena or to gather information useful for pedagogical purposes. Despite the fact that there have been a good number of projects dealing with woodwind or brass instruments, very few of these designs deal with the issue of air flow through the body of the instruments and how to quantify the energy carried by the air.

Gathering data from air flow can be very difficult as disturbing the air column inside a musical instrument results in undesired affectations to the overall acoustics of the instrument. In consequence, a design where all the data collection happens away from the air column running inside the instrument is the only feasible option. This, however, raises different problems on different aerophones as the way the air is blown into the instrument varies. In instruments with a blowhole, such as flutes, the air is blown across a sharp edge of the blowhole. This system allows for external data collection of the airflow. In whistles, single reed instruments, double reed instruments, brass instruments (cup mouthpiece) and pipes, acquiring this information can be difficult as the air travels directly from the mouth to the inside of the instrument. ${ }^{1}$

In research undertaken at McGill University, it was proven how difficult but useful it can be to gather air flow information and reuse it as a musical control variable. In this 2005 project, air pressure sensors are mounted around the mouthpiece gather the data. However, this system can be unreliable since the flute performative practices sometimes require a change of the angle in which the air is blown into the instrument, resulting in variation of the air pressure blown into the sensor (Da Silva, Wanderley, and Scavone 2005).

Other projects aiming to measure the energy of the air stream utilize a microphone which gathers information on the quality and dynamics of noise produced by air to obtain comprehensible data of the energy carried by the air stream. The use of microphones to obtain information on the air blown has been very successful in projects such as Smule, an iOS app which has been successfully used by thousands of users around the globe (Wang 2009).

Projects such as the SABRe (Schiesser and Schacher 2012) deal with problems related to reed instruments. In this case, it becomes impossible to use a microphone or an air pressure sensor inside the body of the instrument or at the end of it since foreign objects inside the instrument would disturb the air column, and placing the data collector at the end of the instrument does not yield accurate results since the air stream's energy is lost through the various holes of the instruments depending on the fingerings. In this particular project, its authors developed a system in which a small hose mounted on top of the instrument redirects a portion of the air stream into an air pressure sensor, obtaining accurate readings in every situation. In this case the sensor, being mounted on top of the mouthpiece, is close enough to the air stream source (player's mouth) to ensure that no energy (pressure) is lost before the sensor reading is done.

Another way to achieve accurate acquisition of data was explored in a joint effort between Universitat Pompeu Fabra and Standford University, where it was demonstrated that modifications to the mouthpiece of an alto recorder to redirect a portion of the air flow into an air pressure sensor was possible (Garcia et al. 2011) by creating conduits in the body of the mouthpiece to redirect a portion of the air stream into a sensor.

Based on these previous developments the authors have modified a 3D model of an alto saxophone mouthpiece (shared on thingiverse.com) to produce a new version that features modifications that allow a portion the air stream to be redirected into an air pressure sensor, thus obtaining accurate readings without disturbing the air column inside the instrument at the same time as it provides a comfortable playing experience.

\section{SYSTEM OVERVIEW}

The hybrid saxophone mouthpiece has been designed with the purpose of offering a tool that can be used both to perform and to collect data about the performance. The collected data can eventually be used in different situations which may include information gathering for performance study or more practical goals such as controlling diverse parameters inside a software capable of communicating via serial or MIDI protocols.

\subsection{D Design}

The first step in developing the mouthpiece was considering the fact that introducing an object inside a saxophone causes disturbance in the air column, therefore changing the quality of the sound, affecting the intonation, producing "squeaks", or completely stopping the sound. Based on past projects, listed above, the authors decided to work with a $3 \mathrm{D}$ design that would feature an alternate conduit in the body of the mouthpiece that would permit data collection without affecting the air stream flowing through the mouthpiece and final air column inside the saxophone.

The mouthpiece is an adaptation of the Alto Saxophone Mouthpiece designed by Thingiverse user Allanarps (Allanrps 2011). The original design is based upon a Yamaha beginner mouthpiece, with similarities including bore length and shape, facing width, tip opening and basic length measurements, featuring a flat baffle and squared throat (bore).

The adaptation keeps the basic shape of the interior with the same dimensions in the chamber of the mouthpiece, facing width and bore shape, which ensures that the air column running through the chamber behaves in the same manner as in the original design. The changes made to the design include a shorter tip opening of $1.8 \mathrm{~mm}$

\footnotetext{
${ }^{1}$ For a more detailed classification of aerophones: (von von Hornbostel, Sachs, and Montagu 2011)
} 
(original is $2 \mathrm{~mm}$ ) and a thicker beak that allocates a small conduit running through the body of the mouthpiece to an added section in the back in which a sensor is mounted to measure the air pressure. See Figure 1 for a comparison between the original design by Allanarps and the adaptations made to it.
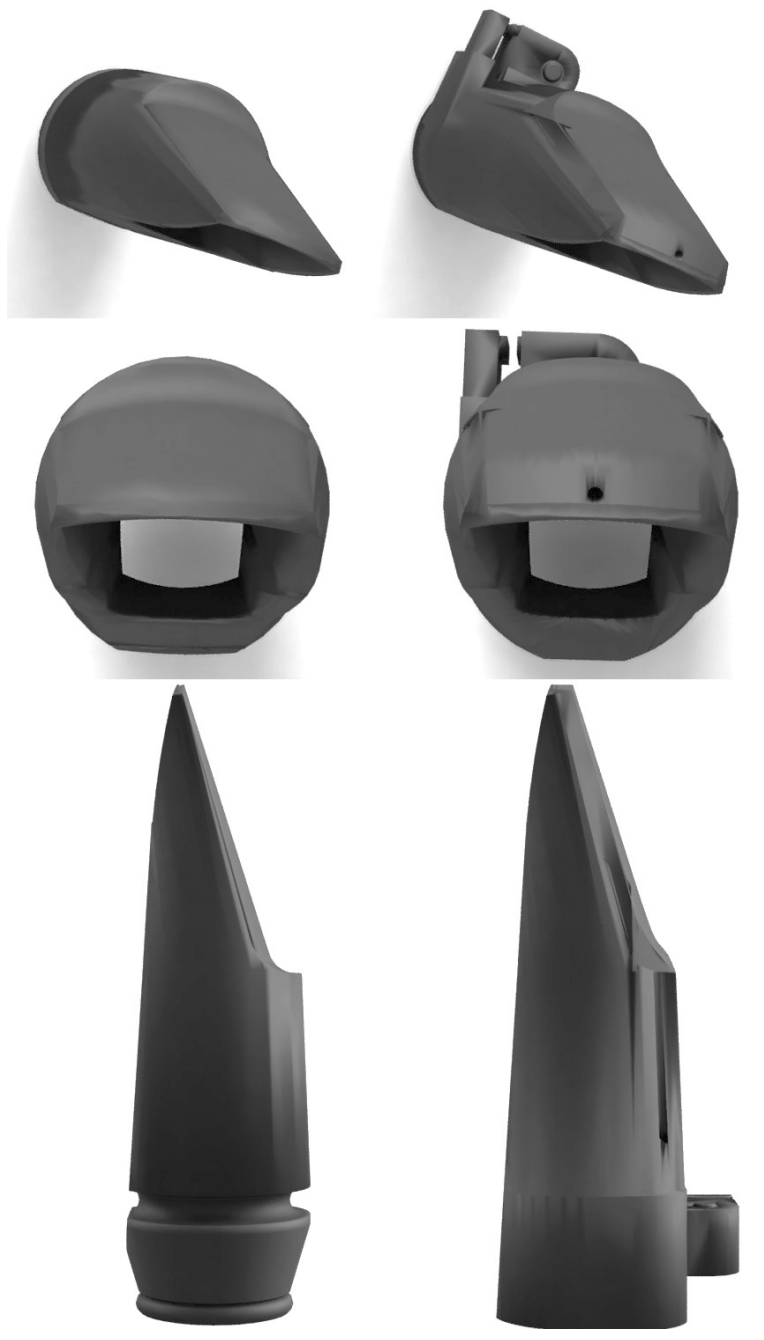

Figure 1. Comparison between the original 3D model (left) and the new version (right) with adaptations to allocate a pressure sensor and an alternate air conduit.

As mentioned before, the conduit inside the body was designed to allow comfortable playing with no disturbance to the air stream and air column, and to achieve this goal a minimal amount of material was added to the top of the beak of the mouthpiece to accommodate the conduit. In total, $2 \mathrm{~mm}$ of thickness was added to the beak, of which a $1 \mathrm{~mm}$ radius cylindrical opening is found at the front/center of the beak, where the structure is weaker because of its dimensions. Further back, the conduit opens more to fit the dimensions of the sensor at its back end (see Figure 2). There is also a small side canal that allows moisture and saliva to escape the system. This opening can be blocked or opened if desired with wax or tape. Further testing will determine whether this side opening is necessary.

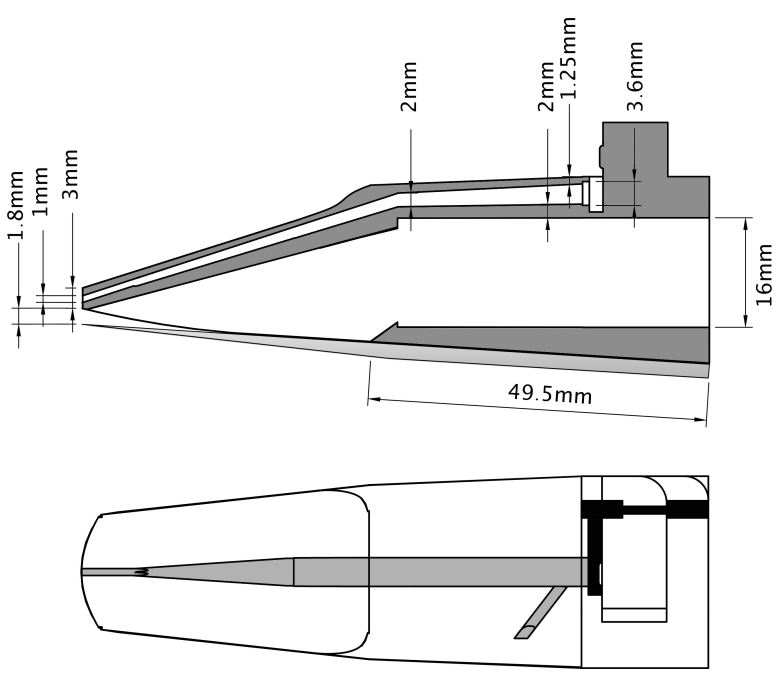

Figure 2. Interior of the mouthpiece. The top image shows a slice of the mouthpiece where the chamber and conduit are shown in white. The bottom image shows the conduit and side canal in gray. The area designed to position the sensor breakout is colored in black.

\subsection{Components}

The Hybrid Saxophone consists of two main components: the 3D printed mouthpiece and an electronic barometer sensor BMP180.

The 3D model, previously described, can be printed in a variety of materials, including organic-based (wood) and other plastic and polymer based materials, many of them resembling ebonite, a common material used in the fabrication of mouthpieces. For the first printing trials, an Up Mini filament deposition modelling 3D printer was used. For the printing material, ABS (acrylonitrile butadiene styrene) has been used due to its durability, ease of use and price.

To measure the air pressure, various kinds of sensors were considered. Unlike previous projects, this one has the advantage of 3D design customization which allows the opportunity to fit any reasonably sized sensor. The chosen sensor is a BMP180 designed by BOSCH as a barometric pressure sensor commonly used in applications such as indoor navigation, GPS-enhancement for dead-reckoning, slope detection, etc., sport devices, (e.g. altitude profile), weather forecast, vertical velocity indication (rise/sink speed) and others ("BMP180" 2017). Considering the applications for which the BMP180 was designed, this is not the most obvious choice of sensor, however, due to the flexibility of design it was easy to fit this sensor in the mouthpiece and match it with the end of the alternate air conduit.

The BMP180's most relevant features are: $300-1100$ $\mathrm{hPa}$ pressure range, an average current consumption of 
$650 \mu \mathrm{A}$ that can go down to $3 \mu \mathrm{A}$, supply voltage VDD of $1.8 \mathrm{~V}$ to $3.6 \mathrm{~V}$, a relative accuracy pressure of $\pm 0.12 \mathrm{hPa}$, and the use of the $\mathrm{I}^{2} \mathrm{C}$ protocol at a $3.4 \mathrm{MHz}$ transfer rate (BOSCH 2015).

Unlike most sensors designed to measure air pressure, the BMP180 doesn't feature a funnel-like structure to redirect the air into it, which becomes unnecessary with the conduit built into the mouthpiece 3D model (see Figure 3). The BMP180 can be purchased on its own or mounted on different breakout boards. For this project, the GY68 breakout has been chosen because on this design the "hole" that captures the air in the sensor is situated close to one of its edges, which makes it convenient to introduce it in the body of the mouthpiece and reach the end of the conduit. Other breakout boards place the sensor on the center of the $\mathrm{PCB}$, and the use of these boards would translate in carving into the model to reach the air conduit, this could compromise the structure of the mouthpiece.
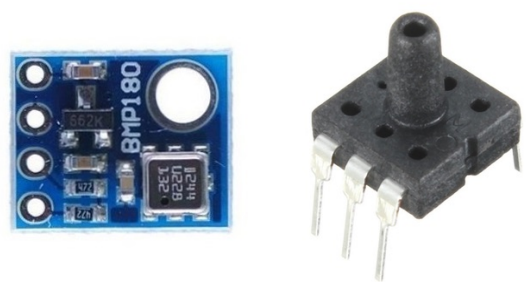

Figure 3. BMP180 Breakout GY68 next to a common air pressure sensor with a funnel-like casing.

The breakout stays in place thanks to a small protuberance on the mouthpiece which matches the screw hole in the breakout. Also, the breakout pins help secure the sensor in place once they are connected to standard $2.54 \mathrm{~mm}$ connectors. In Figure 4, the sensor is mounted on the printed model, showing the way it "locks" in place. The GY68 breakout design is perfect for handling and replacing the sensor if necessary as it only needs to be pushed into the opening in the mouthpiece designed to allocate it. Therefore, any user can replace it without the assistance of a technician and at a very low cost.

\footnotetext{
${ }^{1}$ For more information on the Teensy LC, visit the developer's website at https://www.pjrc.com/teensy/teensyLC.html
}

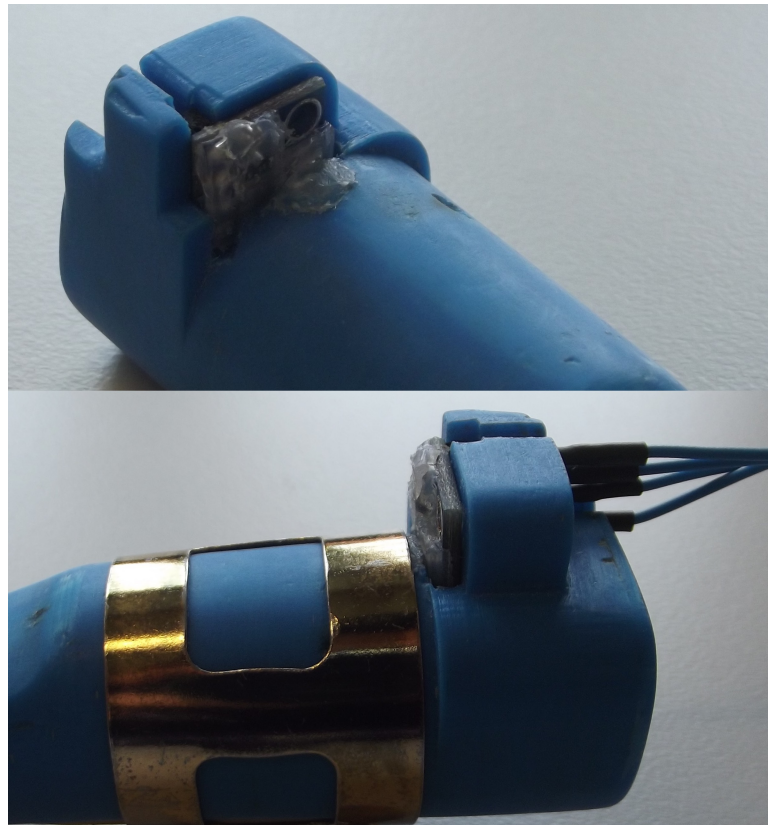

Figure 4. BMP180 Breakout GY68 secured on the mouthpiece. The visible silicone on the breakout protects its components from moisture without affecting the sensor response.

\subsection{Board and On-board software}

At this stage of the project, the barometric sensor is working together with a Teensy LC board ${ }^{1}$, sending data using the $\mathrm{I}^{2} \mathrm{C}$ protocol. This board is also capable of communicating using the MIDI protocol without having to implement complex code or hardware development, which opens up the possibility of utilizing the system as a MIDI controller.

The program loaded on the board executes a selfcalibration when the system is powered. To calibrate, it firstly determines the ambient temperature, and then sets a current pressure (actual atmospheric pressure) as a baseline value, setting that value to 0 . Once these parameters have been established, every iteration of the program loop compares the new results to the baseline value, outputting positive numbers for any value greater than the baseline, and negative numbers to values under the atmospheric pressure. The values are expressed in hectopascals.

The sensor is affected by temperature in a manner similar to woodwind instruments. To address this, the program performs a new calibration every five minutes to compensate for temperature changes that occur due to breathing and blowing into the mouthpiece. The sample rate is at 33 readings per second, but can be changed according to the needs of the project. A faster sample rate could be beneficial to detect attacks more precisely and avoid latency in musical contexts. Another fact to be considered when deciding the sample rate is the delay 
that occurs between the moment when the performer starts blowing and the sonic event, which depends on dynamics, expressivity, the hardness of the reed, the calibration of the instrument's mechanics (to avoid air leaks) and other factors. Further investigation must be done to address this issue.

\section{TESTS AND RESULTS}

As mentioned before, the goal of designing this mouthpiece is to be used as a key element of a hypersaxophone. For this reason, the performance of the mouthpiece has been tested by using it in common musical practices. In order to test the system, a Max7 patch was designed (see Figure 5). This program receives the incoming data using the USB port. The program updates the data 33 times per second in synchronization to the Teensy's firmware. Then, the data is used to create a graphic profile of the measurements in a Cartesian system representing time (in seconds) and pressure (in hectopascals). The object used in Max7 to create the visuals representing the air pressure is [plot ] set to draw a profile with 33 points per second (indicated by the markers on the $\mathrm{X}$ axes) thus creating a representation without smoothing the collected data. The range in both the $\mathrm{X}$ and $\mathrm{Y}$ axes can be resized to draw a profile that meets the needs of the tests.

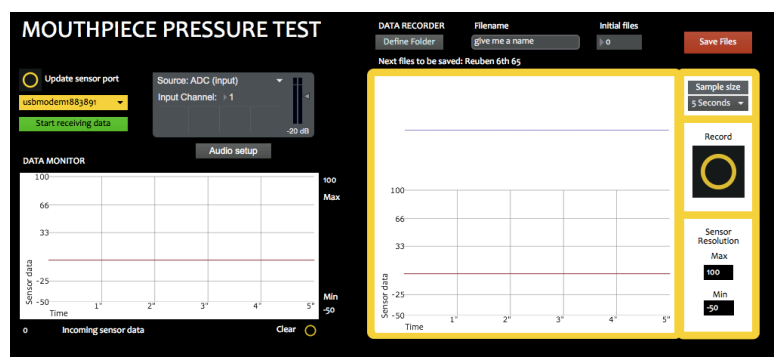

Figure 5. Max patch designed to read incoming data.

The characterization program also features a representation of the audio input to compare the morphology of sound (attack, decay, sustain and release) with the pressure readings. It has a monitoring function as well as a recording function which allows users to save an audio file and a screenshot of the soundwave and pressure profile representations for further analysis.

In an experiment made with the help of saxophonists Reuben Chin and Jasmine Lovell-Smith, the system was tested at the Lilburn Studios at Victoria University of Wellington. The setup consisted of the mouthpiece/sensor mounted on the alto saxophone, along with a Zoom H2 microphone, both connected via USB to a Macbook Pro running Max7 on OSX 10.11. The results demonstrated the efficacy of the system using a sensor that is sensitive enough to capture minimal changes in pressure such those produced by the performer's breathing patterns. In Figure 6 one can see the rapid response of the system and the high pressure produced by preforming the slap tongue technique and a regular note in forte dynamic.

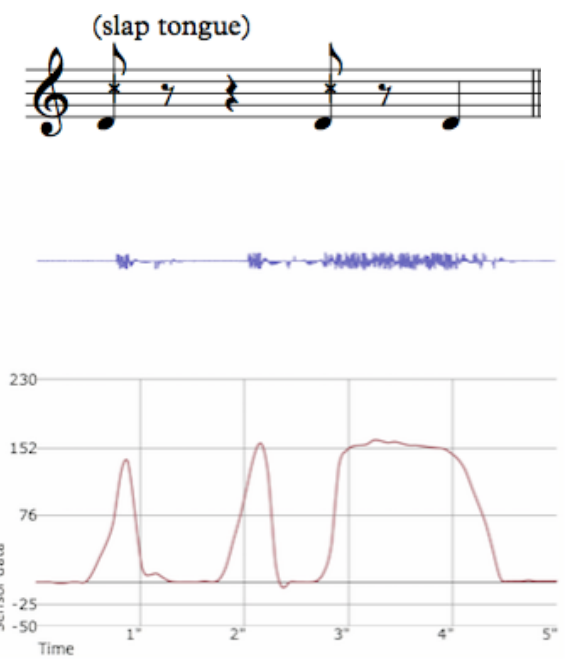

Figure 6. Slap tongue test.

In Figure 7 one can see the pressure change needed to accurately tune notes as the performer ascends or descends within an octave while playing a diatonic scale.
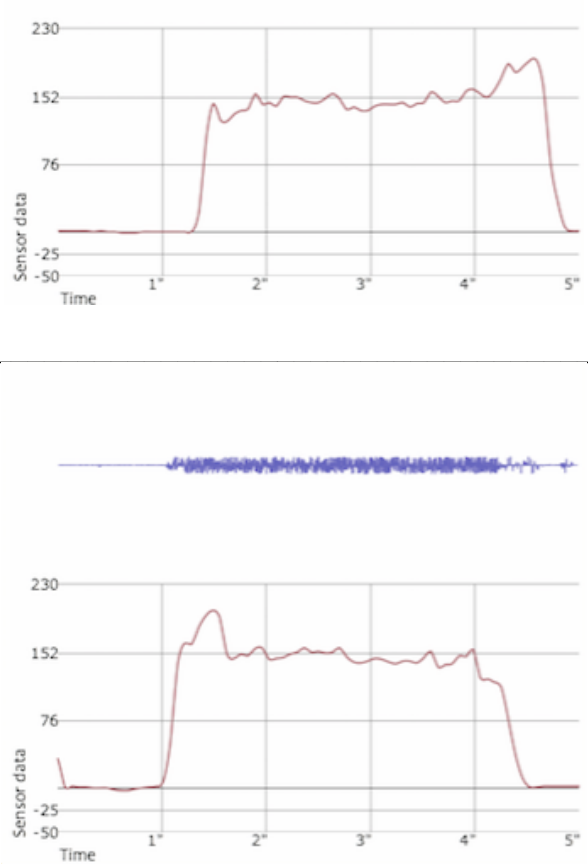

Figure 7. Ascending scale (top) and descending scale (bottom) between B4 and B5. 
The system is also capable of demonstrating performance errors to be corrected, which holds promise for future pedagogical contexts. In Figure 8 the graphic shows a well-executed flutter tonguing technique and a failed execution of the same exercise. This example shows how a greater air pressure does not permit the correct execution of this technique.

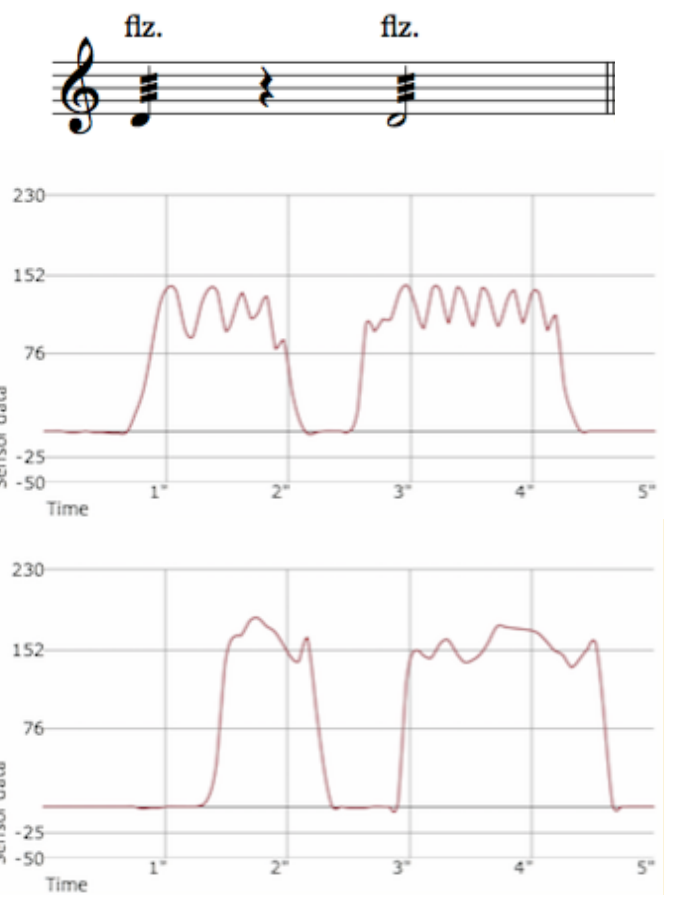

Figure 8. Fluttertonguing exercise (top) and the corresponding sensor reading of good (middle) and poor (bottom) execution of it.

The timbre of the saxophone doesn't seem to be greatly affected when the $3 \mathrm{D}$ printed mouthpiece is used. There are changes in the overall spectral content, however this differences in timbre are common even between two mouthpieces of the same brand and model.

The 3D printed model was compared to a Yamaha beginner mouthpiece (in which the $3 \mathrm{D}$ model is based). The results show variations of the energy on some of the overtones. Despite these differences, the overall overtone structure of the timbre is constant. In the lower register the differences are minimal, resulting in an almost imperceptible change of timbre. In the higher register the 3D printed model tends to present less energy on the overtones, which results in a less bright sound, but still consistent, stable and controllable. These differences are visually represented in Figure 9 and Figure 10, where a comparison of spectrum profiles is presented. These profiles where obtained from recordings of a Conn $6 \mathrm{M}$ (Naked lady) alto saxophone using the 3D printed model and the Yamaha mouthpiece playing a $\mathrm{D}_{b 3}$ (Figure 9) and $\mathrm{D}_{b_{5}}$ (Figure 10).
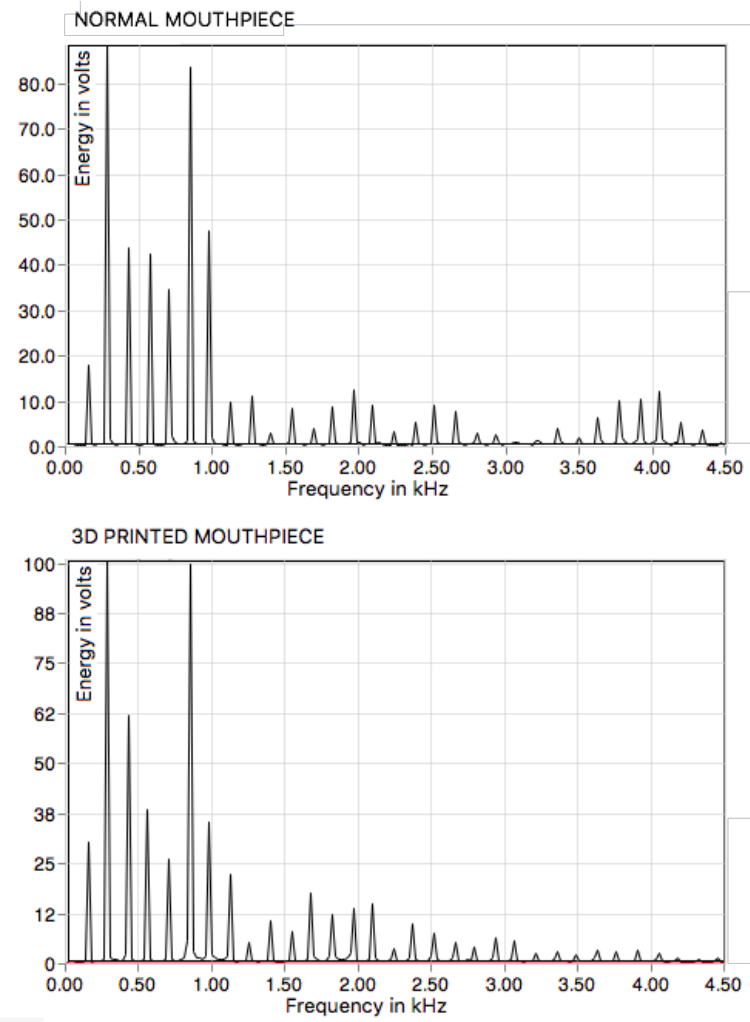

Figure 9. Overtones found in beginner Yamaha and 3D printed mouthpieces playing $\mathrm{D}_{b_{3}}$
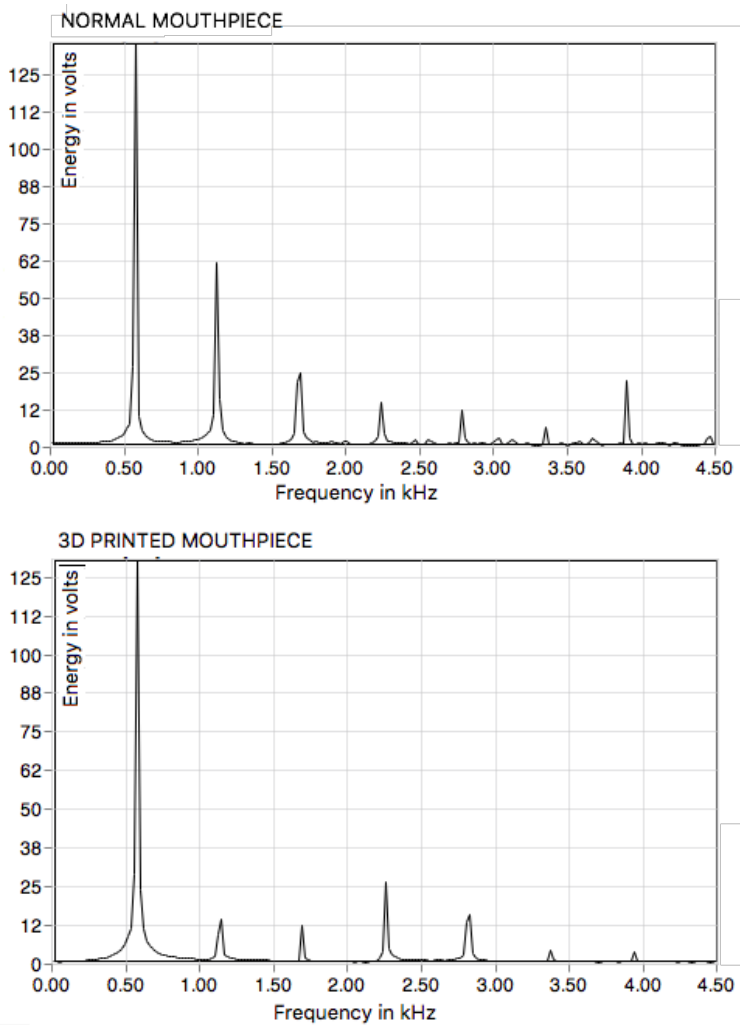

Figure 10. Overtones found in beginner Yamaha and 3D printed mouthpieces playing $\mathrm{D}_{b 5}$ 


\section{EVALUATION AND LIMITATIONS}

Up to this point, the mouthpiece design together with the barometric sensor has been successful. Nevertheless, the project has faced some problems such as poor 3D printing and sensor failure due to moisture.

Achieving a good printing quality can be difficult and it does not only rely on the printer: other factors such as material and, most importantly, 3D model design matter. The resolution at which some $3 \mathrm{D}$ printers can print can make it very difficult to print fine details such as the $1 \mathrm{~mm}$ radius opening of the alternate air conduit. On the first printing attempt, this section of the conduit was completely blocked. To fix this problem it was necessary to do a manual perforation. The texture achieved by the printer was somewhat rough. The major problem with this texture is the fact that it could produce air leaks in between the mouthpiece and the lips of the performer, which not only can be uncomfortable for the instrumentalists but could also make the sound production more difficult. The 3D printed model texture was removed by sanding carefully and adding a coat of bee wax. The wax not only helped smoothing the surface but also gave the mouthpiece a more organic feeling, but most importantly helps reduce any disturbance of the air flow in the interior of the mouthpiece. This coating will eventually wear off, but the mouthpiece can easily be re-coated if necessary.

The preliminary results show a consistency of playing throughout the range of the instrument with a relative ease of sound control. Even though the beak is slightly thicker than the beak on a conventional mouthpiece, according to the 3 performers surveyed, it does not seem to be uncomfortable or to pose any issues for an experienced performer. More tests are needed to assess the repercussions that using this mouthpiece has on tone quality and harmonic content. Further printing attempts will consider these results, as well as the possible differences caused by printing the model with different materials and printing techniques.

The construction of the barometric sensor permits its use despite the presence of moisture. However, the breakout GY68 fails as soon as moisture is in contact with its components as the design does not feature a protective casing. The first tests proved that, after a few minutes of playing, moisture from breathing and saliva make the system fail. To correct this issue, silicone was applied over the entire surface of the breakout and edges of the sensor, leaving the front face of the sensor uncovered to allow the air to reach the internal components of the sensor. Three possible improvements will be explored in the future. The first one will be to attempt a more precise design in which the back opening of the conduit fits perfectly with the sensor face such that no air, moisture or saliva can escape to the breakout components. This solution would be ideal because it would make replacement of the sensor an easy task of pulling out the breakout and pushing in a new one, but it can be very difficult as it depends on the quality of the printing and each new model would be slightly different. A second solution is to use a 3D printer capable of mixing two materials in a model and redesign the model in a way that the back opening of the conduit features a silicone material that can press against the sensor in such way that it prevents moisture ingress. The third solution, and possibly the easiest to achieve, is to also print a silicone casing for the breakout which could be placed on the breakout before it is set into the mouthpiece; if the sensor failed at some point it would be easily replaceable by a new sensor utilizing the same silicone casing.

\section{CONCLUSIONS AND FUTURE WORK}

The project has been successful so far, achieving accuracy greater than anticipated. Although it may need to be further developed, the mouthpiece is at a stage where it can be used to perform and to offer information about the performance. The system has the potential to add a new feature to control live electronics: it can not only detect the air blowing into the mouthpiece, but also is capable of detecting air suction (represented as negative values under the baseline value set on calibration).

This mouthpiece can be used in various applications. The main purpose in the development of this mouthpiece is to use it as a tool to gather data that will be used in performance practices as part of the development of a hypersaxophone. More mouthpieces based on the same idea and design will be produced to fit different instruments of the saxophone family. Changes in the design will explore the tone quality production by changing the characteristics of the baffle, bore, chamber, etc. The data collected from the pressure sensor will be used in conjunction with other sensors to control live electronics and to alter the acoustics of the instrument itself.

A new software to gather information from the data will be created to make the study of performance more effective, so that the project can be used for pedagogical purposes. It will also feature a reed with an integrated sensor to further investigate the relationship between the tongue and air pressure, and the effects that controlling them has on performance.

The use of 3D printed tools combined with the new generation of highly accurate sensors holds promise for further developments, considering the wide variety of materials that can be used to print the models. The ease of manipulation of these models offers a fast and accurate way of prototyping, developing and creating final products that will enhance our interaction with musical instruments, allowing performers and composers to expand and realize their creative ideas beyond the current limits of instrumental techniques.

\section{REFERENCES}

Allanrps. 2011. “Alto Saxophone Mouthpiece by Allanrps Thingiverse.” December 10.

https://www.thingiverse.com/thing:14495. 
"BMP180." 2017. Accessed July 4.

https://www.boschsensortec.com/bst/products/all_products/ bmp180.

BOSCH. 2015. "BMP180 Digital Pressure Sensor Data Sheet."

https://aebst.resource.bosch.com/media/_tech/media/datash eets/BST-BMP180-DS000-121.pdf.

Burtner, Matthew. 2002. "Noisegate 67 for Metasaxophone: Composition and Performance Considerations of a New Computer Music Controller." In Proceedings of the 2002 Conference on New Interfaces for Musical Expression, 1-6. National University of Singapore.

Da Silva, Andrey R., Marcelo M. Wanderley, and Gary Scavone. 2005. "On the Use of Flute Air Jet as a Musical Control Variable." In Proceedings of the 2005 Conference on New Interfaces for Musical Expression, Vancouver, BC, Canada, 2005, pp. 105-108.

M. Dabin, T. Narushima, S. Beirne, C. Ritz, and K. Grady. 2016. "3D modelling and printing of microtonal flutes," in Proceedings of the international conference on new interfaces for musical expression, Brisbane, Australia, 2016, pp. 286-290.

Garcia, Francisco, Leny Vinceslas, Josep Tubau, and Esteban Maestre. 2011. "Acquisition and Study of Blowing Pressure Profiles in Recorder Playing." in Proceedings of the international conference on new interfaces for musical expression, Oslo, Norway, 2011, pp. 124-127.

Hornbostel, E.M. von von, C. Sachs, and J. Montagu. 2011. Revision of the Hornbostel-Sachs Classification of Musical Instruments by the MIMO Consortium. Verlag nicht ermittelbar.

Leitman, Sasha, and John Granzow. 2017. "Music maker: $3 \mathrm{~d}$ printing and acoustics curriculum," in Proceedings of the international conference on new interfaces for musical expression, Brisbane, Australia, 2016, pp. 118-121.

Schiesser, Sébastien, and Jan C. Schacher. 2012. "Sabre: the augmented bass clarinet," in Proceedings of the international conference on new interfaces for musical expression, Ann Arbor, Michigan, 2012.

Wang, Ge. 2009. "Designing Smule's ocarina : the iPhone's magic flute," in Proceedings of the international conference on new interfaces for musical expression, Pittsburgh, PA, United States, 2009, pp. 303-307. 


\section{APPENDIX E: 'HYPESAX. SAXOPHONE ACOUSTIC AUGMENTATION'}




\section{HypeSax: Saxophone acoustic augmentation}

\author{
Cristohper Ramos Flores \\ Victoria University of Wellington \\ NZSM, Room 202, 92 Fairlie Terrace \\ Wellington, New Zealand \\ cris.ramosflores@wuv.ac.nz
}

\author{
Jim Murphy \\ Victoria University of Wellington \\ NZSM, Room 105, 92 Fairlie Terrace \\ Wellington, New Zealand \\ jim.murphy@vuw.ac.nz
}

\author{
Michael Norris \\ Victoria University of Wellington \\ NZSM, Room 107, 90 Fairlie Terrace \\ Wellington, New Zealand \\ michael.norris@vuw.ac.nz
}

\begin{abstract}
New interfaces allow performers to access new possibilities for musical expression. Even though interfaces are often designed to be adaptable to different software, most of them rely on external speakers or similar transducers. This often results in disembodiment and acoustic disengagement from the interface; and, in the case of augmented instruments, from the instruments themselves. This paper describes a project in which a hybrid system allows an acoustic integration between the sound of acoustic saxophone and electronics.
\end{abstract}

\section{Author Keywords}

Hyperinstrument, Saxophone, Sensors, Gesture, Embodiment.

\section{CCS Concepts}

- Hardware $\rightarrow$ Sensor devices and platforms; Humancentered computing $\rightarrow$ Gestural input; $\bullet$ Applied computing $\rightarrow$ Sound and music computing

\section{INTRODUCTION}

Performing with a musical instrument traditionally implies a strong physical connection between performative actions and sound through the interaction of two bodies: the body of the performer (possibly using an extension, e.g. mallet) and the body of the instrument. The action of strumming activates the vibration of the strings, blowing air into a flute at the correct angle produces a column of air that resonates in the body of the instrument, and hitting a drum also has a sounding result. All of these are examples of physical actions that connect the performer and the resulting sound, regardless of the quality of the sound or whether it is organized in any way. Regardless of the spectral structure (pitched or unpitched), the resulting sound corresponds to the action in a natural way: large/energetic movements result in loud sound while minimal/delicate movements produce an almost imperceptible sound.

Today the one-gesture-to-one-acoustic-event [22] paradigm has become blurred with the introduction of electronic systems and signal processing. In the case of augmented instruments, there are still actions that trigger an acoustic event, however these actions don't necessarily correspond gesturally with the output sound: pressing one button or activating a sensor can result in many different outcomes.

Another problem that arises with hyperinstruments is the disembodiment of the audio source, as the sound usually comes out of a speaker that is normally not part of the instrument, hence disengaging the acoustics from their body.

The HypeSax project addresses these issues with the goal of developing a hypersaxophone that reembodies electronics as part of the physicality of the instrument. There are many other issues that could be identified in the use of new technologies for musical creation in live contexts, especially from the point of view of the audience's perception. However, this paper will center on the design features that allow electronics to be inserted in the acoustic body of the saxophone.

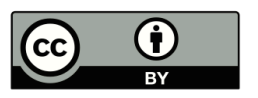

Licensed under a Creative Commons Attribution 4.0 International License (CC BY 4.0). Copyright remains with the author(s).

NIME'19, June 3-6, 2019, Federal University of Rio Grande do Sul, Porto Alegre, Brazil.

\section{PRIOR ART}

Many previous projects have served as inspirations for the design of the HypeSax. The precedents to this work have explored and developed technical accomplishments that have served as models for the design of the HypeSax. Projects such as the SABRe introduce a fully functional air pressure retrieval system [17] using a pipe that redirects air into a sensor, while García et al acquired blowing pressure profiles on a recorder by modifying the mouthpiece's body [5]. Various projects take advantage of push buttons and touch sensors as triggers, as well as gyroscopes and accelerometers [11]. Relatively few projects have focused specifically on saxophone augmentation. However, projects such as those directed by Burtner [2], Schiesser [19], Hong [7] [6], Portovedo [15], and Onozawa's work for Yamaha [14] [13] have served as inspiration in designing the HypeSax. Nevertheless, very few of those projects seek to achieve sound hybridization. The exception is Burtner's Metasax, which deals with feedback control using the mechanics and resonances of the saxophone body.

When it comes to augmenting the sound field of the instruments through combining instrumental acoustic sound and electronically generated sound, interesting research has been conducted at IRCAM, such as the IMAREV project [23] and Smart Intruments [24], Juan Arroyo's hybrid string instruments [8] and the Active Instruments [25] [10]. These projects approach instrumental augmentation as a fully integrated acoustic/electronic hybridization. Inspired by all of these projects, the HypeSax has the goal of achieving hybridization with a standalone system.

\section{SYSTEM OVERVIEW}

The HypeSax is a system designed to be an attachment that can be fitted on any ordinary alto saxophone. The system is modular and can work with some or all of its components attached to the saxophone. It consists of an Un-mute (3.1), Sensor-link (3.2), a customized mouthpiece (3.3), and keycaps with touch sensors and buttons (3.4) (see Figure 1). The system is capable of retrieving data using six touch sensors placed on the saxophone to work as additional keys, three push buttons used normally as triggers or switches, a special mouthpiece that captures air pressure data, gyroscope and accelerometer, a microphone to capture and process audio and a self-contained audio system (soundcard and speaker). The way in which all of these components work within the system is described in detail in the following sections.

\subsection{Un-mute}

Augmented instruments often work with a hemispherical speaker located near the performer, following Cook and Trueman's approach to "reorient the relationship between performer, sound source and listener" in electronic music performance [3]. However, a goal of this project is the integration of the electronics with the acoustics of the instrument. In order to achieve this, the HypeSax includes a speaker inside the saxophone to integrate the acoustic chamber of the instrument into the system. A 3D model of an enclosure was designed to 


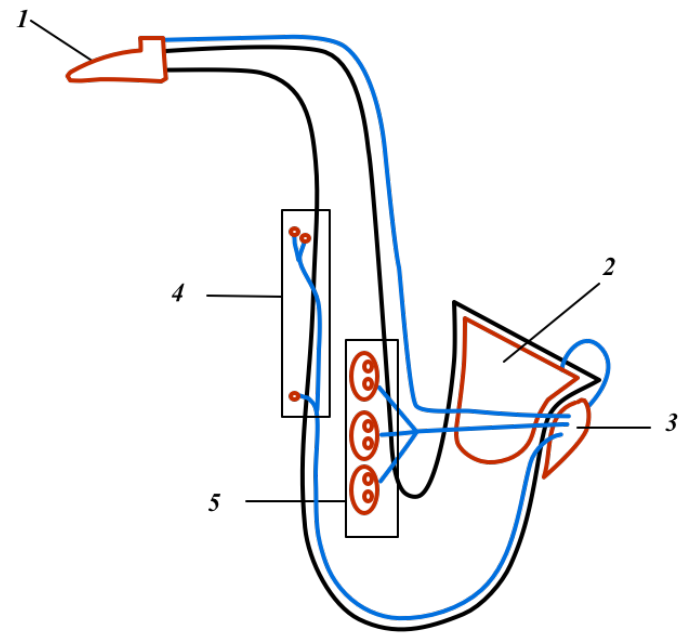

Figure 1. Modular components of the HypeSax. In red: mouthpiece (1), Un-mute (2), Sensor-link (3), new keys for thumbs (4) and new keys situated over custom-designed key caps (5). In blue: representation of the connecting wires.

hold the speaker and electronic components inside the bell of the saxophone, making the Un-mute the heart of the HypeSax. This mute-like enclosure and its components is called Un-mute. It is a device that, rather than muting the sound of the instrument, enriches the spectra by incorporating new components into the final sound.

The enclosure was designed to avoid negative effects on the air column inside the saxophone. A dip was included at the top of the Un-mute to allow air flow while playing in the low register of the instrument with most of the keys closed (see Figure 2). This design is somewhat effective, but it affects the air flow while playing the lowest note of the saxophone. This is due to the fact that the Un-mute is long enough to reach and partially block the opening of the low B b key when inserted in the bell, making it difficult to impossible to play the lowest note.

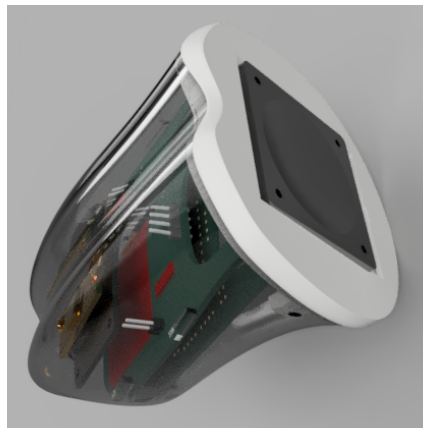

Figure 2. Un-mute for Alto Saxophone.

In the back of the enclosure there is an opening that allows for the free flow of air pressure produced by the back of the cone of the speaker, which is facing the front of the enclosure and out of the bell of the saxophone. The back opening is partially covered to stop moisture from entering the enclosure, potentially damaging the electronic components. Also, in the back, an electret microphone is positioned to capture audio. The close placement of the microphone and the speaker (about $7 \mathrm{~cm}$ away from each other) is one of the reasons why the speaker faces forward and not directly into the opening of the saxophone. However, the back opening is big enough for the speaker's sound to travel back into the instrument in order to introduce new audio to the body of the saxophone. In the same way, the introduced audio is affected (filtered) by the resonance of the saxophone's acoustic chamber, allowing acoustical integration between acoustic sound and synthesis. Undoubtedly feedback can be present if there is a free signal flow and the levels are not calibrated. Nevertheless, feedback can become an interesting feature of the Un-mute which is discussed in section 4.1.3.

Without the Un-mute, the HypeSax cannot work, as it contains inside the most important electronic components of the HypeSax. The complete list of components includes two Teensy boards, an audio board adaptor for teensy, a mono 2.4W Class D audio amplifier PAM8302A, a 40Ohm 5W Speaker, an electret microphone, a capacitive touch sensor board MPR121, an accelerometer and gyroscope board GY-521 MPU6050, and two custom designed PCBs.

The system works with one Teensy LC board (slave) and a Teensy 3.2 board (master). The Teensy 3.2 is connected to the audio board adaptor. Its main function is to retrieve audio using an electret microphone, send the audio to a laptop via USB, and to receive audio signal that is outputted using the speaker mounted on the Un-mute. Currently the audio signal is being processed externally, but a goal is to develop the appropriate code to handle audio analysis and synthesis on board in future iterations. For this reason, a second microcontroller is used to collect data from the multiple sensors mounted on the saxophone. This configuration helps to minimize latency levels.

The slave microcontroller sends data to the master via serial communication. In order to achieve this, as well as to provide the appropriate connections for sensors and visual feedback (LEDs), two PCBs have been designed. This design fits inside the Unmute and features a port which allows connection to the Sensorlink which is located outside the bell of the saxophone.

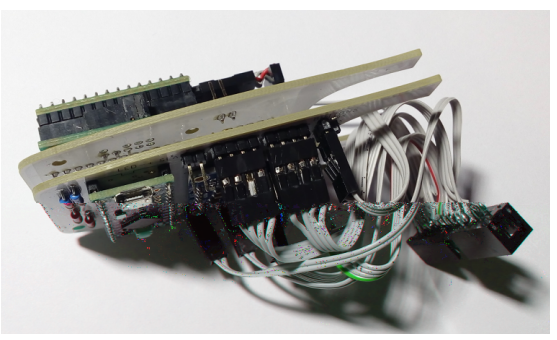

Figure 3. Un-mute's electronic components mounted on custom PCBs.

The HypeSax features only 6 touch keys (see section 3.4), but an Adafruit 12-key capacitive touch sensor board MPR121 is mounted on the PCB. The initial concept featured ten touch keys, but it became very difficult to incorporate all keys to the HypeSax for alto saxophone due to the limited space between the instrument's keys. However, even when only six keys are used, a 12-key breakout board was used in order to facilitate sharing code with future iterations of the HypeSax for bigger saxophones (tenor or baritone) which might feature more keys.

An accelerometer and gyroscope board GY-521 MPU6050 is also located inside the Un-mute, as the saxophone bell is possibly one of the best locations for it since it is right at the center of the instrument. This location helps with keeping track of any movement in $\mathrm{x}$, $\mathrm{y}$ or $\mathrm{z}$ planes more accurately.

\subsection{Sensor-link}

As described in the previous section, the Un-mute holds the slave microcontroller that retrieves data from a variety of sensors. Some of these sensors are located in the mouthpiece, on saxophone keys and near the thumb rests. In order to connect those sensors to the slave microcontroller, the Sensor-link was developed, a custom PCB that features sensor inputs, a type B USB port, LED indicators for visual feedback, a gain knob and a standard $6.3 \mathrm{~mm}$ jack to connect an expression pedal for gain control. The Sensor-link is the connector route where signal and data flows between the HypeSax and computer.

The implementation of the Sensor-link is arguably unnecessary, as the connecting ports could be located directly on the front face of the 


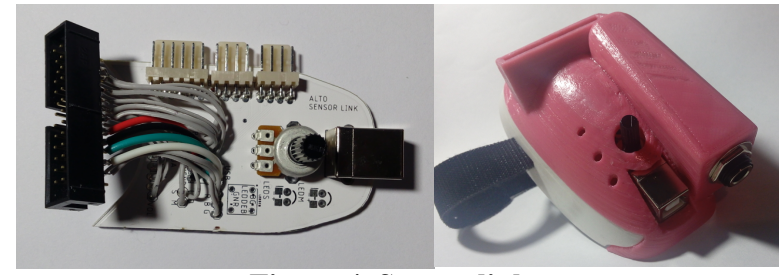

Figure 4. Sensor-link.

Un-mute. However, that design would make it difficult to fit all the components inside the Un-mute, particularly with the alto saxophone version. On the other hand, using some of the area of the Un-mute's front face to allocate sensor ports would result in less space for a speaker, which would have an impact on the audio capabilities of the system. For these reasons the Sensor-link is justifiable. In addition, it provides a better configuration of cable connection such as the USB and pedal jack facing down, preventing potential stress on the cables produced by gravity.

\subsection{Mouthpiece}

Acquiring data relating to the air flow going into the saxophone can be very difficult, but previous projects such as the SABRe [18] or the modified alto recorder mouthpiece by Garcia et al [5] demonstrated that using a tangential conduit can be effective to measure the variations in air pressure being blown into the instrument. A similar approach was used to develop a module for the HypeSax. A 3D model of an Alto Saxophone Mouthpiece designed by Thingiverse user Allanarps [21] obtained online was modified. The original design is based upon a Yamaha beginner mouthpiece, with similarities including bore length and shape, facing width, tip opening and basic length measurements, featuring a flat baffle and squared throat (bore). This model was modified to produce a new version that features a $1 \mathrm{~mm}$ conduit that allows a portion of the air stream to be redirected into a barometer sensor, thus obtaining data about the air pressure without disturbing the air column inside the instrument. At the same time, this configuration provides a comfortable playing experience.

The adaptation keeps the basic shape of the interior with the same dimensions in the chamber of the mouthpiece, facing width and bore shape, which ensures that the air column running through the chamber behaves in the same manner as in the original design. The changes made to the design include a shorter tip opening of $1.8 \mathrm{~mm}$ (original is $2 \mathrm{~mm}$ ) and a thicker beak that allocates a small conduit running through the body of the mouthpiece to an added section in the back in which a sensor is mounted to measure the air pressure. A minimal amount of material has been added to the top of the beak of the mouthpiece to accommodate the conduit. In total, $2 \mathrm{~mm}$ of thickness has been added to the beak, within which a $1 \mathrm{~mm}$ radius cylindrical opening is contained at the front/centre of the beak, where the structure is weaker because of its dimensions. Further back, the conduit opens more to fit the dimensions of the sensor at its back end (see Figure 5). There is also a small side canal that allows moisture and saliva to escape the system. This opening is usually blocked by the ligature during performance.

A BMP180 barometric sensor is mounted on the mouthpiece. Unlike most sensors designed to measure air pressure, the BMP180 doesn't feature a funnel-like structure to redirect the air into it, which becomes unnecessary with the conduit built into the mouthpiece 3D model. For this project, the GY68 breakout has been chosen because in this design the "hole" that captures the air in the sensor is situated close to one of its edges, which makes it convenient to introduce it in the body of the mouthpiece and reach the end of the conduit. Other breakout boards place the sensor on the center of the PCB, and the use of these boards would translate in carving into the model to reach the air conduit, which could compromise the structure of the mouthpiece. This breakout sends data via $\mathrm{I}^{2} \mathrm{C}$. More details of this mouthpiece can be found in $A 3 D$ printed hybrid saxophone mouthpiece for data collection [16].
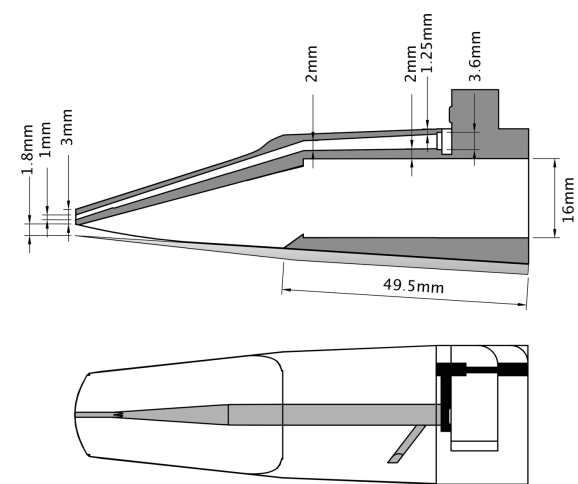

Figure 5. Interior of the mouthpiece. The top image shows a cross section of the mouthpiece where the chamber and conduit are shown in white. The bottom image shows the conduit and side canal in gray. The area designed to position the sensor breakout is colored in black.

\subsection{New keys}

Complex systems, such as the one implemented in the SABRe, monitor all keys to identify fingering by using Hall Effect sensors [17]. Such a design is highly effective but requires a complex setup process which makes the design inconvenient, which is possibly the reason why the commercial version of the SABRe (Multi-Sensor) doesn't include this fingering tracking system [26].

In the case of the HypeSax, easy setup and high compatibility with many saxophone models is a priority. To address this, a series of $3 \mathrm{D}$ models of saxophone key-caps were designed to allocate terminals coming from the MPR121 capacitive touch sensor board installed inside the Un-mute (see section 3.1). There are six terminals mounted on three key-caps which fit over the F, E and D keys. The 3D models are parametric and can be adapted to fit any size and configuration of saxophone keys.

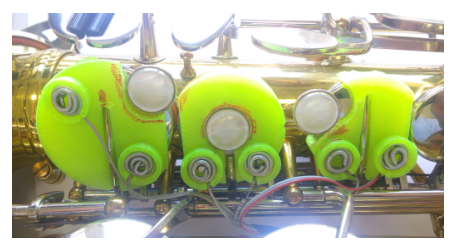

Figure 6. First iteration of 3D printed key-caps.

The position of the terminals mounted on the keycaps is close enough to the resting buttons of the keys, which allows for a comfortable experience while performing. With this configuration the performer can either close the keys by pressing the key buttons or by pressing on the touch pads. Depending on the shape of the performer's fingers and/or the angle of the finger in reference to the saxophone body, up to two touch pads can be touched by one finger at a time. The protuberances on the key-caps holding the terminals also help the player to avoid touching them by mistake. There is also a possibility of activating the touch sensors by only touching the terminals without pressing and closing the keys.

Three push buttons are included in the HypeSax design. Two of these buttons are mounted on the saxophone bellow the left thumb rest, and the third button is mounted next to the thumb hook to be easily accessible with the right thumb. Unlike the case of the terminals mounted on the saxophone keys, the thumbs interact with push buttons as the weight of the saxophone pushes against the thumbs, which produces unwanted interactions with touch sensors. This makes the mechanical system of the push buttons a more reliable approach.

\section{SOFTWARE}

The HypeSax has a USB port which allows communication with a computer or a MIDI device thanks to the high flexibility of Teensy boards. For this project, the slave board is set up as a Serial device since 
its only function is to gather information from the sensors, organize it and send it to the master board. The master board is set up to work as a Serial/MIDI/Audio device. With this configuration, the HypeSax is able to send MIDI messages for a full compatibility with MIDI devices or commercial software. It can work as an audio card to record and output audio (using the audio board adaptor), as well as send and receive audio signals through the USB port to a computer. It also communicates with a computer using the Serial protocol.

To take advantage of the latter aspect, a server application was developed in Max to receive, decode and organize the messages sent from the HypeSax. Once the communication is established, the application shows a visual representation of the sensors' activity. The user can then re-route the data using either MIDI, OSC, Serial or MAX's send/receive messages (when using the patch version of the application).

The potential of the HypeSax as a controller allows for adaptability for the creative needs of a composer or performer. However, this is the common approach of most hyperinstruments, where the hardware augmentations usually only work as controllers mounted on an instrument. This approach does not demerit a hyperinstrument, but at the same time there is not a real integration between the electronics and the instrument, as the synthesis (no matter how good it can be) remains as an addition output through an external source (speaker) rather than becoming integrated with the acoustics of the instrument.

Considering the idea of integration, the ideal goal for the HypeSax project is for it to be able to work as a standalone device without the need to communicate with a computer (in the same manner as devices like guitar pedals). However, at the moment the onboard software is being developed and it will take some time before the HypeSax can be fully independent. Nevertheless, computer software is being developed to achieve integration and will be featured in future releases.

\subsection{Acoustic integration through use of software}

As described in section 3.1, the Un-mute features a speaker that is mounted inside the bell of the saxophone. This allows for a new approach to hybridization of acoustic and electronic sound. Recent developments such as Juan Arroyo's hybrid string quartet [8] or the IMAREV project [23] have demonstrated the way in which active instruments allow for an interesting approach to sound treatment from an acoustical augmentation point of view. To achieve such augmentation, special software capable of real time audio analysis, sensor data analysis and audio processing has to be developed.

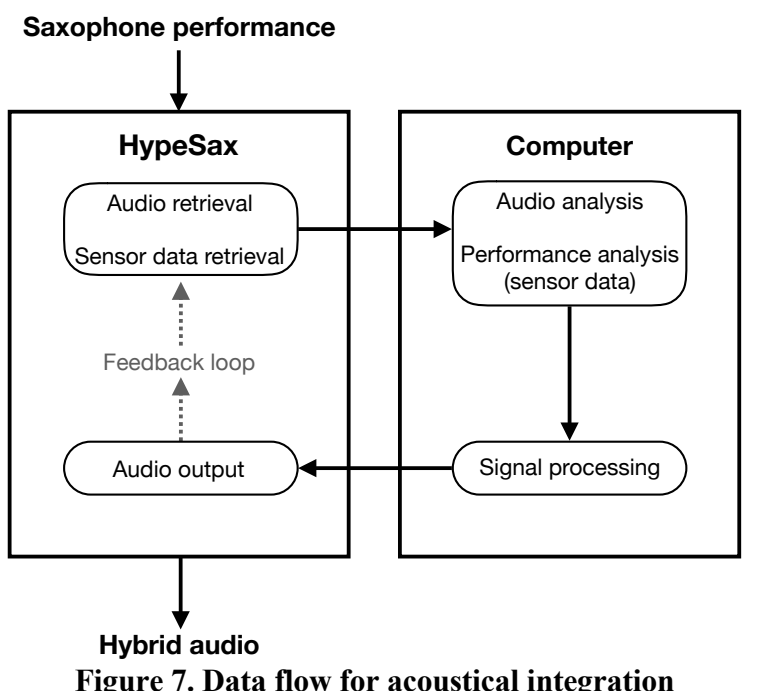

Any software developed to work with the HypeSax must follow the following data flow: First data is collected from the performance via HypeSax sensors. This data is sent to the computer using the built-in
USB port. Software analyzes audio and sensor data to process signal. Signal is sent back to the HypeSax and inserted in the body of the saxophone to achieve audio hybridization. In some cases, the software might allow feedback for a special audio treatment (see Figure 7).

Currently experimental software seeking to achieve hybrid sound and musical expressivity through gestural embodiment is being developed. In the following sections some examples of experimental software are discussed in detail.

\subsubsection{Experiments with sound morphology manipulation}

Timbre manipulation is one of the most exciting possibilities of audio treatment using new software. In the case of the HypeSax software, two approaches to sound morphology manipulation have been explored, one in the time domain and the other in the frequency domain.

To work in the time-domain, a Max patch records audio continuously in a ten-minute long buffer, which rolls over to continue recording indefinitely. A timer keeps track of elapsed time to be able to match the exact moment of any event with the audio being recorded. Previous to the performance, a calibration process of the gyroscope is necessary. This process consists of holding the saxophone in a natural playing position and pressing the calibration button on the Max patch. This sets the $\mathrm{X}, \mathrm{Y}$ and $\mathrm{Z}$ axes to 0 . Using the touch pads or the push buttons (this can be set up according to the preference of the performer or composer) the performer triggers a granulation relative to the time position of the buffer at that moment, which creates the effect of freezing the pitch. This effect has an envelope which varies in reference to the $\mathrm{Y}$ axis of the saxophone position while performing (see figure 8). If $Y>0$ (lifting the bell) a fast attack and release operate when activating or deactivating the granulation. If $\mathrm{Y}<0$ the attack and release are slow. The gain of the granular synthesis is controlled by the air pressure measured by the mouthpiece, effectively having a similar variation to that of the acoustic saxophone sound. There is no real-time unprocessed signal being outputted to avoid feedback. In this way, the performer can introduce a second voice whose envelope can be manipulated to create a complex timbre or multiphonic effect when mixed with the acoustic sound. The performer can then create different effects overlapping pitch changes of the acoustic sound with the granular synthesis, creating microtonal effects, beating, multiphonics, etc., which can be manipulated over time.

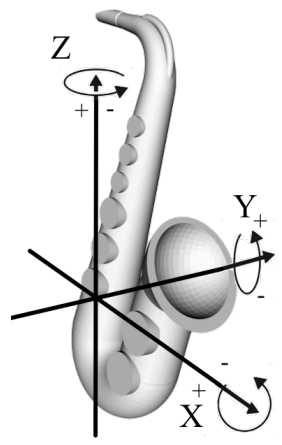

Figure 8. $X, Y$ and $Z$ Axes of the HypeSax's gyroscope in reference to the saxophone's natural performing position

In the frequency-domain, the authors have the intention to transform the timbre of the saxophone by reinforcing the energy of selected overtones in order to emulate the unique timbres of other instruments. A Max patch analyses the audio to obtain the fundamental frequency. It also tracks the relative energy of the overtones in the harmonic series. Then, based on data obtained from previous analysis of other instruments (i.e., a clarinet) playing the same pitch, the patch makes a comparison of the energy of each of the first 15 overtones in the harmonic series of the saxophone and the analyzed instrument. Then, using an active multi-band filter with a high $\mathrm{Q}$ on each of the 15 overtones, the software reintroduces the original audio which has now 
been filtered to add more energy or no energy on the overtones, simulating the timbre of the analyzed instrument. This approach is effective, but further work on the patch is necessary as the current version is resource-intensive and produces constant crashes.

A second approach with similar results was also explored. In this case, the patch only tracks the fundamental frequency. Then the software uses data from previous analysis and comparisons of saxophone recordings and other instruments. In this case, the data only tells the software how much gain it needs to control the components of an additive synthesizer. Finally, the synthesized sound is introduced into the saxophone.

The quality of audio resulted from the first approach is better, but unreliable. Perceptively, the second approach is good enough to achieve the effect of timbre modification. However, the system is limited to the power of the audio system, which means that manipulating the timbre is only possible when performing with a dynamic range between $\boldsymbol{p p p}$ and $\boldsymbol{m} \boldsymbol{f}$. Louder dynamics are possible but not completely effective as the saxophone can overpower the capabilities of the audio system. Also, timbre transformation is not very convincing when using the models of instruments with defined envelopes such as plucked strings, piano, percussion, etc. Using models of fiddle instruments, woodwind, brass and particularly double reed instruments and clarinet (possibly due to its unusual harmonic series) are very convincing.

\subsubsection{Gestural-instrumental-technique: an approach to instrumental-gesture embodiment.}

Every instrument or family of instruments has a characteristic set of performing techniques. Strings, for instance, can be plucked and bowed, however it is not common to bow some instruments such a harp (although it is possible using extended techniques). The difference between bowed and plucked techniques is mainly the envelope control. A high degree of envelop control is possible to achieve with woodwind instruments thanks to a combination of articulation and breath control.

Some techniques are more specific to an instrument. The ricochet is a technique unique to fiddle instruments, which again can be imitated using extended techniques with other string instruments. Nevertheless, the ricochet is impossible to achieve with woodwind instruments due to its characteristic echo-like fast series of attacks with a loss of energy, which cannot be replicated with articulation and breath control.

These kinds of instrumental techniques require a specific physical gesture. In the case of the ricochet, the physical gesture is a strike on the string followed by natural bouncing of the bow over the string. This is what we call gestural-instrumentaltechnique, a physical action that results in a specific instrumental technique. Some gestural-instrumental-techniques are transferable between instruments. The ricochet for instance can be achieved on the piano using a mallet directly on the strings, but no similar action can be performed with brass instruments to achieve a similar effect.

The HypeSax can potentially extend the limits of the saxophone gestural-instrumental-techniques, through the addition of techniques previously unique to other instruments. Using a mix of gesture recognition techniques and audio analysis we have been able to bring ricochet, sul pont/sul tasto, and string subharmonic techniques to the saxophone.

The ricochet requires the use of two sensors and slap tongue. The first step is to perform a slap tongue which is monitored by the mouthpiece's air pressure sensor. The air pressure changes produced by this technique follow a distinctive curve (see figure 10) which begins with a negative reading resulting from sucking and pulling the reed, followed by a rapid increase of pressure and a quick release back to negative, and then zero pressure. The performer must also press quickly two buttons or touch pads (configurable by the user). The order or events must be then as follows: 1) slap tongue, 2) trigger 1, and 3) trigger 2. If these three events occur in a lapse of less than 500 milliseconds, the software triggers a short loop of $200 \mathrm{~ms}$ since the last peak (of the last $500 \mathrm{~ms}$ ) with a decaying envelope of 1.5 seconds. The result outputted through the HypeSax into the saxophone resembles that of the string ricochet.

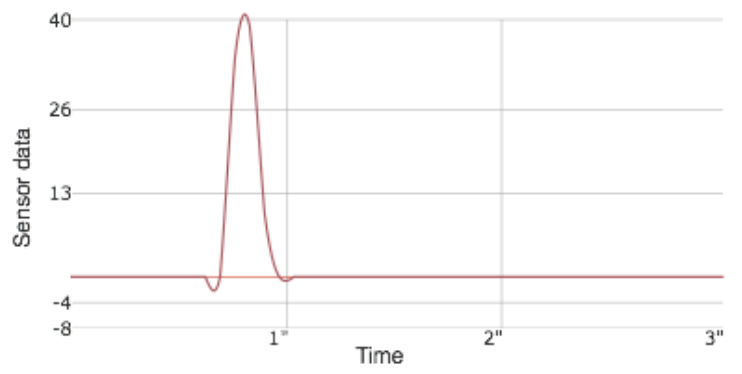

Figure 10. Typical data curve of air pressure changes in slap tongue technique

Sul ponticello string technique is achieved by placing the string exciter (bow in the case of fiddles, plectrum or fingers in plucked string instruments) close to one of the ends of the string, while for the sul tasto technique the exciter is placed near the center of the length of the string. The sounding result is an accentuation on the higher harmonics or the lower ones. In the case of the cello, the bow is typically taken down to the lower part of the string, near the bridge for sul pont sound, and up for sul tasto. Following this model, in the HypeSax, the user activates the touch pads in a consecutive way going from the highest to the lowest (top F-key to low D-key touch pads) imitating the cello sul pont action, or in the opposite direction for sul tasto, in a sweeping movement, usually with one finger. When the software detects this physical gesture, the incoming audio signal is filtered (low or high pass according to the gesture) and reintroduces the filtered sound into the system.

The string subharmonic technique as utilized in works such as George Crumb's Black Angels (called pedal tones) [4] or the work of Mary Kimura [9] is useful in extending the range of instruments. In the specific case of bowed strings, three factors seem to be the most crucial: Bow pressure, bow speed, and bow position. Considering this, the HypeSax software looks for a similar combination of three factors: the mouthpiece must read a data of high pressure (imitating bow pressure), the pressure must remain somewhat constant for two seconds (imitating bow speed), and the saxophone must be tilted over the $\mathrm{X}$ axis to read negative numbers (see figure 8), taking the bell to the right and mouth piece to the left in a counter-clockwise direction from the perspective of the performer. During these events, the fundamental frequency is being tracked. When the three factors are in play, the first subharmonic (an 8ve lower) is introduced. Augmenting the inclination on the $\mathrm{X}$ axis produces a second subharmonic $(8 \mathrm{ve}+\mathrm{p} 5)$ and replaces the first subharmonic. The synthesis is constructed with sine waves using the frequency of the interval of the subharmonic (an $8 \mathrm{ve}$ or $8 \mathrm{ve}+\mathrm{p} 5$ lower) mixed with a real time transposition of the original sound, filtered with a high $\mathrm{Q}$ over the fundamental frequency of the transposed sound, which adds a natural aspect to the synthesis.

\subsubsection{Acoustic electronics: feedback as a musical element}

Introducing audio in the body of the saxophone using the HypeSax system can produce unwanted feedback due to the closeness between the microphone and the speaker. However, it is very exciting to discover that the feedback can be controlled by the mechanics of the saxophone. Opening and closing keys has the effect of changing the length of the tube and the resonance of the internal space, which has a direct effect on the feedback, a phenomenon previously explored in composition by 
Burtner [1] and further explored by Snyder et al [20]. The pitch of the feedback can be controlled with the saxophone keys, as well as by introducing air pressure or playing pitches with the saxophone. Also, by changing the gain using the built-in pedal effect jack, the introduction or amount of feedback can be controlled. This is a remarkable hybridization between electronic sound and mechanical control which is worth exploring further.

\section{EVALUATION AND LIMITATIONS}

The HypeSax as a controller works without issues at the moment. However, we are aware of potential for improvement to the design, especially from the technical point of view, through re-testing and curating the best sensor and electronic components to achieve optimization. Nevertheless, the ergonomics of the design do not interfere with the normal instrumental techniques.

In the specific case of the alto HypeSax, a major downside is the loss of the lowest note the saxophone's range, due to the disturbance of the air column by the Un-mute. Another issue is the fact that the HypeSax cannot equal the volume of the saxophone, meaning that hybridization can only be achieved at restrained dynamic levels.

Further evaluation is required to assess the software in musical contexts.

\section{CONCLUSIONS AND FUTURE WORK}

The HypeSax, in its developing phase, allows the user to aproach acoustical and musical gesture in a unique way. Although further research and development is necessary, the potential to transform timbre and to enrich the capabilities of the saxophone through adapting techniques borrowed from other instruments are possibly the two most important affordances of the project. From the perspective of the composer and performer, the openness of software integration (custom or commercial) using the server or MIDI is a strong point in favor of the HypeSax, and it is the intention of the authors to maintain this aspect in further iterations of the HypeSax.

The next two goals of the project are to achieve complete independence from the computer, and to develop a more powerful audio system. The Un-mute can potentially be adapted to fit in different instruments, and its acoustic impact will depend on many aspects including the shape of the instrument, the acoustic response of the speaker, the position of the speaker (facing inwards or outwards from the body of the instrument), use of multiple speakers, audio capture system and processing, microphones, etc. Further study of its effect on the resulting sound of any adaptation is necessary, and at the moment the concept resembles an expansion of the active mute designed by Meurisse et al. [12]

Future iterations of the HypeSax will include new designs for other instruments in the saxophone family. Most importantly, future work will focus on refining the built-in audio system and ergonomic aspect of the design, as it is the wish of the authors that the HypeSax be highly efficient and functional without negatively affecting the performer/instrument relationship or the capabilities of the instrument, truly providing a comfortable and effective hybrid instrument experience.

\section{REFERENCES}

[1] Chris Burns and Matthew Burtner. 2004. Recursive Audio Systems: Acoustic Feedback in Composition. Leonardo Music Journal Electronic Almanac (2004).

[2] Matthew Burtner. The Metasaxophone: concept, implementation, and mapping strategies for a new computer music instrument. Organised Sound 7, 02 , 201-213.

[3] Perry Cook and Dan Trueman. 2000. BoSSA: The Deconstructed Violin Reconstructed. Journal of New Music Research 29, 2 (June 2000), 121-130.

[4] George Crumb. 1971. Black angels (images 1). Peters, New York.

[5] Francisco García, Vinceslas Leny, Tubau Josep, and Maestre Esteban. 2011. Acquisition and study of blowing pressure profiles in recorder playing. (2011).
[6] Euyshick Hong and Jun Kim. 2017. Telesaxophone: Hybrid saxophone Interface. In Proceedings of the International Conference on Algorithms, Computing and Systems - ICACS '17, 83-87. Retrieved January 5, 2019 from http://dl.acm.org/citation.cfm?doid=3127942.3127955

[7] Euyshick Hong, Guanyu Zhu, and Wendel Moreira Duarte. 2016. Dr. Saxophone: Hybrid saxophone interface. 2016 3rd International Conference on Systems and Informatics (ICSAI) (2016), 1149-1153.

[8] Fabien Houlès. 2017. Le premier quatuor à cordes hybride, l'example de Smaqra de Juan Arroyo (L'Harmattan ed.). Paris.

[9] Mari Kimura. 1999. How to Produce Subharmonics on the Violin. Journal of New Music Research 28, 2 (June 1999), 178184.

[10] Otso Lähdeoja. 2016. Active Acoustic Instruments for Electronic Chamber Music. In Proceedings of the International Conference on New Interfaces for Musical Expression (22204806), 132-136.

[11] Carolina Medeiros and Marcelo Wanderley. 2014. A Comprehensive Review of Sensors and Instrumentation Methods in Devices for Musical Expression. Sensors 14, 8 (July 2014), 13556-13591.

[12] Thibaut Meurisse, Adrien Mamou-Mani, René Caussé, Benny Sluchin, and David B. Sharp. 2015. An active mute for the trombone. The Journal of the Acoustical Society of America 138, 6 (December 2015), 3539-3548.

[13] N. Onozawa. 2010. Hybrid wind musical instrument and electric system for the same. Google Patents. Retrieved from https://www.google.com/patents/US7741555

[14] N. Onozawa and K. Fujita. 2006. Hybrid wind instrument selectively producing acoustic tones and electric tones and electronic system used therein. Google Patents. Retrieved from https://www.google.com/patents/US7049503

[15] Henrique Portovedo, Paulo Ferreira Lopes, and Ricardo Mendes. Saxophone Augmentation: An hybrid augment system of gestural symbiosis. 4 .

[16] Cristohper Ramos Flores, Jim Murphy, and Michael Norris. 2017. A 3d printed hybrid saxophone mouthpiece for data collection. In Do androids dream of computer music?, 1926.

[17] Sébastien Schiesser and Jan C. Schacher. 2012. SABRe: The Augmented Bass Clarinet. In NIME.

[18] Sébastien Schiesser and Jan C. Schacher. 2012. Sabre: affordances, realizations and Perspectives. In ICMC. Retrieved September 3, 2017 from http://www.augmentedinstruments.net/_media/schiesser-

sabre affordances realizations and perspectives.pdf

[19] Sébastien Schiesser and Caroline Traube. 2006. On Making and Playing an Electronically-augmented Saxophone. In Proceedings of the International Conference on New Interfaces for Musical Expression, 308-313.

[20] Jeff Snyder, Rajeev Erramilli, and Mike Mulshine. 2018. The Feedback Trombone: Controlling Feedback in Brass Instruments.

[21] Thingiverse.com. Alto Saxophone Mouthpiece by Allanrps. Retrieved September 3, 2017 from https://www.thingiverse.com/thing: 14495

[22] David Wessel and Matthew Wright. 2002. Problems and Prospects for Intimate Musical Control of Computers. Computer Music Journal 26, 3 (2002), 11-22.

[23] IMAREV $\mid$ Acoustique Instrumentale. Retrieved January 15, 2019 from http://instrum.ircam.fr/imarev/

[24] SmartInstruments $\mid$ Acoustique Instrumentale. Retrieved January 15, 2019 from http://instrum.ircam.fr/smartinstruments/ [25] Acoustically Active Augmented Instruments. Retrieved January 15, 2019 from /en/inspirational-projects/acousticallyactive-augmented-instruments/

[26] SABRE | SABRE multisensor. SABRe. Retrieved January 9, 2019 from https://www.sabre-mt.com/sabremultisensor 


\section{APPENDIX F: LIST OF INSTRUMENTAL TECHNIQUES FOR GESTURAL-INSTRUMENTAL TECHNIQUE DEVELOPMENT}

This appendix list a set of instrumental techniques and their availability in different instrumental families. A second list presents a guide useful in approaching the development of gestural-instrumental techniques for the HypeSax. The techniques are organised according to their effect on characteristics of spectromorphology of sound. The symbols ' $\mathrm{X}$ ', '/, and '-' are used to represent 'available', 'similar is available' and 'not available' respectively.

\begin{tabular}{|c|c|c|c|c|c|}
\hline \multicolumn{6}{|c|}{ TECHNIQUES: MULTIPLE INSTRUMENTS } \\
\hline TECHNIQUE & IDIOPHONE & MEMBRAPH. & CHORDOPH. & AEROPH. & ELECTROPH. \\
\hline \multicolumn{6}{|c|}{ onset } \\
\hline Articulation & $\bar{X}$ & - & $\mathrm{X}$ & $\bar{X}$ & $\mathrm{X}$ \\
\hline Pizzicato & - & - & $\mathrm{X}$ & $\mathrm{X}$ & $\mathrm{X}$ \\
\hline Bartók pizz & - & - & $\mathrm{X}$ & - & $\mathrm{X}$ \\
\hline Key clicks & - & - & - & $\mathrm{x}$ & $\mathrm{X}$ \\
\hline Ricochet & $\mathrm{X}$ & $\mathrm{X}$ & $\mathrm{X}$ & - & $\mathrm{X}$ \\
\hline $\begin{array}{l}\text { Legno } \\
\text { battuto }\end{array}$ & $\mathrm{X}$ & $\mathrm{X}$ & $\mathrm{X}$ & - & $\mathrm{X}$ \\
\hline Slap tongue & - & - & - & $\bar{X}$ & $\mathrm{X}$ \\
\hline $\begin{array}{l}\text { Mult.Attack- } \\
\text { Tremolo }\end{array}$ & $\mathrm{X}$ & $\mathrm{X}$ & $\mathrm{X}$ & $\mathrm{X}$ & $\mathrm{X}$ \\
\hline Hammering & $\mathrm{X}$ & $\mathrm{X}$ & $\mathrm{X}$ & - & $\mathrm{X}$ \\
\hline Knocking & $\mathrm{X}$ & $\mathrm{X}$ & $\mathrm{X}$ & - & $\mathrm{X}$ \\
\hline $\begin{array}{l}\text { Spiccato } \\
\end{array}$ & 7 & 1 & $\mathrm{X}$ & 1 & $\mathrm{X}$ \\
\hline Tongue ram & - & - & - & $\mathrm{X}$ & $\mathrm{X}$ \\
\hline \multicolumn{6}{|c|}{ continuant } \\
\hline Dynamics & $\mathrm{X}$ & $\mathrm{X}$ & $\mathrm{X}$ & $\mathrm{X}$ & $\mathrm{X}$ \\
\hline Vibrato & - & - & $\mathrm{X}$ & $\mathrm{X}$ & $\mathrm{X}$ \\
\hline Wide vibrato & - & - & $\mathrm{X}$ & - & $\bar{X}$ \\
\hline Air & - & - & - & $\mathrm{X}$ & $\bar{X}$ \\
\hline Jet whistle & - & - & - & $\mathrm{X}$ & - \\
\hline $\mathrm{Sp}, \mathrm{St}$ & $\mathrm{X}$ & $\mathrm{X}$ & $\bar{X}$ & - & $\mathrm{X}$ \\
\hline Overpressure & - & - & $\bar{X}$ & - & $\bar{X}$ \\
\hline
\end{tabular}


APPENDIX F: list of instrumental techniques for gestural-instrumental technique development

\begin{tabular}{|c|c|c|c|c|c|}
\hline Mutes & $\mathrm{X}$ & $\mathrm{X}$ & $\mathrm{X}$ & $\mathrm{X}$ & - \\
\hline Cuivré & - & - & - & $\mathrm{X}$ & $\bar{X}$ \\
\hline Col legno & $\mathrm{X}$ & $\mathrm{X}$ & $\mathrm{X}$ & - & - \\
\hline $\begin{array}{l}\text { Dampers/ } \\
\text { pedals }\end{array}$ & $\mathrm{X}$ & $\mathrm{X}$ & $\mathrm{X}$ & $\mathrm{X}$ & $\mathrm{X}$ \\
\hline $\begin{array}{l}\text { Growling/int } \\
\text { erference }\end{array}$ & & & & & \\
\hline \multicolumn{6}{|c|}{ termination } \\
\hline Staccatto & $\bar{X}$ & $\mathrm{X}$ & $\mathrm{X}$ & $\mathrm{X}$ & $\mathrm{X}$ \\
\hline \multicolumn{6}{|l|}{$\begin{array}{l}\text { Continuous } \\
\text { Dynamics }\end{array}$} \\
\hline \multicolumn{6}{|l|}{ let ring } \\
\hline \multicolumn{6}{|l|}{ Damping } \\
\hline \multicolumn{6}{|c|}{ pitch } \\
\hline $\begin{array}{l}\text { Gliss/portam } \\
\text { ento/bend }\end{array}$ & - & $\mathrm{X}$ & $\bar{X}$ & $\bar{X}$ & $\mathrm{X}$ \\
\hline Multiphonic & - & - & $\bar{X}$ & $\mathrm{X}$ & $\mathrm{X}$ \\
\hline Microtones & - & - & $\mathrm{X}$ & $\bar{X}$ & $\mathrm{X}$ \\
\hline Harmonics & - & $\bar{X}$ & $\mathrm{X}$ & $\bar{X}$ & $\mathrm{X}$ \\
\hline Subharmoni & - & - & $\mathrm{X}$ & $\bar{X}$ & $\mathrm{X}$ \\
\hline $\begin{array}{l}\text { Trills/ } \\
\text { Mult.Note- } \\
\text { Tremolo }\end{array}$ & $\mathrm{X}$ & $\mathrm{X}$ & $\bar{X}$ & $\mathrm{X}$ & $\mathrm{X}$ \\
\hline $\begin{array}{l}\text { Hold note/ } \\
\text { play others }\end{array}$ & $\bar{X}$ & - & $\bar{X}$ & - & $\bar{X}$ \\
\hline \multicolumn{6}{|l|}{ Noise } \\
\hline \multicolumn{6}{|c|}{ miscellaneous } \\
\hline Bells up & - & - & - & $\bar{X}$ & - \\
\hline Directionalit & - & - & $\mathrm{X}$ & $\mathrm{X}$ & - \\
\hline Walk & & & & & \\
\hline
\end{tabular}


TECHNIQUES: SAXOPHONE

\begin{tabular}{|c|c|c|c|c|c|}
\hline TECHNIQUE & $\begin{array}{c}\text { POSSIBLE } \\
\text { ON SAX }\end{array}$ & $\begin{array}{c}\text { POSSIBLE ON } \\
\text { HYPESAX, } \\
\text { HOW? }\end{array}$ & $\begin{array}{l}\text { PHYSICAL } \\
\text { GESTURE ON } \\
\text { HYPESAX }\end{array}$ & MAPPING & $\begin{array}{c}\text { PROGRAM } \\
\text { NAME }\end{array}$ \\
\hline \multicolumn{6}{|c|}{ onset } \\
\hline Articulation & $\mathrm{X}$ & & & & \\
\hline Pizzicato & X & & & & \\
\hline Bartók pizz & - & $\begin{array}{l}\text { Synthesised 'hit' } \\
\text { synchronised } \\
\text { with attack }\end{array}$ & $\begin{array}{l}\text { Play a loud- } \\
\text { short note and at } \\
\text { the same time } \\
\text { push/ press } \\
\text { button }\end{array}$ & Use any button & Bartok \\
\hline Key clicks & $\mathrm{X}$ & & & & \\
\hline Ricochet & - & $\begin{array}{l}\text { Record buffer, } \\
\text { detect attack } \\
\text { and repeat the } \\
\text { last attack } \\
\text { multiple times } \\
\text { with envelope }\end{array}$ & $\begin{array}{l}\text { play slap tongue } \\
\text { or forceful } \\
\text { attack, and } \\
\text { quickly press/ } \\
\text { touch } 2 \text { buttons, } \\
\text { one after } \\
\text { another. The } \\
\text { entire event } \\
\text { should take no } \\
\text { longer than } \\
300 \mathrm{~ms} \\
\end{array}$ & $\begin{array}{l}\text { Air pressure to } \\
\text { detect attack, } \\
\text { use any } \\
\text { combination of } \\
\text { buttons. }\end{array}$ & Ricochet \\
\hline Legno battuto & - & $\begin{array}{l}\text { Play staccato, } \\
\text { add synthesised } \\
\text { attack with } \\
\text { dynamics based } \\
\text { on air pressure. } \\
\text { Use a button to } \\
\text { activate function }\end{array}$ & $\begin{array}{l}\text { Play staccato } \\
\text { normally and } \\
\text { synchronise } \\
\text { touching/ } \\
\text { pressing a } \\
\text { button }\end{array}$ & Use any button & Legno_b \\
\hline Slap tongue & $\mathrm{X}$ & & & & \\
\hline $\begin{array}{l}\text { Mult.Attack- } \\
\text { Tremolo }\end{array}$ & $\mathrm{X}$ & & & & \\
\hline Hammering & l & $\begin{array}{l}\text { amplify audio } \\
\text { signal, add } \\
\text { contact mic }\end{array}$ & activate function & Use any button & Martellato \\
\hline Knocking & - & $\begin{array}{l}\text { amplify audio } \\
\text { signal, add } \\
\text { contact mic }\end{array}$ & activate function & Use any button & Knock \\
\hline Spiccato & / & NA & & & \\
\hline Tongue ram & $X$ & & & & \\
\hline & & & & & \\
\hline
\end{tabular}


APPENDIX F: list of instrumental techniques for gestural-instrumental technique development

\begin{tabular}{|c|c|c|c|c|c|}
\hline \multicolumn{6}{|c|}{ continuant } \\
\hline Dynamics & X & & & & \\
\hline Vibrato & X & & & & \\
\hline Wide vibrato & $\mathrm{X}$ & & & & \\
\hline Air & X & & & & \\
\hline Jet whistle & l & & & & \\
\hline $\mathrm{Sp}, \mathrm{St}$ & - & $\begin{array}{l}\text { Amplify and } \\
\text { saxophone and } \\
\text { boost certain } \\
\text { zones of the } \\
\text { spectrum }\end{array}$ & $\begin{array}{l}\text { Right hand } \\
\text { slides up or } \\
\text { down over the } \\
\text { saxophone } \\
\text { body }\end{array}$ & $\begin{array}{l}\text { look for } \\
\text { sequence of } \\
\text { events/touch } \\
\text { sensors }\end{array}$ & $\mathrm{Sp} \_\mathrm{St}$ \\
\hline Overpressure & - & $\begin{array}{l}\text { distort } \\
\text { saxophone } \\
\text { signal with } \\
\text { intermittent } \\
\text { sound. } \\
\text { Introduce noise }\end{array}$ & $\begin{array}{l}\text { Overblow, or } \\
\text { inhale (for quiet } \\
\text { overpressure) }\end{array}$ & $\begin{array}{l}\text { Combination } \\
\text { of trigger to } \\
\text { activate } \\
\text { function and } \\
\text { use extreme air } \\
\text { pressure on } \\
\text { mouthpiece }\end{array}$ & $\begin{array}{c}\text { Not available } \\
\text { yet }\end{array}$ \\
\hline Mutes & - & $\begin{array}{c}\text { Track } \\
\text { fundamental } \\
\text { pitch and } \\
\text { introduce } \\
\text { synthesised } \\
\text { overtones } \\
\end{array}$ & Normal playing & $\begin{array}{l}\text { Use a trigger } \\
\text { to activate }\end{array}$ & $\begin{array}{c}\text { Not available } \\
\text { yet }\end{array}$ \\
\hline Cuivré & l & NA & & & \\
\hline Col legno & - & $\begin{array}{c}\text { Similar to } \\
\text { mutes }\end{array}$ & $\begin{array}{l}\text { Similar to } \\
\text { mutes }\end{array}$ & $\begin{array}{c}\text { Similar to } \\
\text { mutes }\end{array}$ & $\begin{array}{c}\text { Not available } \\
\text { yet }\end{array}$ \\
\hline $\begin{array}{l}\text { Dampers/ } \\
\text { pedals }\end{array}$ & - & Not possible & & & \\
\hline $\begin{array}{l}\text { Growling/ } \\
\text { interference }\end{array}$ & $\mathrm{X}$ & & & & \\
\hline \multicolumn{6}{|c|}{ termination } \\
\hline Staccatto & $\mathrm{X}$ & & & & \\
\hline $\begin{array}{l}\text { Continuous } \\
\text { Dynamics }\end{array}$ & $\mathrm{X}$ & & & & \\
\hline let ring & - & Add reverb & Normal playing & $\begin{array}{l}\text { Activate } \\
\text { function }\end{array}$ & lv \\
\hline Damping & NA & & & & \\
\hline \multicolumn{6}{|c|}{ pitch } \\
\hline $\begin{array}{l}\text { Gliss/ } \\
\text { portamento/ } \\
\text { bend }\end{array}$ & $\mathrm{X}$ & & & & \\
\hline
\end{tabular}




\begin{tabular}{|c|c|c|c|c|c|}
\hline Multiphonics & $\mathrm{X}$ & & & & \\
\hline Microtones & $\mathrm{X}$ & & & & \\
\hline Harmonics & $\mathrm{X}$ & & & & \\
\hline Subharmonics & - & $\begin{array}{l}\text { Use octaver. } \\
\text { Also, some } \\
\text { subharmonics ca } \\
\mathrm{n} \text { be achieved } \\
\text { by tracking the } \\
\text { fundamental } \\
\text { pitch and } \\
\text { introducing a } \\
\text { specific interval } \\
\text { to achieve } \\
\text { 'combination } \\
\text { tones' }\end{array}$ & $\begin{array}{l}\text { Normal playing } \\
\text { + use a button }\end{array}$ & $\begin{array}{l}\text { Transpose } \\
\text { pitch, or } \\
\text { generate a sine }\end{array}$ & $\begin{array}{c}\text { Not available } \\
\text { yet }\end{array}$ \\
\hline $\begin{array}{l}\text { Trills/ } \\
\text { Mult.Note- } \\
\text { Tremolo }\end{array}$ & $\mathrm{X}$ & & & & \\
\hline $\begin{array}{l}\text { Hold note/ play } \\
\text { others }\end{array}$ & - & $\begin{array}{l}\text { Use 'freeze' } \\
\text { effect using } \\
\text { granular } \\
\text { synthesis }\end{array}$ & $\begin{array}{l}\text { Normal playing } \\
+ \text { use touch } \\
\text { button to hold } \\
\text { note }\end{array}$ & $\begin{array}{l}\text { Combination } \\
\text { of live } \\
\text { recording and } \\
\text { granular } \\
\text { synthesis } \\
\end{array}$ & multivoice \\
\hline Noise & $\mathrm{X}$ & & & & \\
\hline \multicolumn{6}{|c|}{ miscellaneous } \\
\hline Bells up & $\mathrm{X}$ & & & & \\
\hline Directionality & $X$ & & & & \\
\hline Walk & $X$ & & & & \\
\hline
\end{tabular}


APPENDIX G: HYPESAX ÉTUDES 
3 HypeSax Études Cristohper Ramos Flores 


\title{
3 HypeSax Études
}

\author{
Performing notes
}

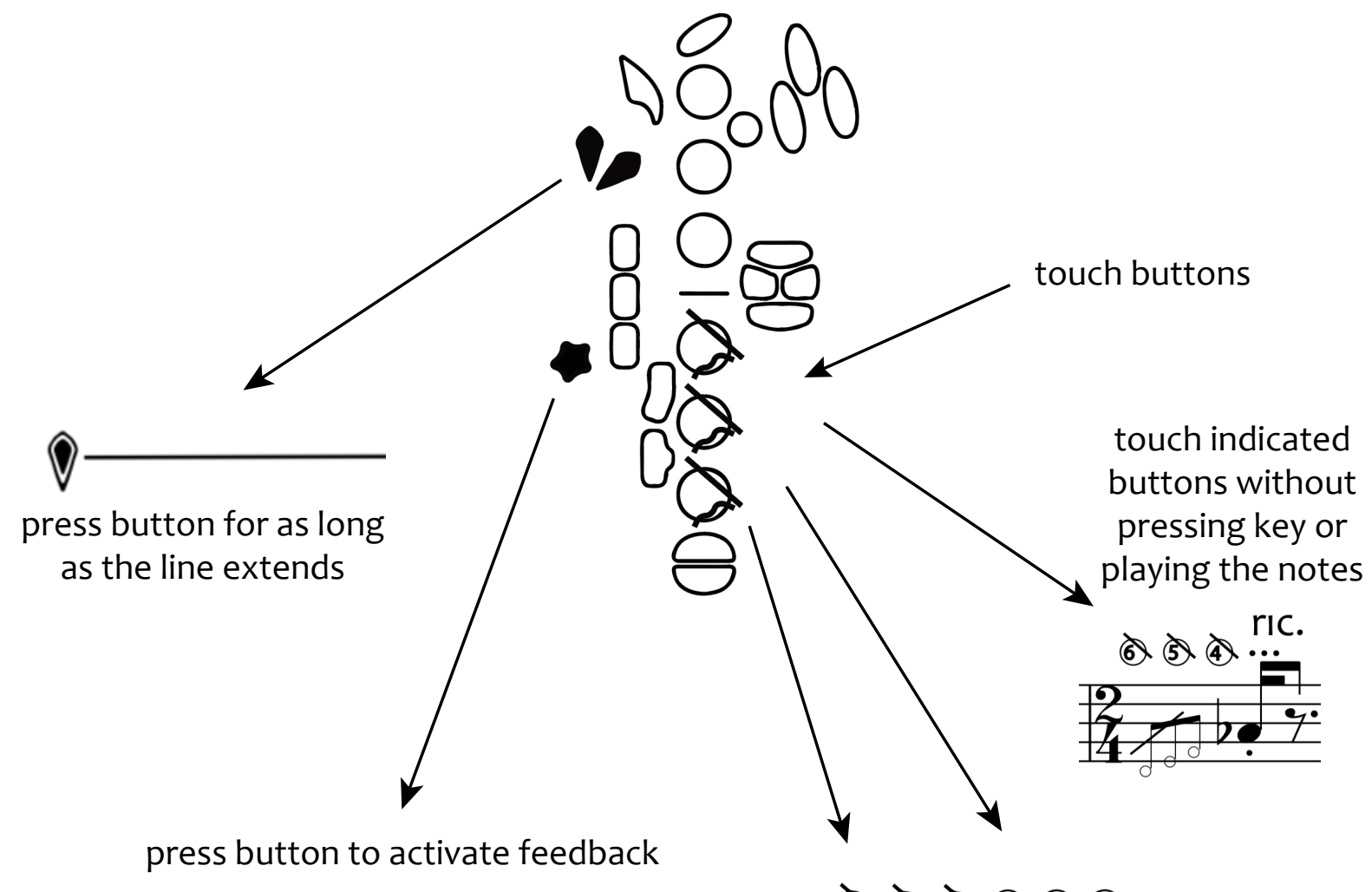

(4) (5) (6) (4) (5) (6)

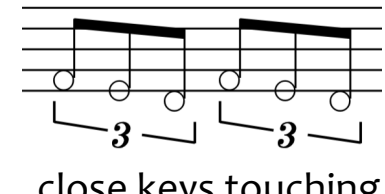

close keys touching indicated buttons without playing the notes

Use the accompanying Max patch Instructions included in the patch 


\section{HypeSax Étude No.1}

for soprano saxophone

Cristohper Ramos Flores

Tempo and dynamics ad libitum
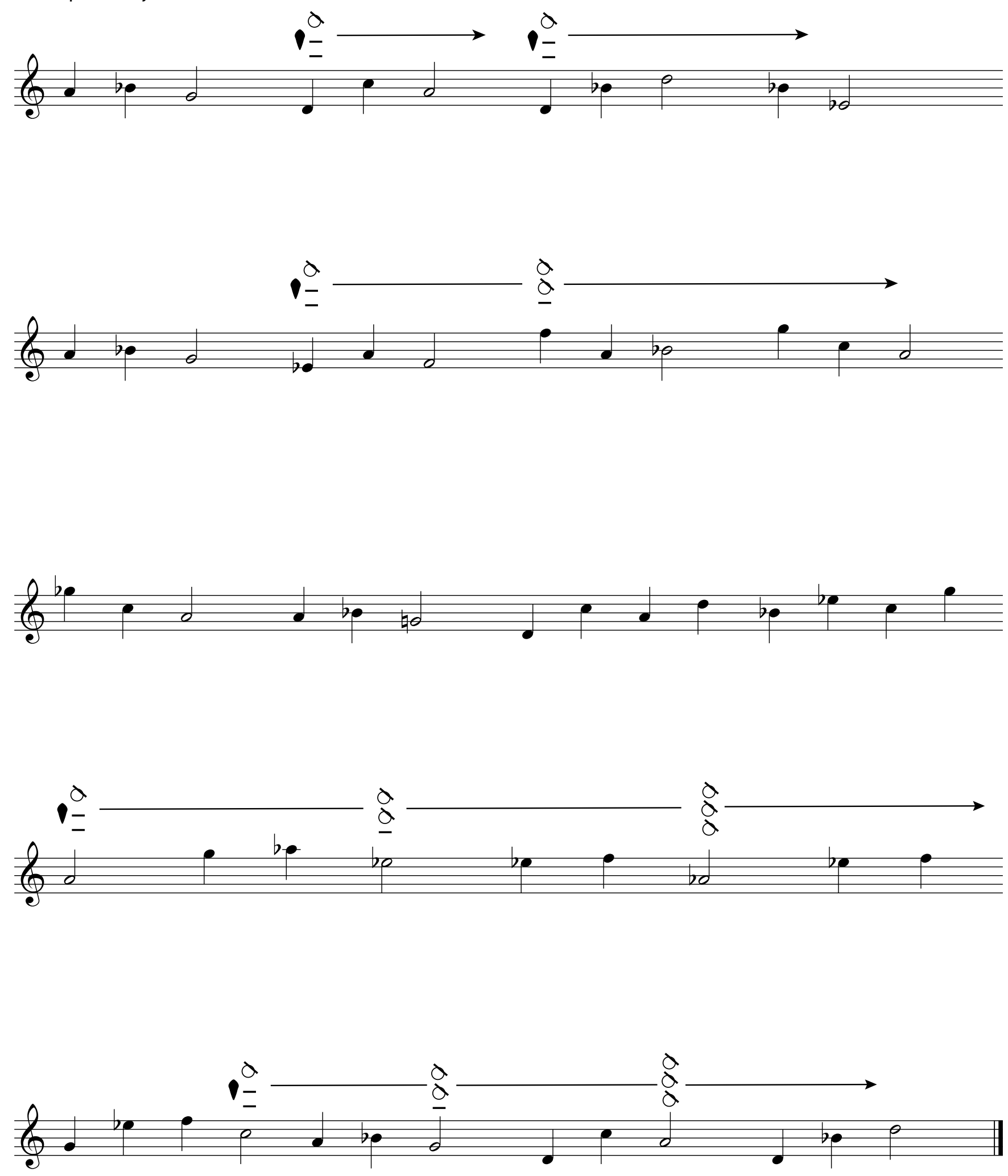


\section{HypeSax Étude No.2}

for alto saxophone
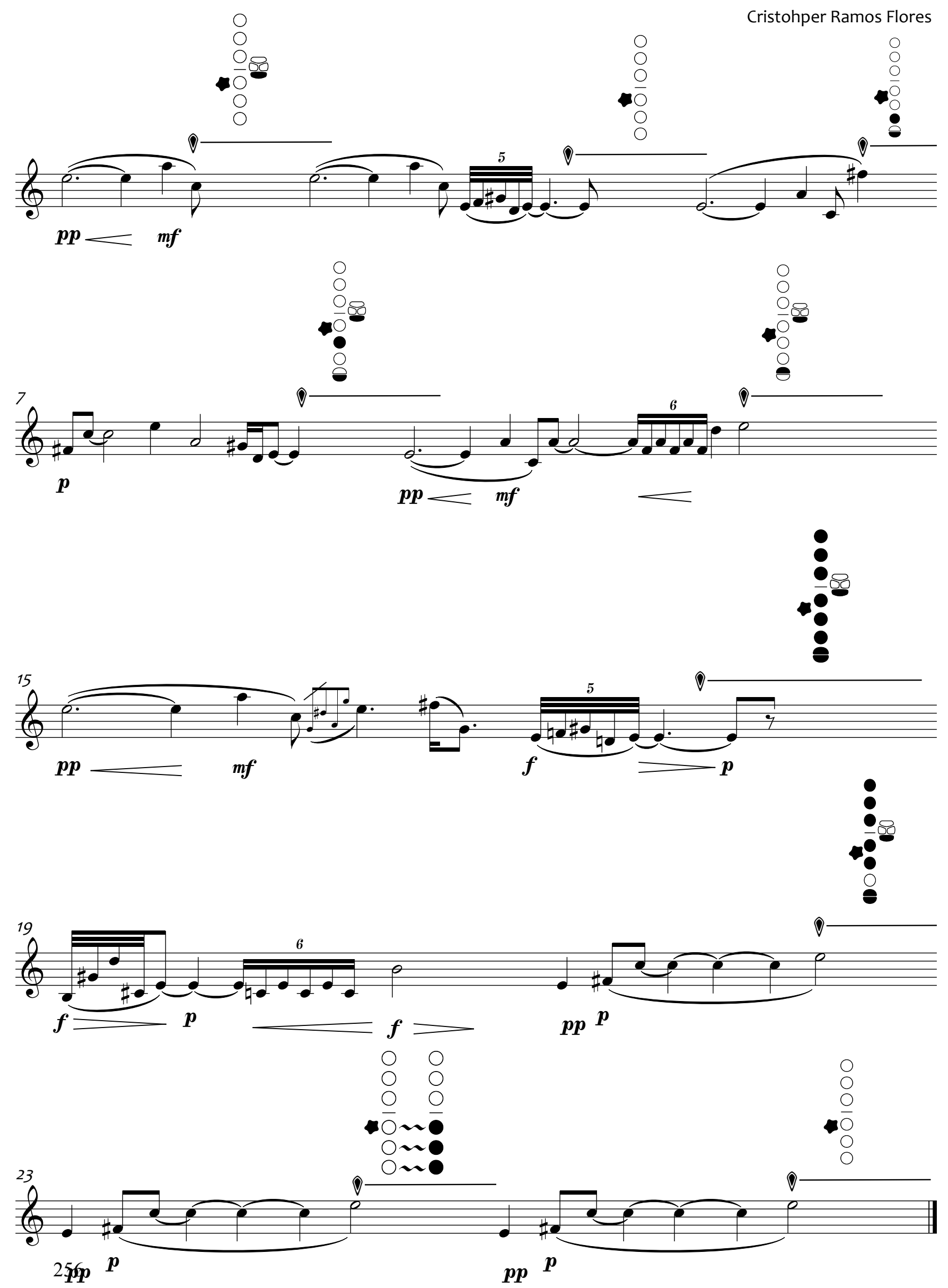

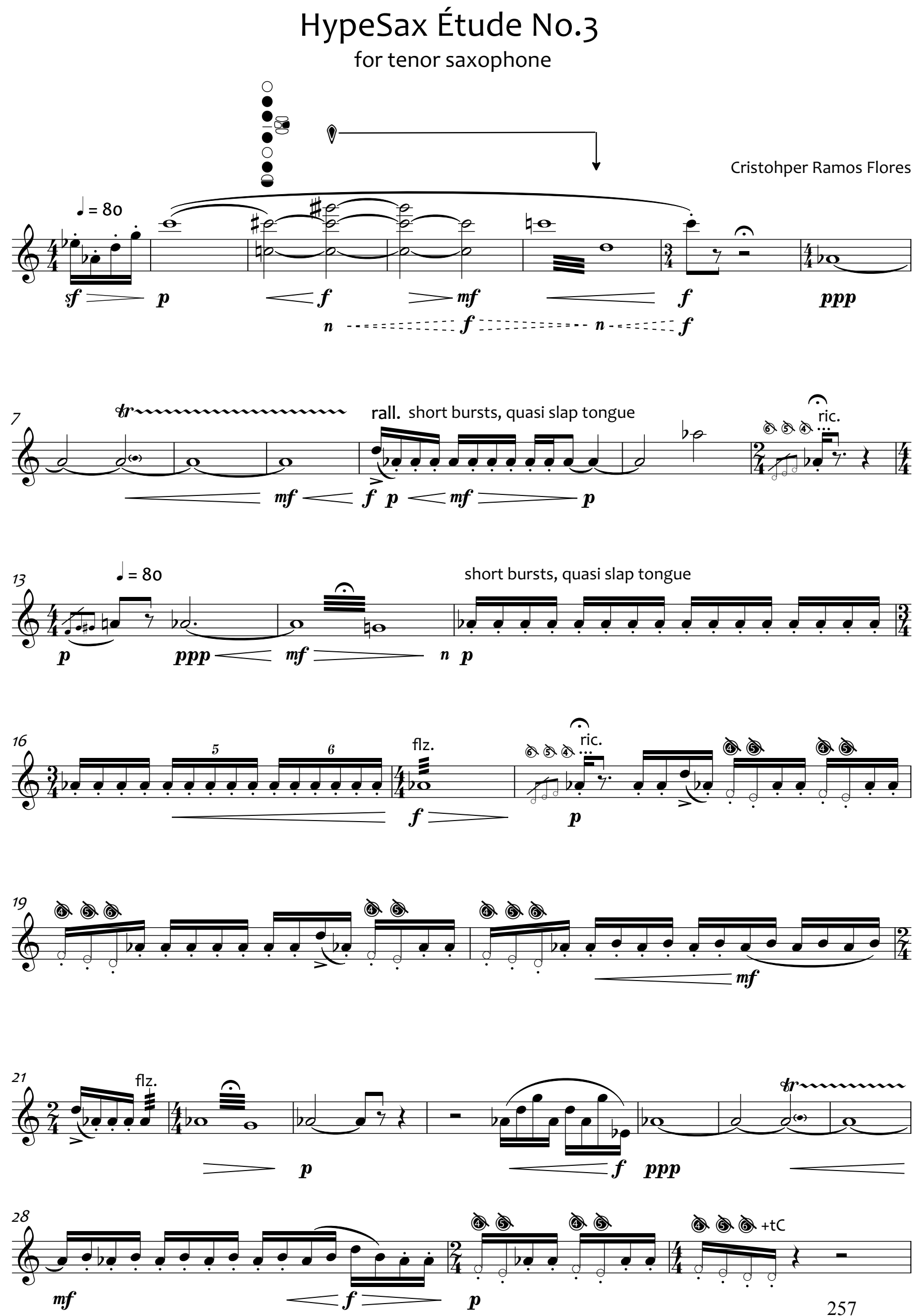
2 (4)!?!?!? ! ? ?

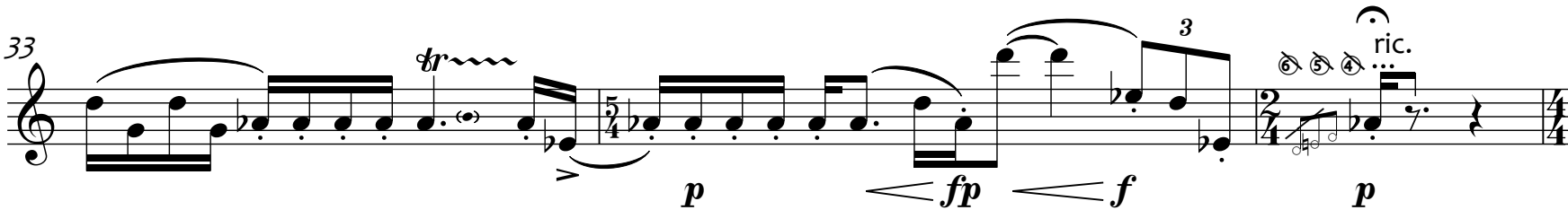
(6) 39 rall. ${ }^{\text {key noise }}$
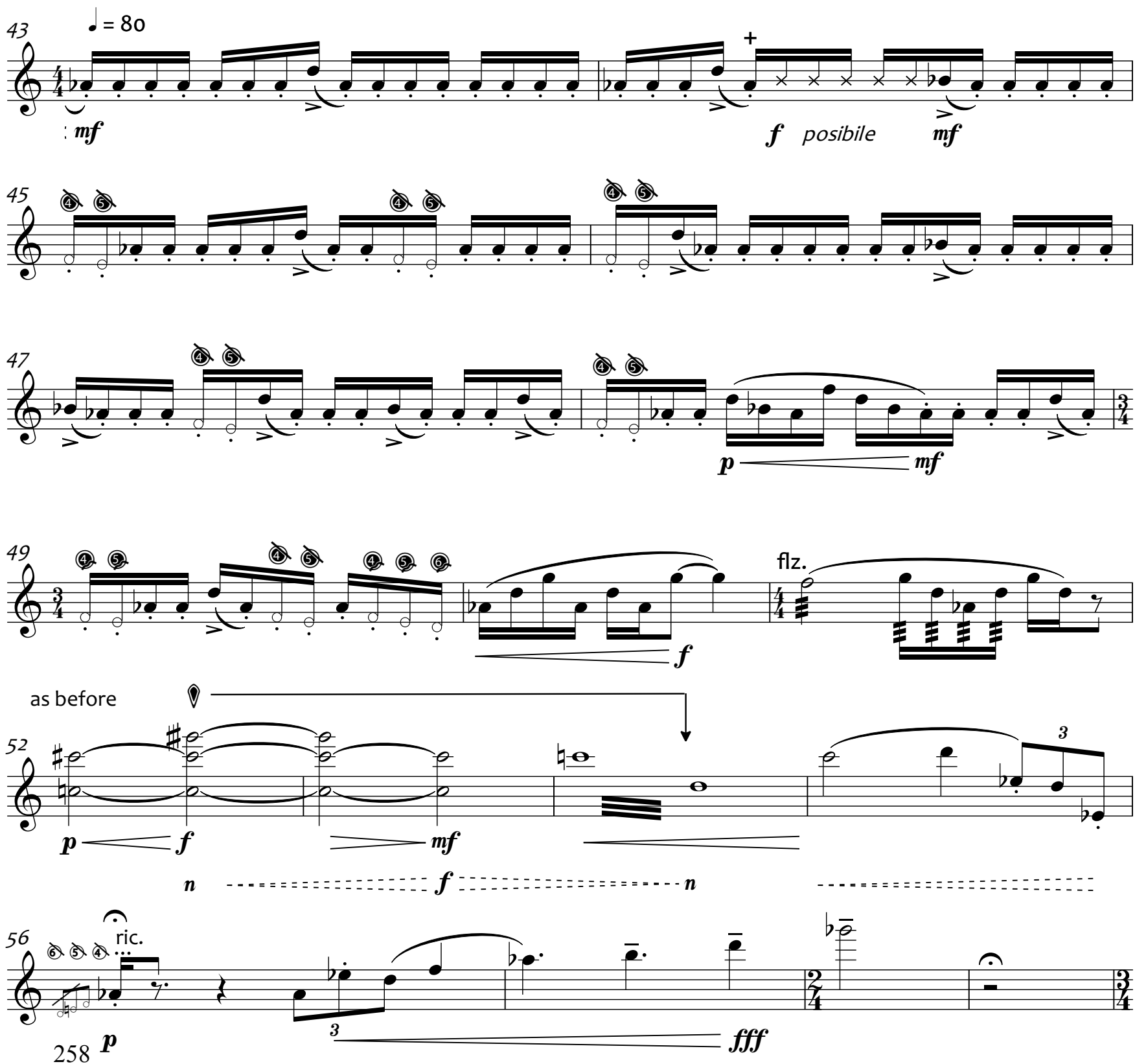

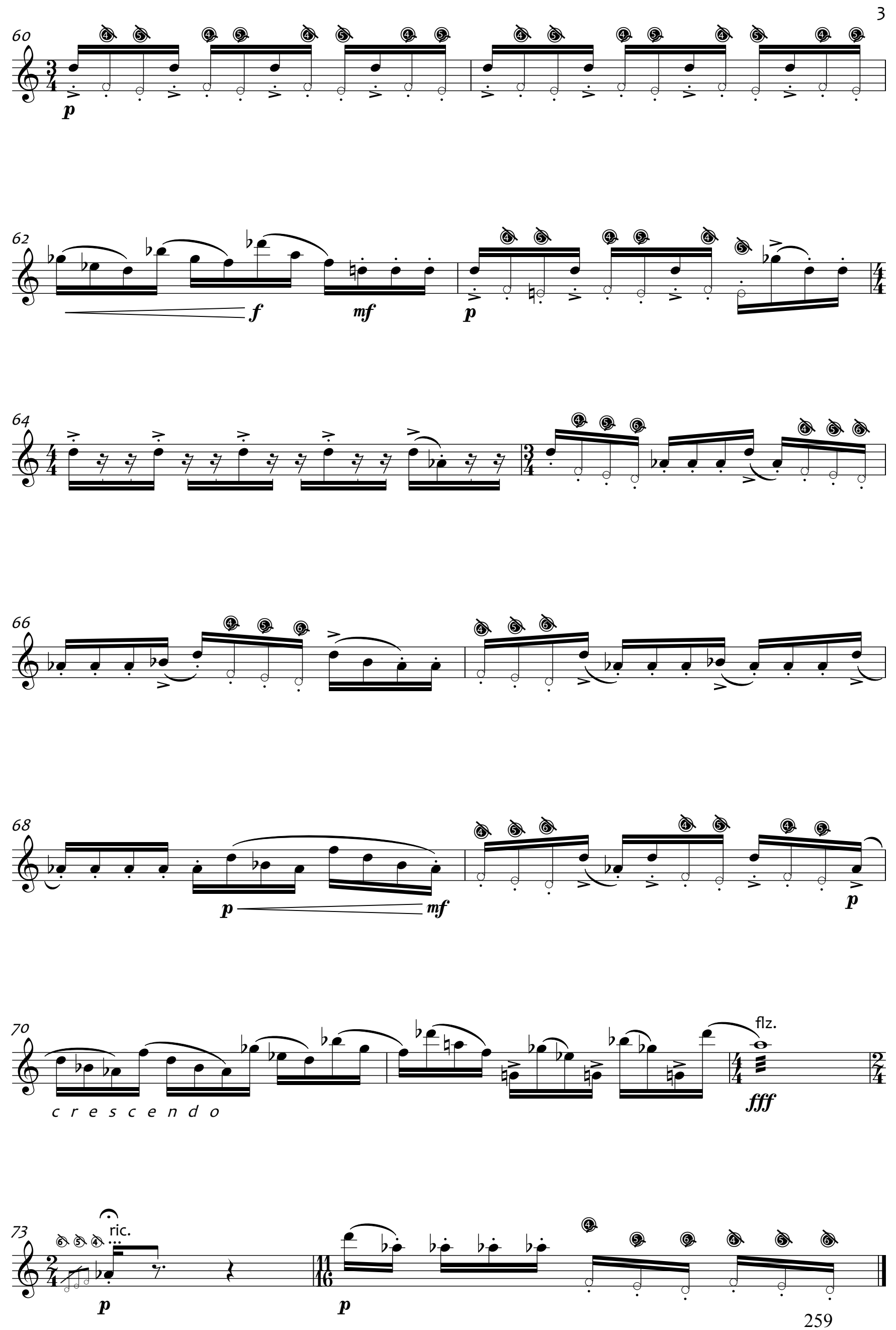
APPENDIX H: MÁS ALLÁ DEL DELIRIO 
Más allá del delirio...

en tu alcoba bipolar

Cristohper Ramos Flores

improvise synth dyanmics with pedal
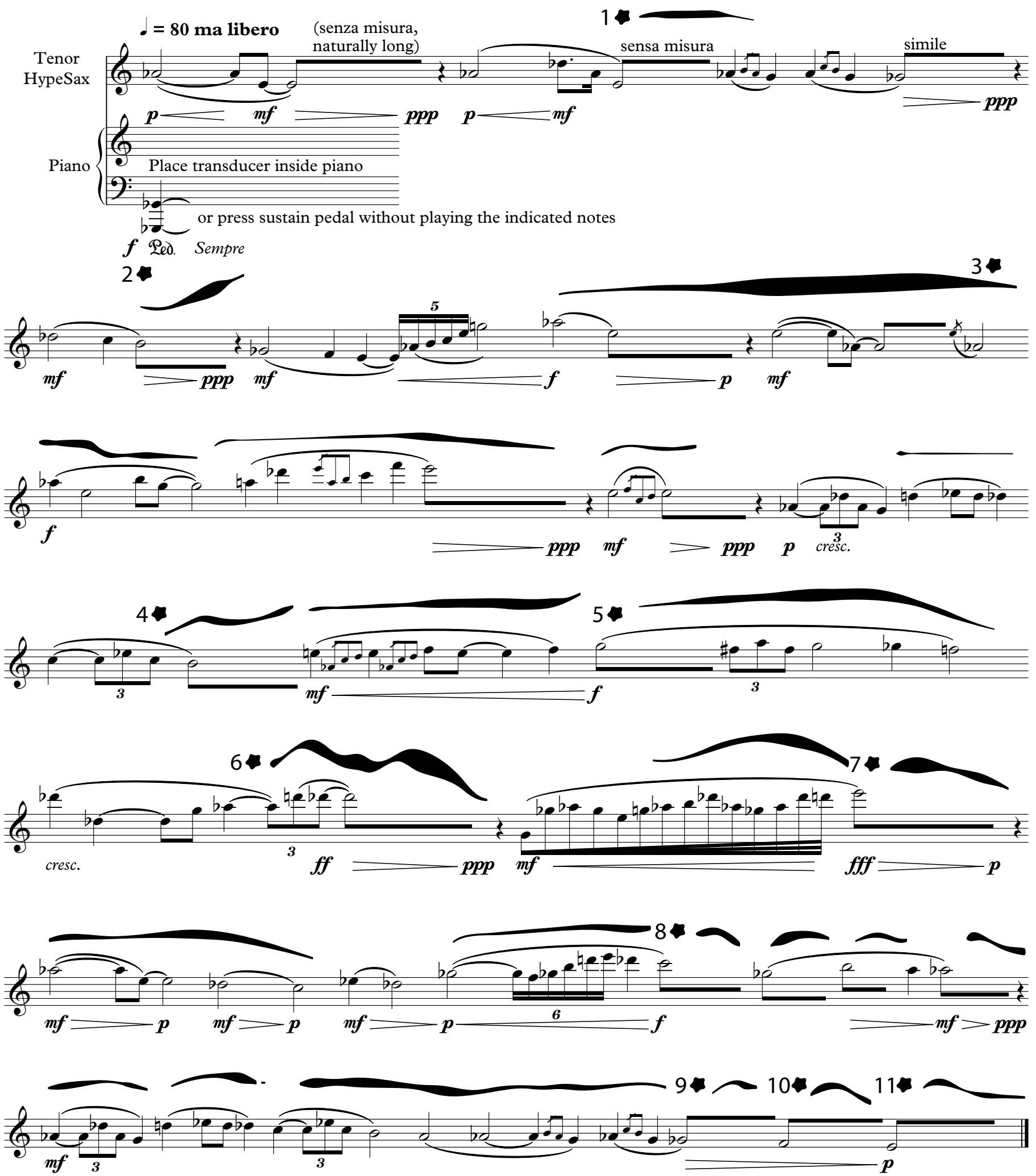


\section{Más allá del delirio... \\ gime un sabor a pena}

Cristohper Ramos Flores $\approx 8 "$. $\approx 8 "$

\section{. $\approx \mathbf{5 0} \mathbf{~ m a}$ ad libitum}
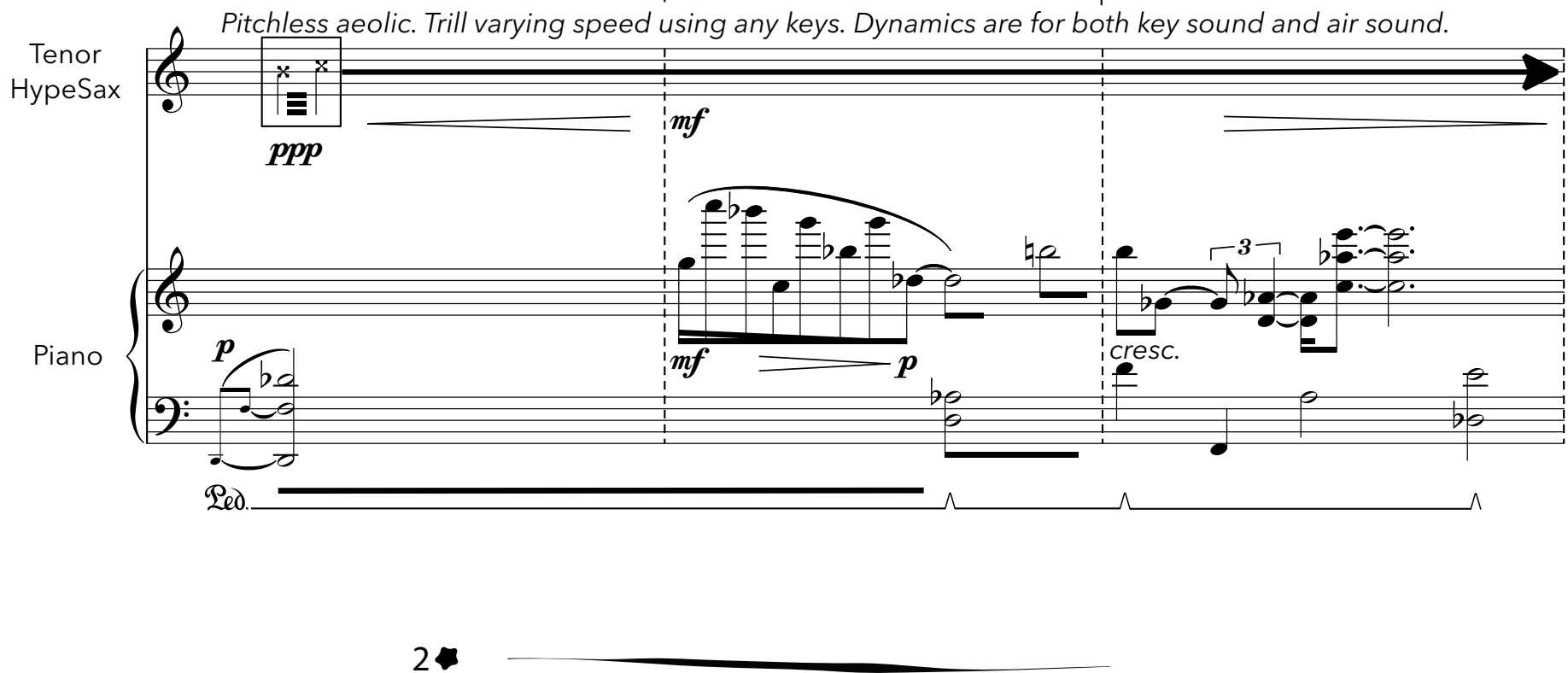

$\approx 10 "$. _ _ _ _ _ _ $\approx 12 "$

$\bullet \approx 50$ ma ad libitum

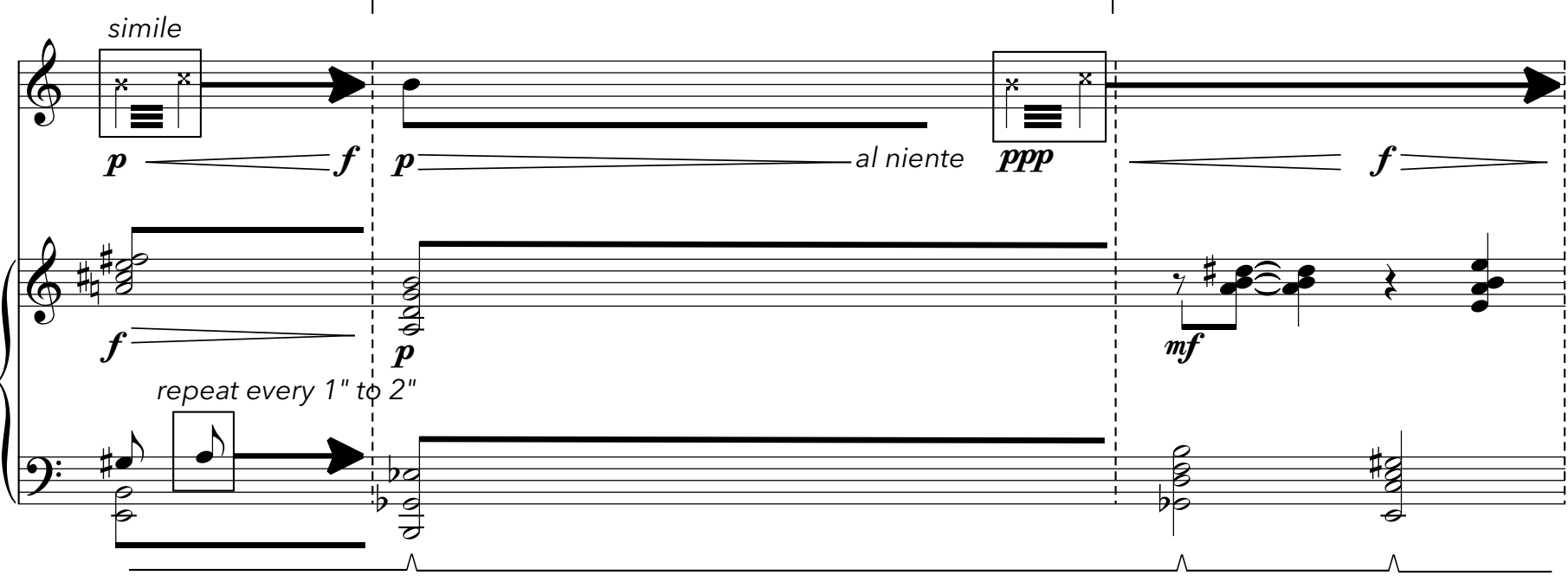

$\approx 12 "$

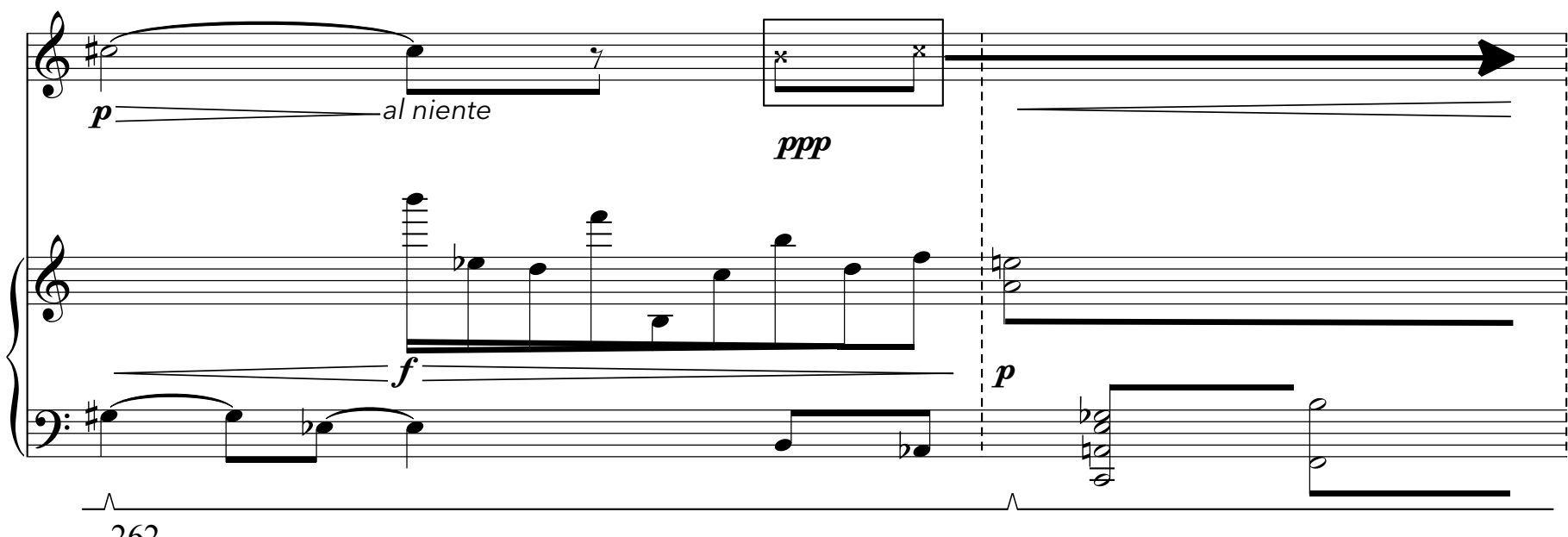



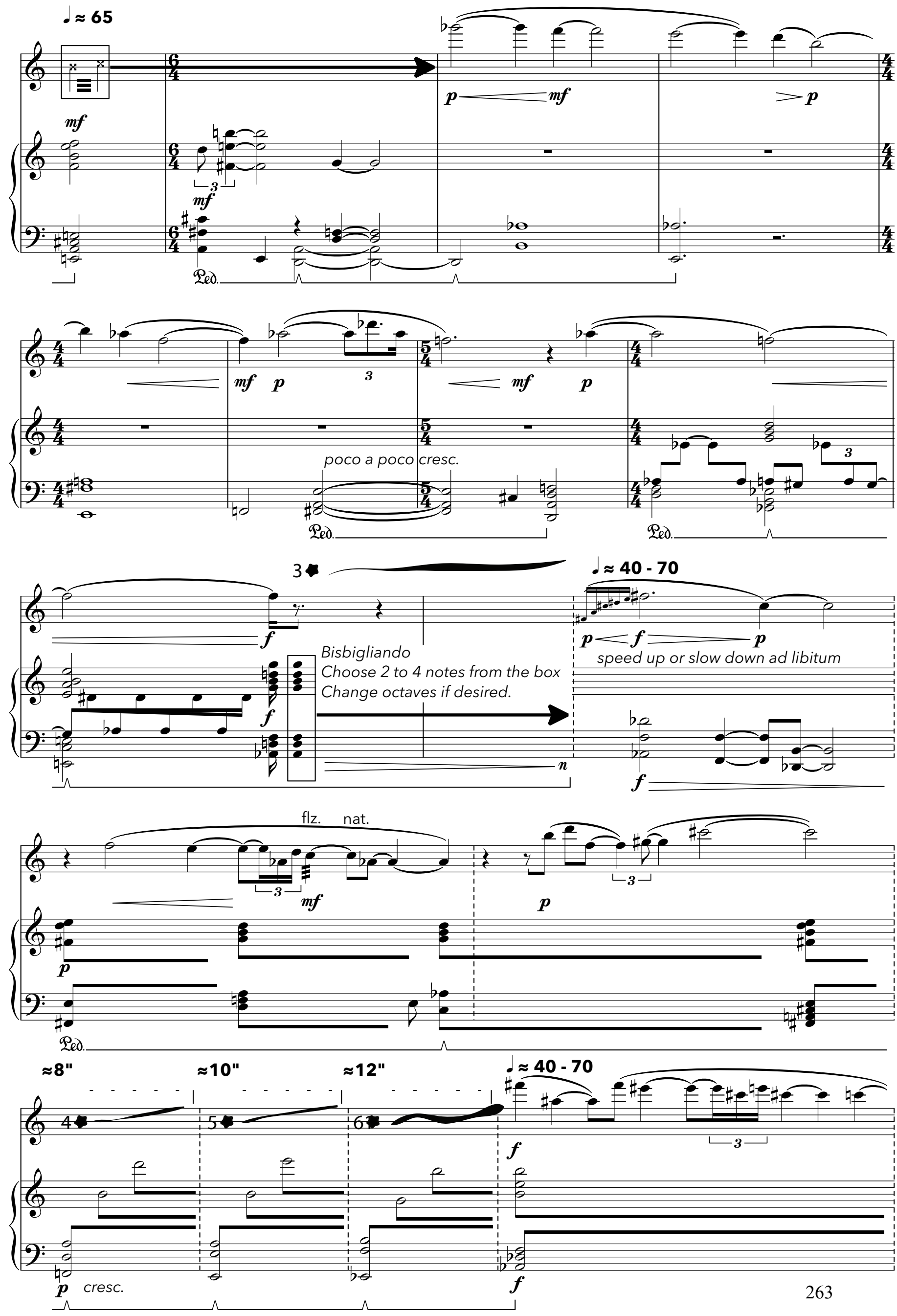

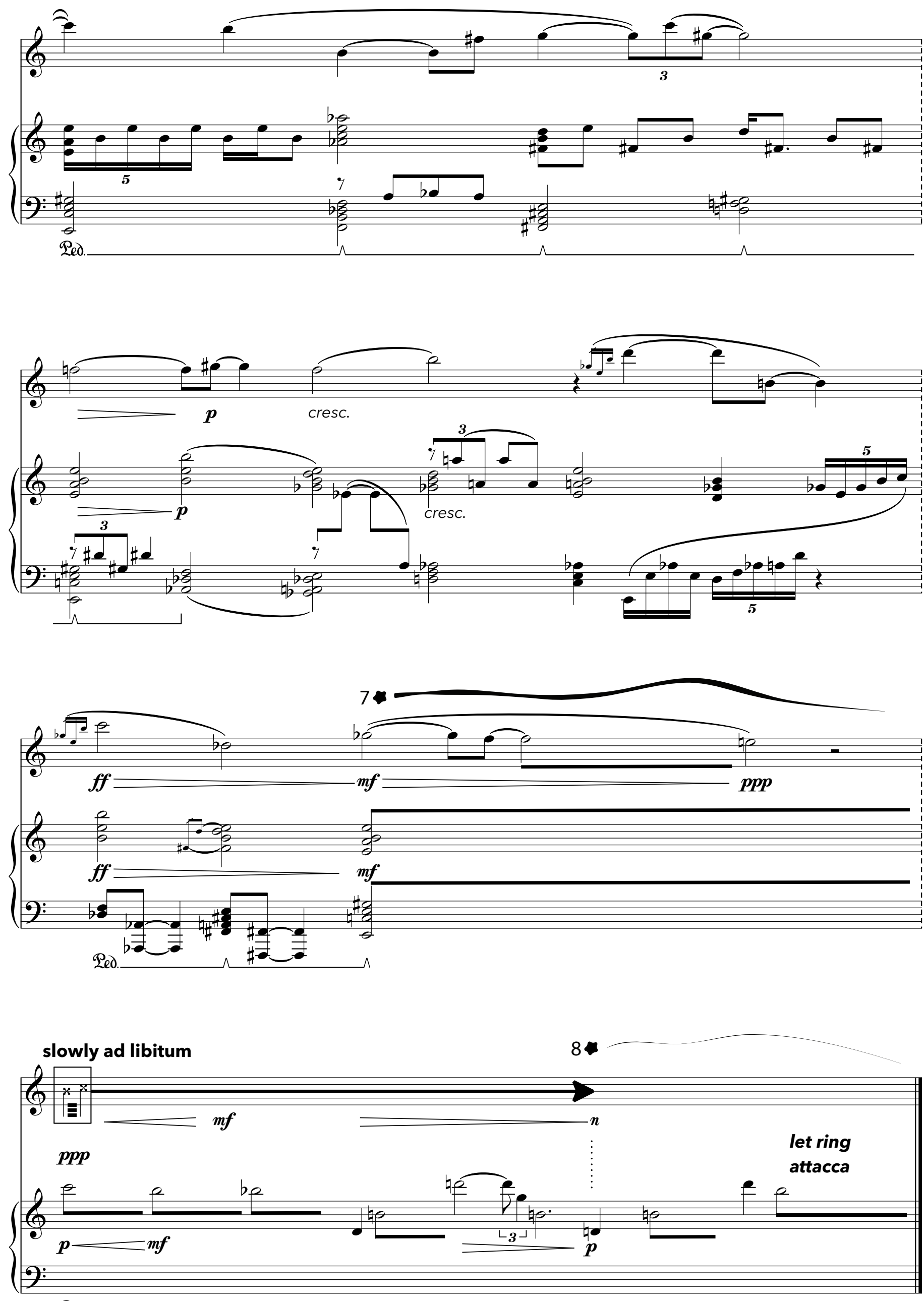


\section{Más allá del delirio... desde un lugar secreto}

Cristohper Ramos Flores
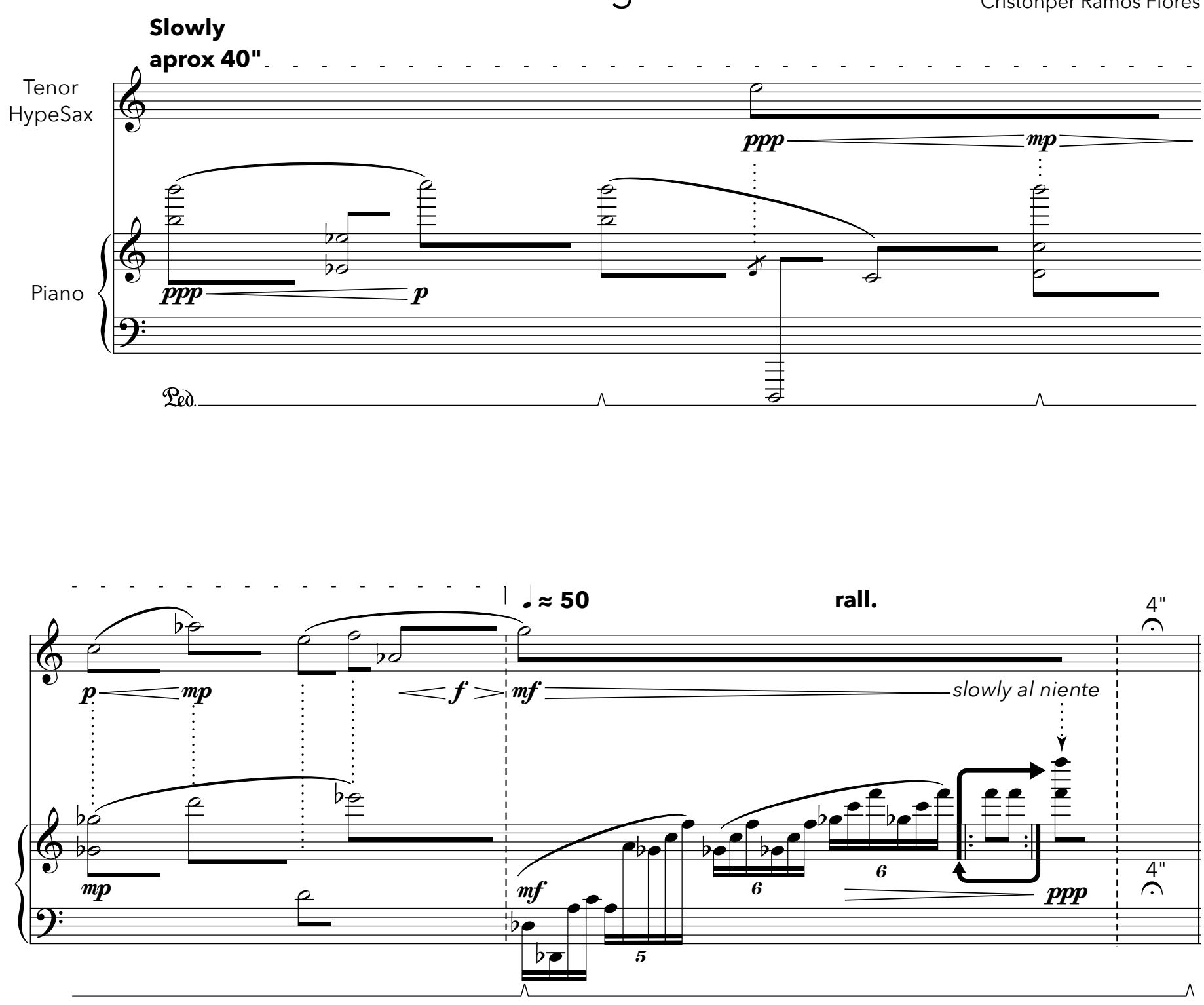

Slowly

aprox. 10".

rall.

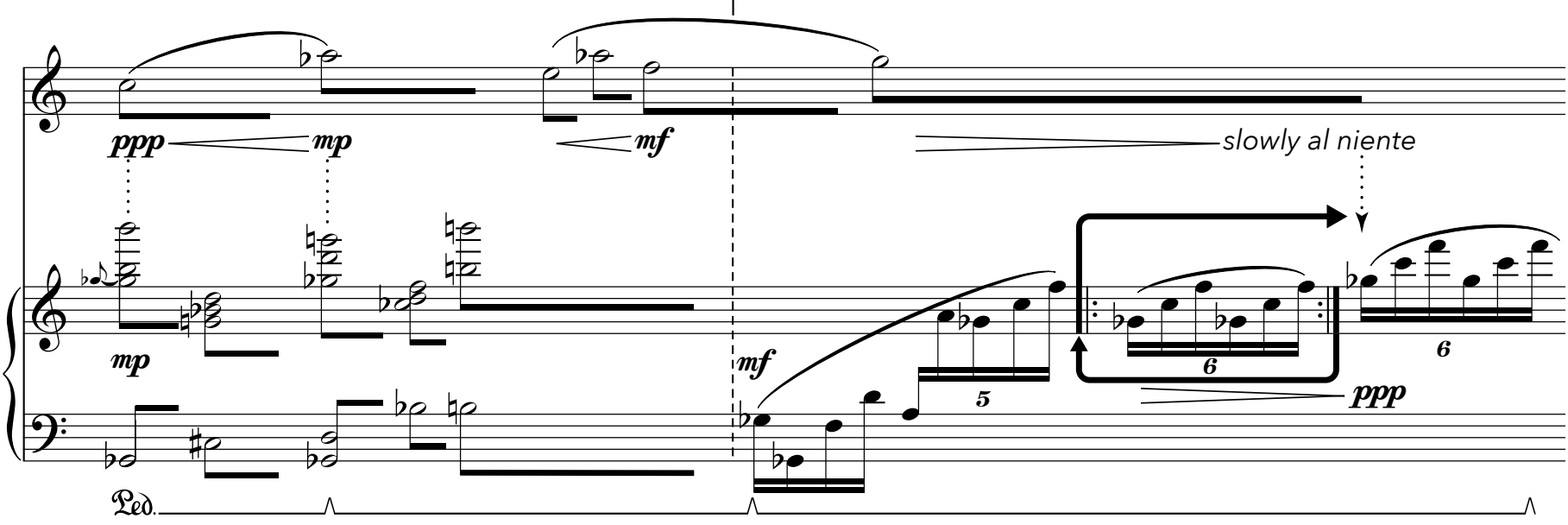



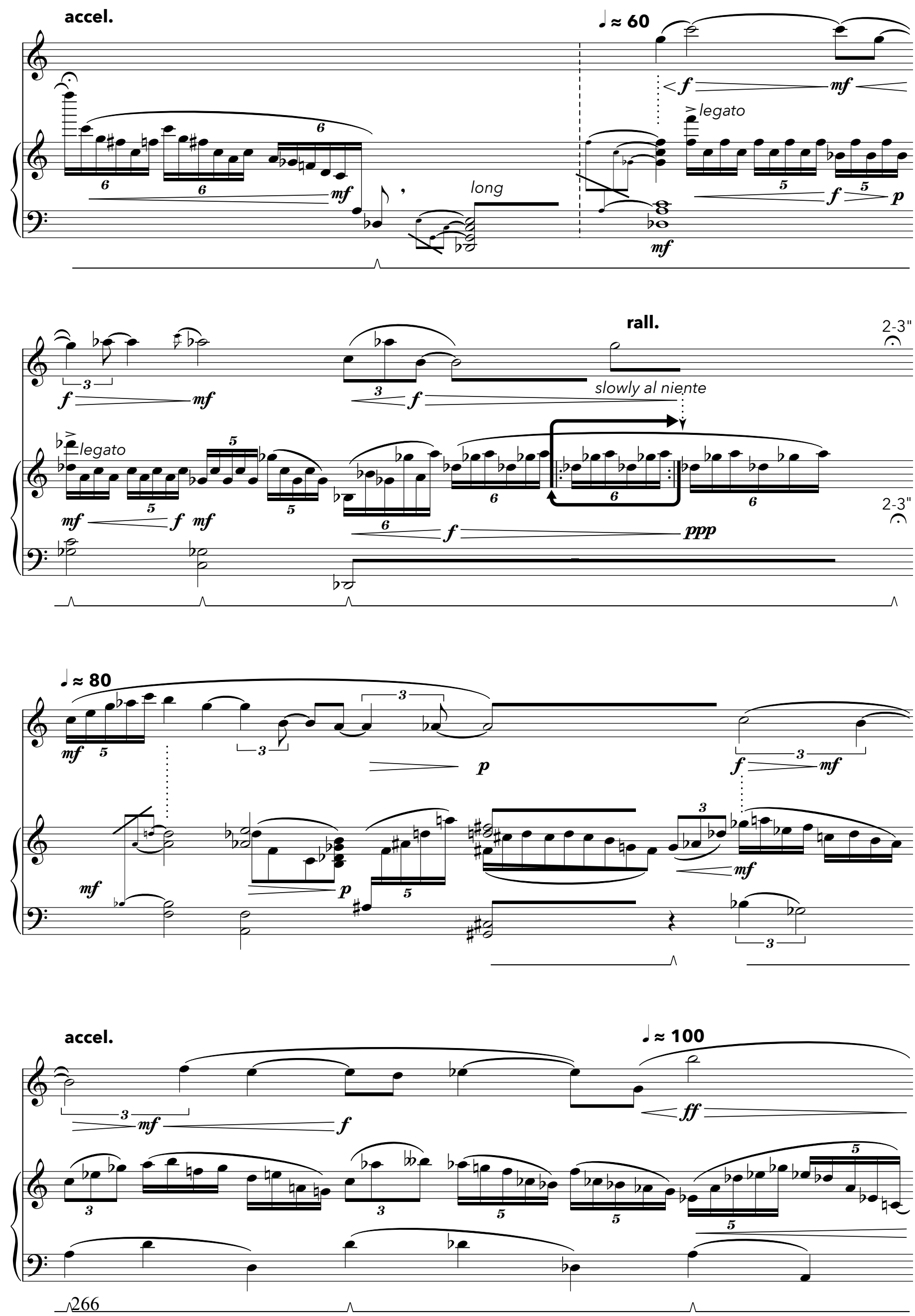

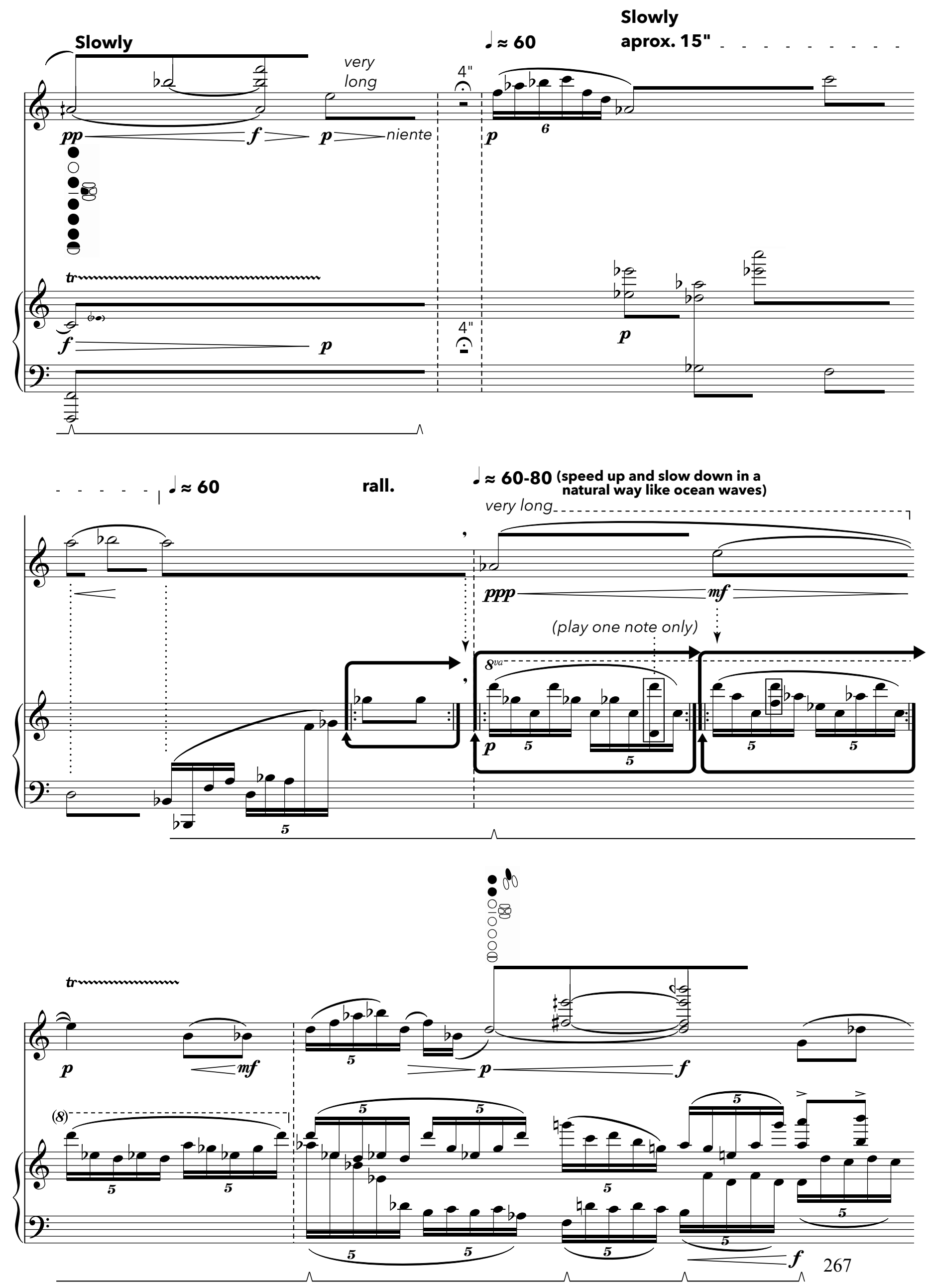
very long.

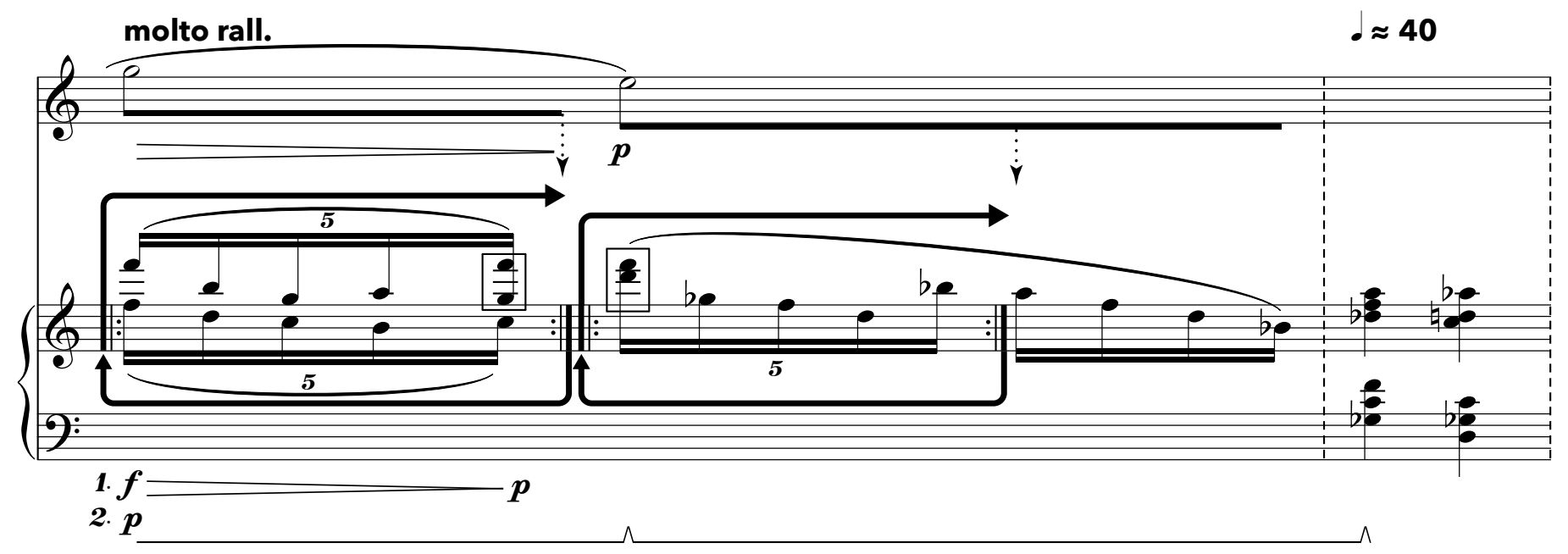

Slowly (long tones in sax, with movement on piano) aprox 40" -
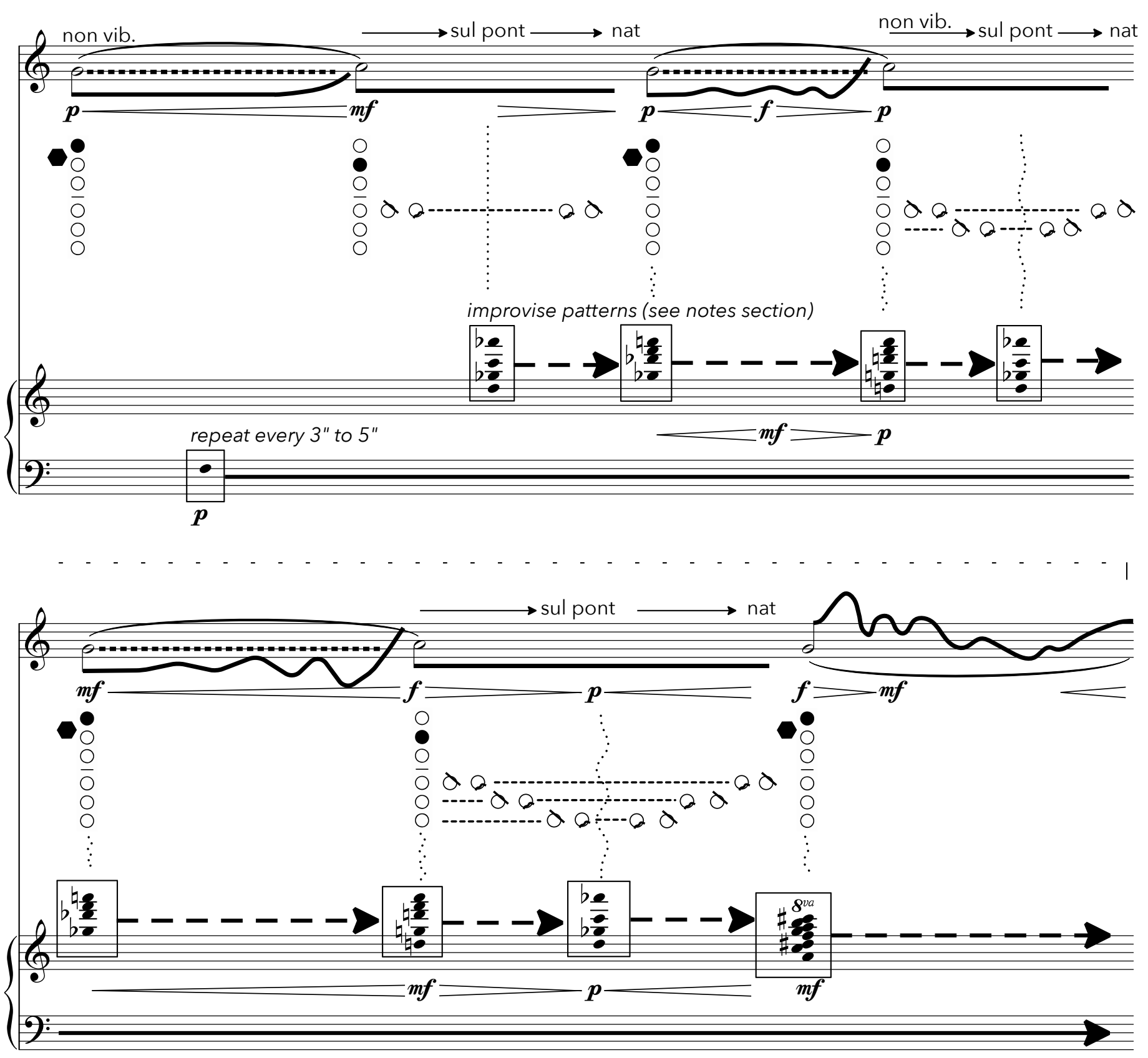


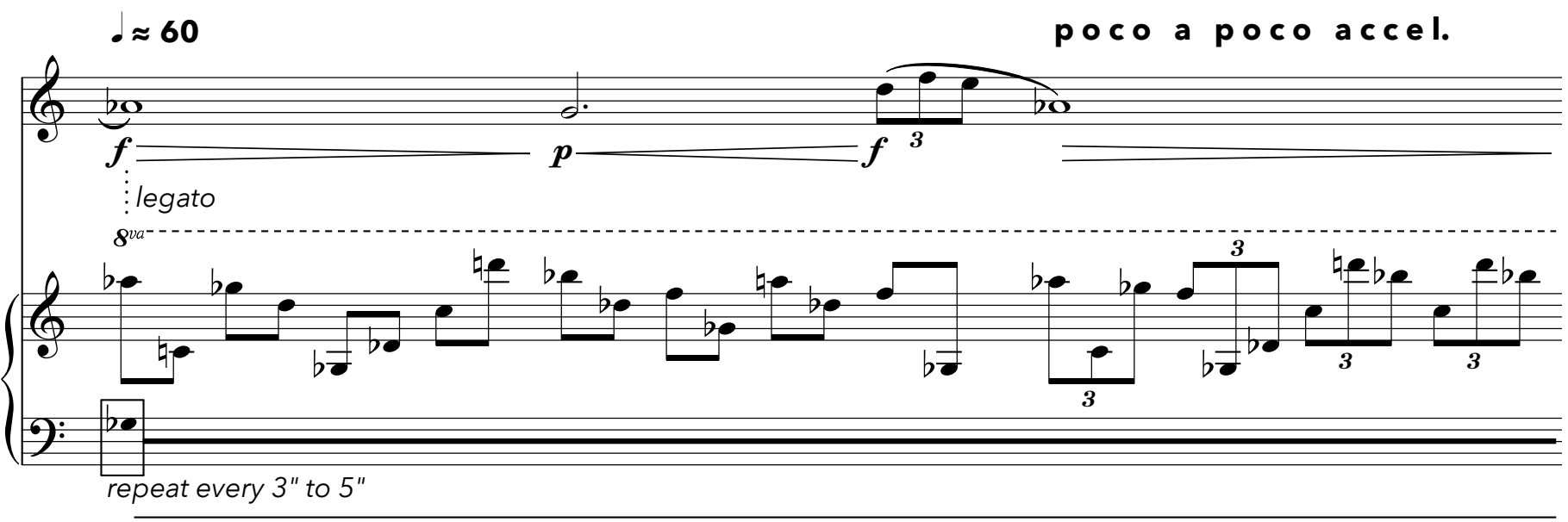

(almost chromatic, non diatonic, as fast as possible)
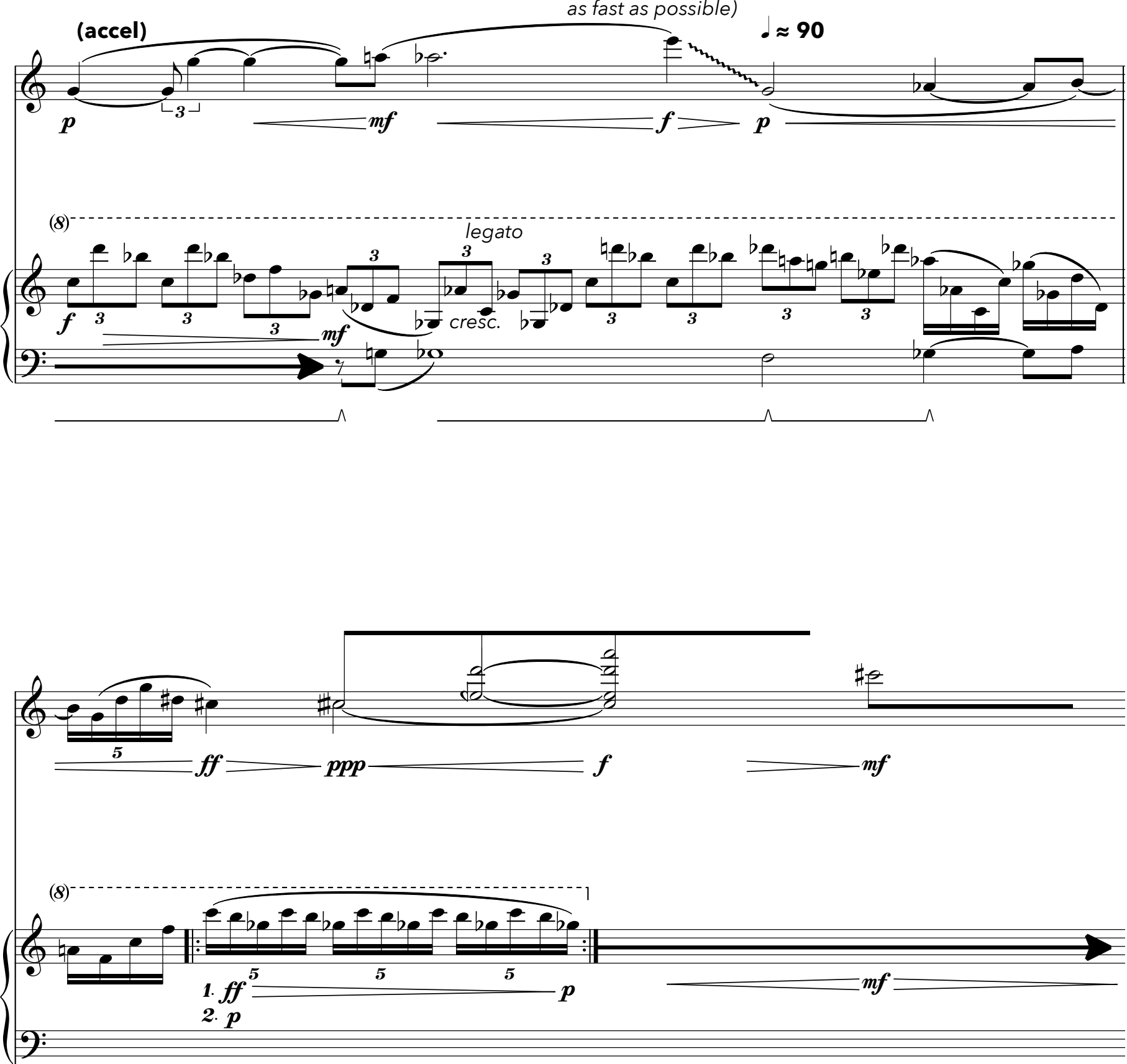

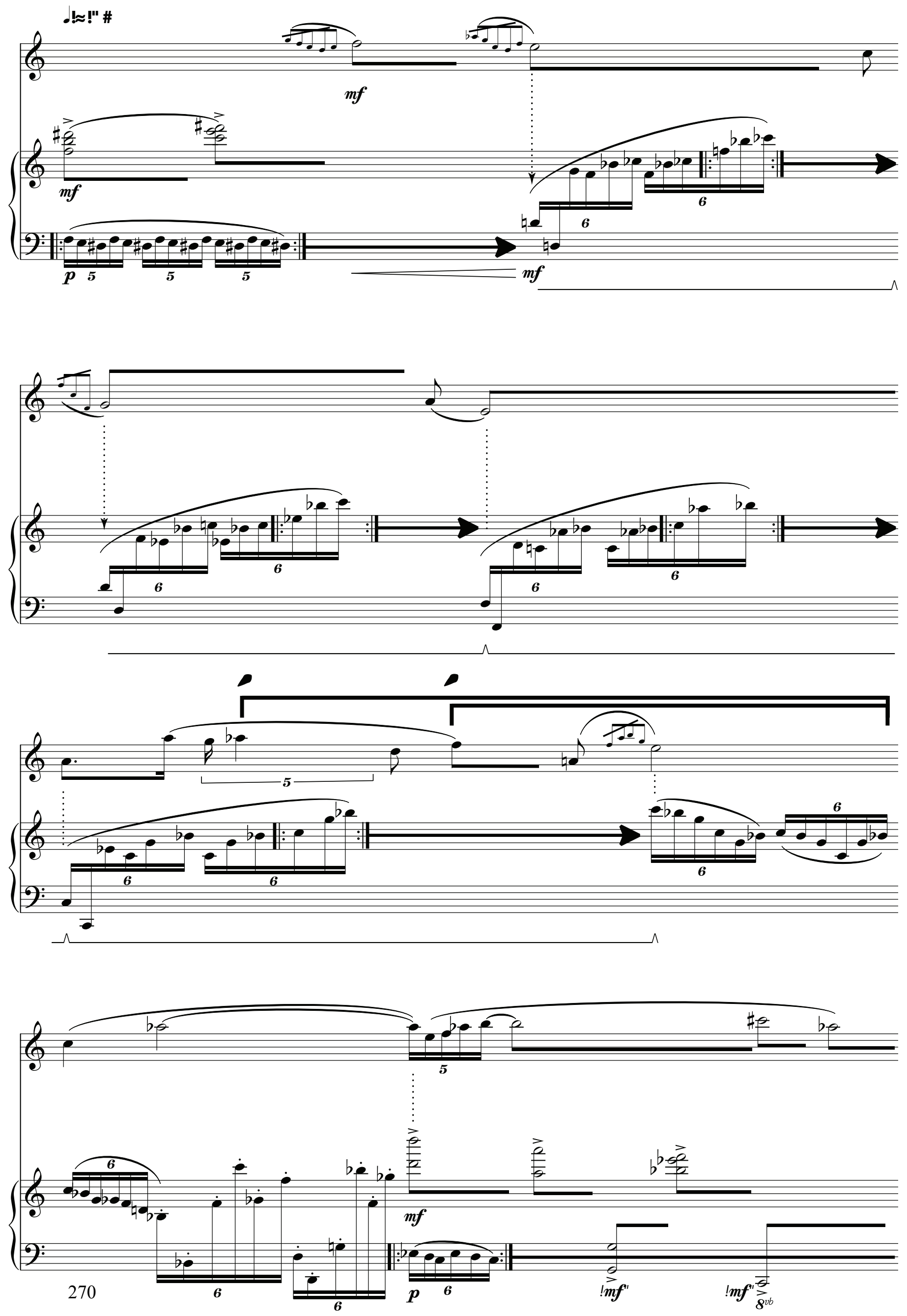

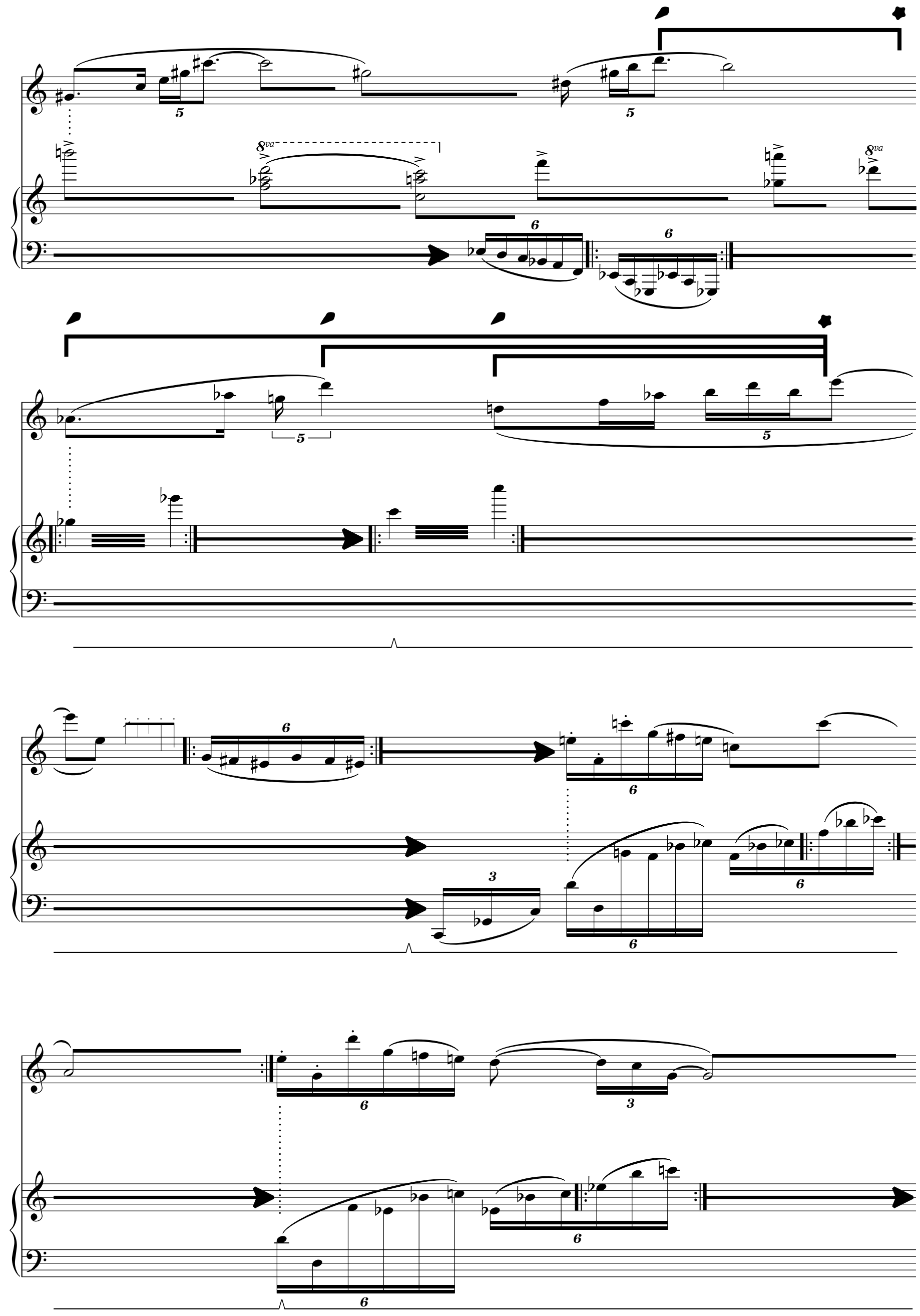


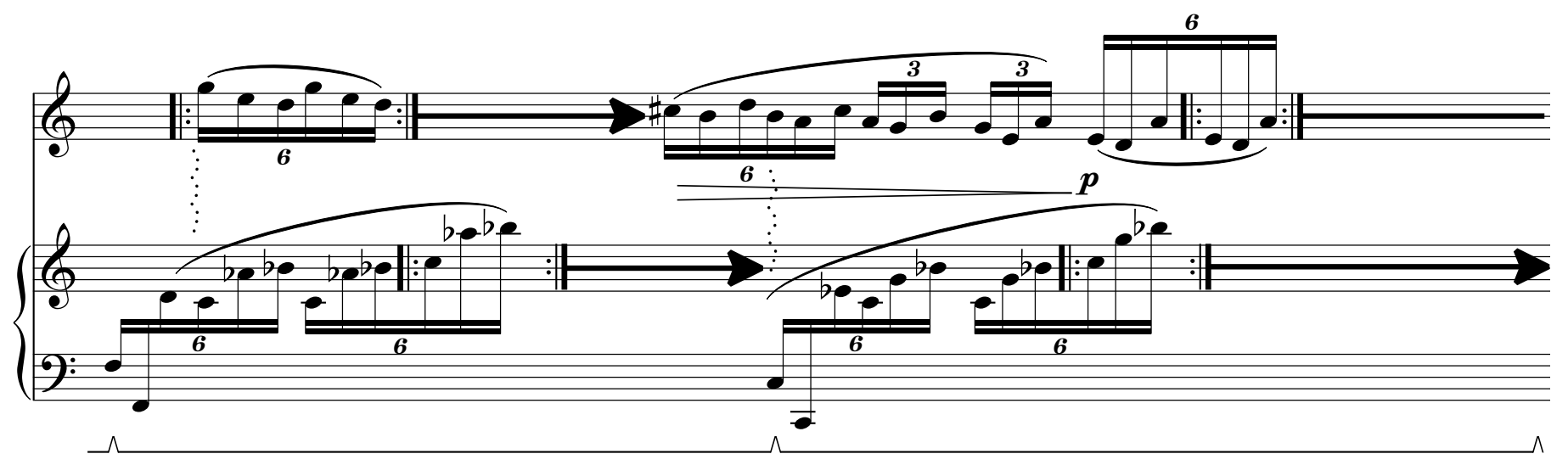

Slowly (piano maintains same speed)

aprox. 25" -

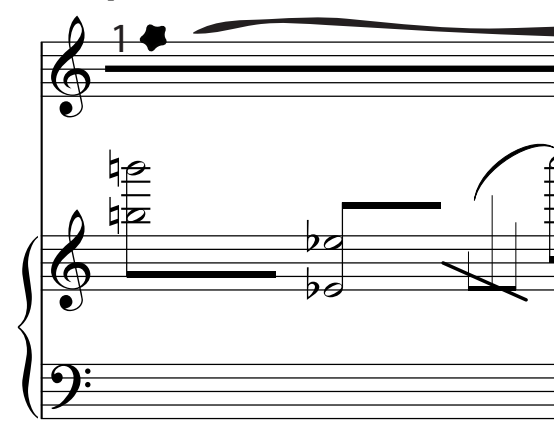

Ted. sempre

()




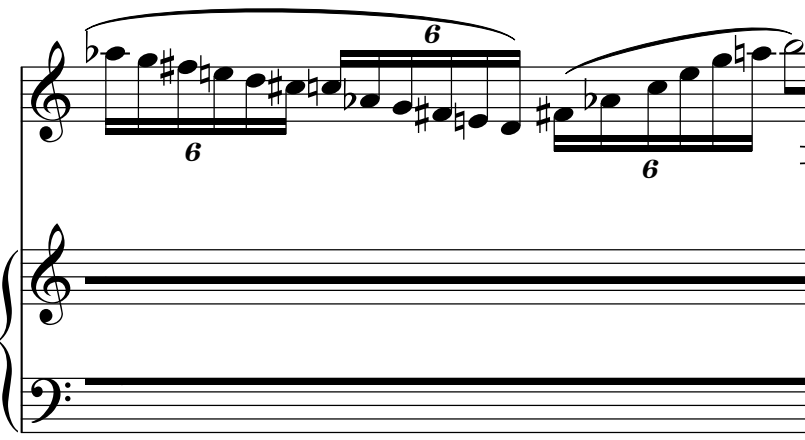

, aprox. 20"
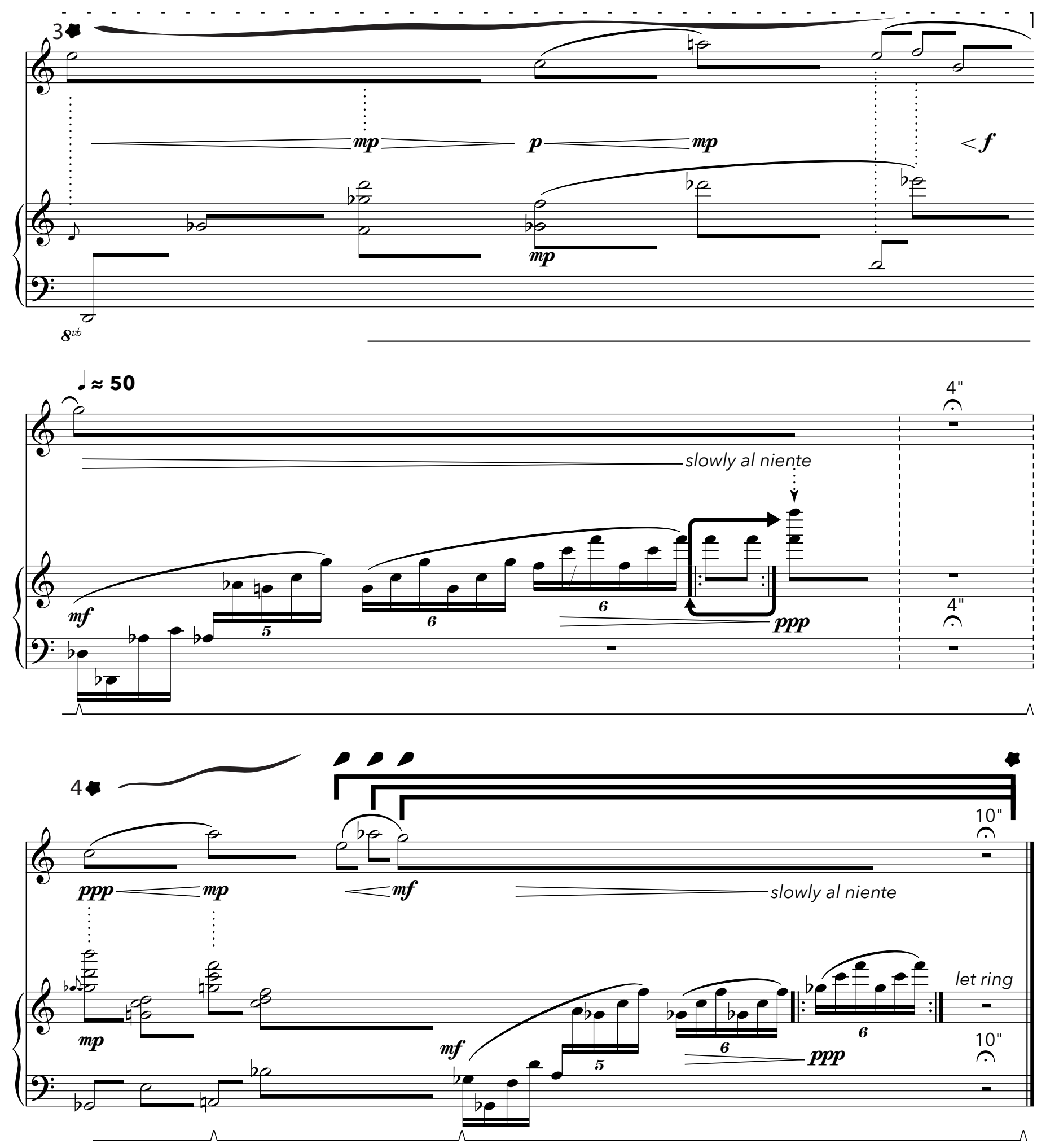
APPENDIX I: LOOK OUT! 


\section{LOOK OUT!}

For HypeSax and three performers on any instrument

\section{INSTRUCTIONS}

\section{Saxophone player}

According to your decisions, the system will propose in which direction the piece should go by displaying images that suggest musical events.

Once you decide you've explored the system's suggestion, press thumb button 1 to indicate the system that you are ready to move to a second module.

The system is tracking your sound and your movements, make sure you feel like having fun and explore what happens when you interact with the HypeSax.

\section{Ensemble}

Improvise freely!

The HypeSax play will lead throughout the performance.

To view the score on a secondary device such as a laptop, tablet, cellular phone or $\mathrm{TV}$, visit the indicated website.

Be free to perform the suggested graphics in any way you want. 
Screenshot of the Max patch

\section{LOOK OUT!}
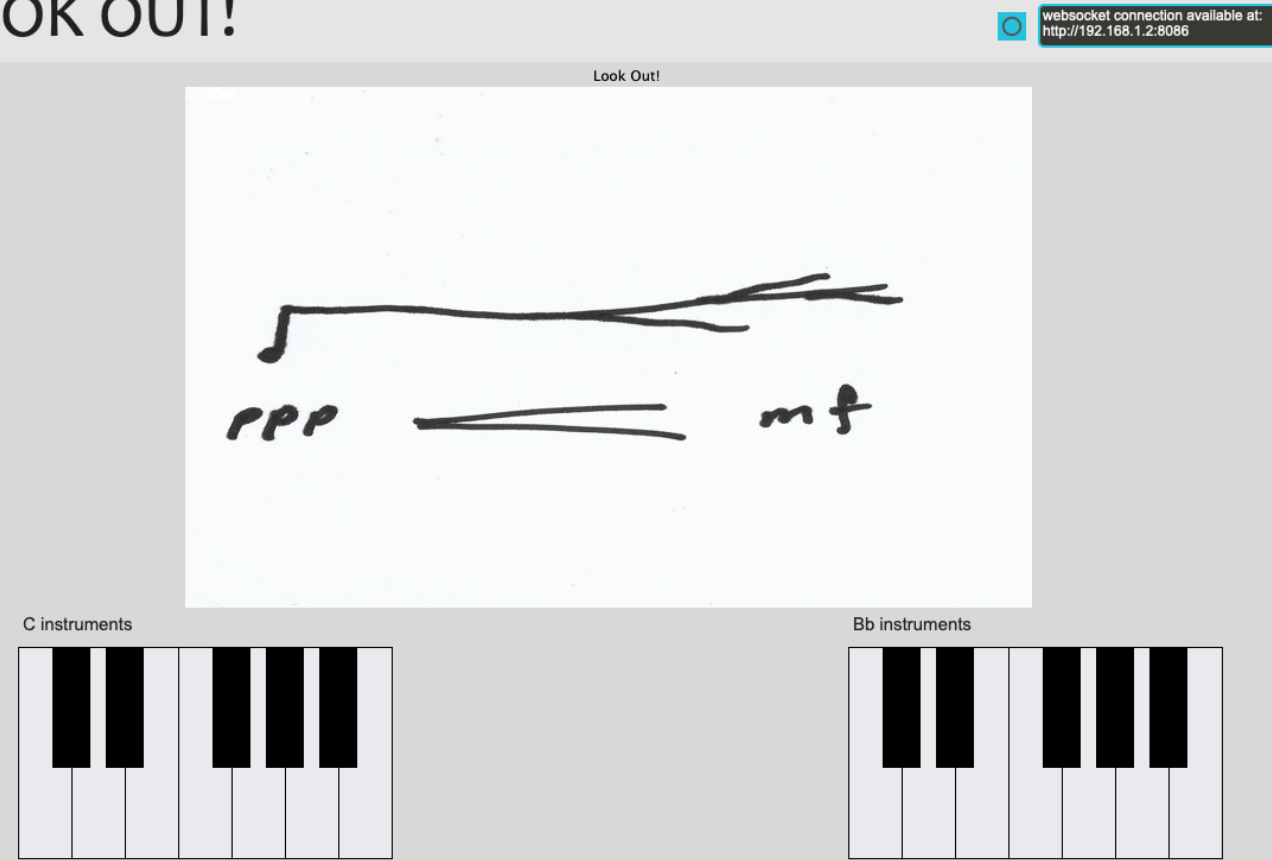

$\mathrm{Bb}$ instruments

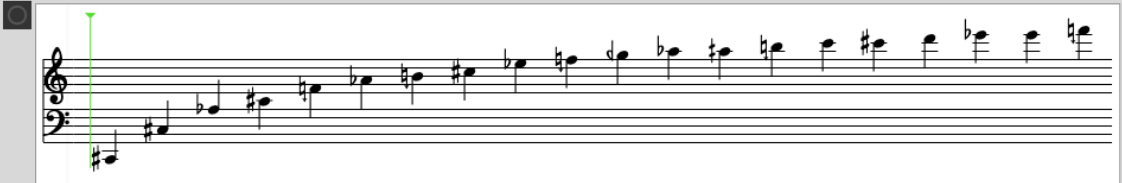


APPENDIX J: BREATHE IN, BREATHE OUT 


\section{Performance notes}

\section{THESE NOTES AND SCORE ARE SUGGESTIONS, DISREGARD THEM AT WILL.}

Performers should synchronise their breathing pulse as much as posible. One player leads the tempo but the ensemble should gesture the breathing pulse together whenever possible.

$=$ Play for a long as the line extends.

$=$ Gradually change to the indicated technique.

$=$ Breathing cycle clef. The duration of the bar is the same as the duration of the breathing cycle of the leading performer. This duration can be variable and the rhythmic figures are represent aproximate proportions of the bar.

\section{Saxophone}

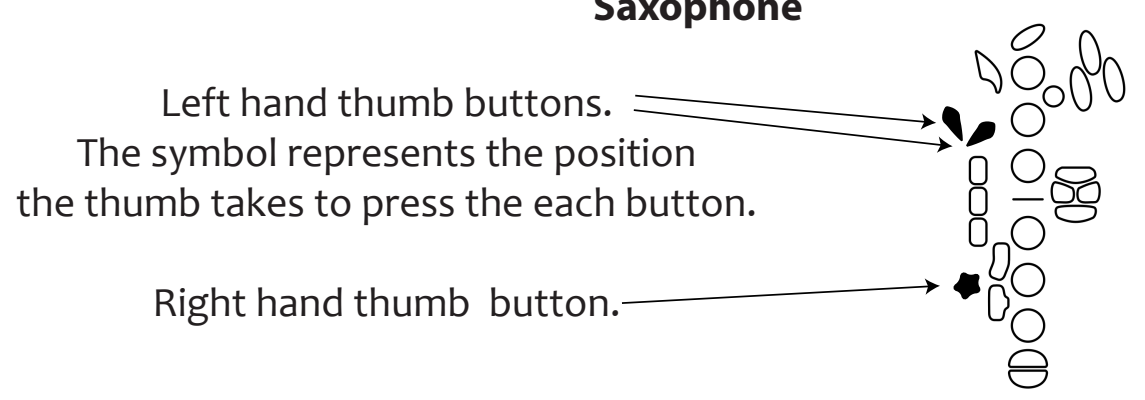

Thumb buttons should only be pressed once to activate or deactivate a function.

Fingering to produce feedback using pedal dynamics
Approximate feedback pitch. Concert pitch in score, transposed in part matching saxophone.

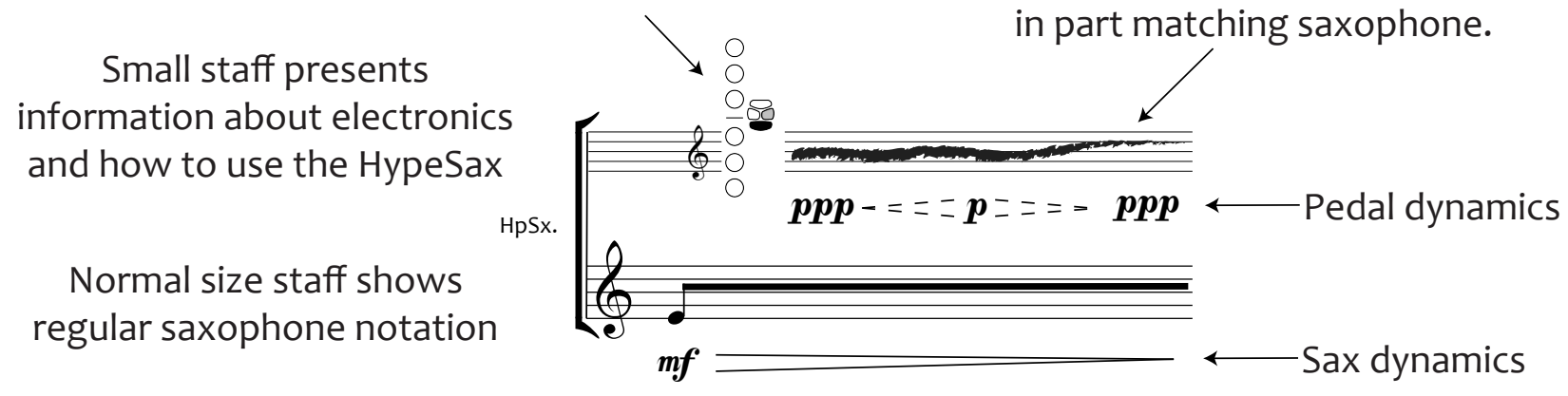

or 
= Short attack with combined with percussion sound (i.e. staccatto + key click, slap tongue, tongue ram, kissing) Change technique freely. It's ok to avoid pitch but, if there is pitch, it should be what is asked for.

Rapidly close keys next to arrow.

= Keys marked closed or opened in previous fingering are not affected.
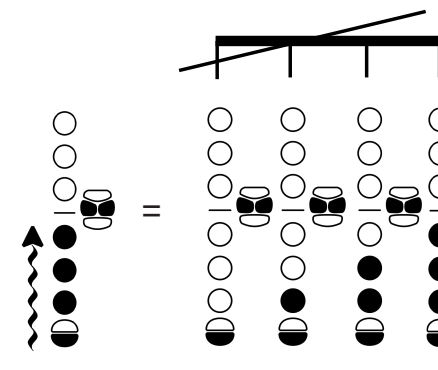

\}

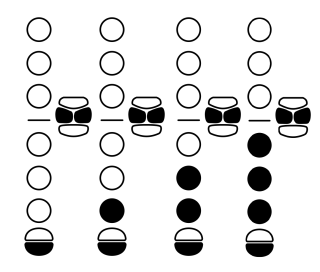

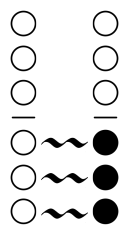

= Key tremolo. Open and close indicated key rapidly to create a natural sound or feedback sound trill effect.

HypeSax setup information can be found in the Breath in, Breath out Max patch

\section{Strings}

ric. = Ricochet. Let bow bouce freely, fast or slow.

RicCLB = Ricochet col legno battuto hitting the string around the indicated zone without using left hand.

$\Pi=$ = Over pressure, allow for scratch tone

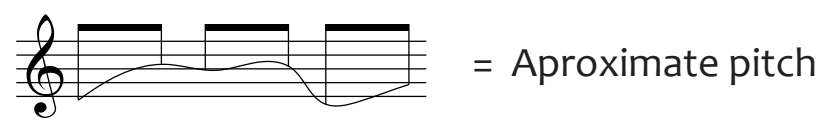

String staff. This staff represents the lenght of the strings, where the lower line indicates the top of the fingerboard and the higher line indicates the bridge. The clef indicates the string or strings to be used.

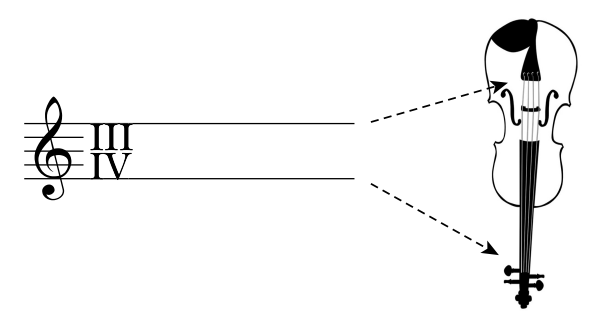




\section{Breathe in, breathe out}

Slowly (deep breathing cycle)

Cristohper Ramos Flores

Saxophone player cues tempo
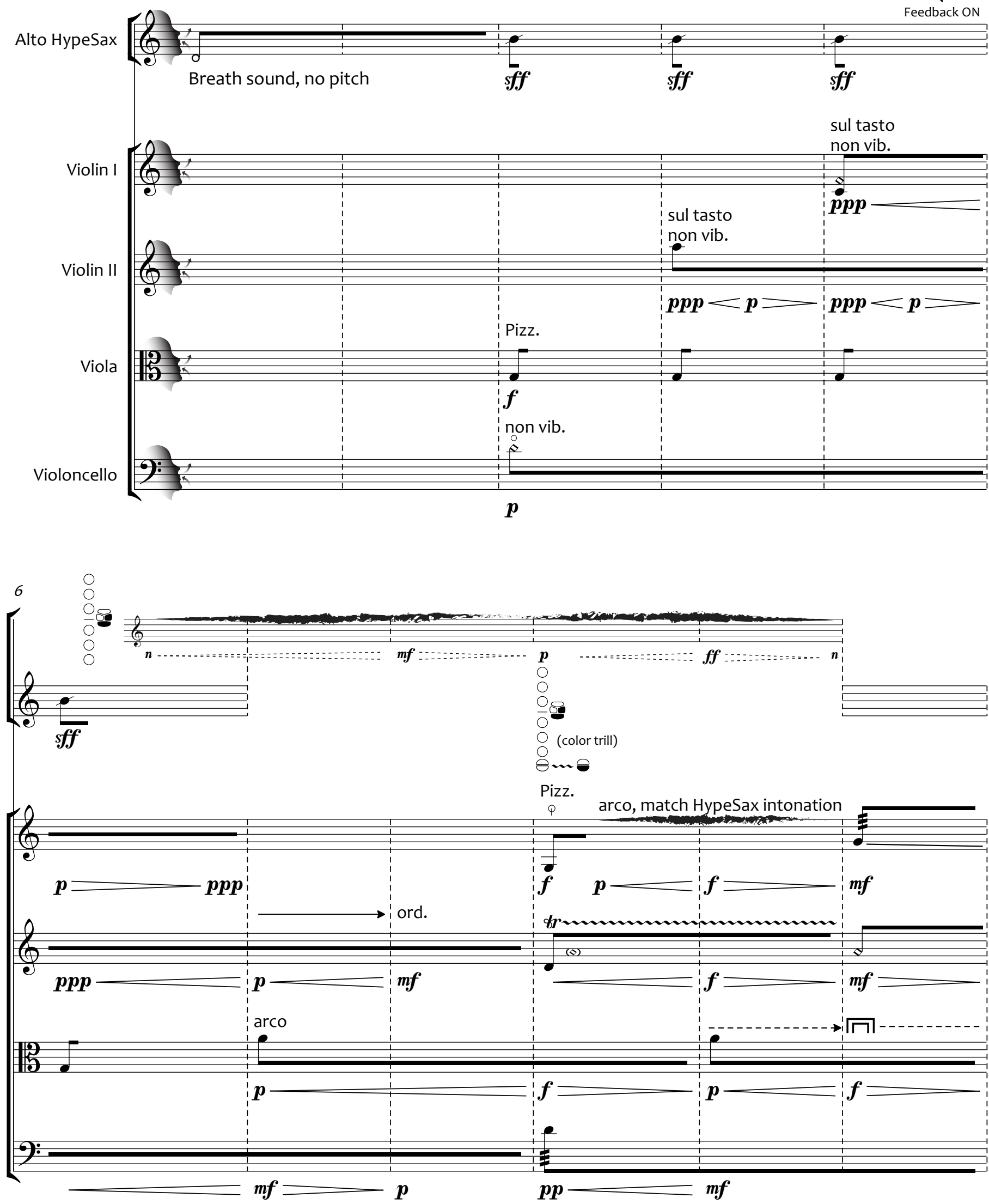

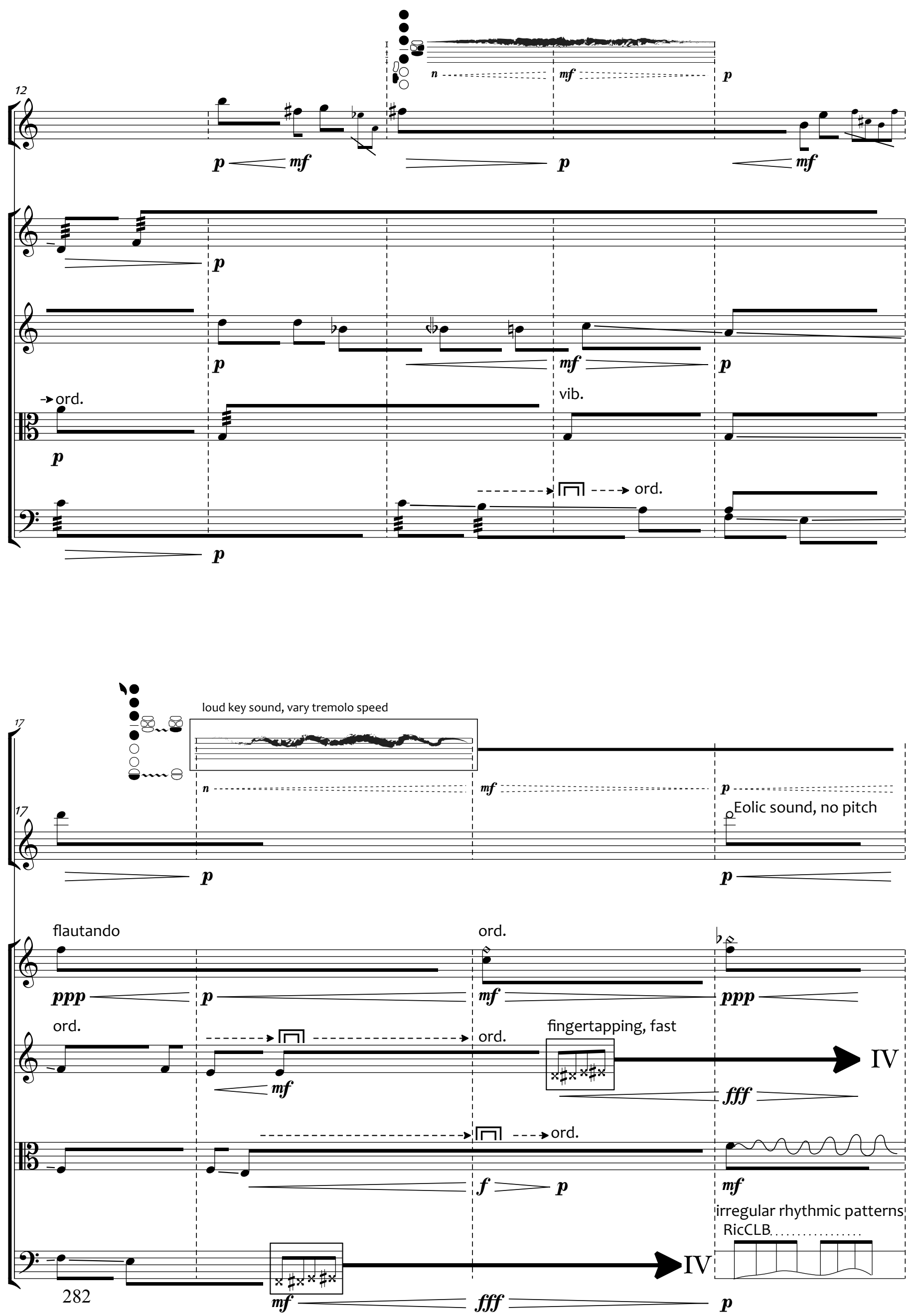

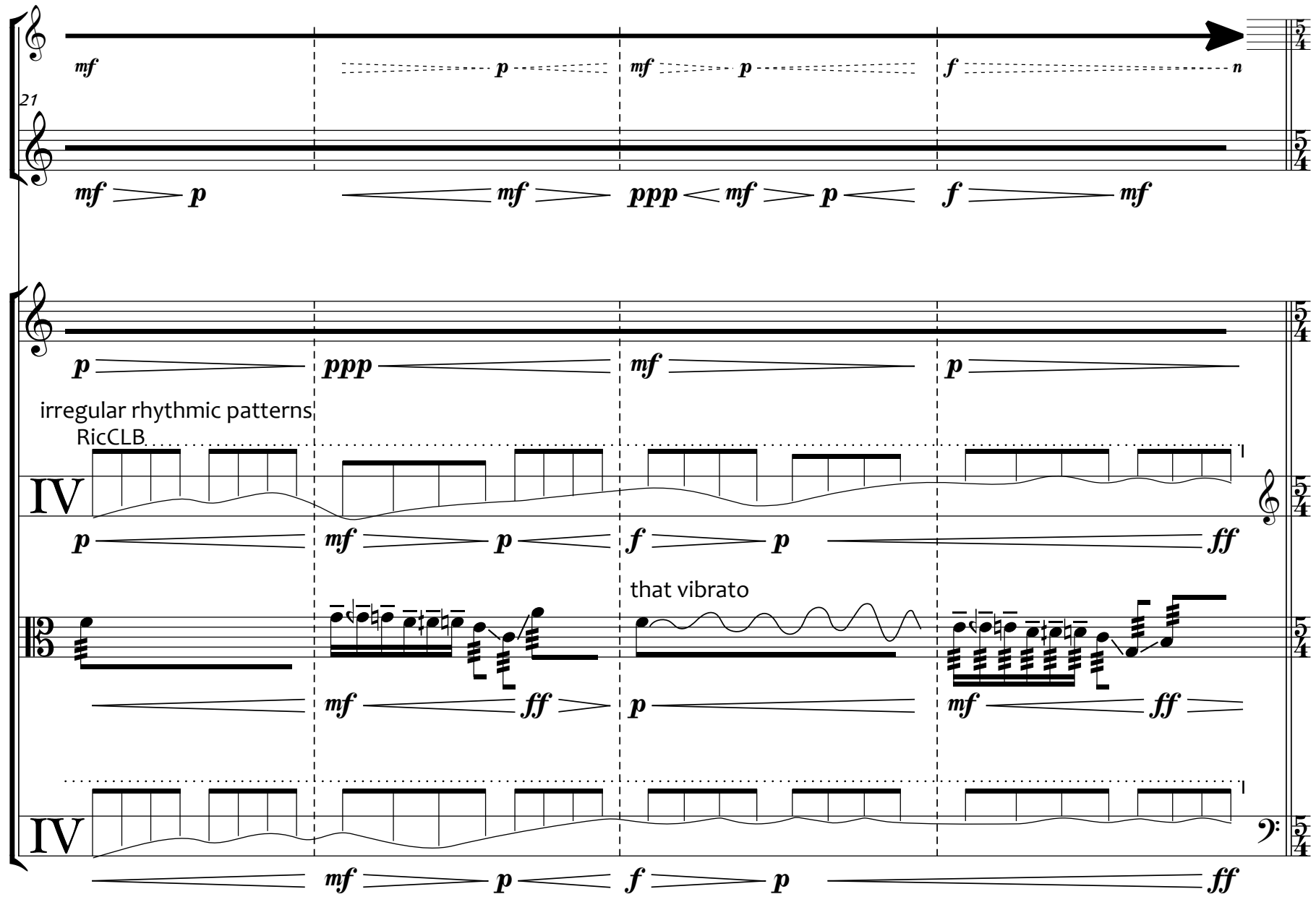

$\downarrow=60$ or faster

1. $\mathrm{O}$

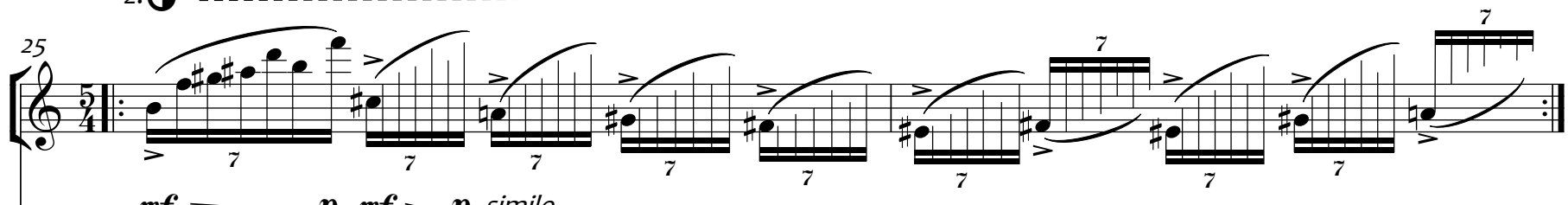
$\boldsymbol{m f}=\boldsymbol{m} \boldsymbol{f}>\boldsymbol{p}$ simile

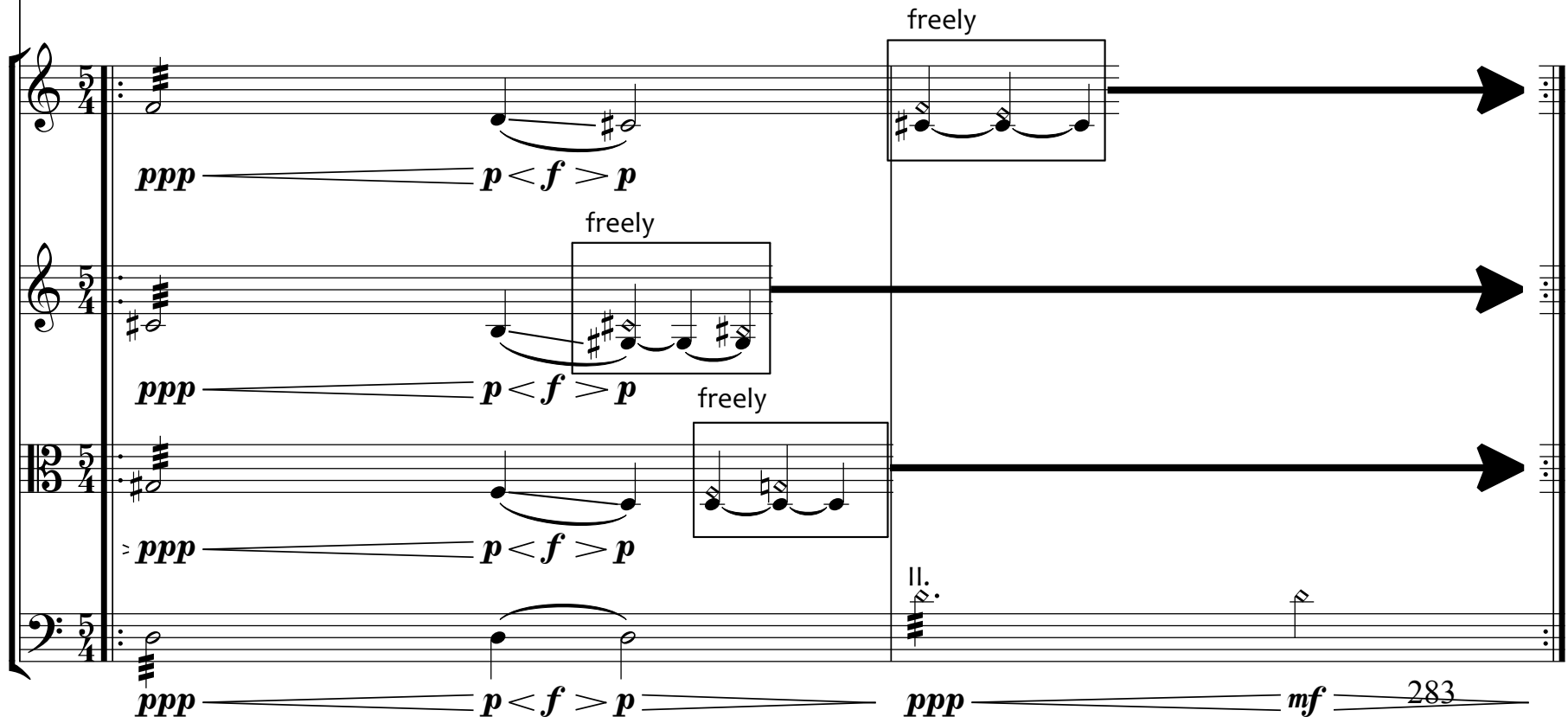


6

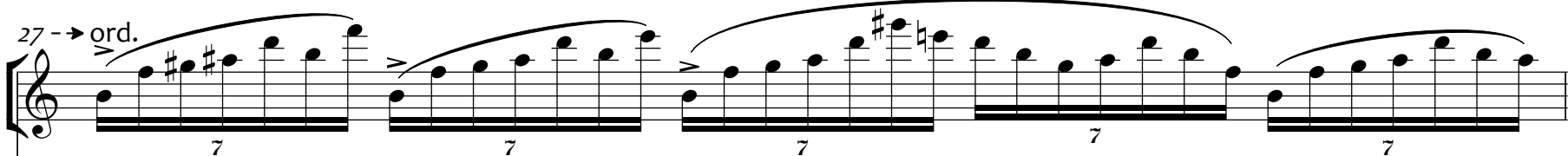

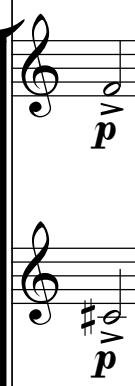

\$0

雌

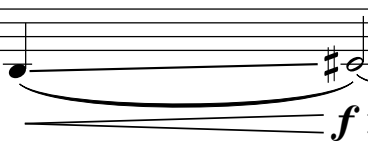

$\frac{9:>}{p}$

$f$
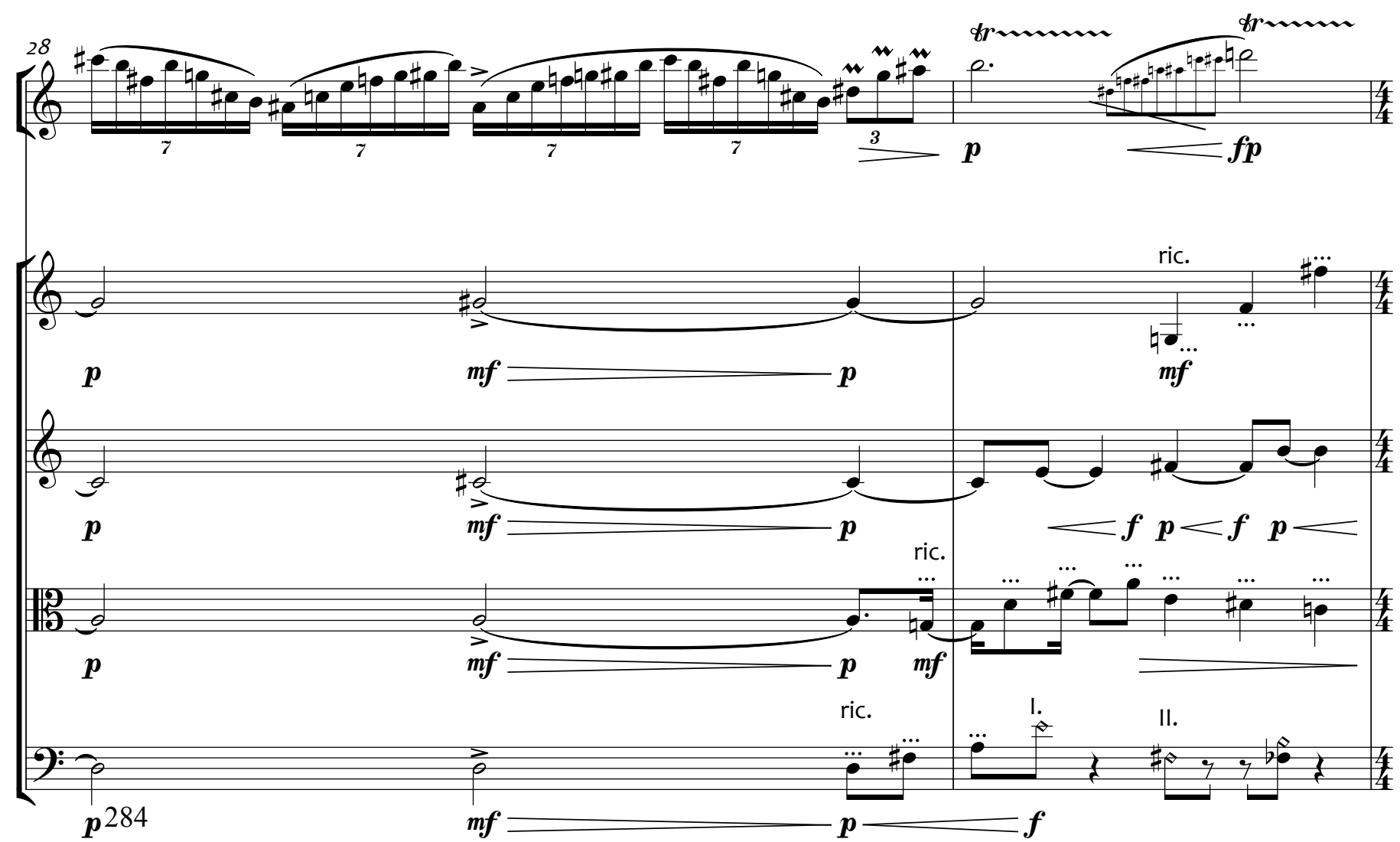
Slow (deep breathing cycle) Saxophone player cues tempo
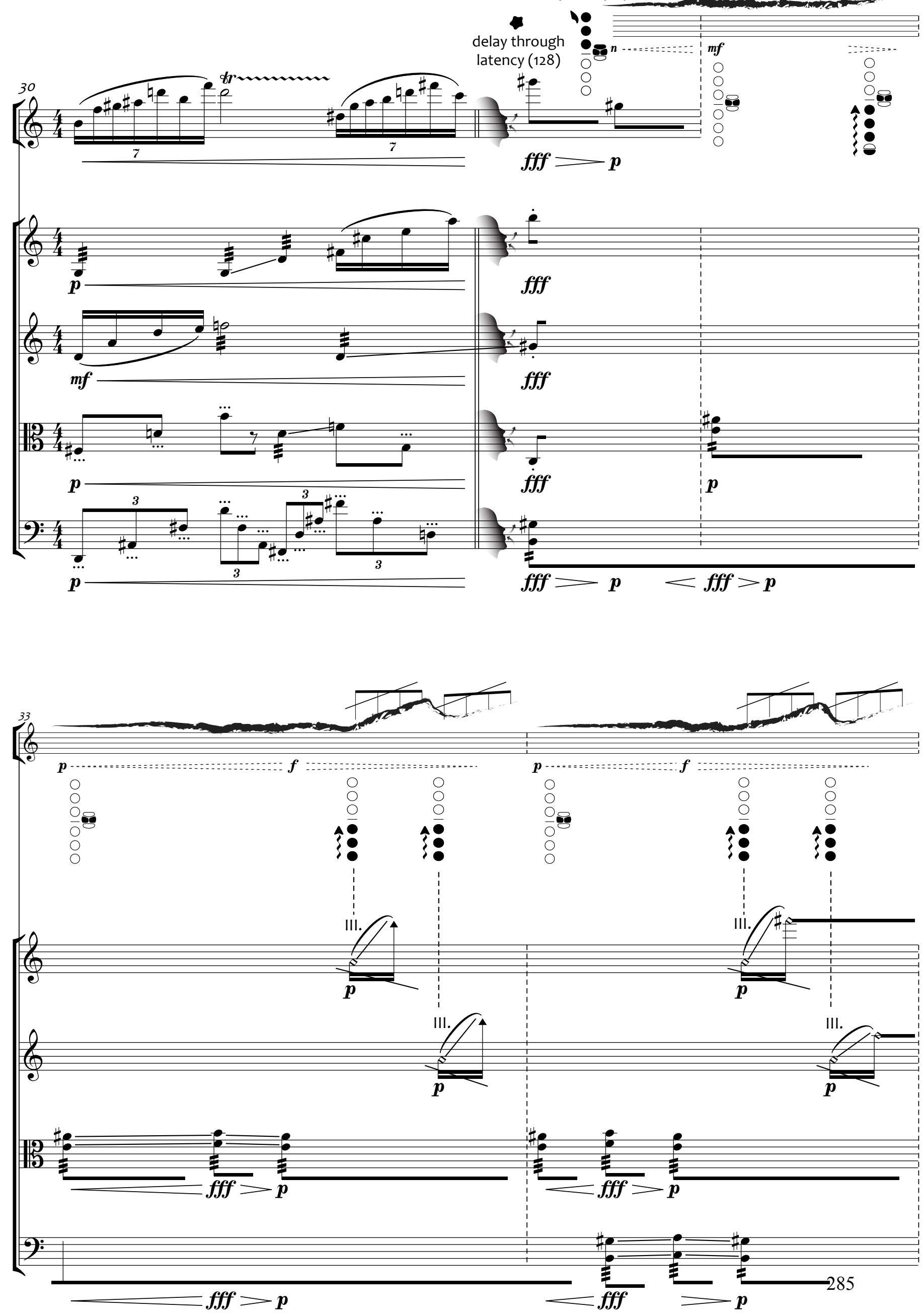

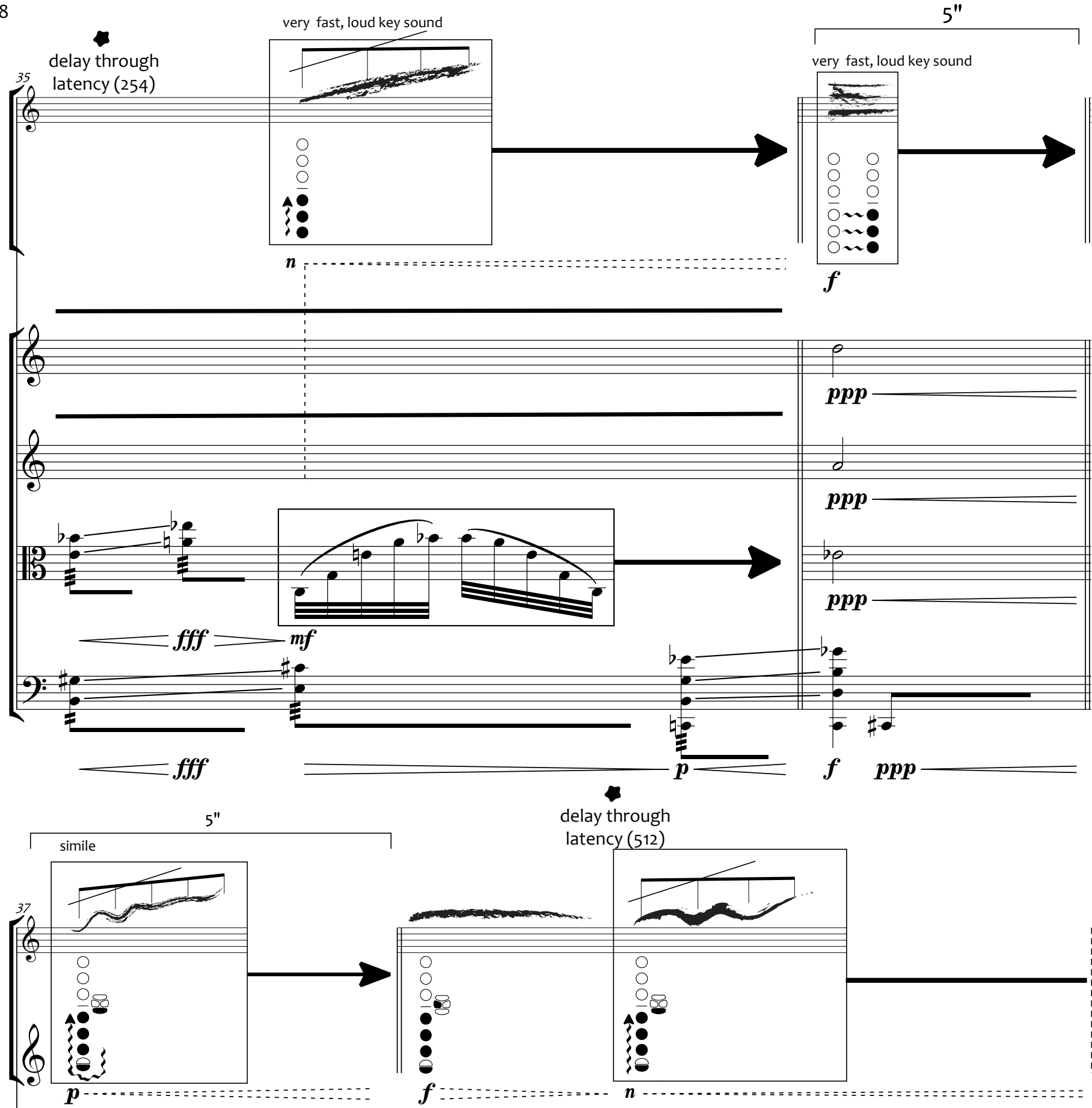

RicCLB.

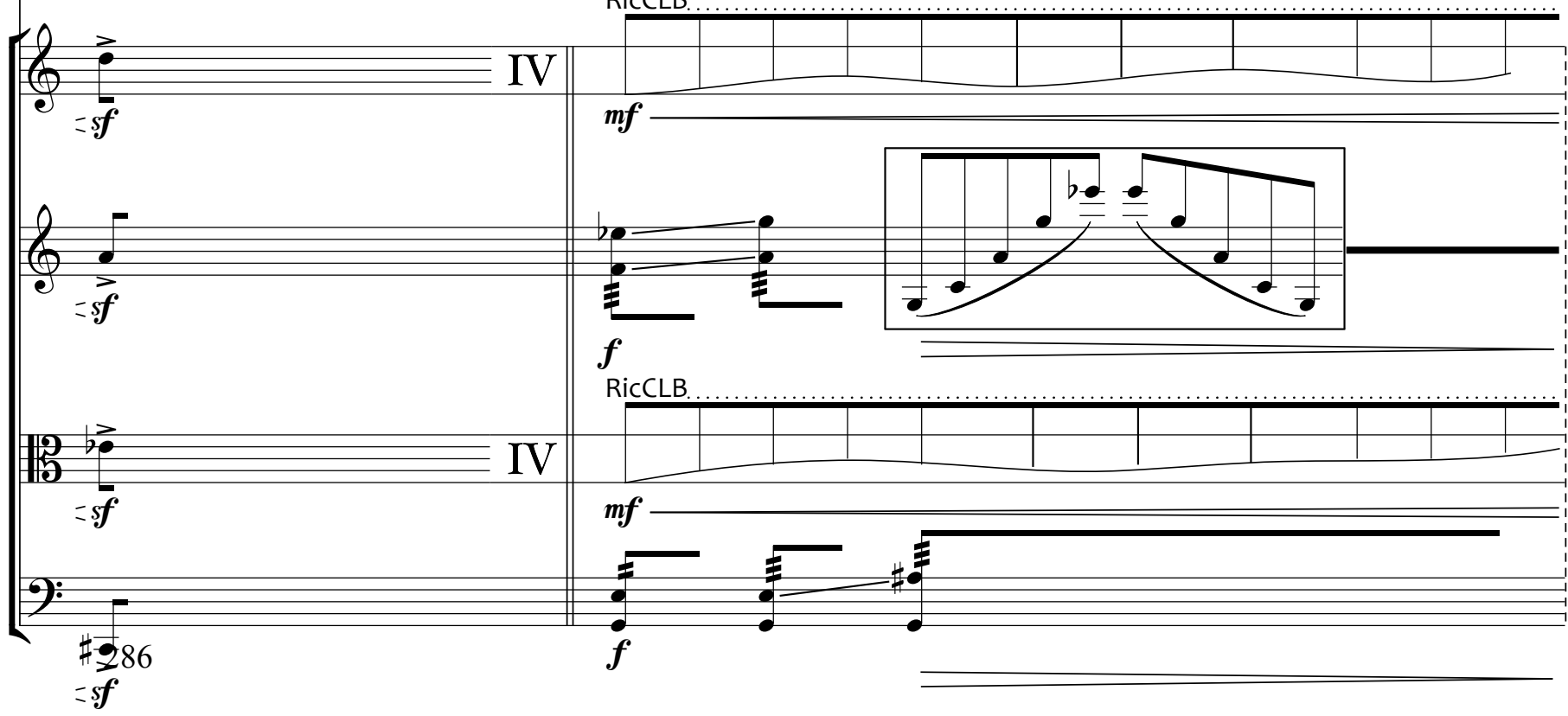



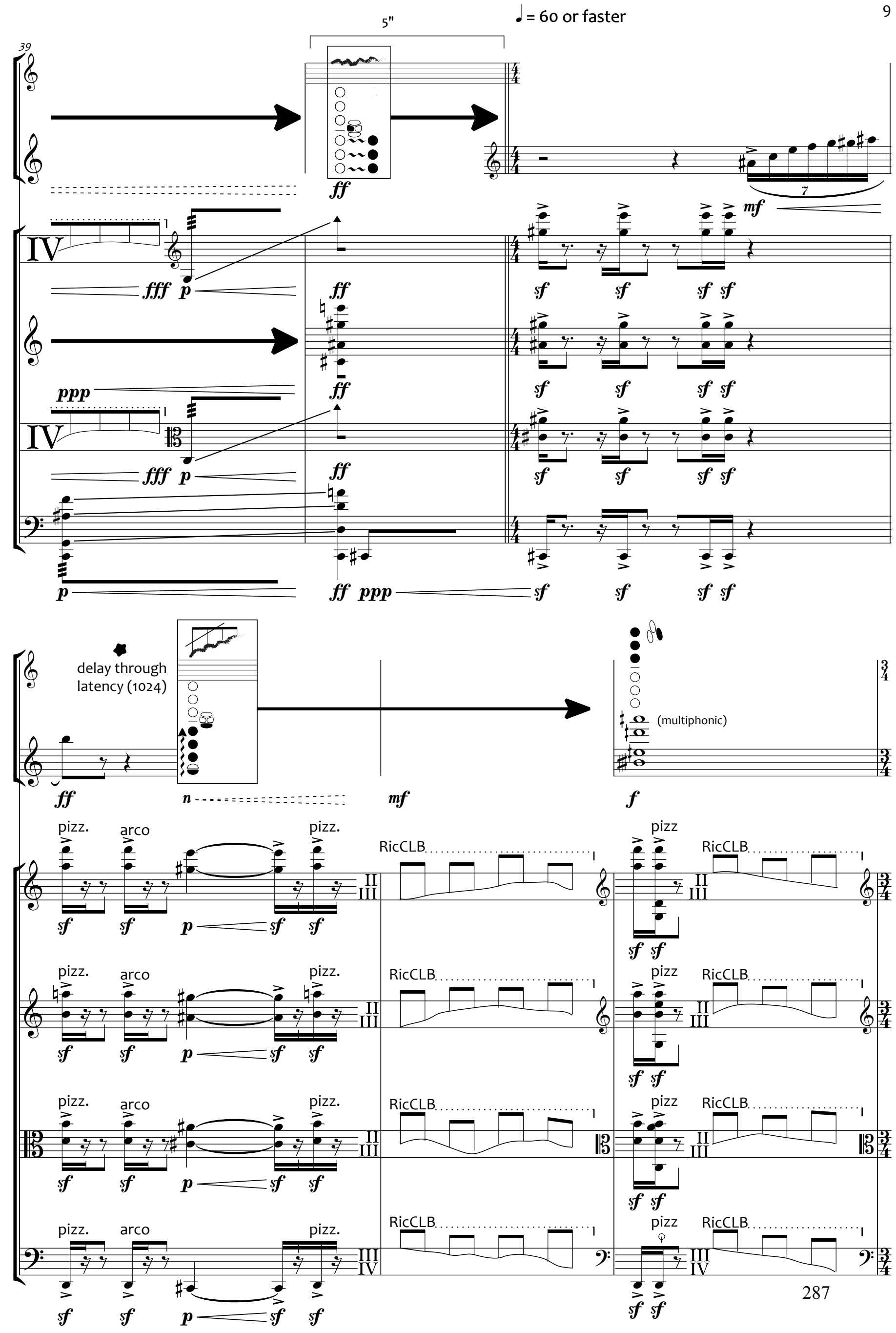

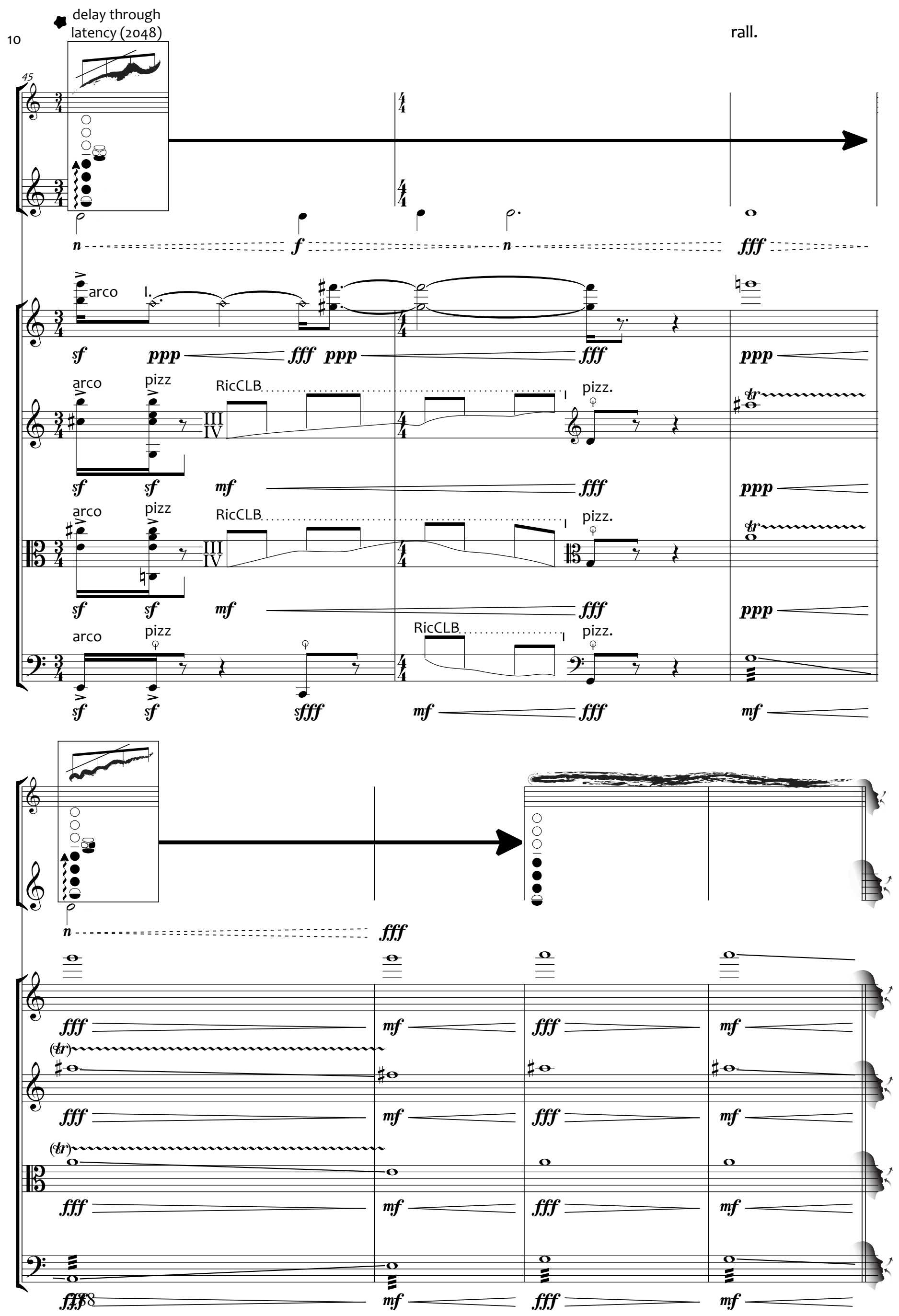
Slowly (deep breathing cycle)

1st Violin player cues

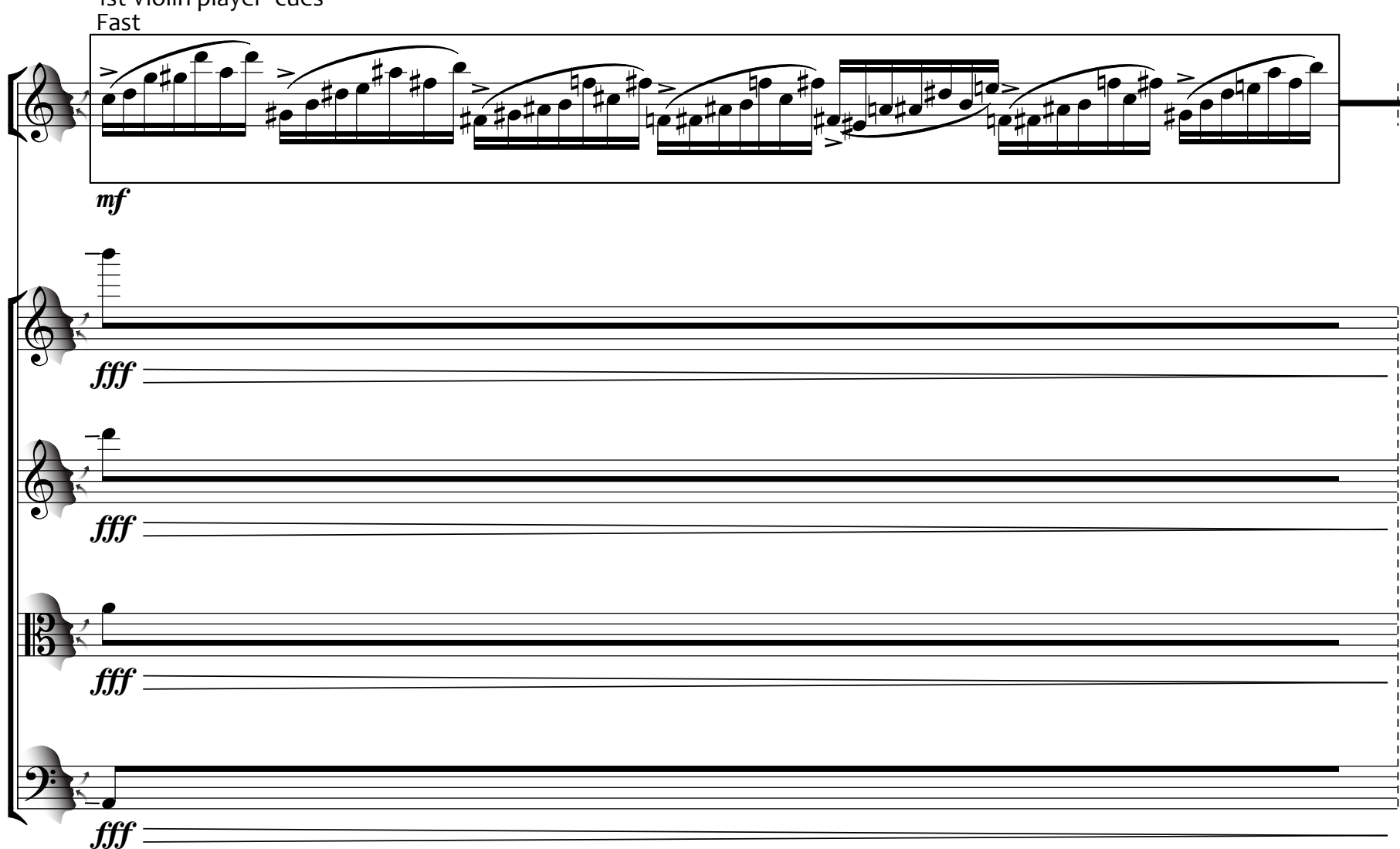

s lowing down the pattern
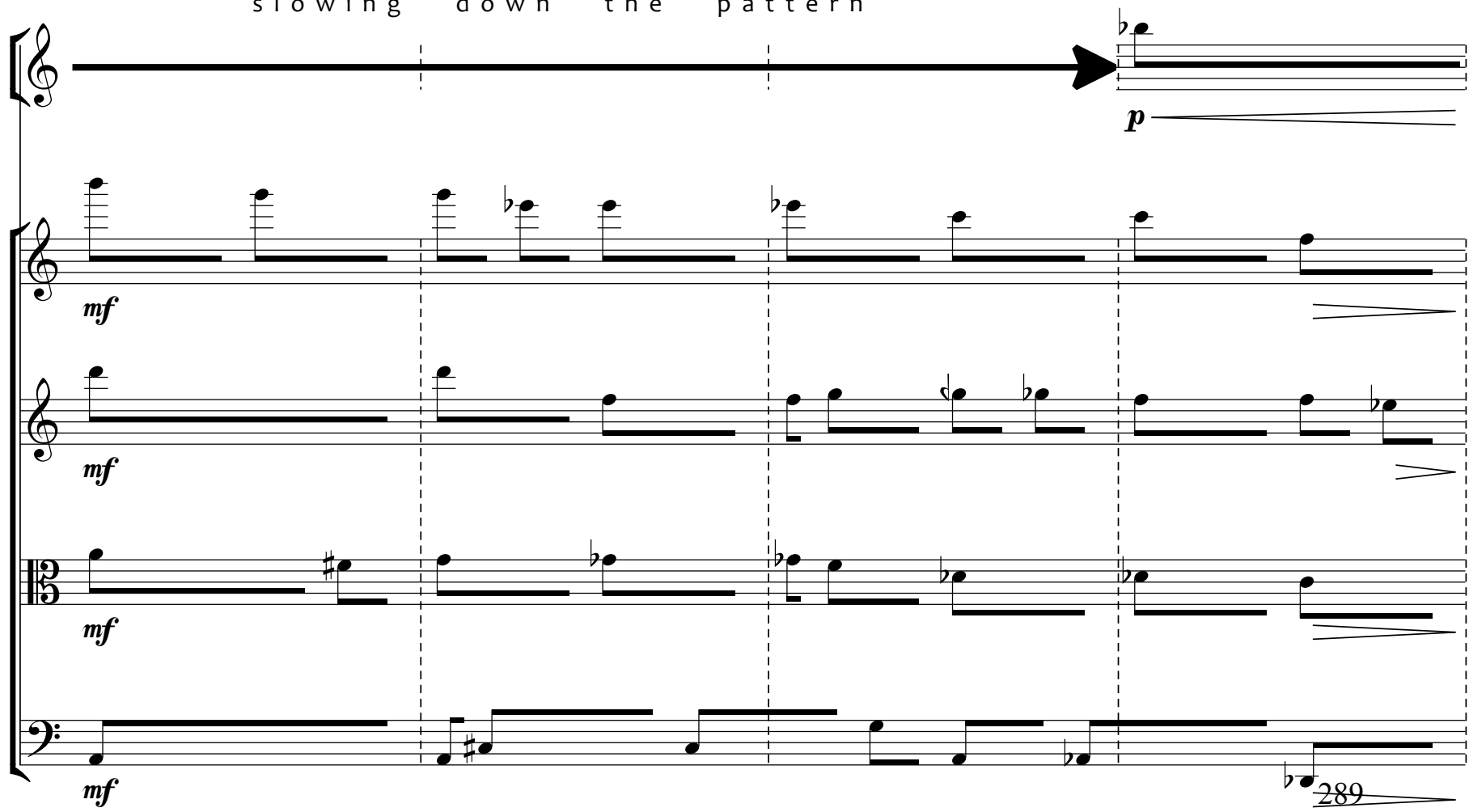

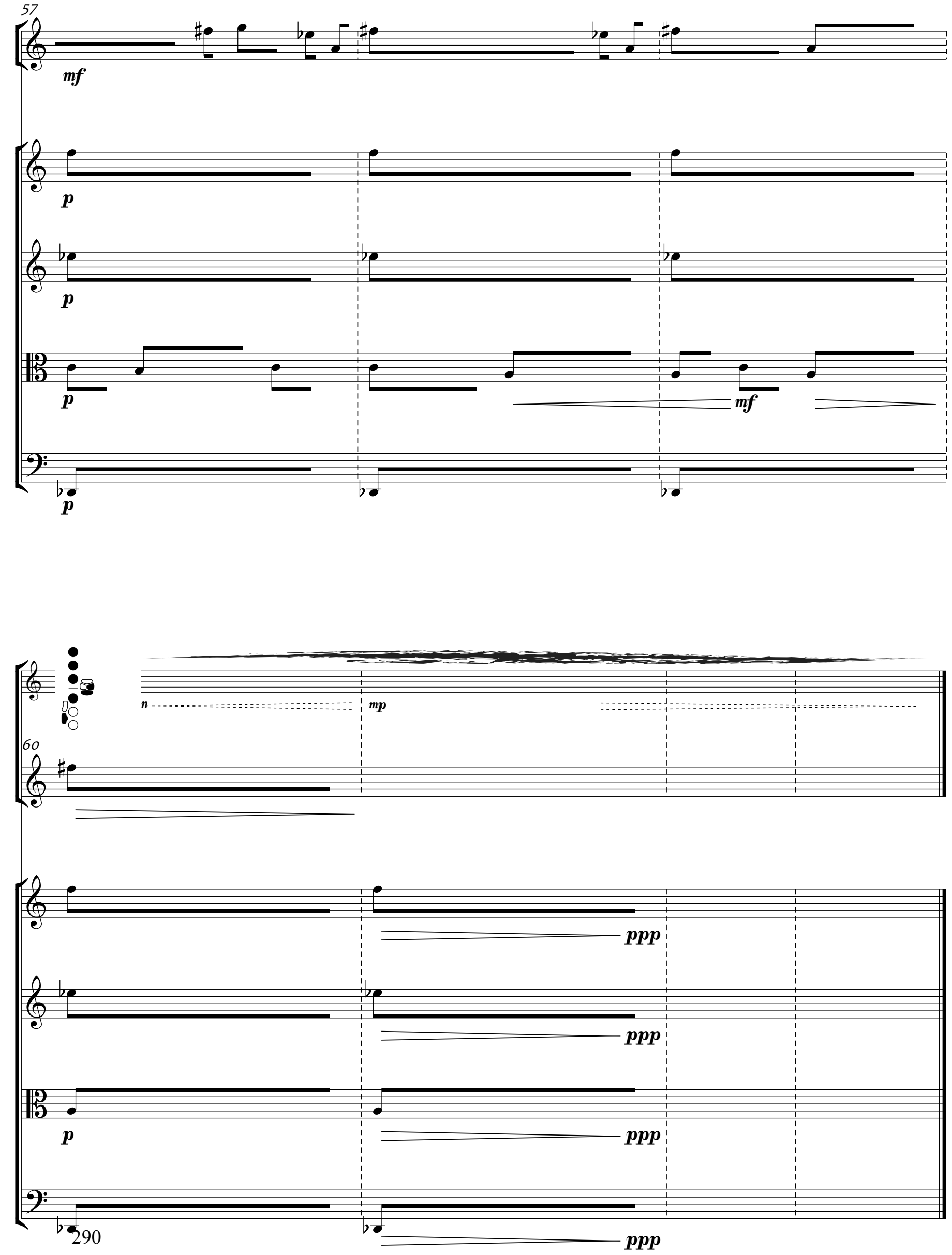
APPENDIX K: COUNTLESS SOULS 


\section{Countless Souls}

For HypeSax and laptop ensemble

Instructions

Play freely using the following score:

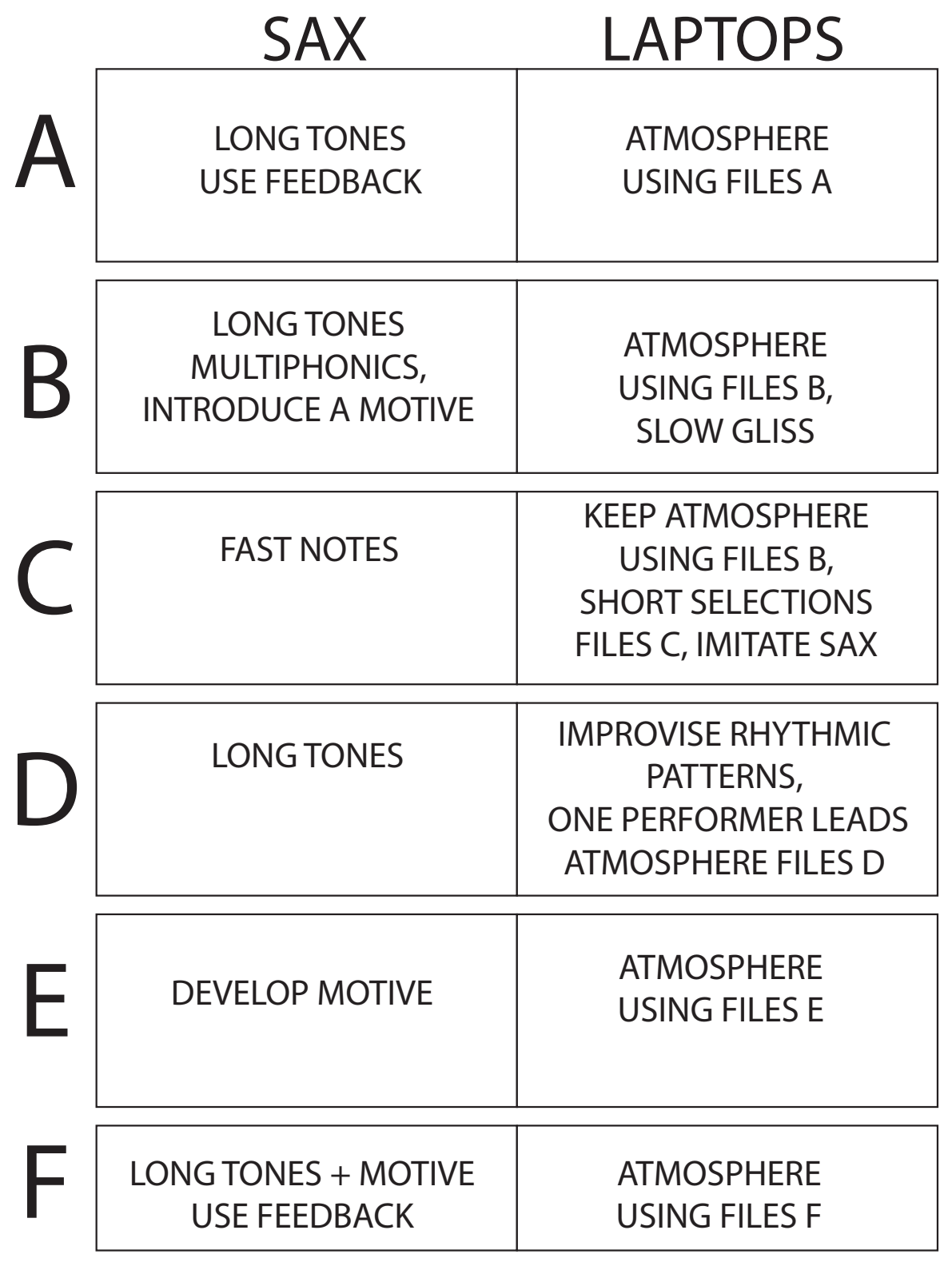

BACK TO A AND END 
HypeSax must use the Hub to connect to any live processing software.

Be free to decide how to use it.

Laptops play using the Countless Souls Max patch.

Instructions included in the patch.

(Screenshot of Countless Souls.maxpat)

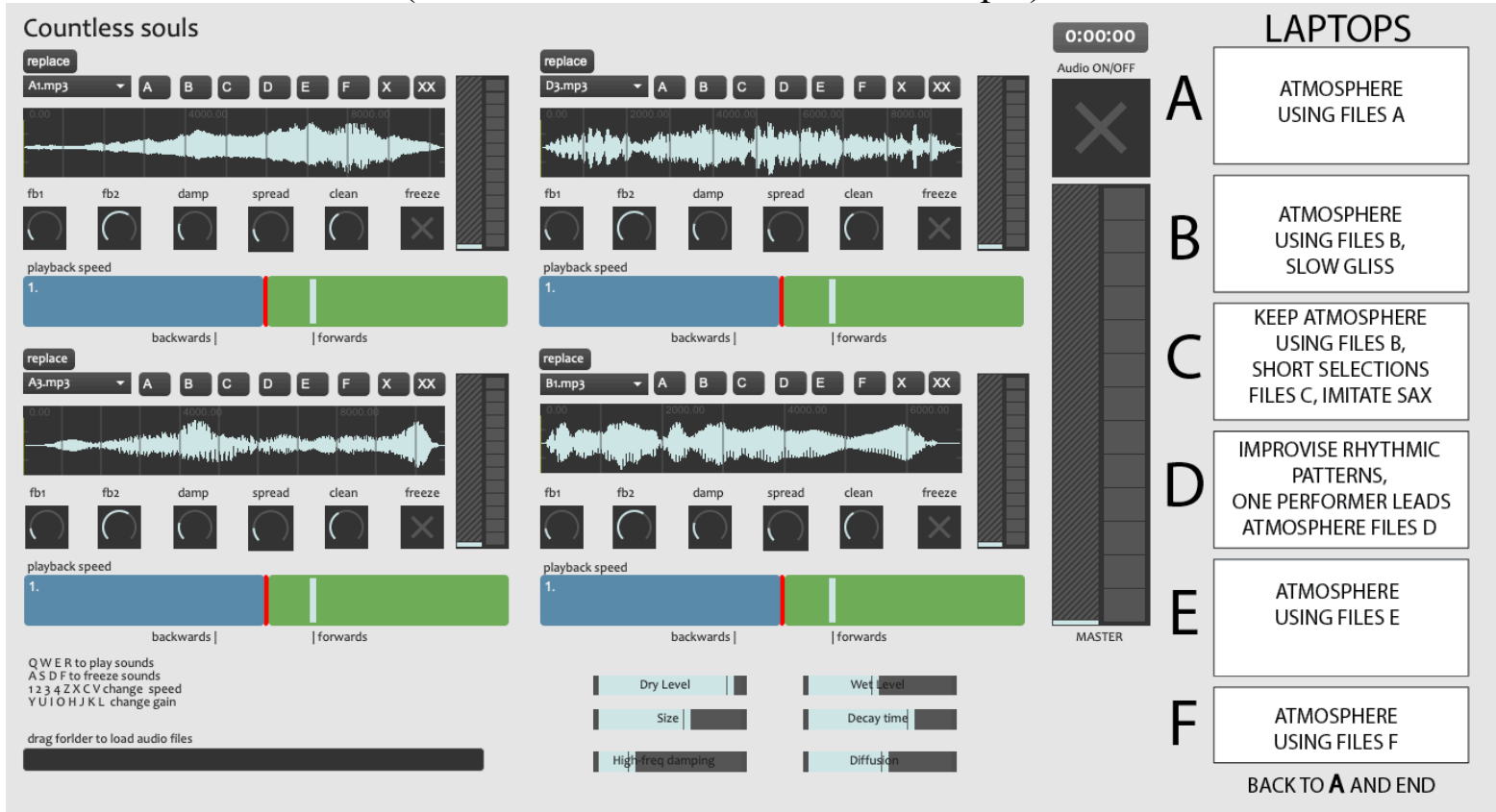


APPENDIX L: COR PLE 


\section{COR PLE}

Cristohper Ramos Flores
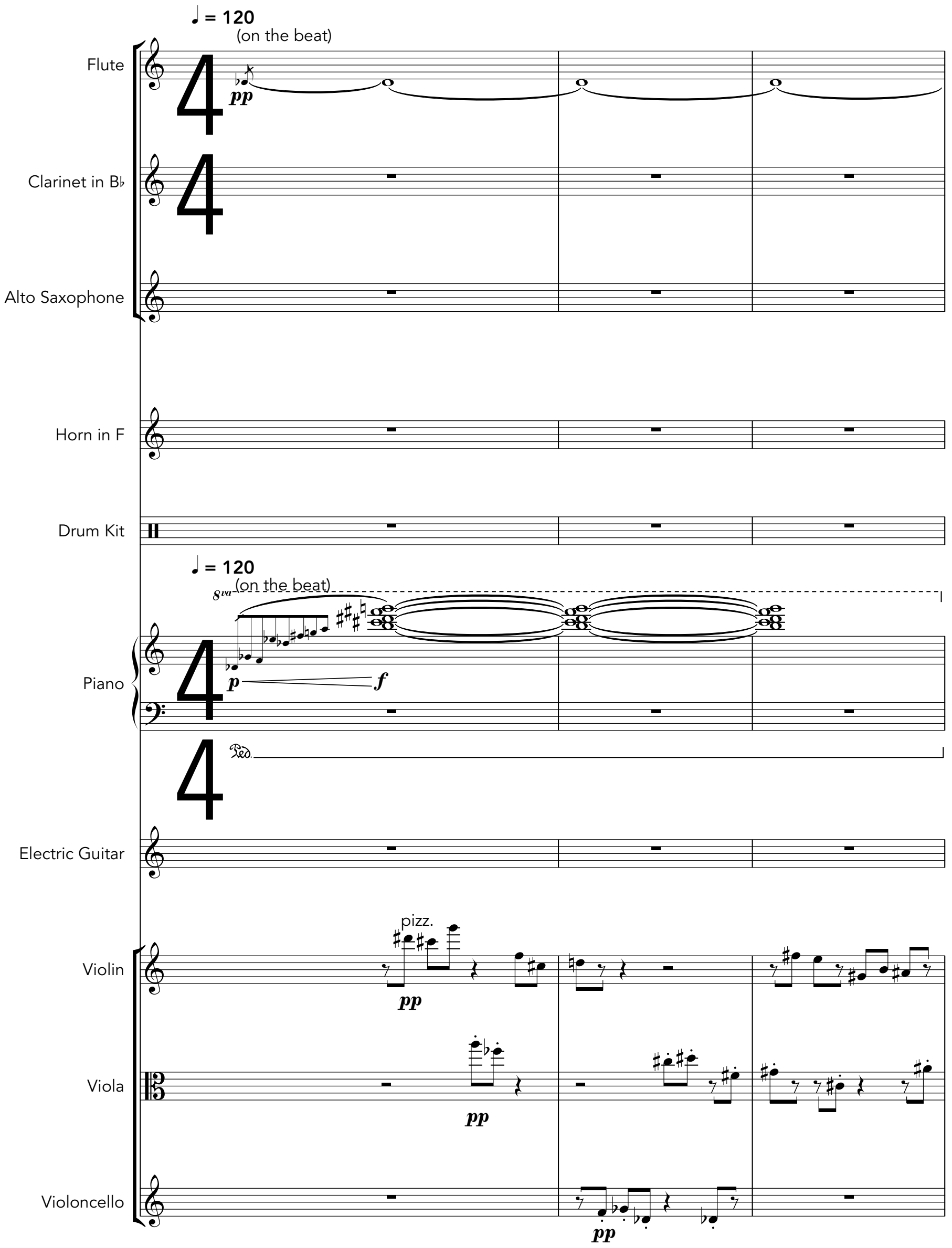

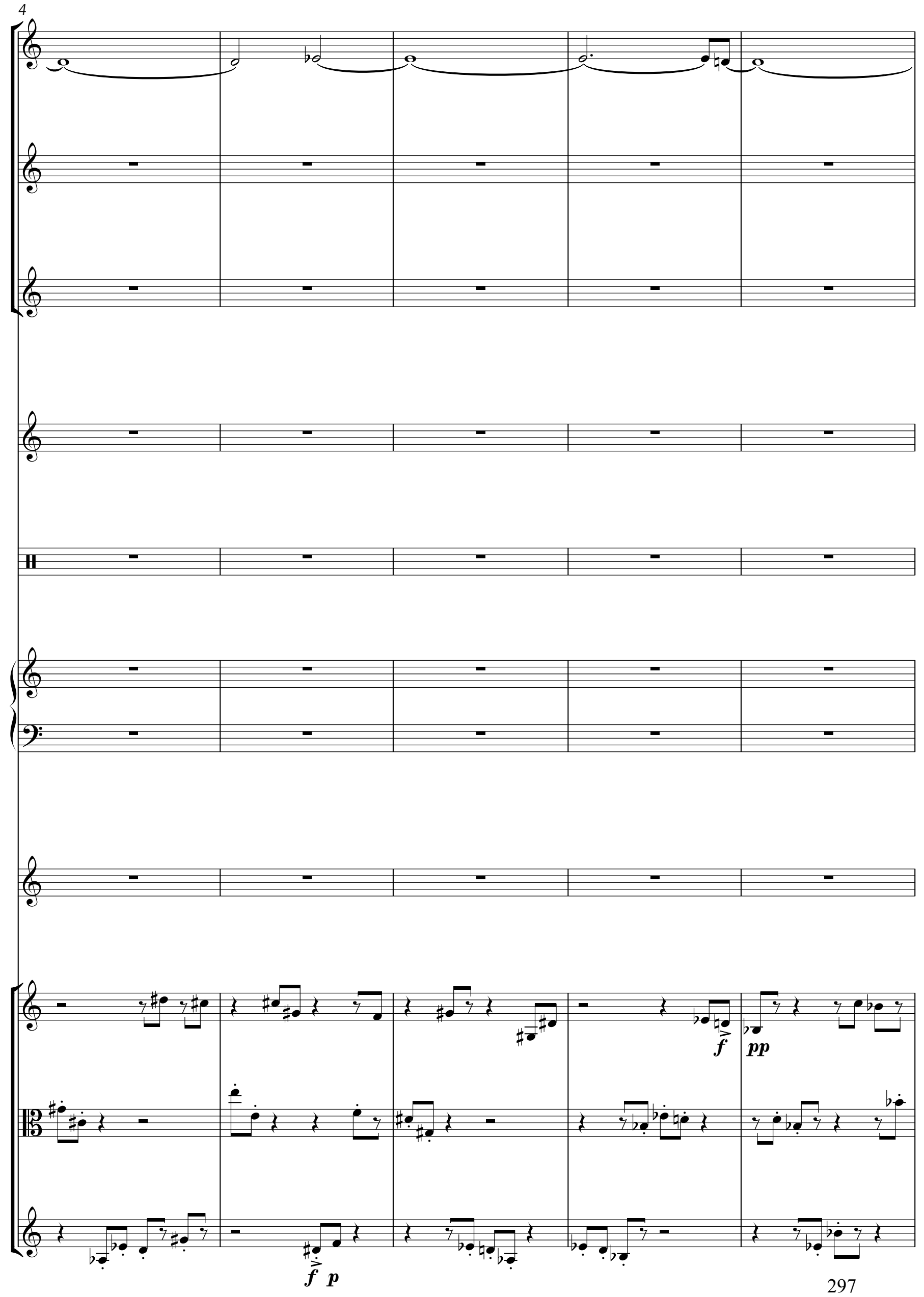

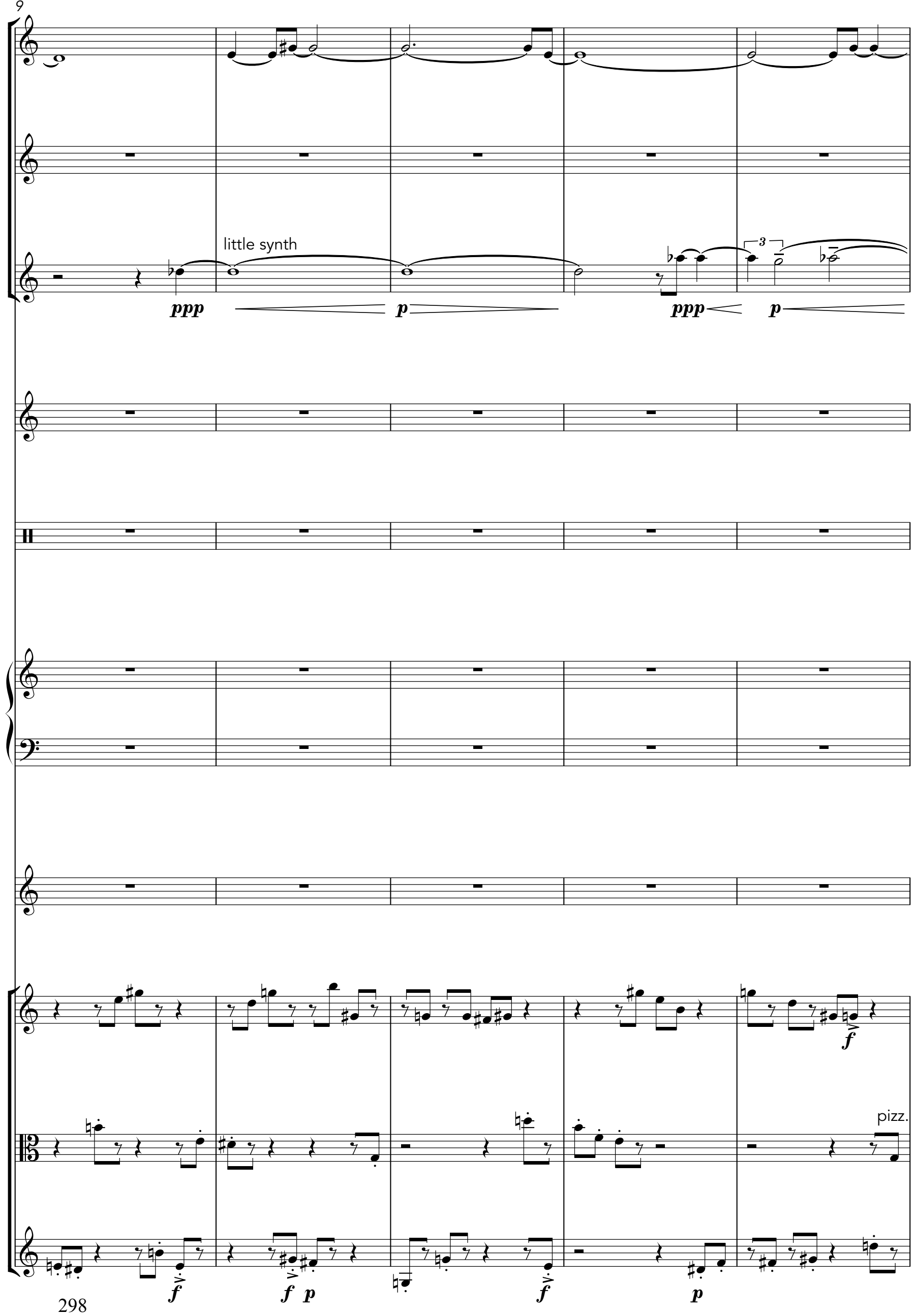

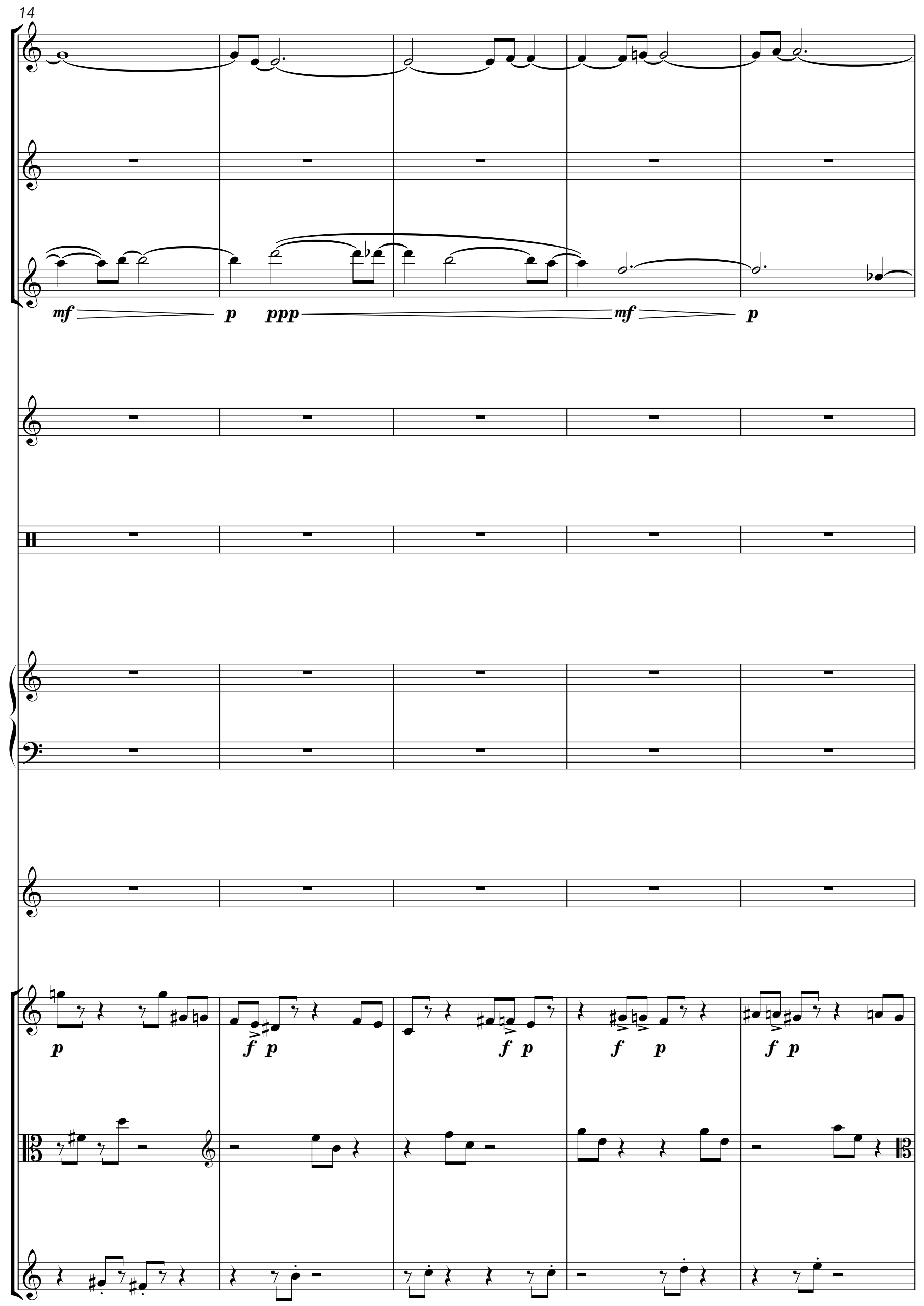

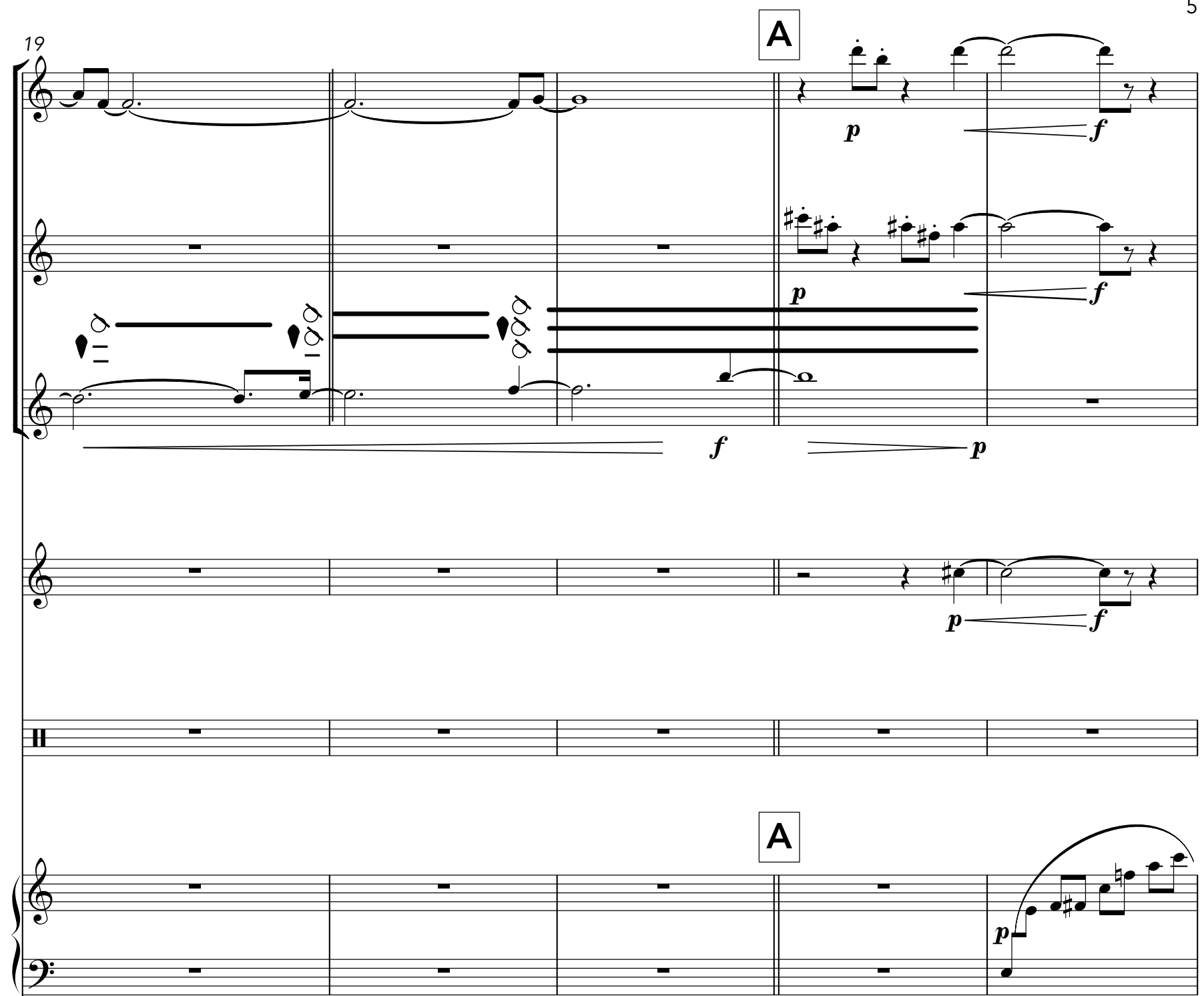

92

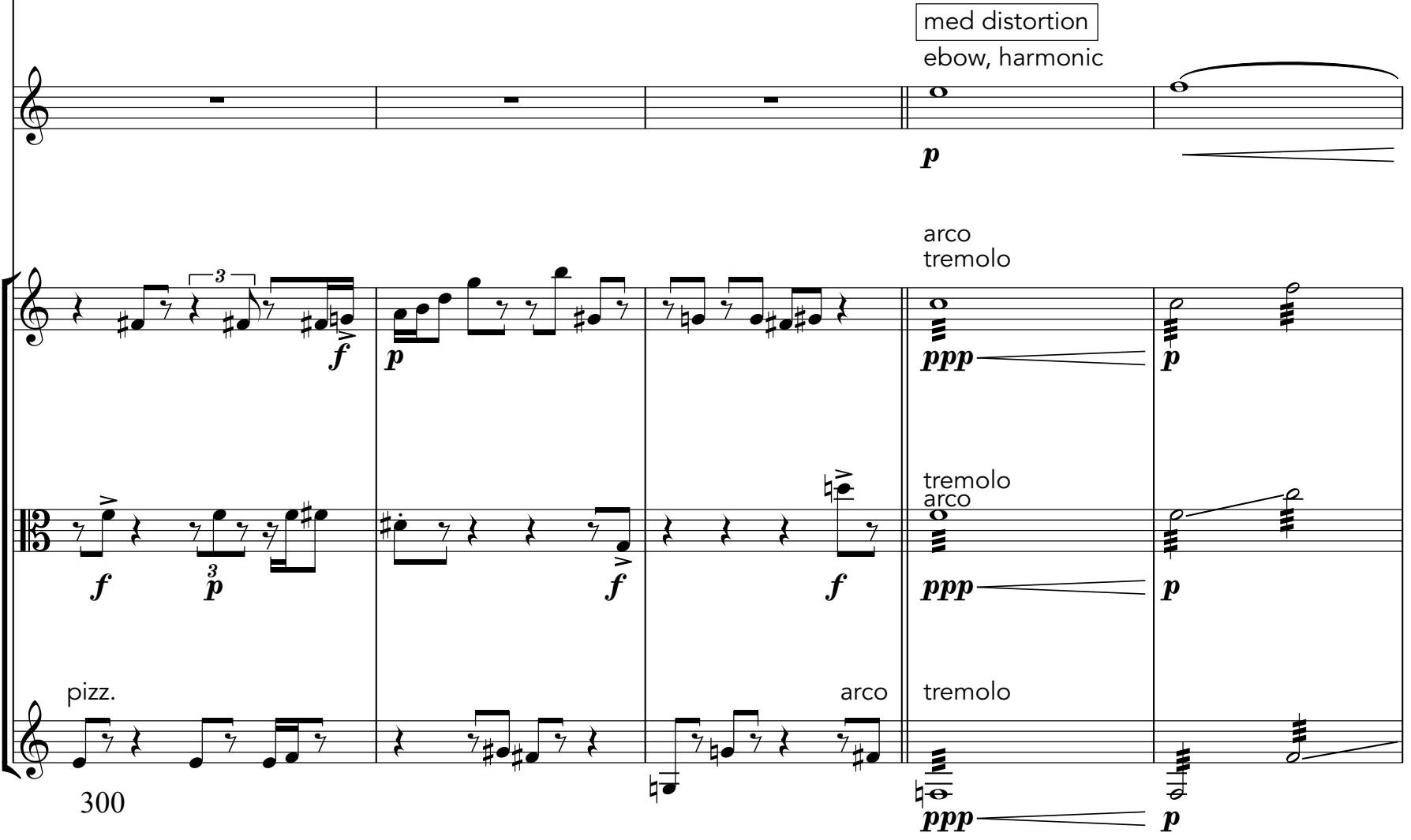




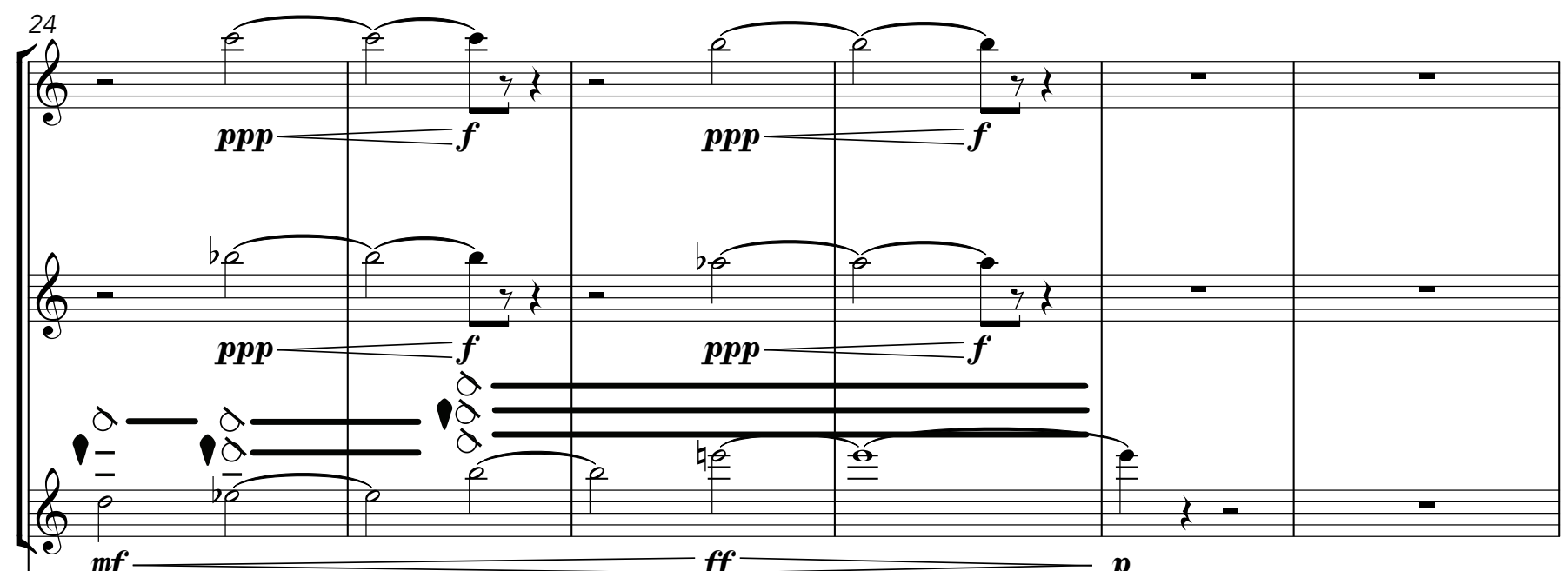

$\boldsymbol{m} \boldsymbol{f}$

$f f$

$\boldsymbol{p}$
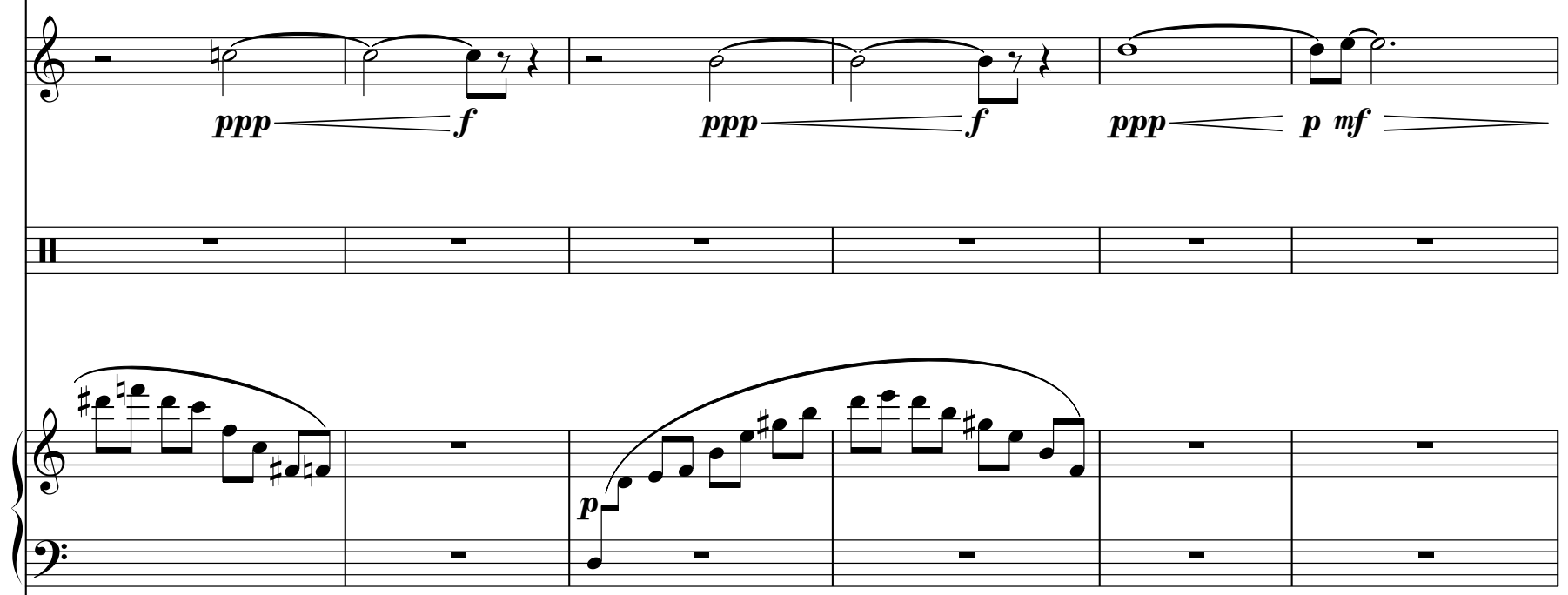

200.
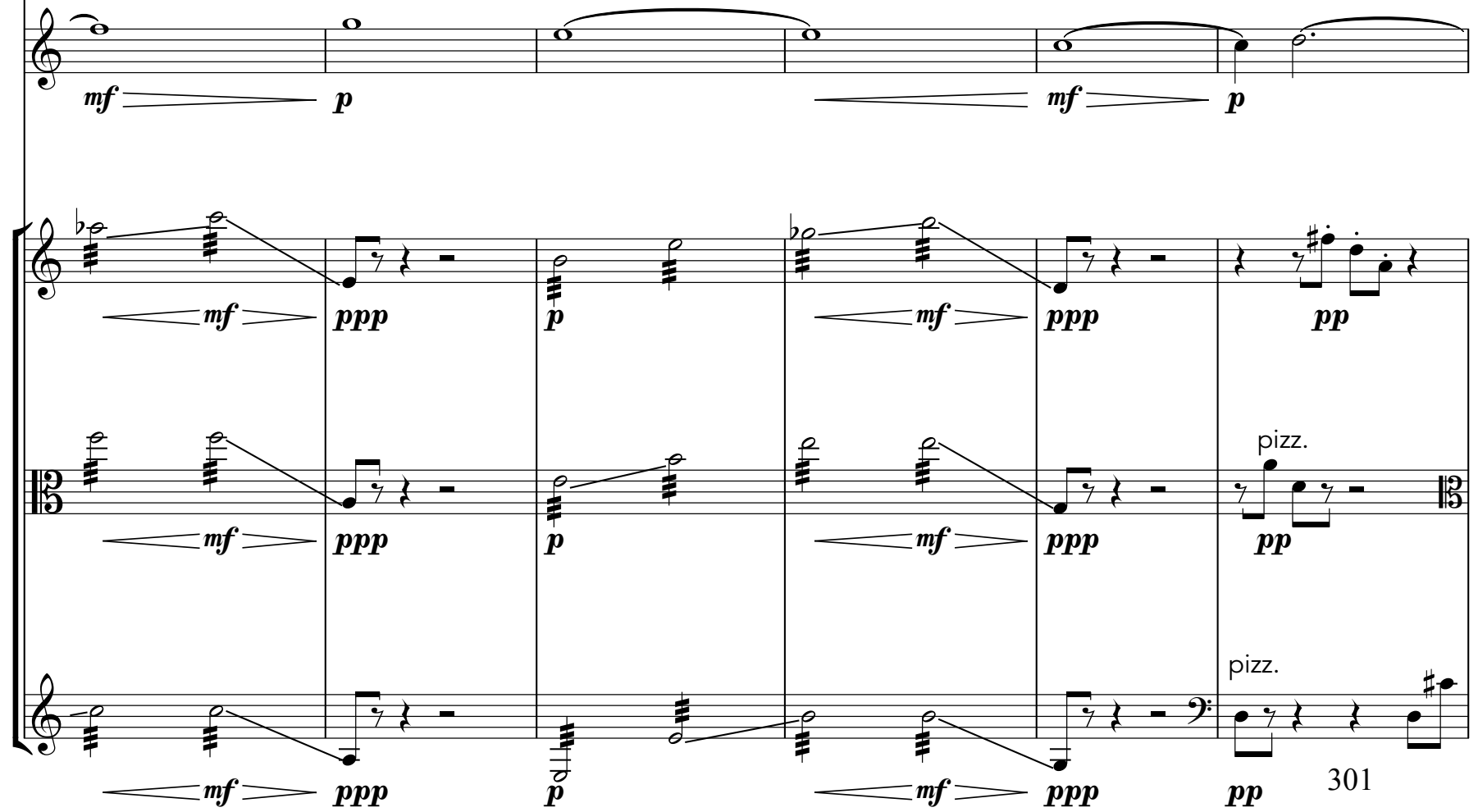


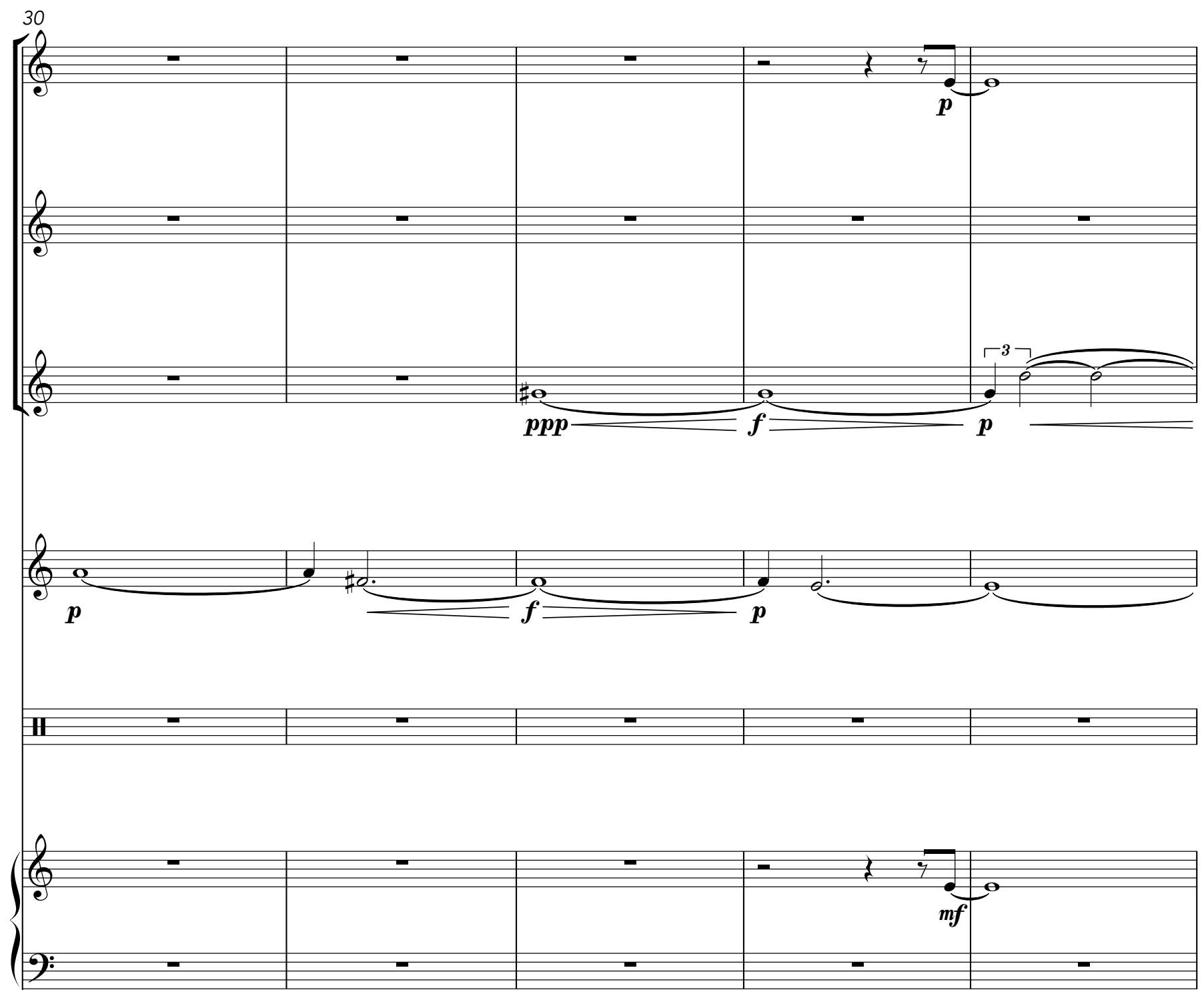

Ted
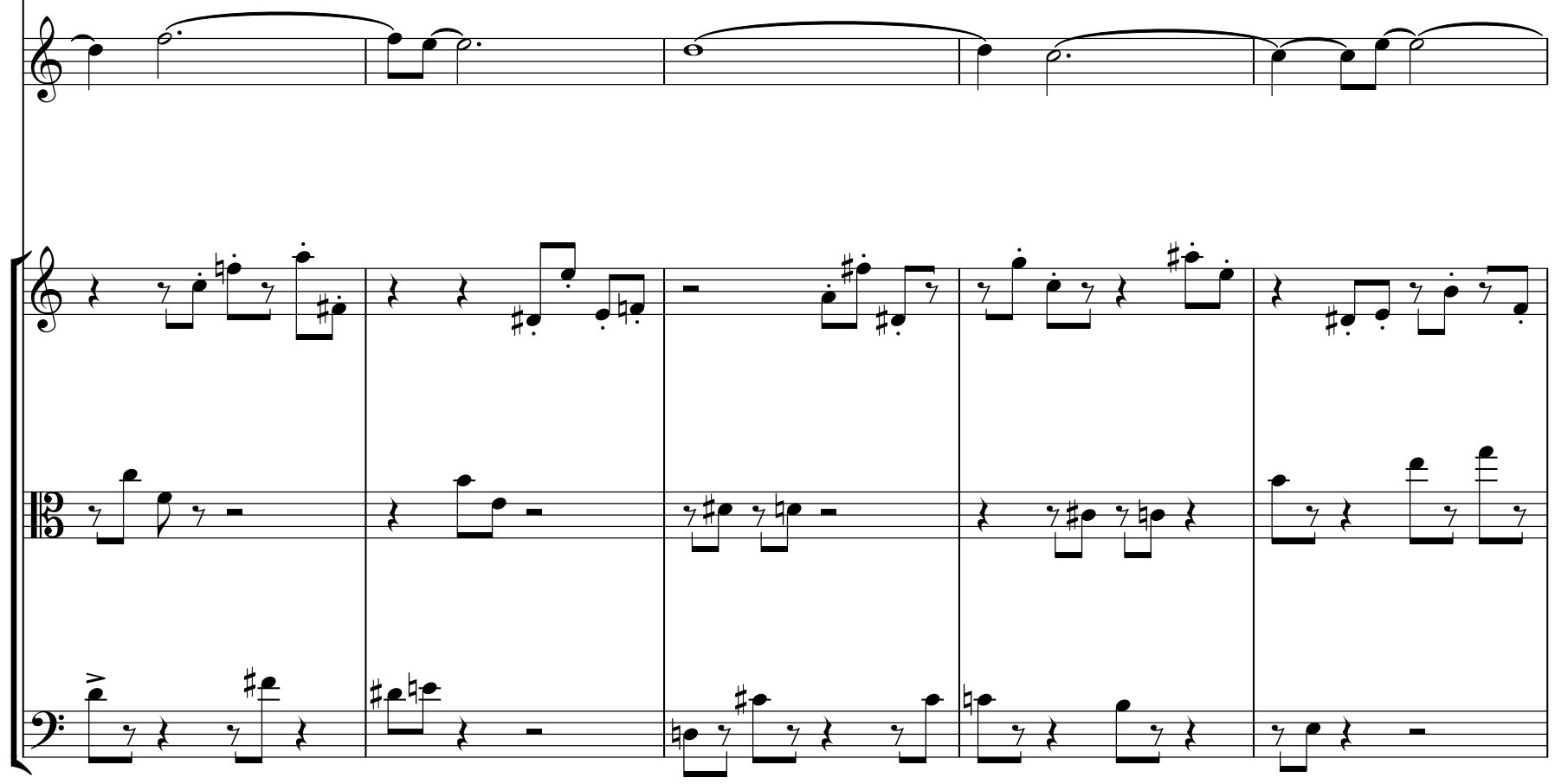

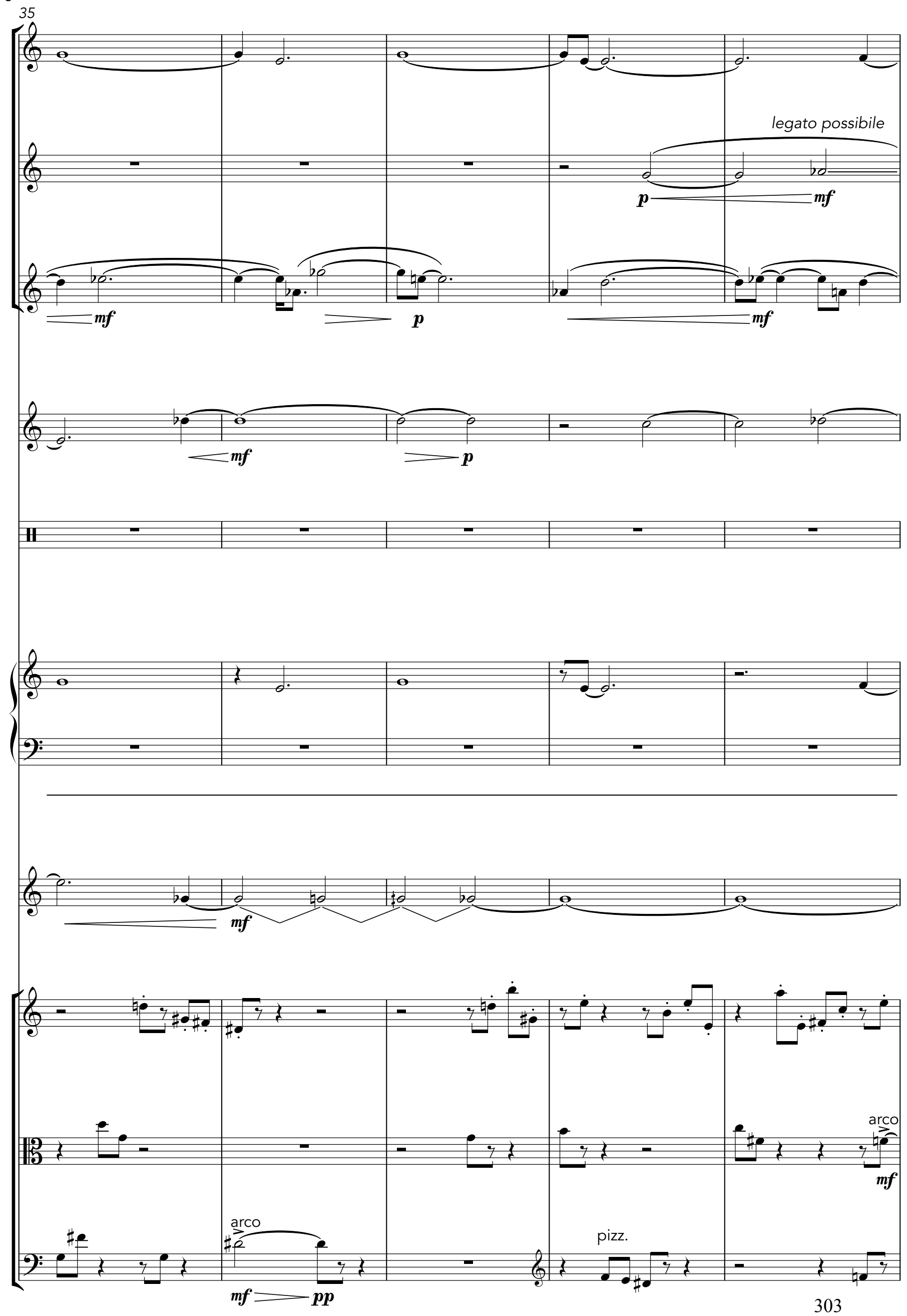


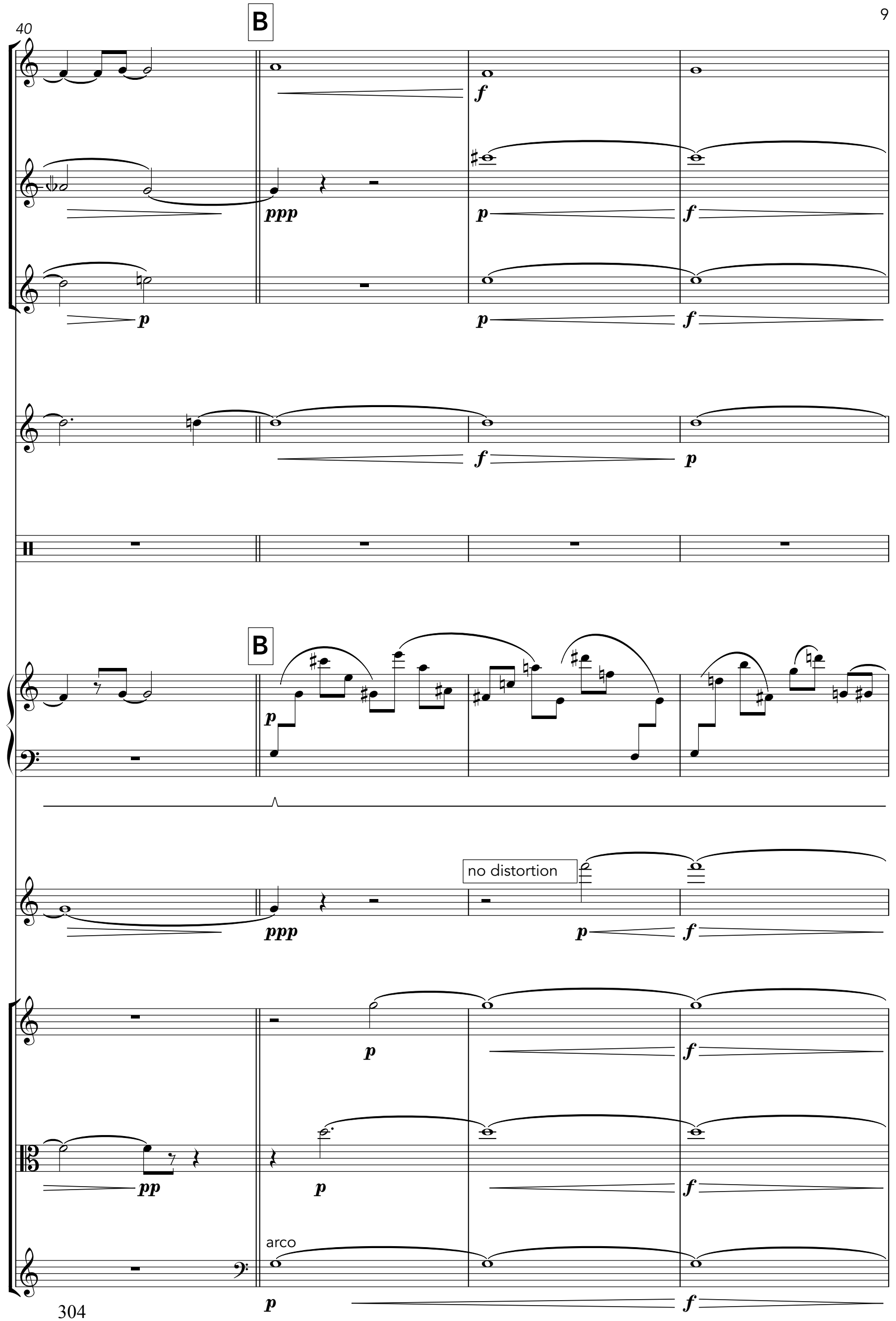




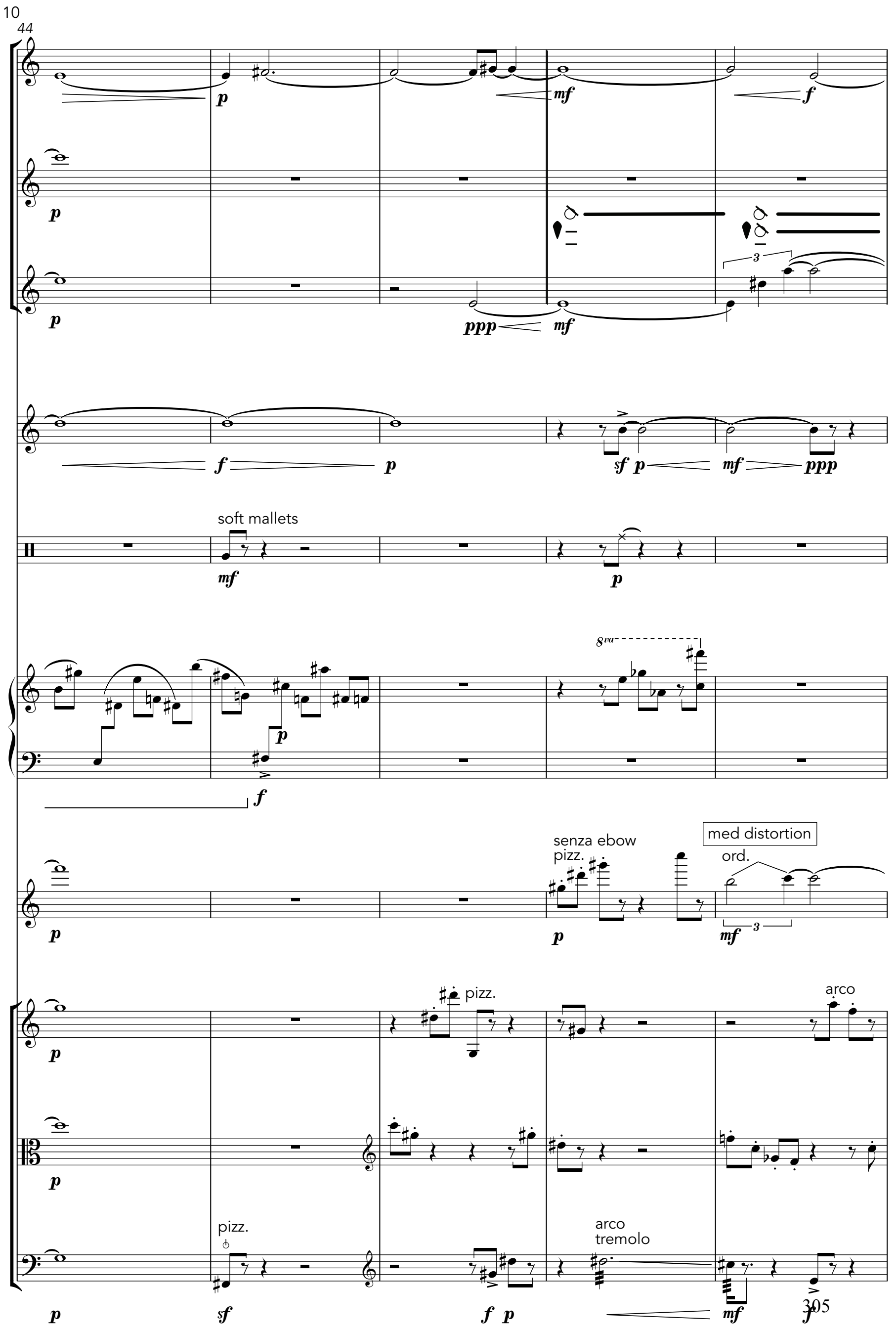



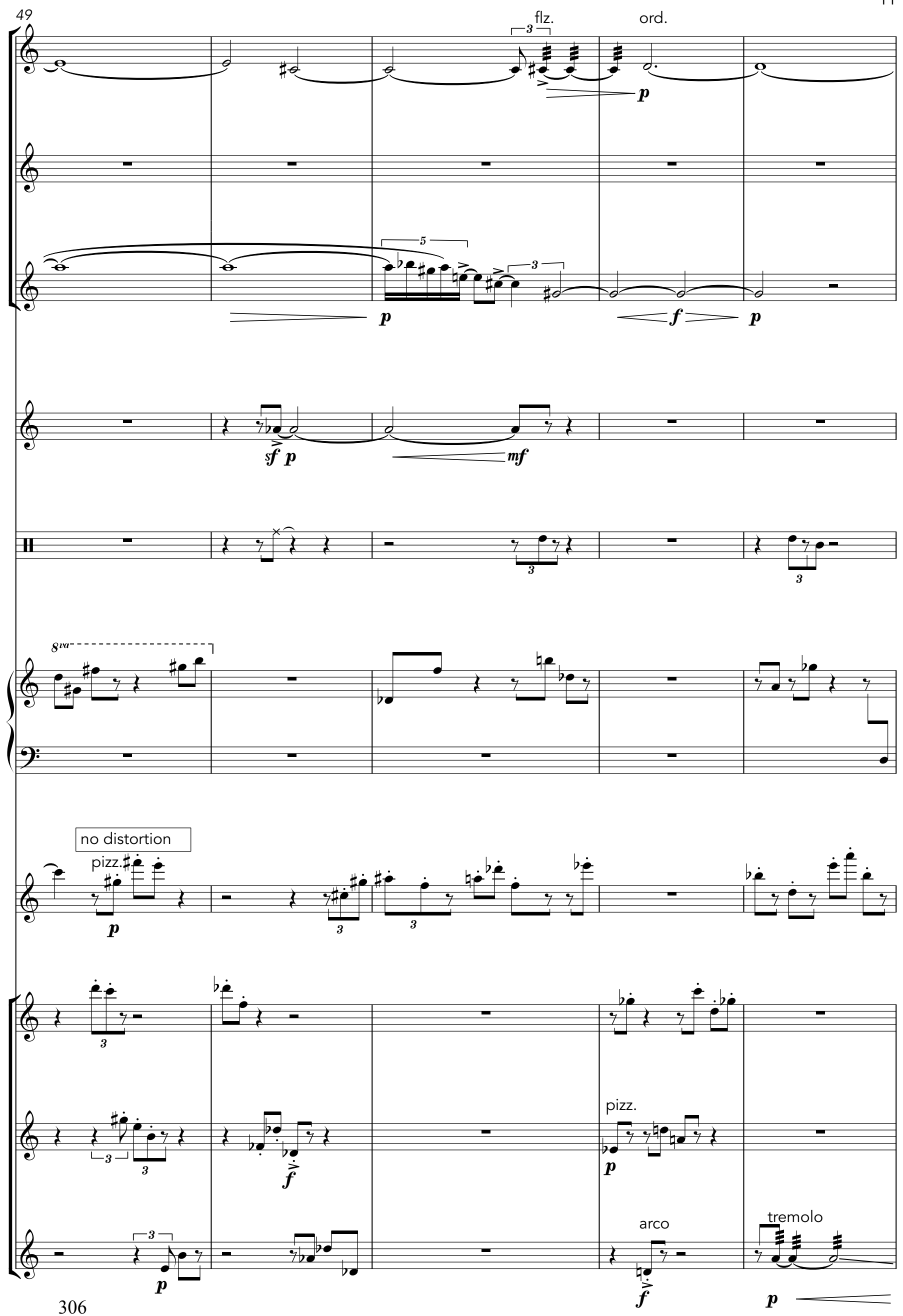


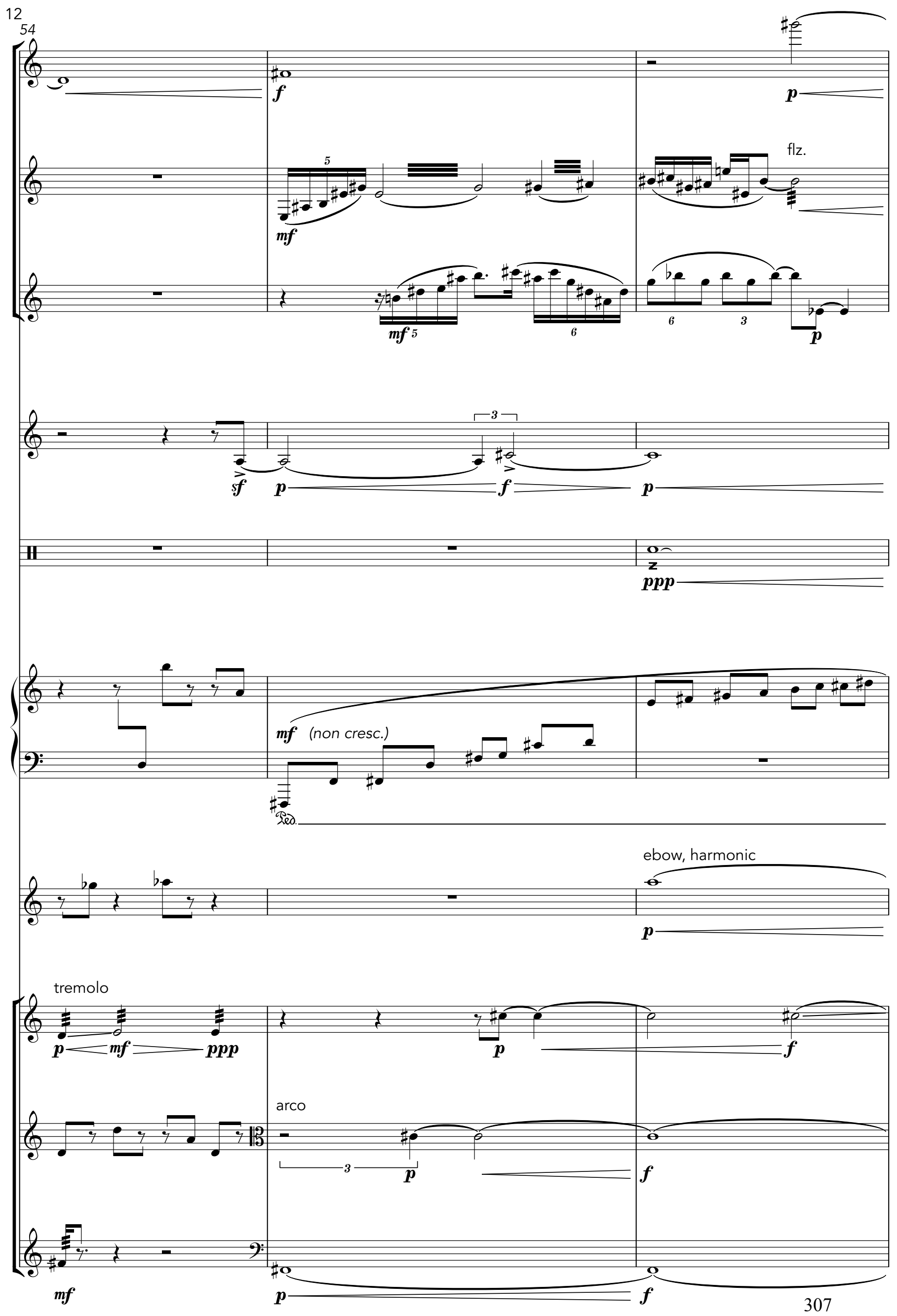




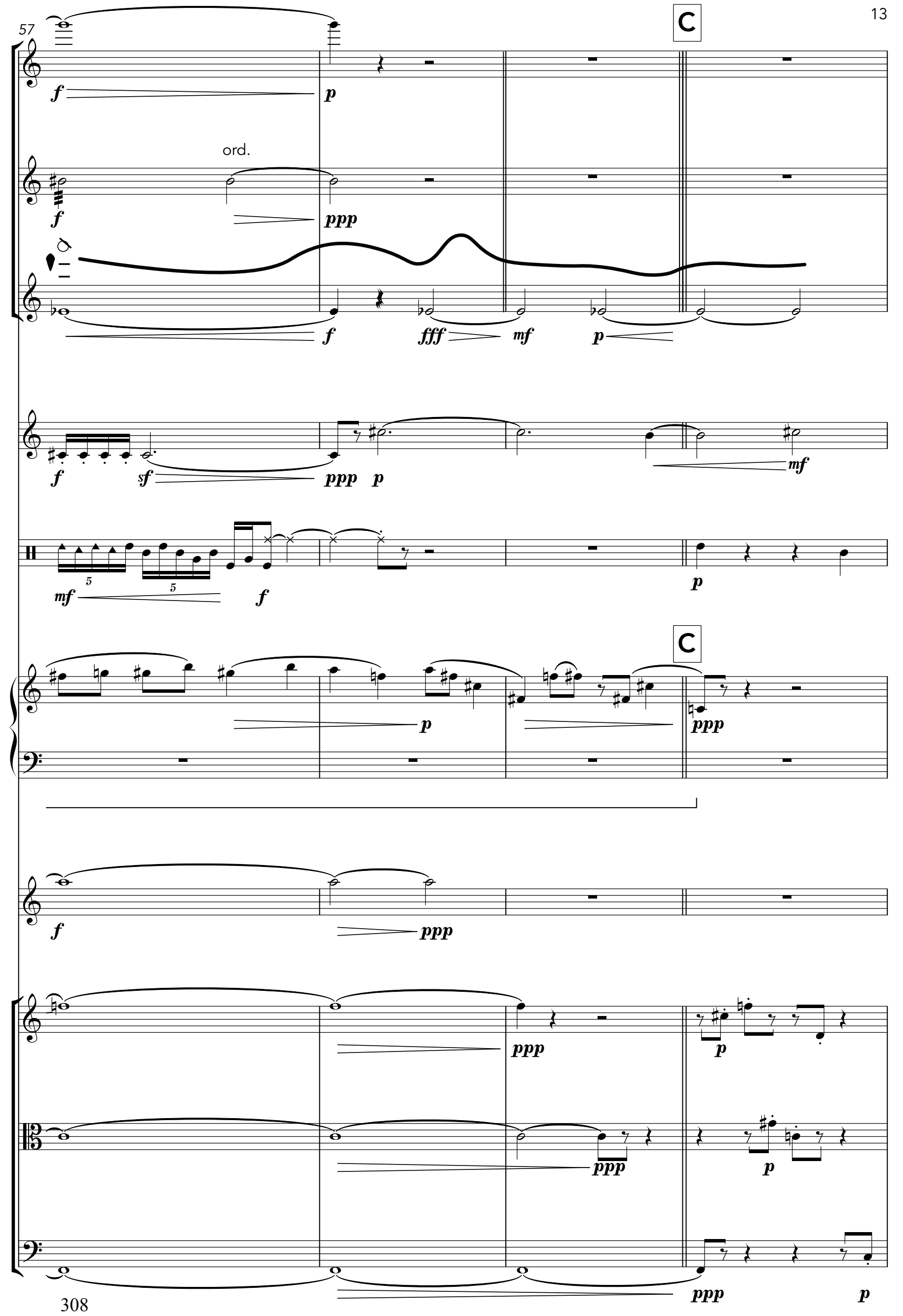



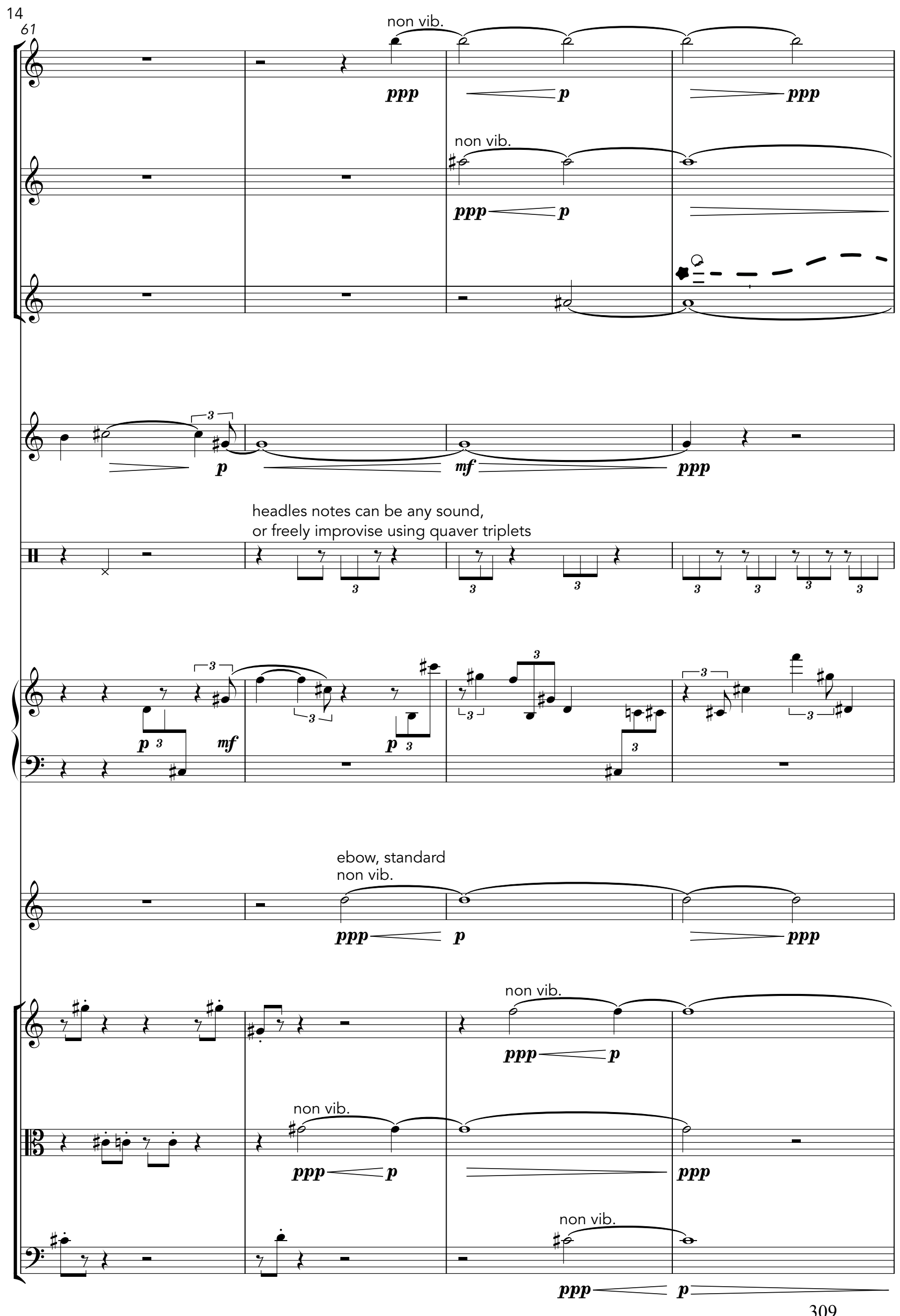


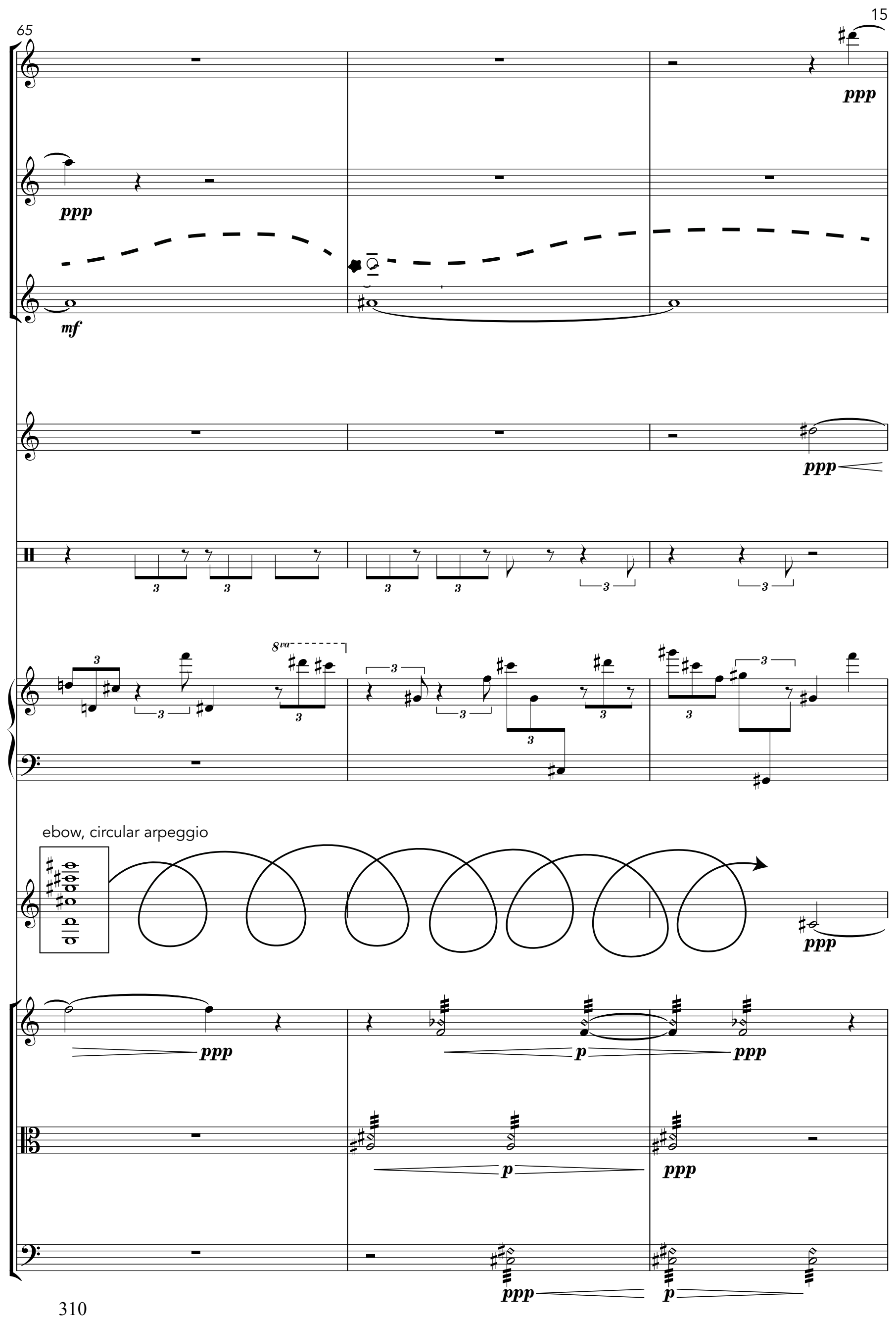




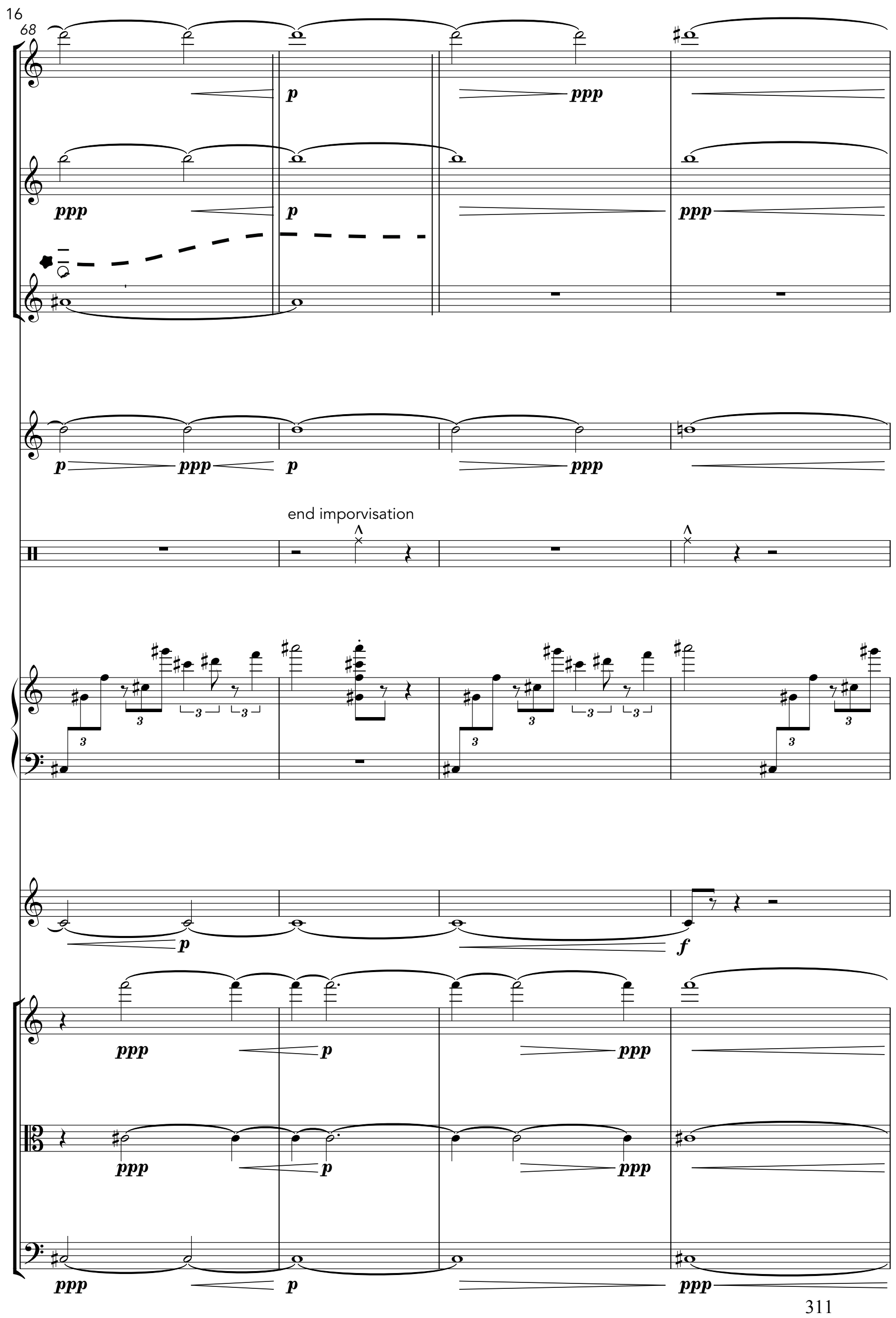




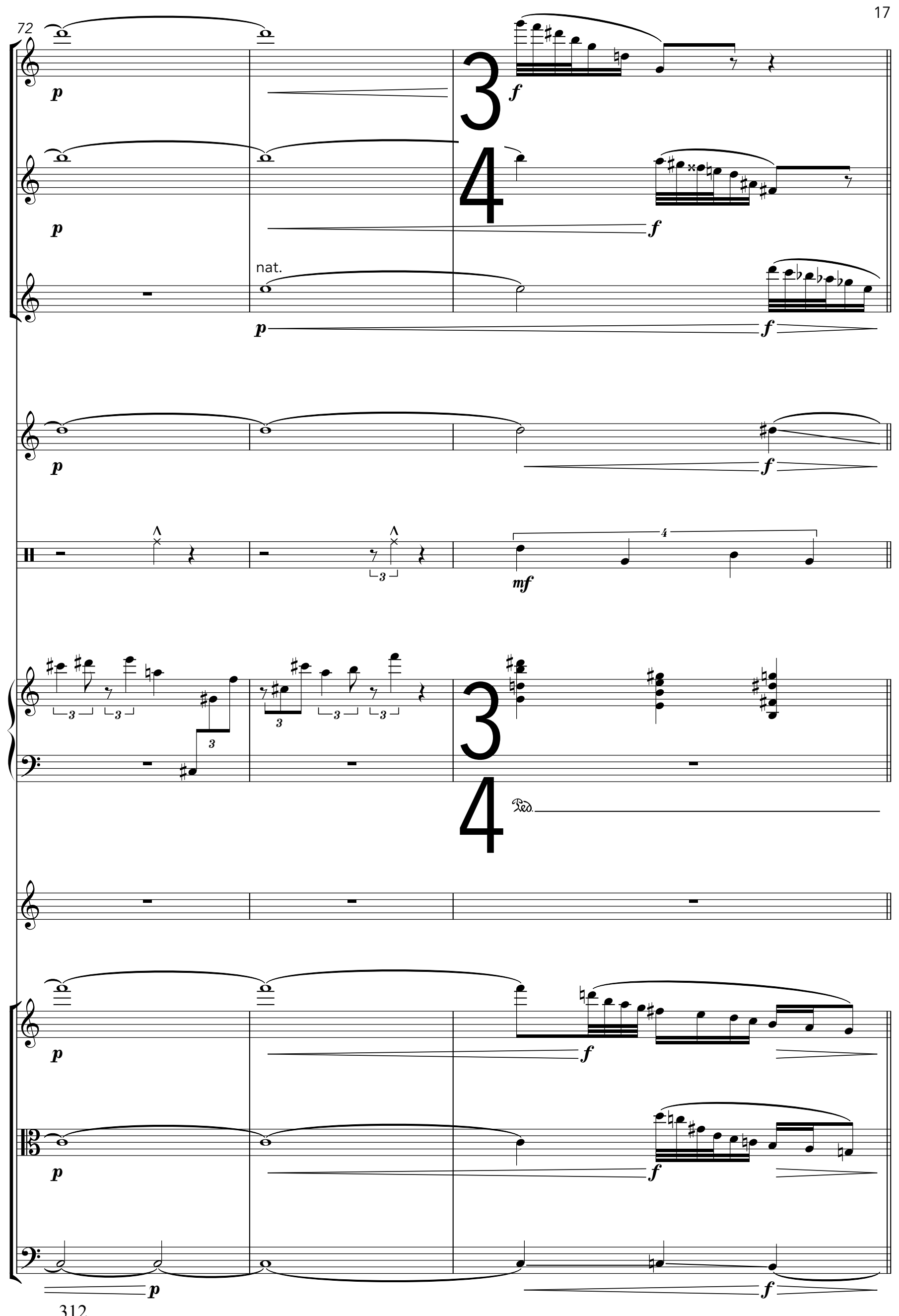




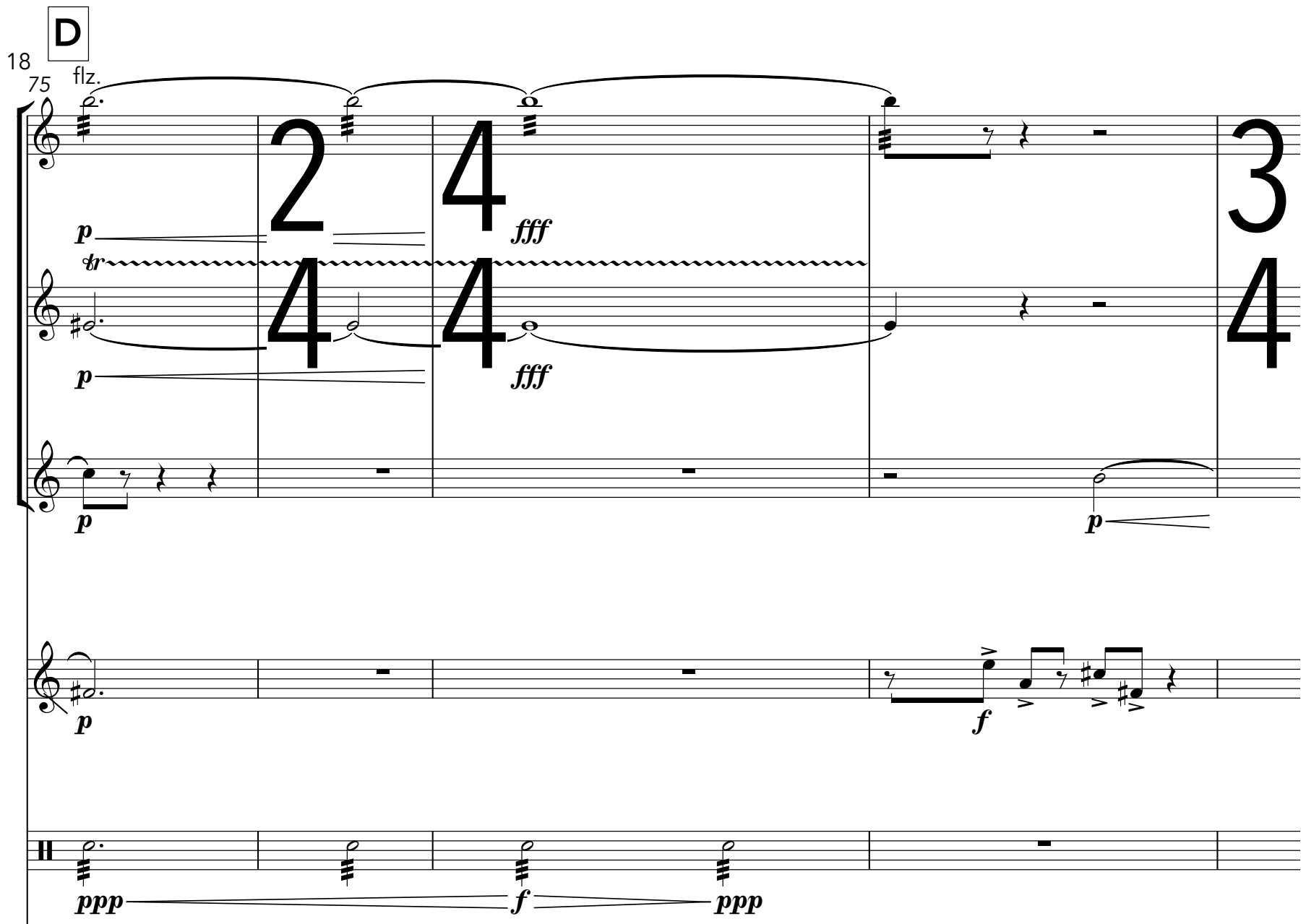

D
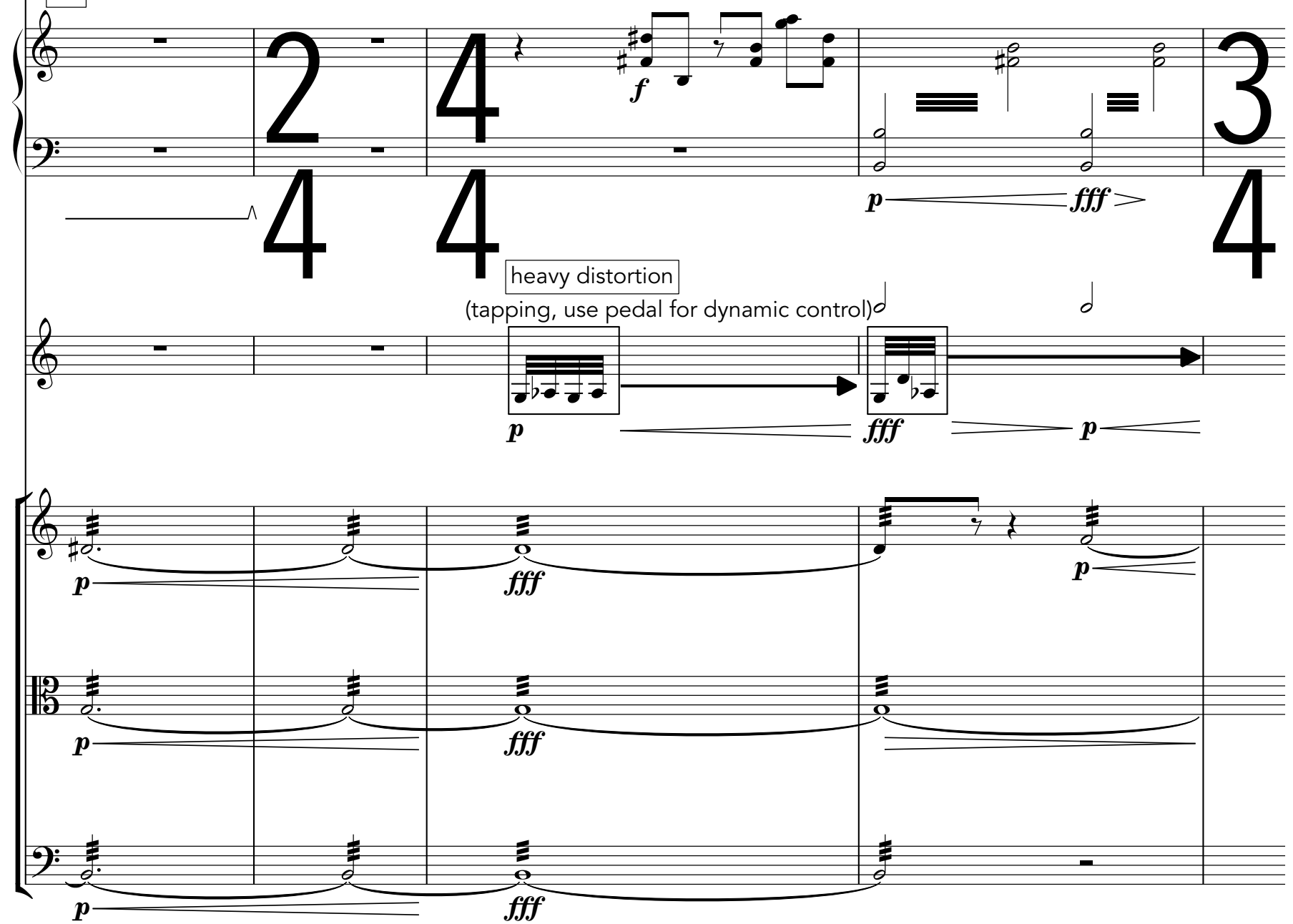


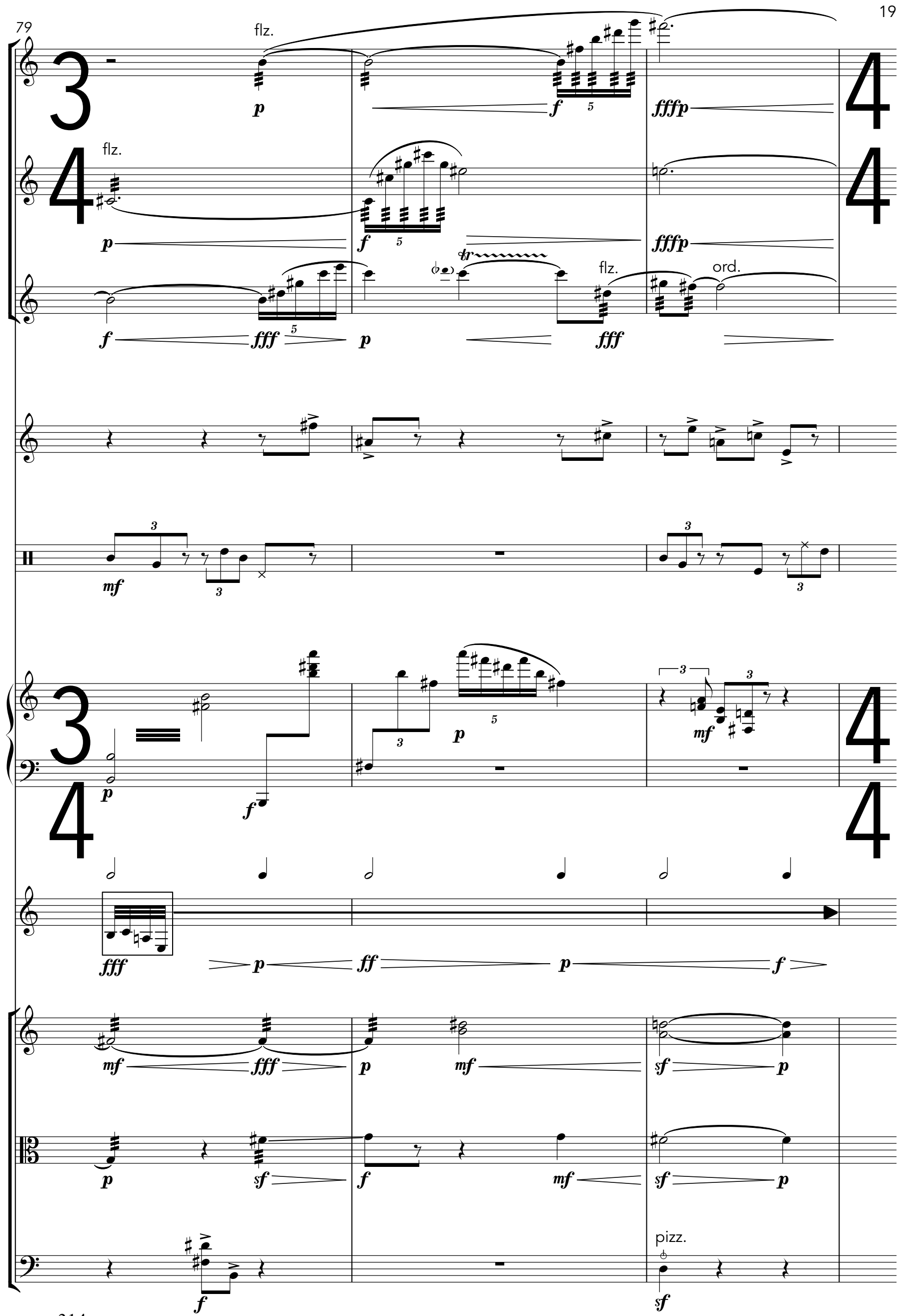




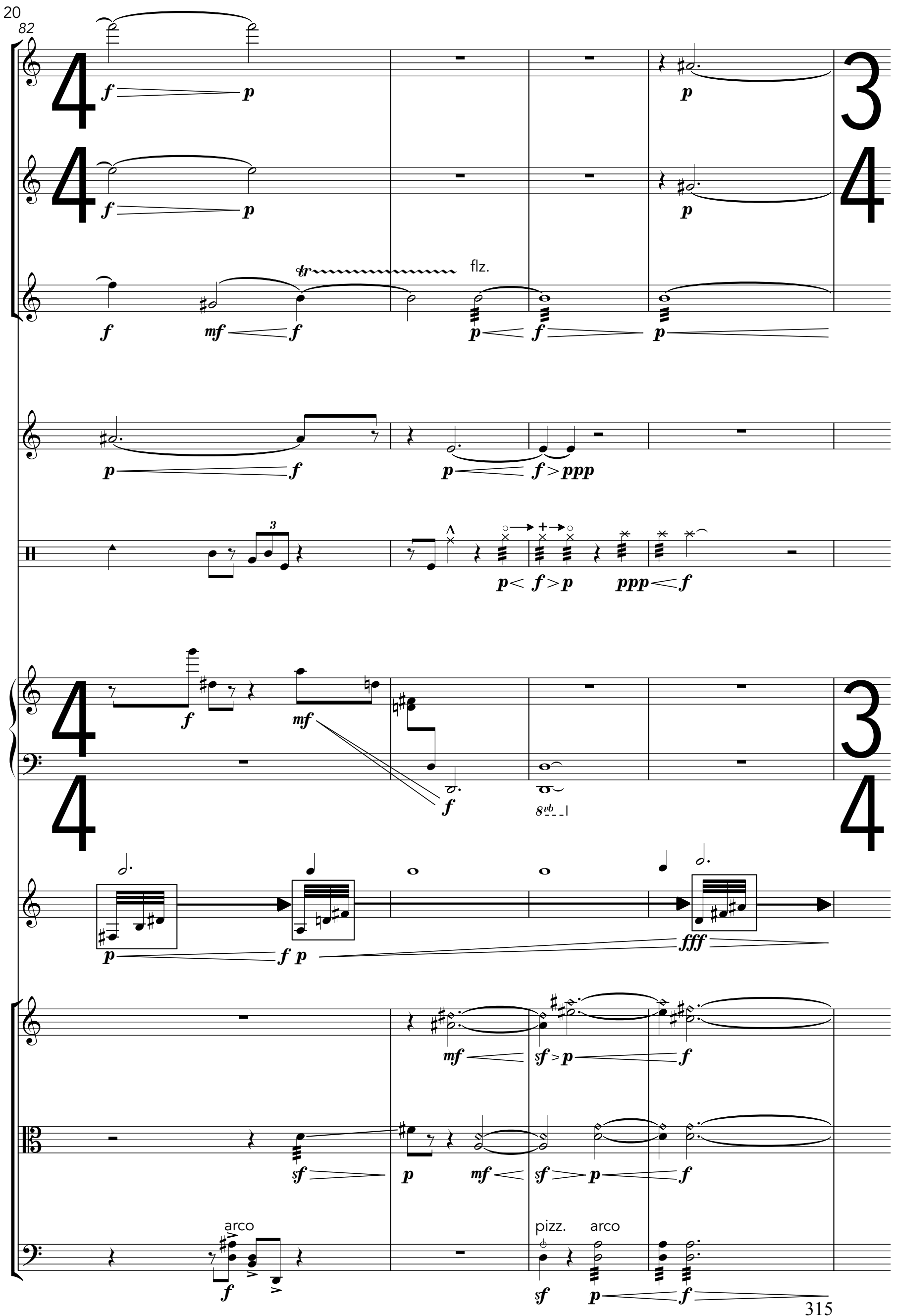



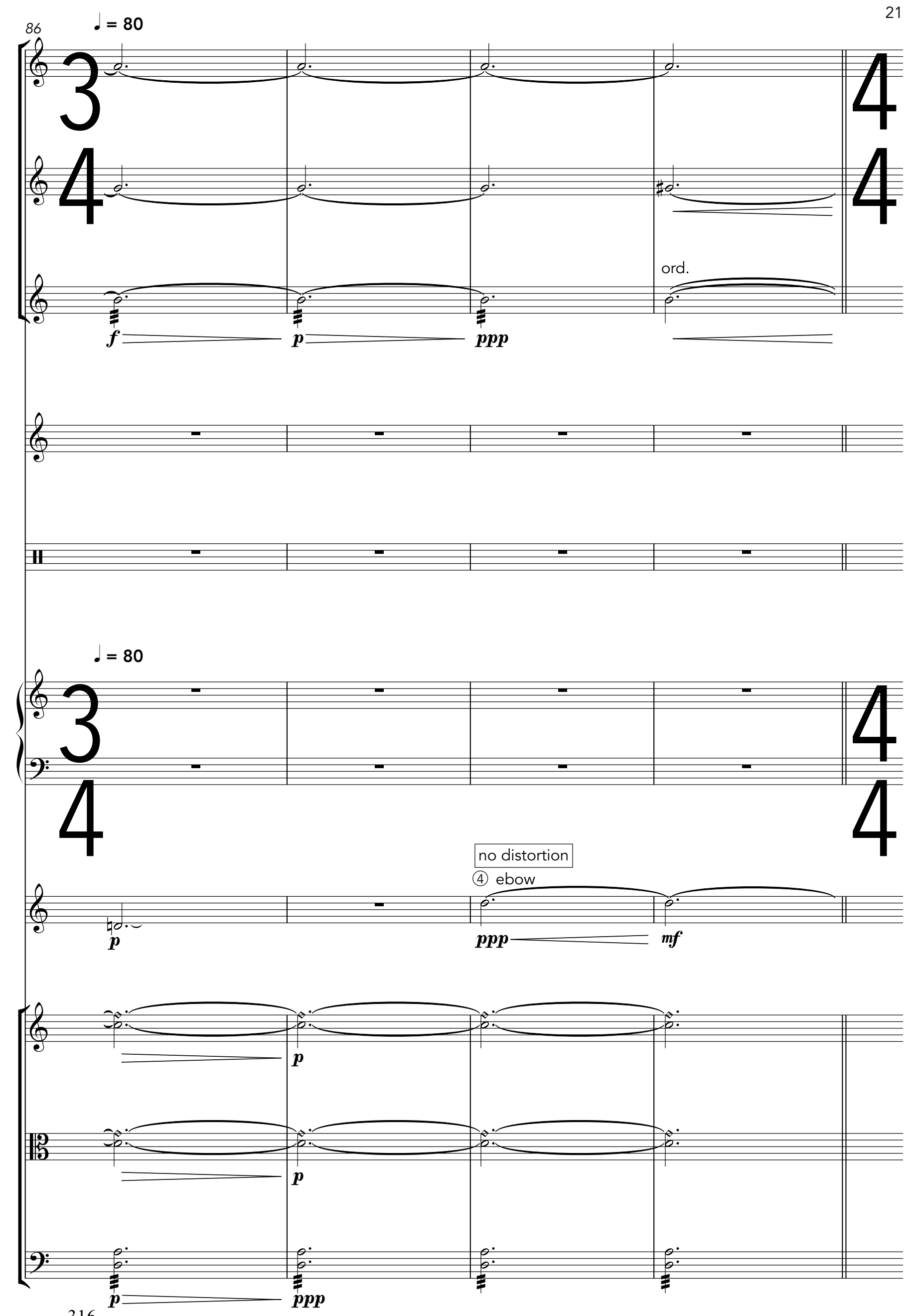


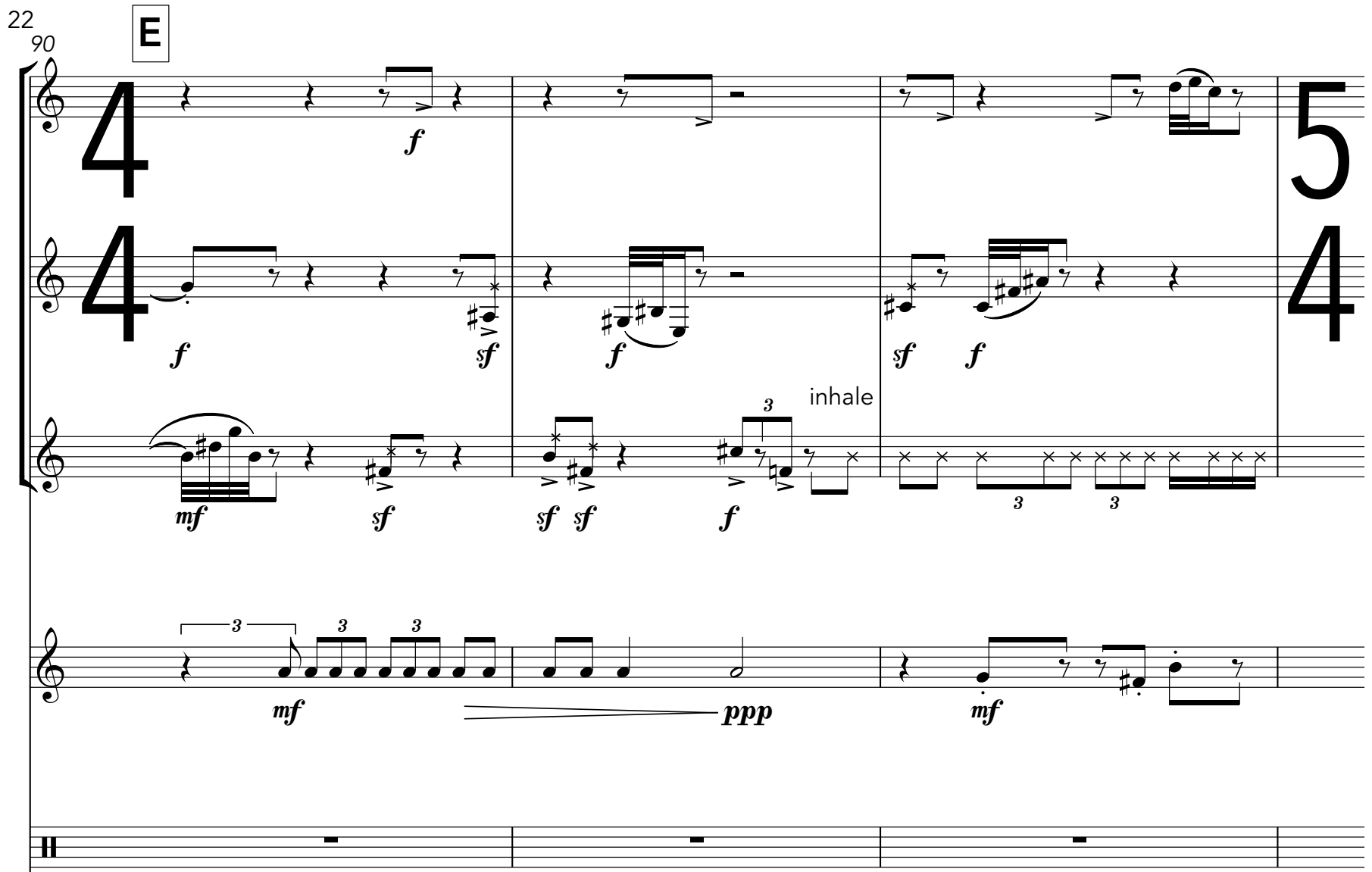

E
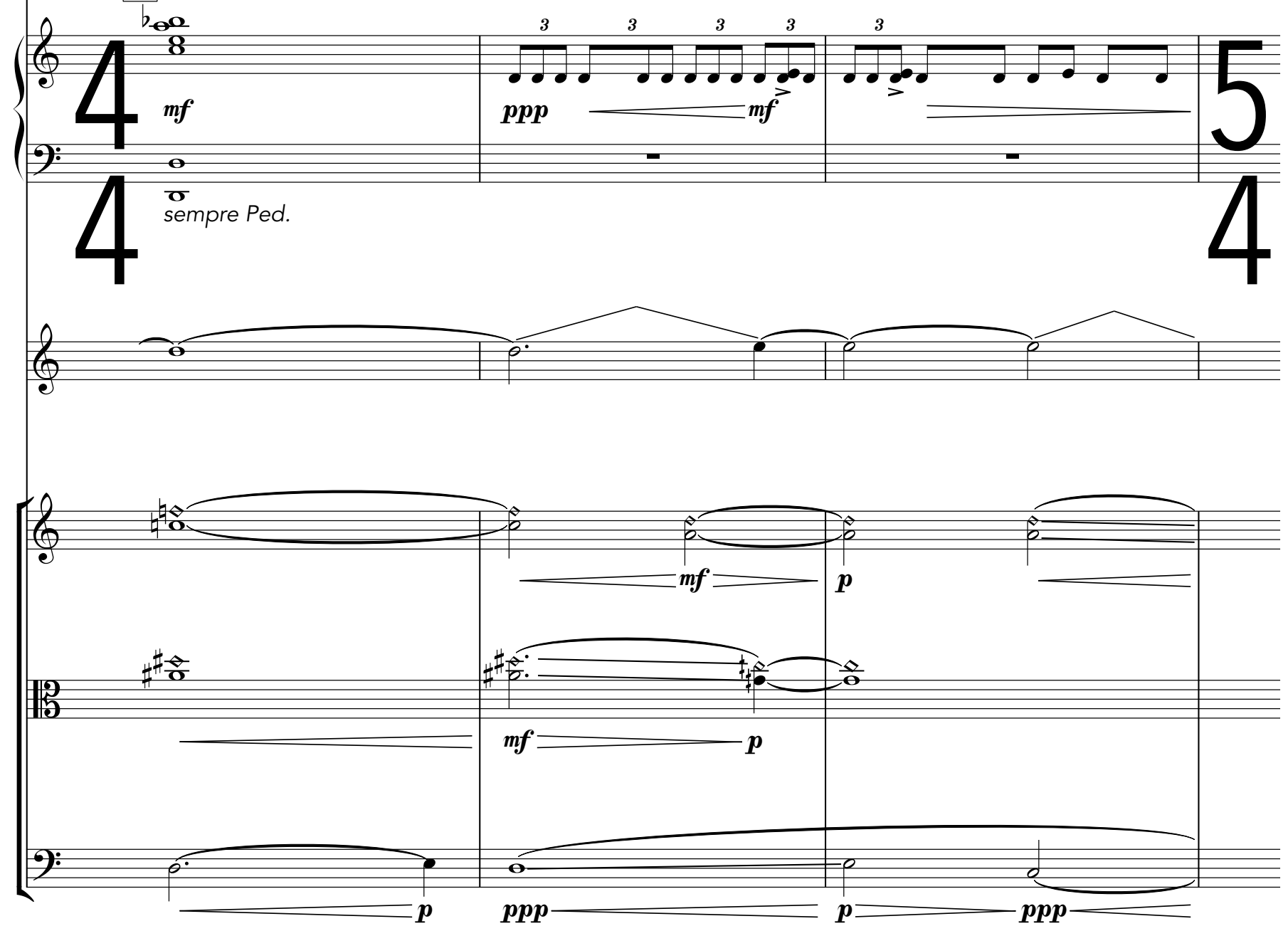

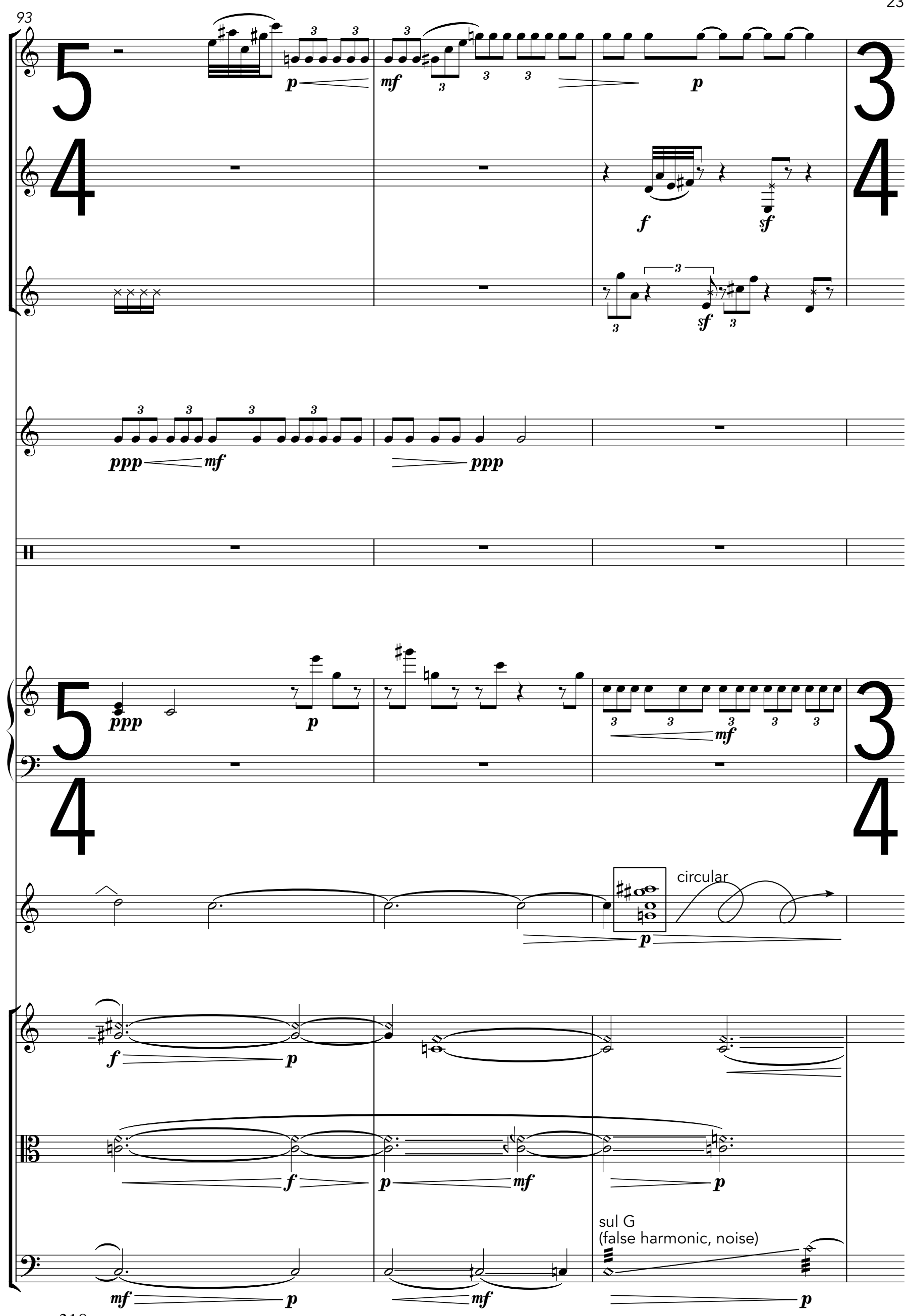


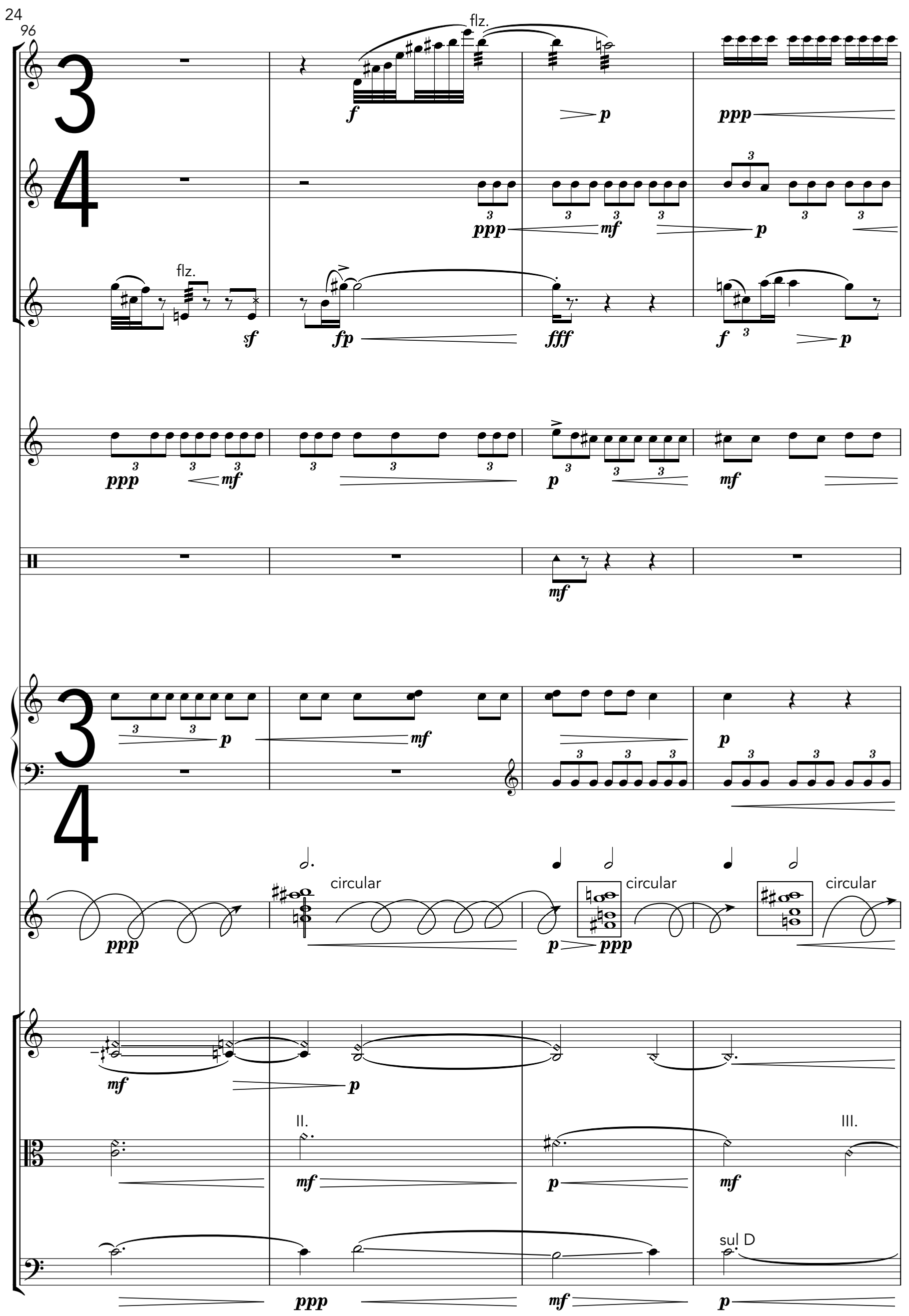



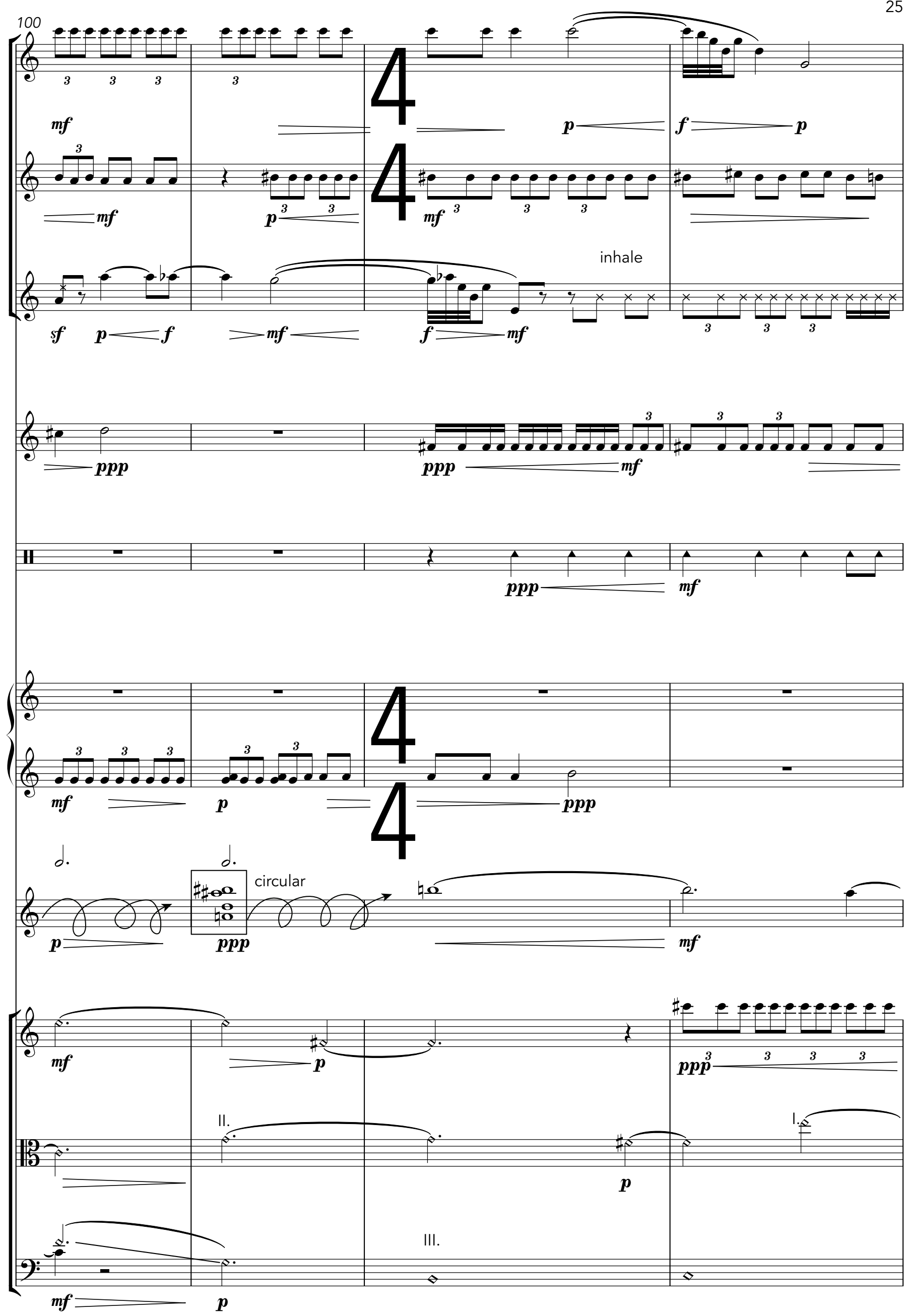


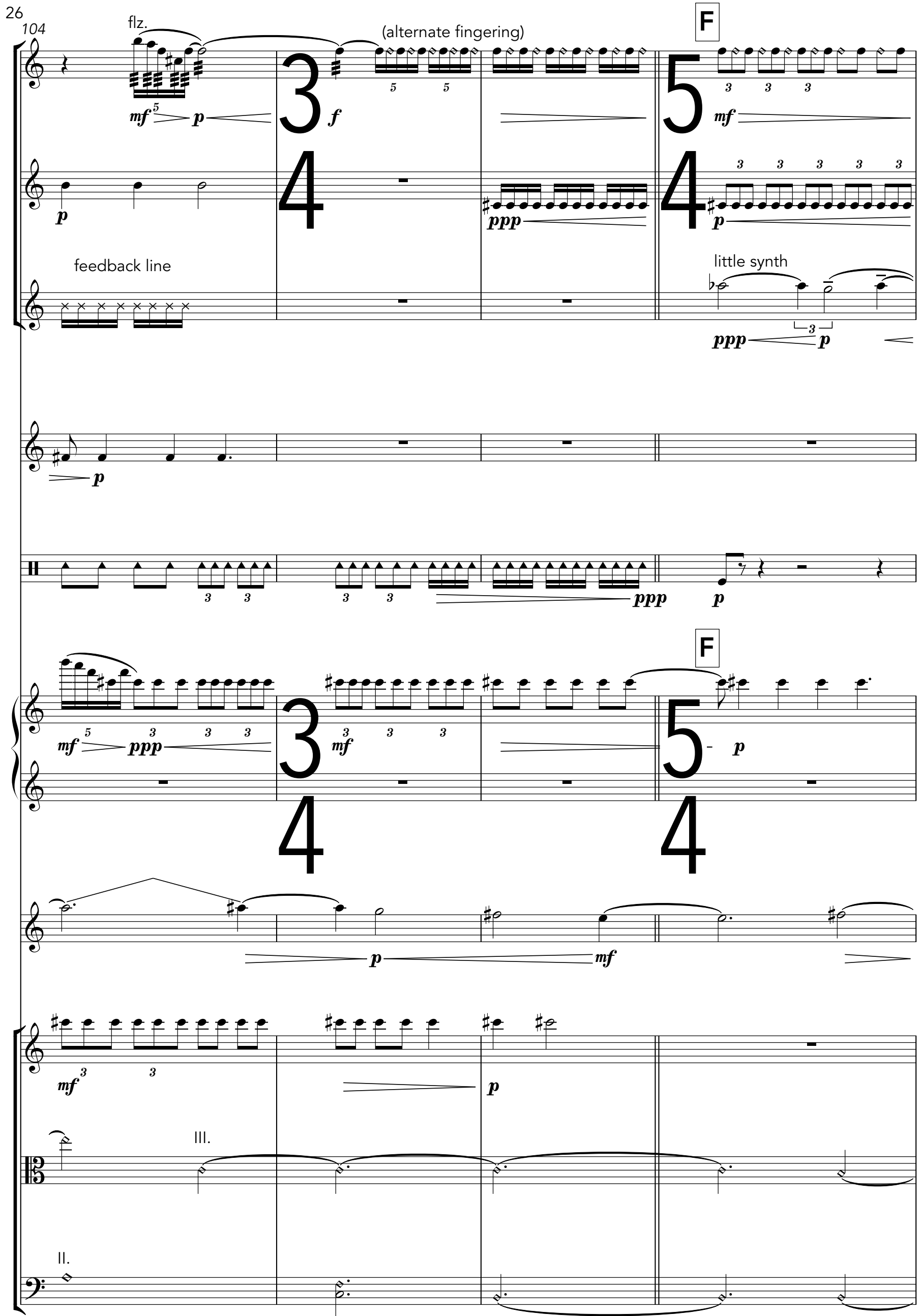



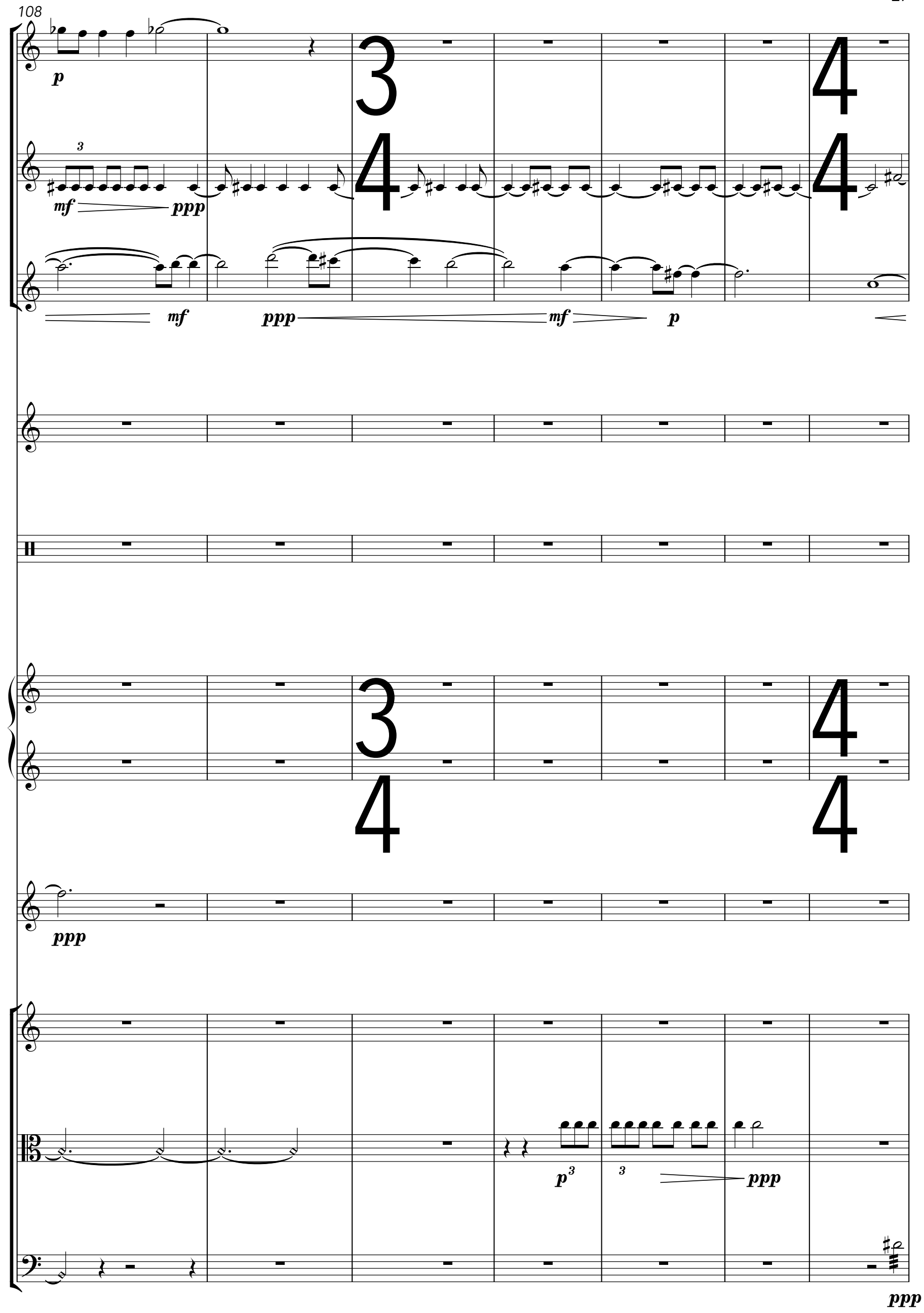


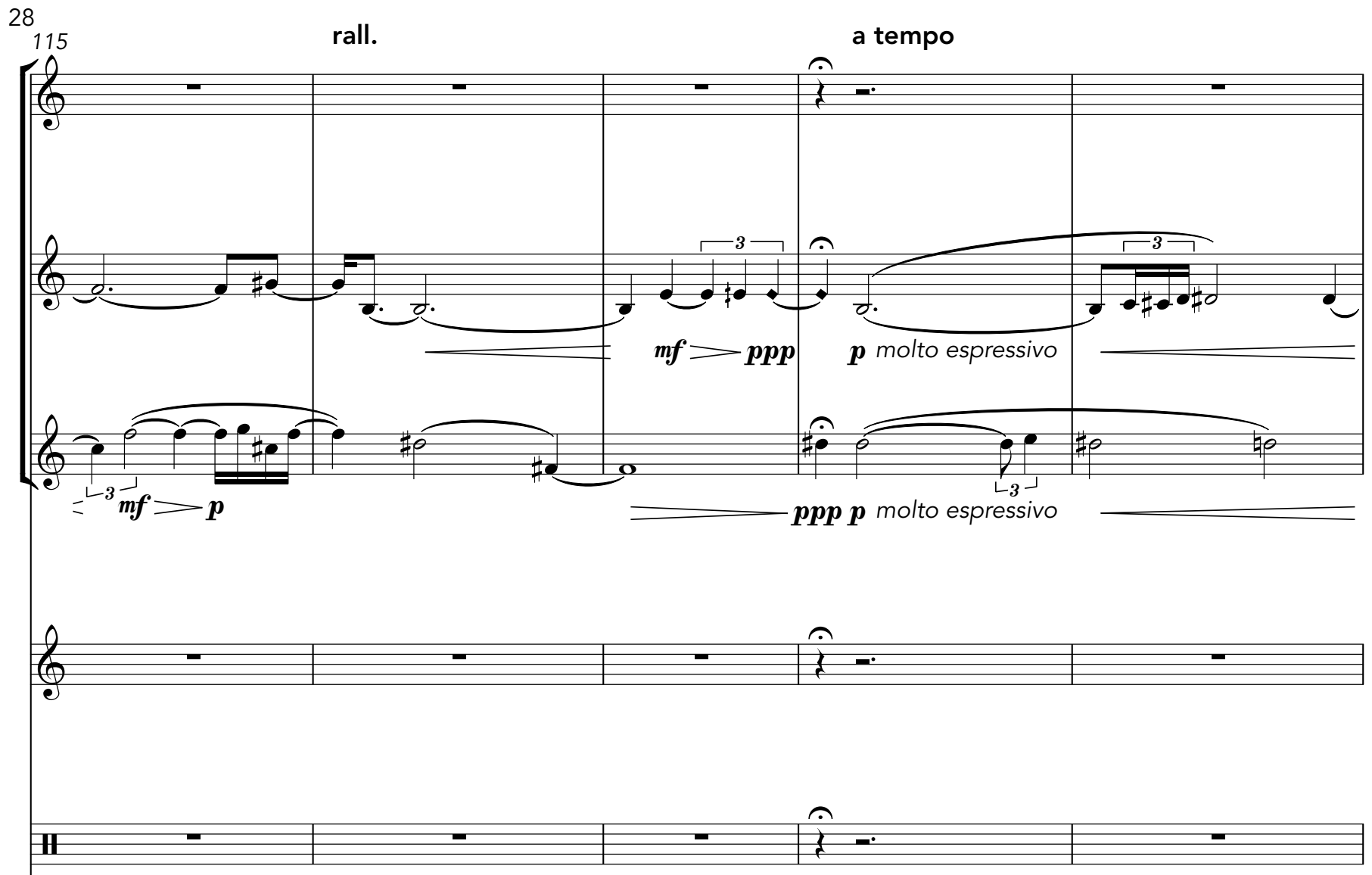

rall. a tempo
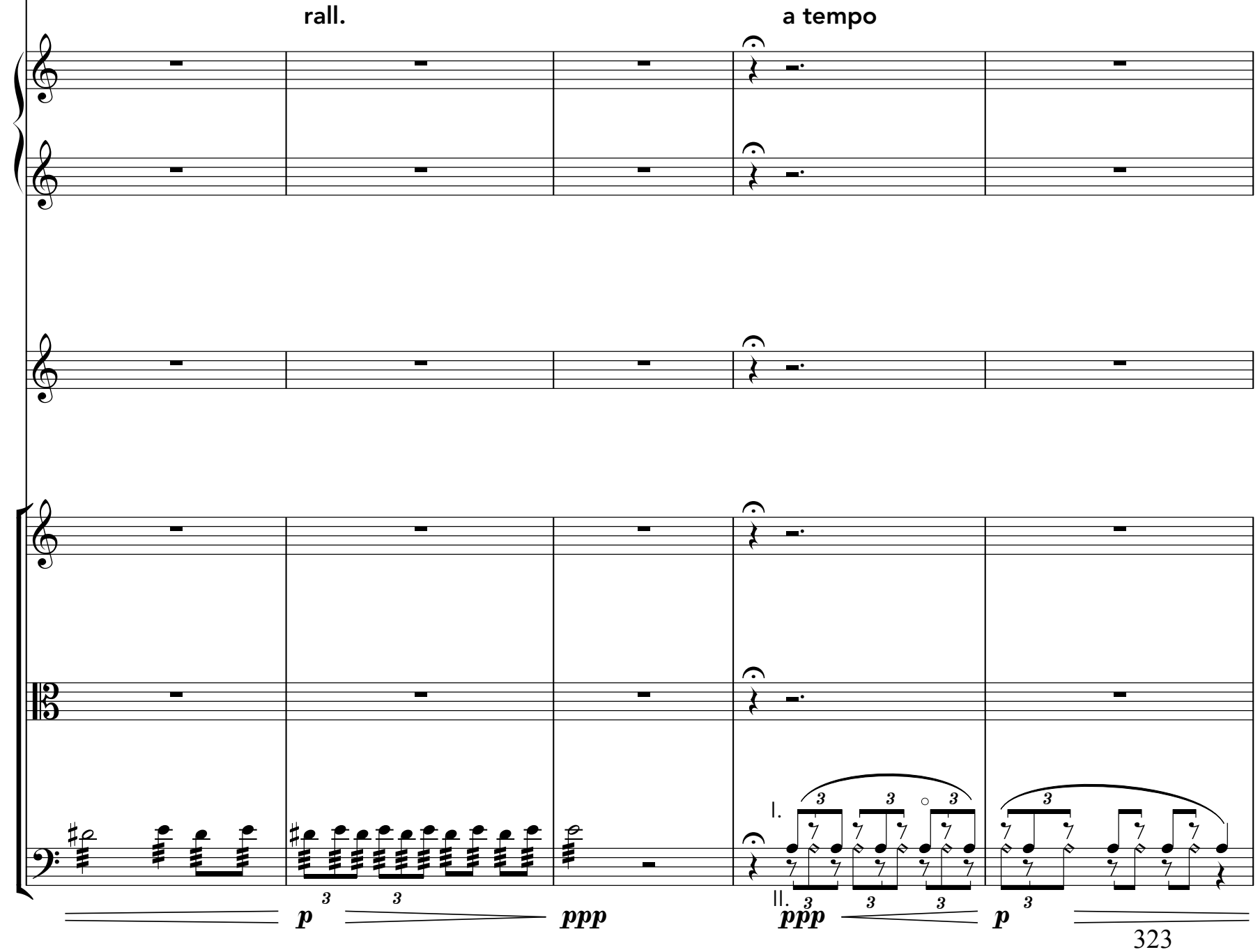


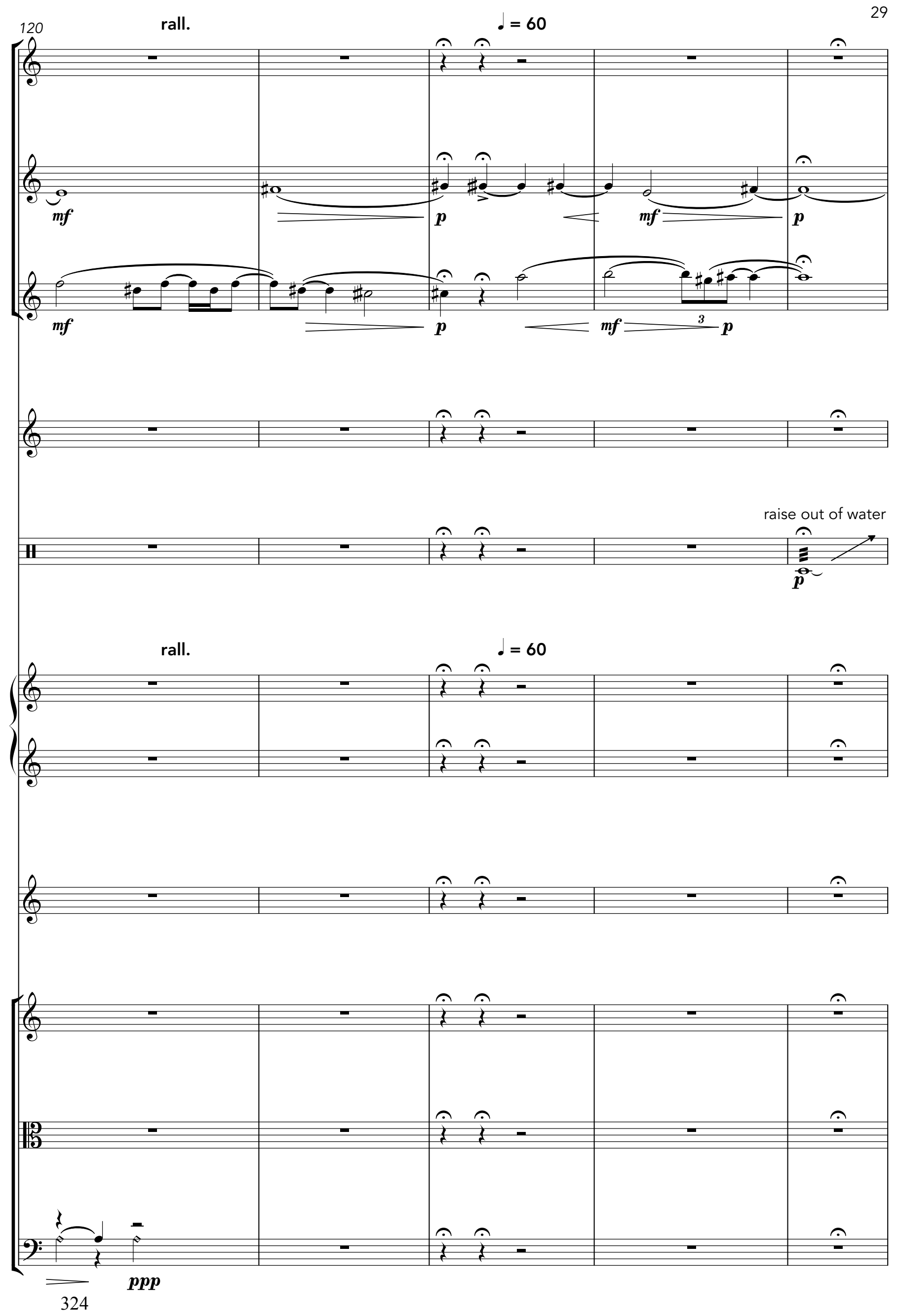




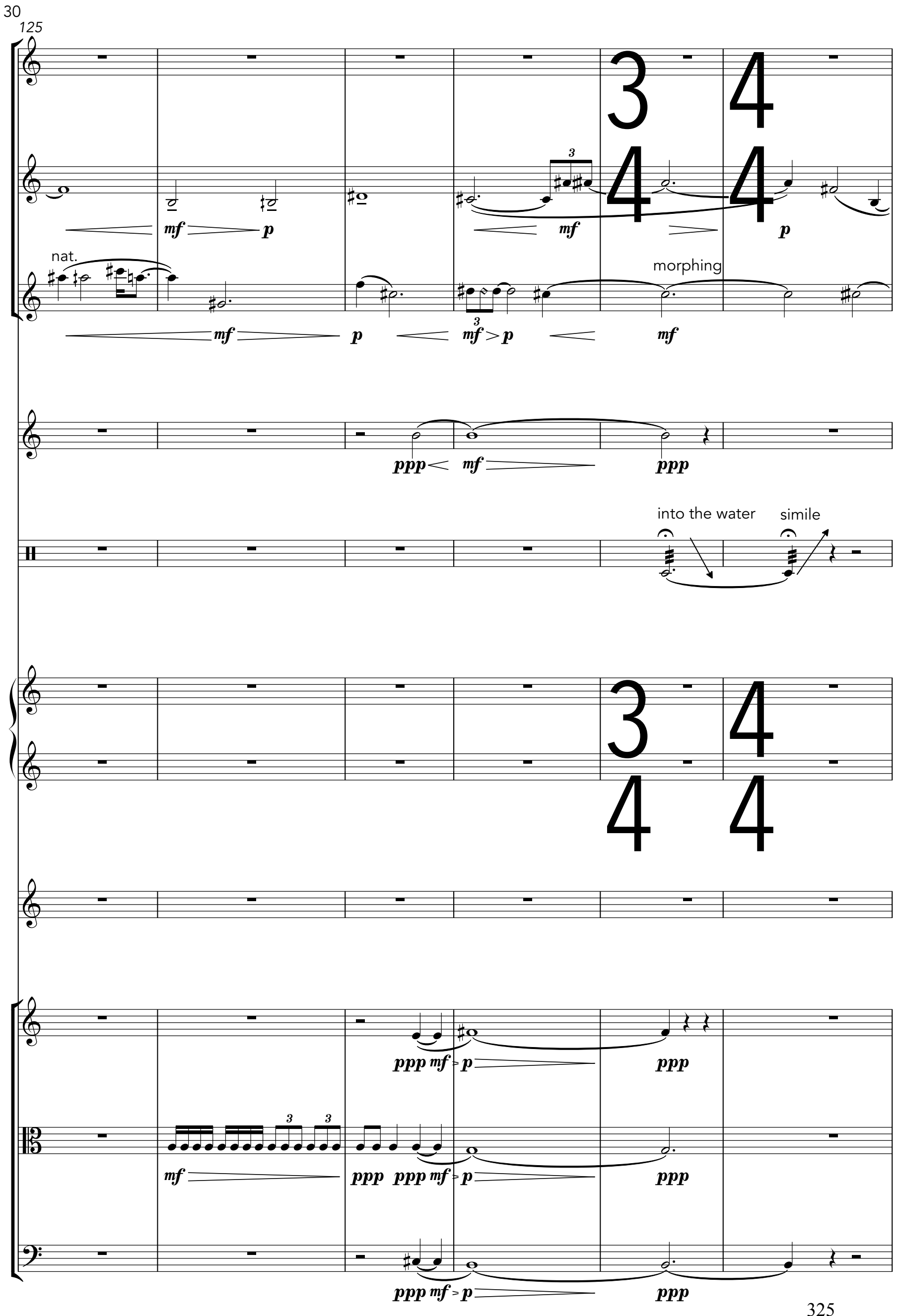



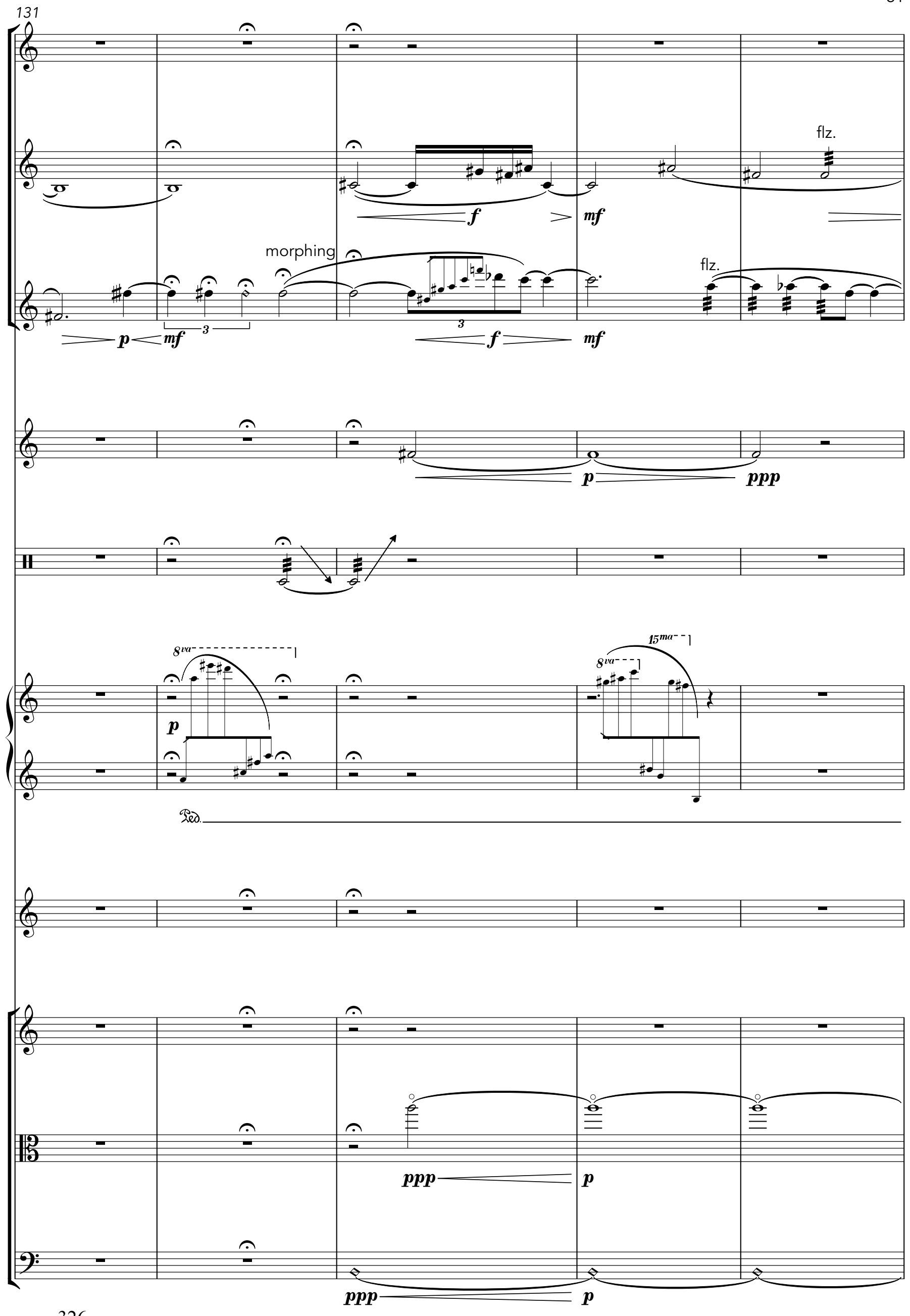


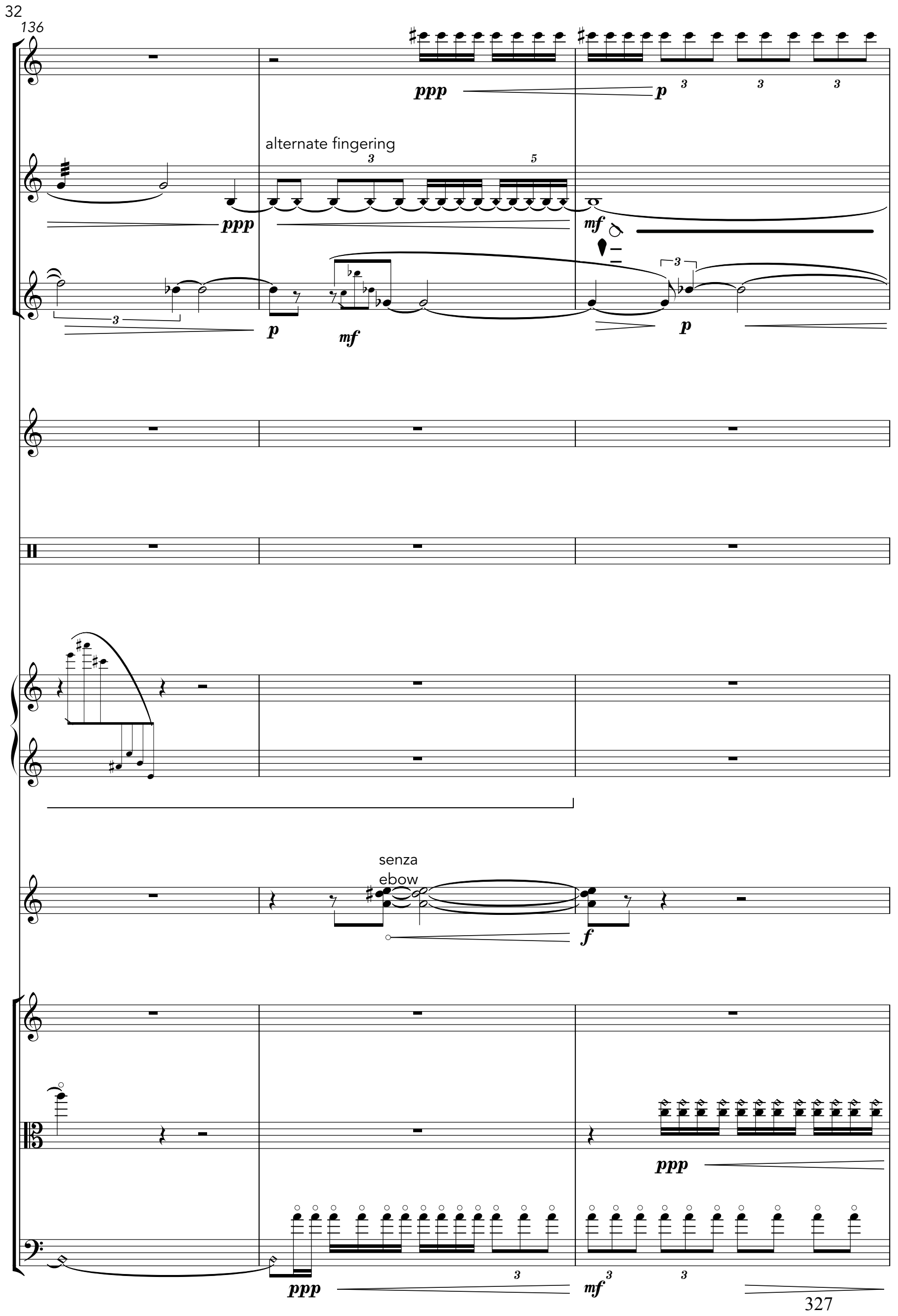



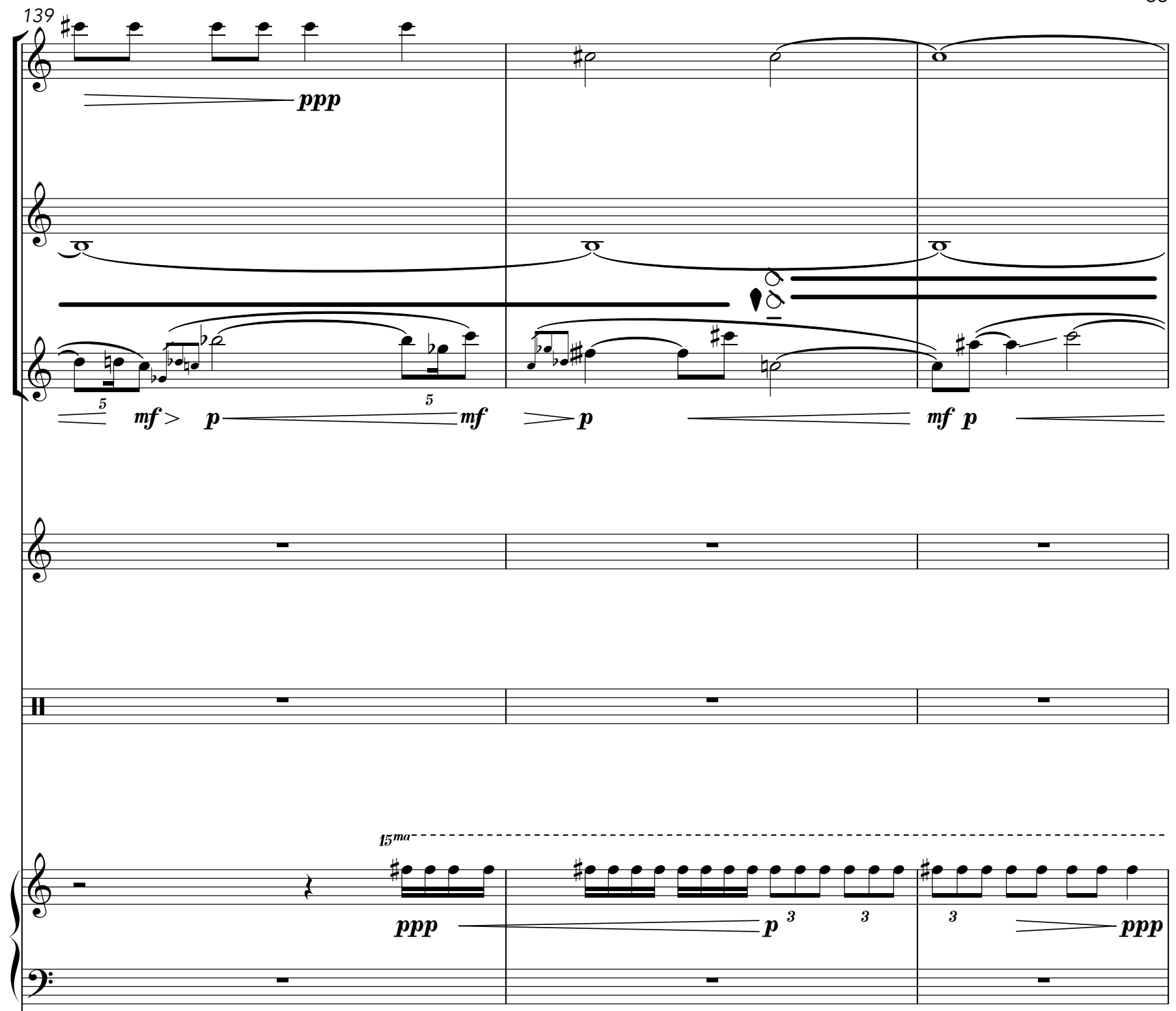
2ed.
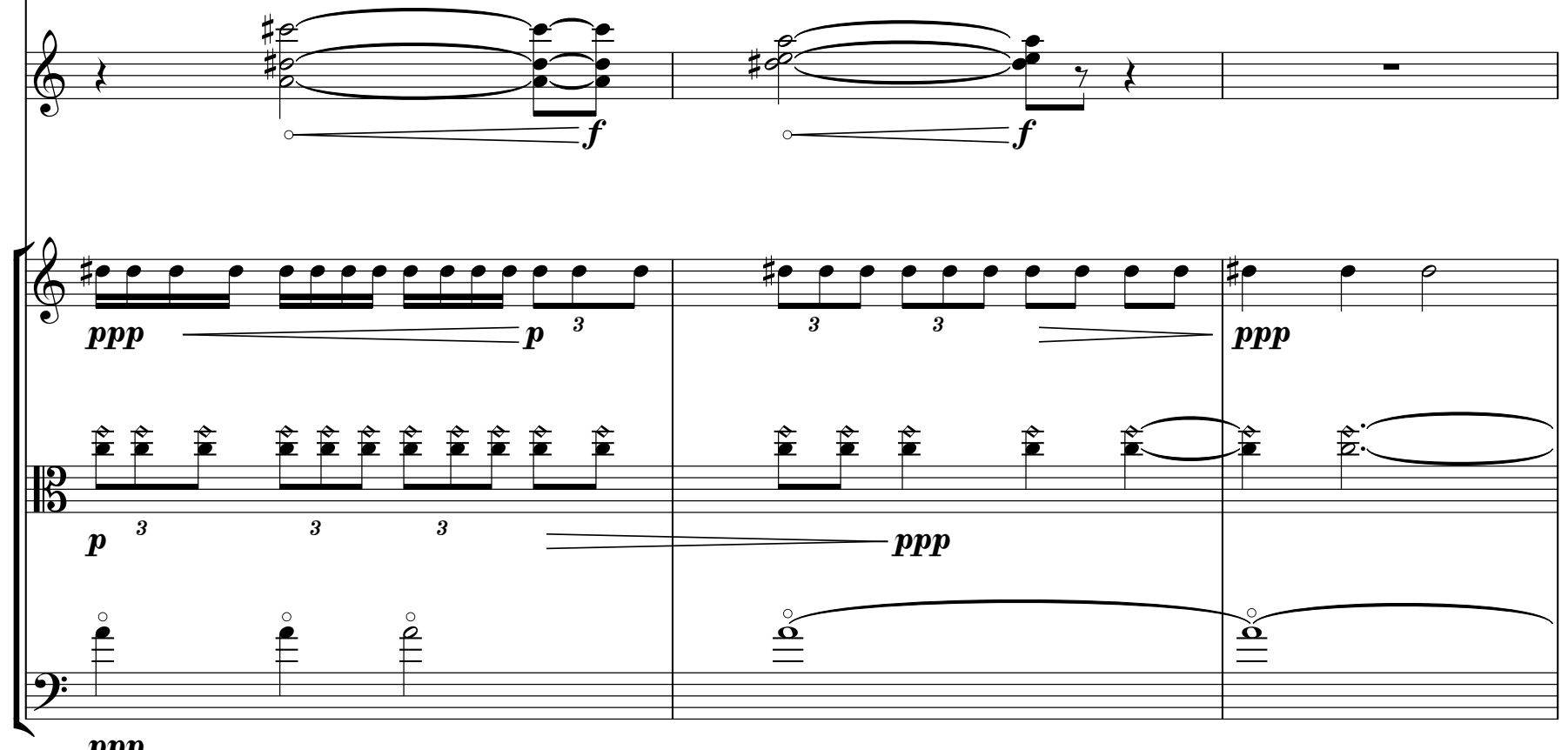


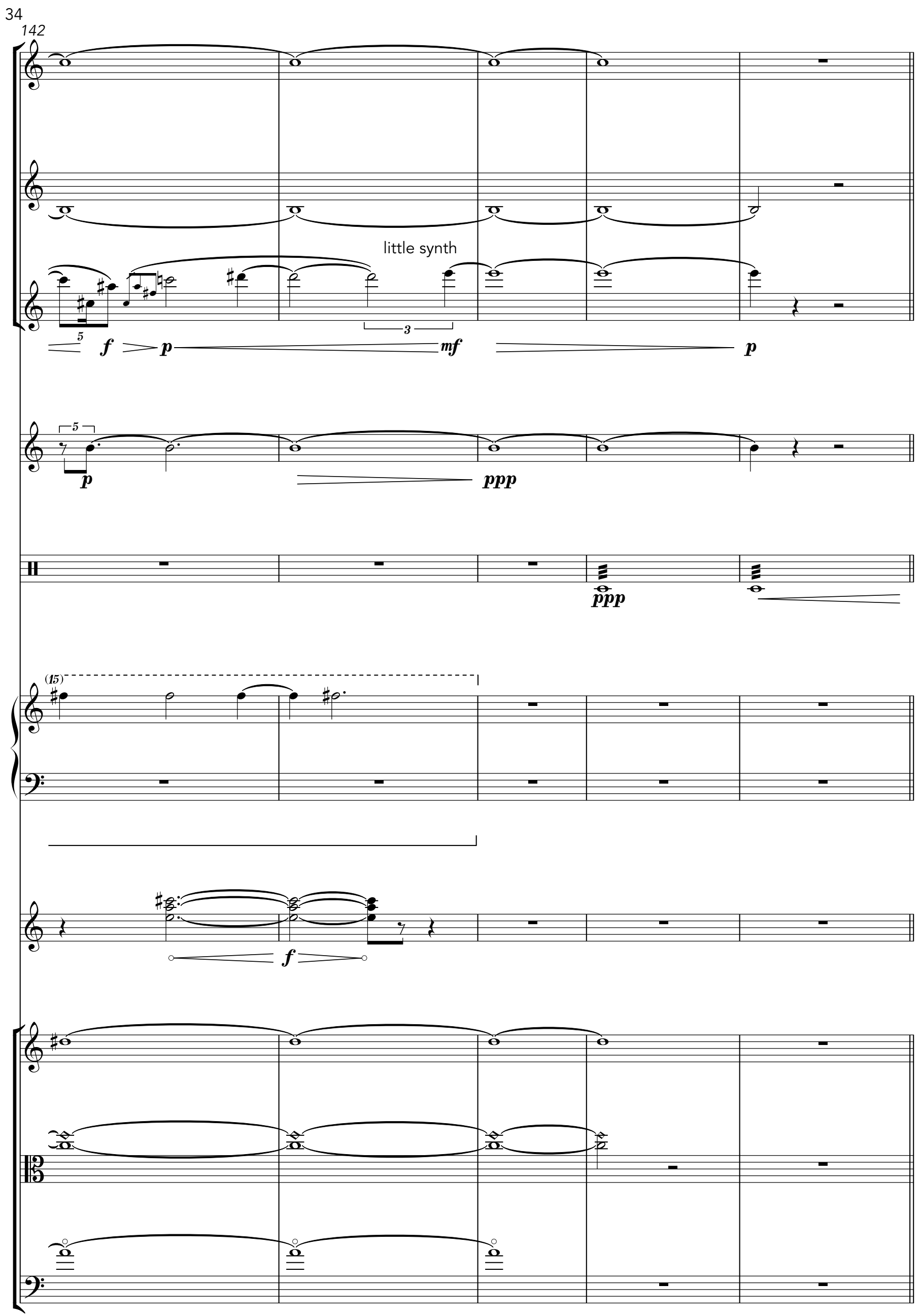




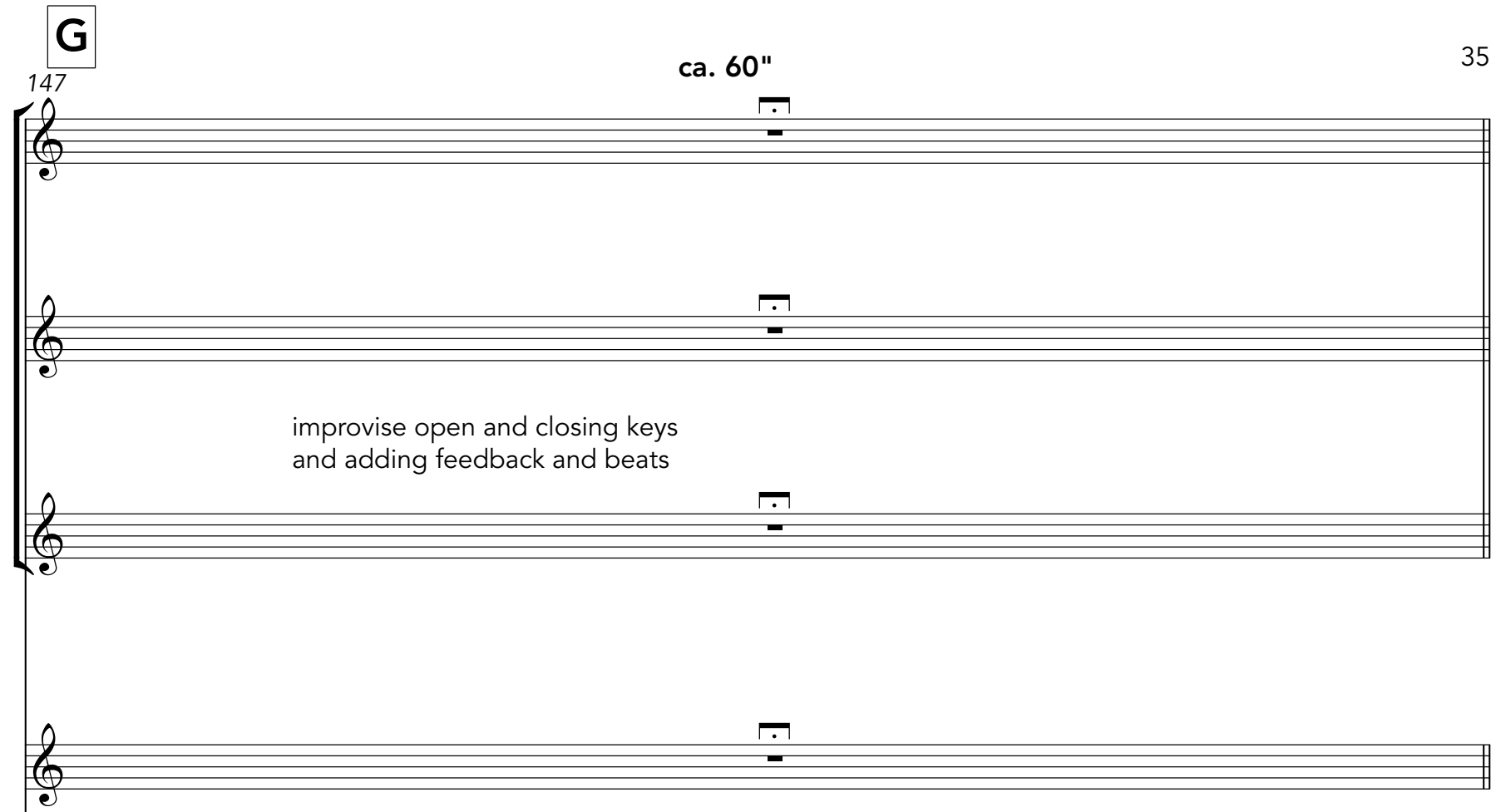

improvise dynamics and pitch bend with the sax

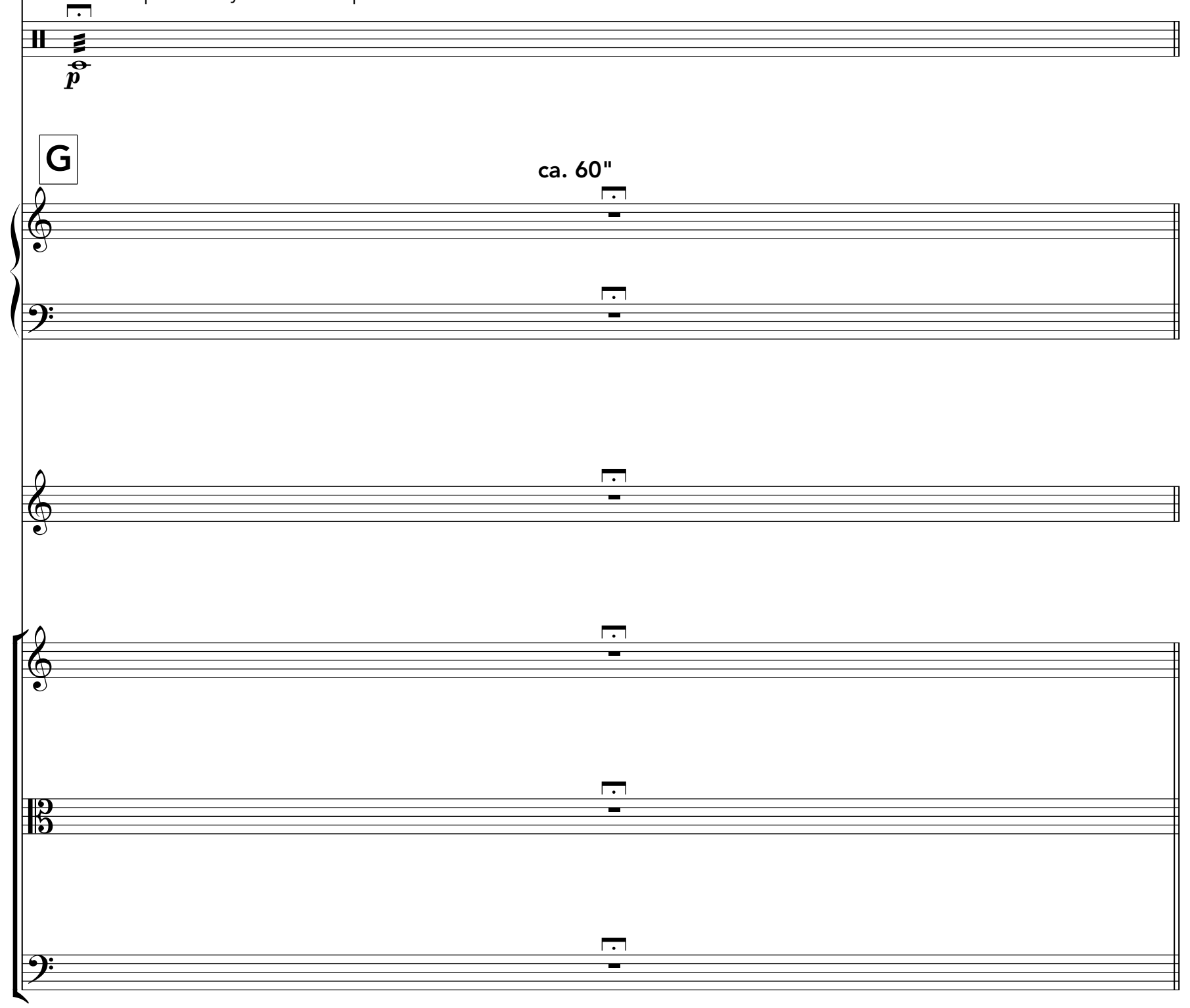




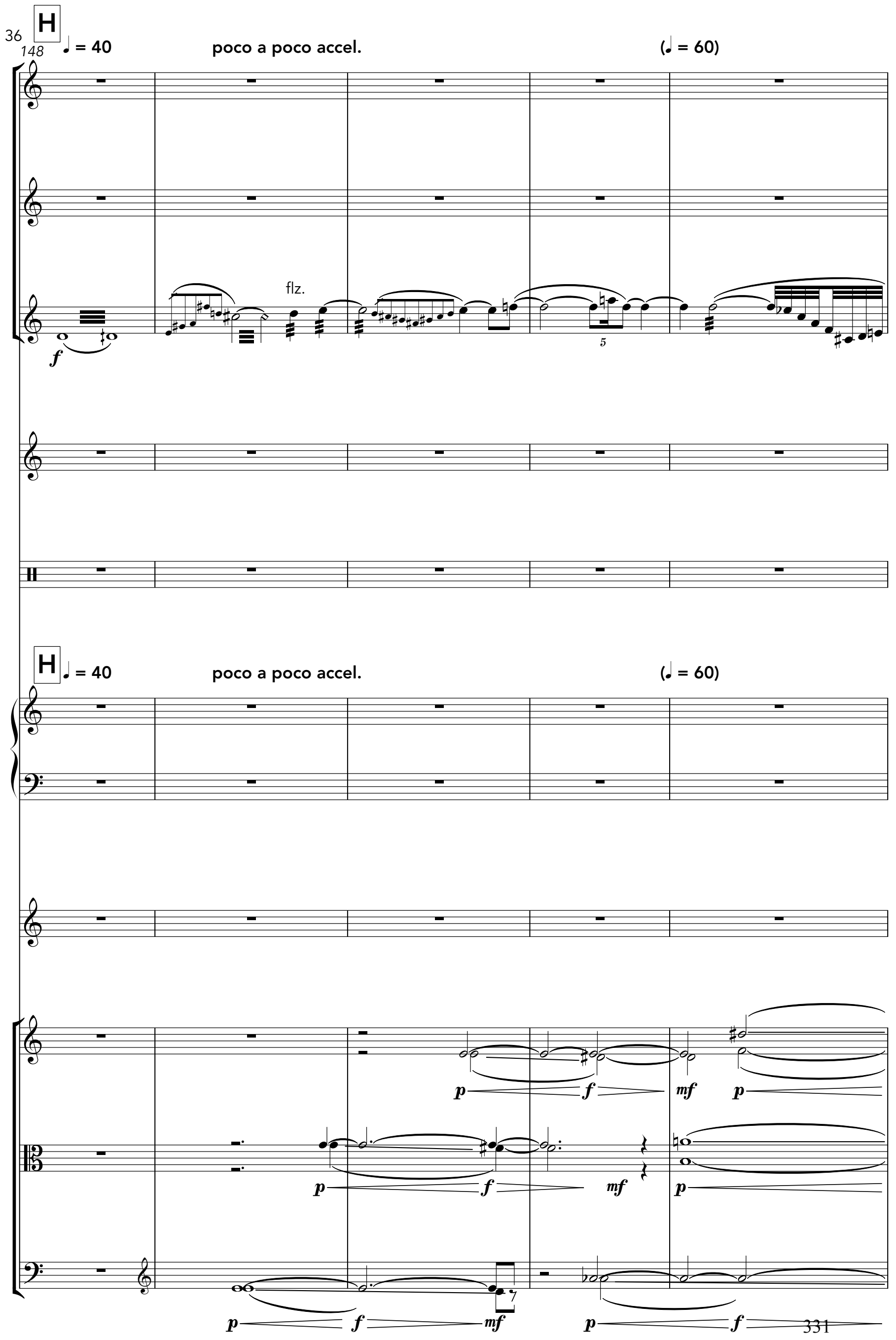



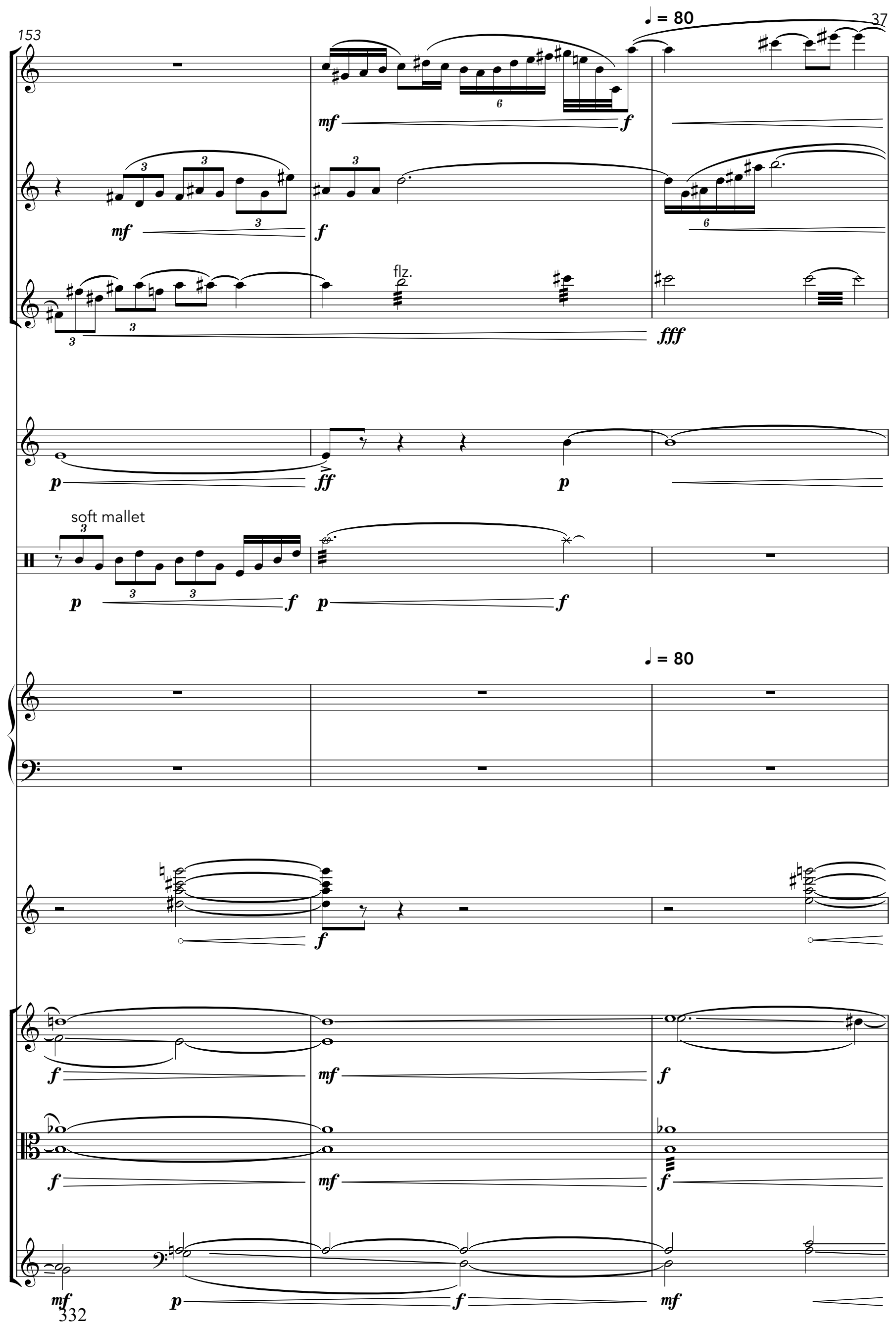


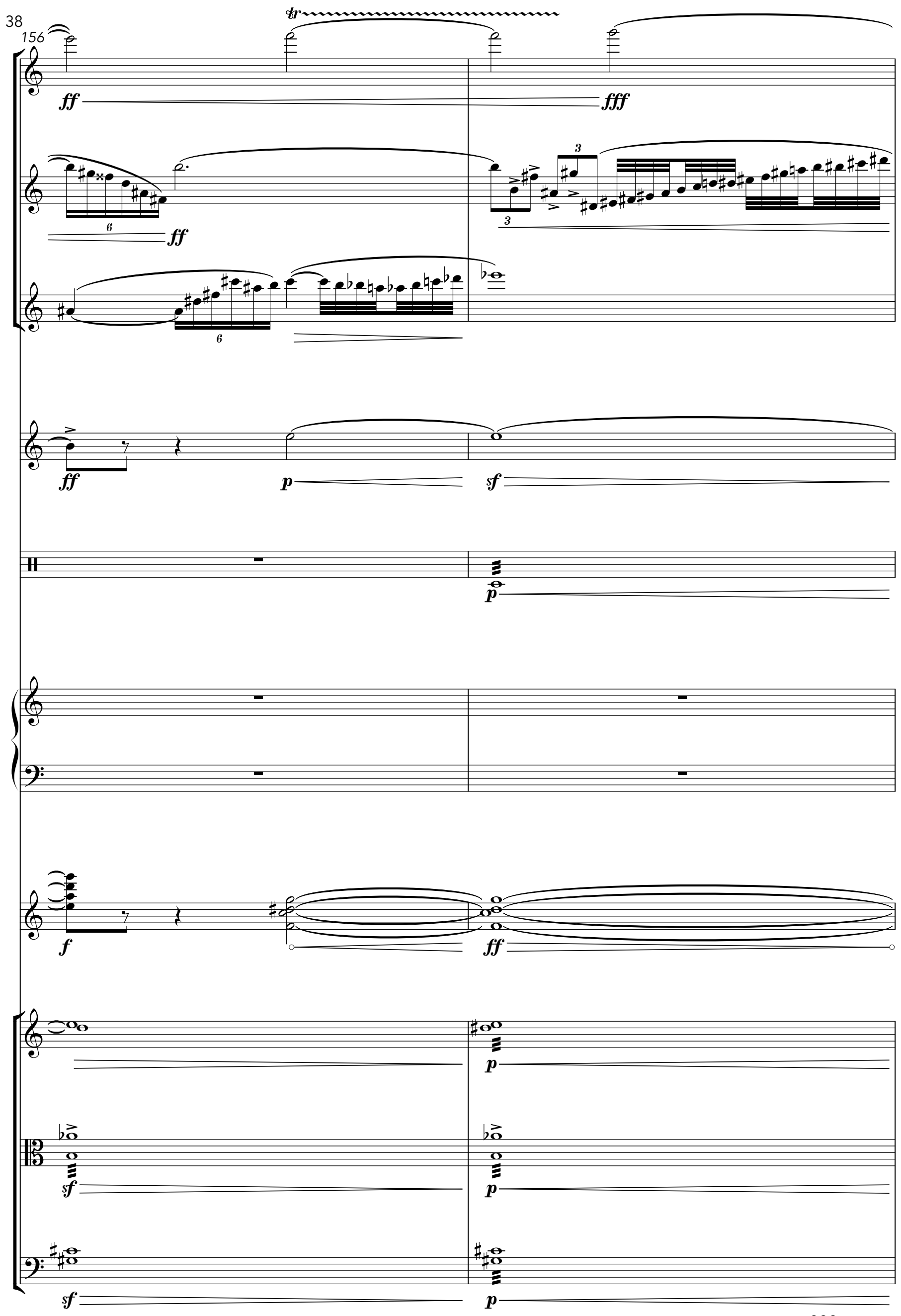



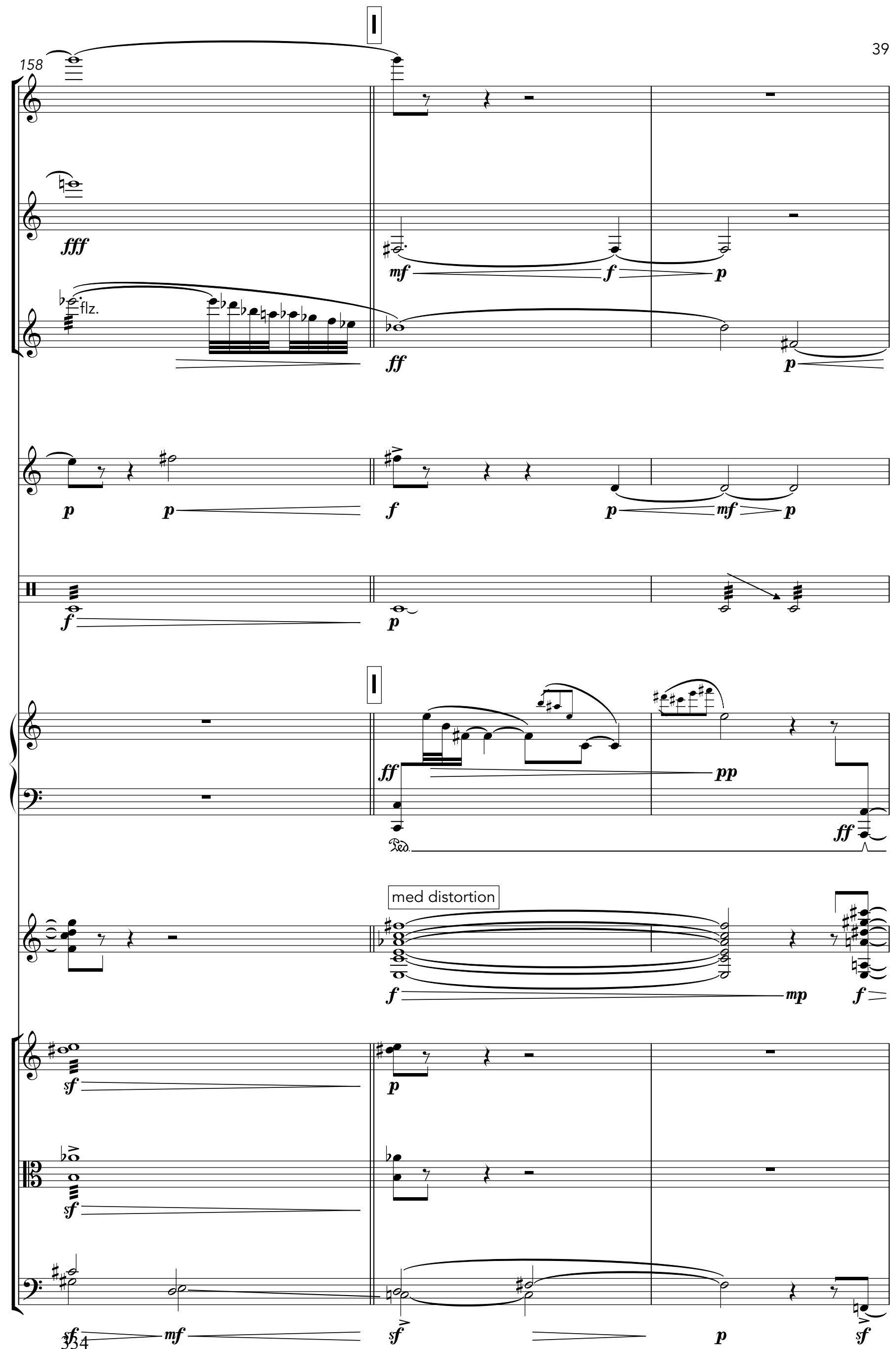


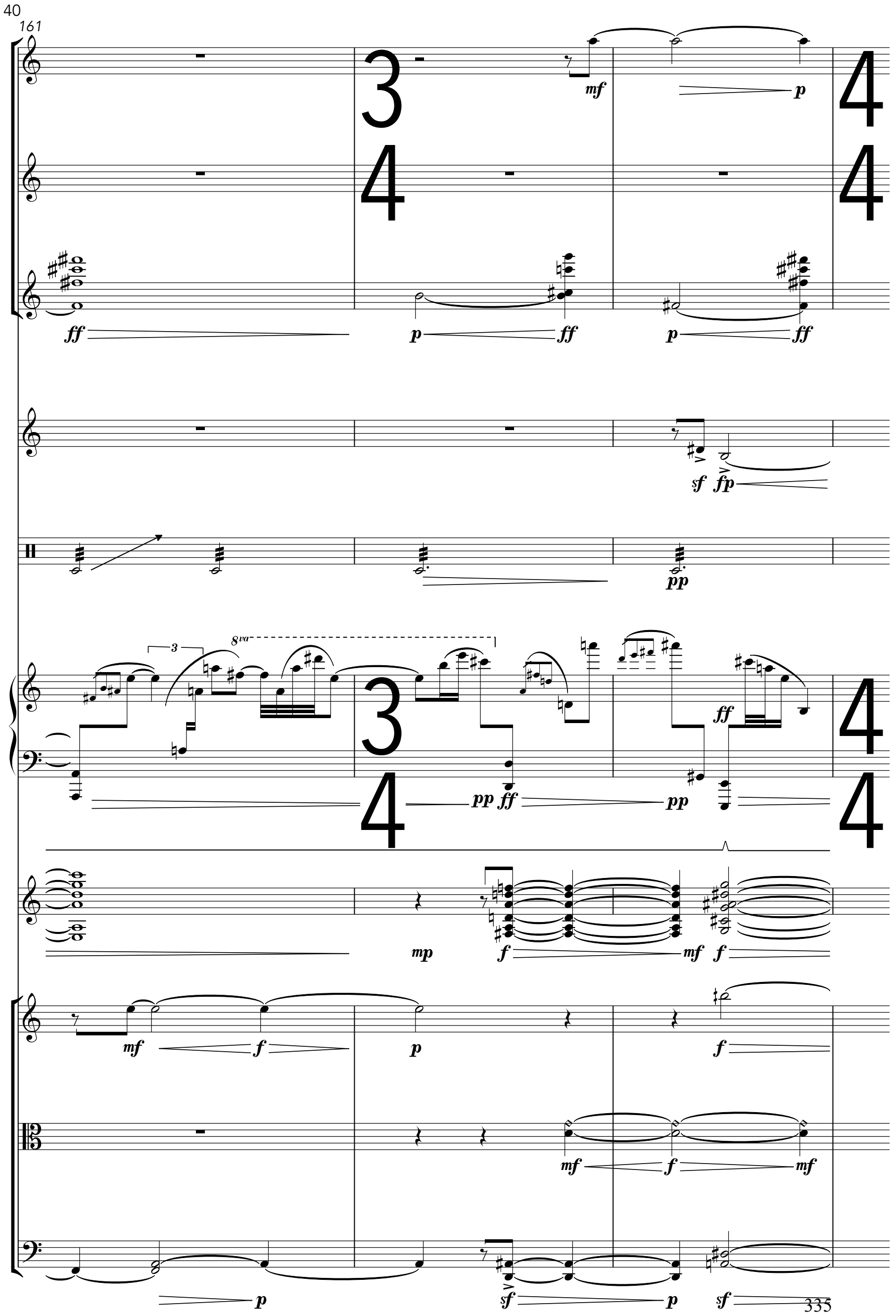



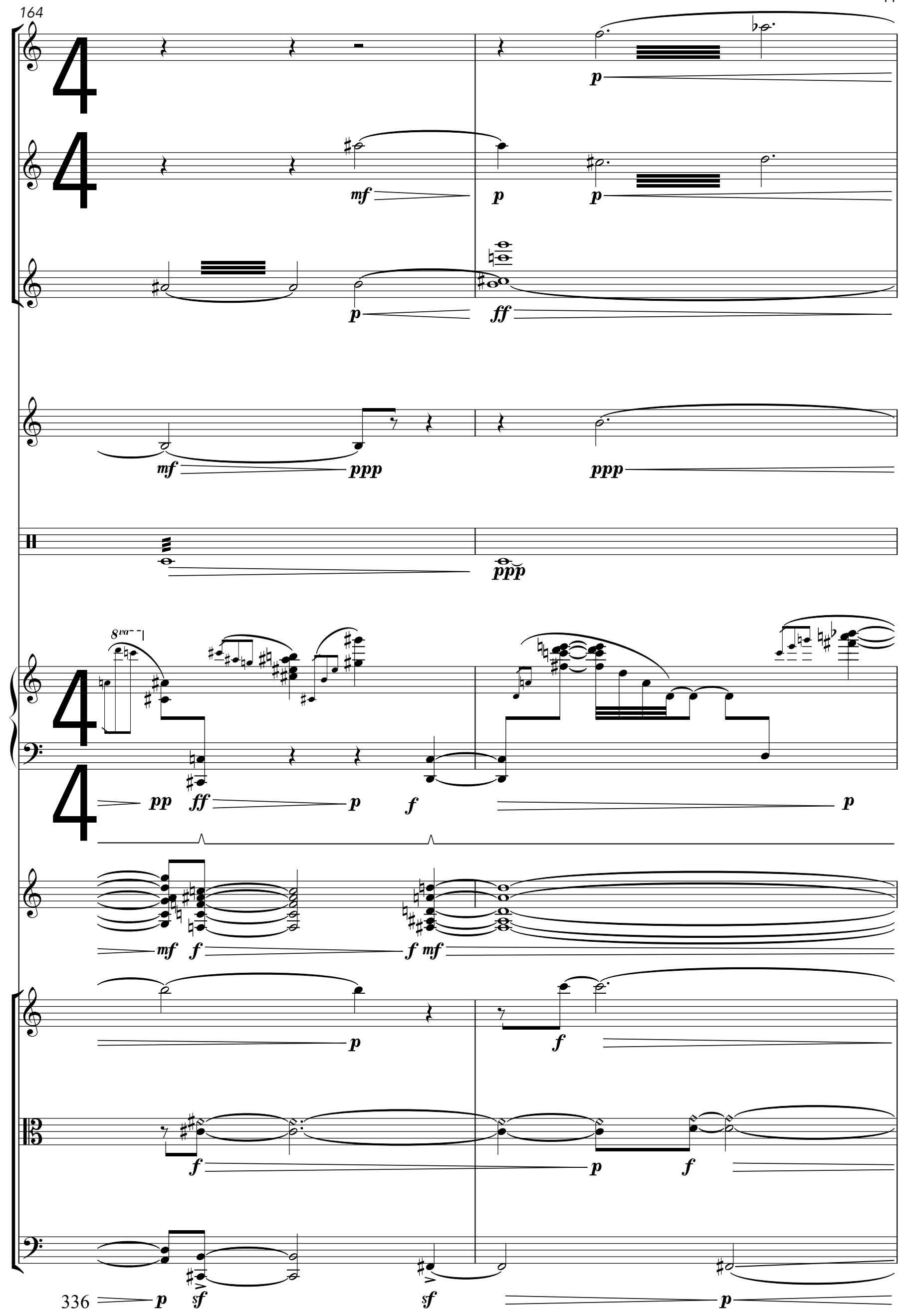


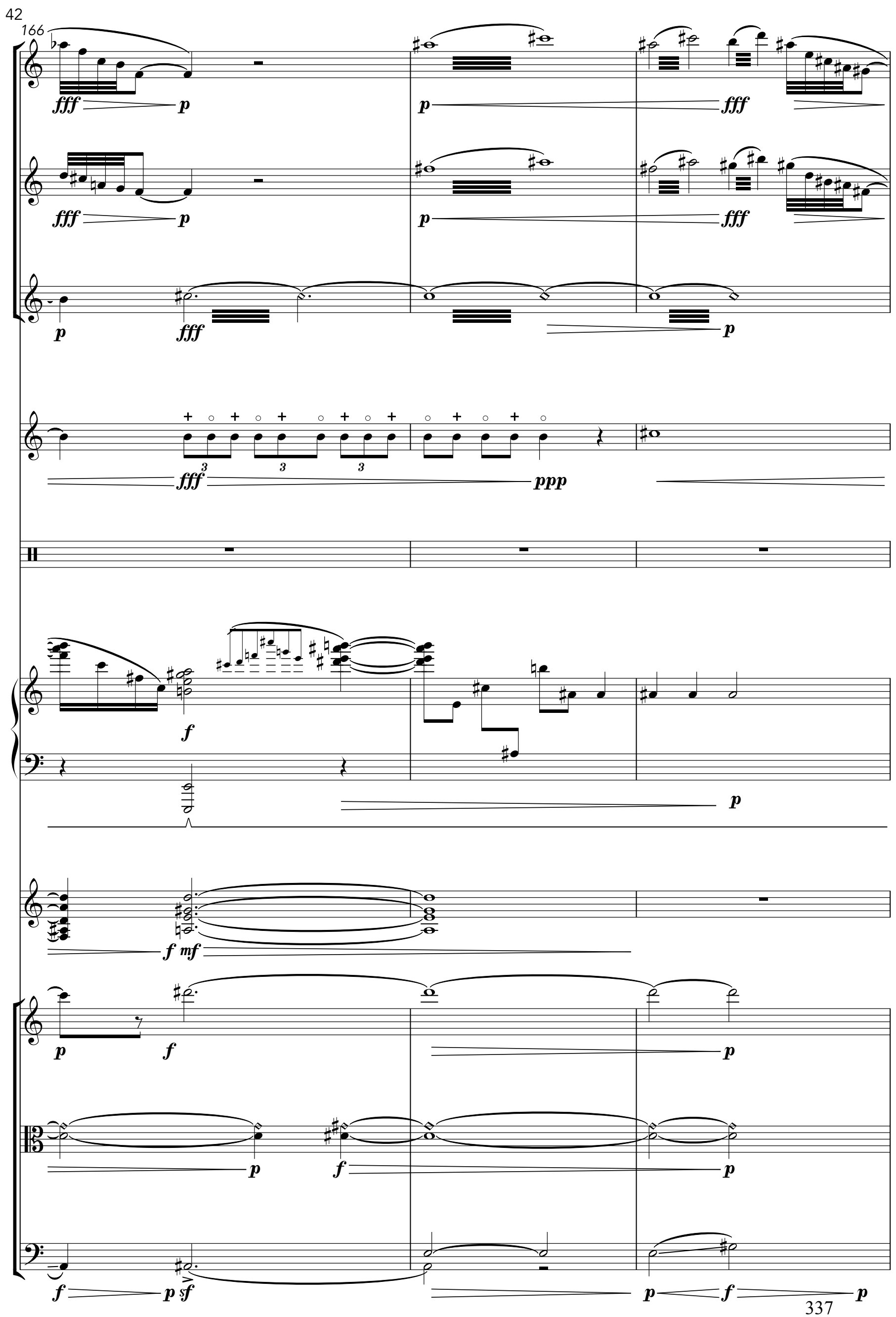




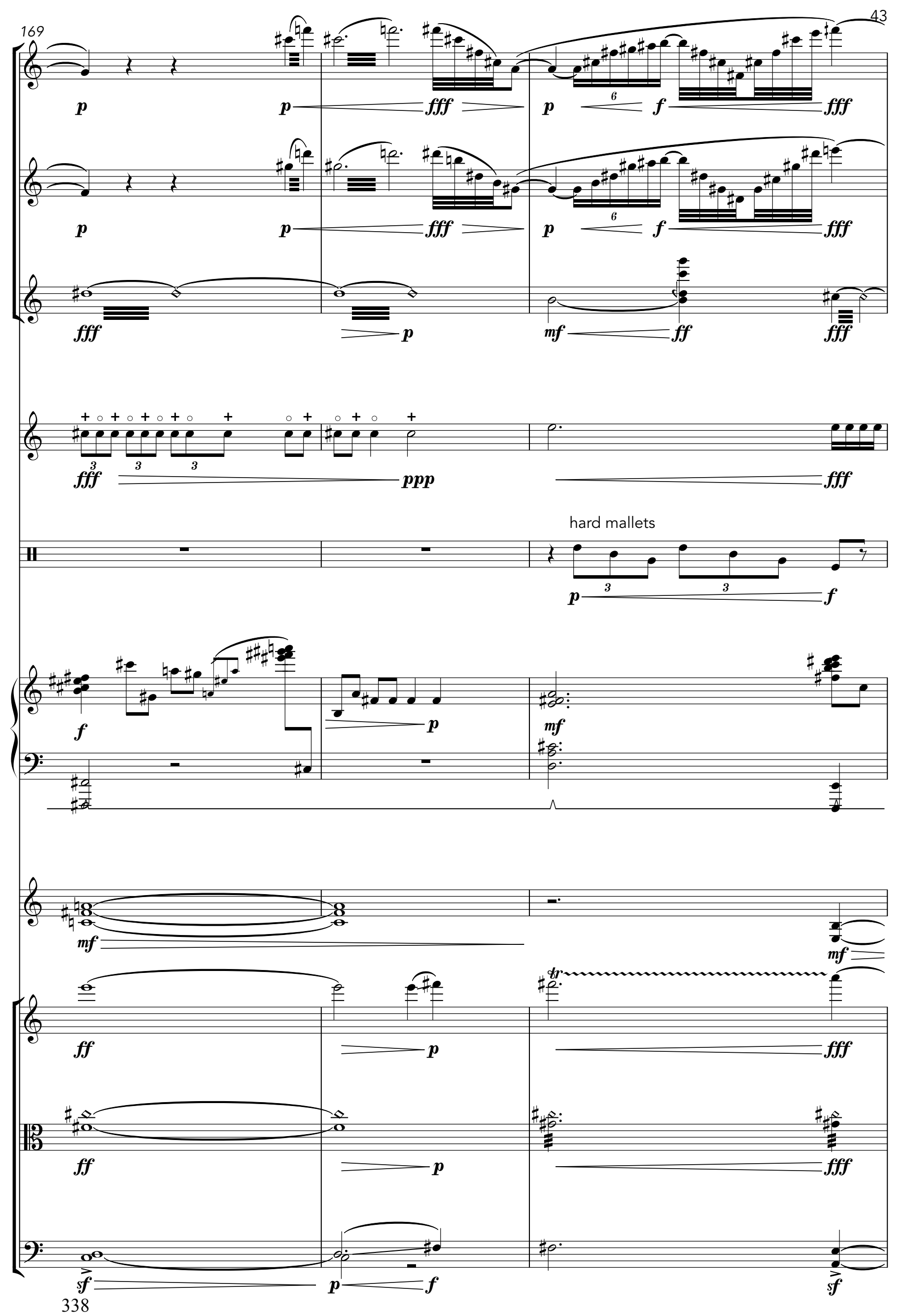




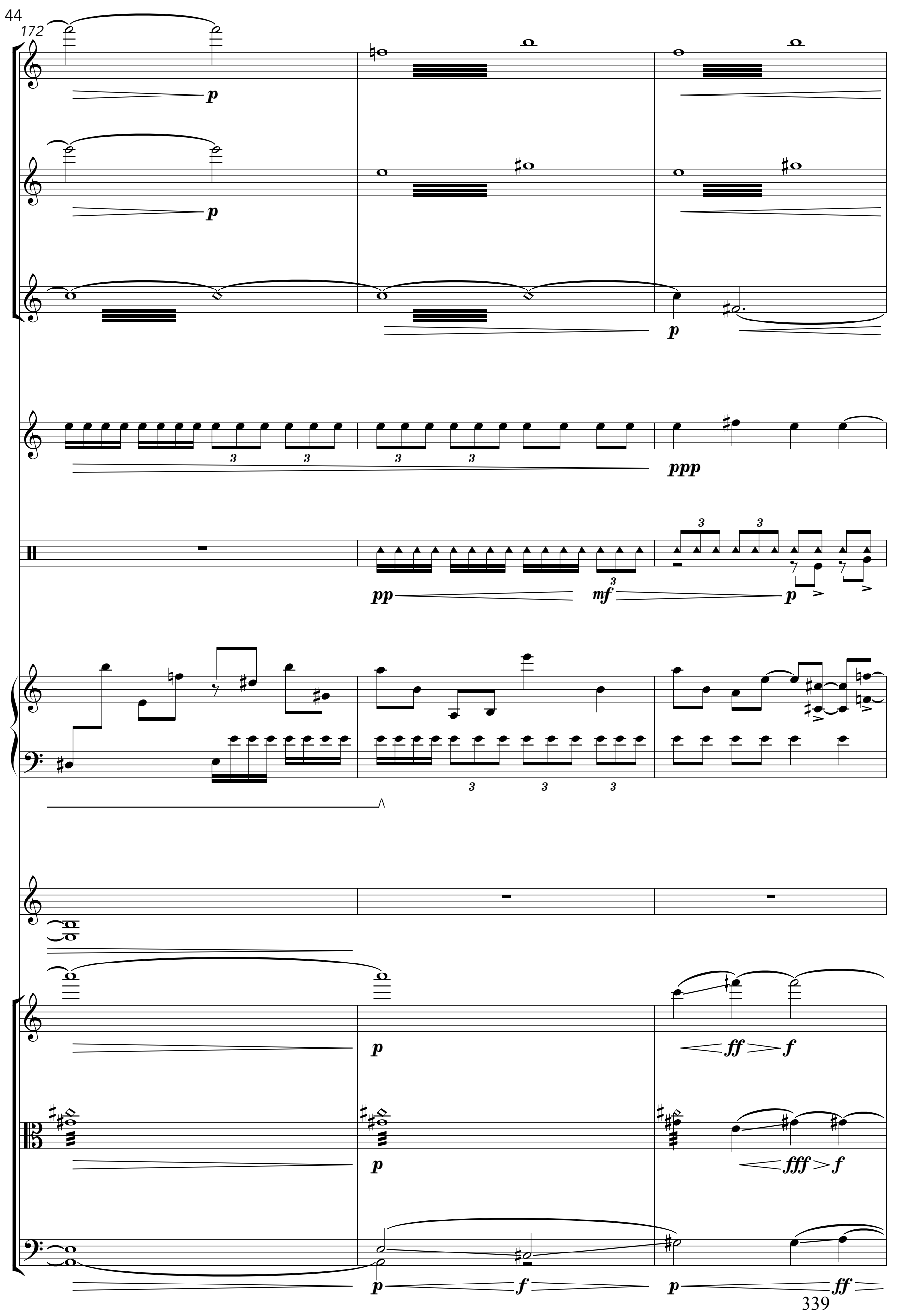




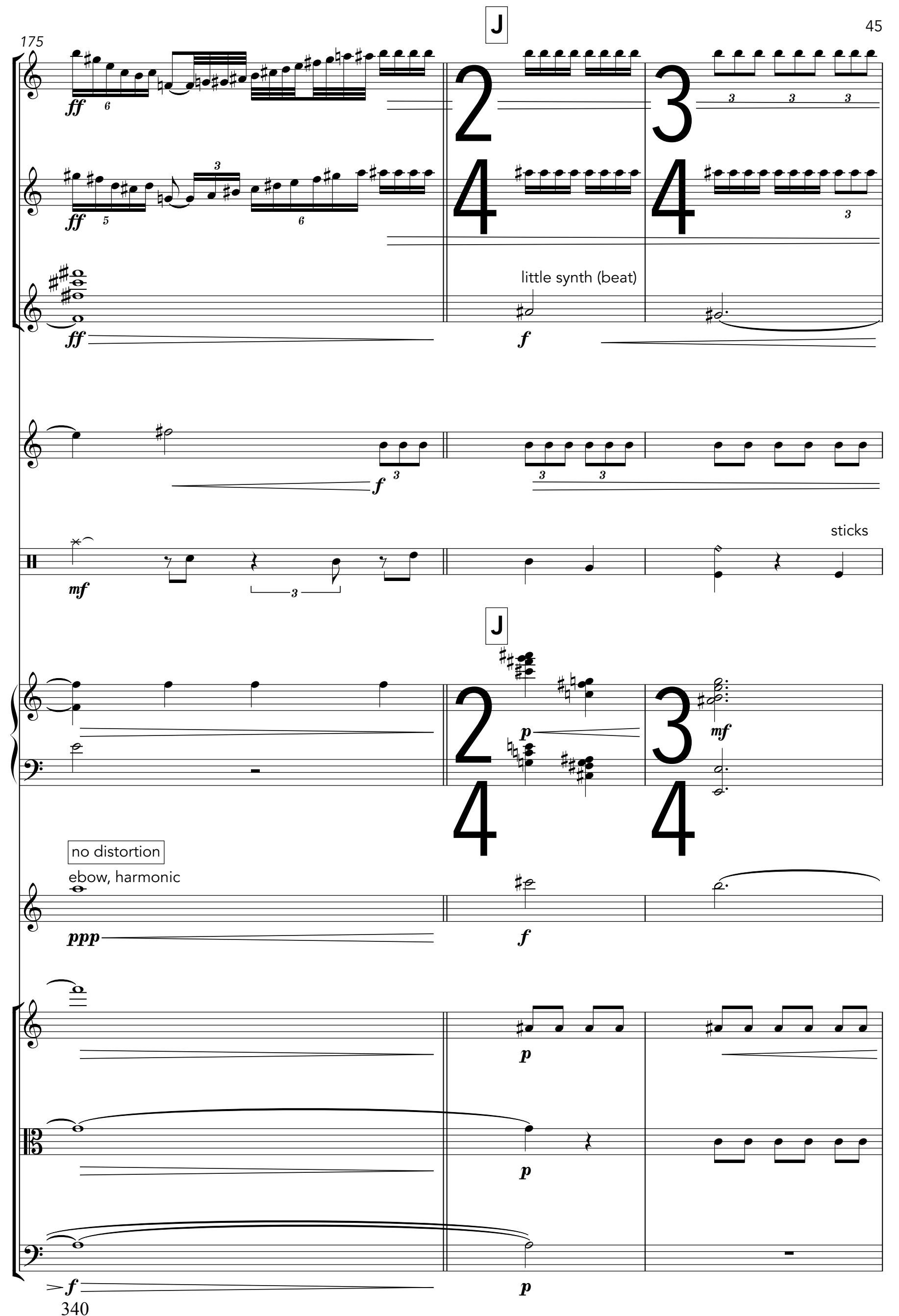




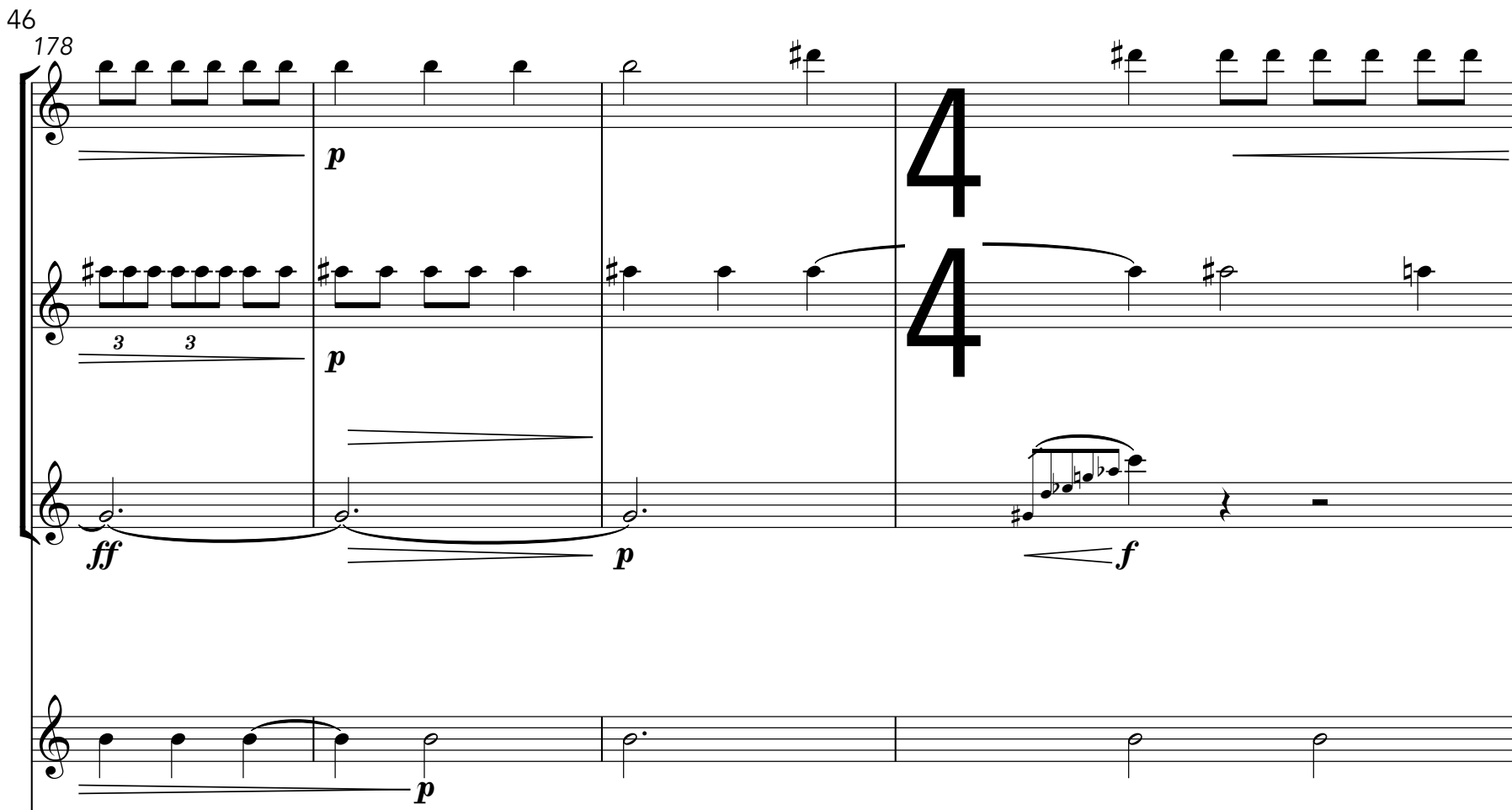

play snare and hi-hat hits, fill out texture slowly introducing a groove

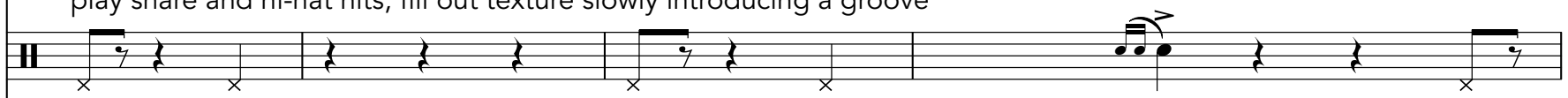
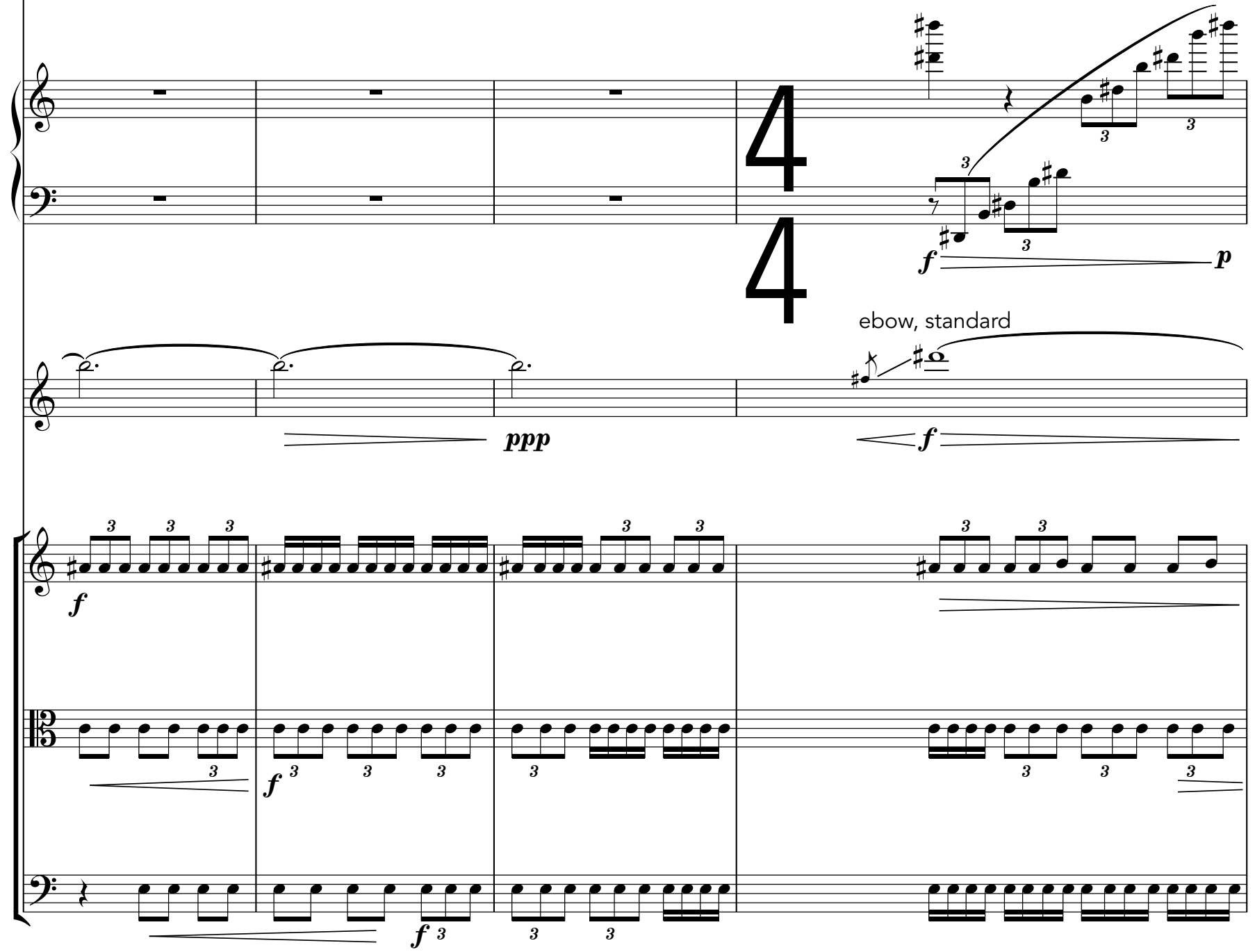

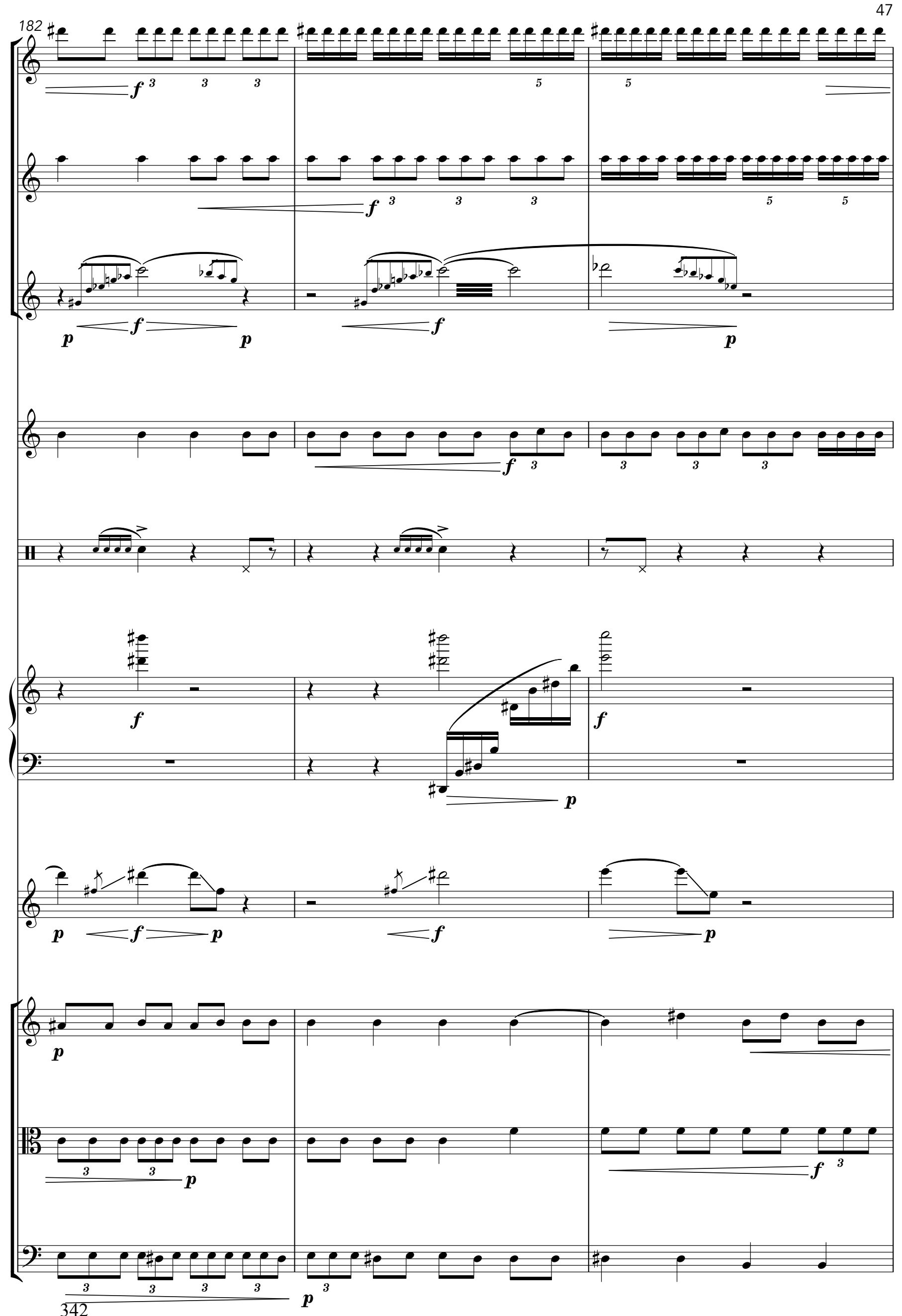


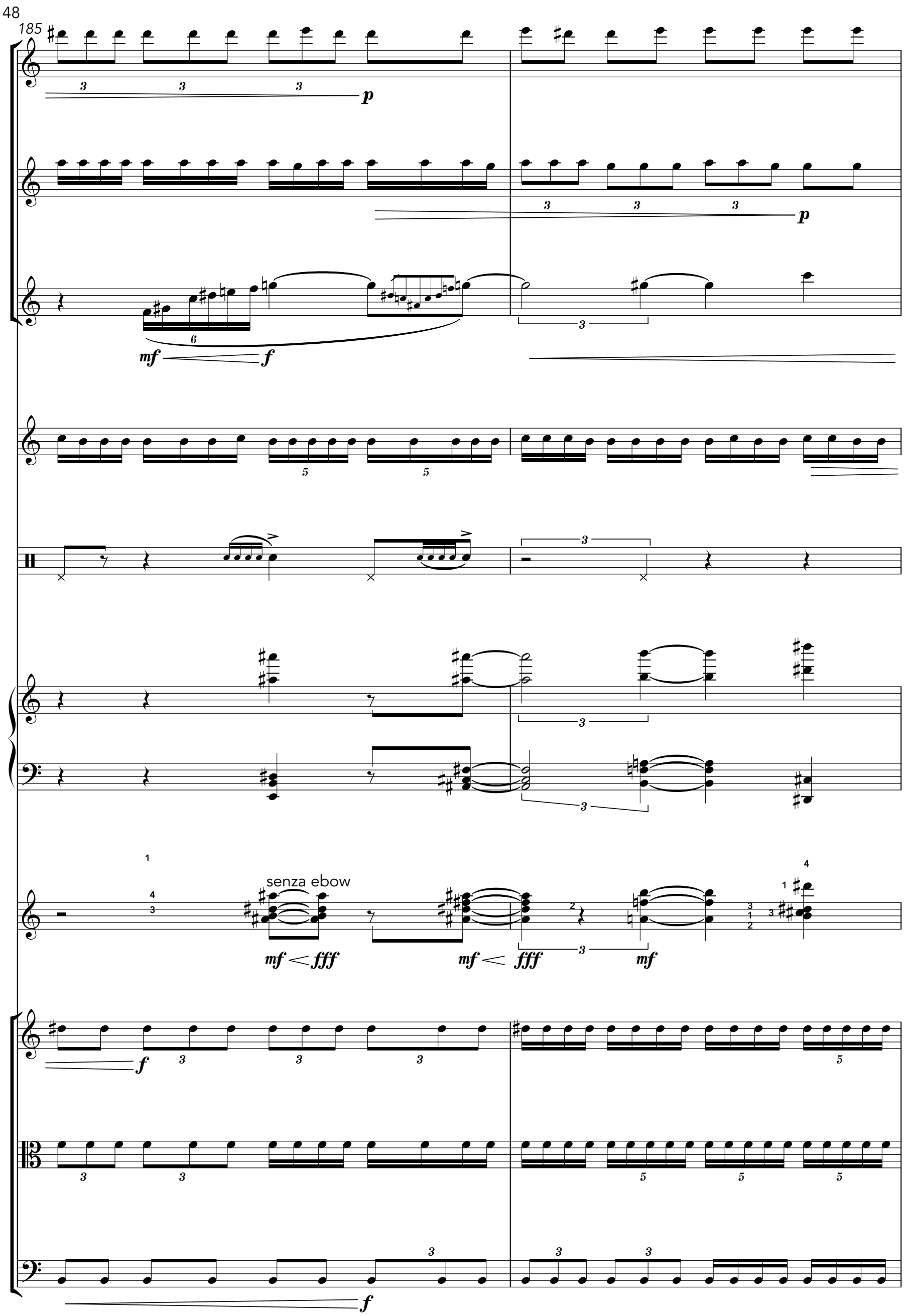



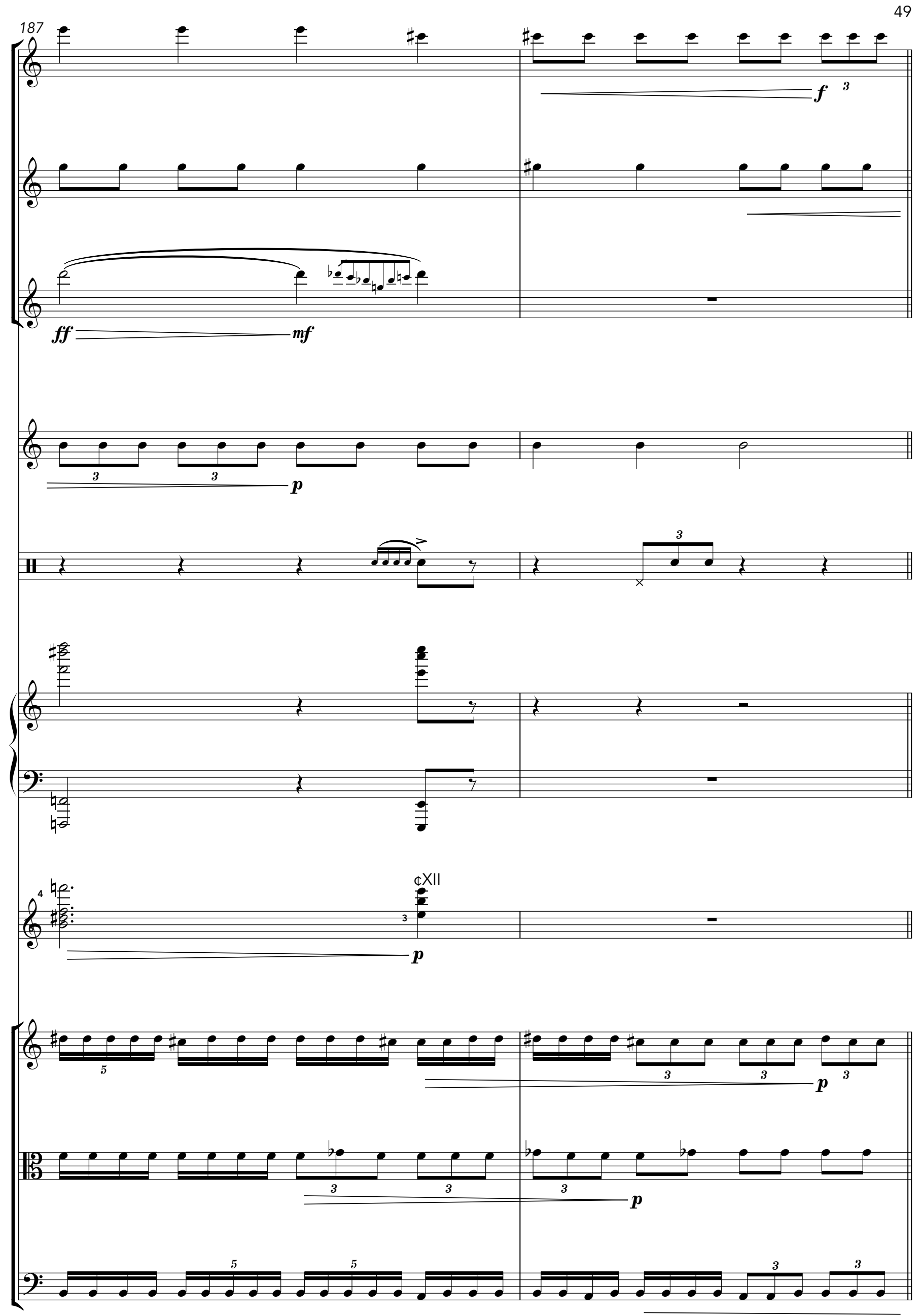


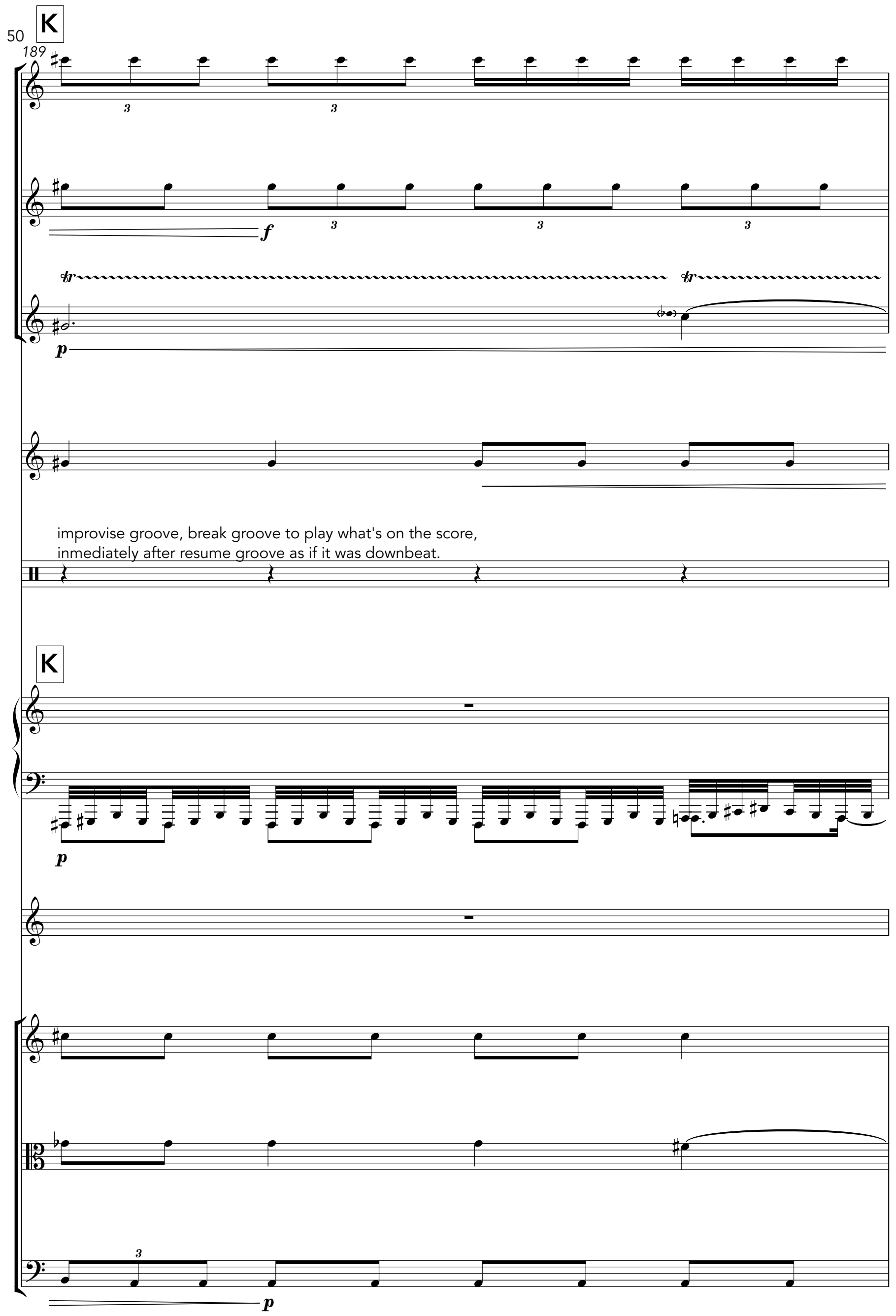



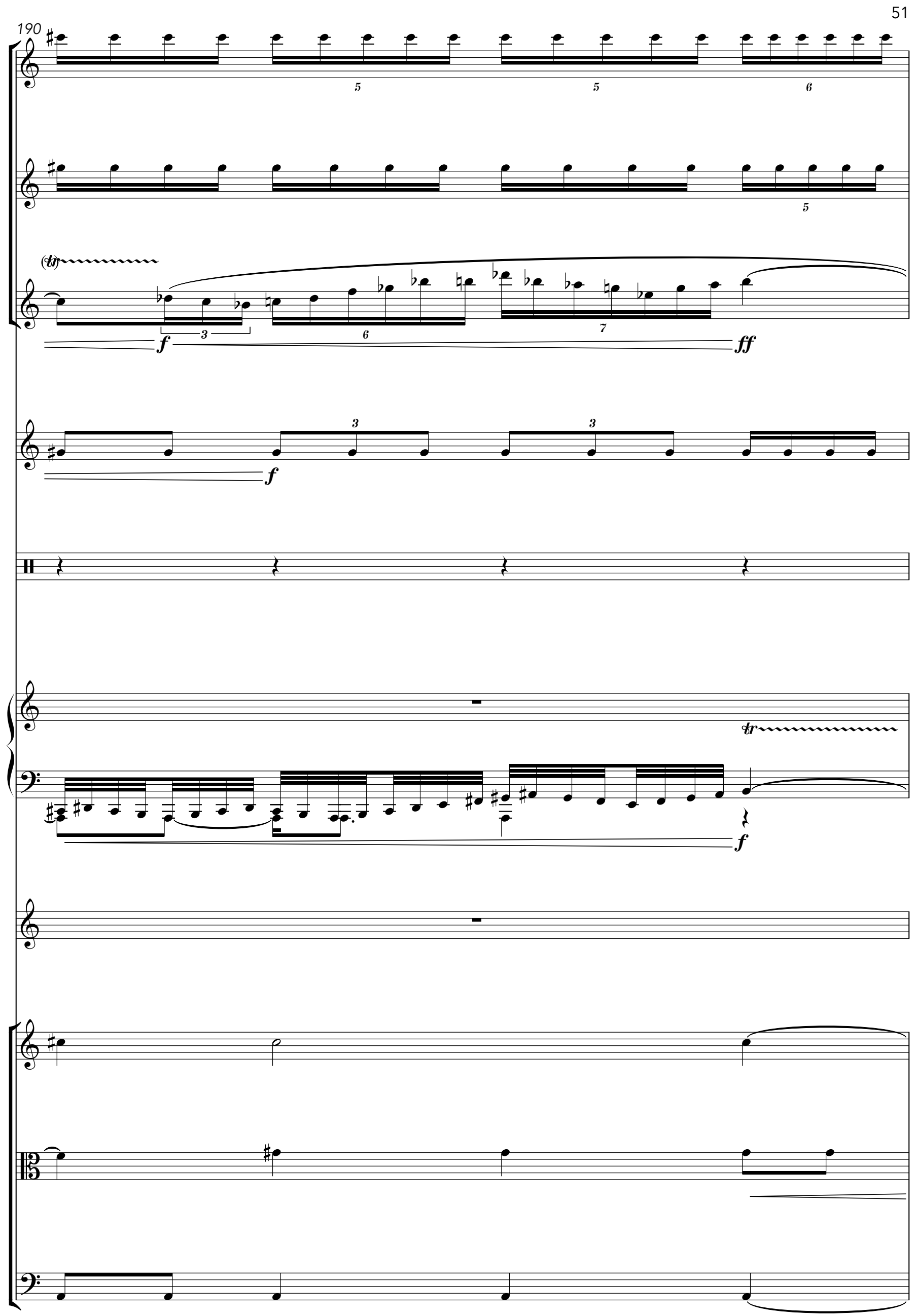


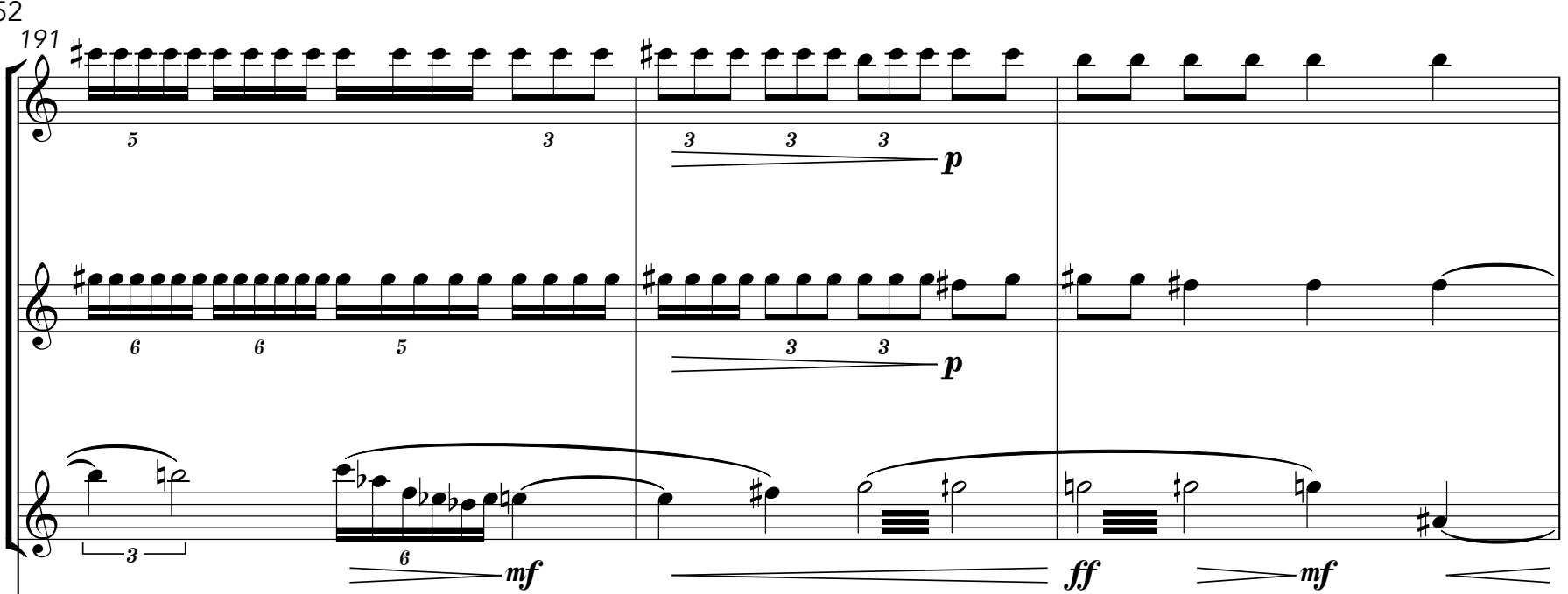

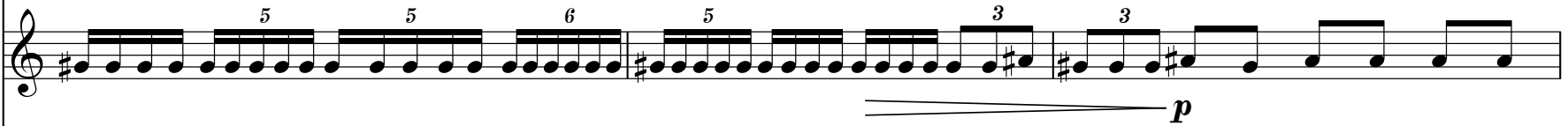
(groove)
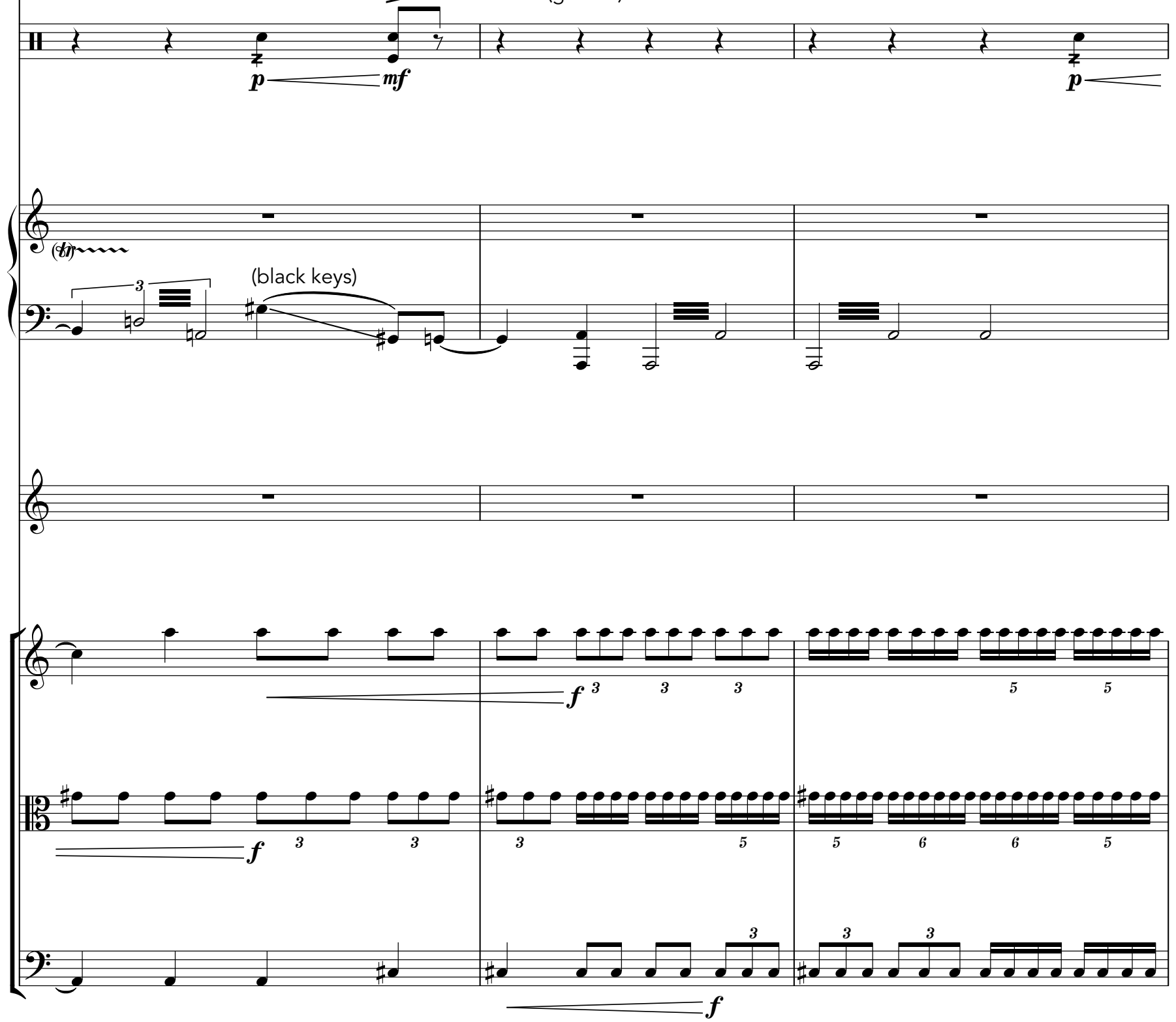

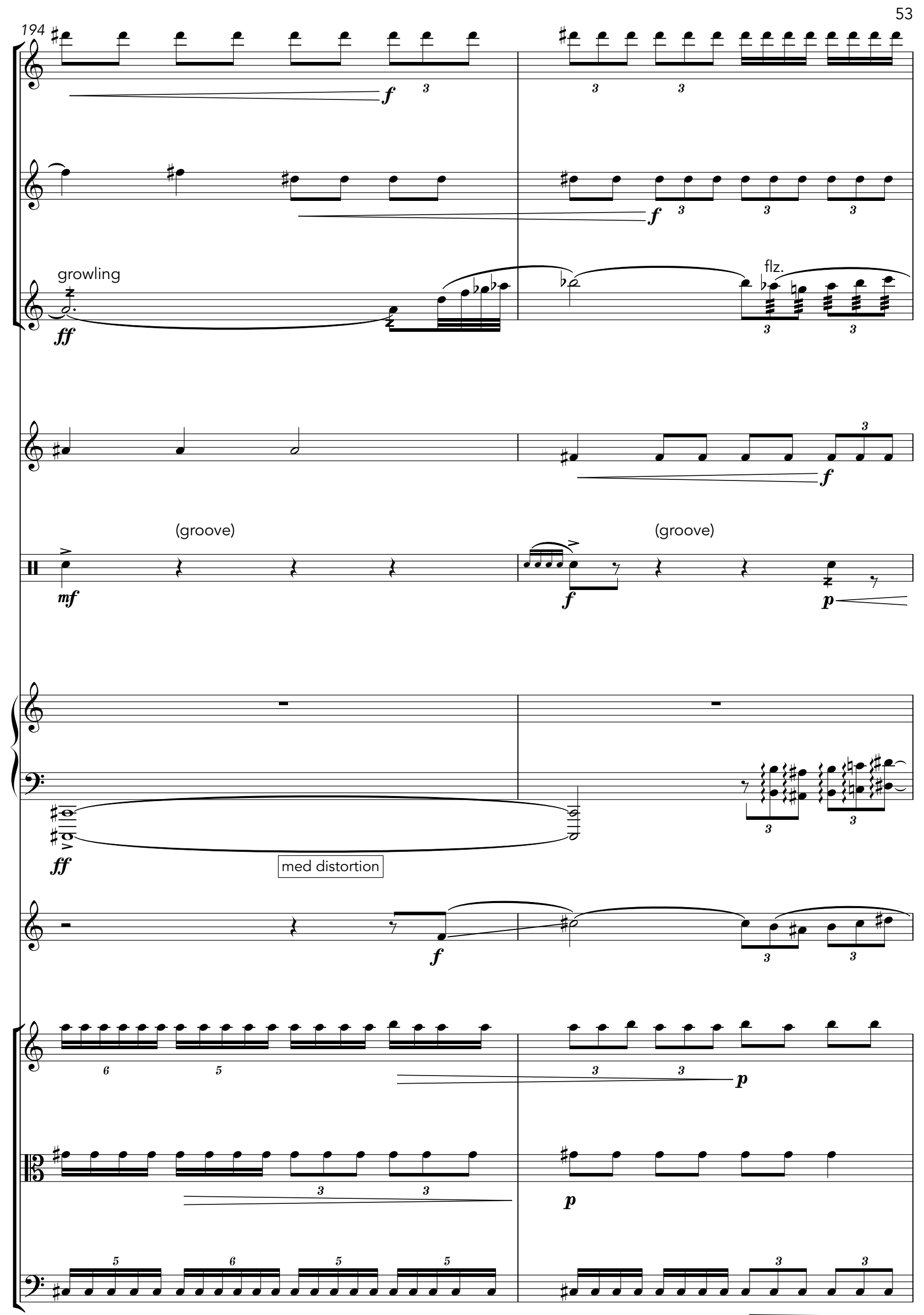


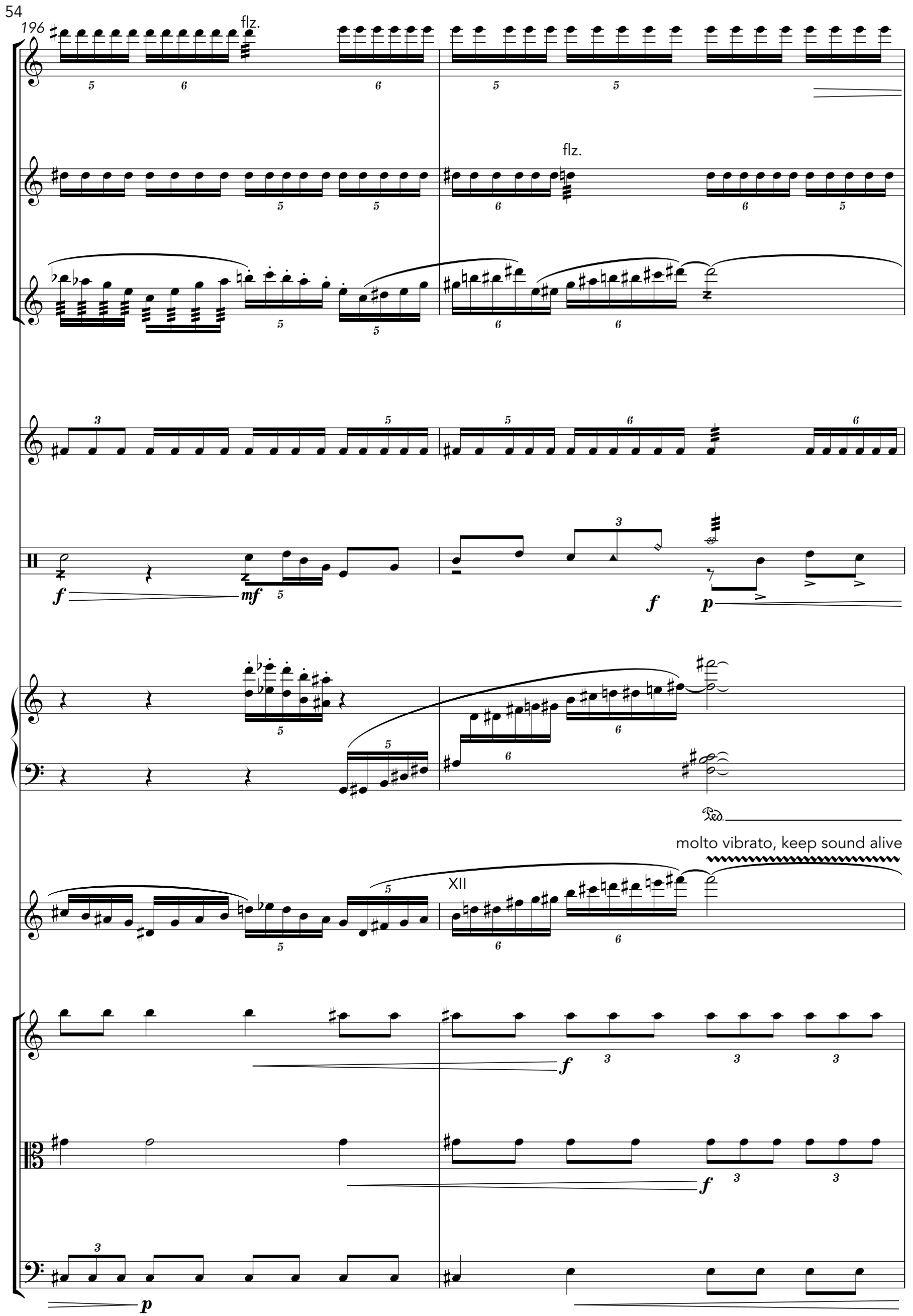




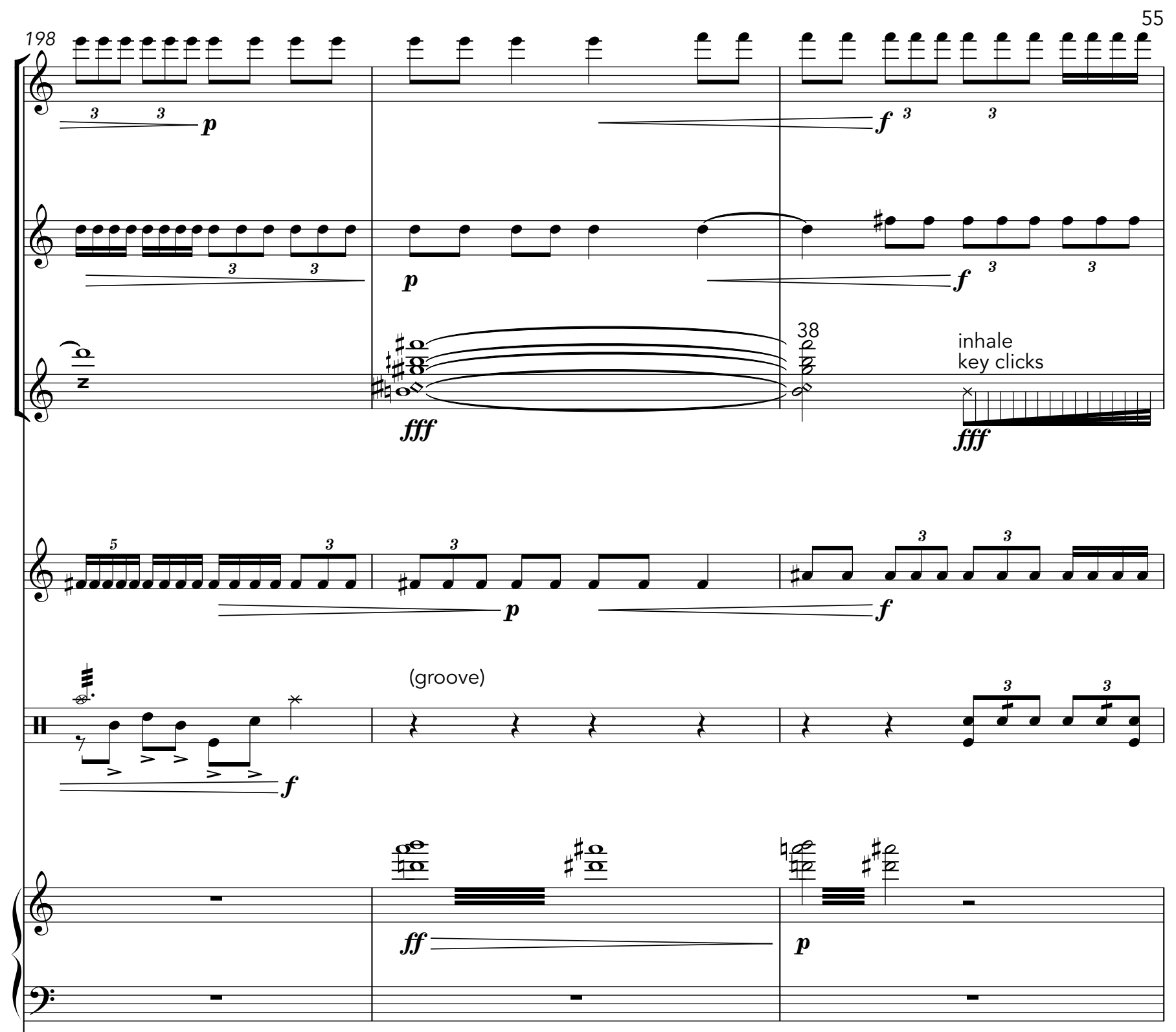

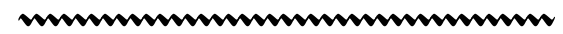
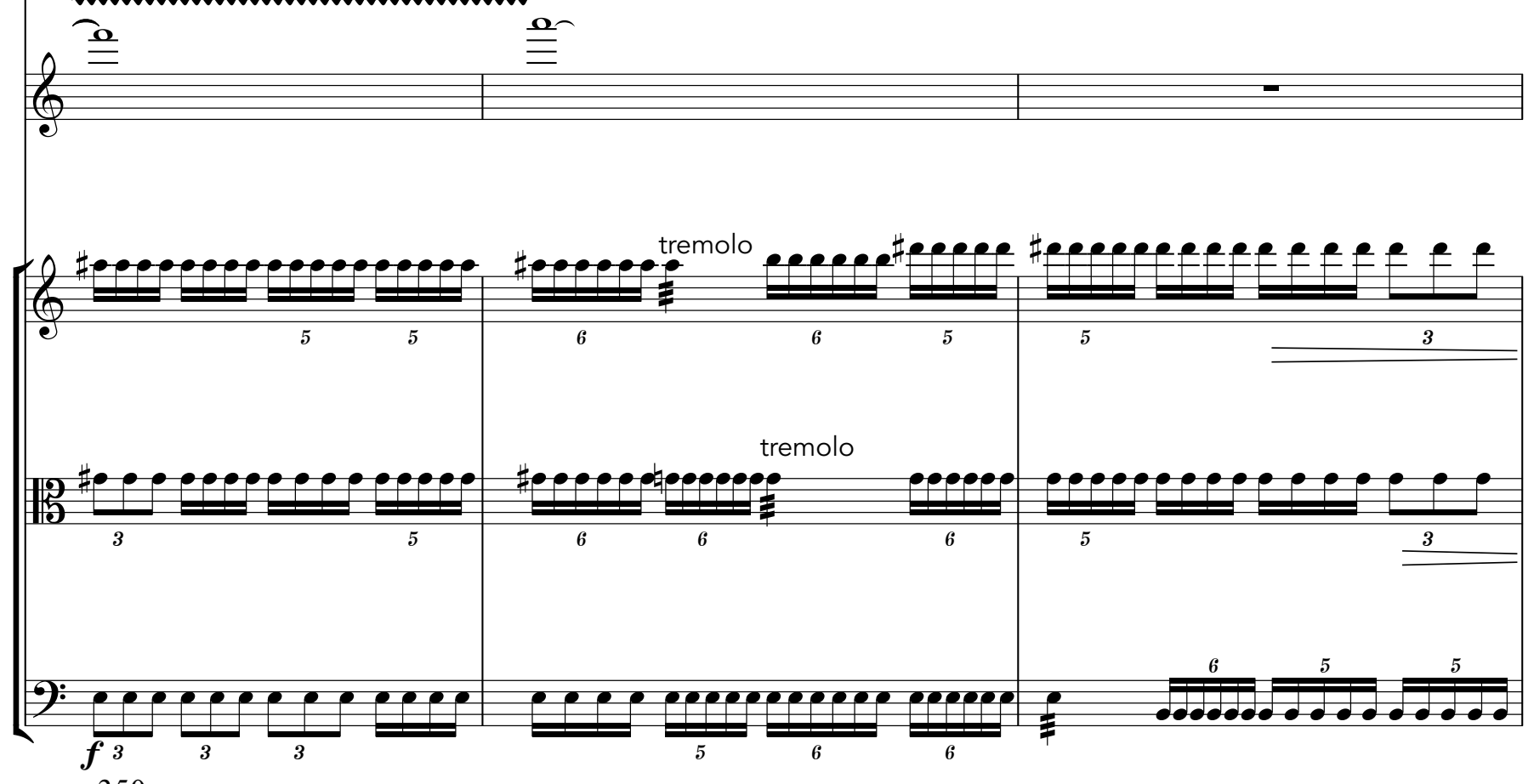


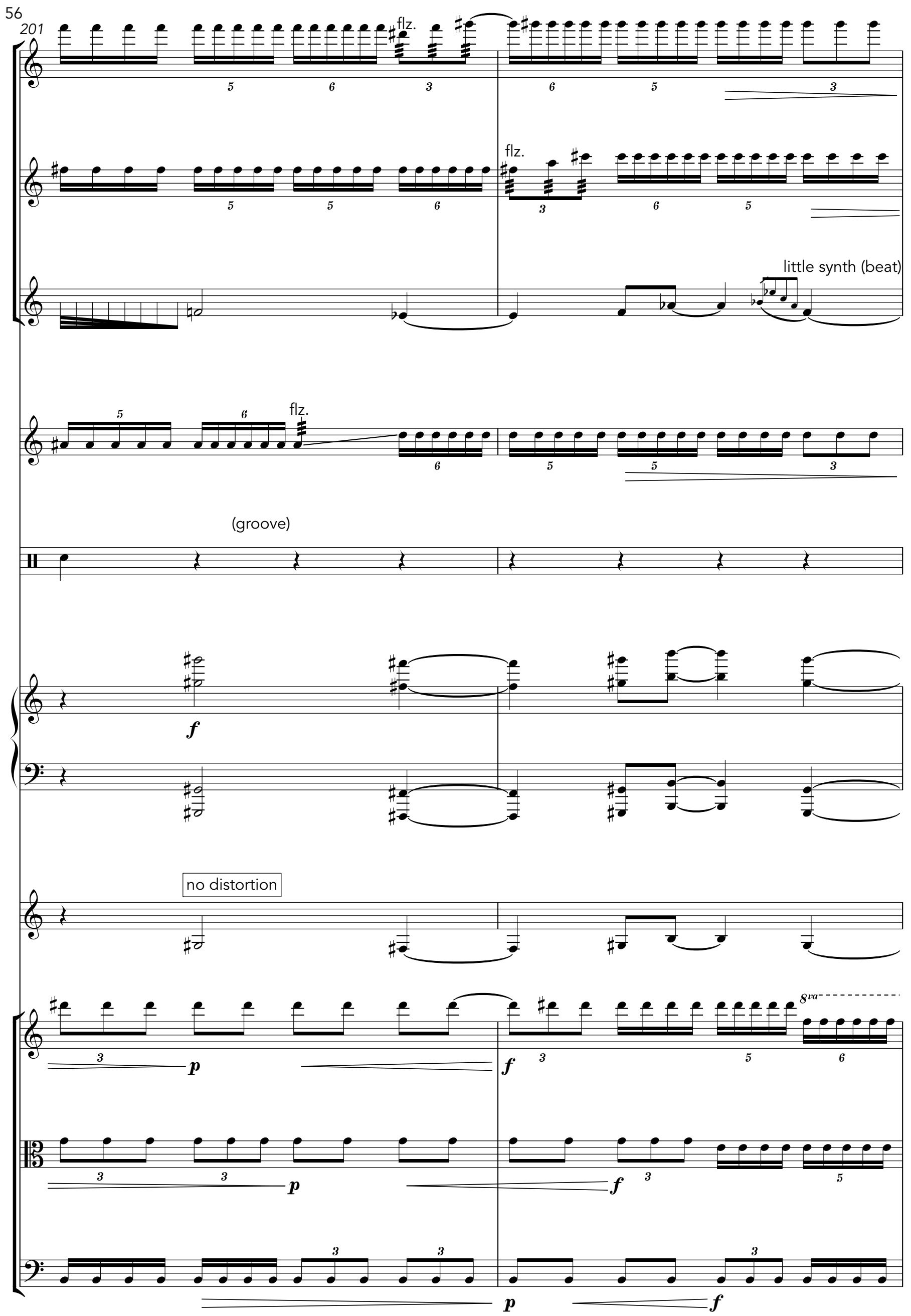




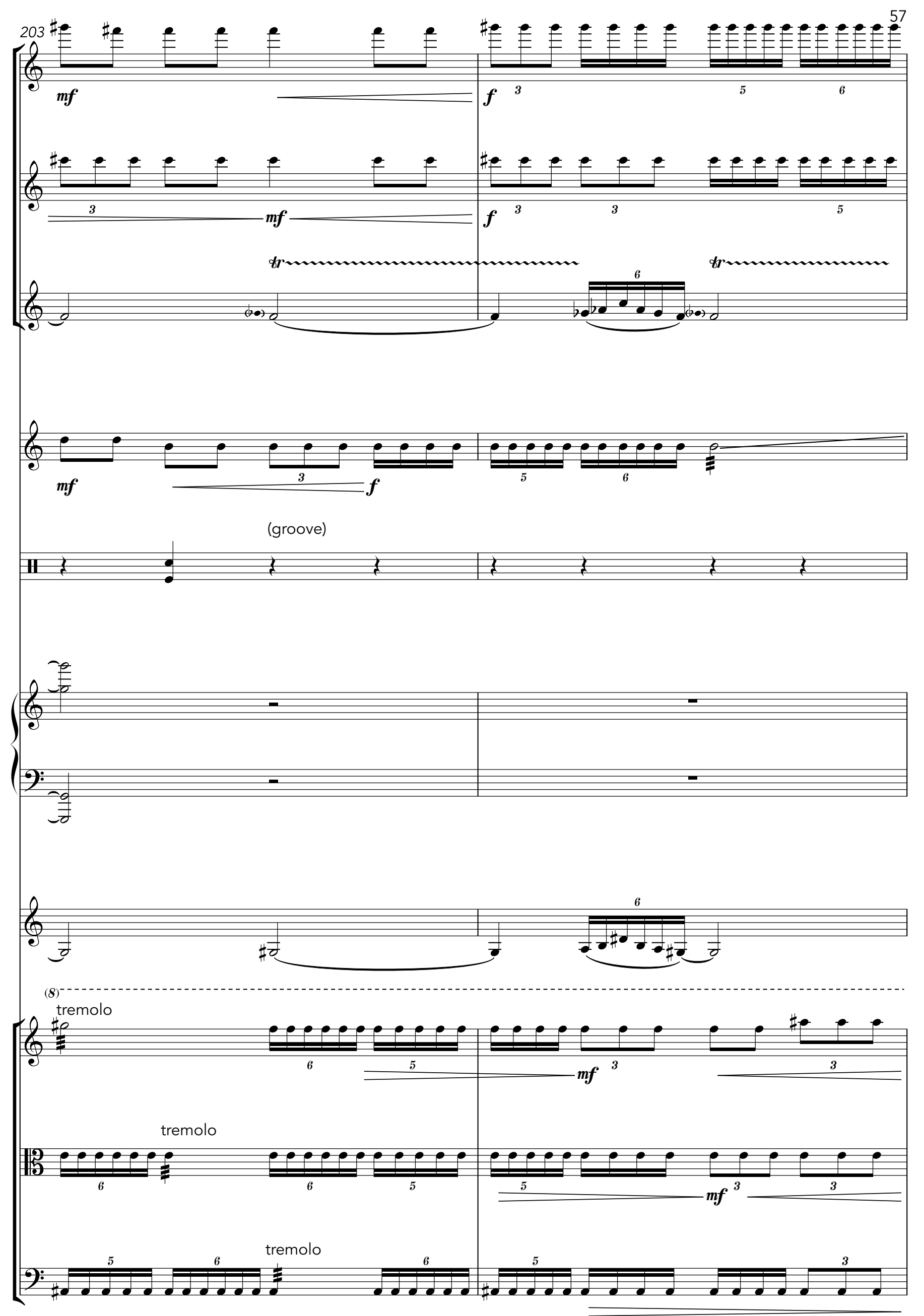




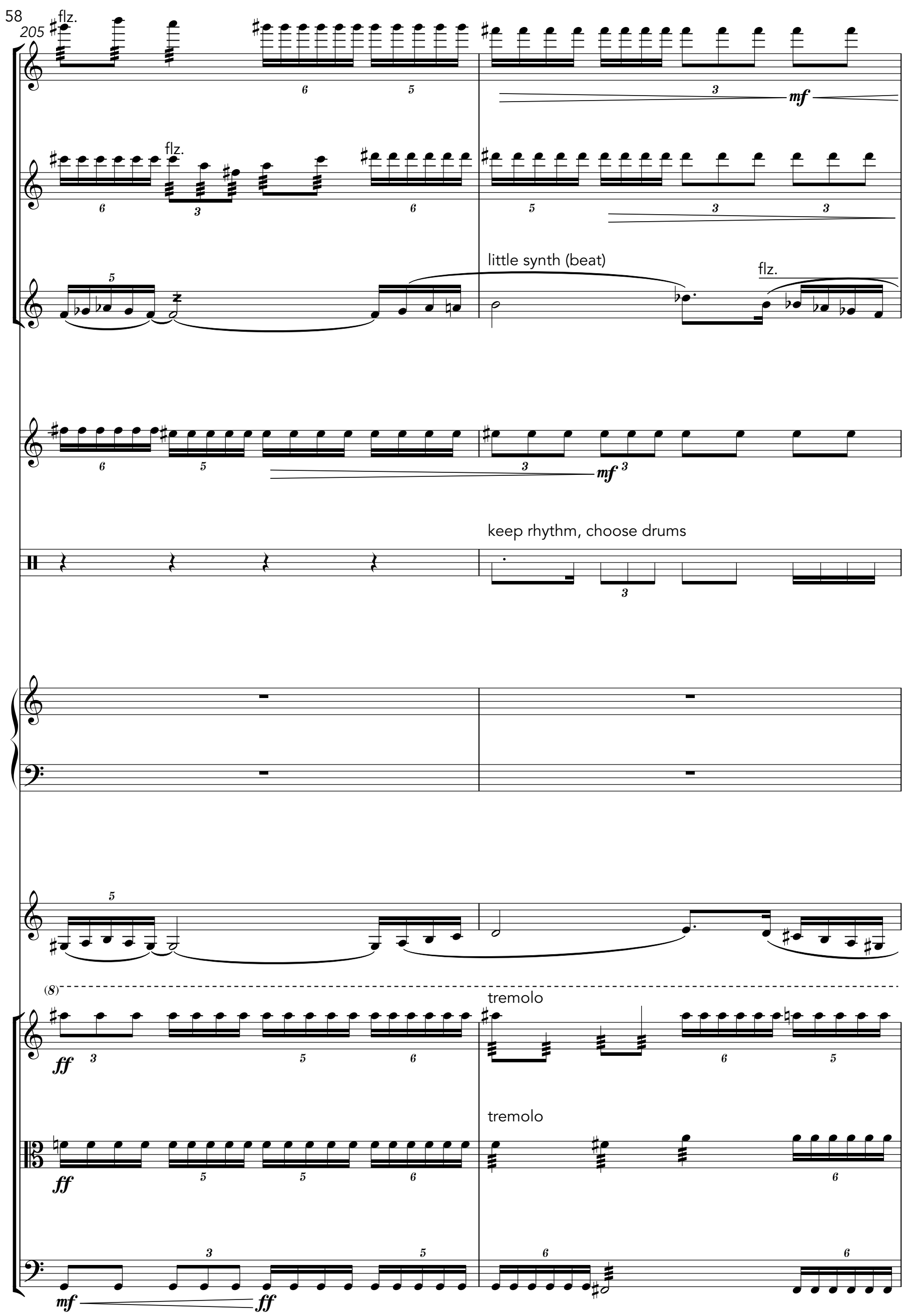




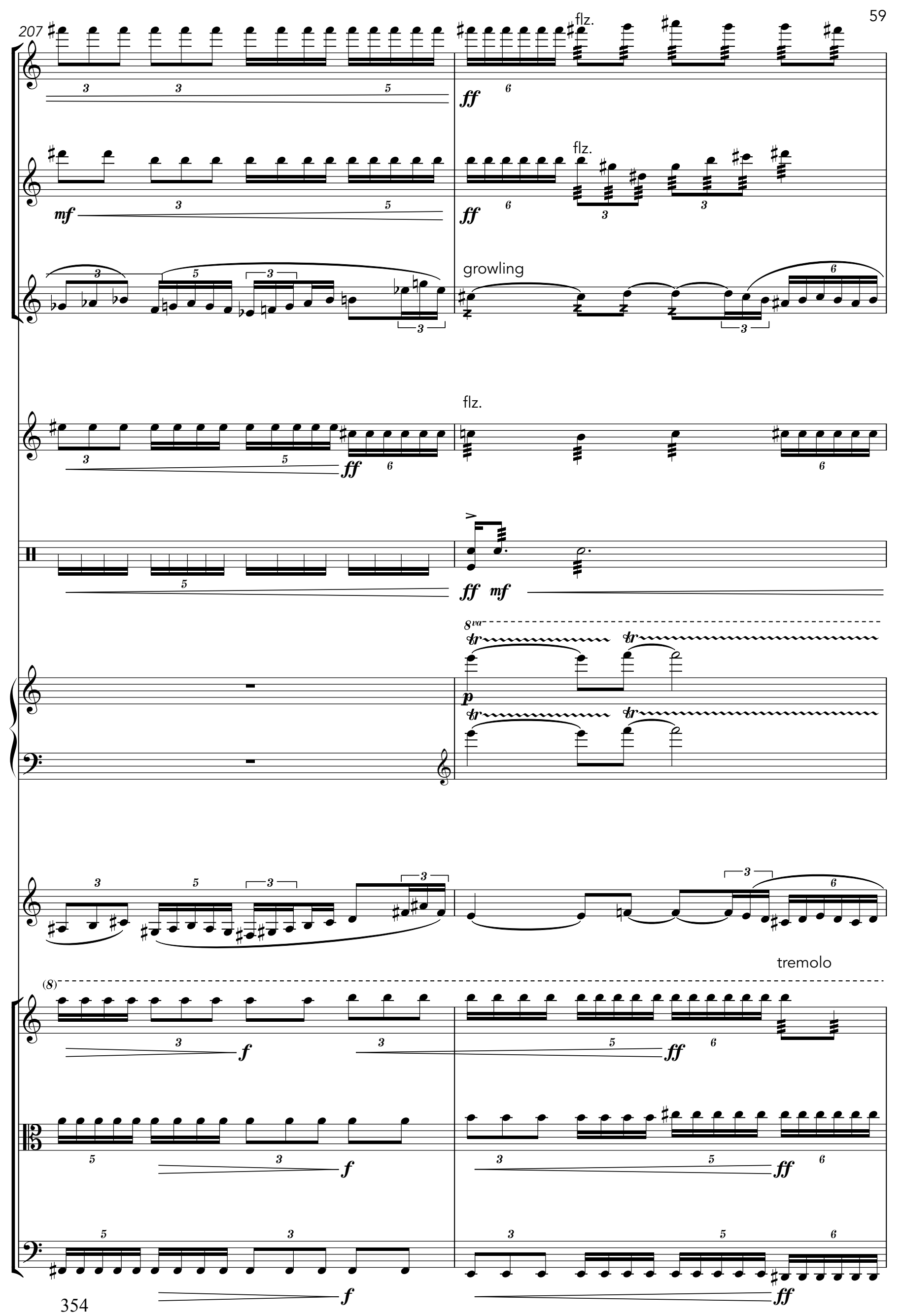




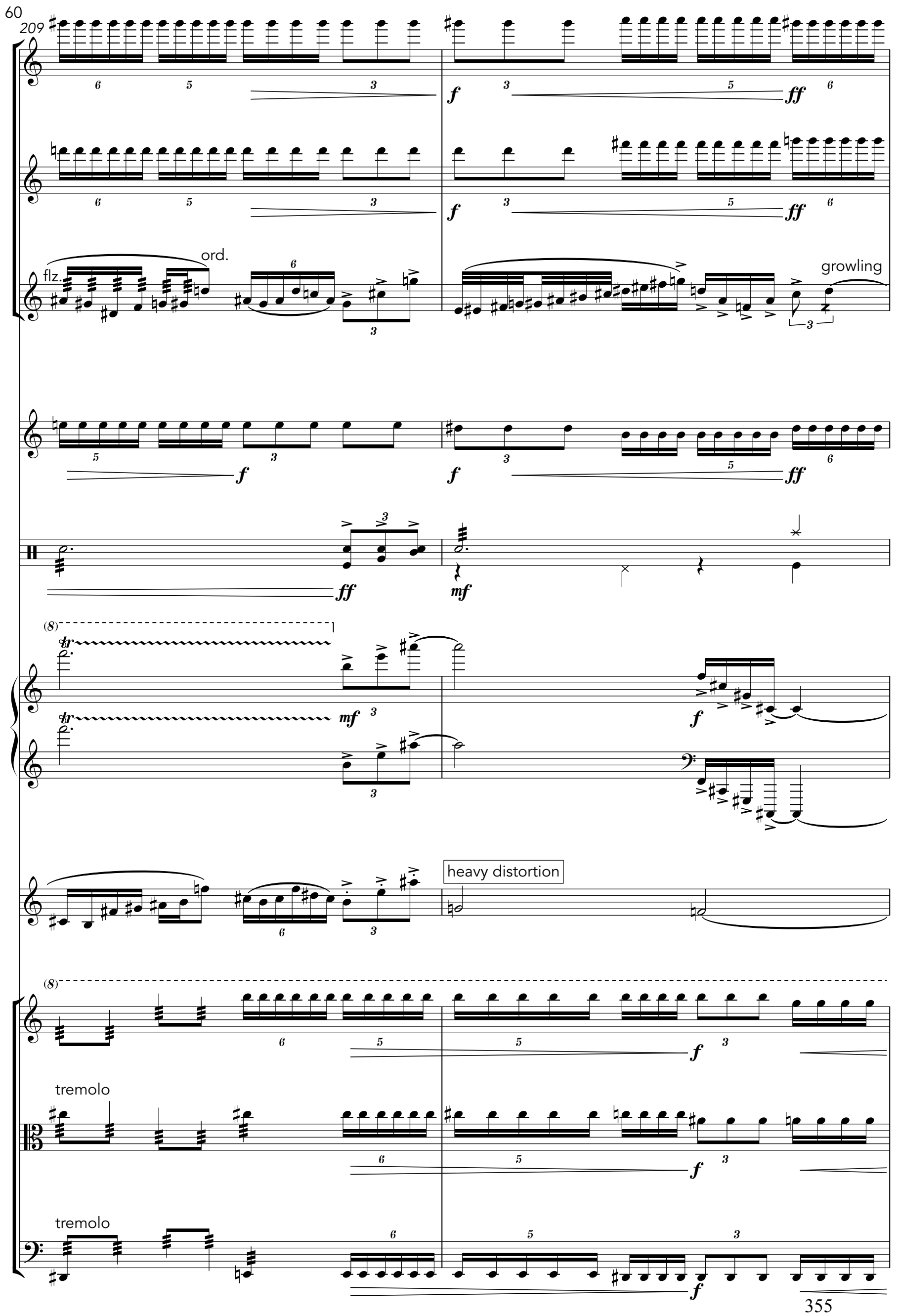



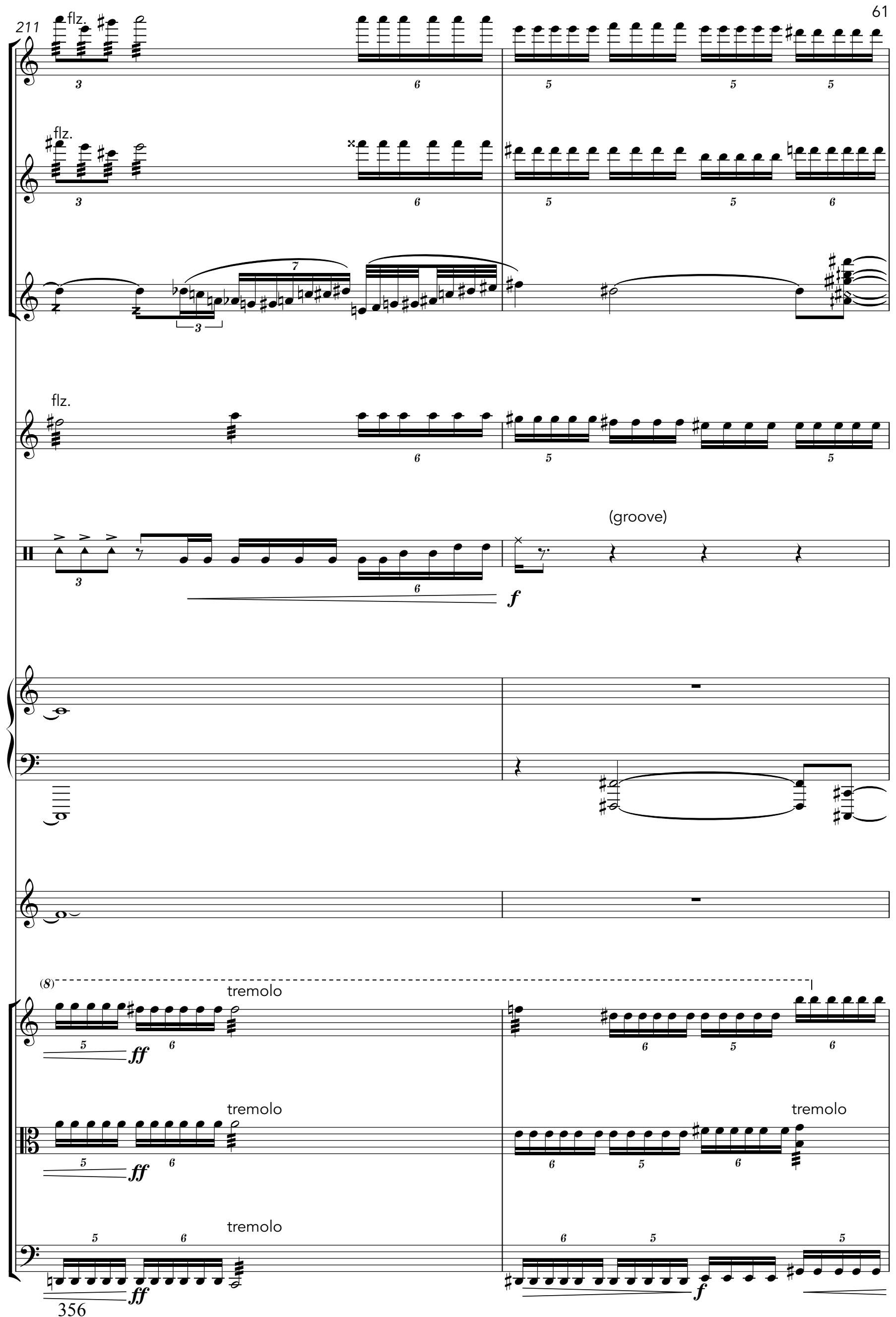


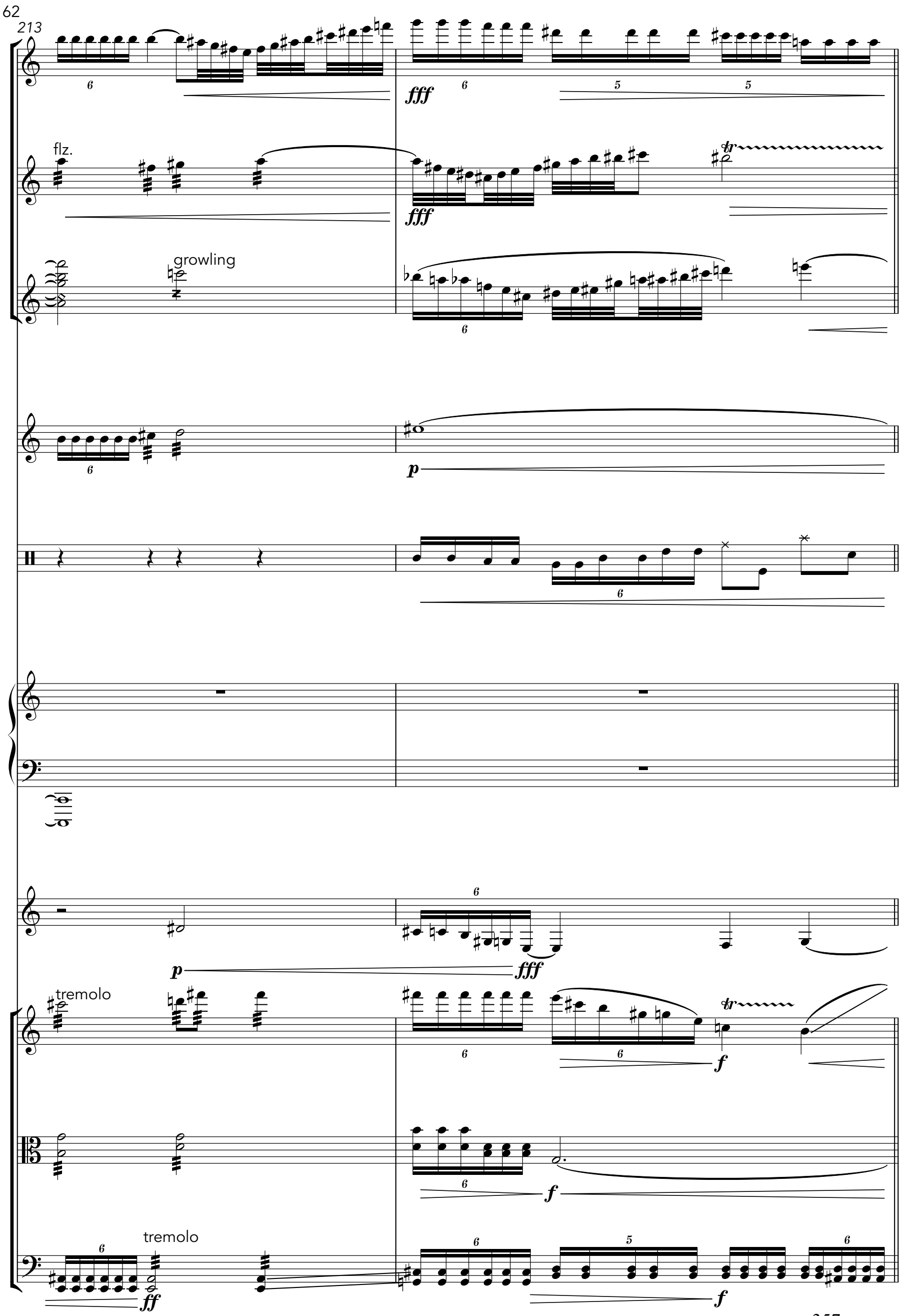




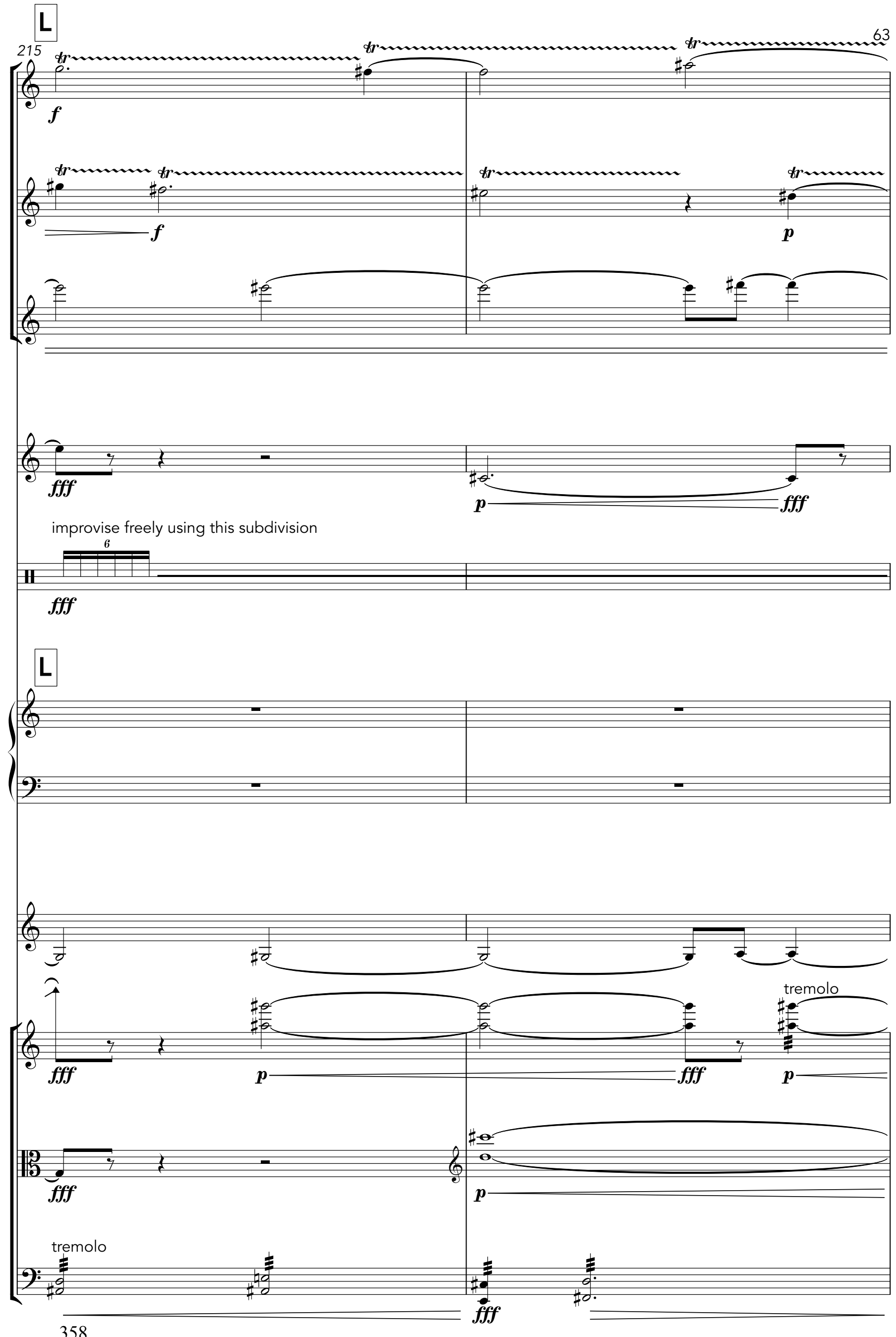




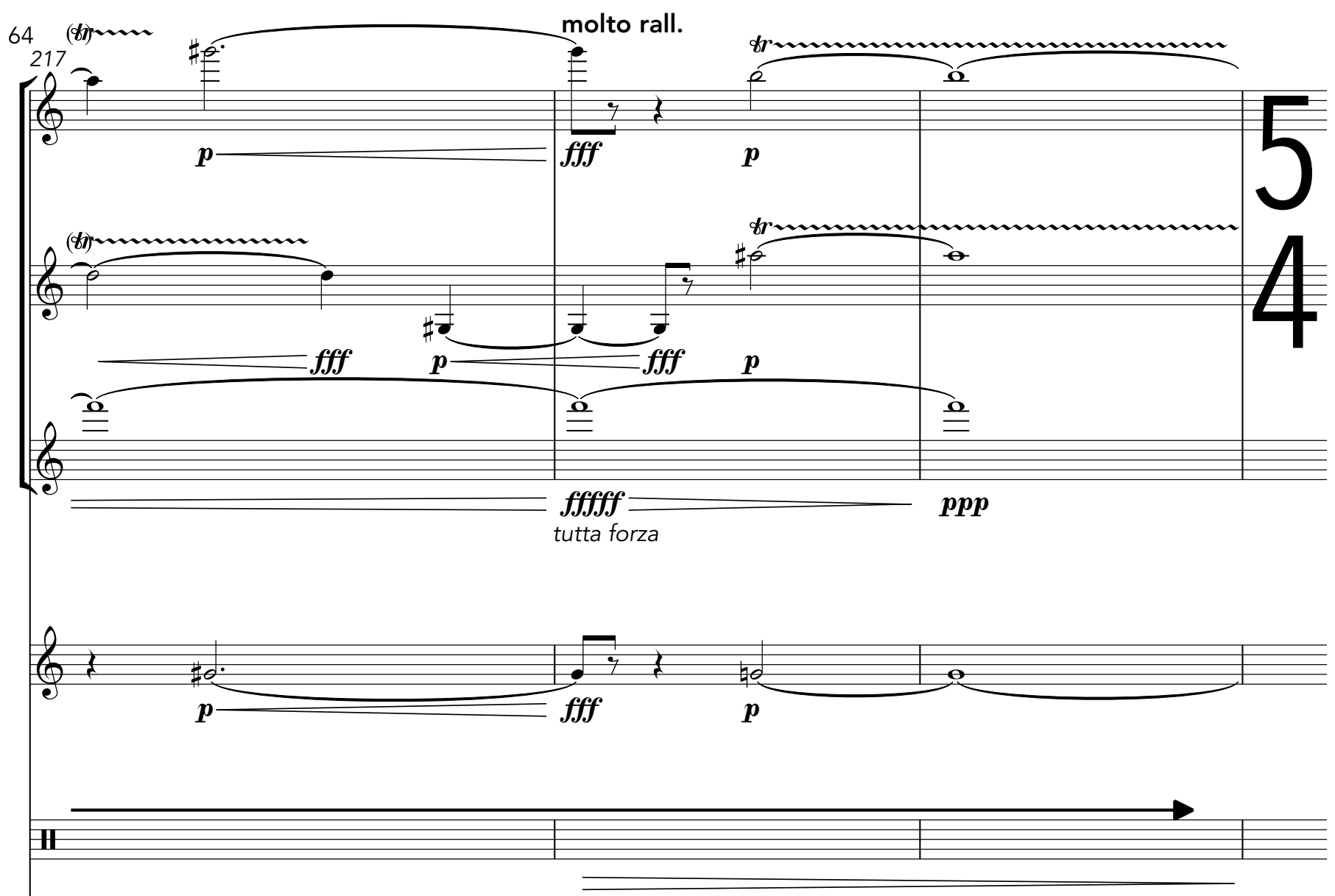

molto rall.
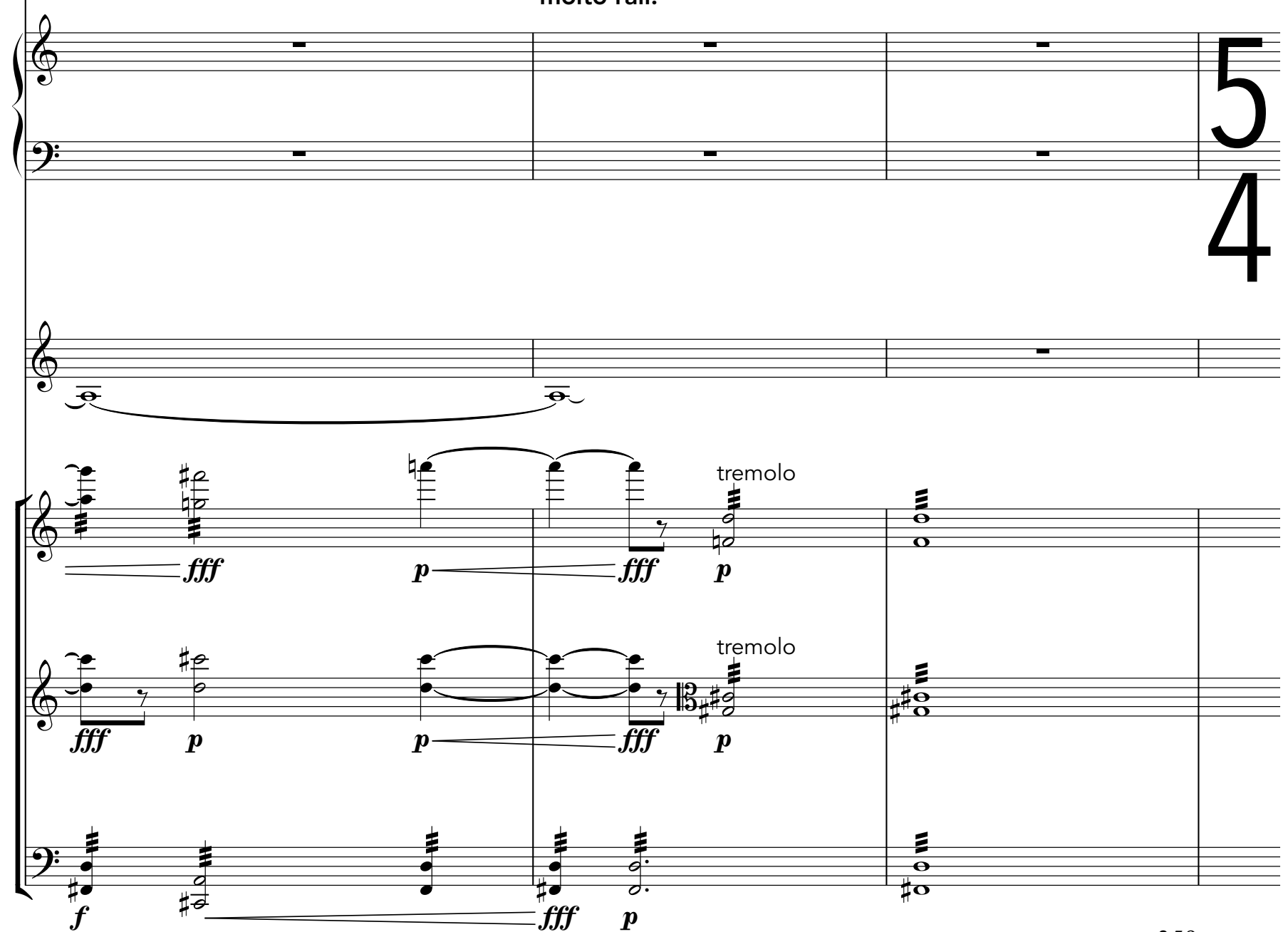


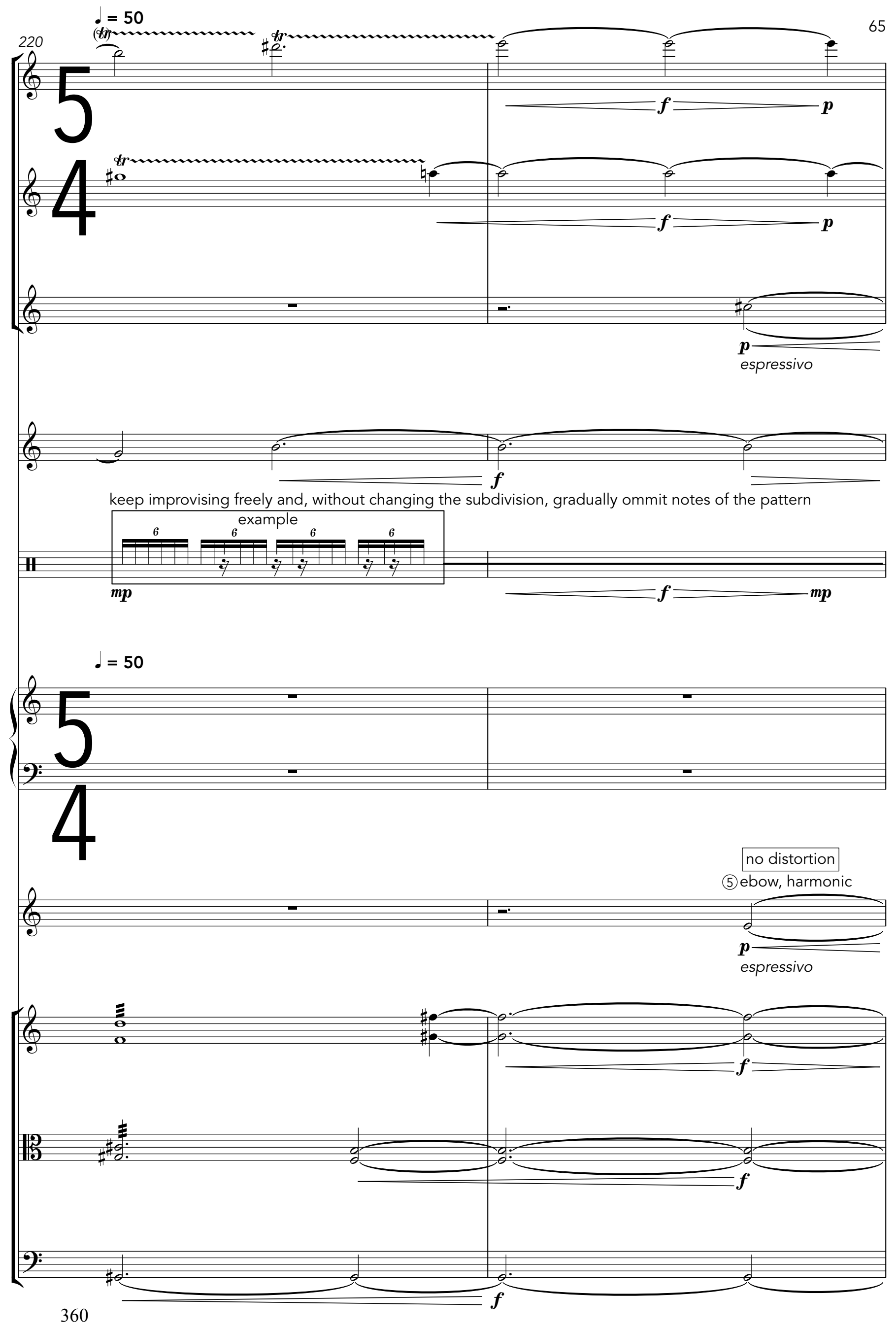




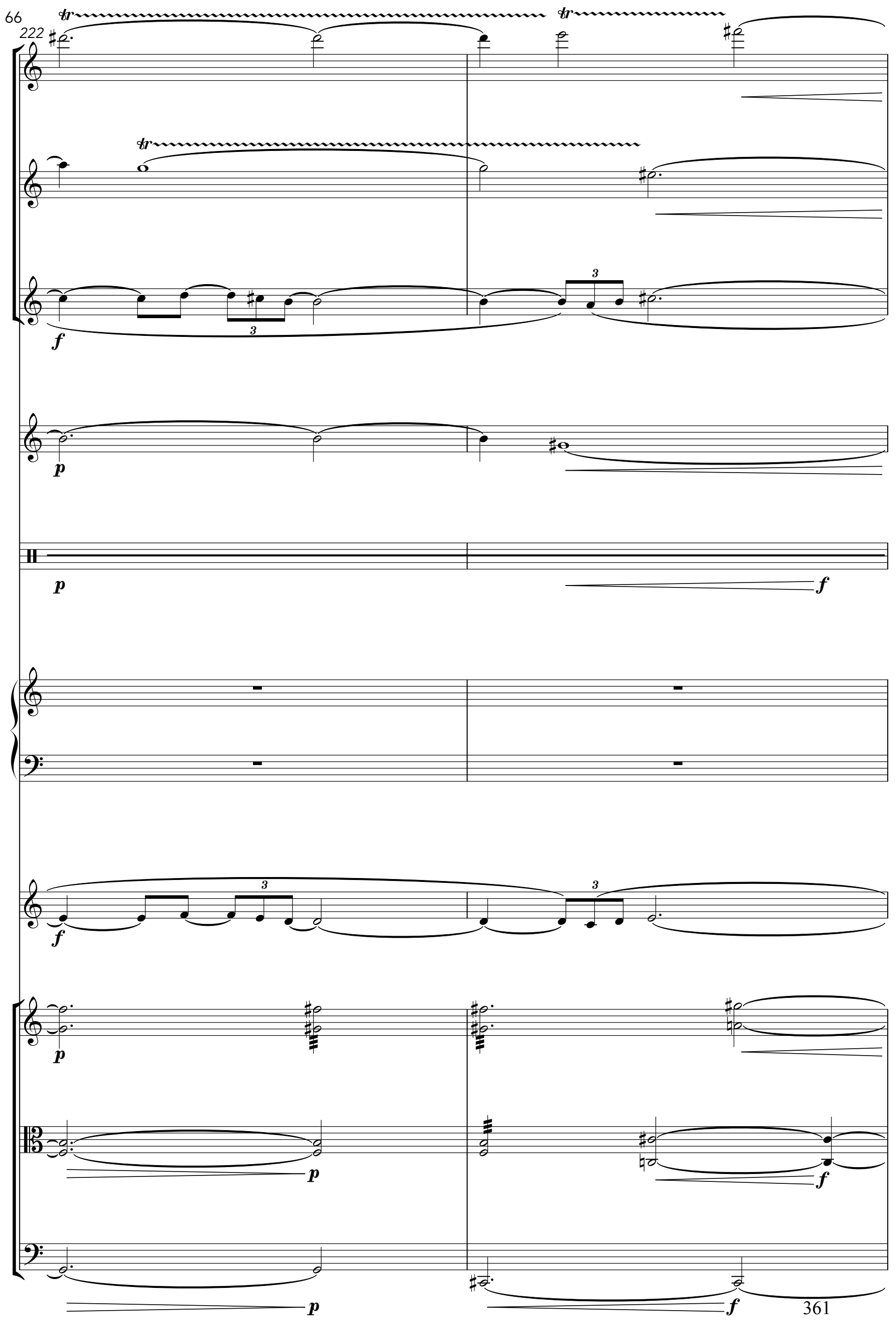




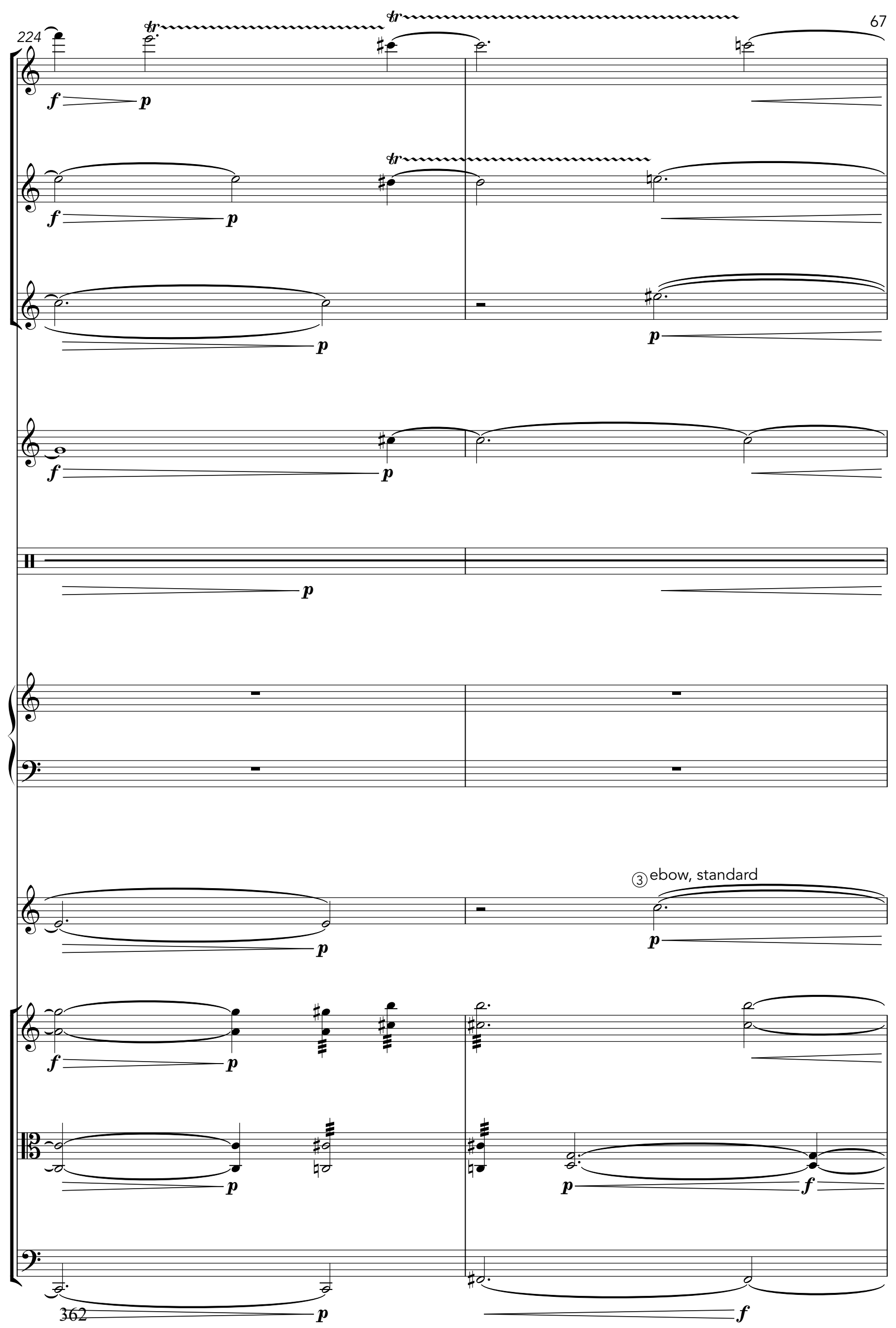




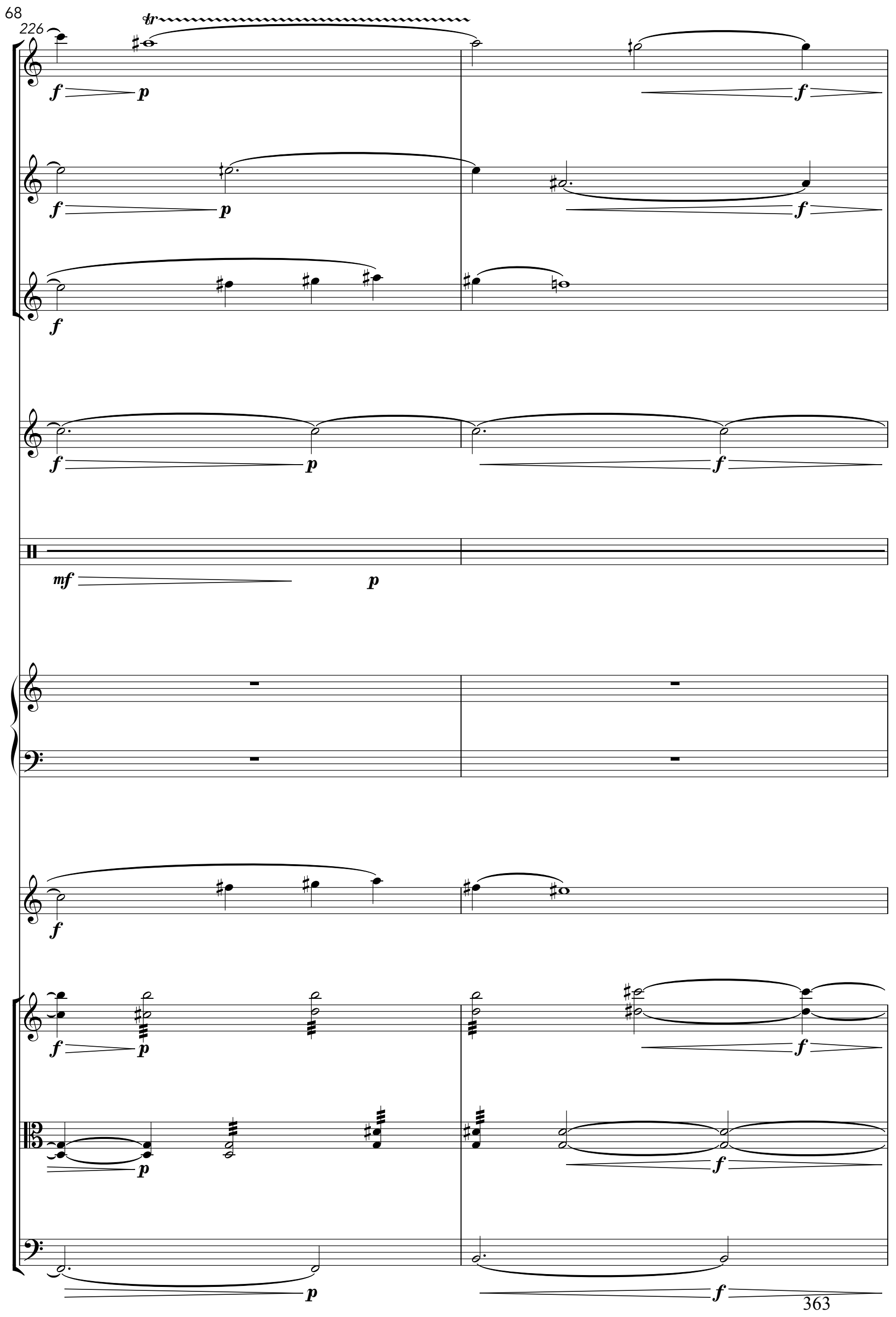



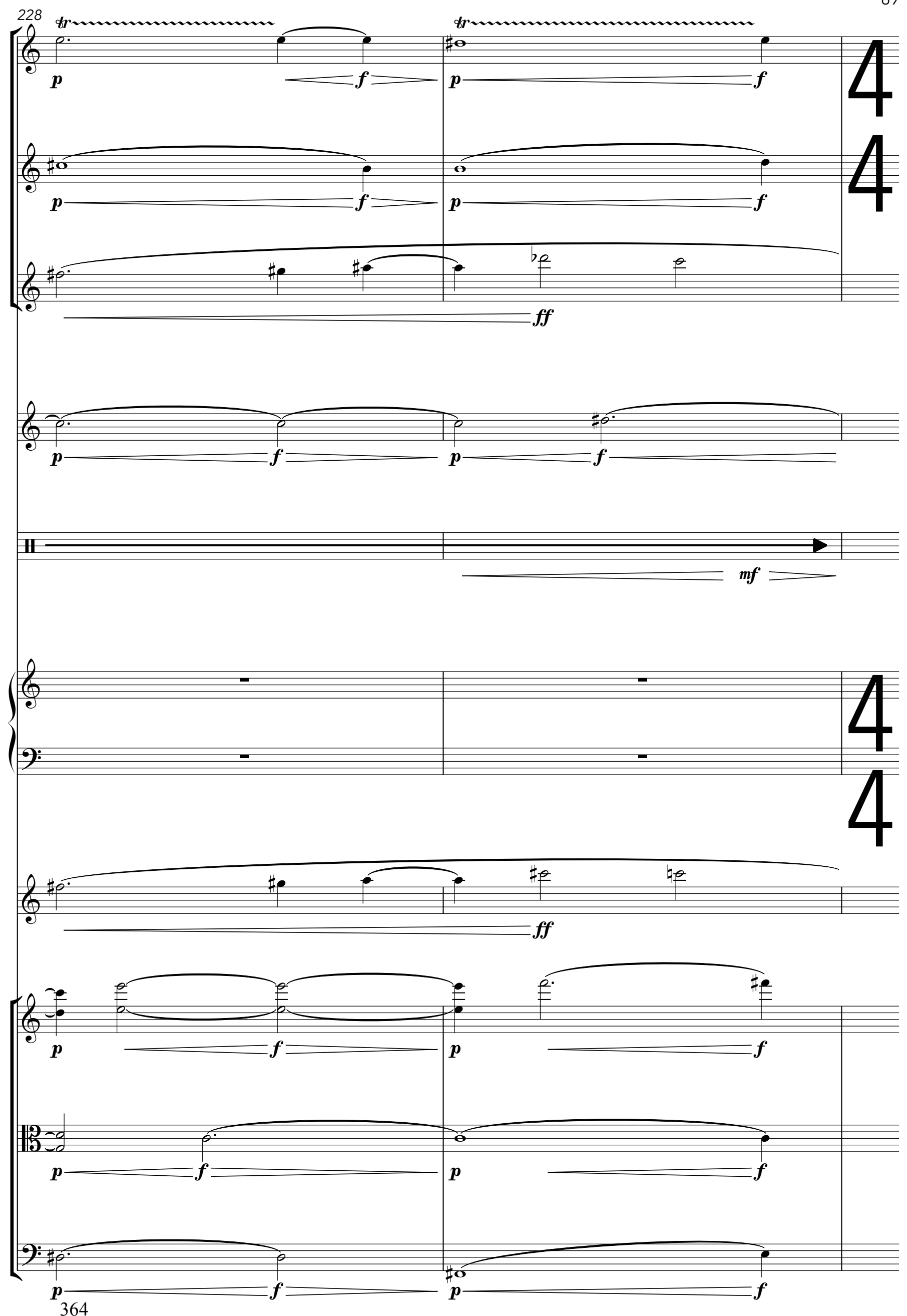


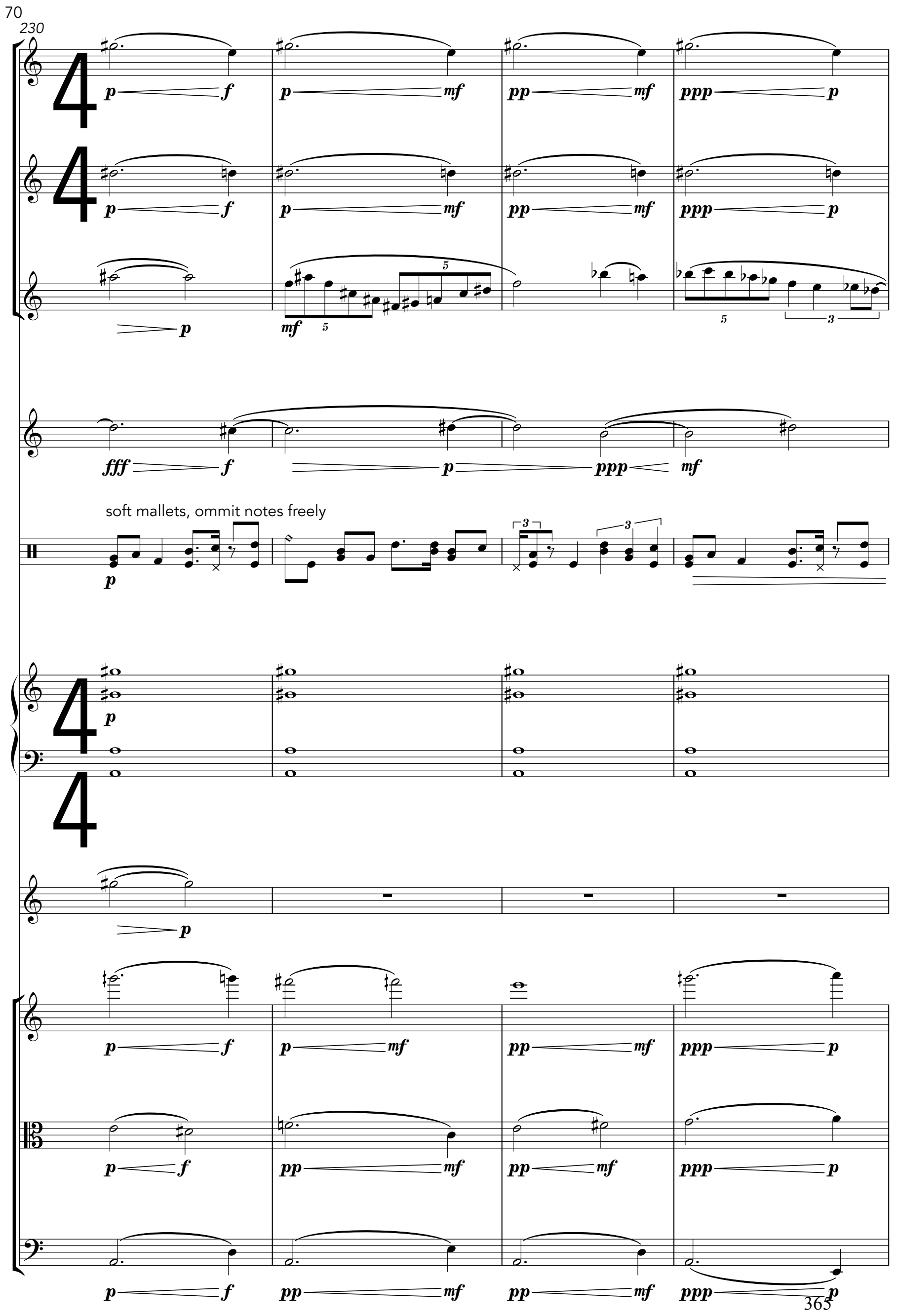



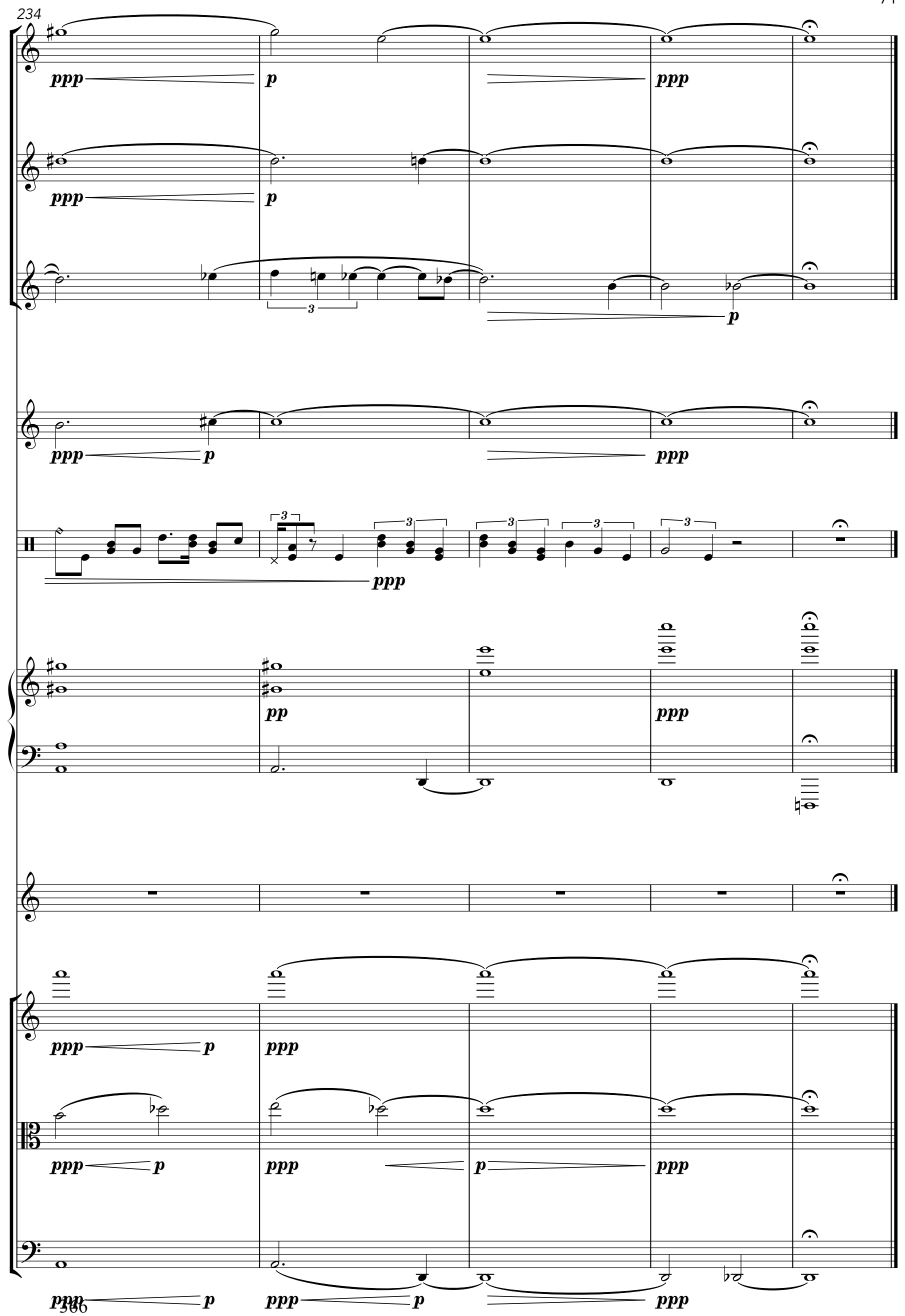
APPENDIX M: BERESHITH 
Bereshith

Cristohper Ramos Flores 


\section{Instrumentation:}

3 Flutes (I doubling Piccolo, III doubling Alto Flute if available using alernative part)

3 Oboes (III doubling English Horn)

3 Clarinets in $\mathrm{Bb}$ (III doubling Bass Clarinet)

3 Bassoons (III doubling Contrabassoon)

\section{Horns \\ 3 Trumpets in $\mathrm{C}$ \\ 3 Trombones \\ 1 Tuba}

\section{Percussionists}

I. 3 Tom-toms (S, M \& L), Tam-tam (L), Nipple Gong (S), Tubular Bells, and snare drum

II. Tambourine, 2 Suspended Cymbals (S \& L), and Glockenspiel (also Vibraphone shared with percussionist III)

III. Vibraphone, Cymbales Antiques (Crotales - high E, F,G, G\#, A, A\#),

and Xilophone (or marimba transsposed to the appropiate octave)

IV. Bass Drum, Timpani

\section{Harp \\ 1 Piano}

Violins I: Divisi in 3 groups: A, B and C

Violins II: Divisi in 3 groups: $D, E$ and $F$

Violas: Divisi in 2 groups

Violoncellos: Divisi in 2 groups

Double Basses

Duration: Aprox. 18'30" Minutes 


\section{Notes:}

This composition features musical gestures moving around the orchestra. The following sitting chart is recommended.

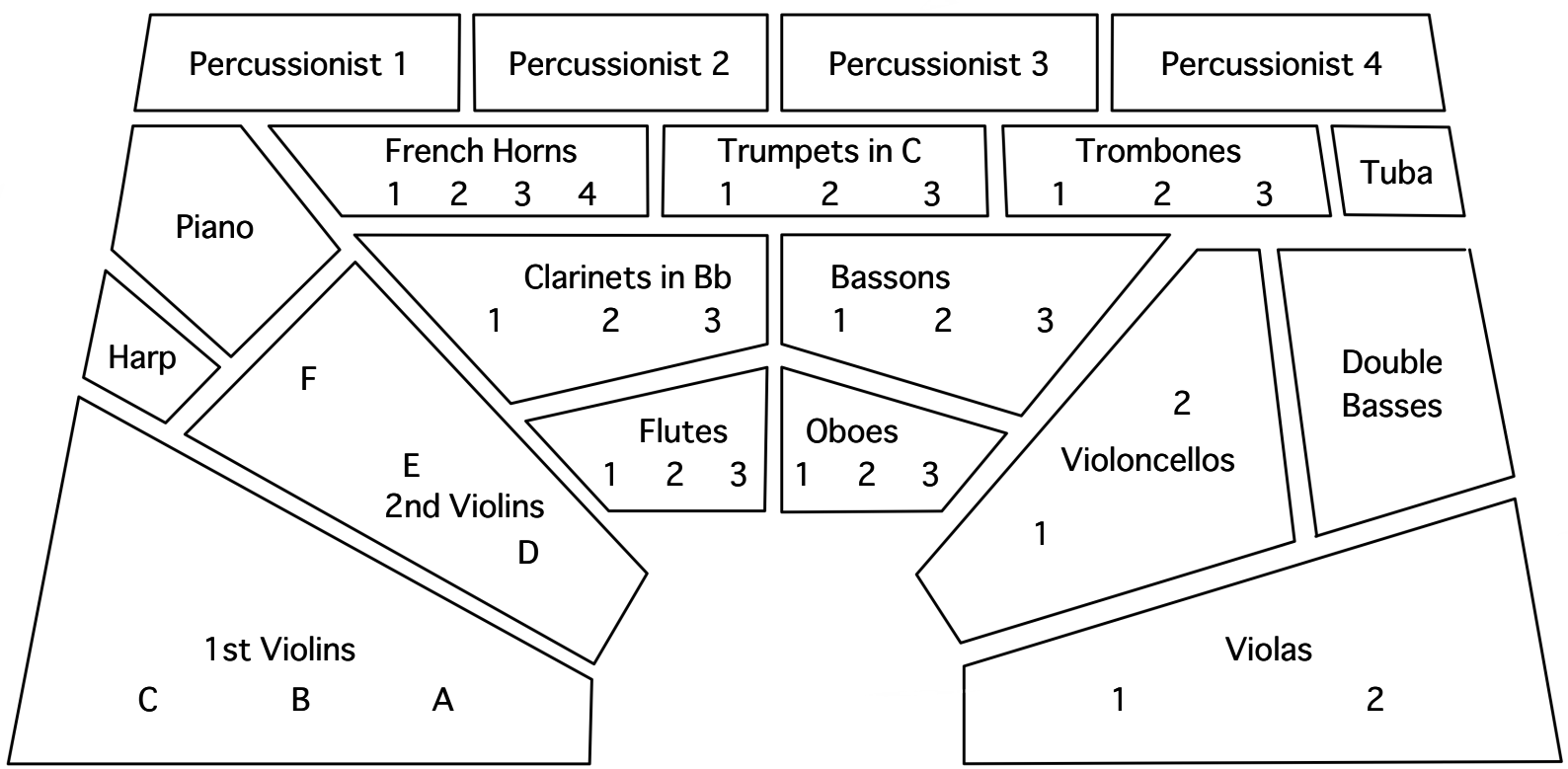

The score is transposed.

Accidentals affect all notes in the bar. Courtesy accidentals may be added.

Trills are to be played up until the point the trill line dissapears.

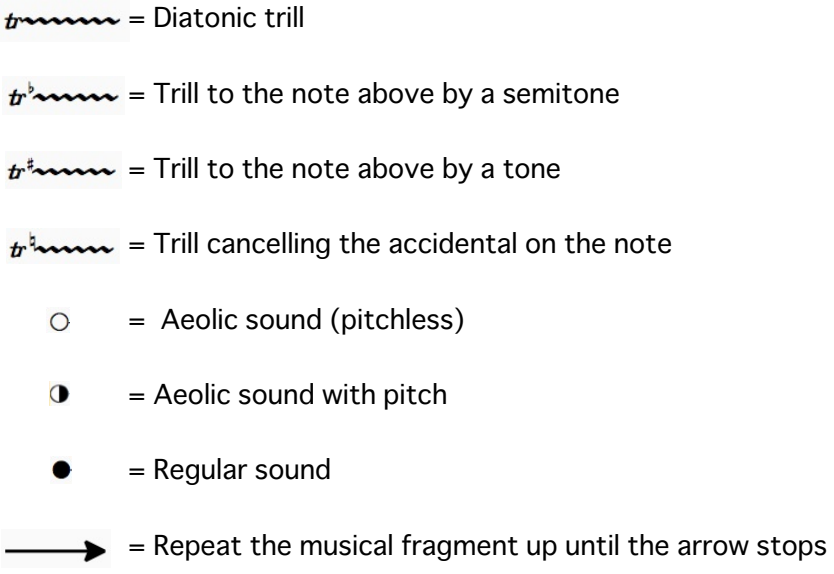


Bereshith

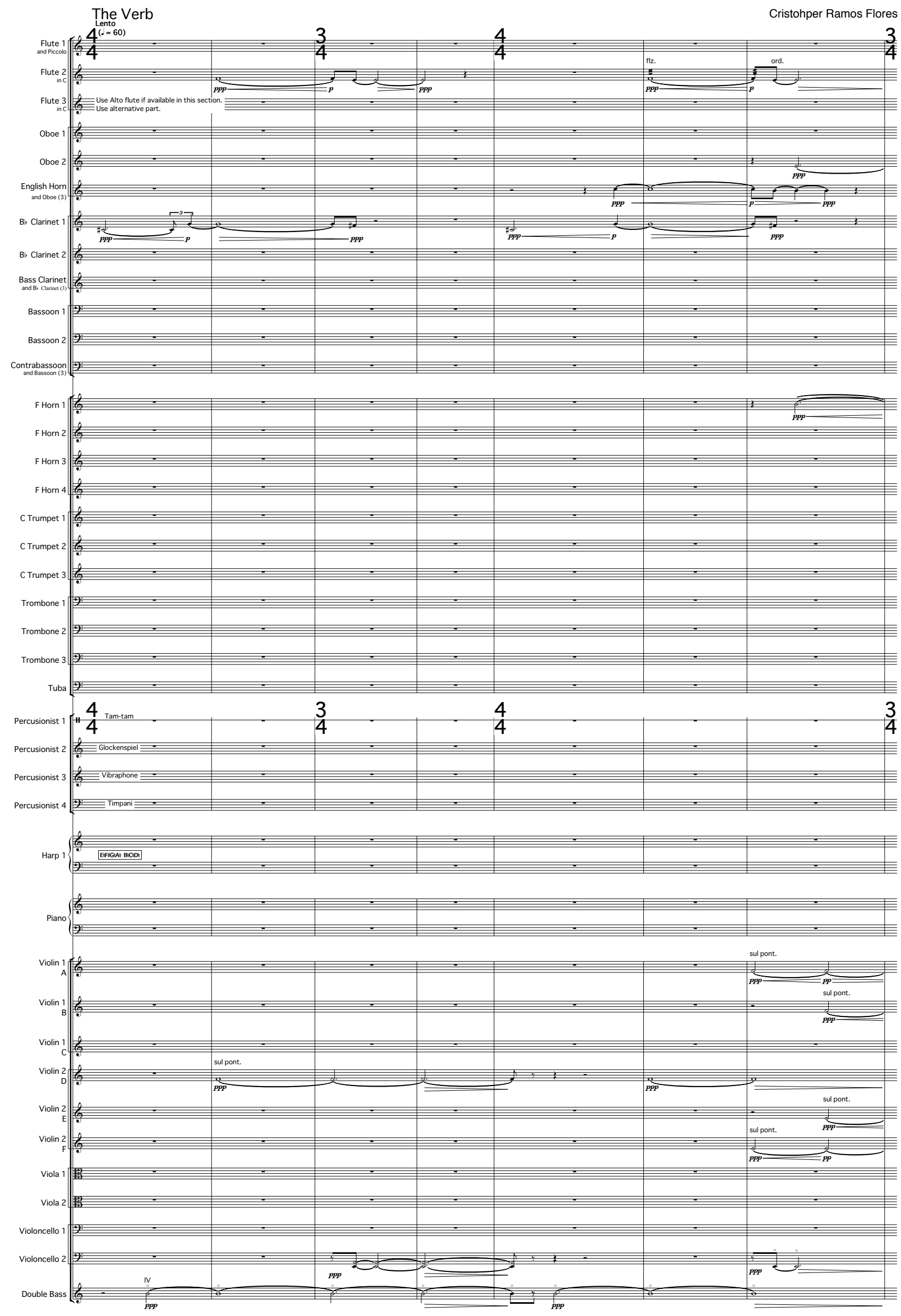




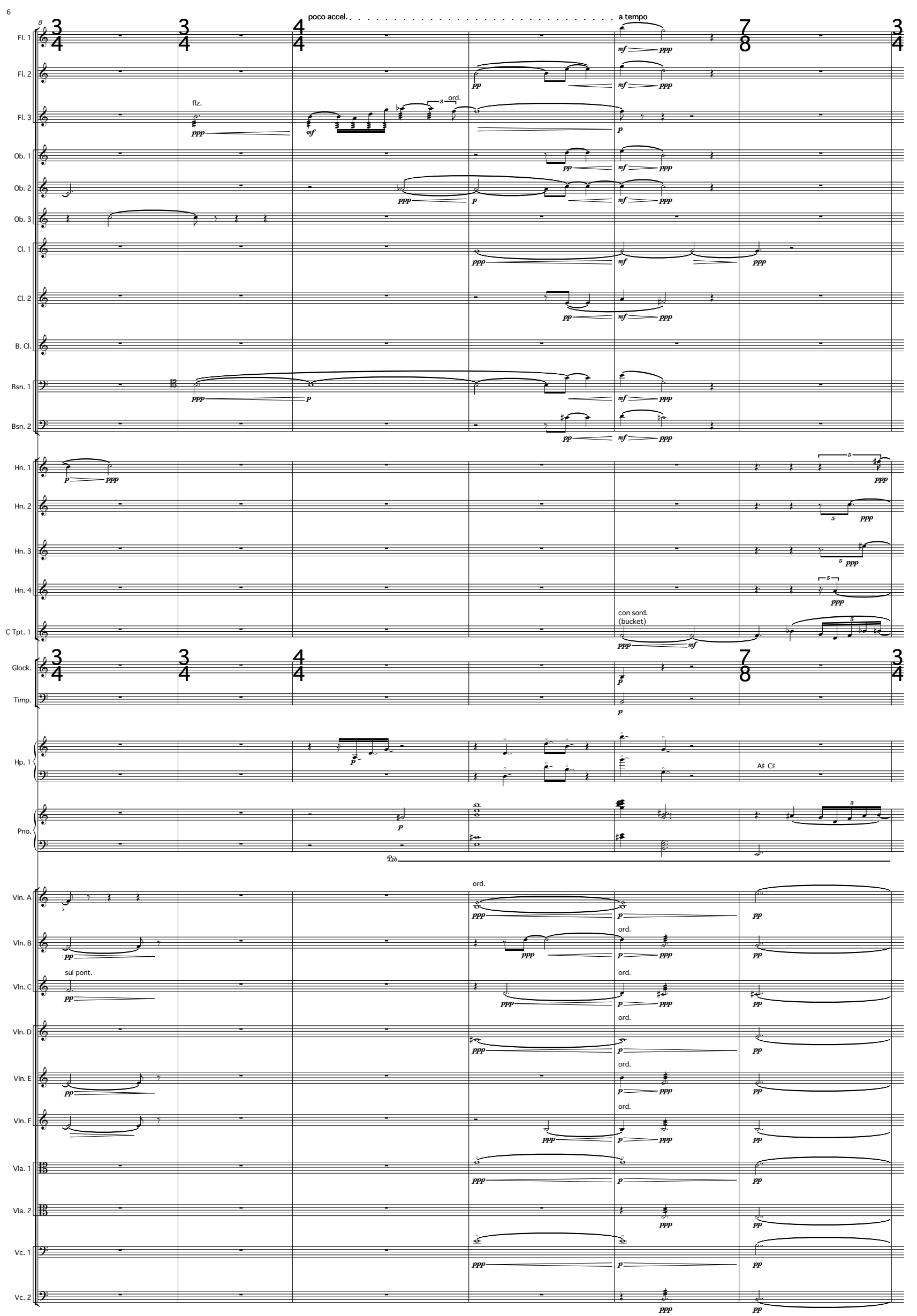




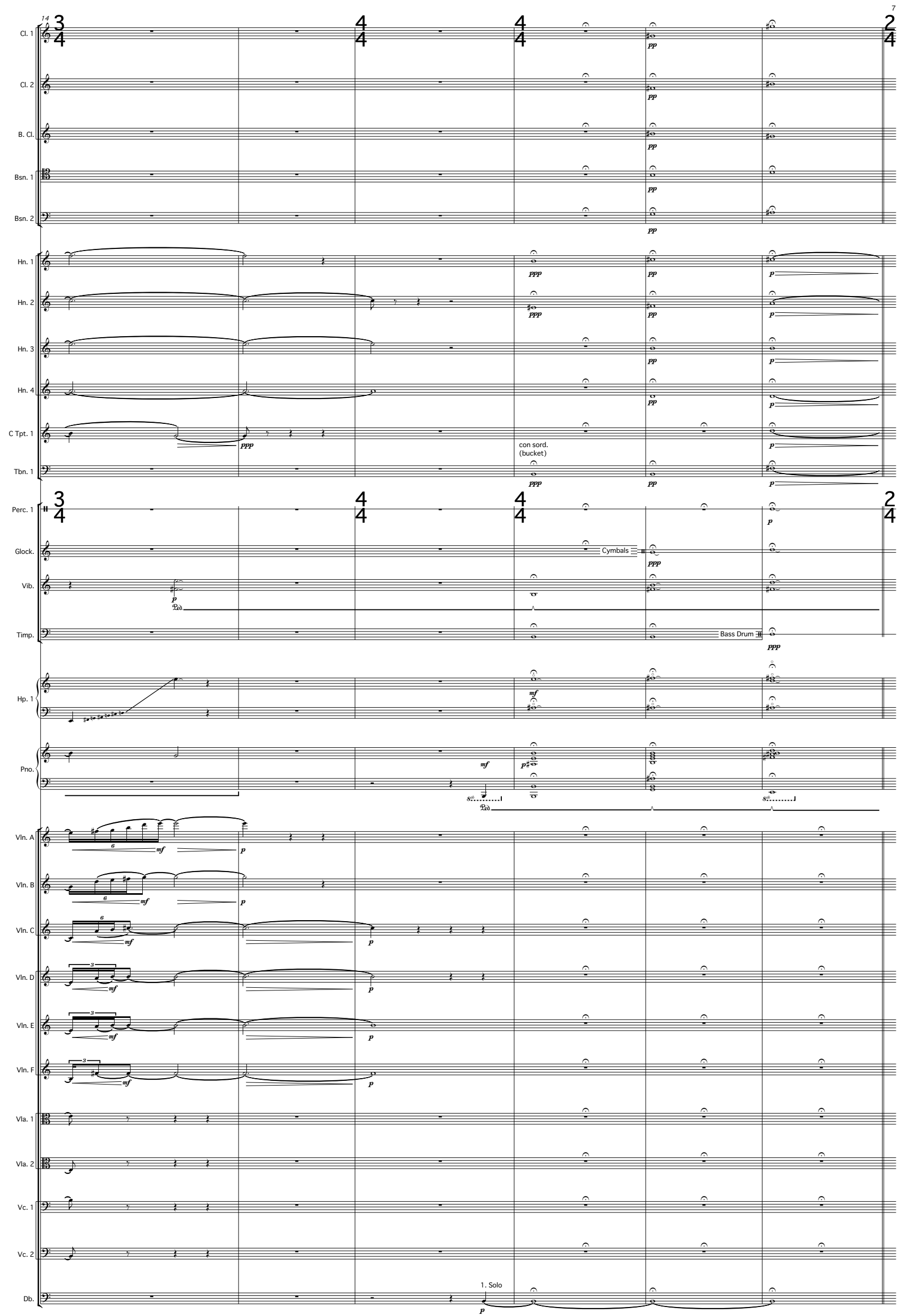


Day 1
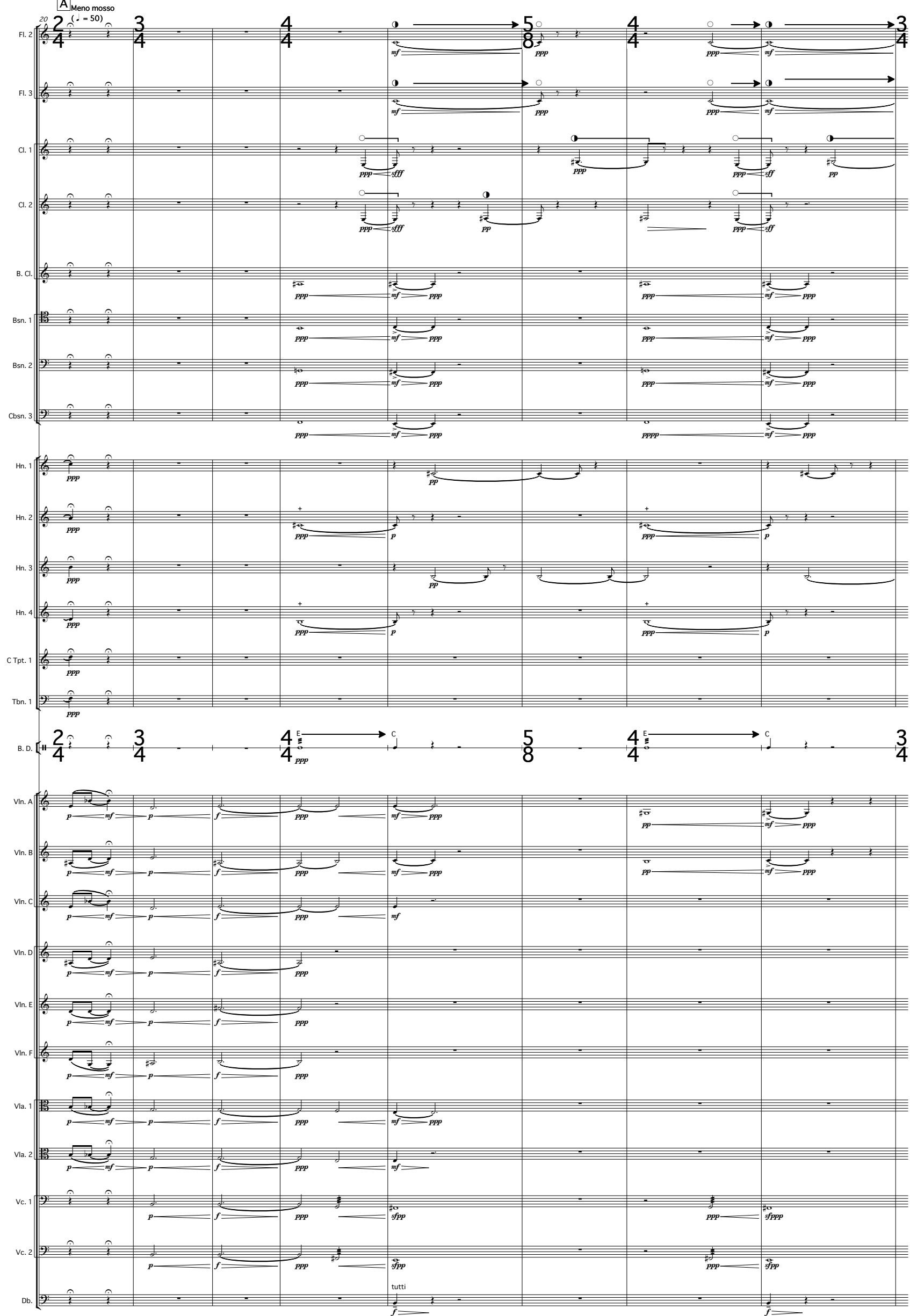


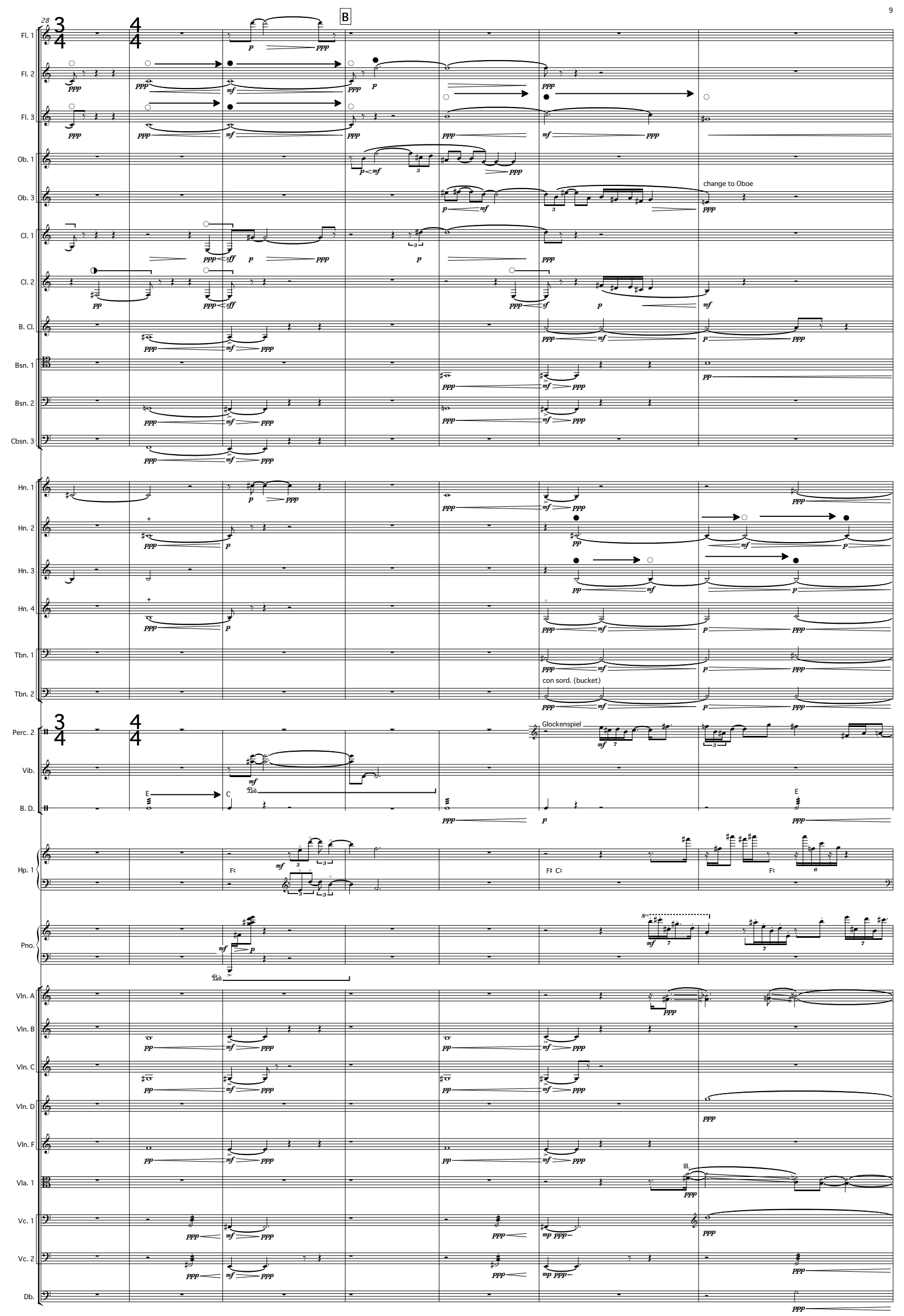




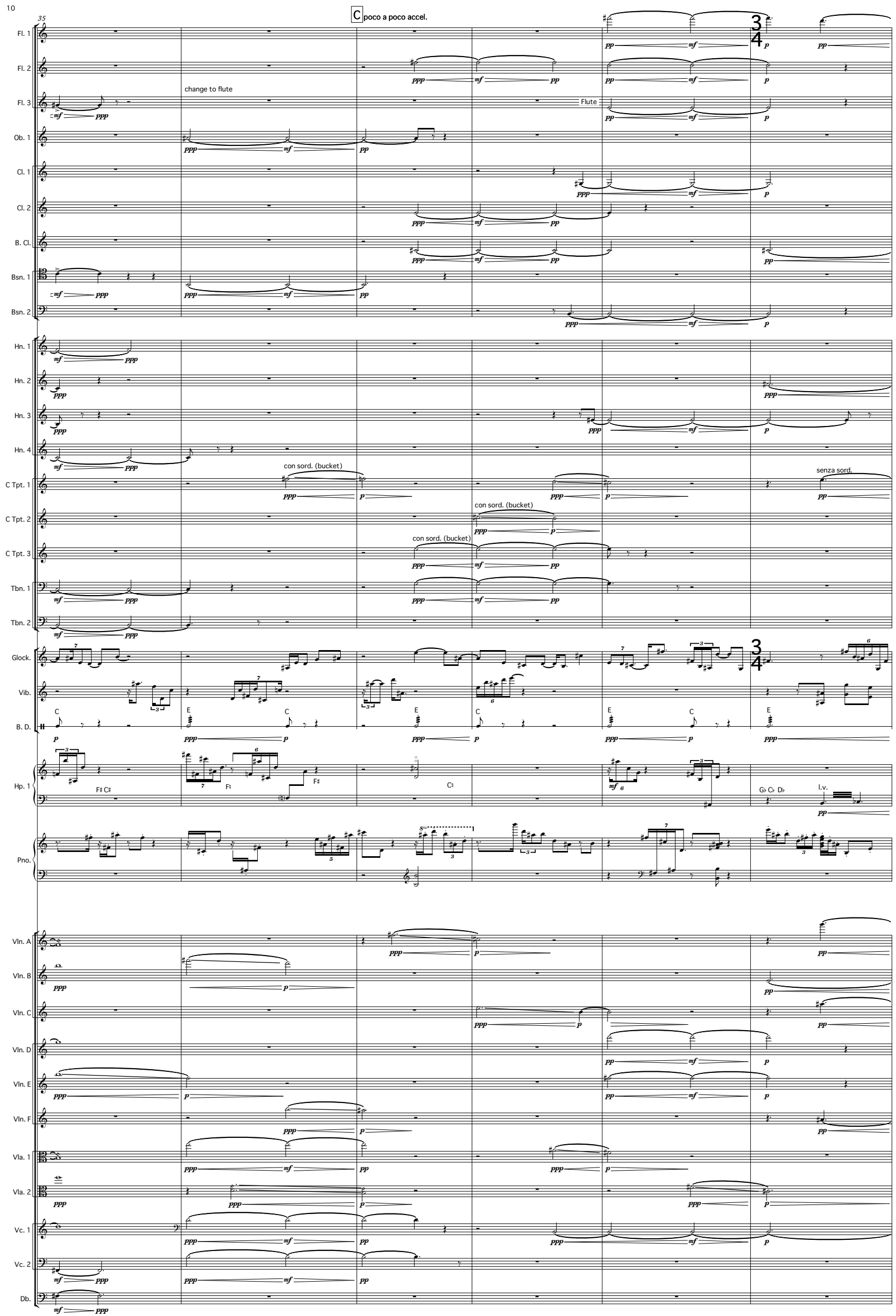




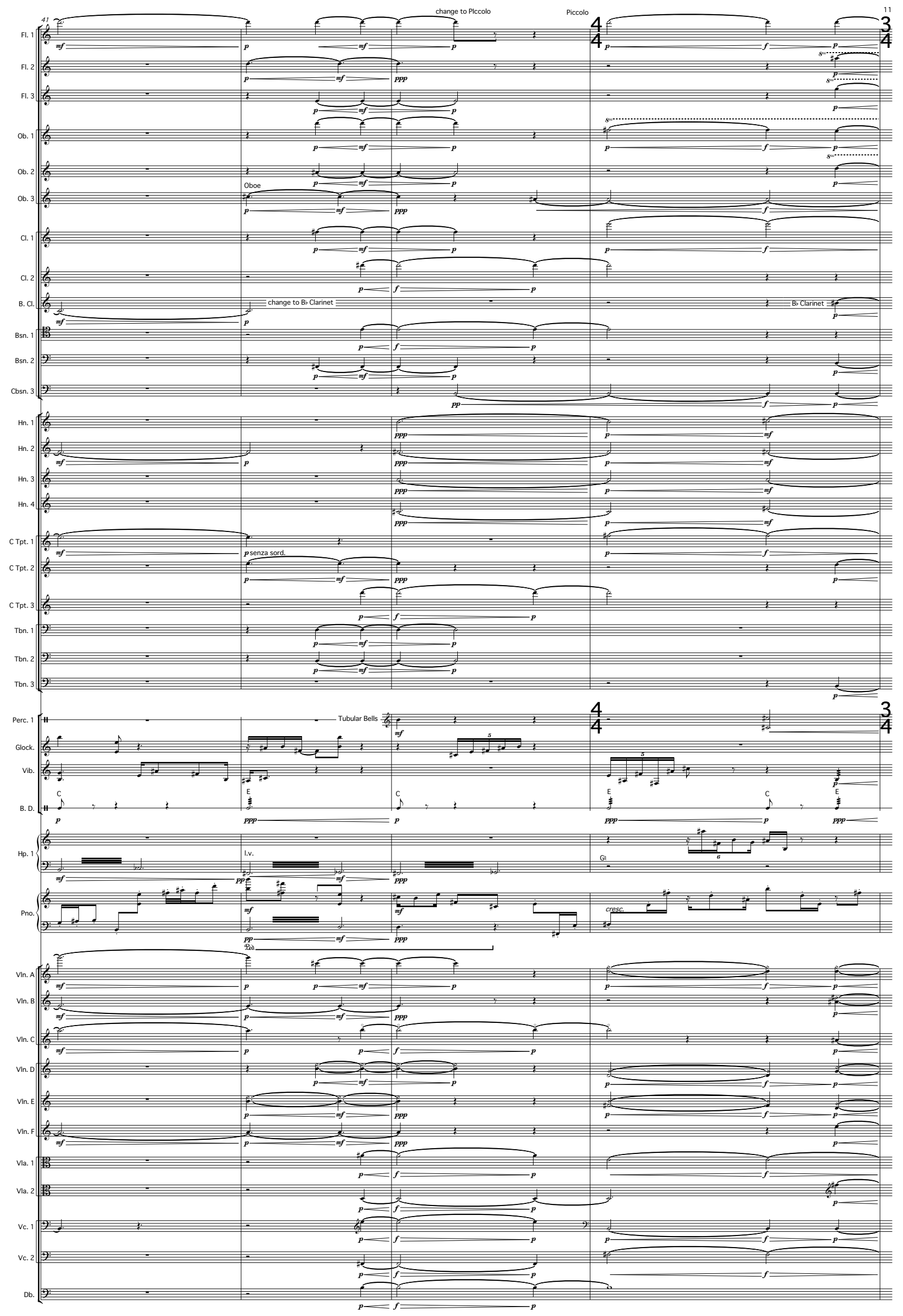




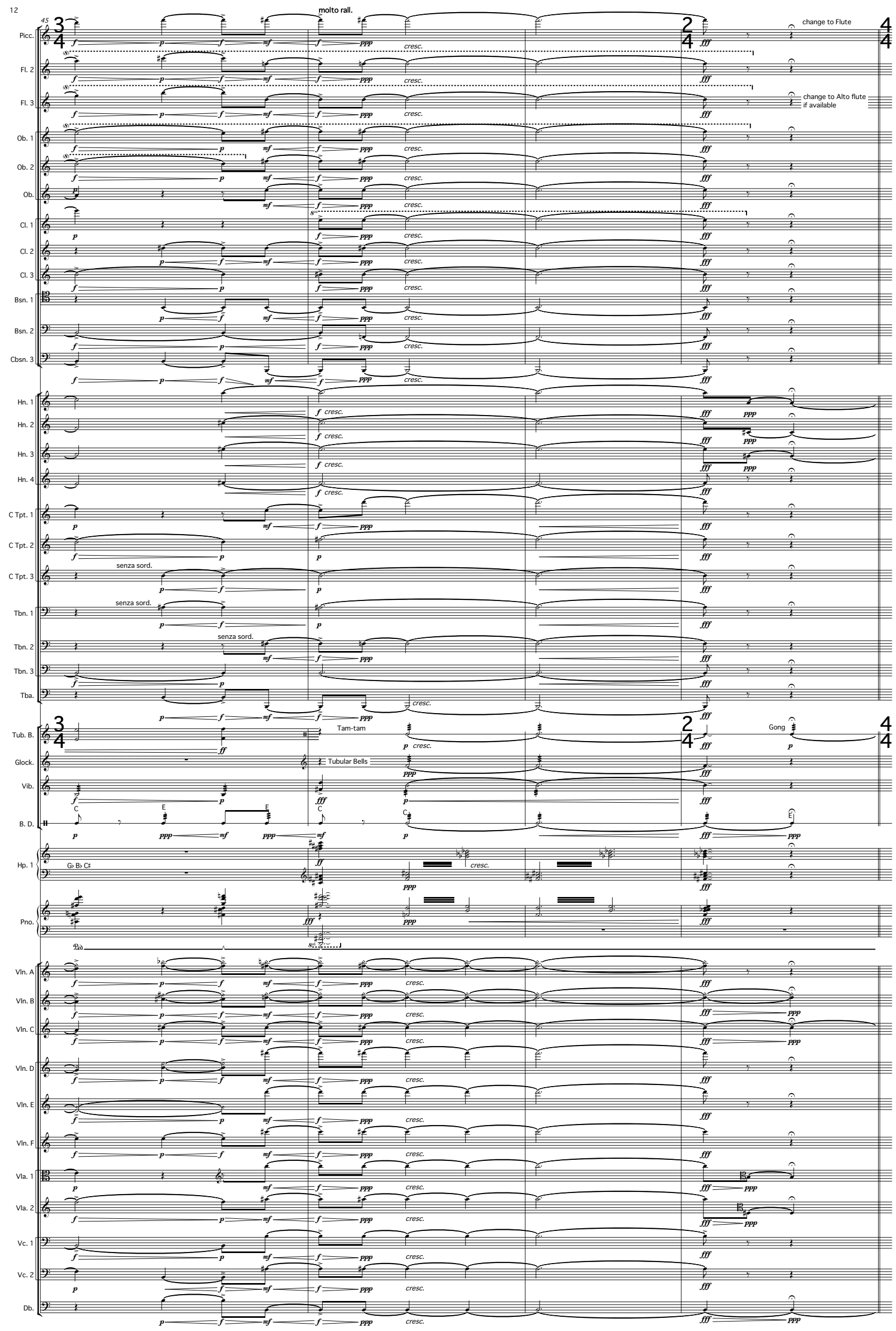


Day 2

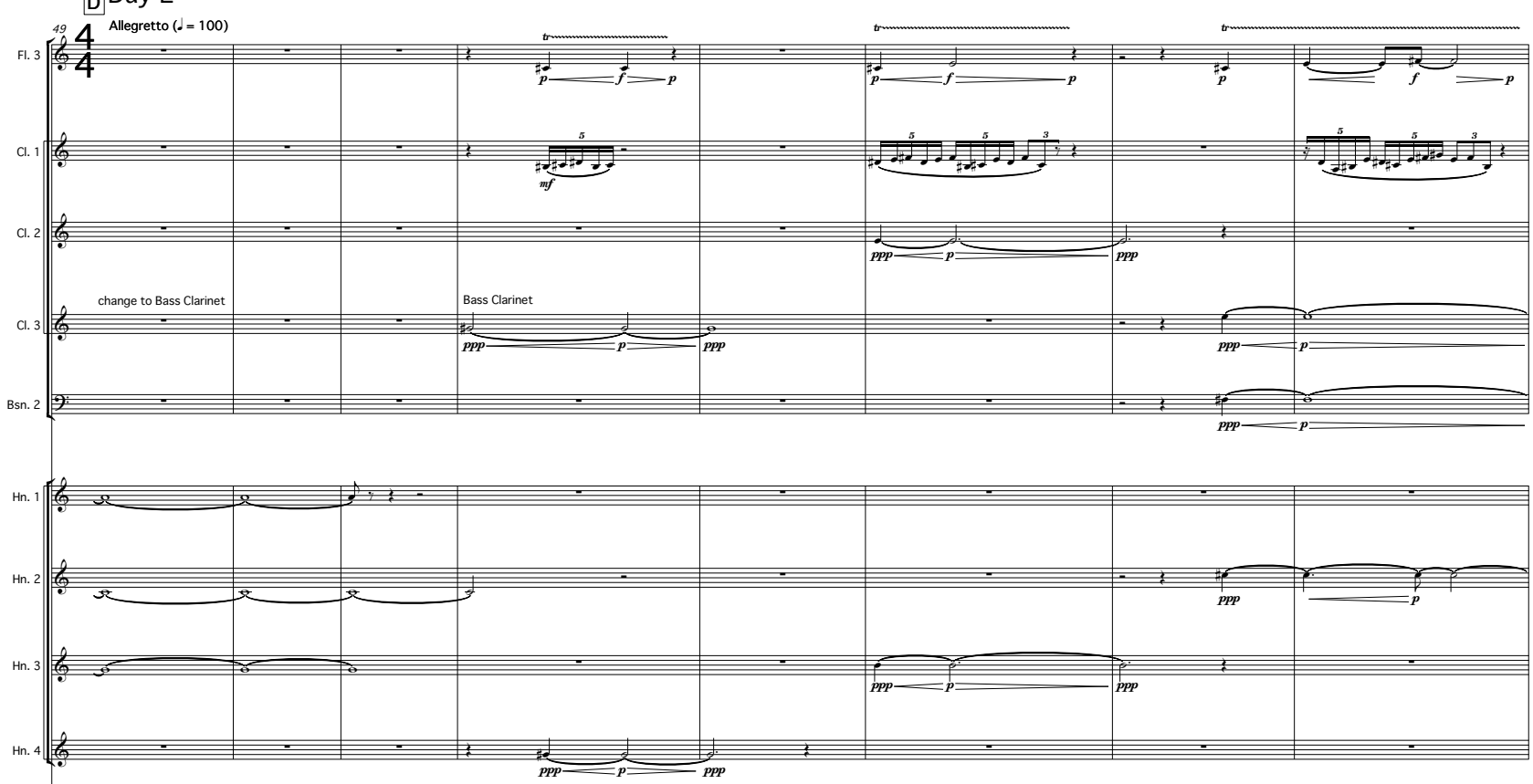

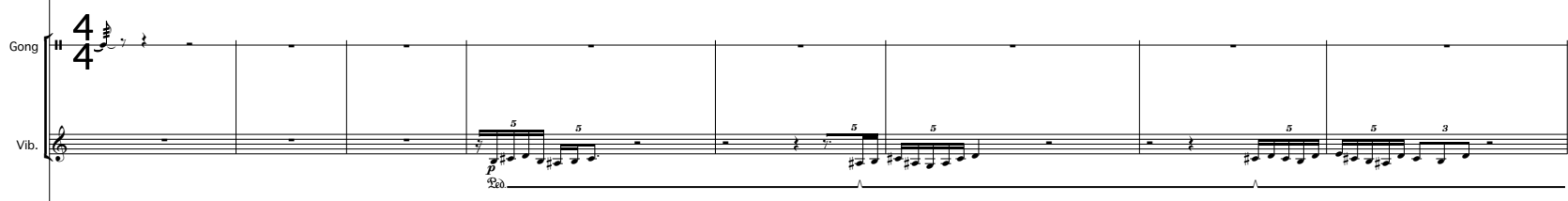

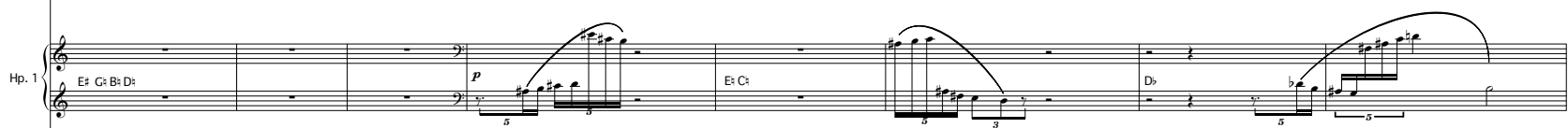

Pno.

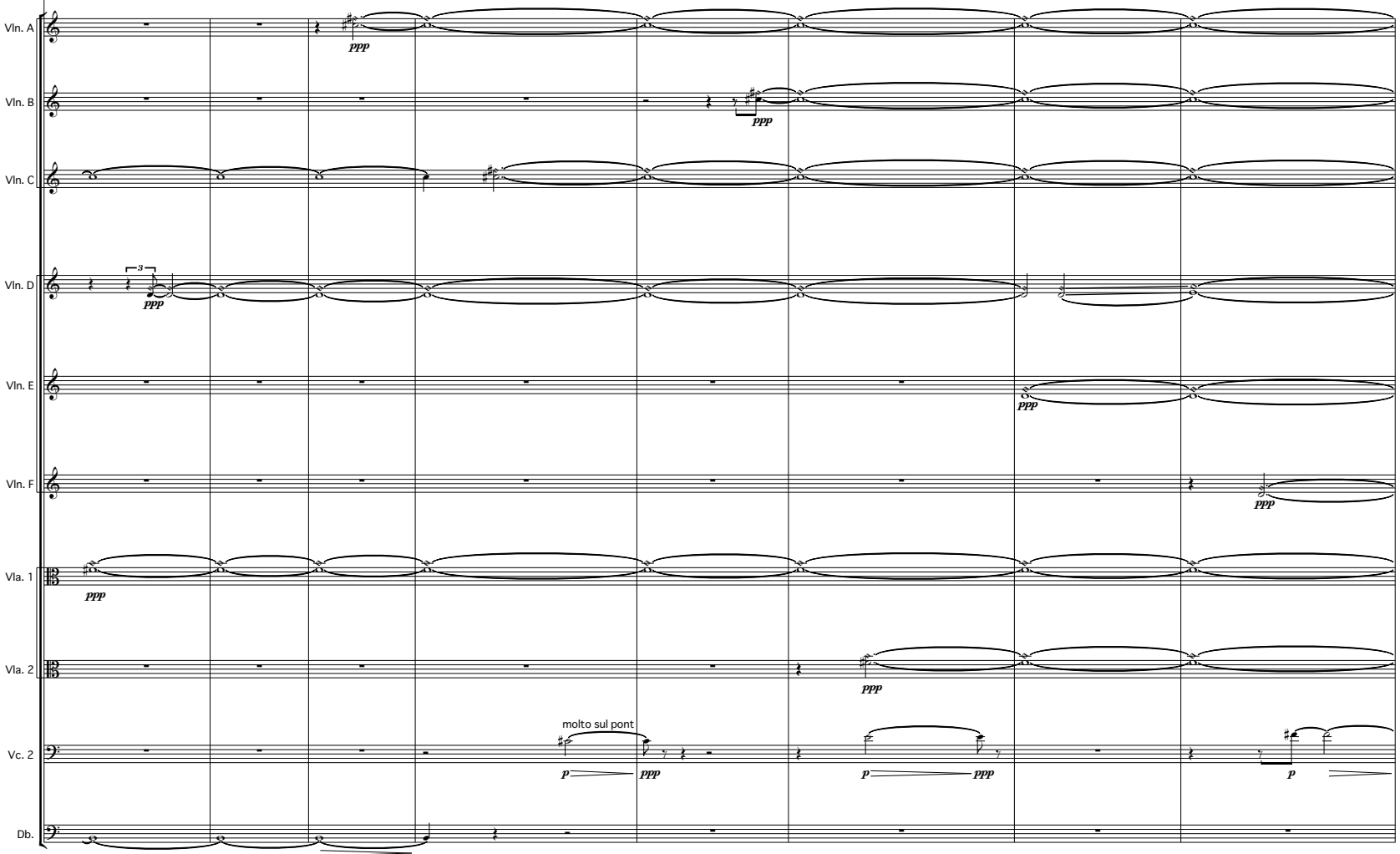




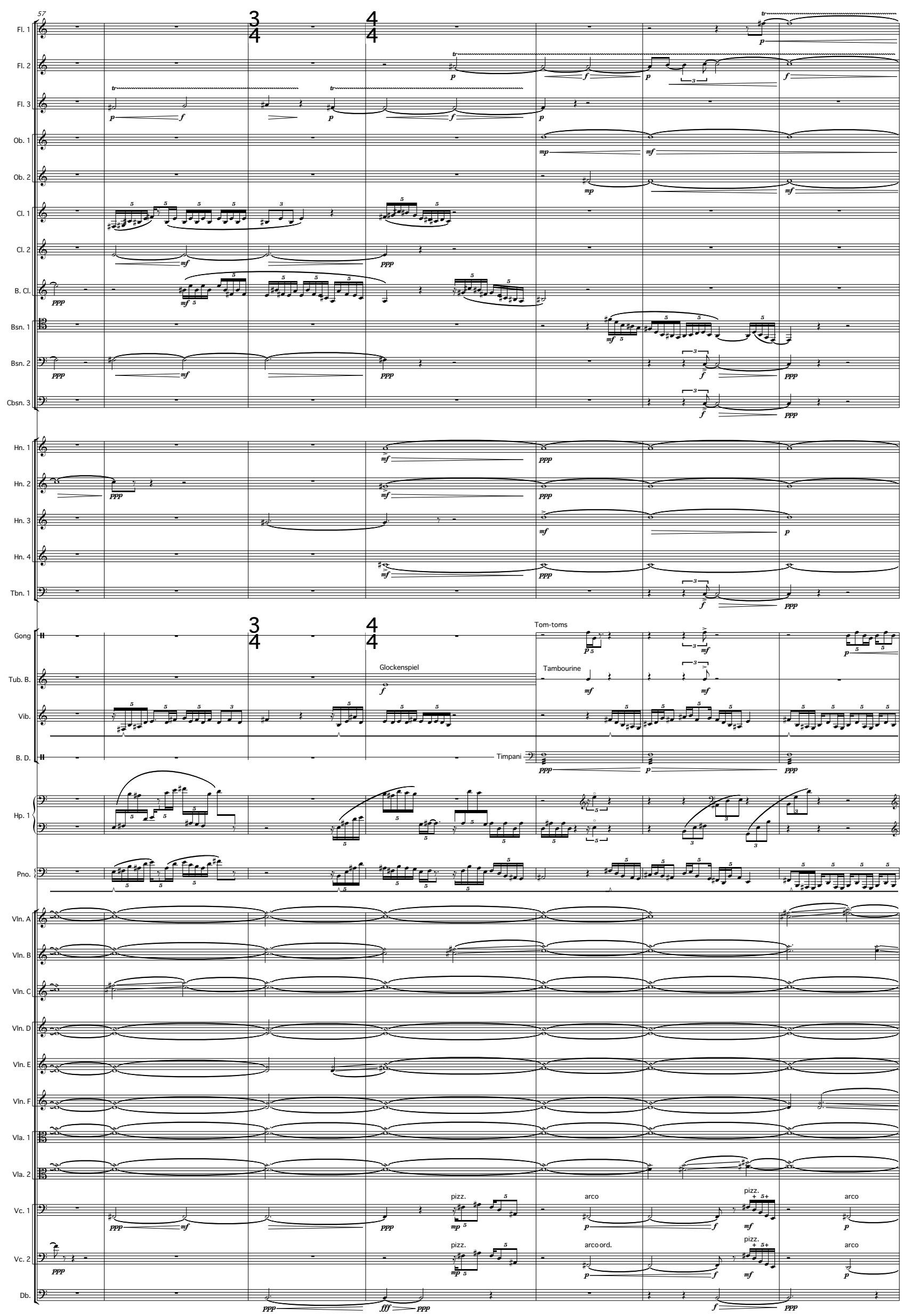



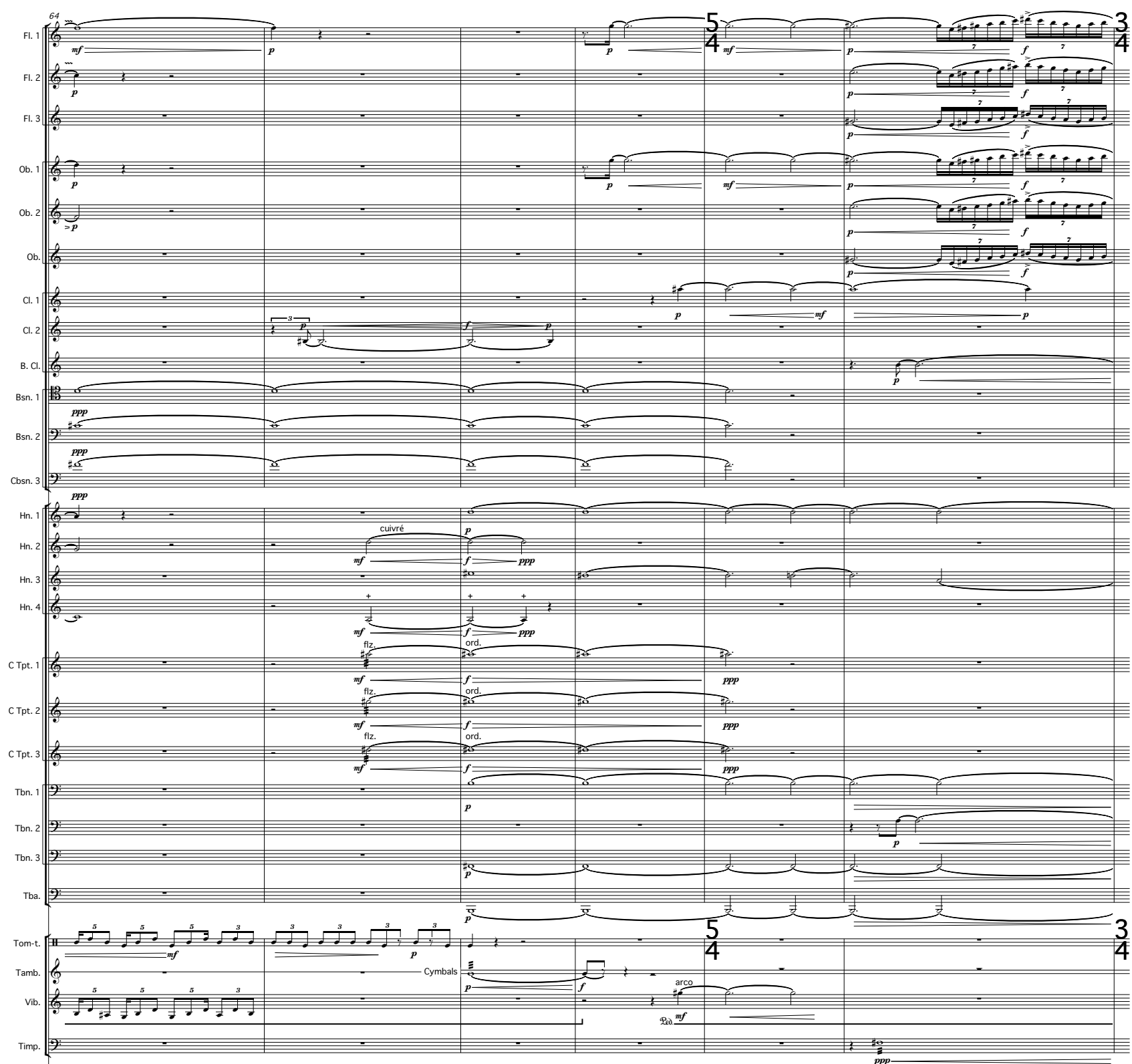

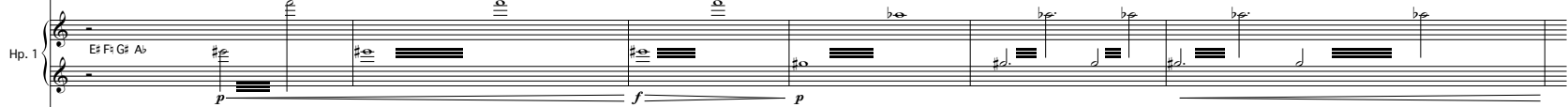

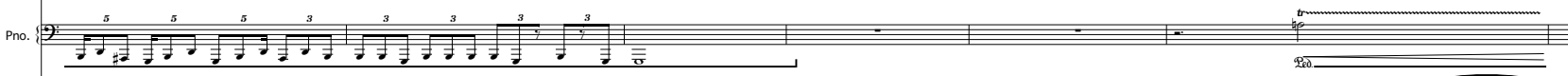

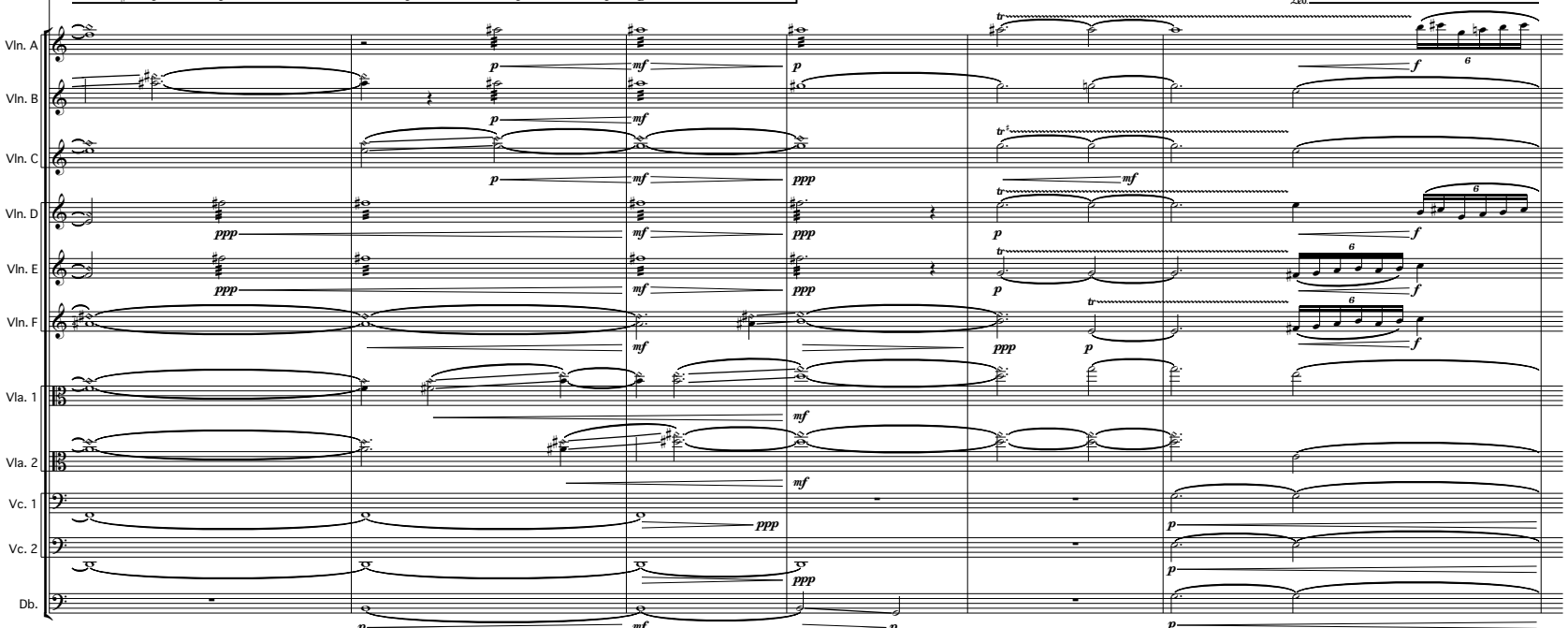




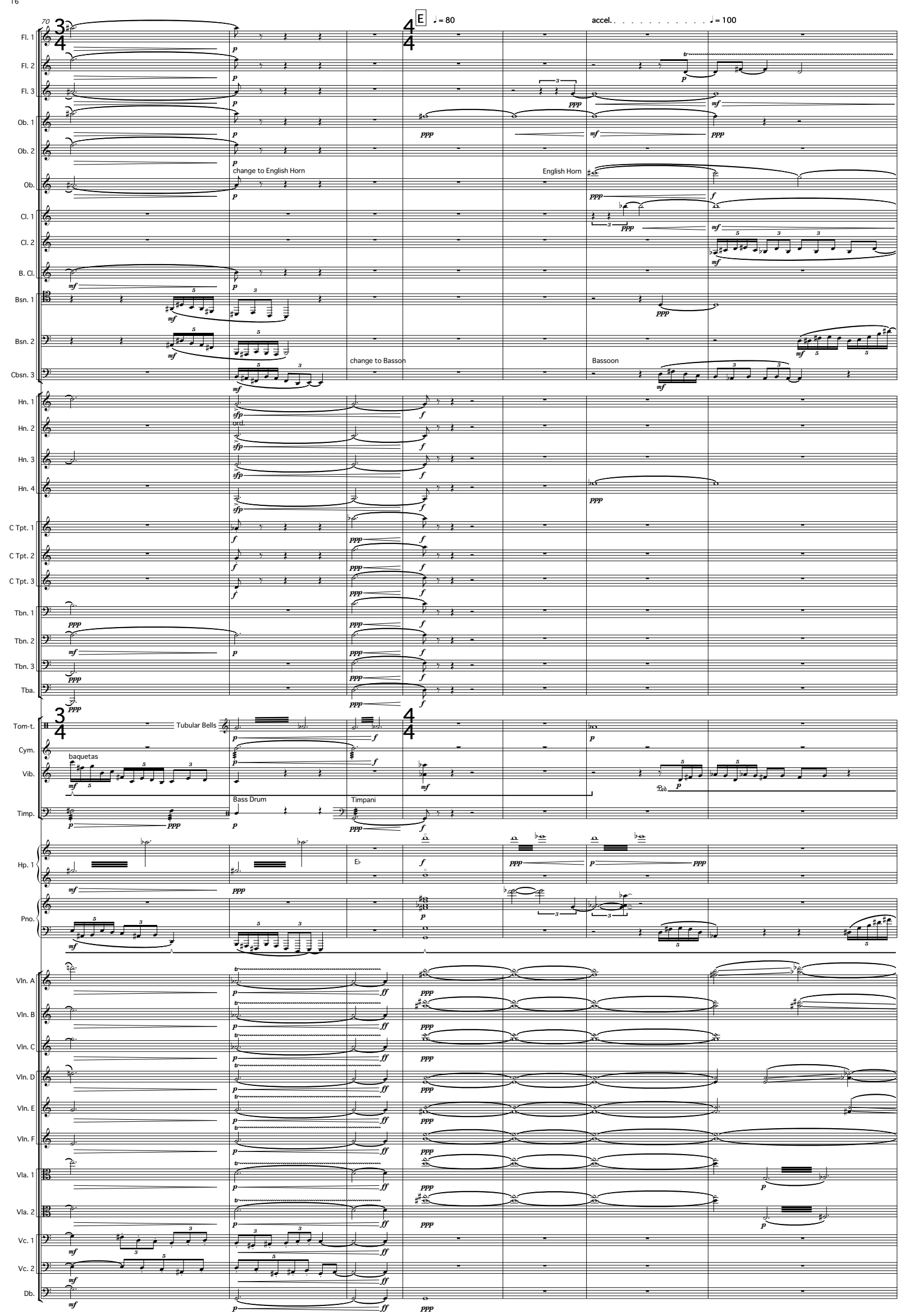



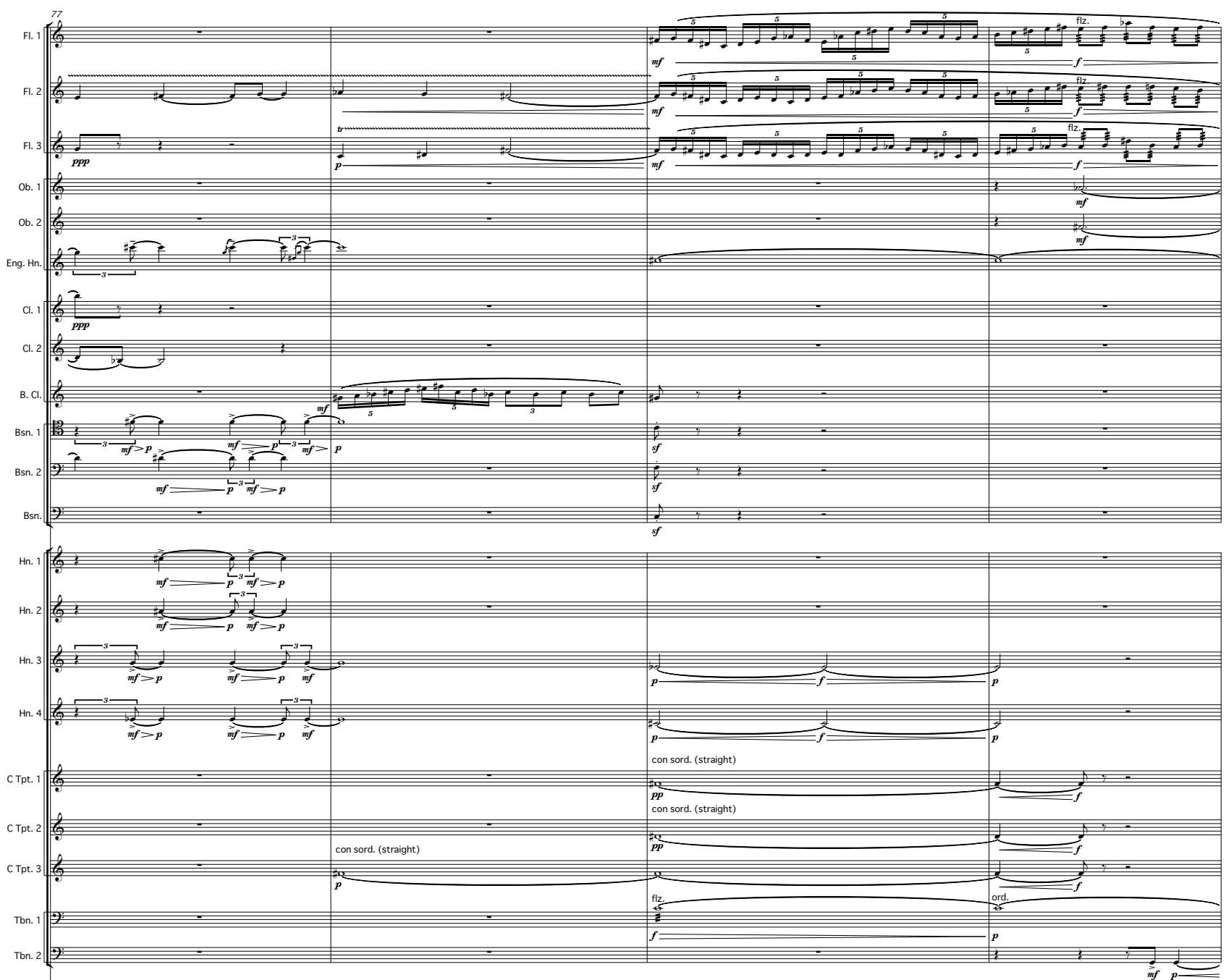

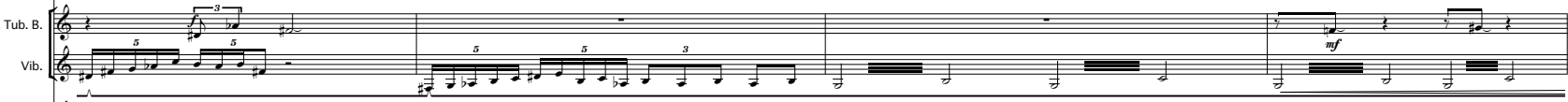

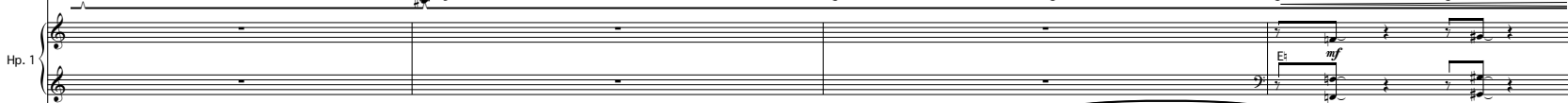

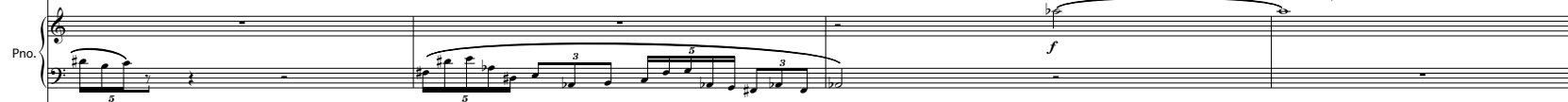

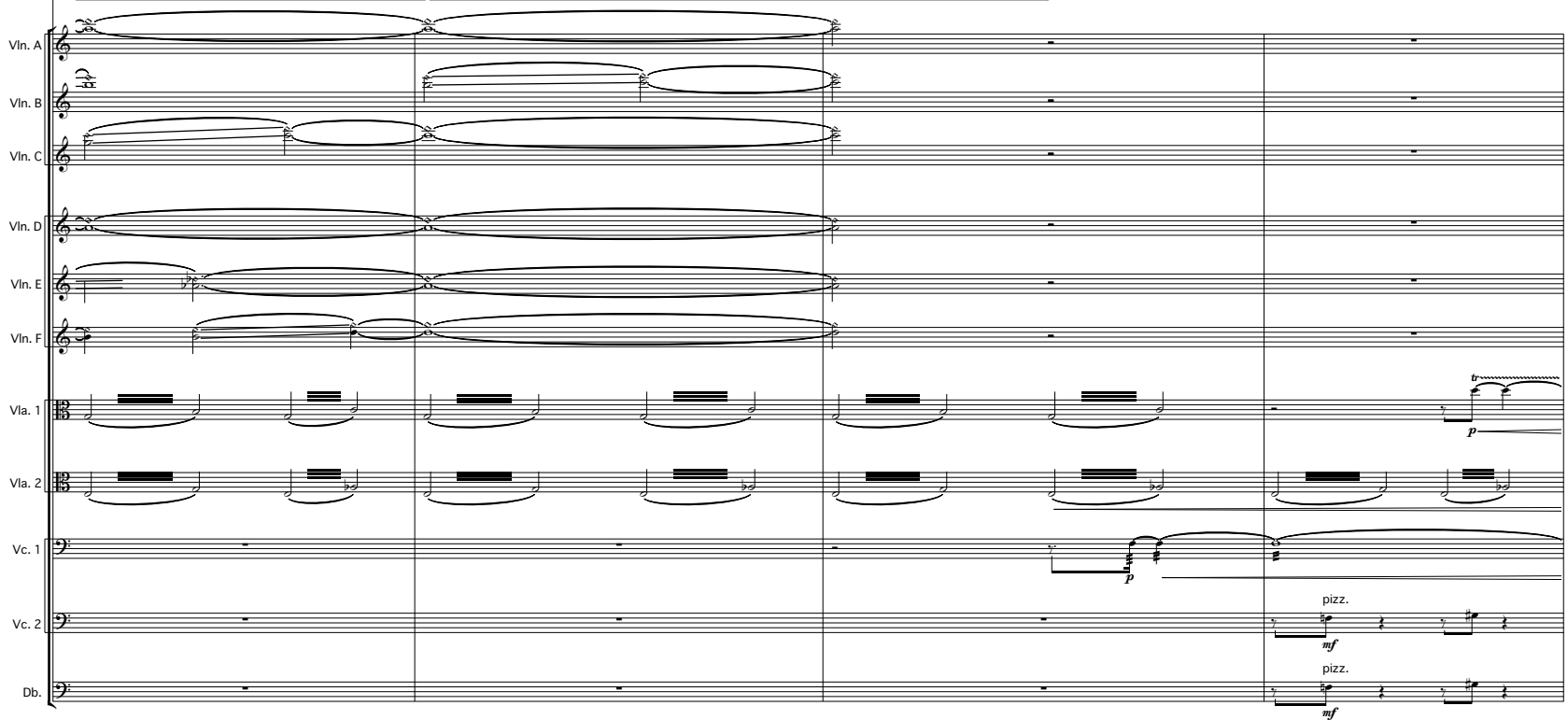




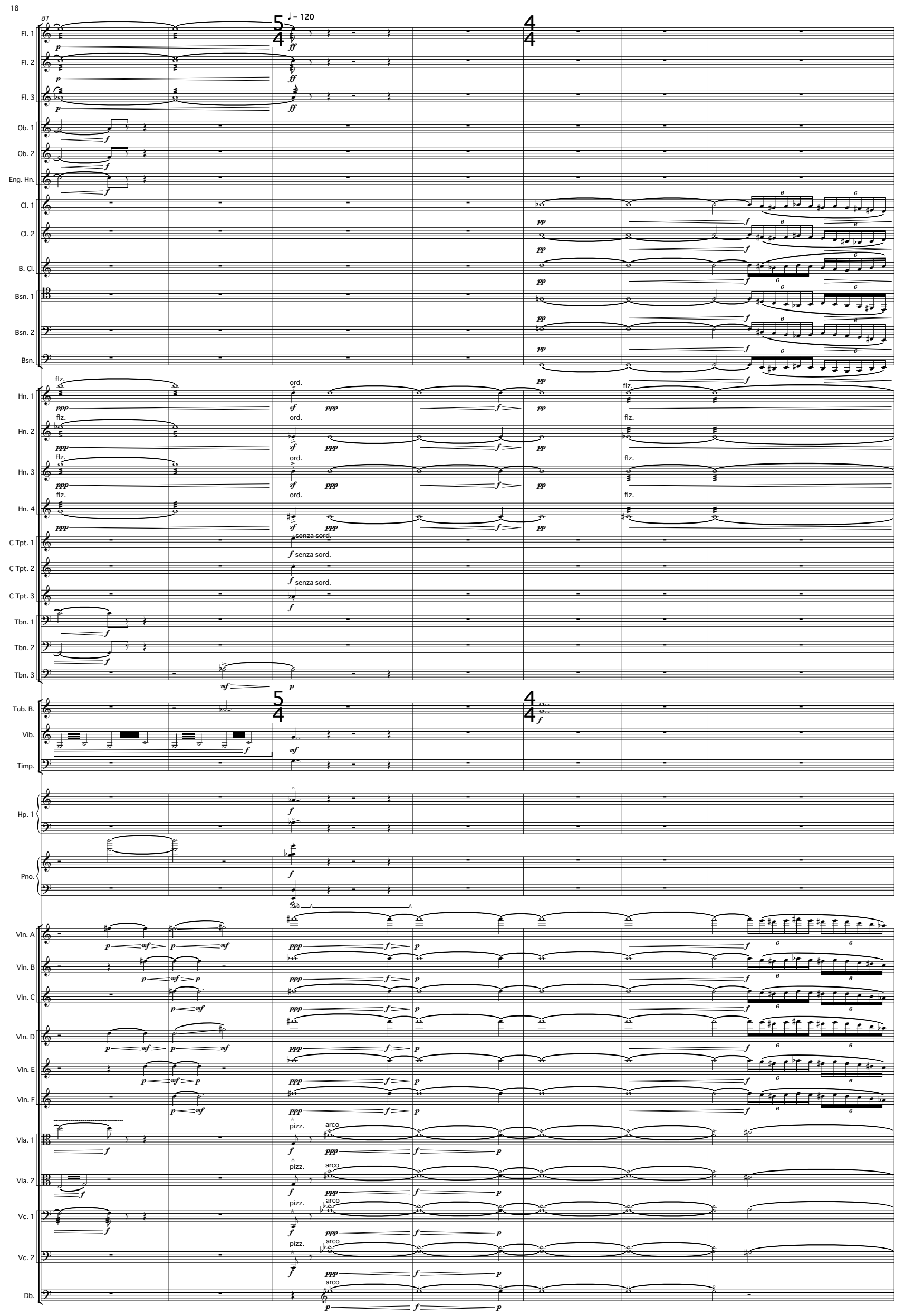


F
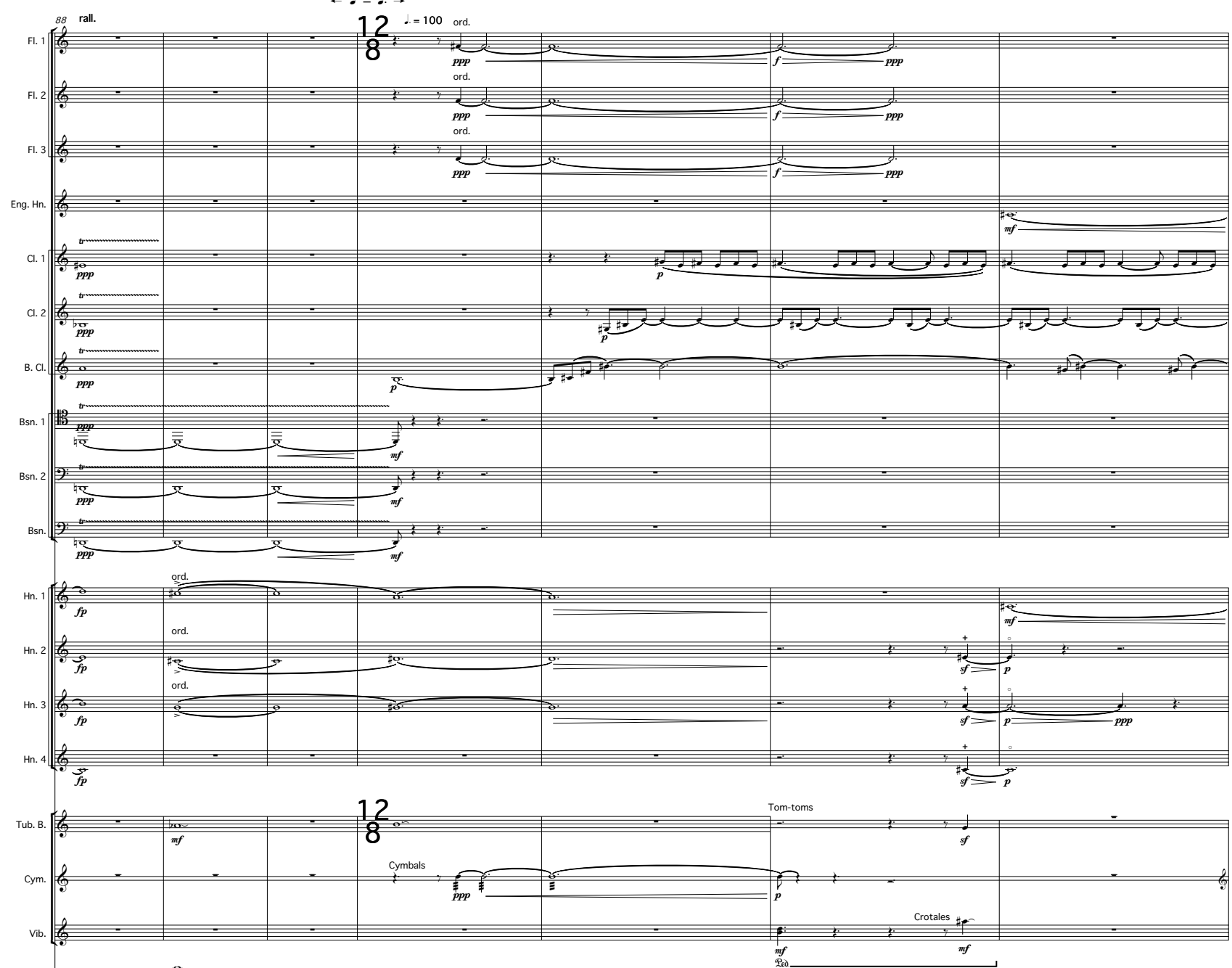

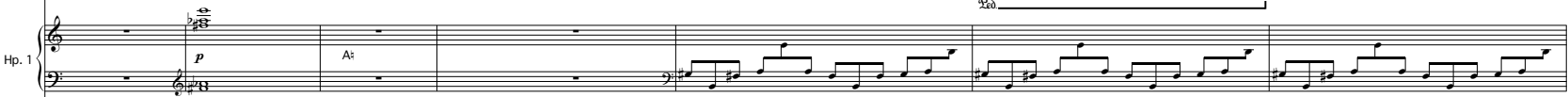

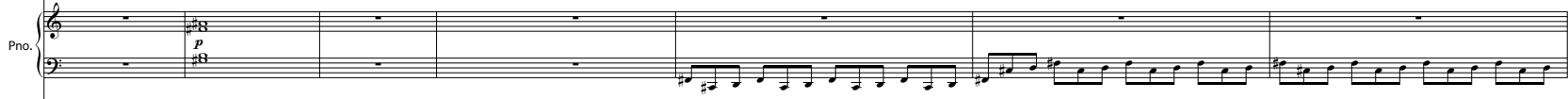

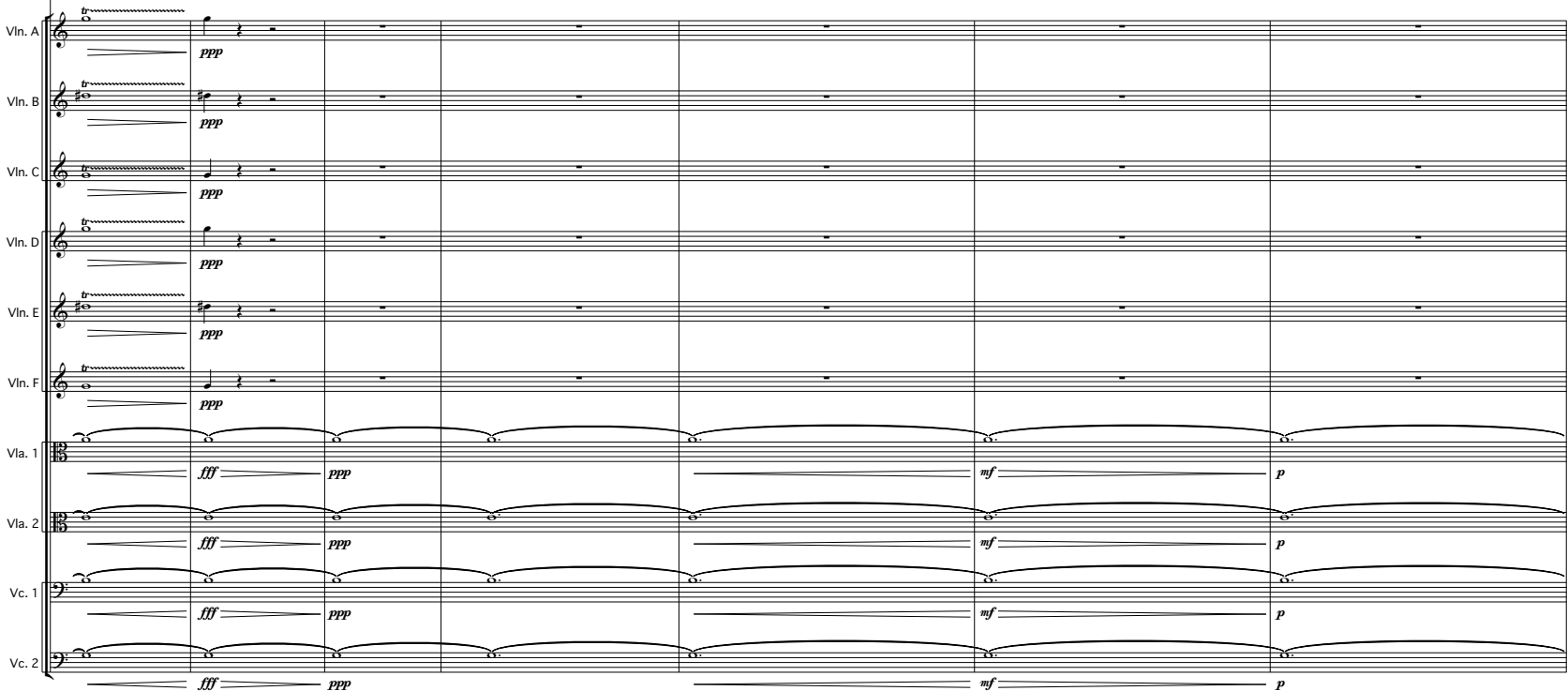




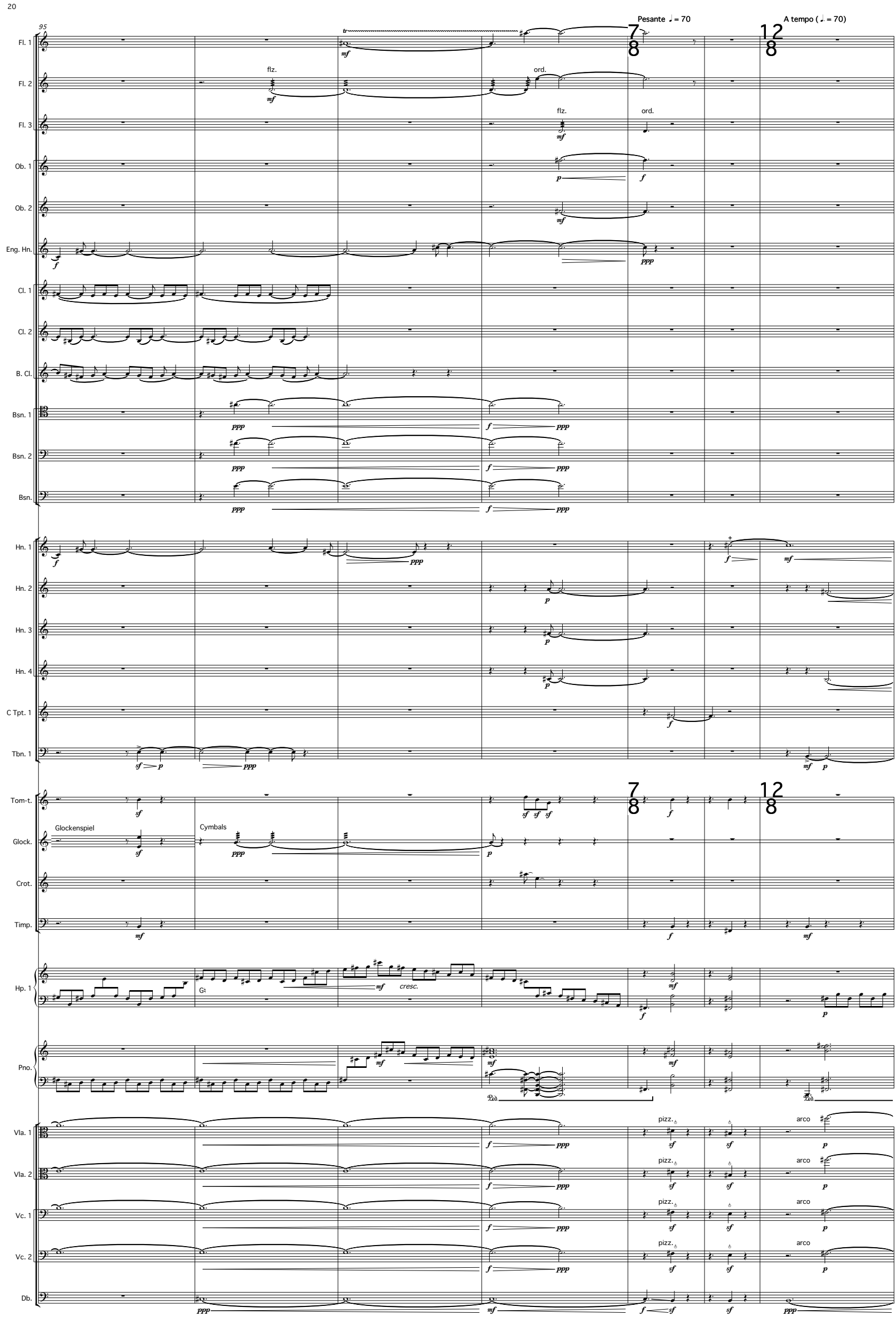




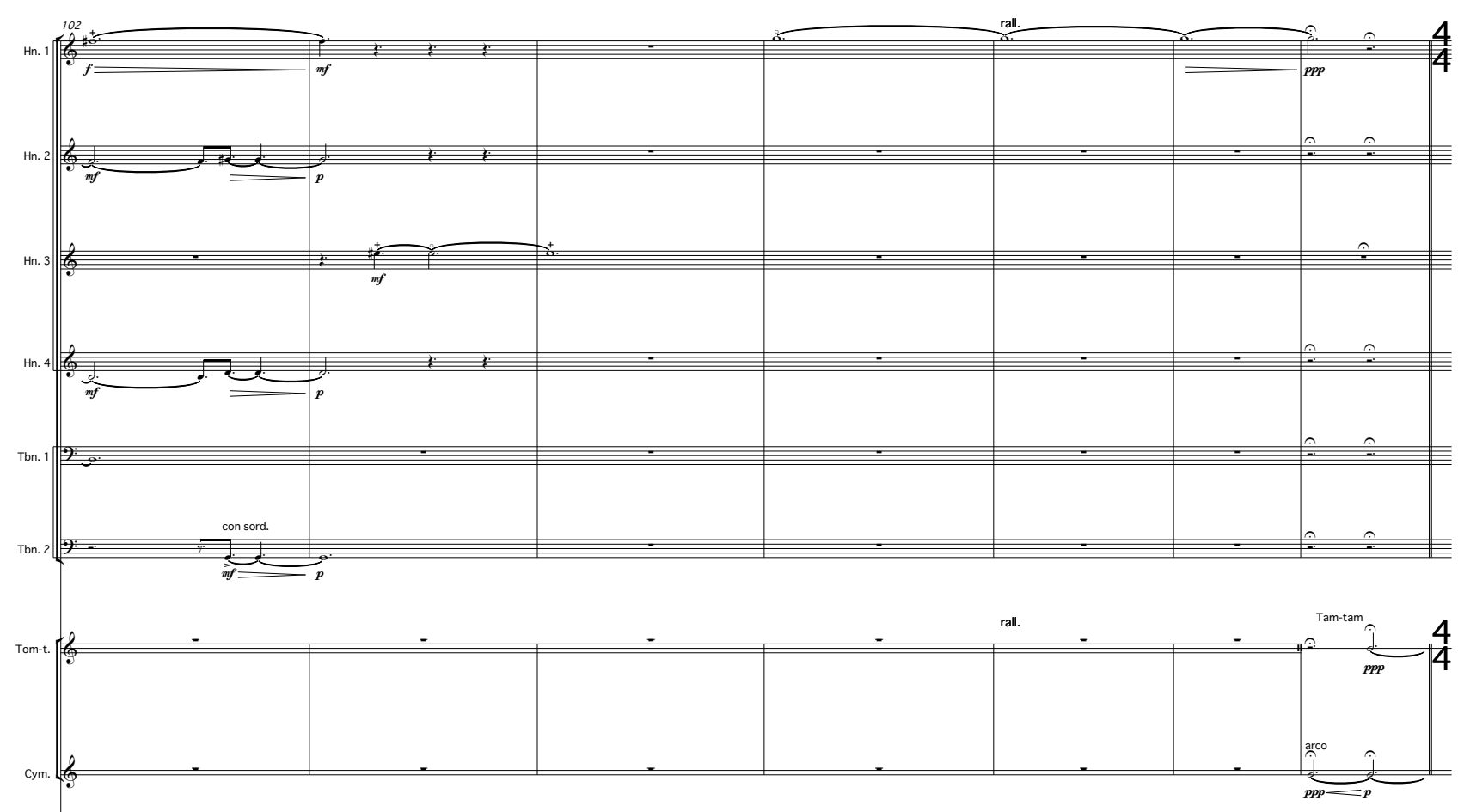

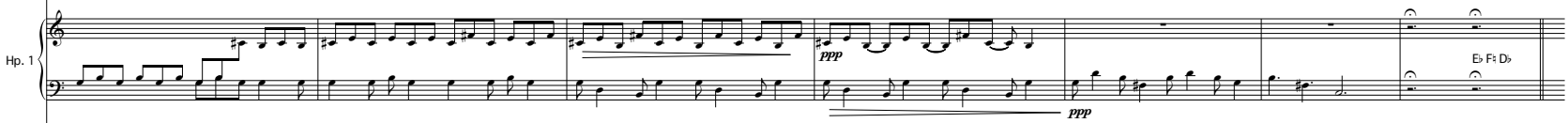
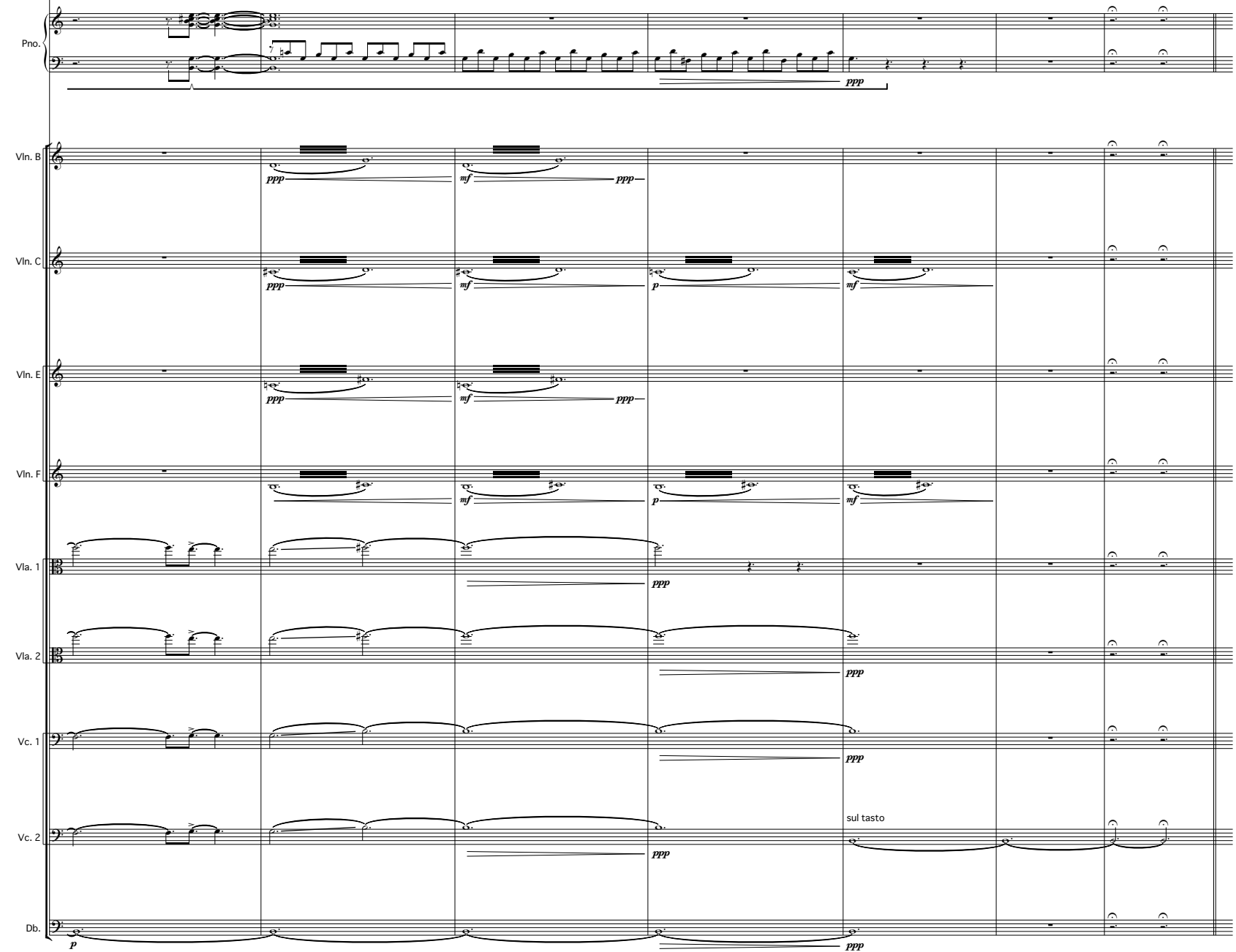
Day 3

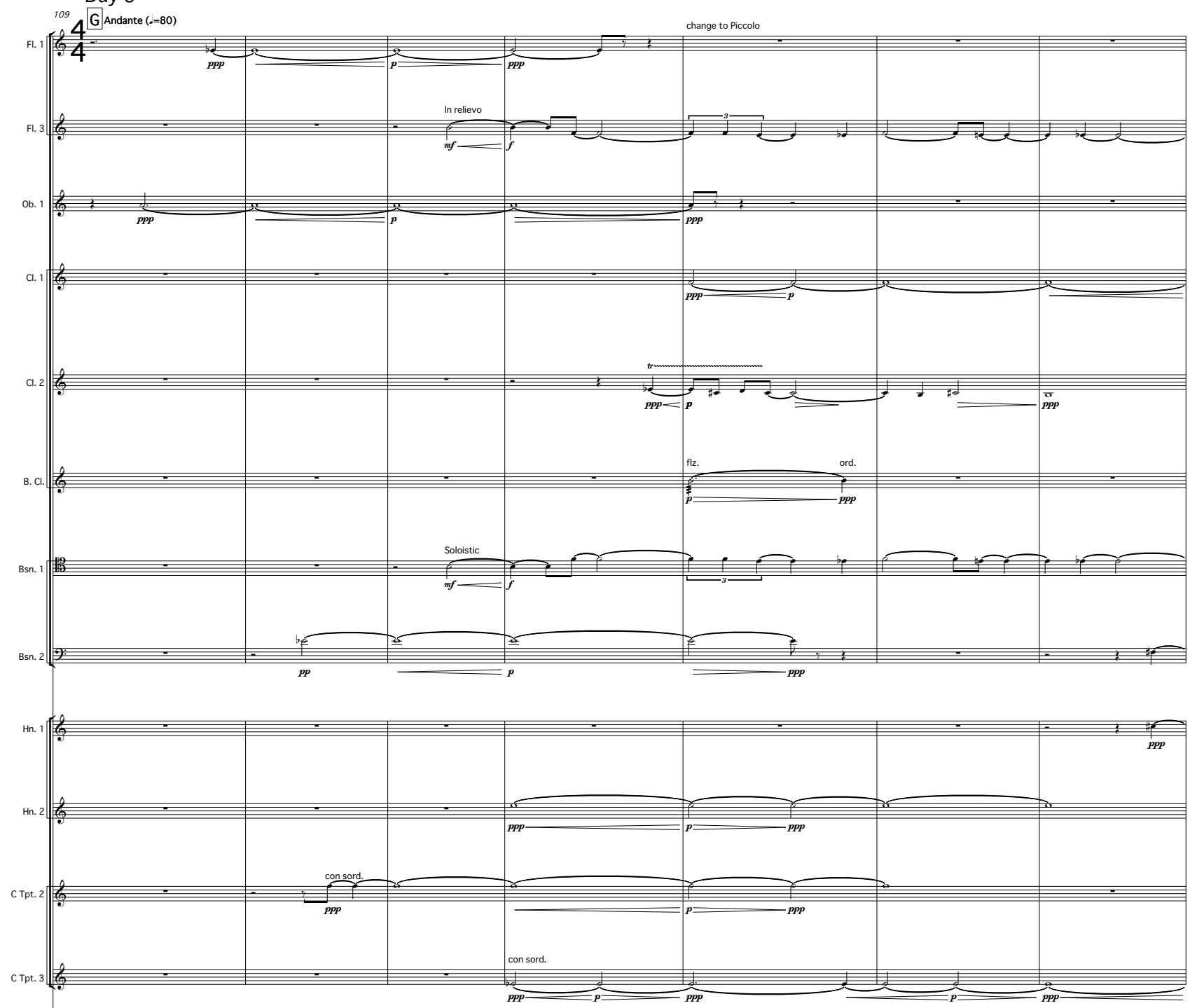

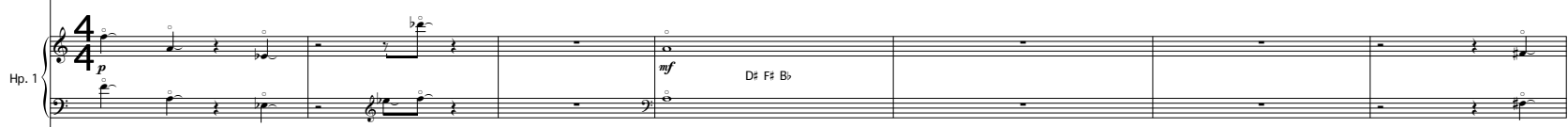
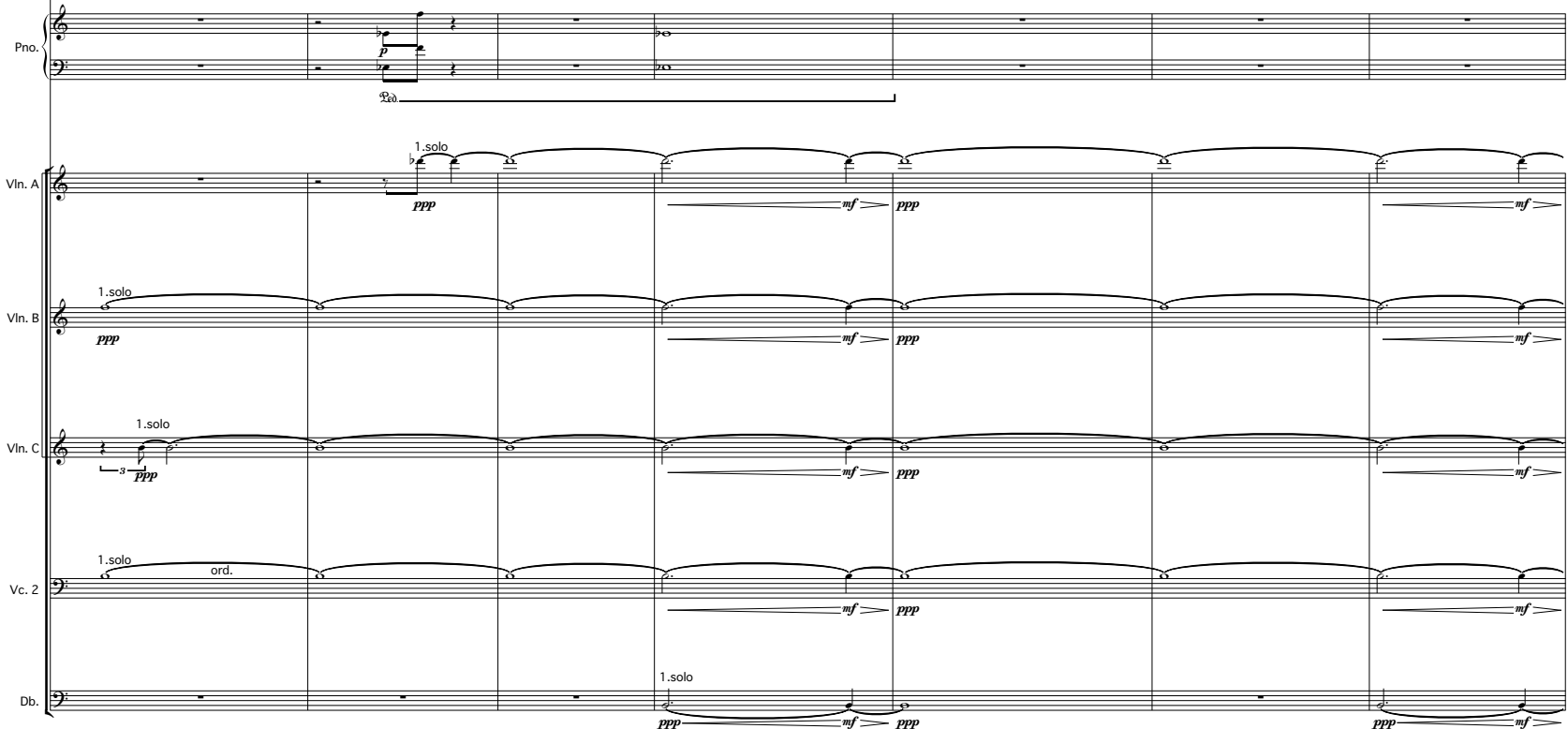


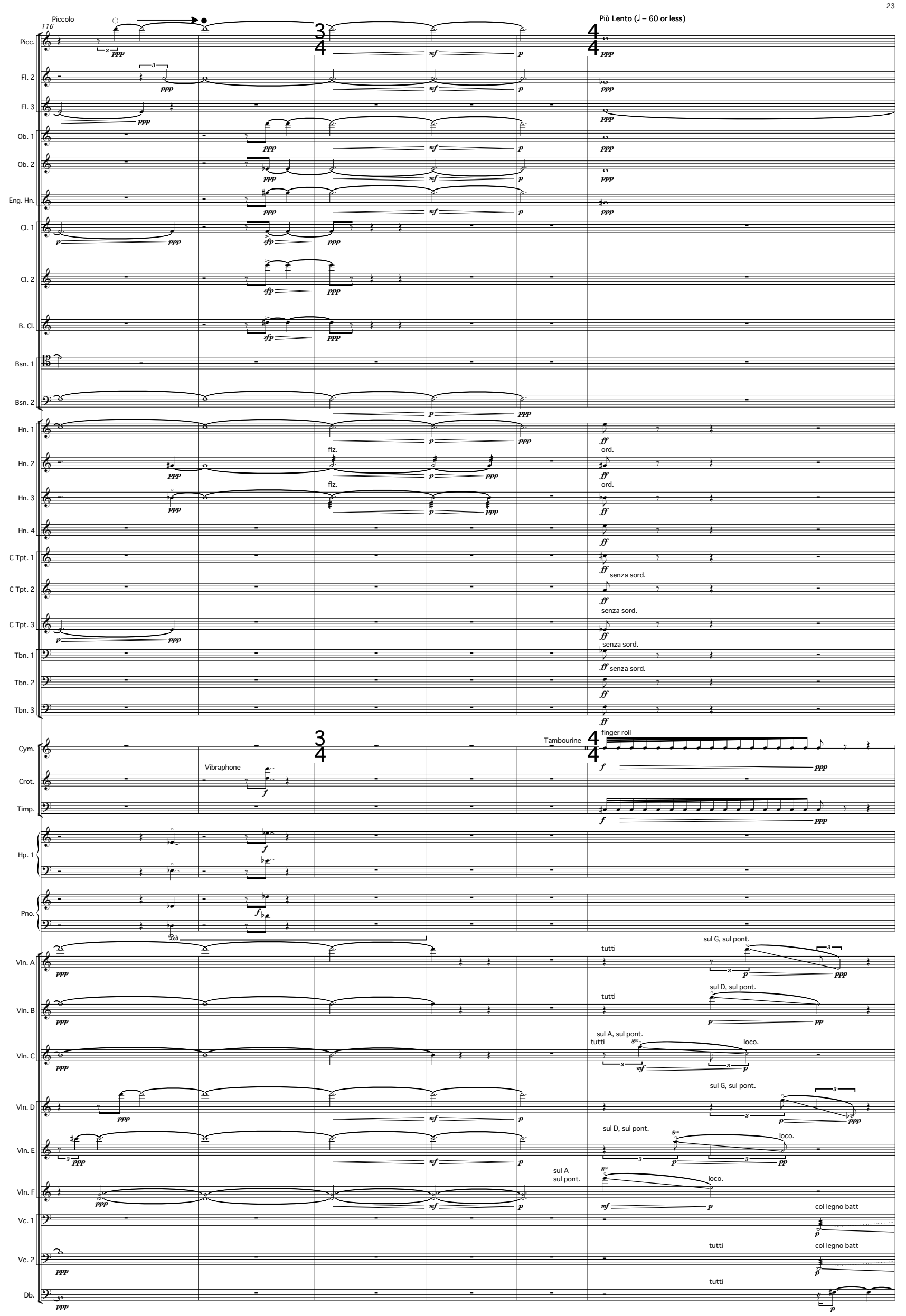




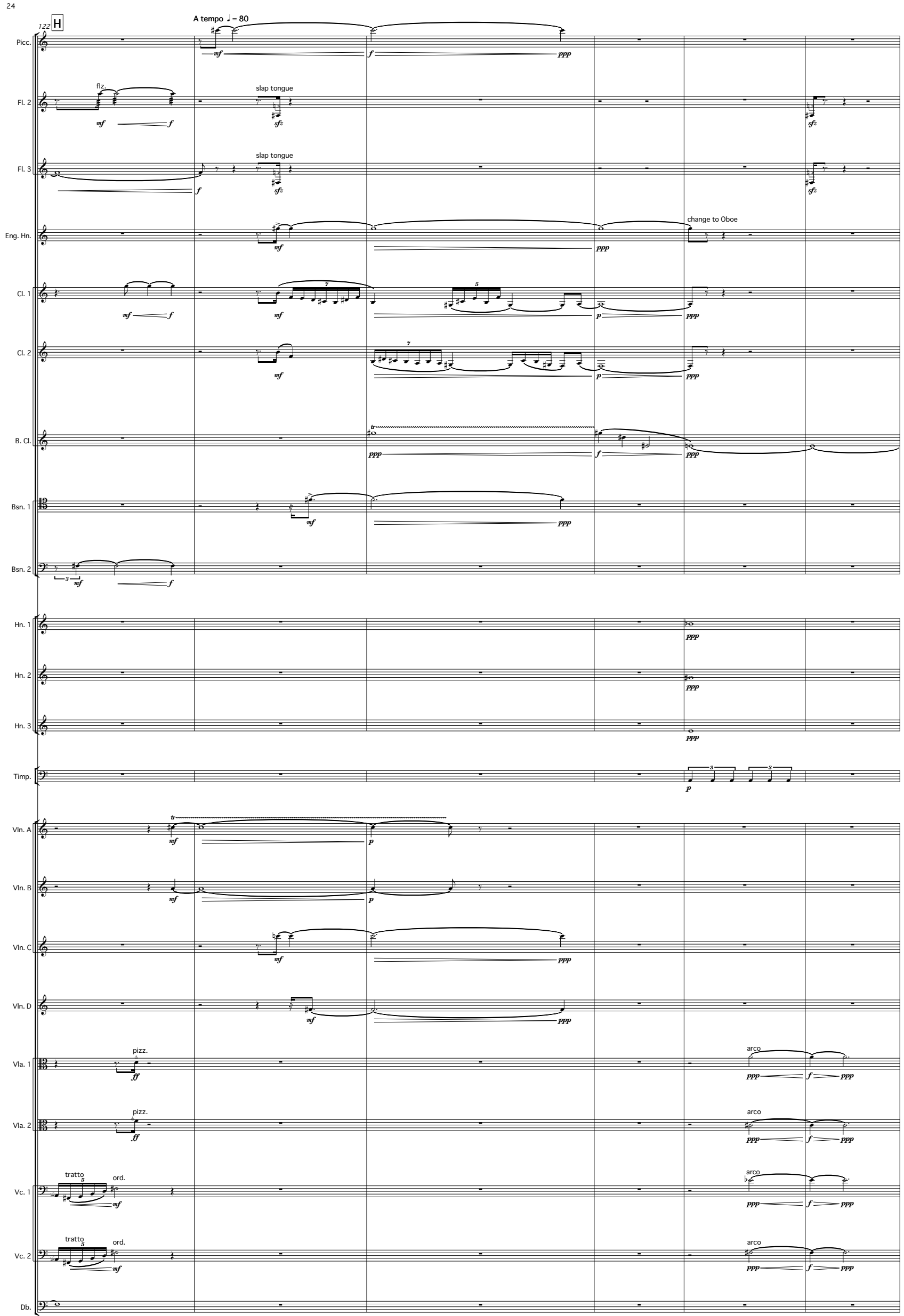



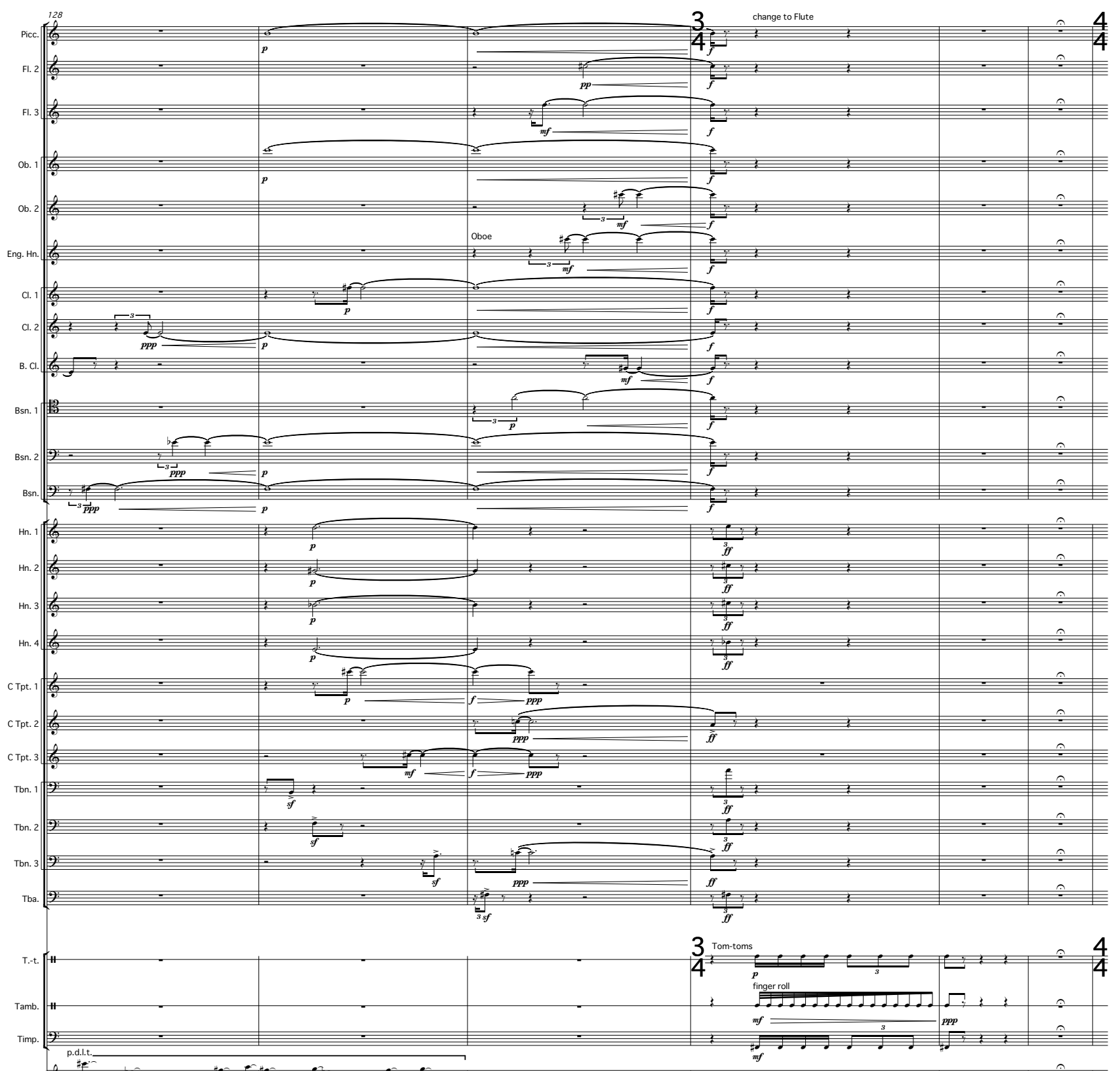

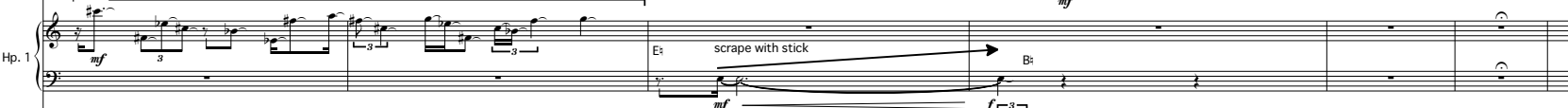
P

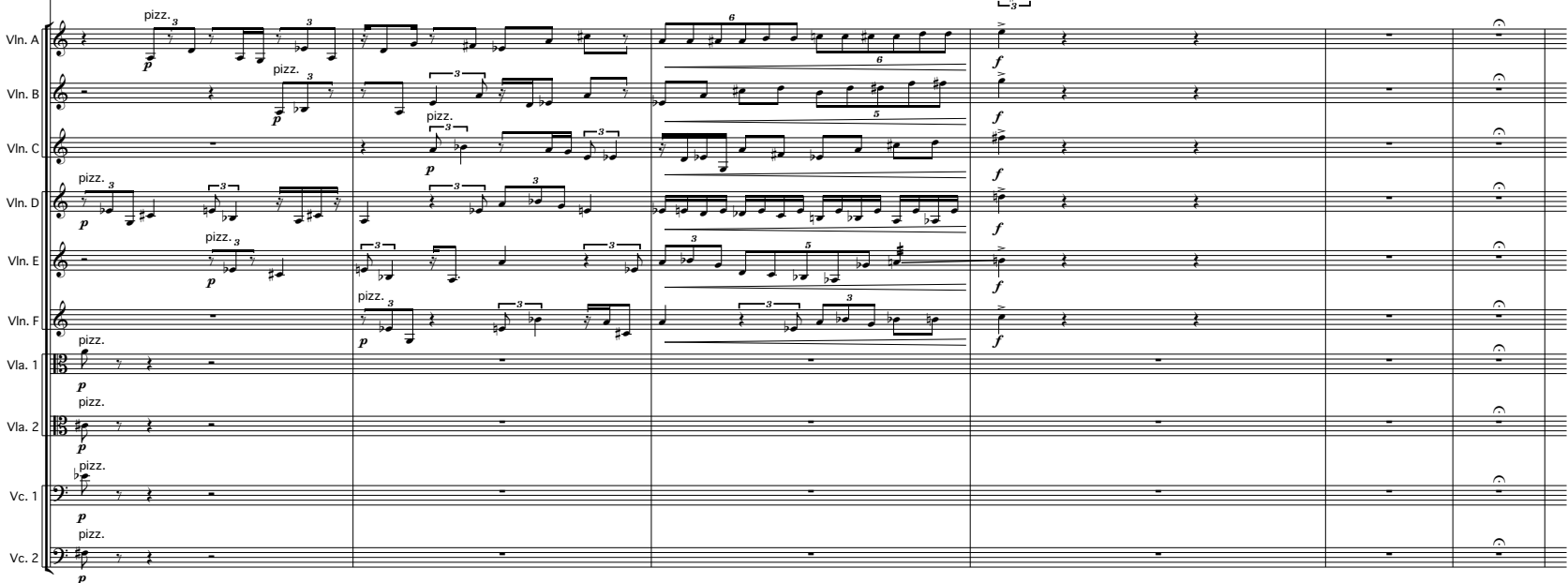




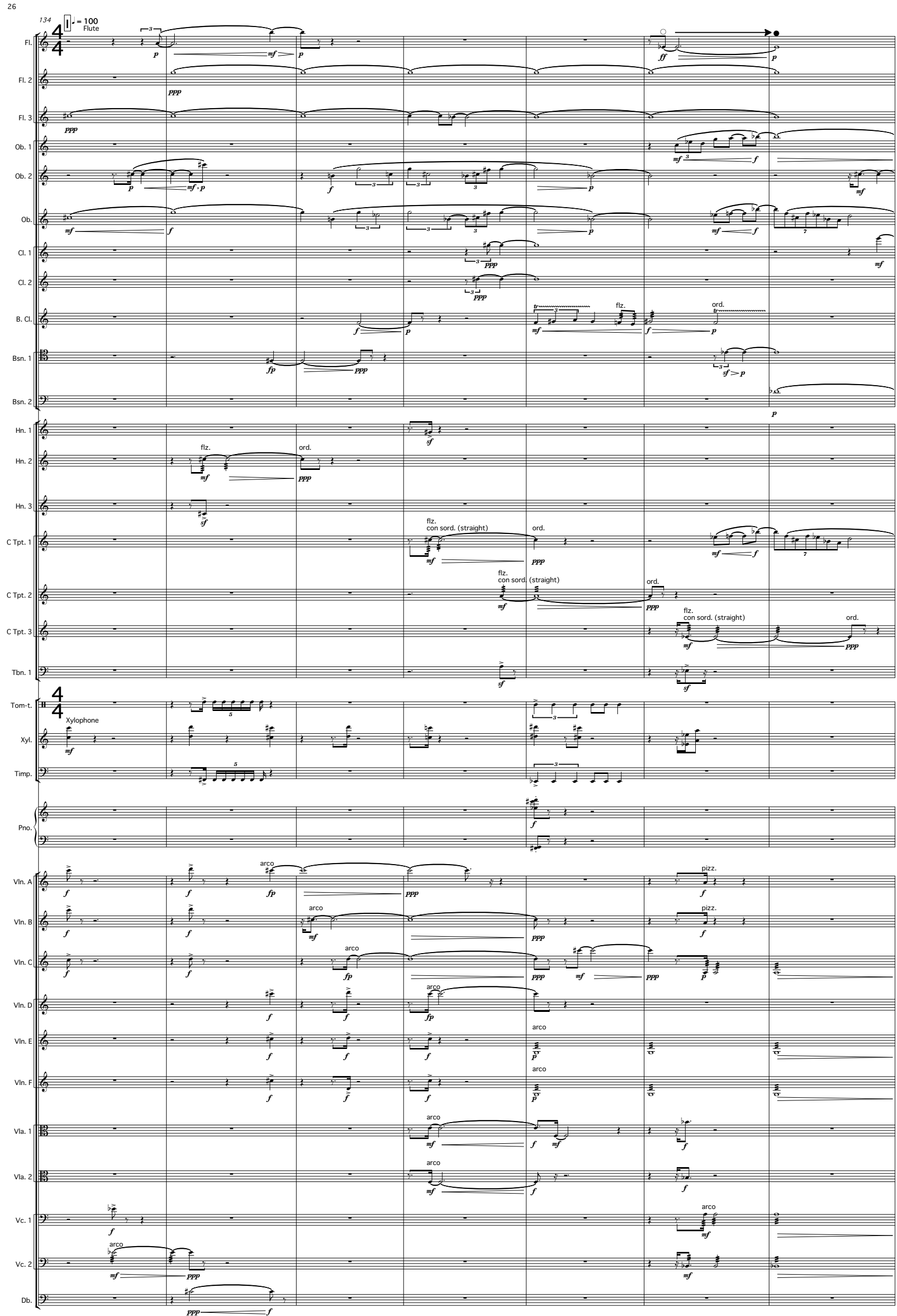




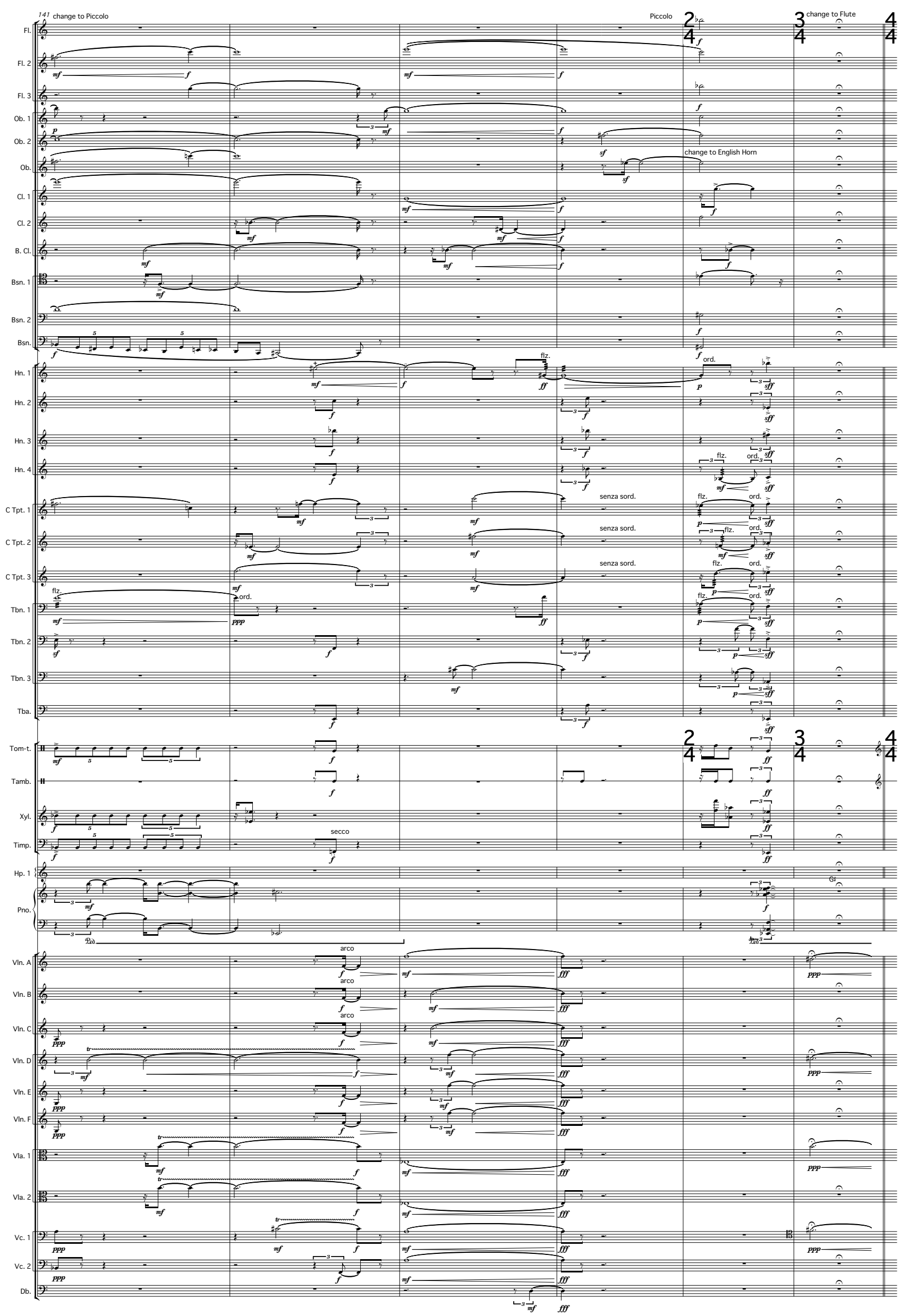



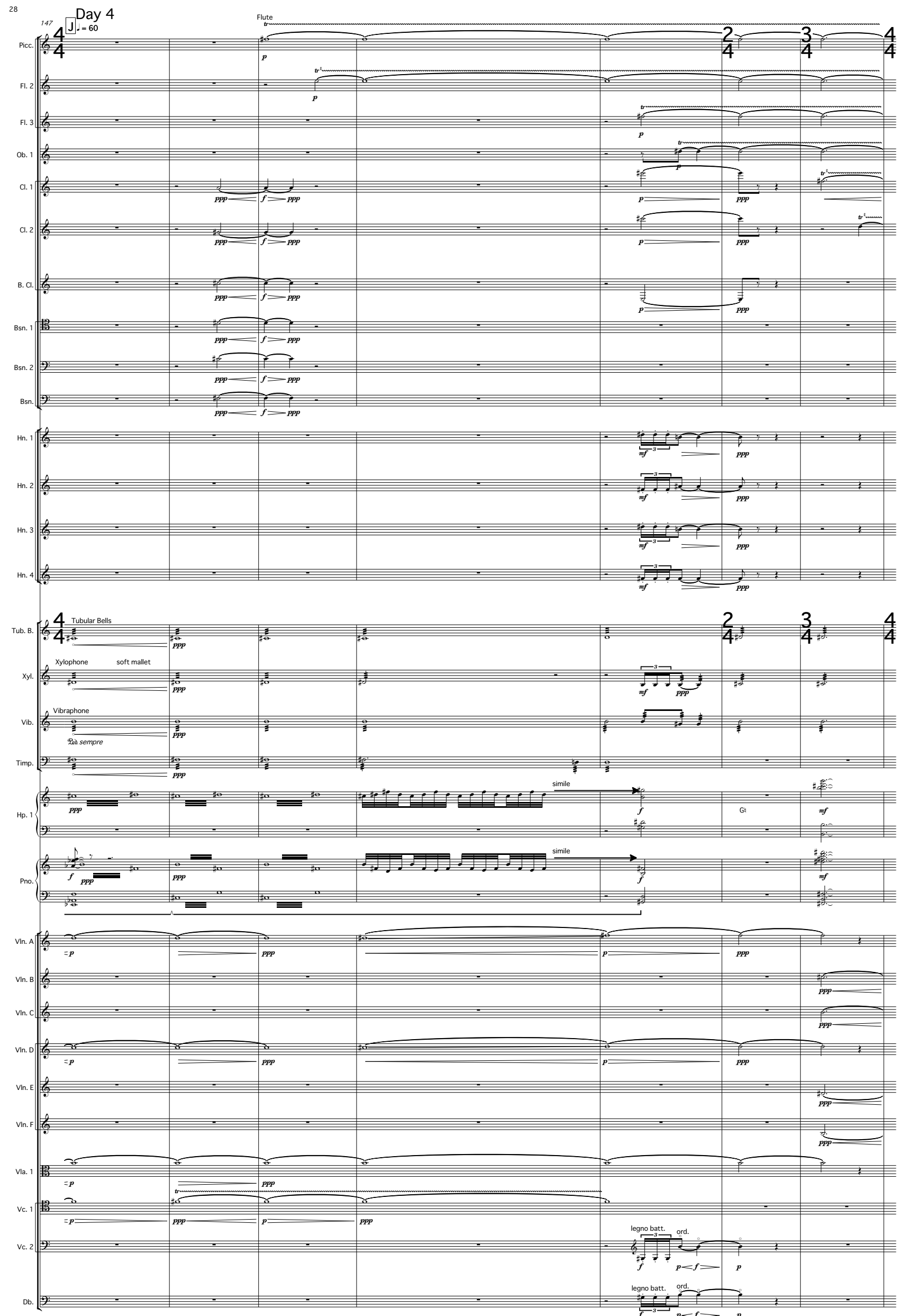

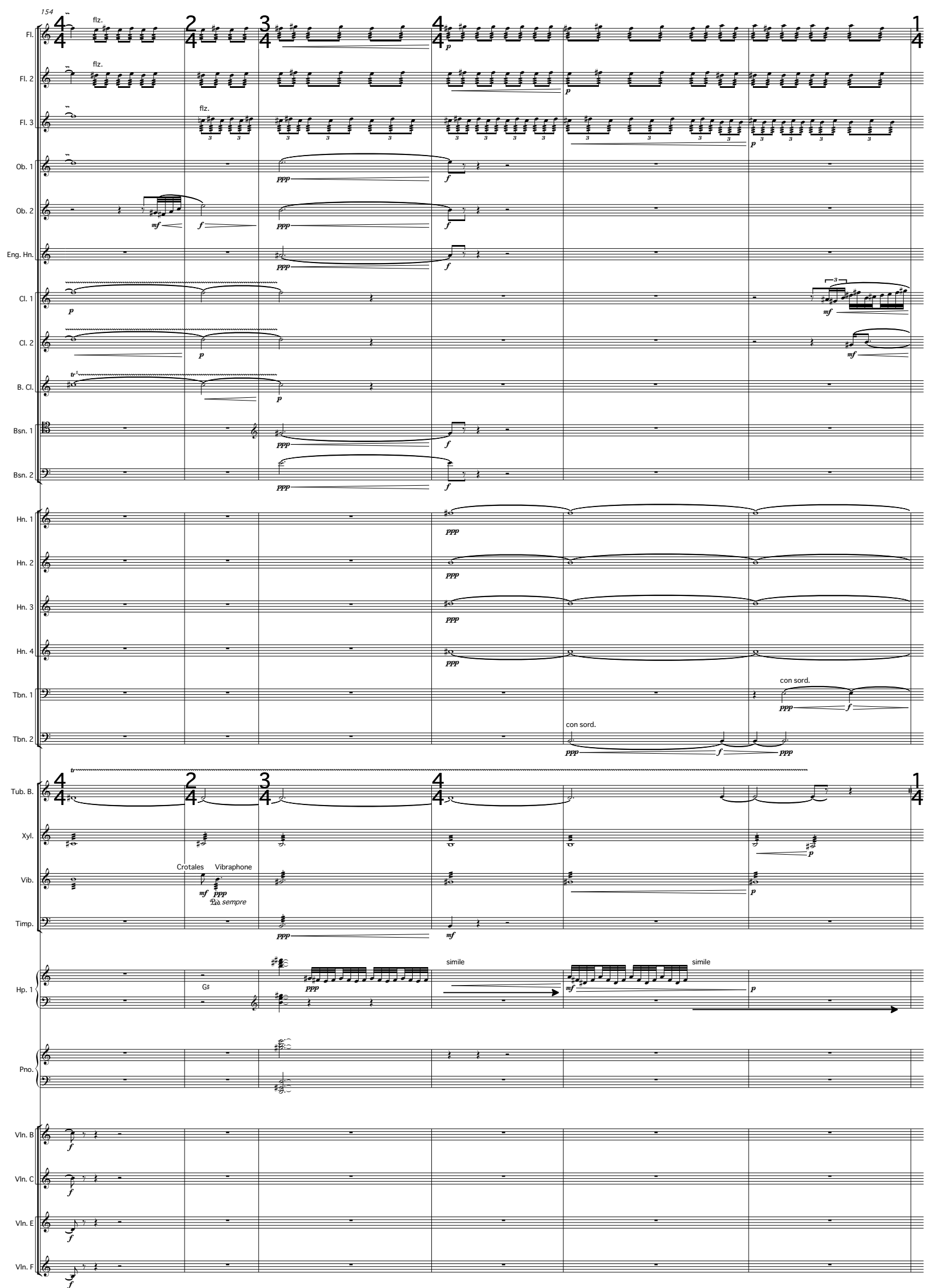
30
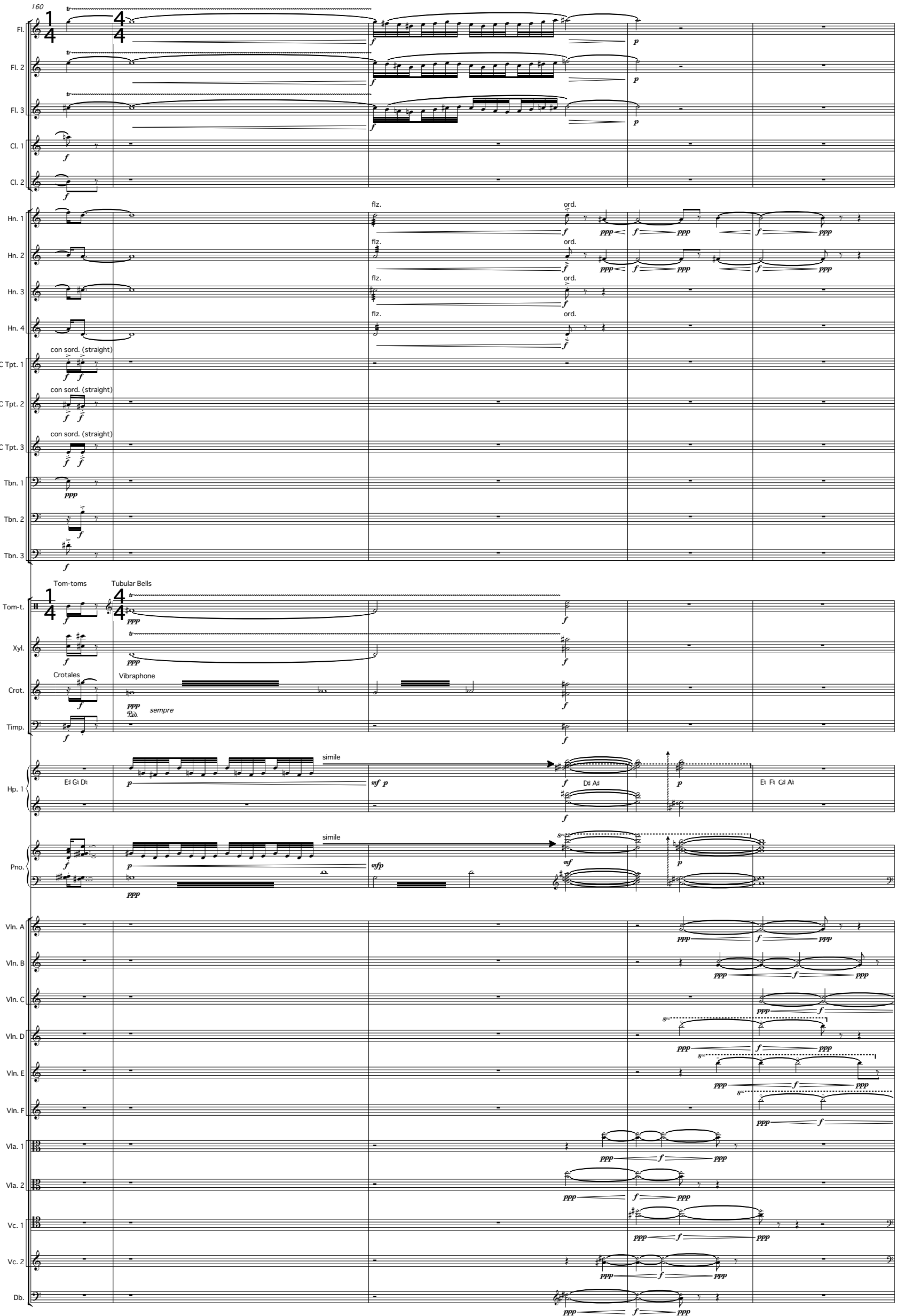


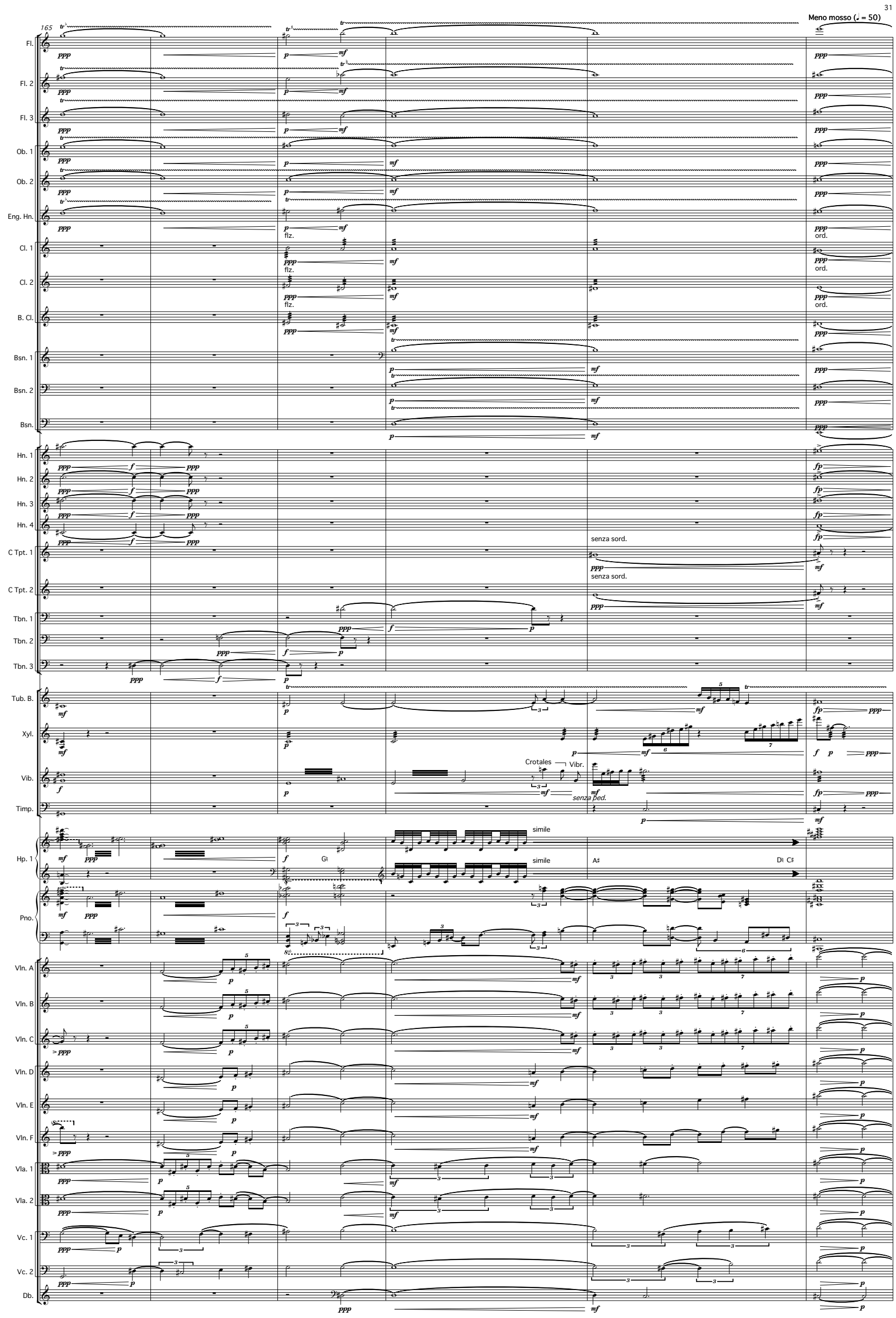




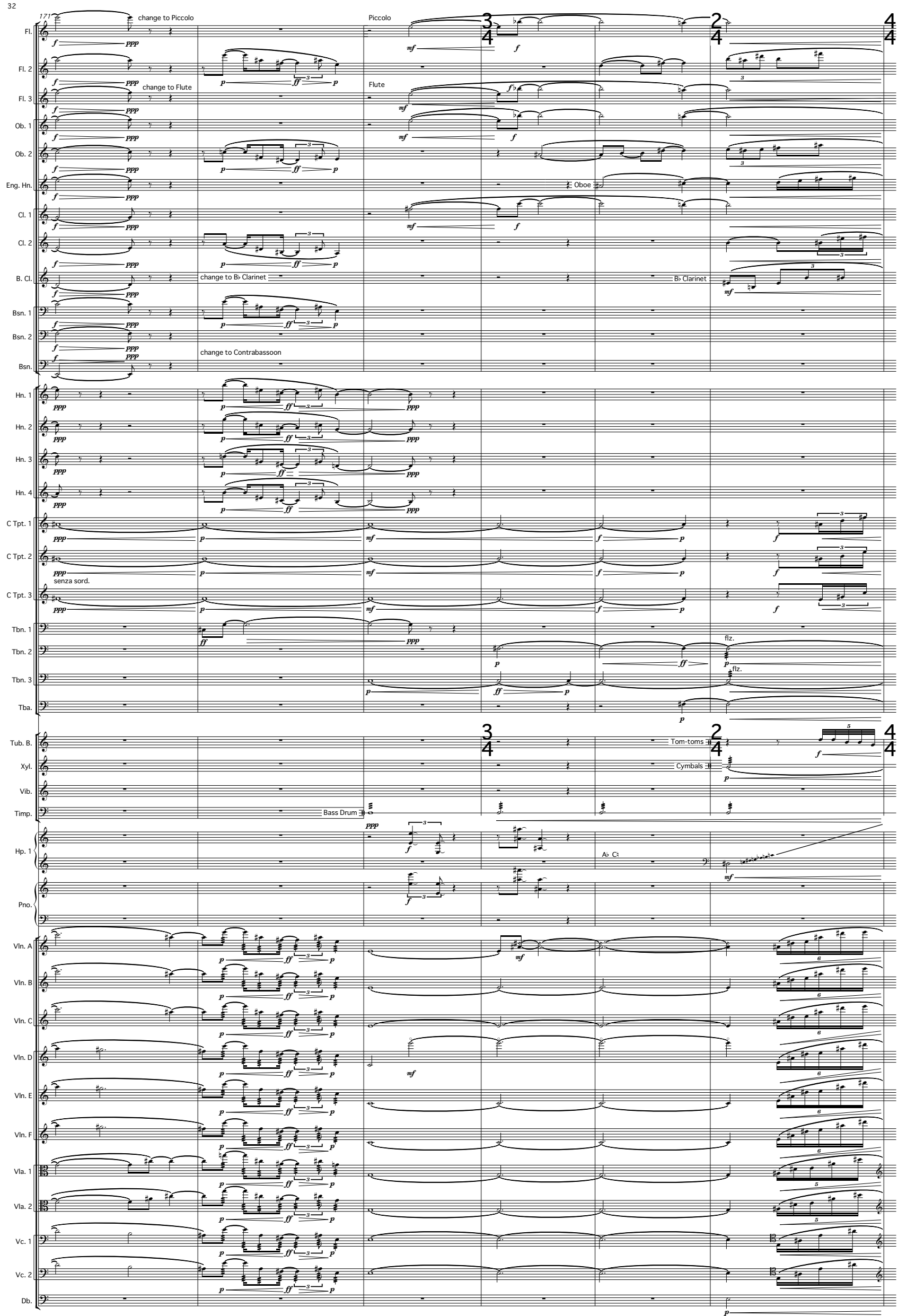



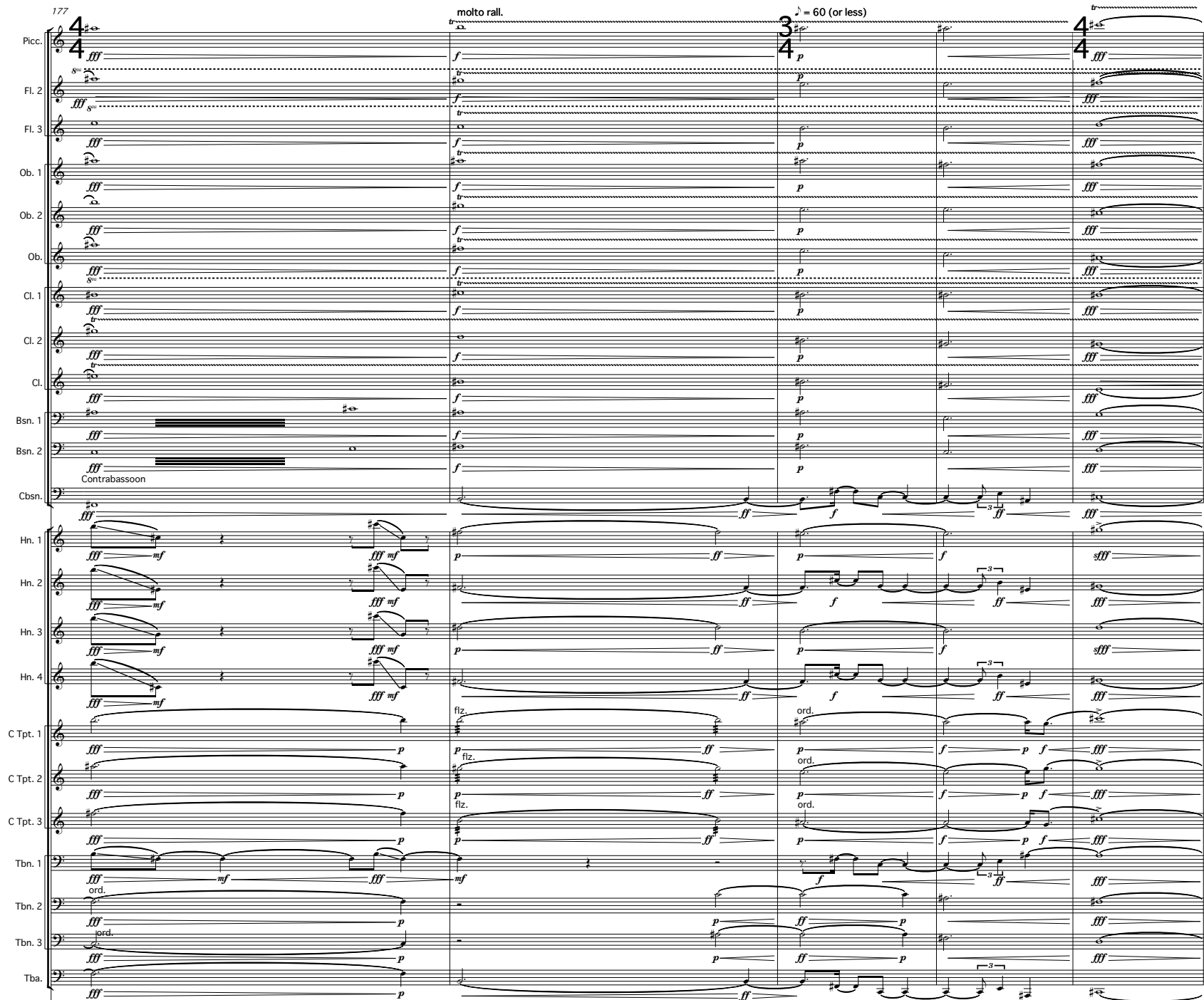
34
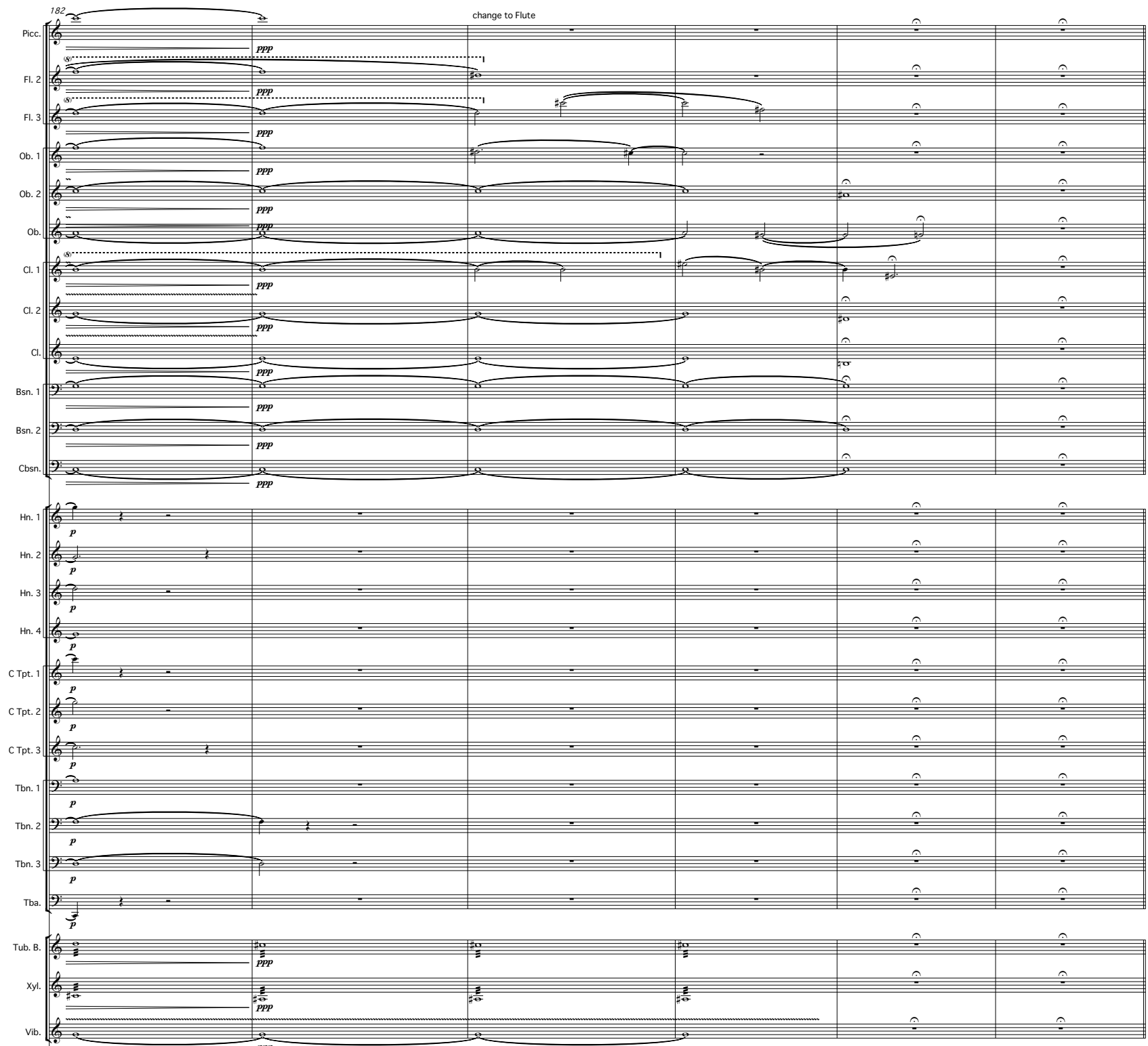

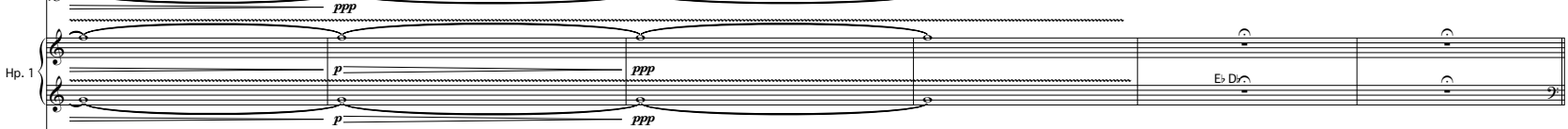

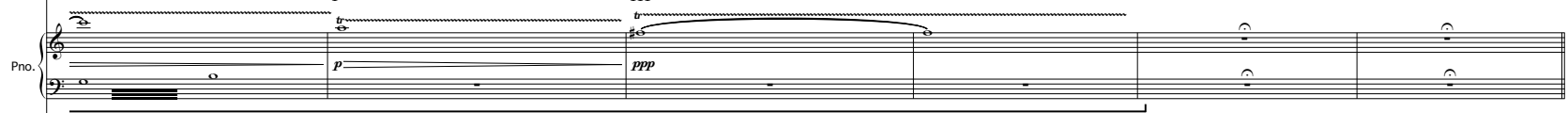

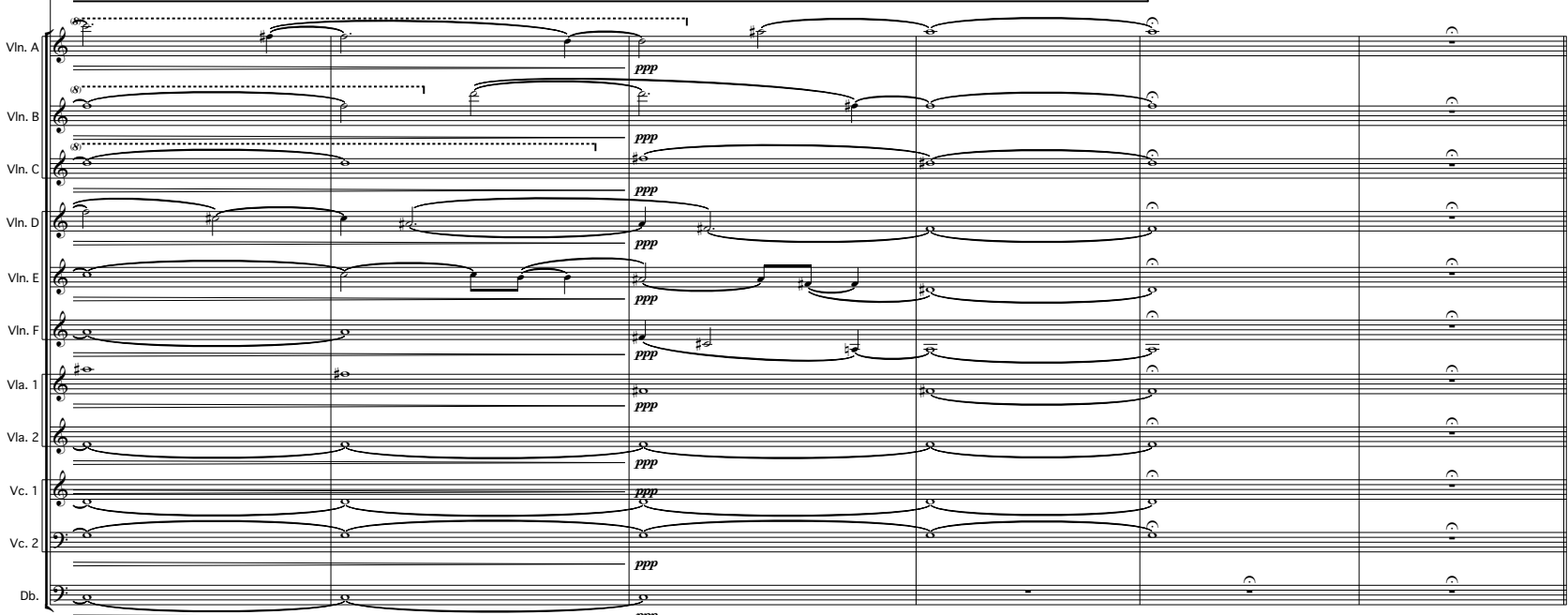



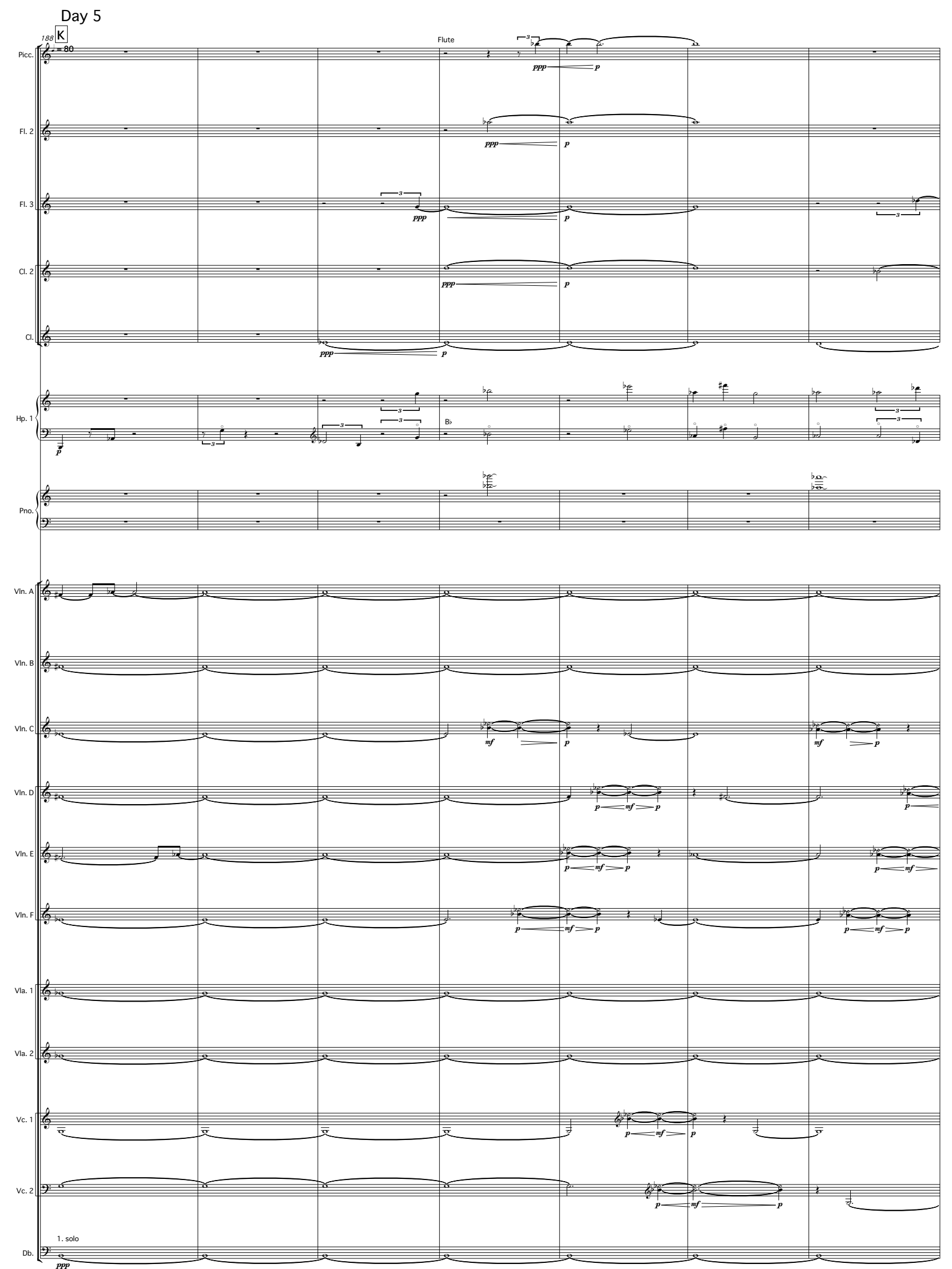


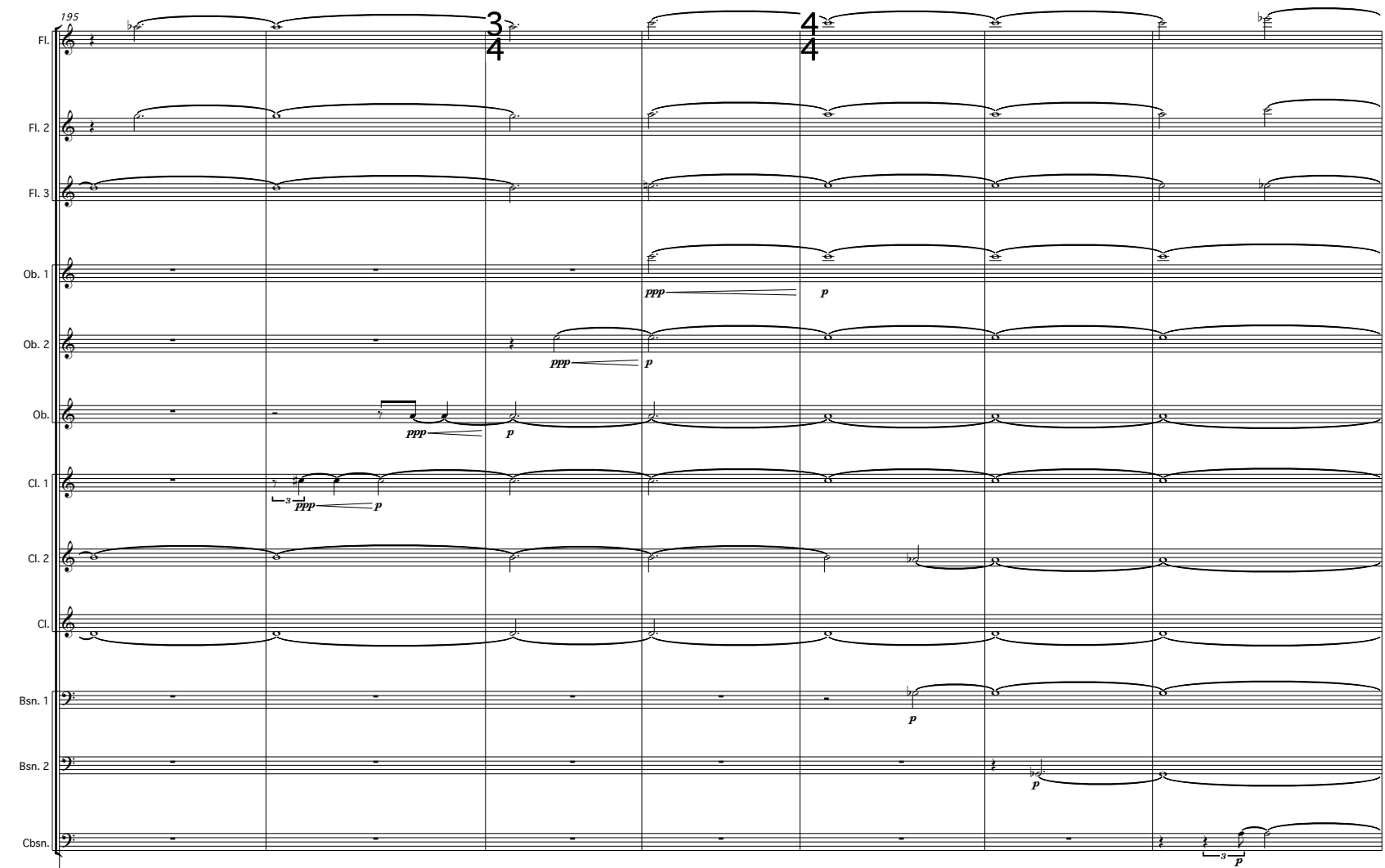

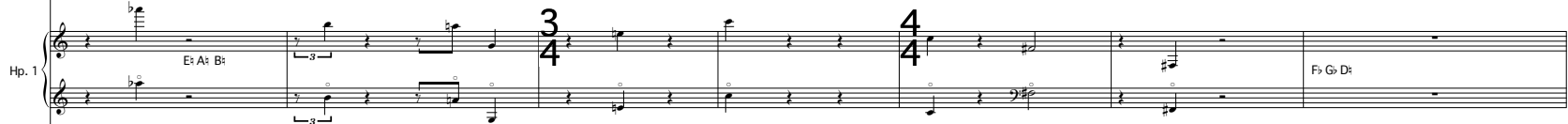
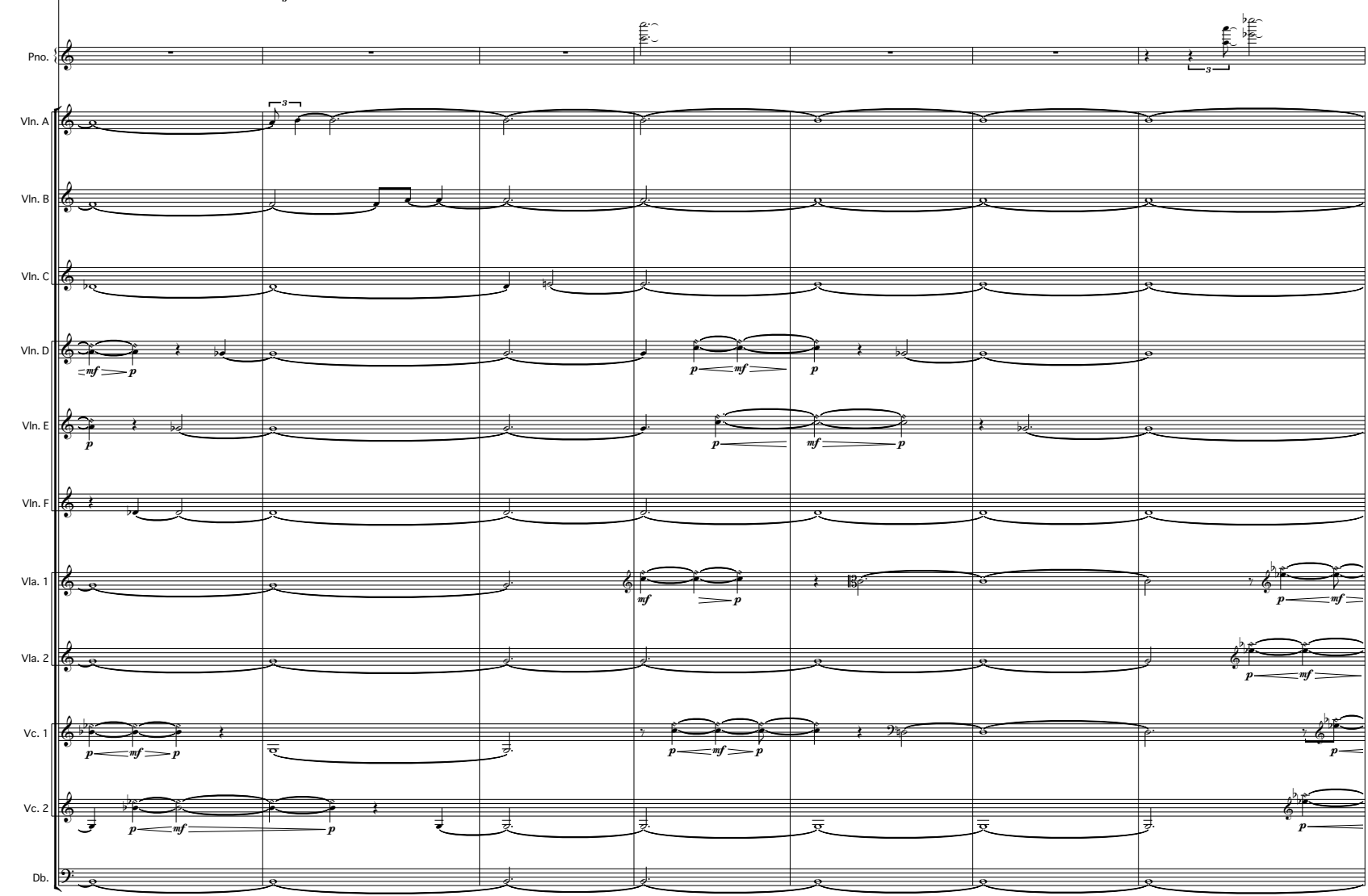

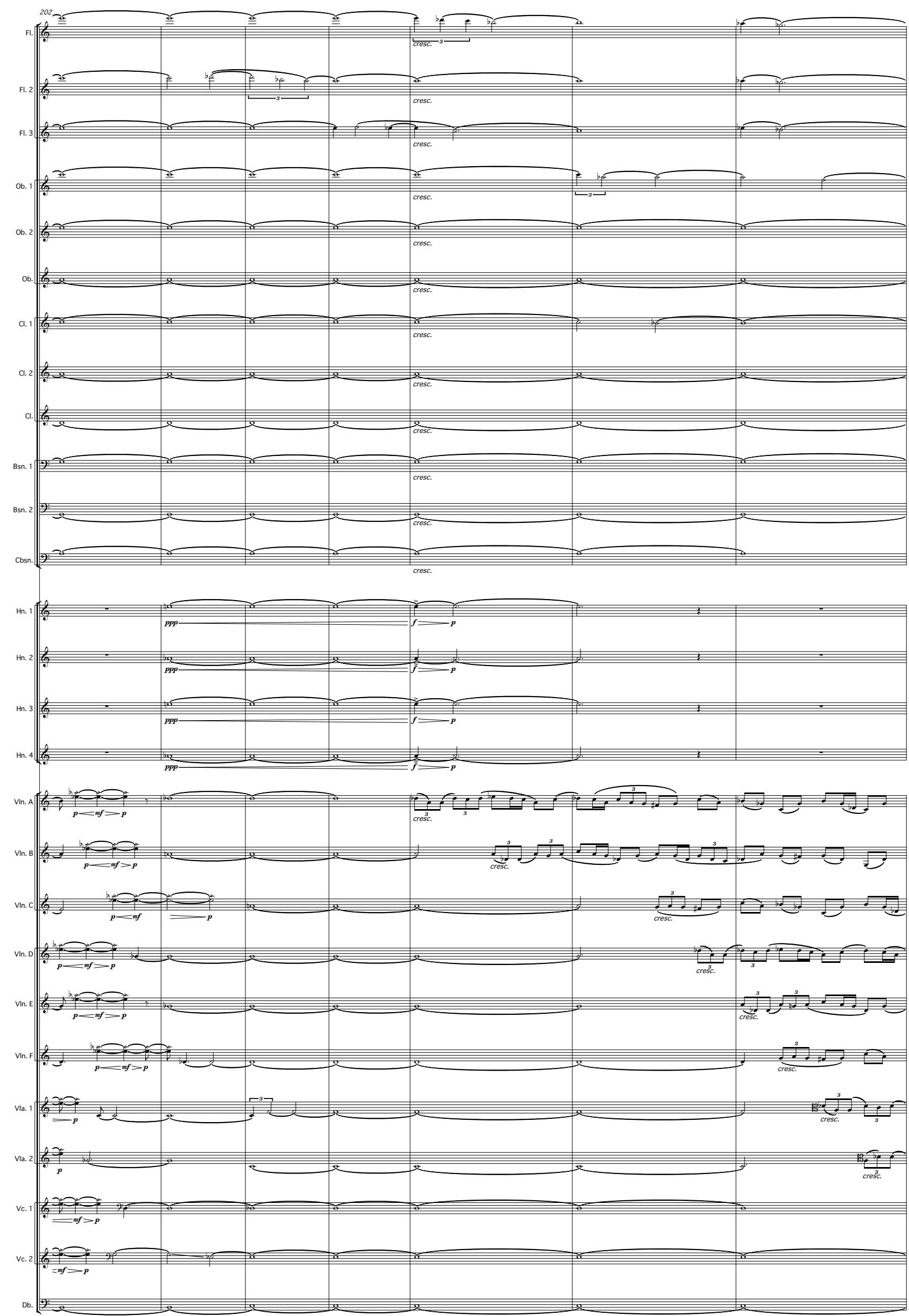


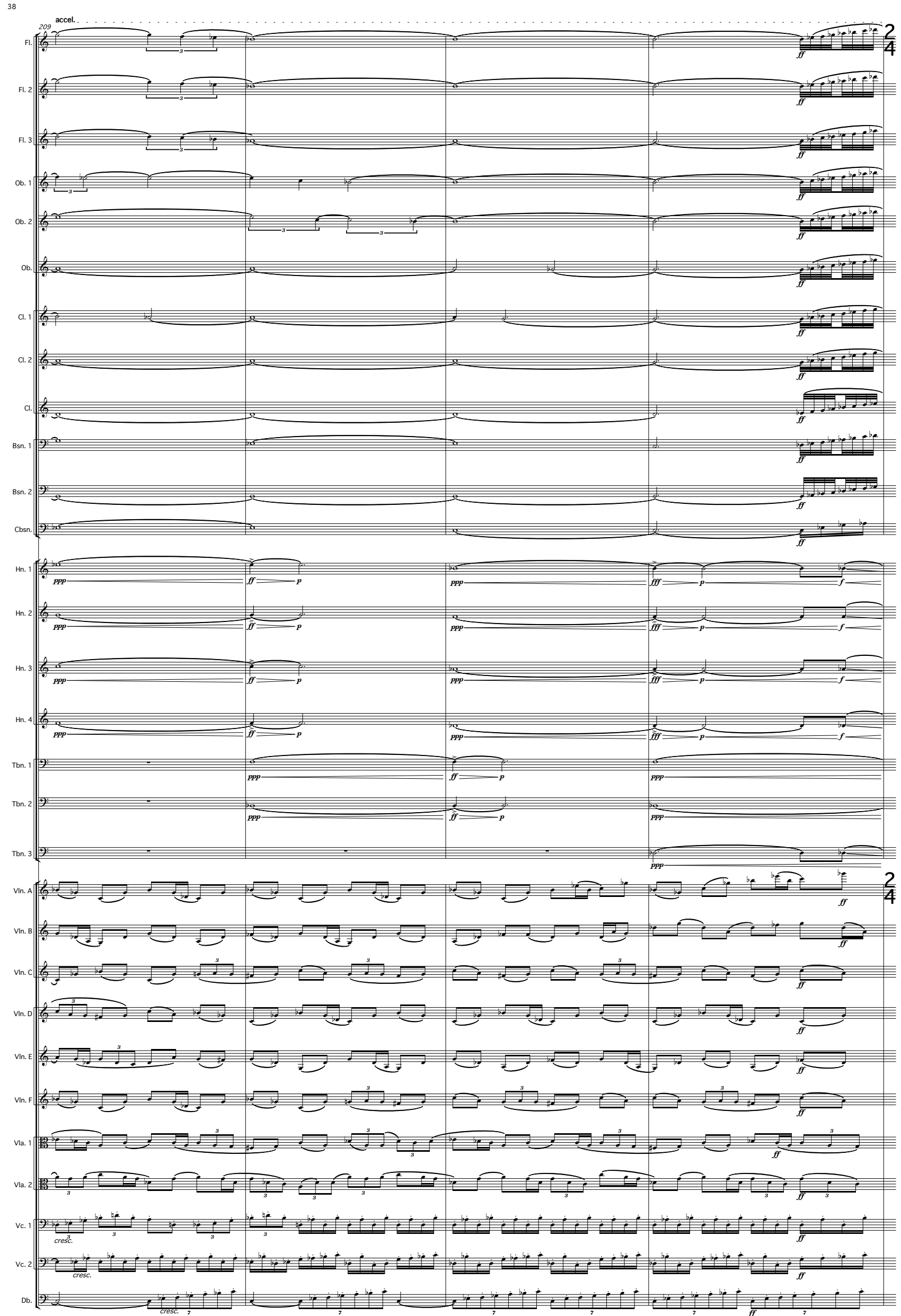




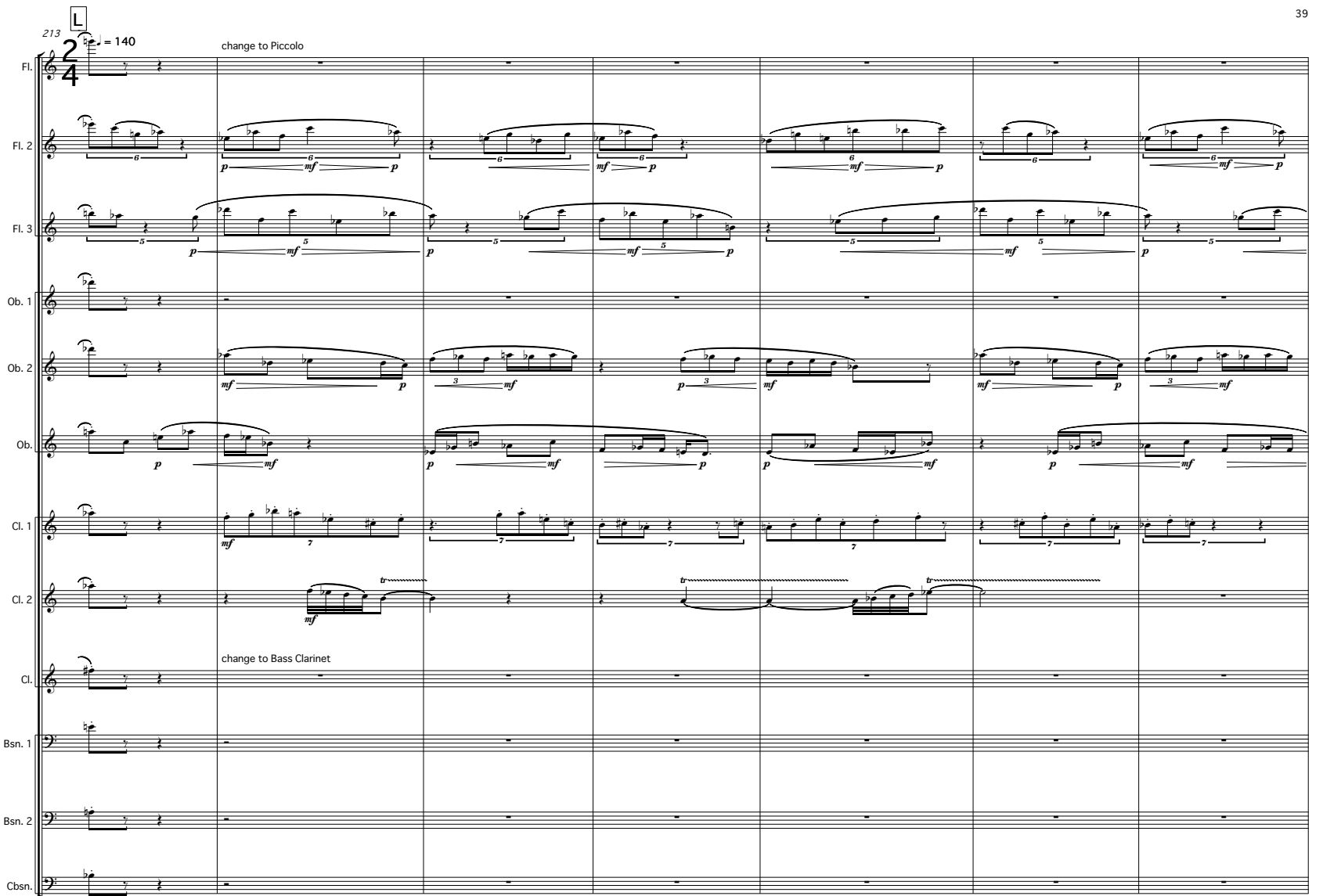

(1)

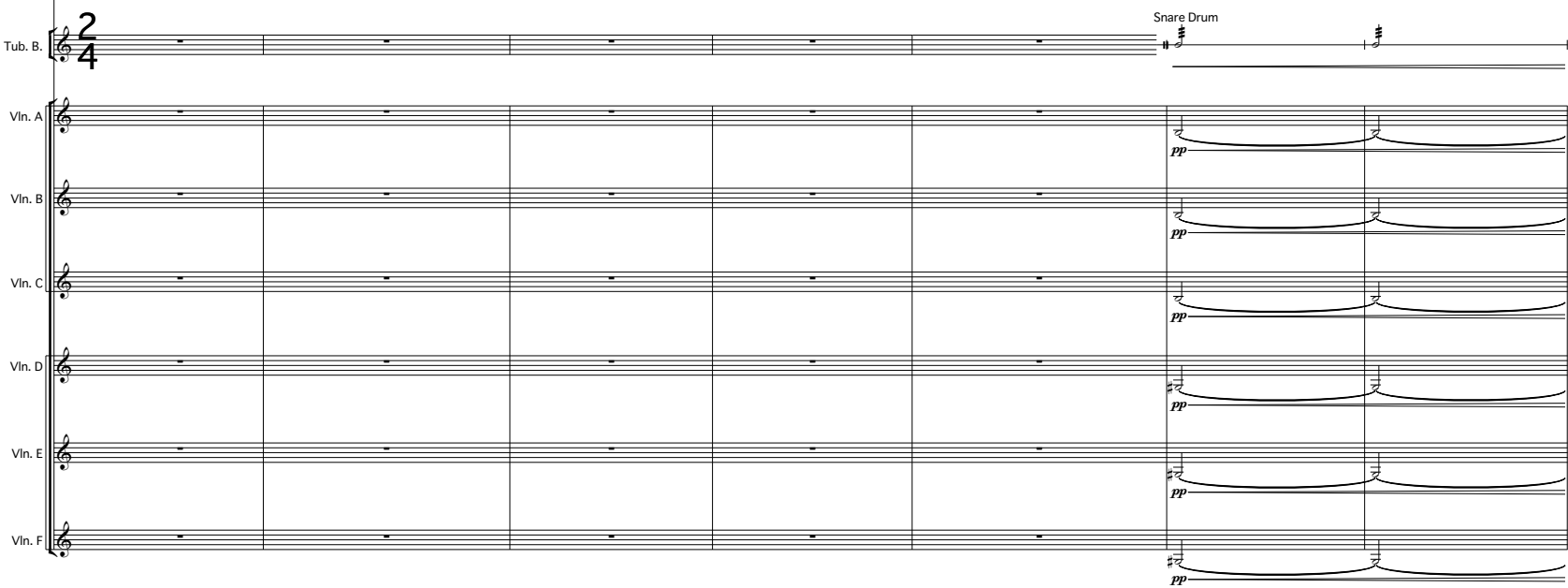




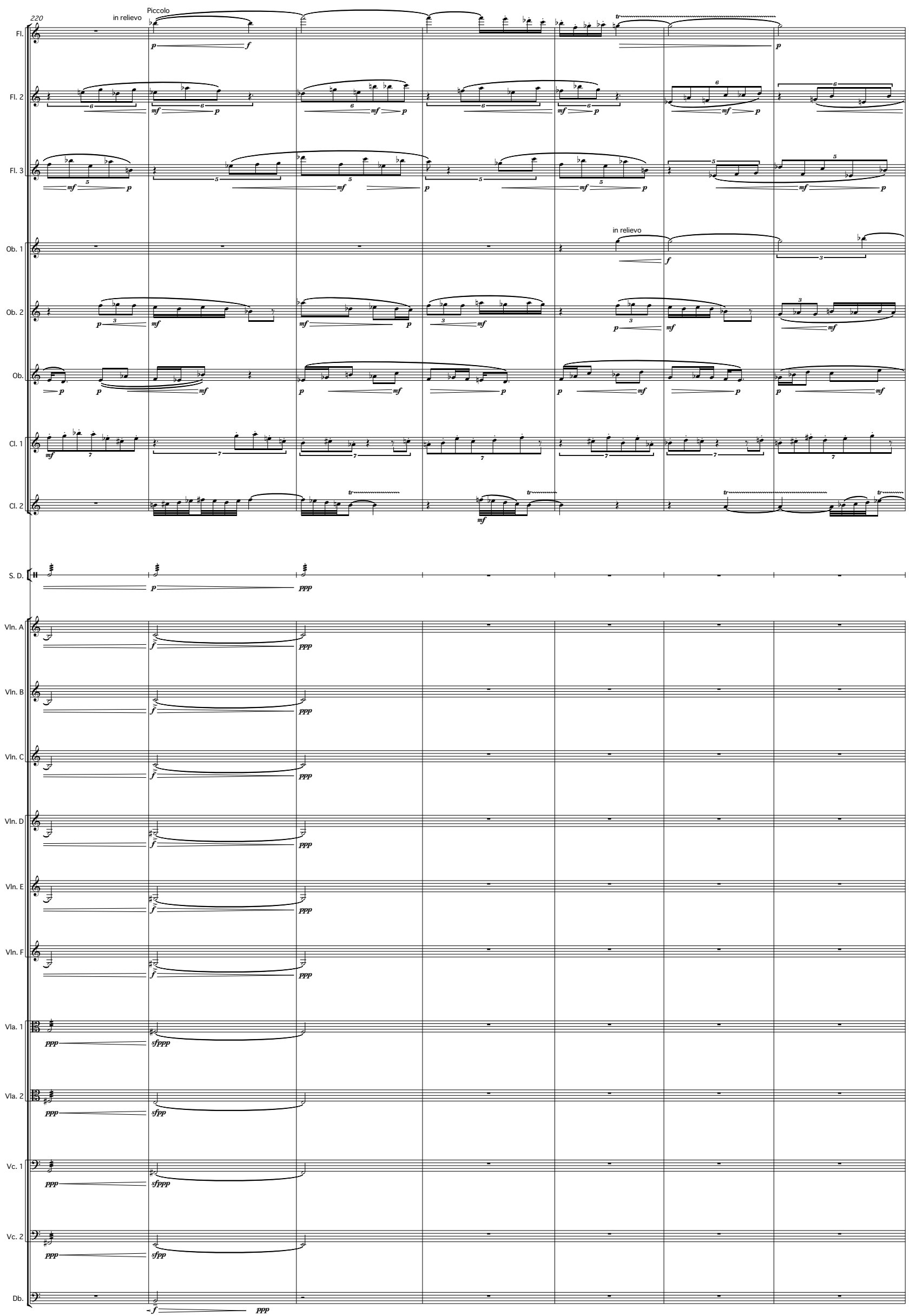




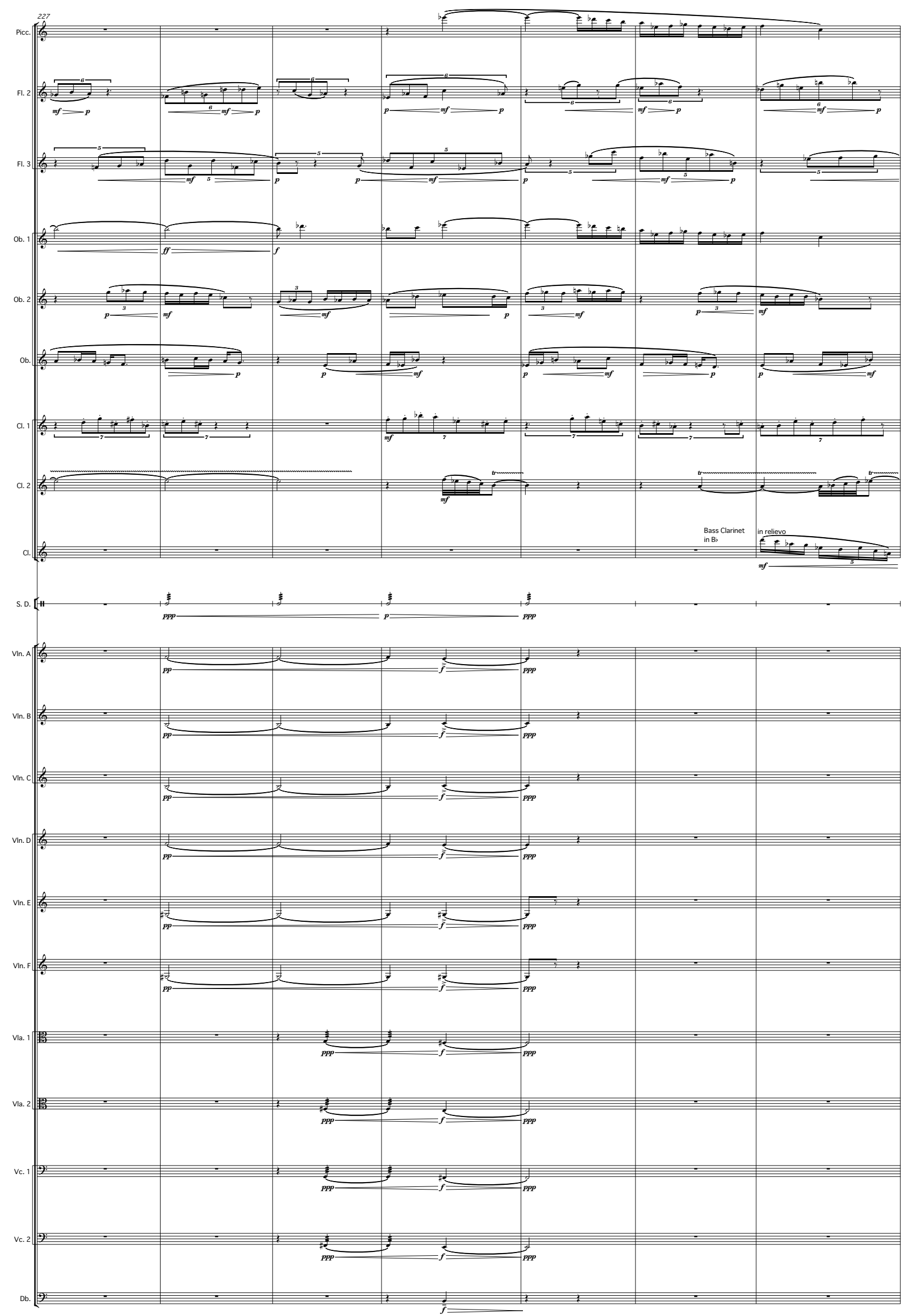




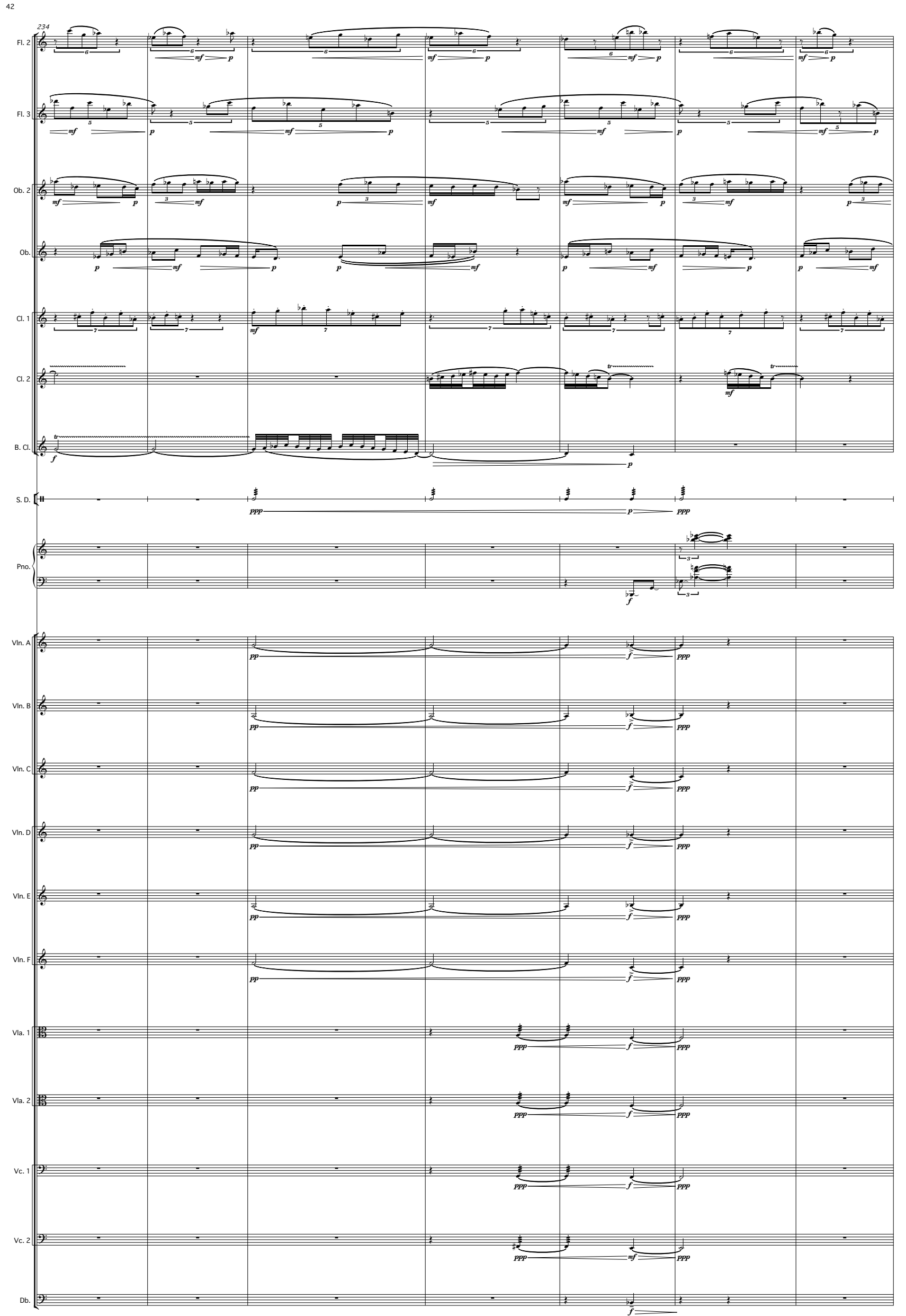



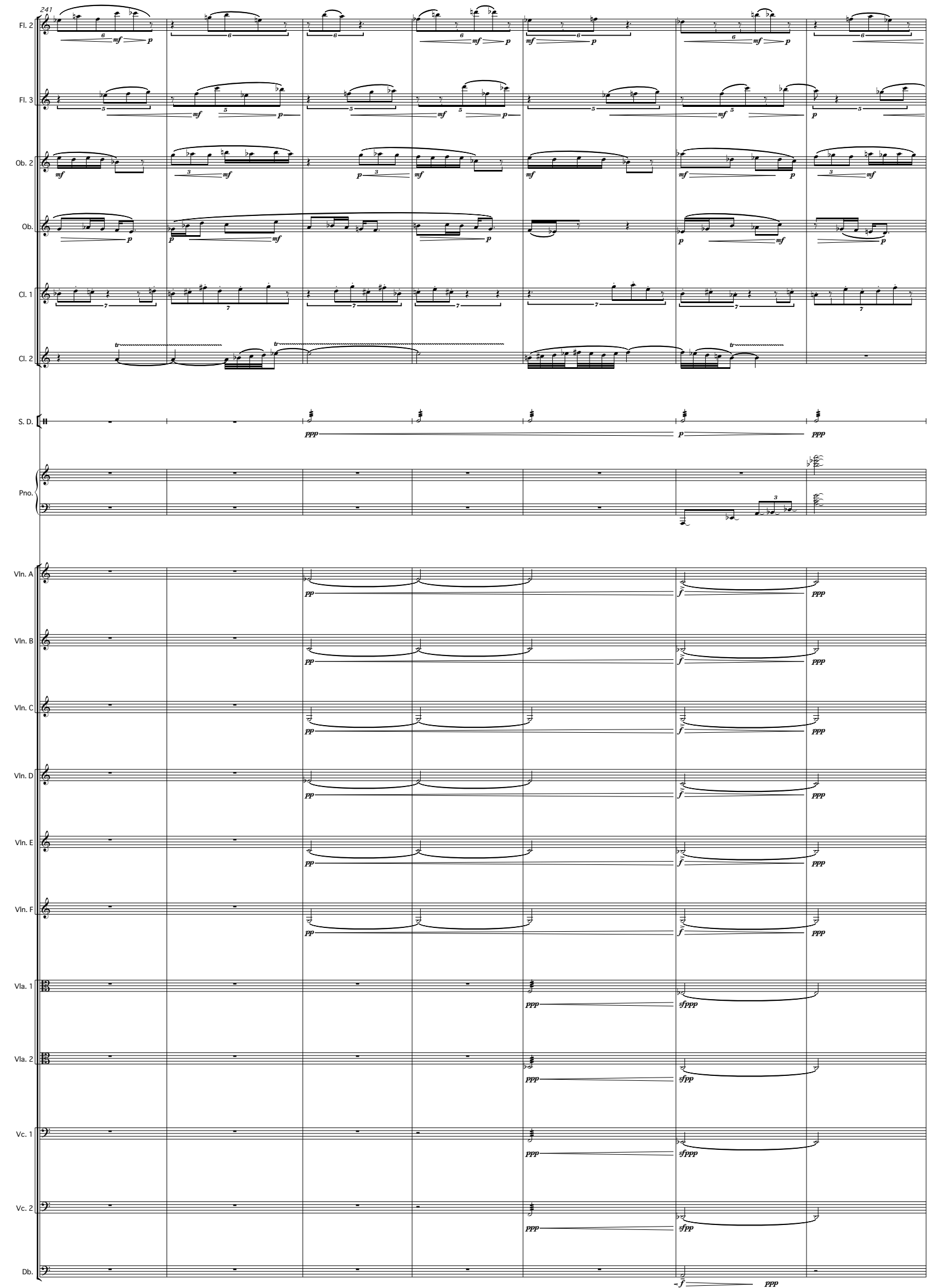

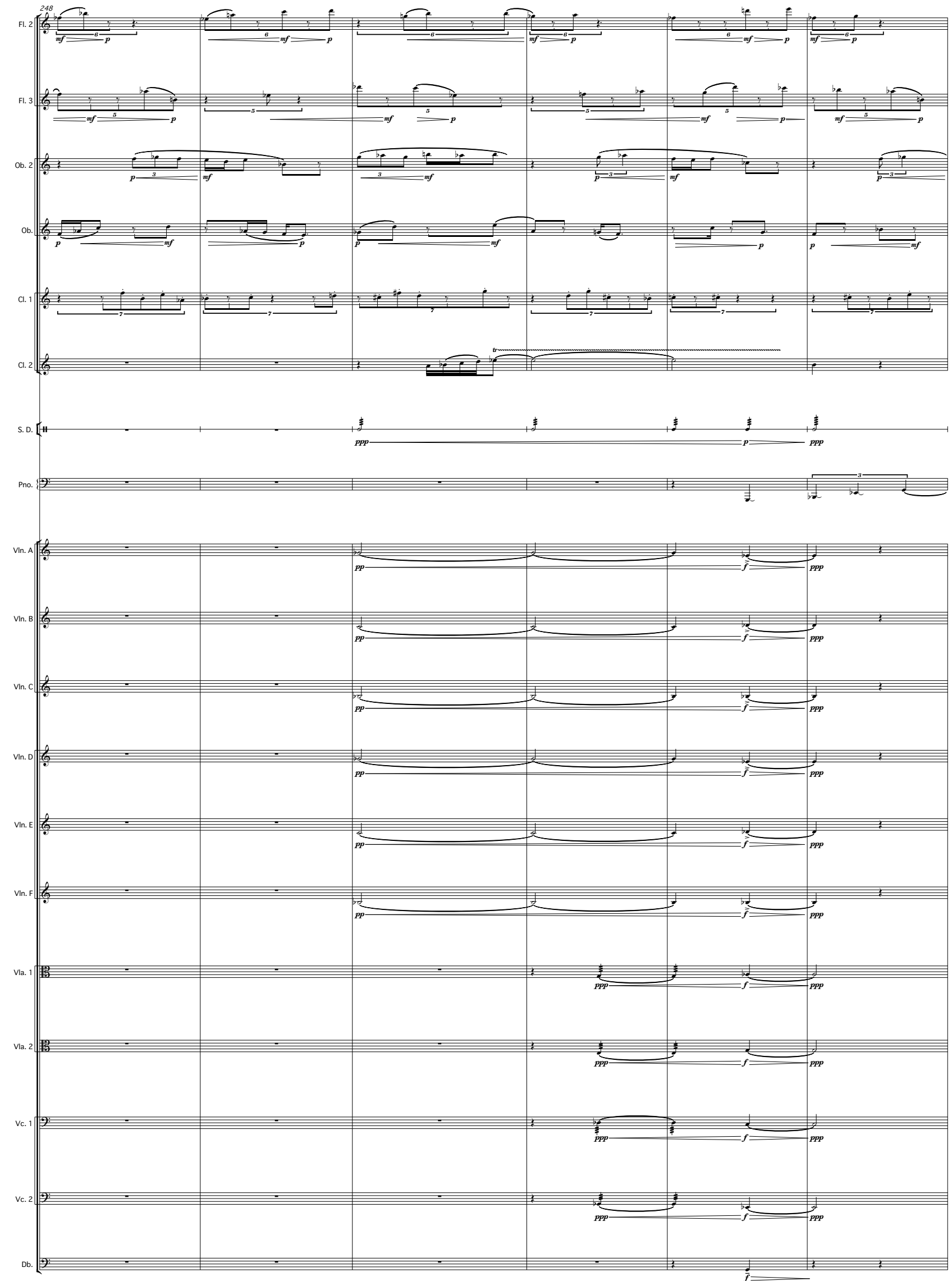


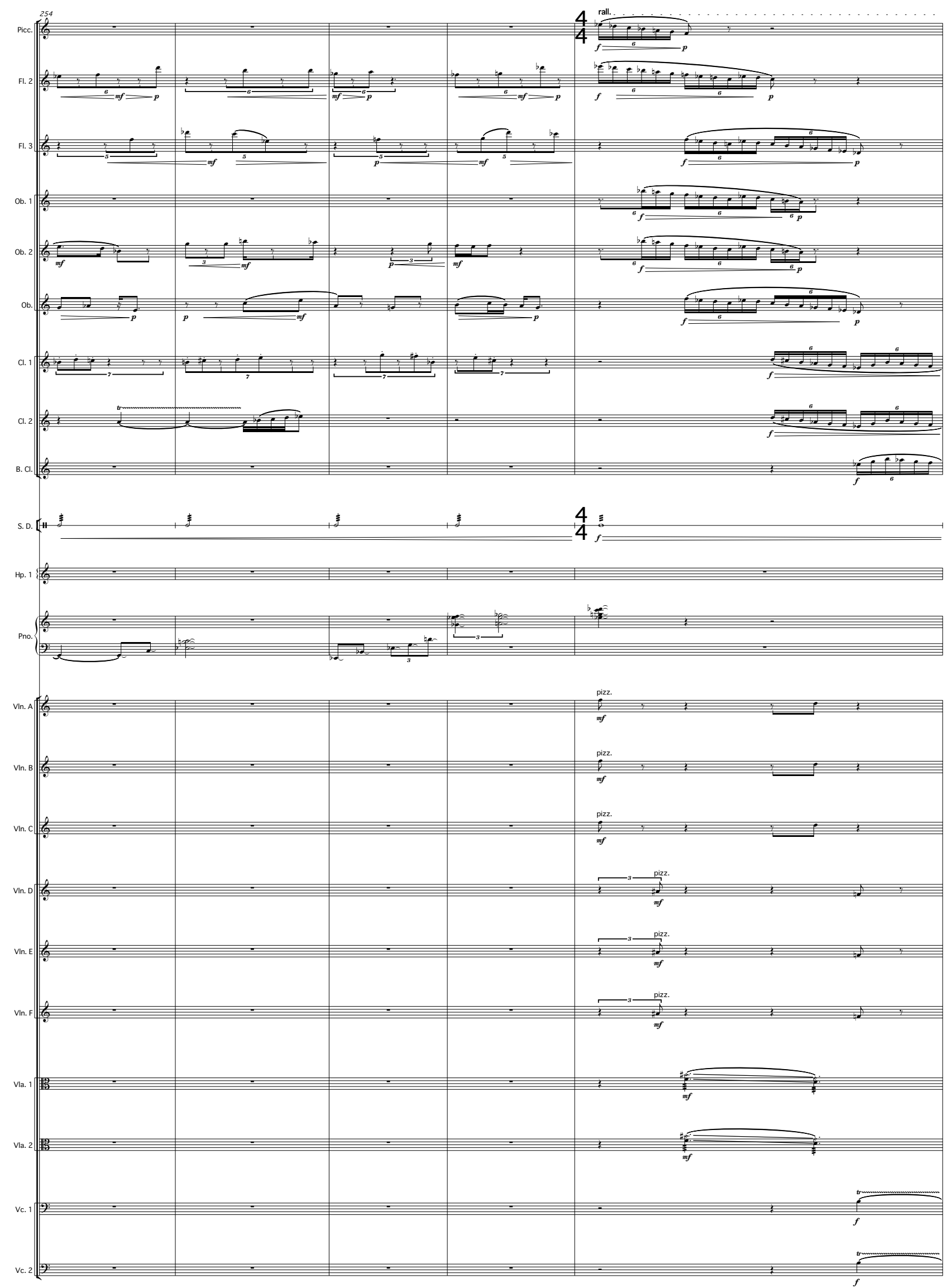




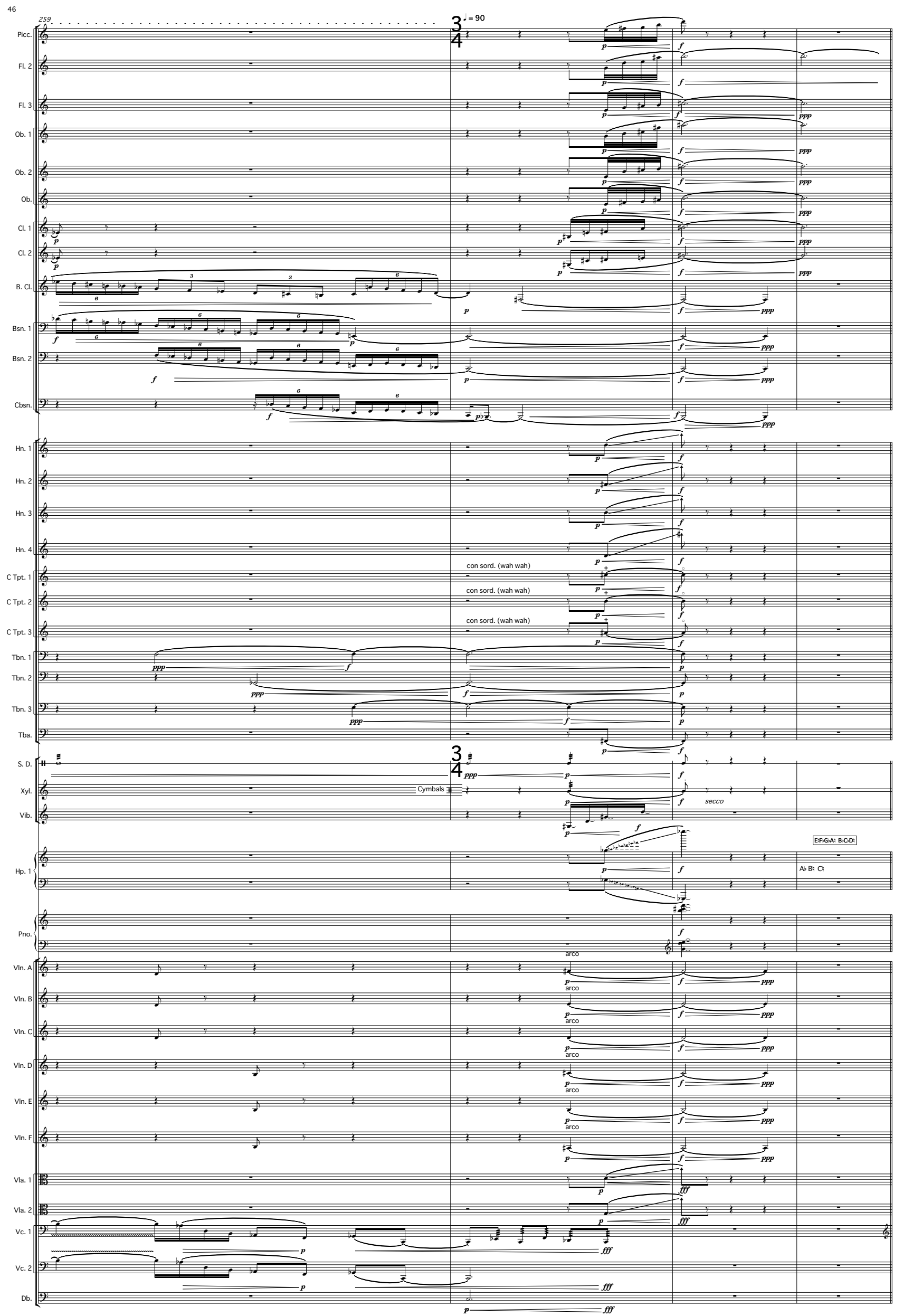


Day 6
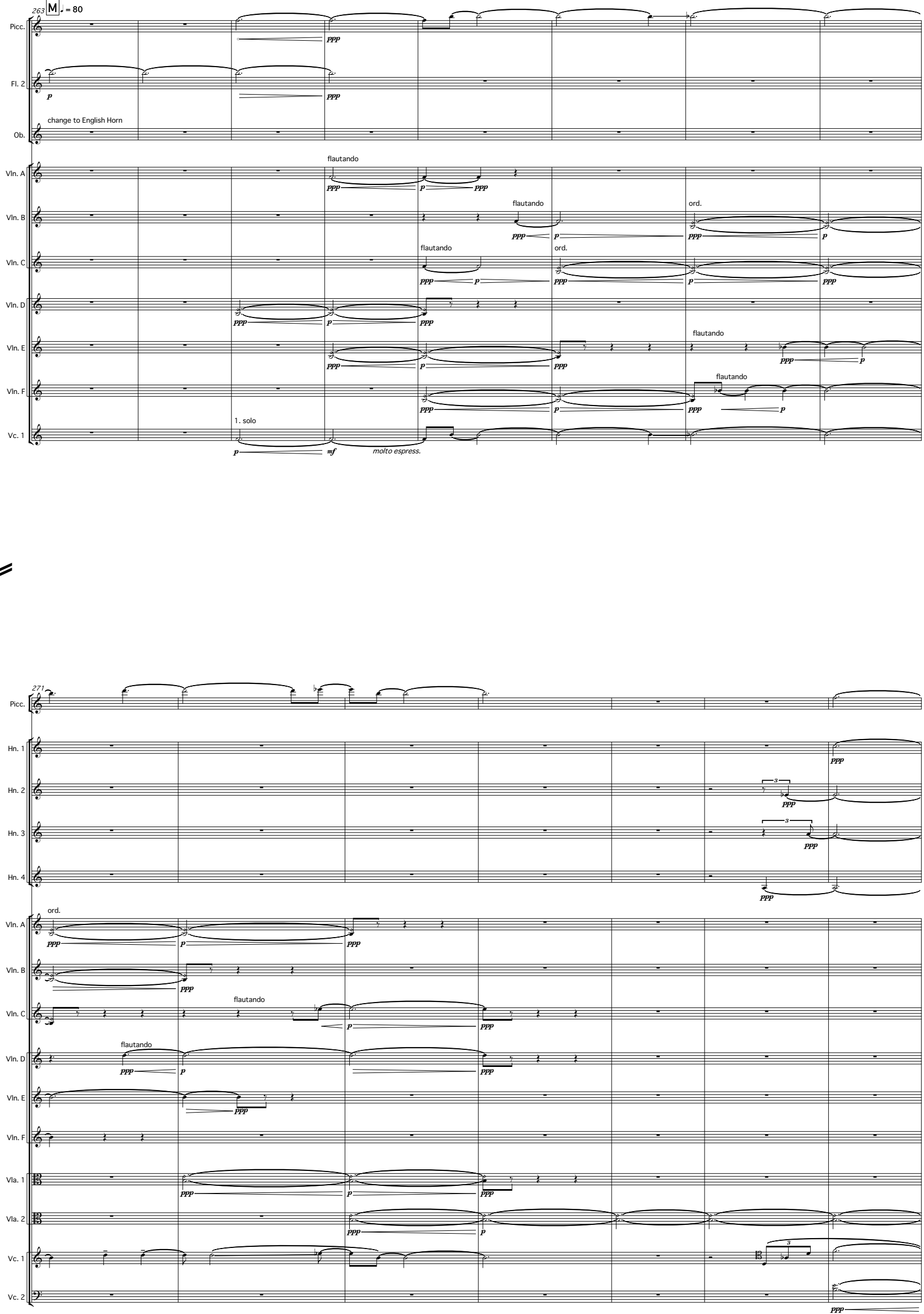

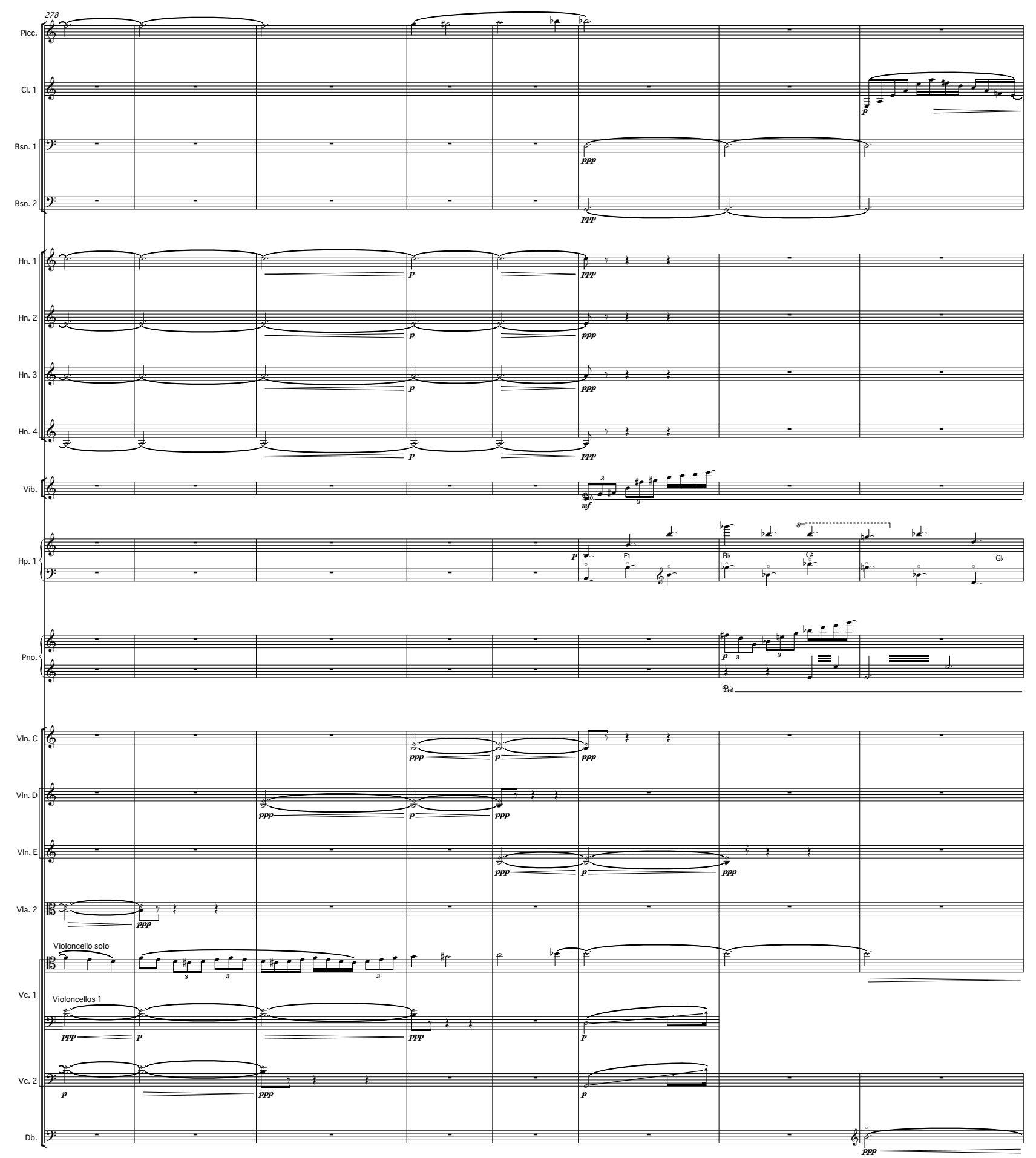

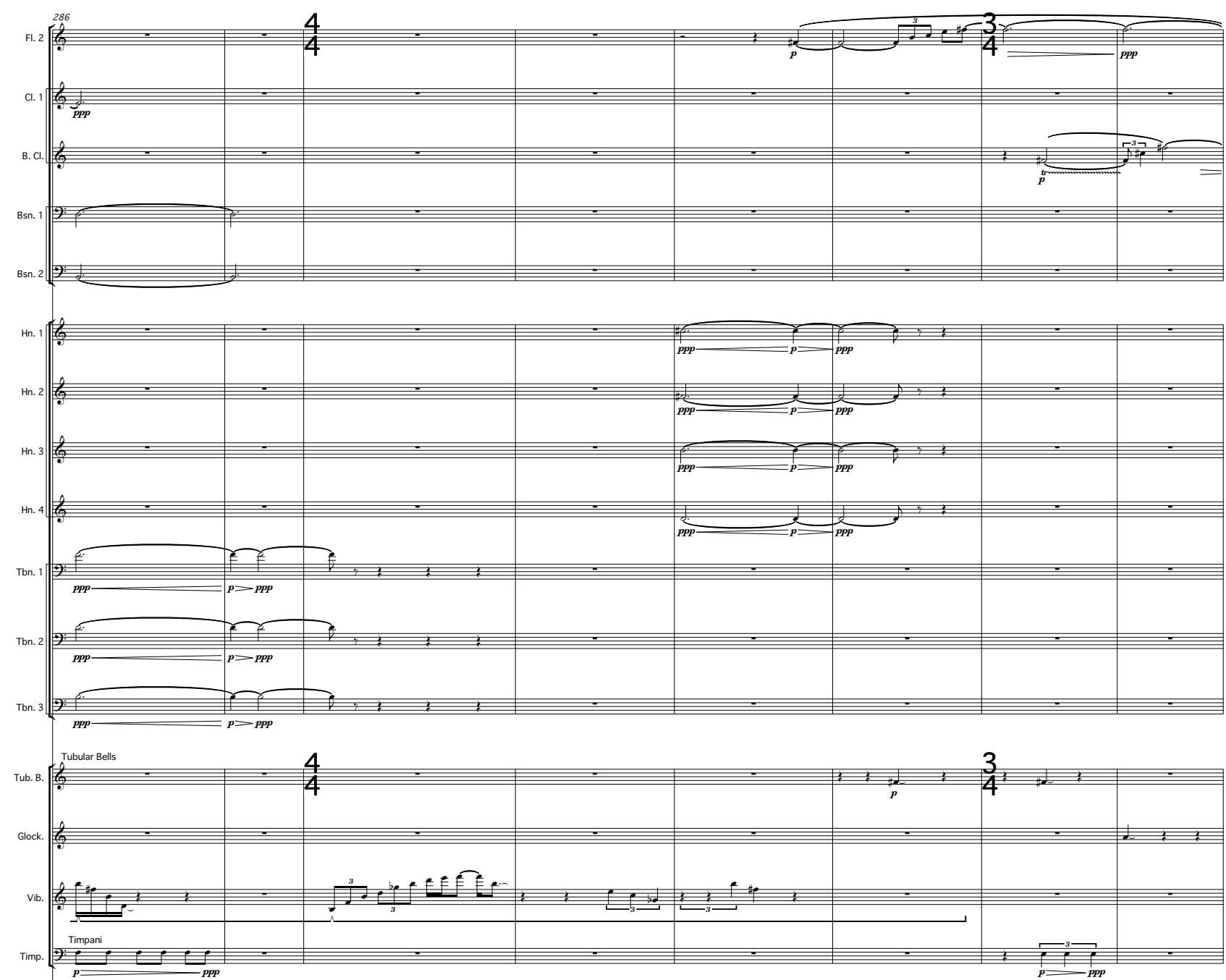

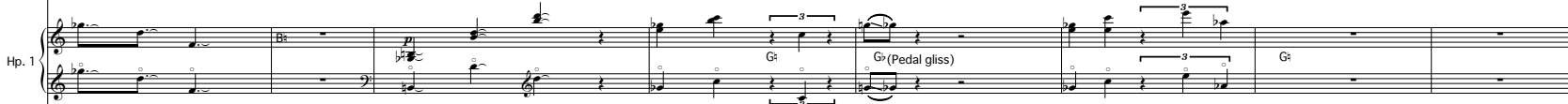

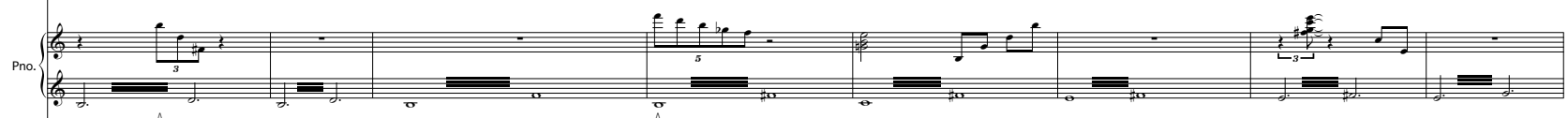

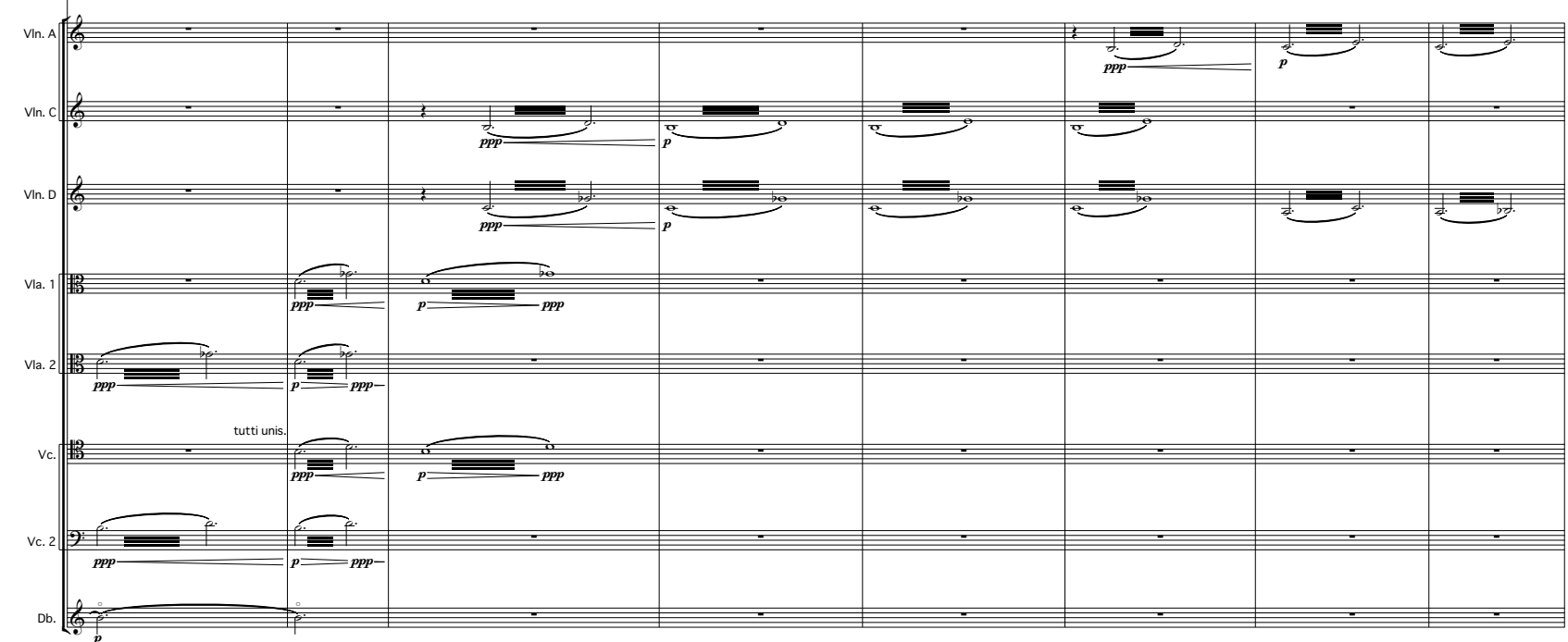



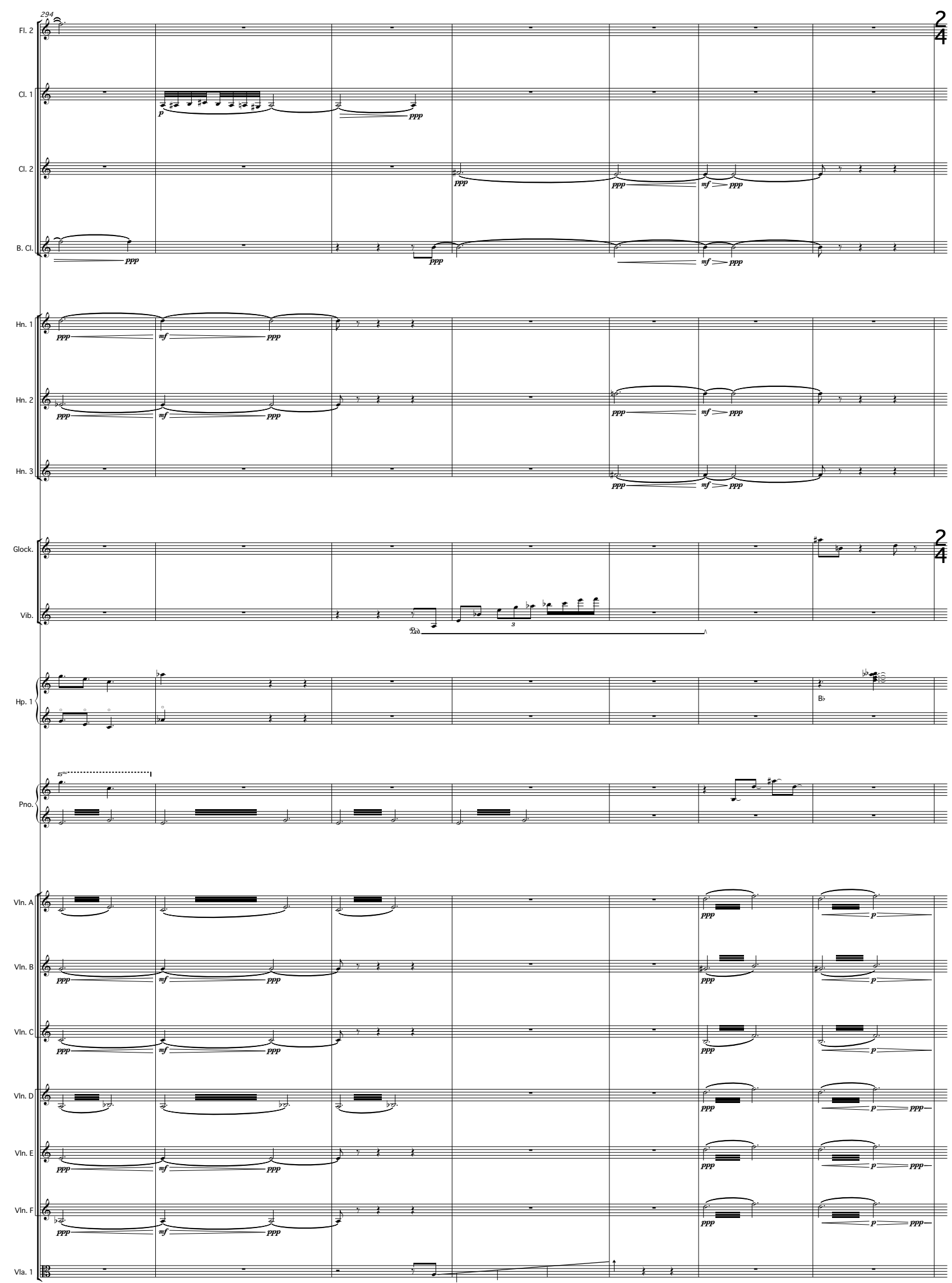

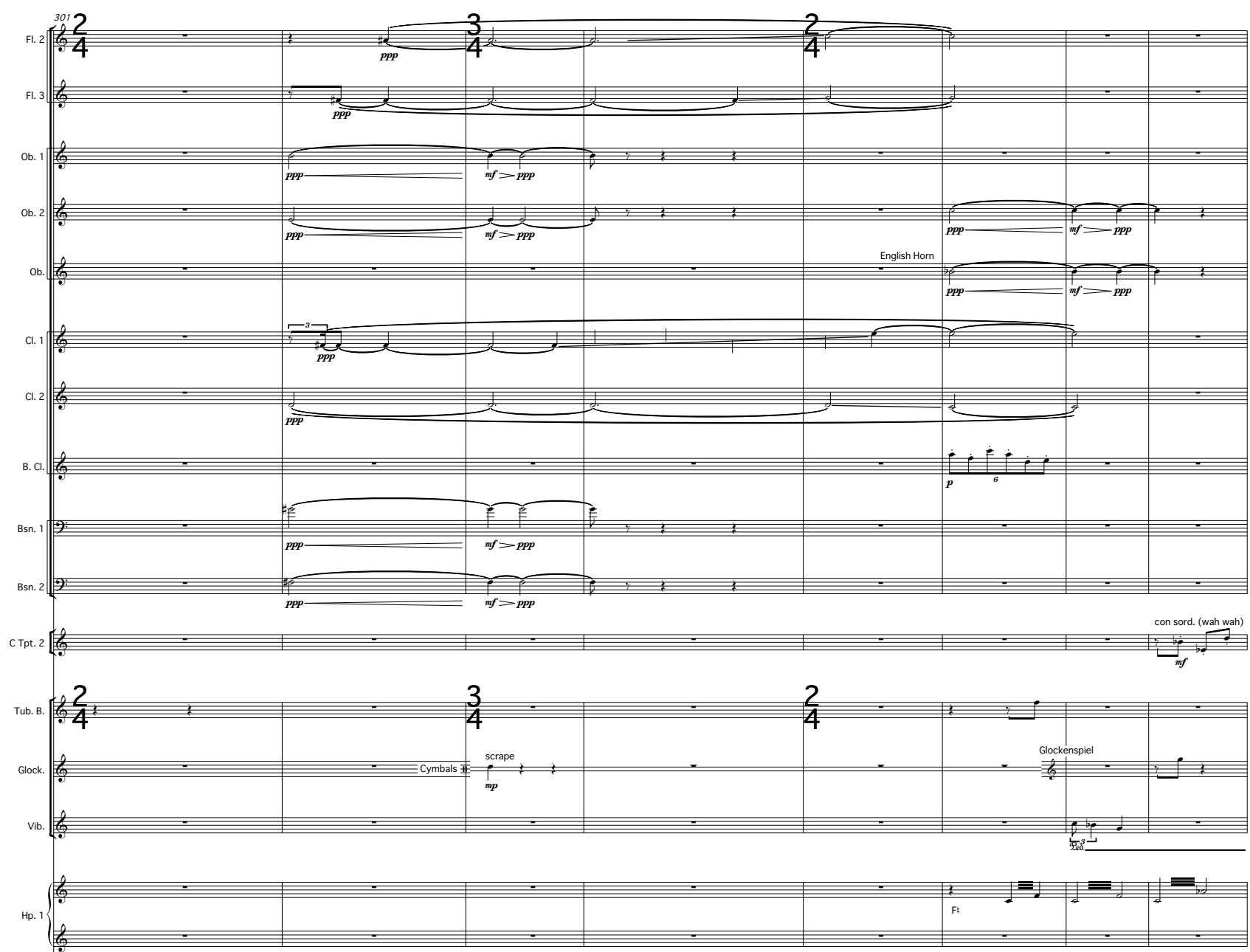

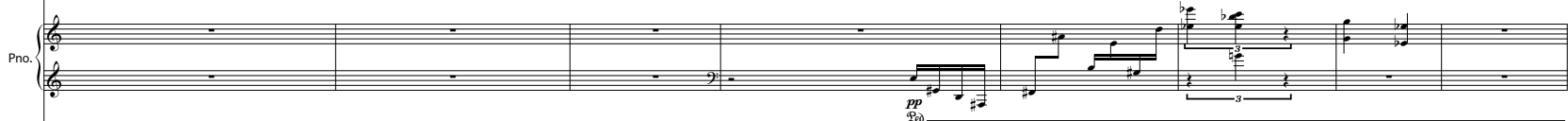

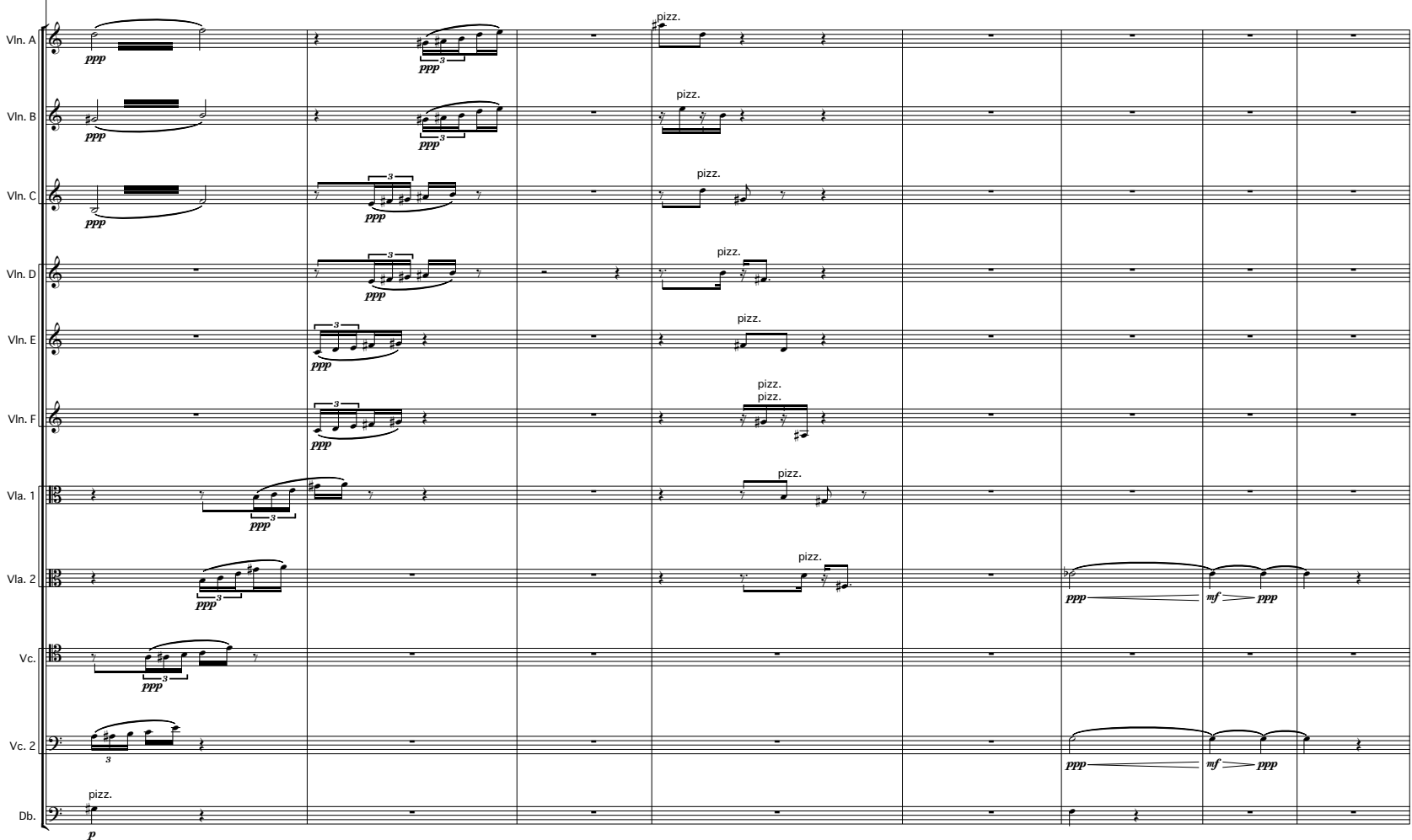


52

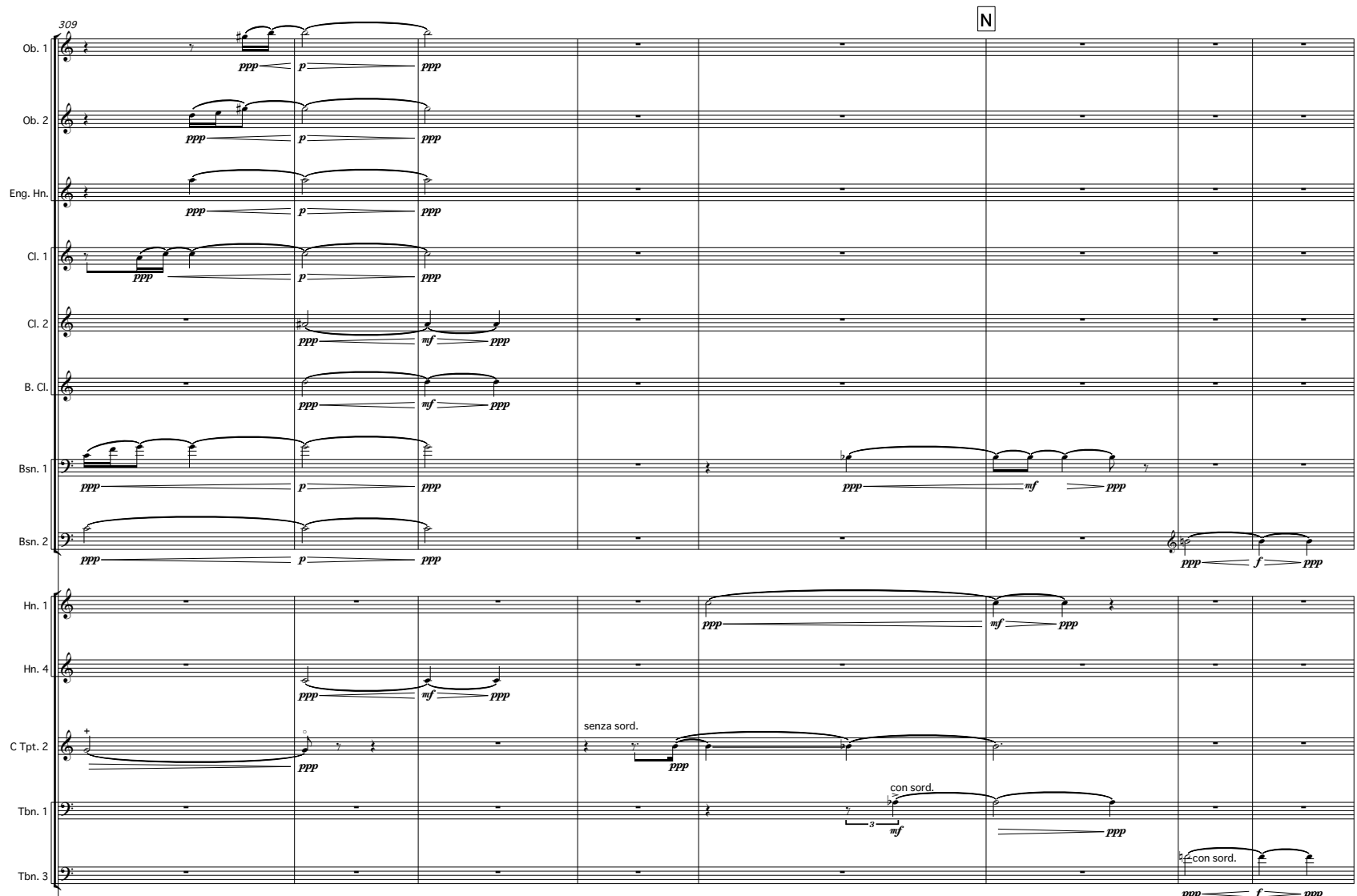

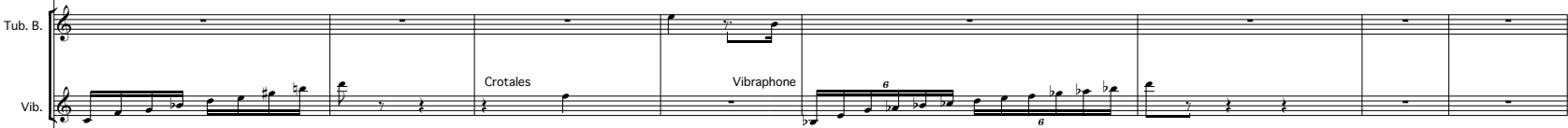

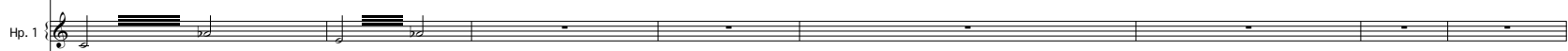

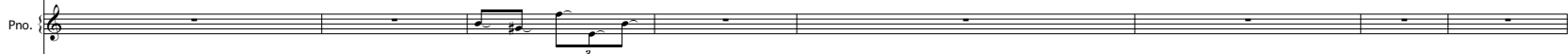

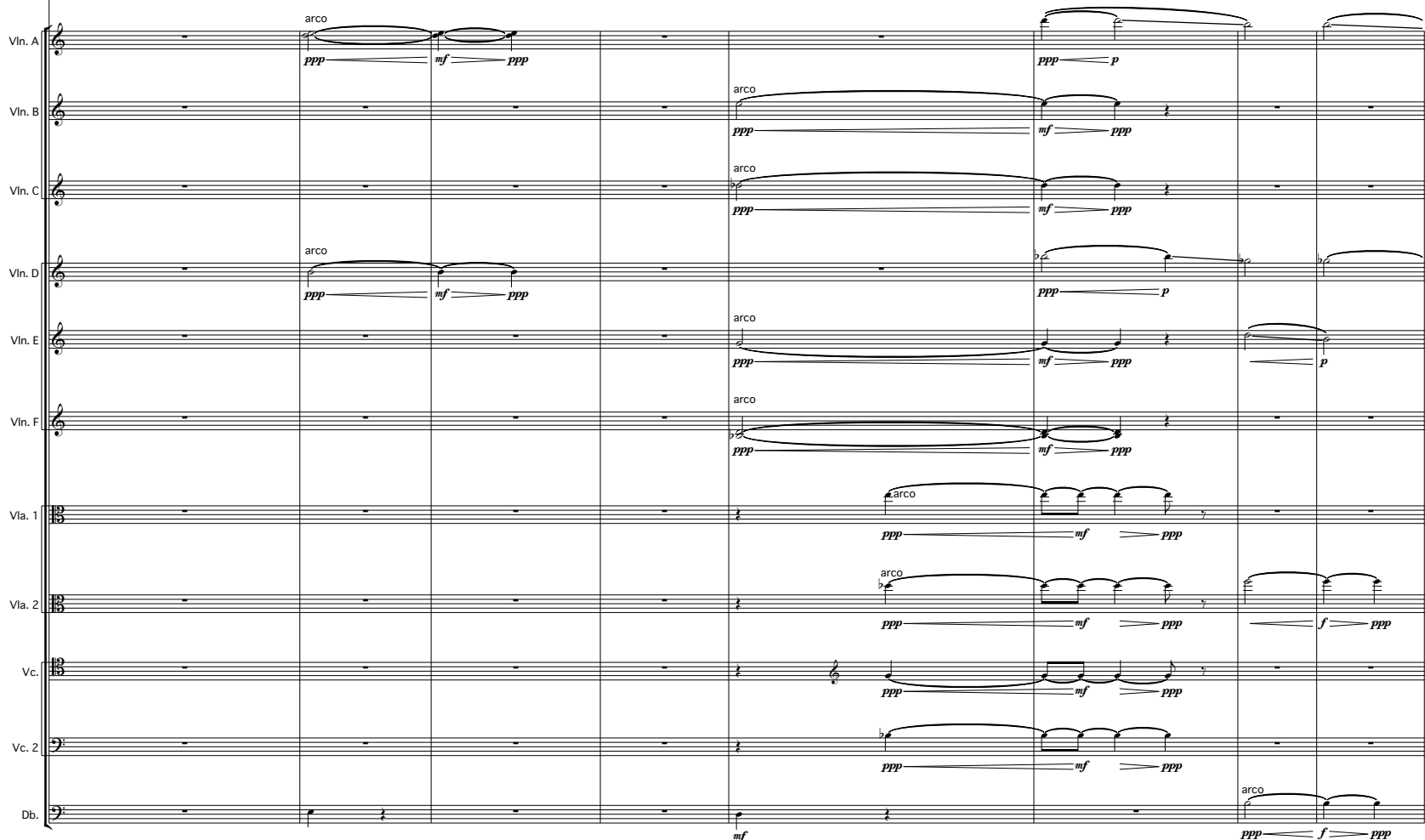



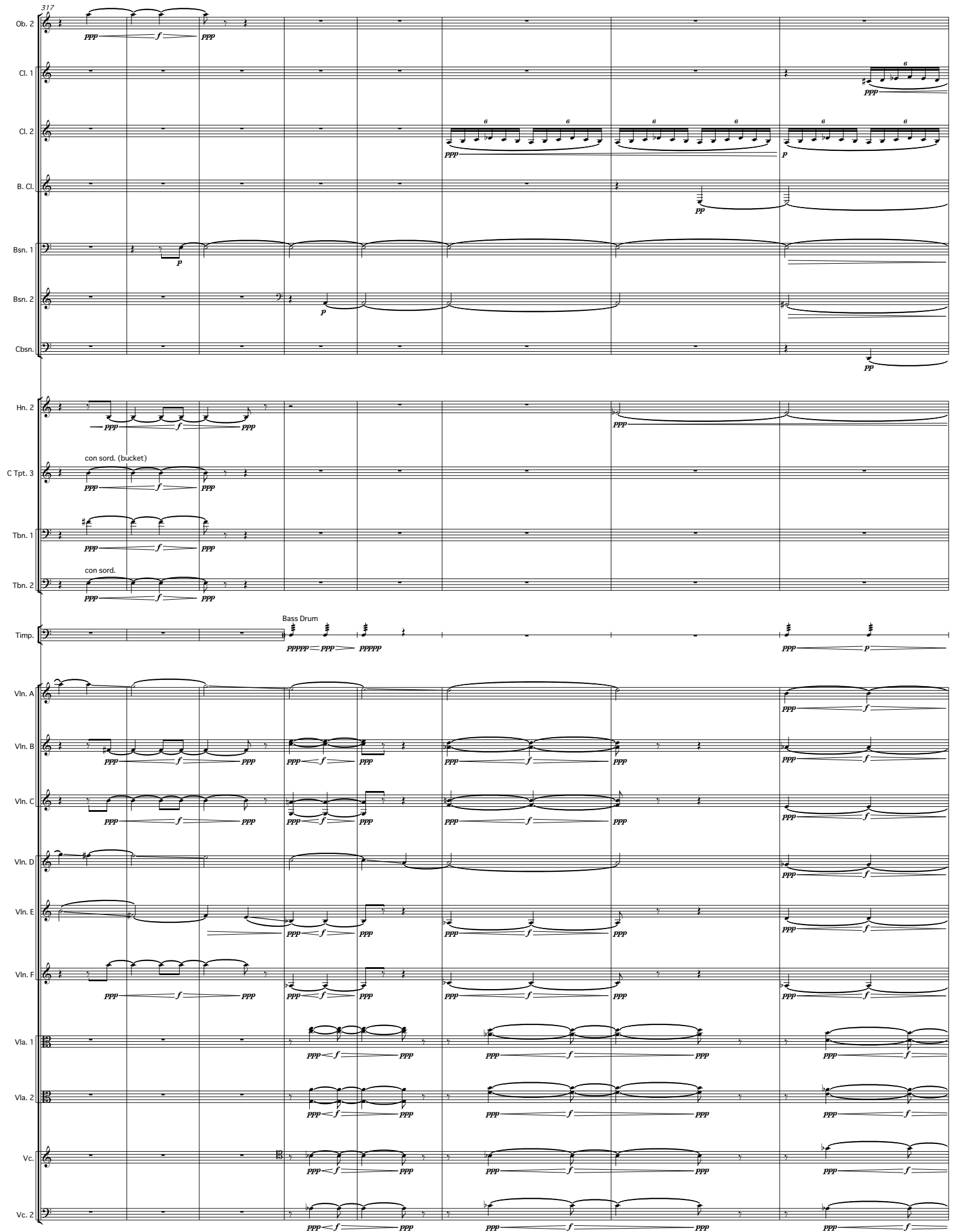


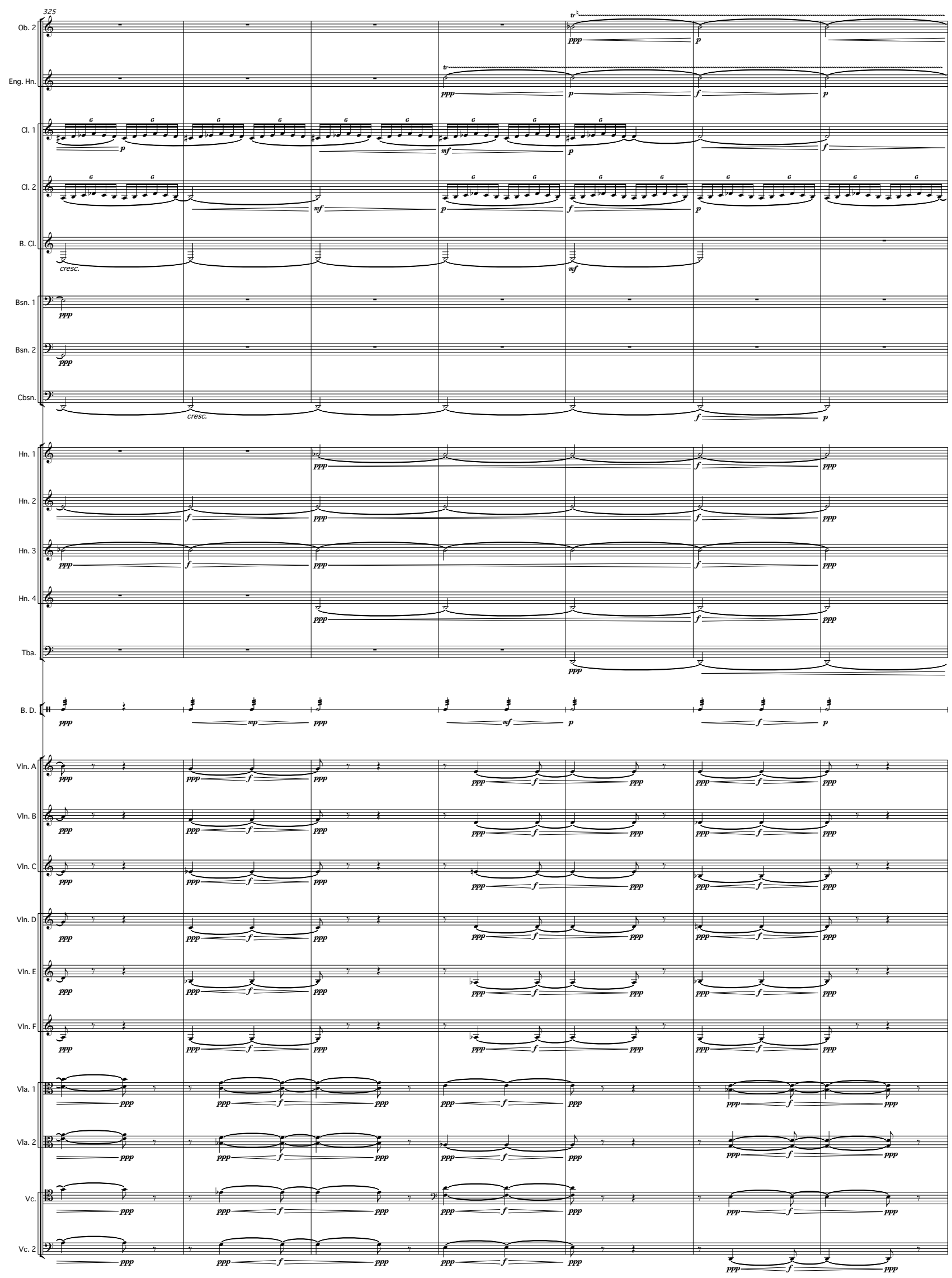



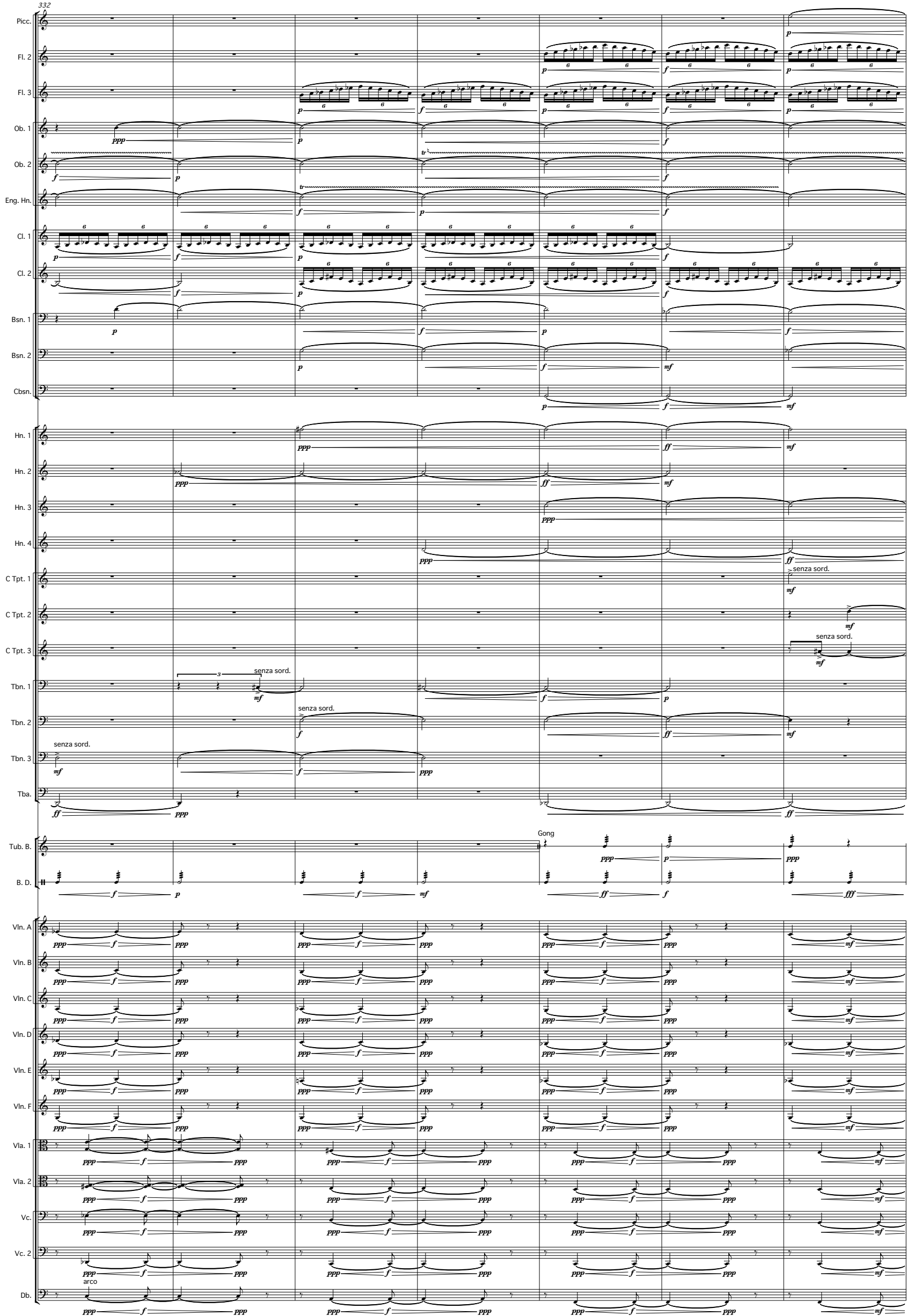


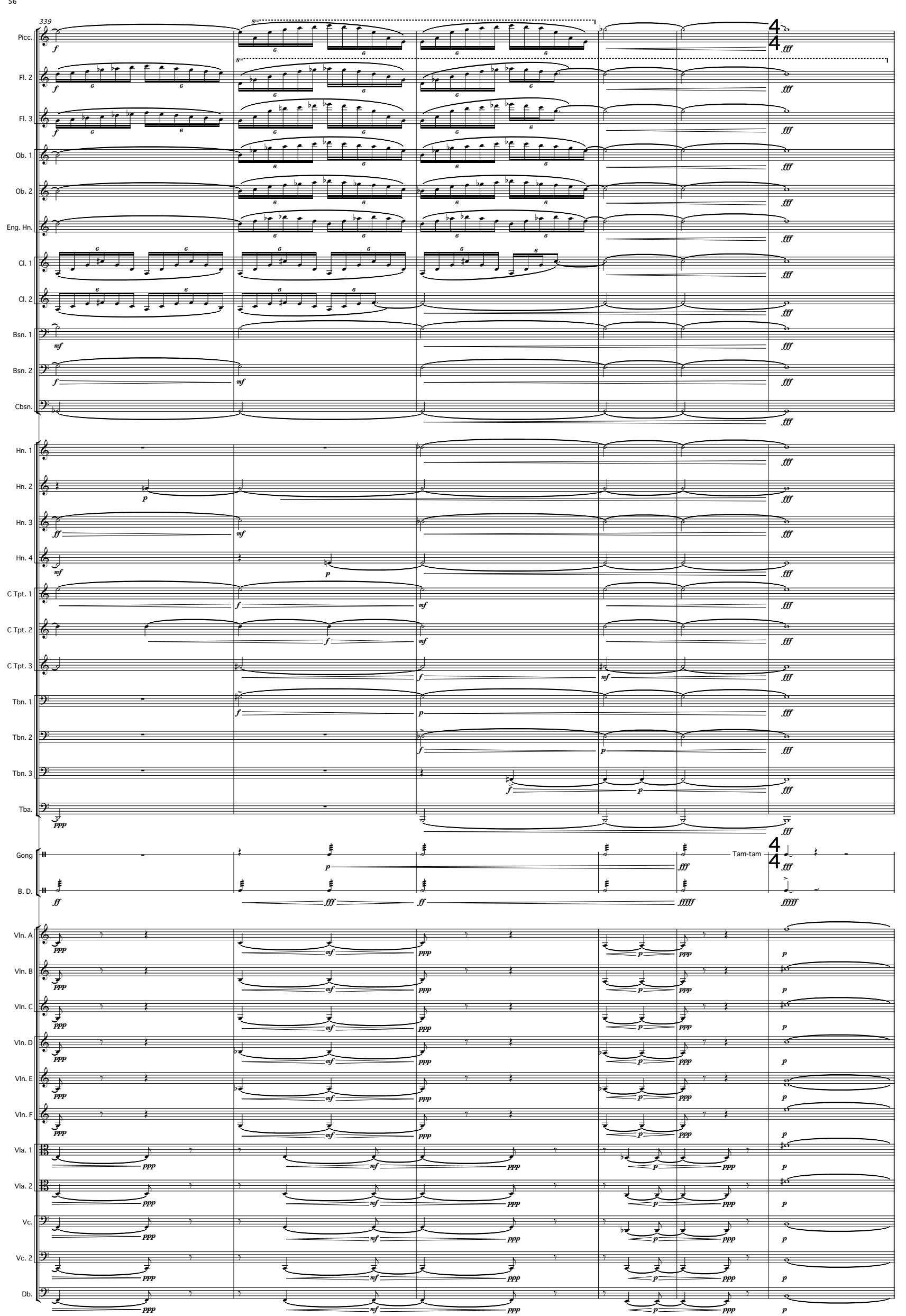




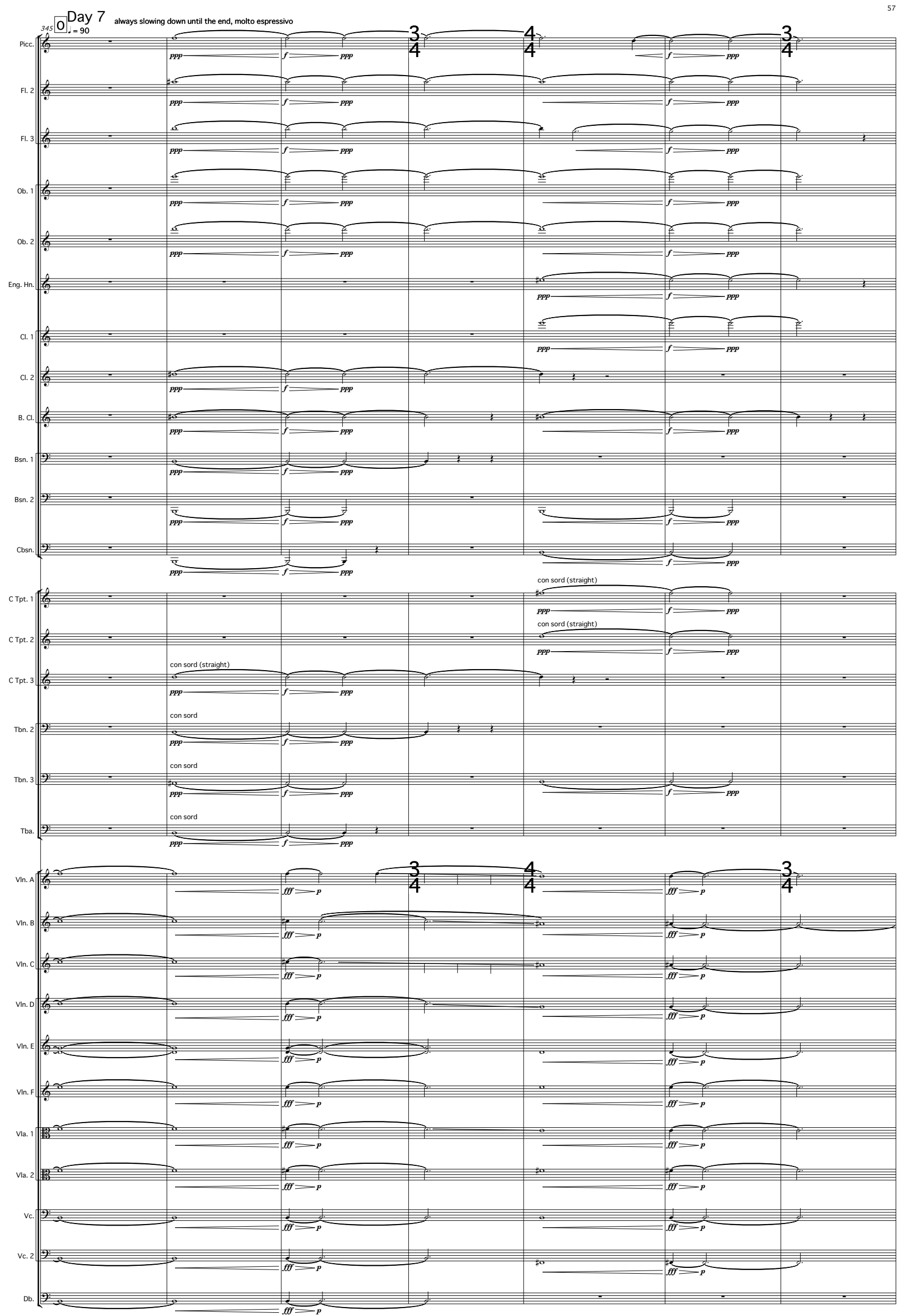



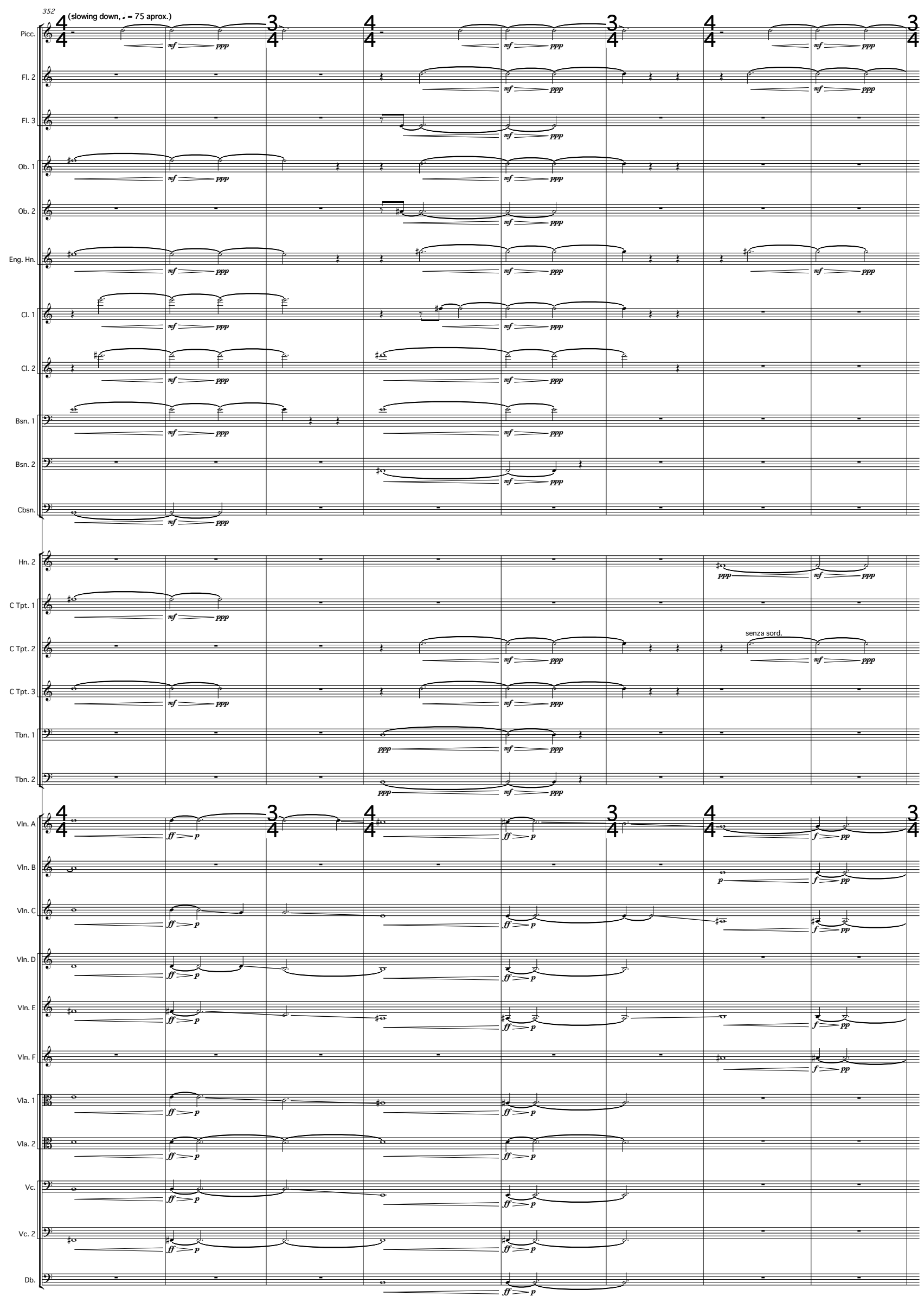

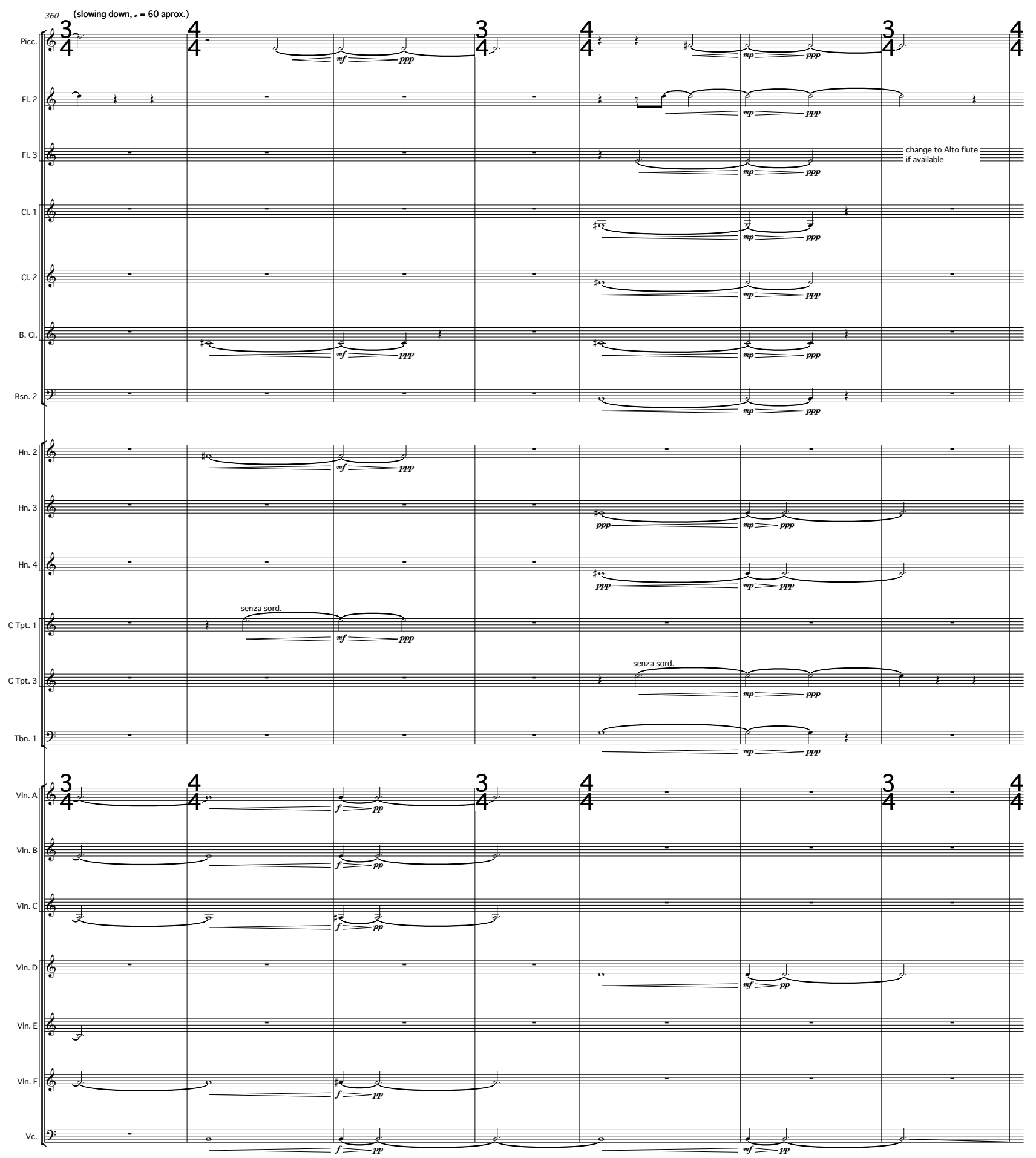


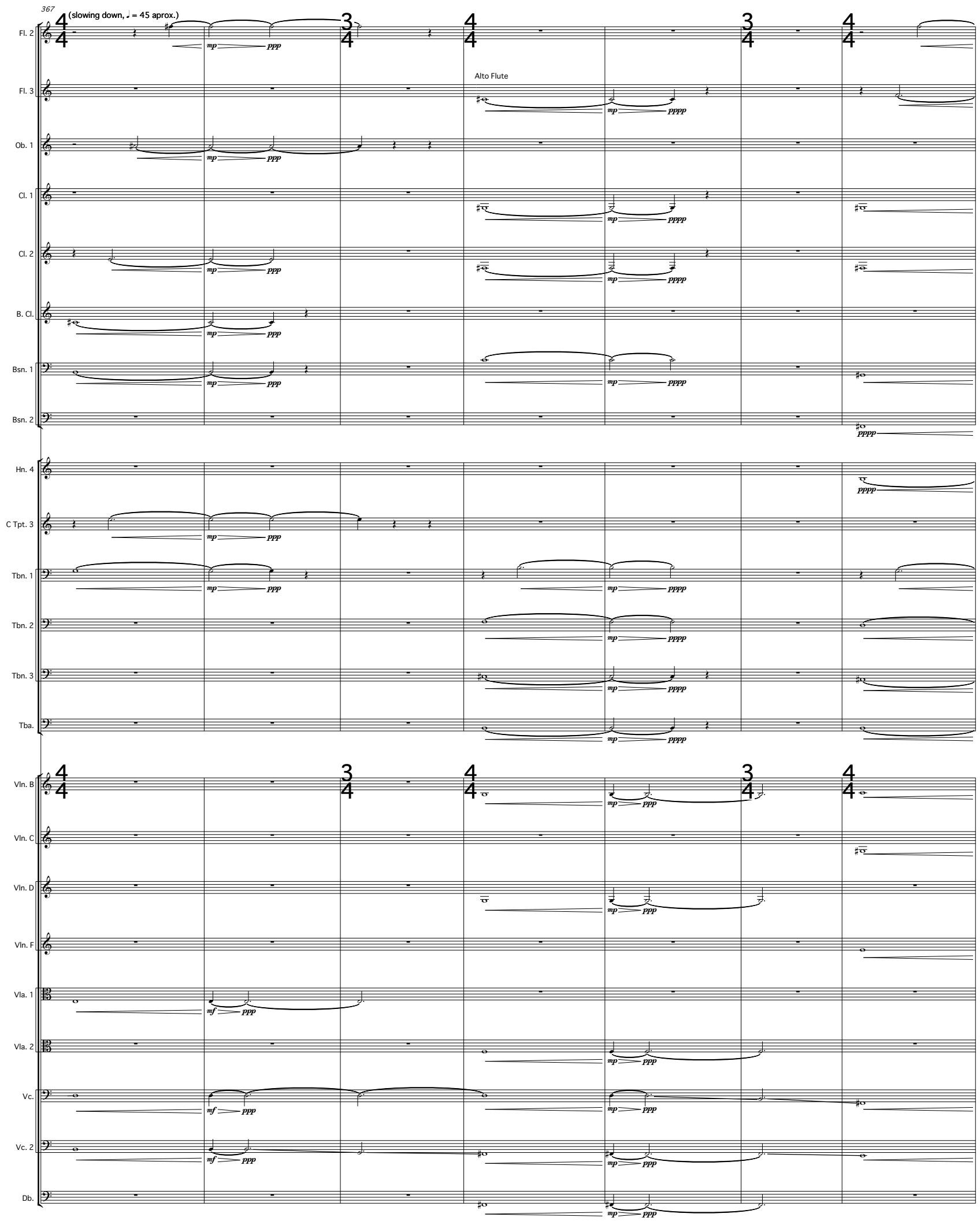



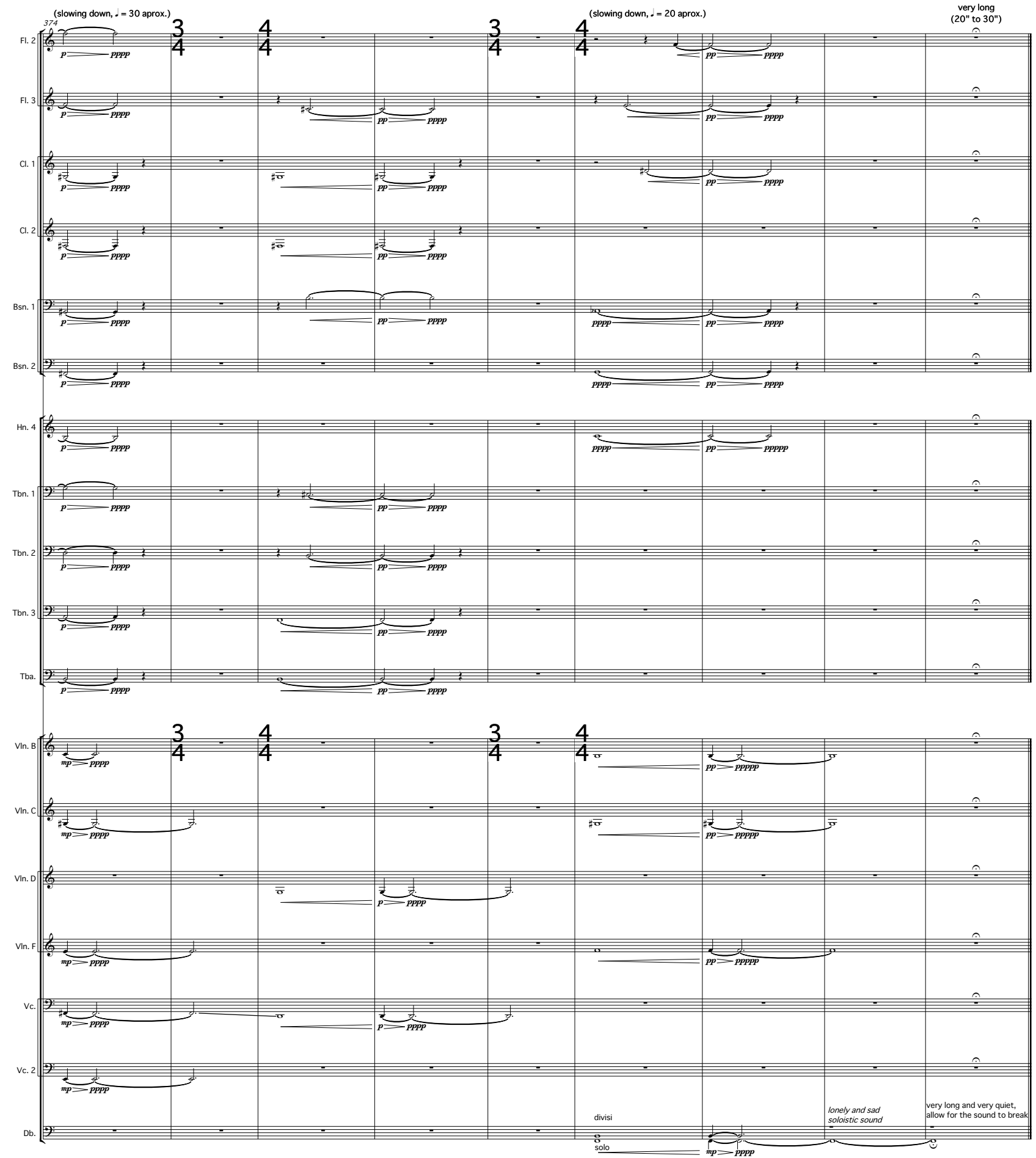


\section{APPENDIX N: SUPPLEMENTAL MEDIA}

The supplemental media accompanying this dissertation includes:

Audio tracks

- Étude for HypeSax No1.wav

- Étude for HypeSax No2.wav

- Étude for HypeSax No3.wav

- Más allá del delirio - en tu alcoba bipolar.wav

- Más allá del delirio - gime un sabor a pena.wav

- Más allá del delirio - desde un lugar secreto.wav

- Look Out.wav

- Breathe In breathe Out.wav

- Countless Souls.wav

- Cor Ple.wav

- Bereshith.wav

Webpage

- www.hypesax.com 